\title{
Anatomy 204L: Laboratory Manual (First Edition)
}

Ethan Snow

University of North Dakota, ethan.snow@und.edu

\section{How does access to this work benefit you? Let us know!}

Follow this and additional works at: https://commons.und.edu/oers

Part of the Anatomy Commons

\section{Recommended Citation}

Snow, Ethan, "Anatomy 204L: Laboratory Manual (First Edition)" (2017). Open Educational Resources. 4. https://commons.und.edu/oers/4

This Course Material is brought to you for free and open access by UND Scholarly Commons. It has been accepted for inclusion in Open Educational Resources by an authorized administrator of UND Scholarly Commons. For more information, please contact und.commons@library.und.edu. 


\section{LABORATORY MANUAL}

Anatomy 204L University of North Dakota

\section{Snow}

Name:

Email:

Lab Time:

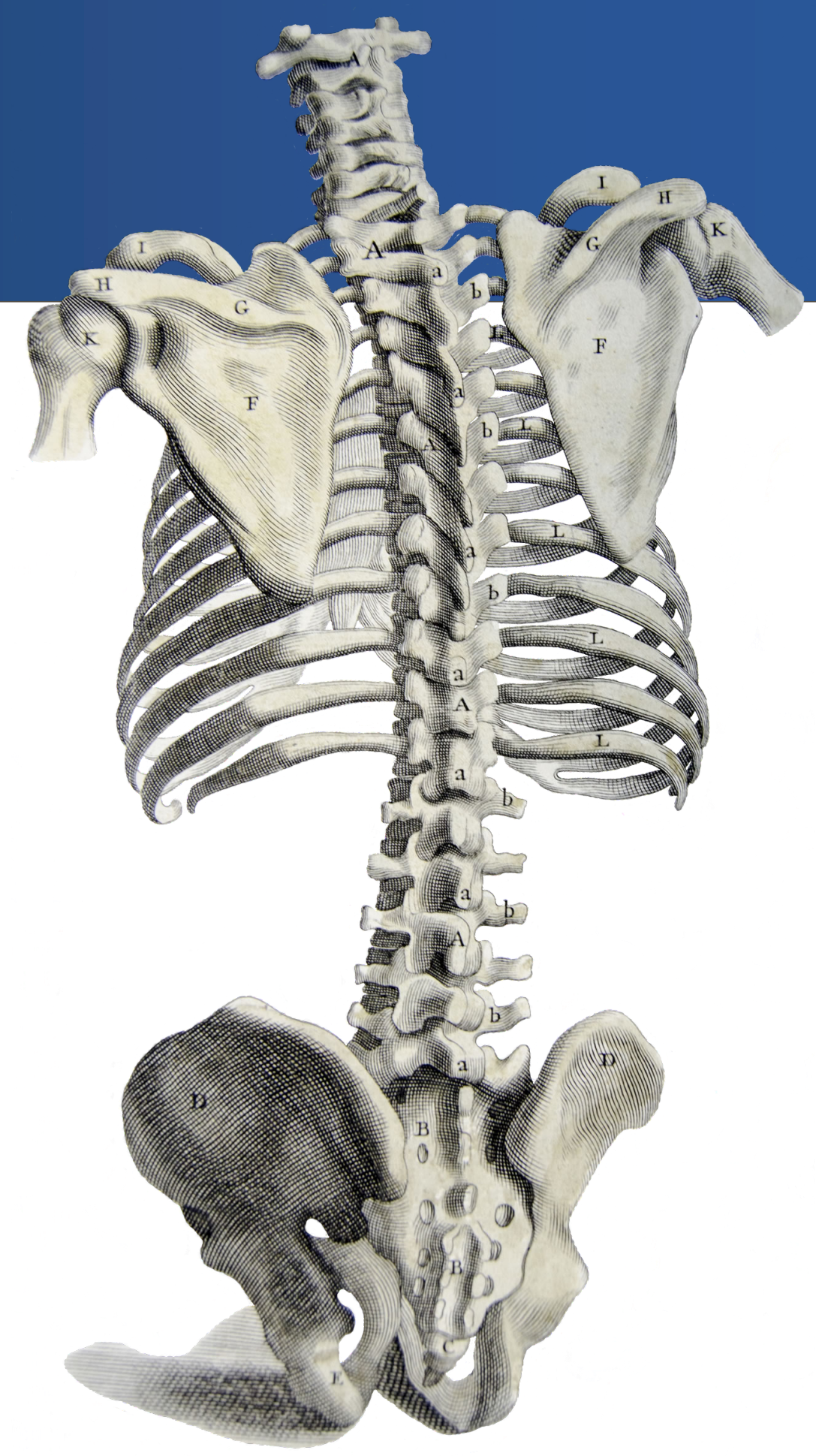




\section{About This Manual}

Course Level: This laboratory manual is designed for undergraduate anatomy labs.

Instructor Access: This manual can be found in the UND repository or obtained directly from Ethan Snow (ethan.snow@und.edu).

Making this Resource Available to Students: This manual may be downloaded and made available for students to access at no cost to them. This manual may be used electronically (free) or in print (printing costs may apply). This manual is designed as an Open Educational Resource (OER) to eliminate the need for students to purchase costly and excessive resources. Although a traditional anatomy atlas may not be required for the students to purchase with the manual, the author recommends having a few anatomy atlases in the lab as an available resource for students.

Examination Set Up Guides: The included examination set-up guides (Appendix B) provide flexibility for choosing examination items while linking those examination items back to course learning objectives and ensuring the examination material is proportionate to the material in the manual. Learning objectives can be found throughout the manual at the beginning of each lab.

Images: All copyrighted images used in this manual are under a Creative Commons license that allows them to be used, edited, and distributed in this manual. See Appendix $\mathrm{C}$ for details about each image.

Video Links: In the manual, video links connect students to mini-lectures about respective content. Some videos may need to be accessed to understand and label some figures. Videos are published to YouTube under a Creative Commons license.

Support: This laboratory manual was created with the support of an Open Educational Resource (OER) Implementation Initiative grant funded by the University of North Dakota Student Senate, Provost, and Vice President of Academic Affairs.

Author Contact Information: Please contact Ethan Snow with any questions, comments, requests, or concerns. You may contact him by calling 402-870-0976 or by sending email correspondence to ethan.snow@und.edu. 


\section{Table of Contents (Tittes are hyperlinked to content)}

Table of Contents.

How to Use This Manual.

Introductory Material

\section{Block I: The Musculoskeletal System}

Lab 1: Axial Skeleton (Head).... 8

Lab 2: Axial Skeleton (Neck, Thorax, \& Abdomen). 16

Lab 3: Appendicular Skeleton (Pectoral Girdle \& Upper Limb).........24

Lab 4: Appendicular Skeleton (Pelvic Girdle \& Lower Limb)..............30

Lab 5: Axial Muscles .37

Lab 6: Appendicular Muscles (Pectoral Girdle \& Upper Limb)

Lab 7: Appendicular Muscles (Pelvic Girdle \& Lower Limb). .51

\section{Block II: The Nervous System and Special Senses}

Lab 8: Spinal Cord \& Peripheral Nerves. 60

Lab 9: Brain. .68

Lab 10: Cranial Nerves. .75

Lab 11: Special Senses.

\section{Block III: The Cardiovascular and Respiratory Systems}

Lab 12: Heart

Lab 13: Vasculature of the Head, Neck, Thorax, \& Abdomen.

Lab 14: Vasculature of the Upper \& Lower Limbs 105

Lab 15: Respiratory System 110

\section{Block IV: The Lymphatic, Digestive, Urinary, and Reproductive}

\section{Systems}

Lab 16: Primary Digestive Organs 117

Lab 17: Secondary Digestive Organs \& Lymphatic System. 125

Lab 18: Urinary System. 136

Lab 19: Male and Female Reproductive Systems 143

\section{Appendices}

Appendix A: Answers to Self-Assessment Questions. 152

Appendix B: Lab Exam Set Up Guides. 162

Appendix C: Images Sources. 


\section{How to Use This Manual}

This manual may be used in electronic or print formats, or both. While note-taking capabilities are available for both formats, hyperlinks and video links can only be used in the electronic format. The videos contain important information about the content and unlabeled diagrams. The video links can be identified by the following symbol:

Video

All of the content in this manual is testable content. Students are expected to learn the content as outlined by the learning objectives (LOs) which can be found throughout the manual before each portion of respective content. They are abbreviated as "LO" followed by the LO number.

Examination items will be determined by the learning objectives and the amount of material covered by each learning objective. Students should use the learning objectives to guide them as they learn the material, self-assess their knowledge, and prepare for examination. Lab Exam Set Up Guides (found in Appendix B) may be used to self-assess learning and prepare for examination. According to the set up guides, $80 \%$ of Lab Exam questions will be primary-type questions (i.e. "Identify this structure" or "Identify this muscle") while $20 \%$ will be secondary-type questions (i.e. "Identify where blood will flow next" or "Identify the action(s) of this muscle").

Yellow boxes like this are found throughout the manual. They contain important, helpful (and testable) information relevant to the nearby content. This information includes many terminology explanations and is intended to help you learn the content in a more efficient, effective, and complete way. You are strongly encouraged to add your own notes to this manual and look up term definitions that aren't listed.
Blue boxes like this are found throughout the manual, and they contain clinical application material related to nearby content. Beginning to understand how structural anatomy translates to clinical settings is important for every healthcare professional.

To best learn this material, you should first identify the listed gross structures and then describe their purposes in the human body. The learning objectives will guide your efforts in doing this. Use the images, video links, and informational boxes presented in this manual to aid your learning and understanding of the content. You should do this to the best of your ability before each lab so that you can use your time in the lab more efficiently.

If at any time you determine the information presented in this manual insufficient for you to learn the structures, refer to internet searches, anatomy atlases, anatomy lab staff, or another resource to help you understand and better learn the content. Some terms that are not labeled in the figures are marked with an asterisk $\left({ }^{*}\right)$.

When you see

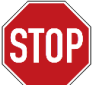

you should stop and self-assess your knowledge by answering the following questions to the best of your ability and without referencing any resources (as if you were taking an examination). After you record an answer for each question, you can then look up the answers to check for correctness. Answers to the self-assessment questions can be found in Appendix A. The respective LO is listed after each selfassessment question. 


\section{Introductory Material}

L01: Identify and describe major anatomical regions, anatomical positions, anatomical planes, and directional terms.

\section{Anatomical Regions}

Knowing the regions of the body is the first step in learning human anatomy. Below are named anatomical regions that will routinely be used throughout the course. The specific anatomical regions can be organized into two groups: axial regions which are associated with the midline of the body and appendicular regions which are associated with the upper and lower limbs.

\section{Major axial anatomical regions}

Head

$\square$ Neck

$\square$ Thorax

$\square$ Abdomen

$\square$ Pelvis

\section{Major appendicular anatomical regions of the upper limb}

Shoulder (joint)

Arm (or brachium)

Elbow (joint)

Forearm (or antebrachium)

$\square$ Wrist (joint)

$\square$ Hand

\section{Major appendicular anatomical regions of the lower limb}

$\square$ Hip (joint)

$\square$ Thigh

Knee (joint)

Leg

Ankle (joint)

$\square$ Foot

The entire upper limb is commonly (incorrectly) called the "arm." Anatomically, however, "arm" and "upper limb" are NOT synonymous terms. Anatomically, the arm is only the region of the upper limb between the shoulder joint and elbow joint.

Similarly, "leg" and "lower limb" are NOT synonymous terms, despite the common misusage of "leg." Anatomically, the leg is only the region of the lower limb between the knee joint and the ankle joint.

Often, when learning the anatomy in the axial and appendicular regions, you may need to specify if you are referring to the right or left side. Throughout this manual, you will find lists of acceptable abbreviations, including those for right and left: $\mathrm{R} .=$ Right and $\mathrm{L} .=$ Left. 


\section{Anatomical Positions}

In anatomy, we will always refer to the body and its components as being in anatomical position. A person in anatomical position is standing up straight, facing forward, has their hands at their sides with palms facing forward, and has their feet together. Other terms are used to describe positions of a person lying down. A person is said to be supine when lying face up and prone when lying face down.

Anatomical position

Supine

Prone

\section{Anatomical Planes}

We use anatomical planes to better understand the relative location of structures in the human body. Anatomical planes are flat, two-dimensional sectional views of the inner body. There are three main anatomical planes: a midsagittal plane divides the body into right and left portions, a coronal plane divides the body into anterior and posterior portions, and a transverse plane divides the body into superior and inferior portions.

\section{Midsagittal plane}

Coronal plane

Transverse plane

\section{Directional Terms}

From anatomical position, we can describe locations of anatomical structures in relation to one another. Below is a list of directional terms you will need to understand and use throughout this course. Your anatomy lab staff will use these terms as they teach, and they will expect that you already know them.

Right $=$ on the right side of the body

Left $=$ on the left side of the body

Remember that right and left always refers to the subject's right and left - not yours!

Anterior $=$ front; in front of

$\square$ Posterior $=$ back; behind

Superior = above; upper

$\square$ Inferior = below; lower

Medial = toward the midline

Lateral = away from the midline

Structures are not always single directions from one another. The terms anterior \& posterior, superior \& inferior, and medial \& lateral can be combined to more accurately describe locations of structures (i.e. anterosuperior, posterolateral, inferomedial, etc.).

Proximal $=$ closer to the reference point $/$ mid-point

Distal $=$ further from the reference point/mid-point 
1) Label the following image with the anatomical regions you've learned. The blue circles represent major joints. (LO1)

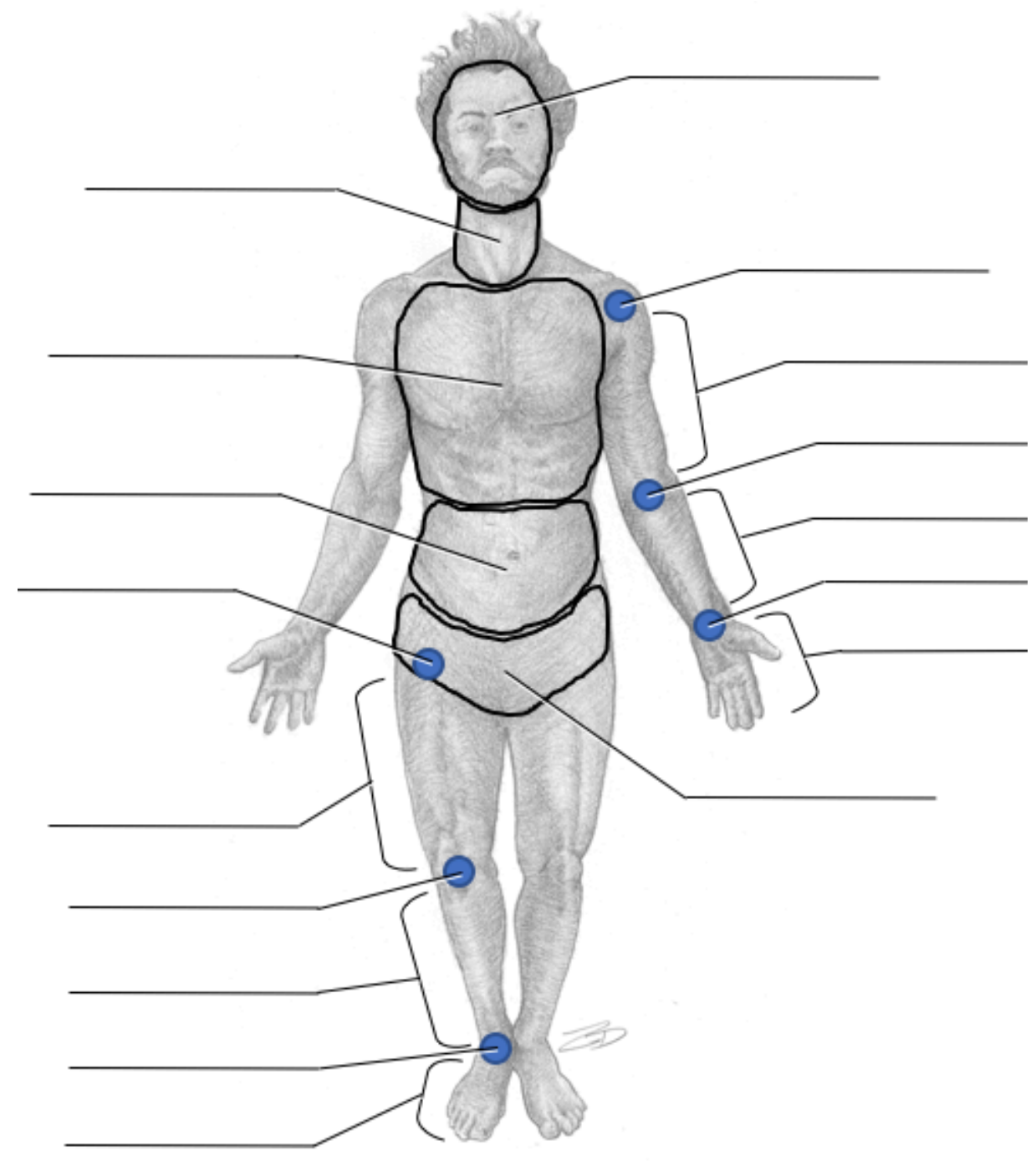

Anatomical Position, Anterior View
2) In anatomy, we always refer to the body as if it were in what position? (LO1)

3) What term is used to describe the position of a person lying face down? (LO1)

4) What does the term lateral mean? (LO1)

5) Choose the correct choices to fill in this statement: The thorax is ( Inferior / Superior ) to the abdomen. (LO1)

6) True or False: Arm and upper limb are synonymous terms. (LO1)

7) True or False: Right and left always refer to the subject's right and left. (LO1)

8) What anatomical plane would pass through both the upper and lower limbs? (LO1)

9) Describe the difference between supine and prone. (LO1)

10)Label the two images on following page with the anatomical planes and directional terms you've learned. (LO1) 
$\overline{\text { (side/direction) }}$

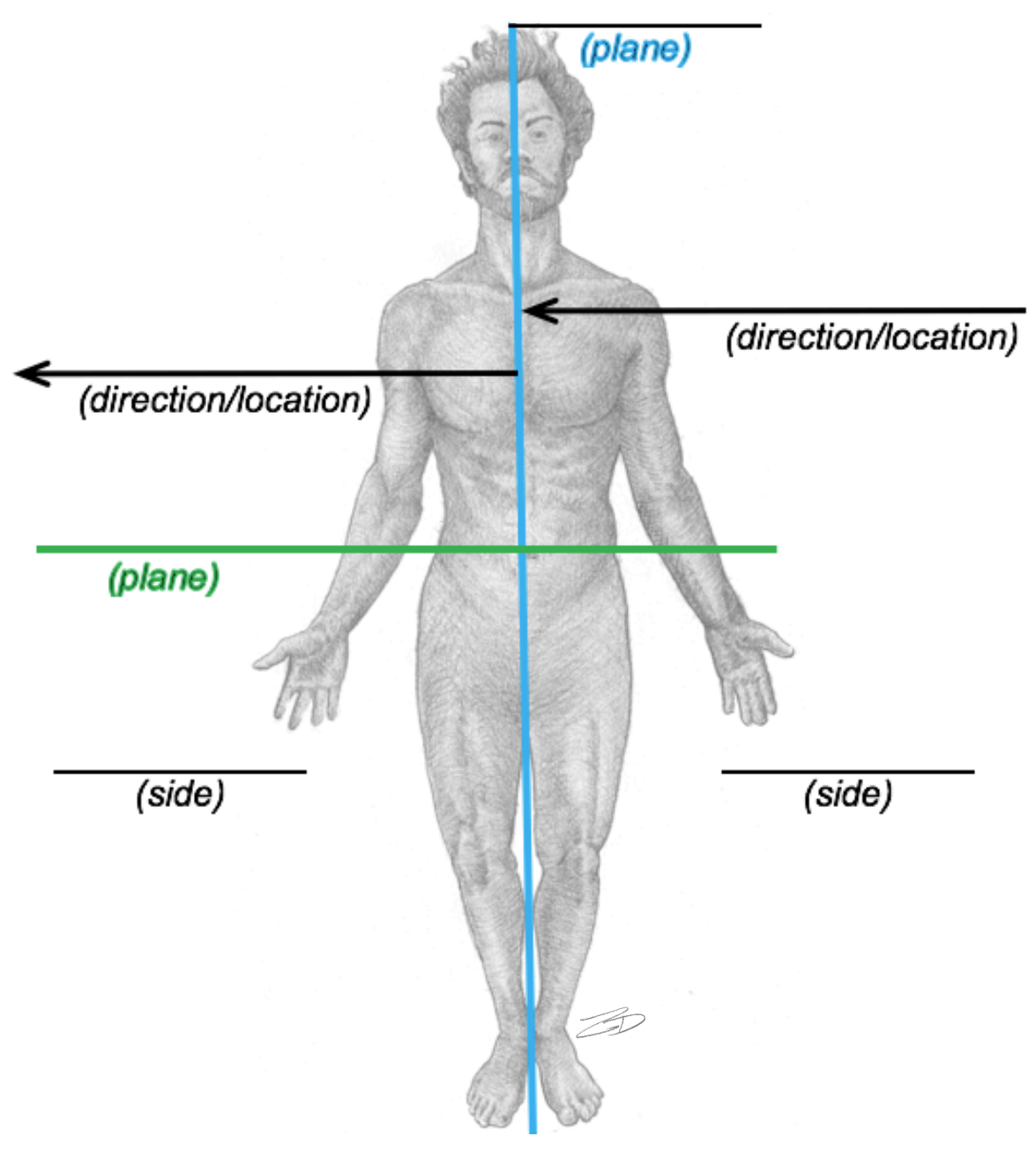

$\overline{\text { (side/direction) }}$

Anatomical Position,

Anterior View $\overline{\text { (side/direction) }}$

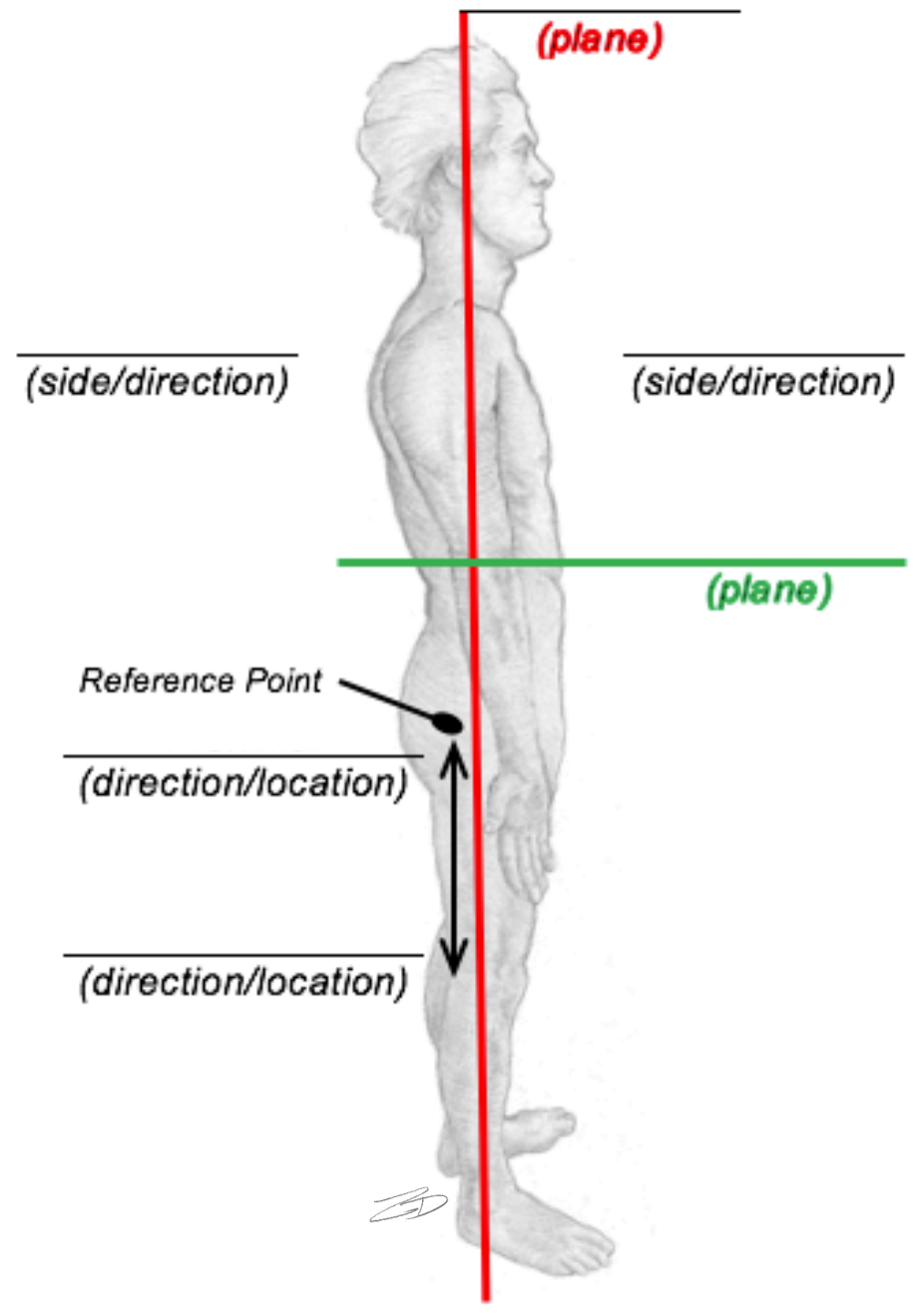

(side/direction)

Anatomical Position,

Right Lateral View 


\section{Lab 1: Axial Skeleton (Head)}

Instructions: Learn the listed terms using the listed learning objectives, labeled images, lab specimens, and other resources.

LO2: Describe the terminology used to identify structures of the axial skeleton in the head.

LO3: Identify the bones and associated structures of the axial skeleton (head).

\section{Frontal bone}

$\square$ Supraorbital foramen

Supra $=$ above; Orbit $=$ eye socket; $\underline{\text { Foramen }}=$ hole or opening Therefore, the supraorbital foramen is a hole above the eye socket. Use the terminology to help you identify the structures.

\section{Maxilla bones (2)}

$\square$ Infraorbital foramen

Infra $=$ below

\section{Nasal bones (2)}

\section{Lacrimal bones (2)}

$\square$ Lacrimal foramen

$\underline{\text { Lacrimal }}=$ concerning tears

\section{Zygomatic bones (2)}

$\square$ Temporal process

$\square$ Zygomatic arch

$\underline{\text { Zygom }}=$ joining bar

Zygomatic Arch

\section{Mandible bone}

$\square$ Condylar process

Mandibular condyle

$\underline{\text { Condyle }}=$ large, rounded, smooth articular surface

$\square$ Coronoid process

$\underline{\text { Process }}=$ projection; outgrowth

$\square$ Mental foramen

$\square$ Mandibular foramen*

$\square$ Alveolus (or Tooth socket)

Palatine bones (2)

Inferior nasal concha bones (2) 


\section{Ethmoid bone}

$\square$ Crista galli

$\square$ Cribriform plate

Olfactory foramina

Olfaction $=$ the sense of smell

Perpendicular plate

Superior nasal concha

$\square$ Middle nasal concha

Notice that the superior and middle nasal conchae are bony structures found on the ethmoid bone, whereas the inferior nasal conchae are their own bones.

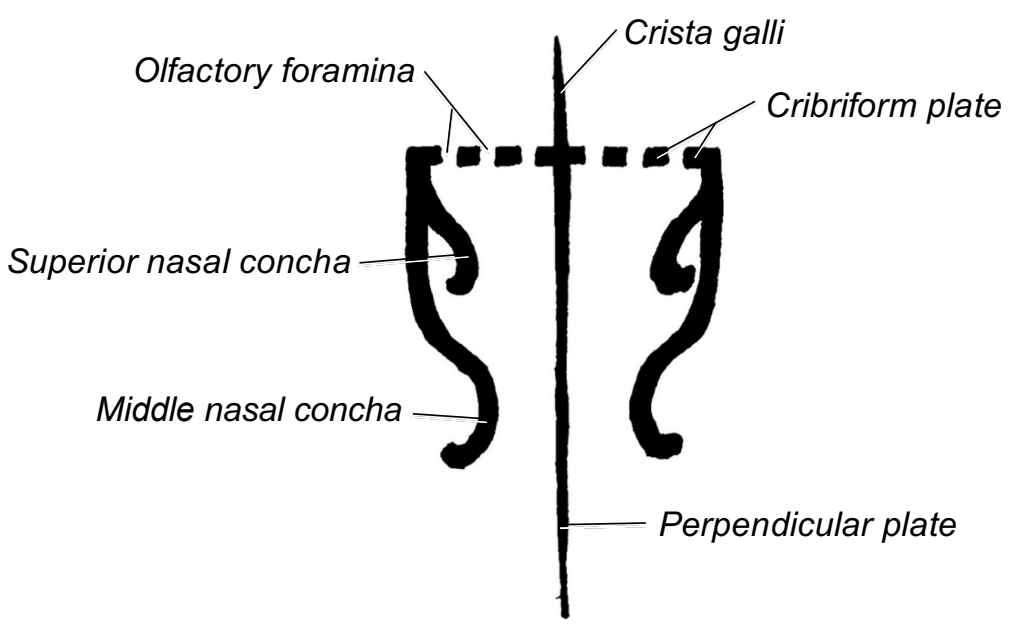

Figure 1.1. Simplified Diagrammatic Relationship of Bony Structures of the Ethmoid Bone, Anterior View

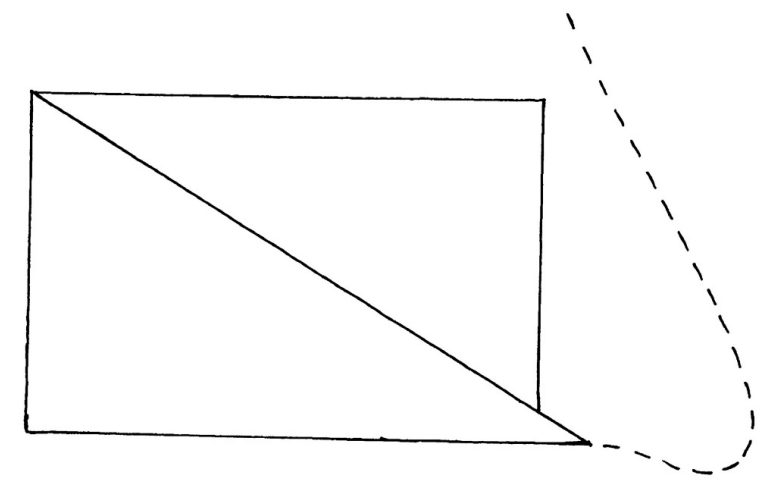

Figure 1.2. Relationship of Perpendicular Plate (of the Ethmoid Bone) to Vomer Bone in the Nasal Septum, Lateral View

\section{Clinical Application: Deviated Septum}
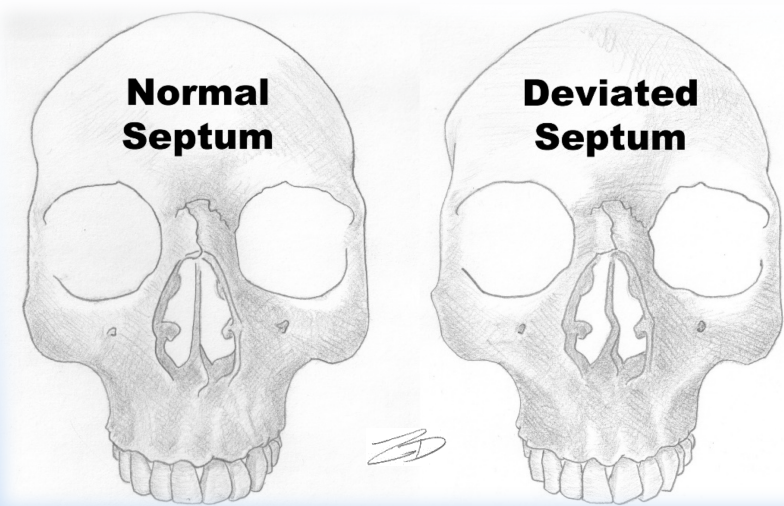

Deviated septa are common. In more severe cases, a deviated septum can cause difficulty breathing, sinus infections, sleep apnea, snoring, repetitive sneezing, facial pain, nosebleeds, and mild to severe loss of the ability to smell. Although most commonly caused by impact trauma, it can also be a congenital disorder caused by compression of the nose during childbirth. Medications can temporarily relieve symptoms, but a minor surgical procedure known as septoplasty is necessary to completely cure the symptoms related to septal deviations.

\section{Vomer bone}




\section{Temporal bones (2)}

\section{Squamous part}

$\underline{\text { Squamous }}=$ flat; sheet-like. The squamous part of the temporal bone forms part of the lateral wall of the cranium.

\section{Petrous part}

Petrous $=$ dense; rock-like. The petrous part of the temporal bone encases and protects the delicate structures in the inner ear.

$\square$ Zygomatic process

$\square$ Mandibular fossa

Fossa $=$ shallow depression. The mandibular fossa is where the mandibular condyle articulates to form the temporomandibular joint. $\underline{\text { Articulate }}=$ to form a joint.

\section{$\square$ Styloid process \\ $\square$ Mastoid process \\ $\square$ Stylomastoid foramen \\ $\square$ Jugular foramen}

The jugular foramen is formed anteriorly by the temporal bone and posteriorly by the occipital bone.

\section{$\square$ Internal acoustic meatus}

Internal = inside; $\underline{\text { Acoustic }}=$ relating to sound or hearing; $\underline{\text { Meatus }}$ $=$ an opening or passageway to a large canal

$\square$ External acoustic meatus

\section{Carotid canal}

\section{Foramen lacerum}

The foramen lacerum is formed between the temporal, sphenoid, and occipital bones, making its edges quite jagged. In Latin, foramen lacerum = lacerated opening.

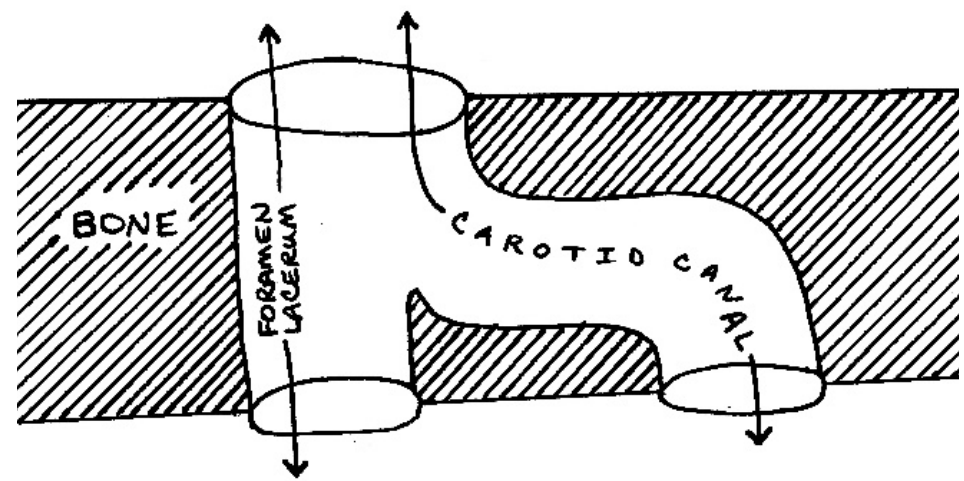

Figure 1.3. Simplified Diagrammatic Relationship Between Foramen Lacerum and Carotid Canal

Notice how the foramen lacerum and the carotid canal share an opening on the inside of the cranium but they have separate openings on the outside of the cranium.

\section{Sphenoid bone}

$\square$ Hypophyseal fossa (or Sella turcica)

Pterygoid processes

$\underline{\text { Ptery }}=$ wing-like

$\square$ Foramen rotundum

$\square$ Foramen ovale

Optic canal

Superior orbital fissure

$\underline{\text { Fissure }}=$ deep, elongated cleft

$\square$ Inferior orbital fissure

\section{Parietal bones (2)}




\section{Occipital bone}

\section{External occipital protuberance}

$\underline{\text { External }}=$ outside $; \underline{\text { Protuberance }}=$ projection or prominence

\section{Occipital condyle}

Hypoglossal canal

Foramen magnum

$\underline{\text { Magnum }}=$ great or large

\section{Sutures}

Sutures are the line-like structures that formed as a result of two bones fusing together.

\section{Coronal suture}

Coronal $=$ crown

$\square$ Sagittal suture

$\square$ Lambdoid suture

$\underline{\text { Lambdoid }}=$ resembling the Greek letter Lambda $(\Lambda)$ in form.

$\square$ Squamous suture

\section{Paranasal sinuses}

Sinuses are large air cavities in bone. Paranasal sinuses are sinuses found around the nasal cavity (hense the name). The paranasal sinuses are named according to which bone they are found within.
$\square$ Frontal sinus
Ethmoid sinus
$\square$ Maxillary sinus
$\square$ Sphenoid sinus

\section{Clinical Application: Fontanelles}

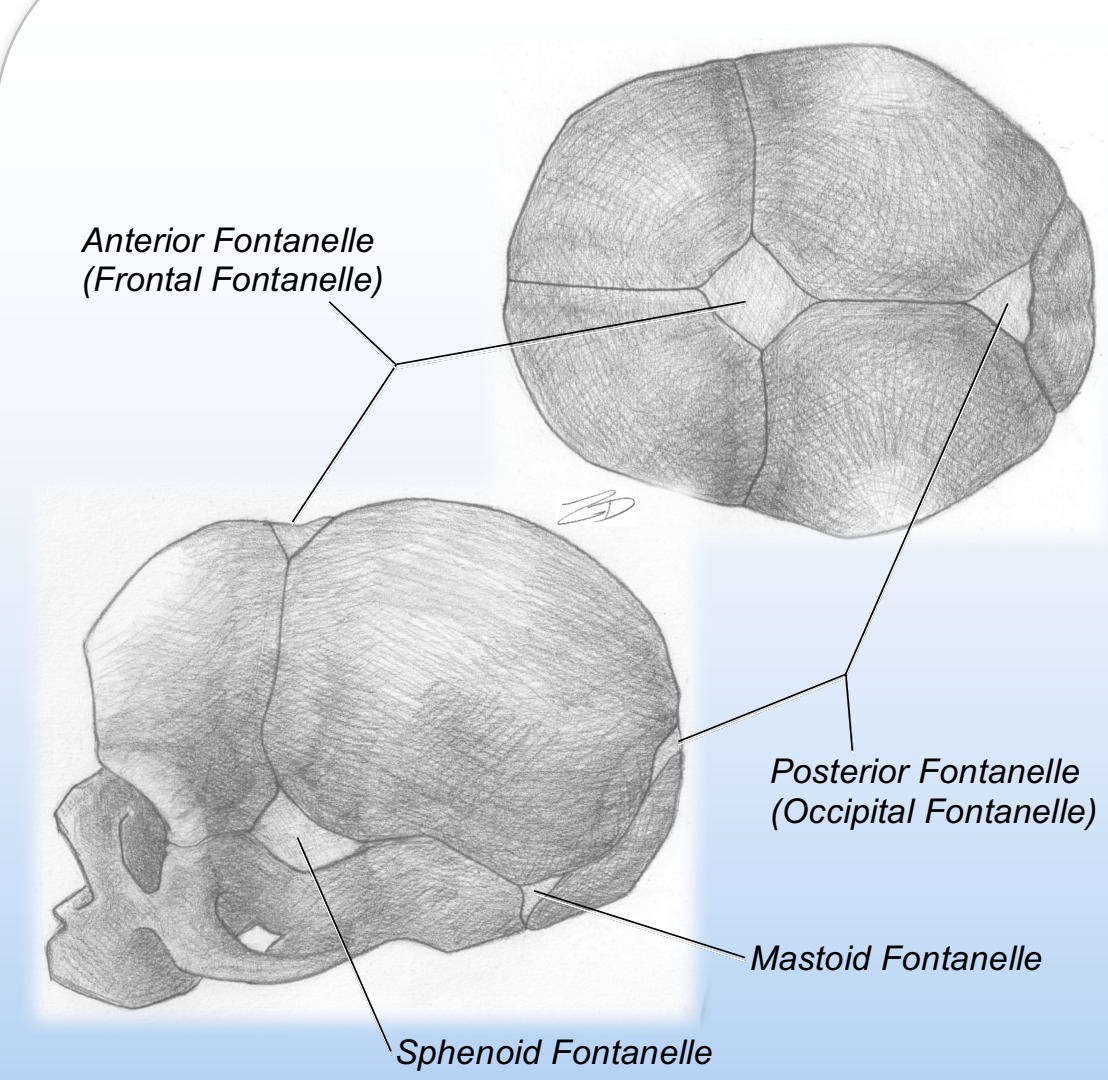

It is common to hear people talking about "soft spots" on the heads of newborn babies. These "soft spots," otherwise called fontanelles, are areas between skull bones that have not yet fully ossified. The major fontanelles can be found where multiple cranial bones come together. Instead of protective bone, only a membrane and the scalp protect the newborn's brain at the fontanelles until they ossify. These areas especially must be protected from blunt force until ossification is complete. In the above images, you can see the names fontanelles from a superior view (top right) and a lateral view (bottom left) on a newborn's skull. 


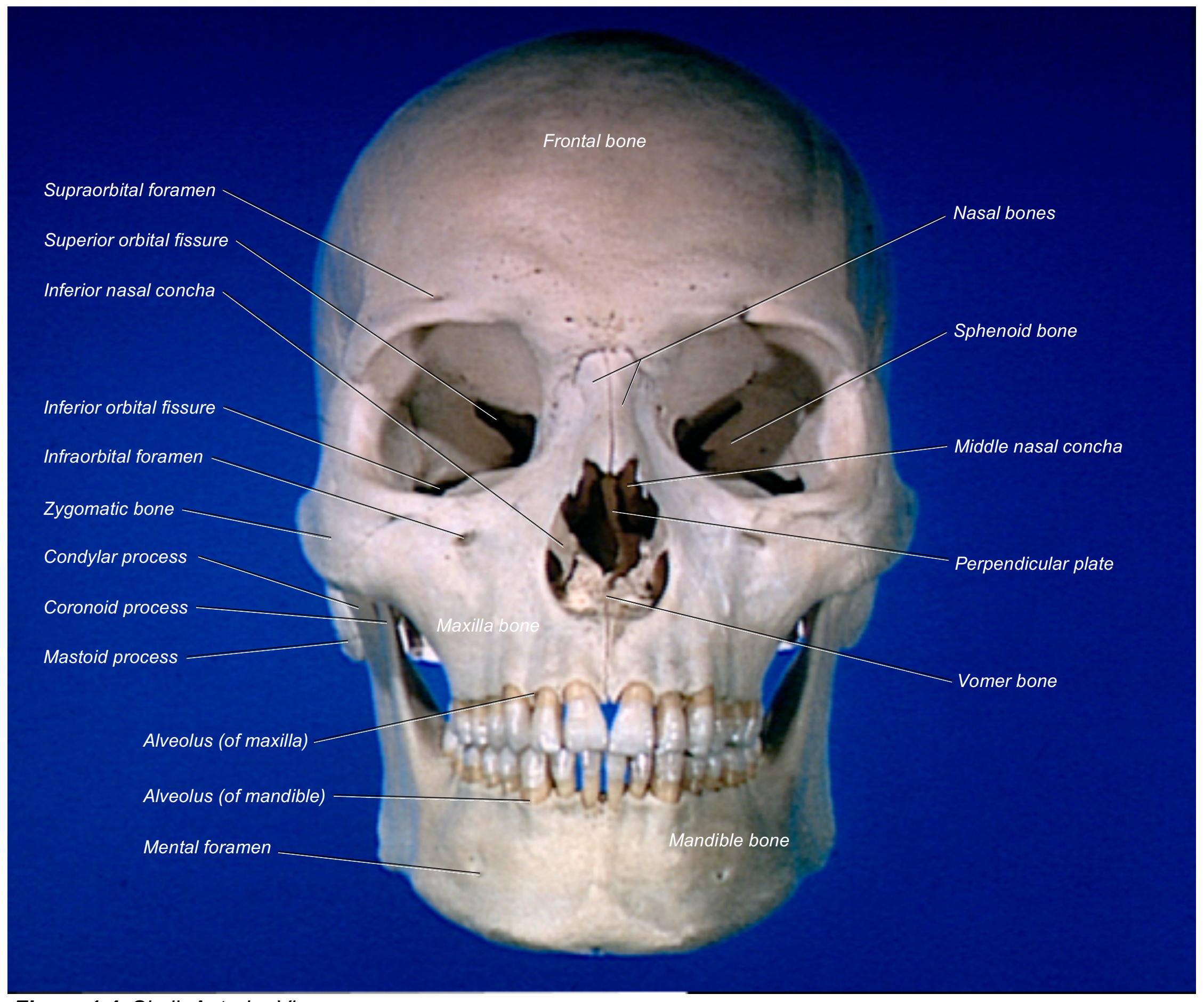

Figure 1.4. Skull, Anterior View 


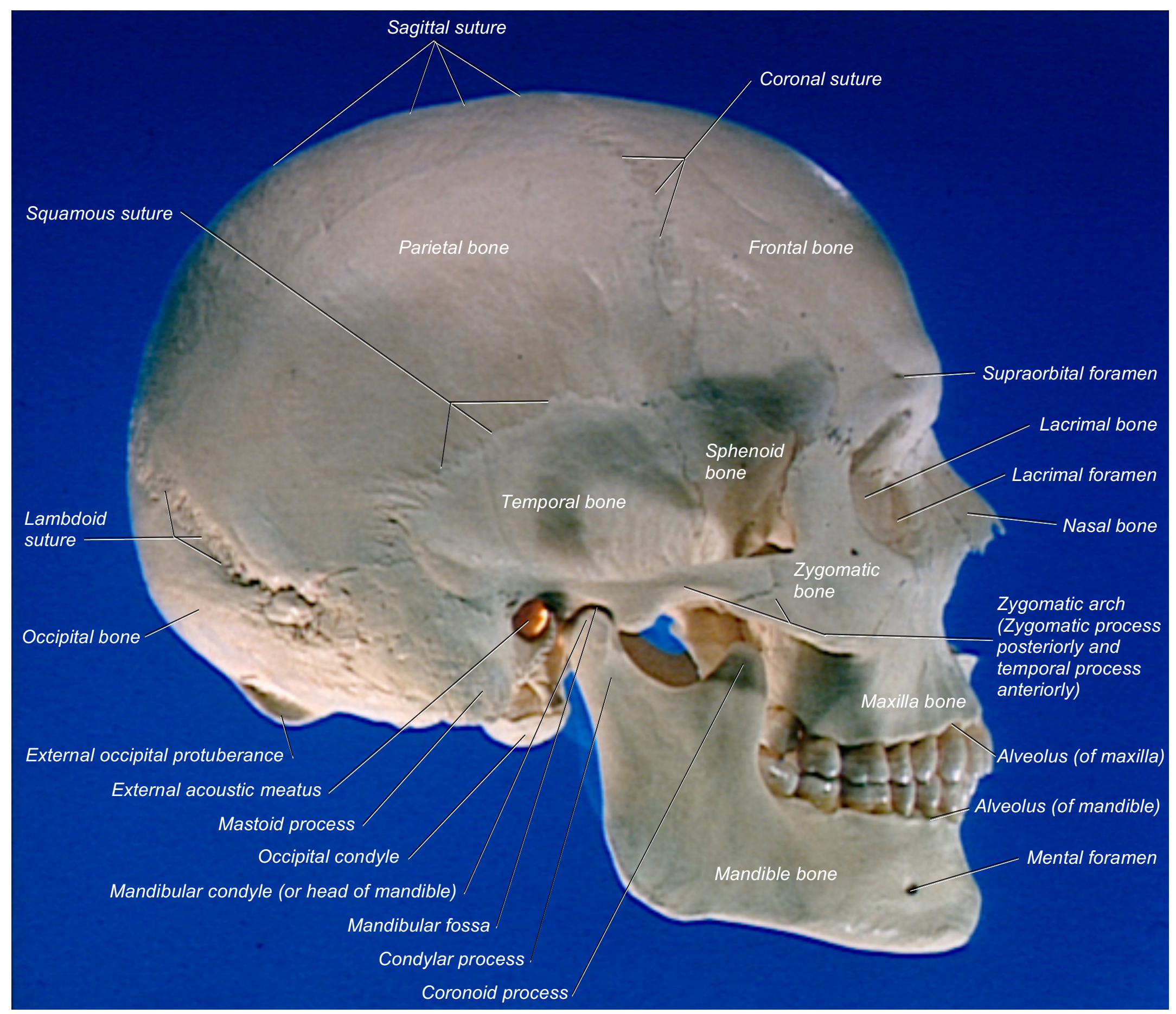

Figure 1.5. Skull, Right Lateral View 


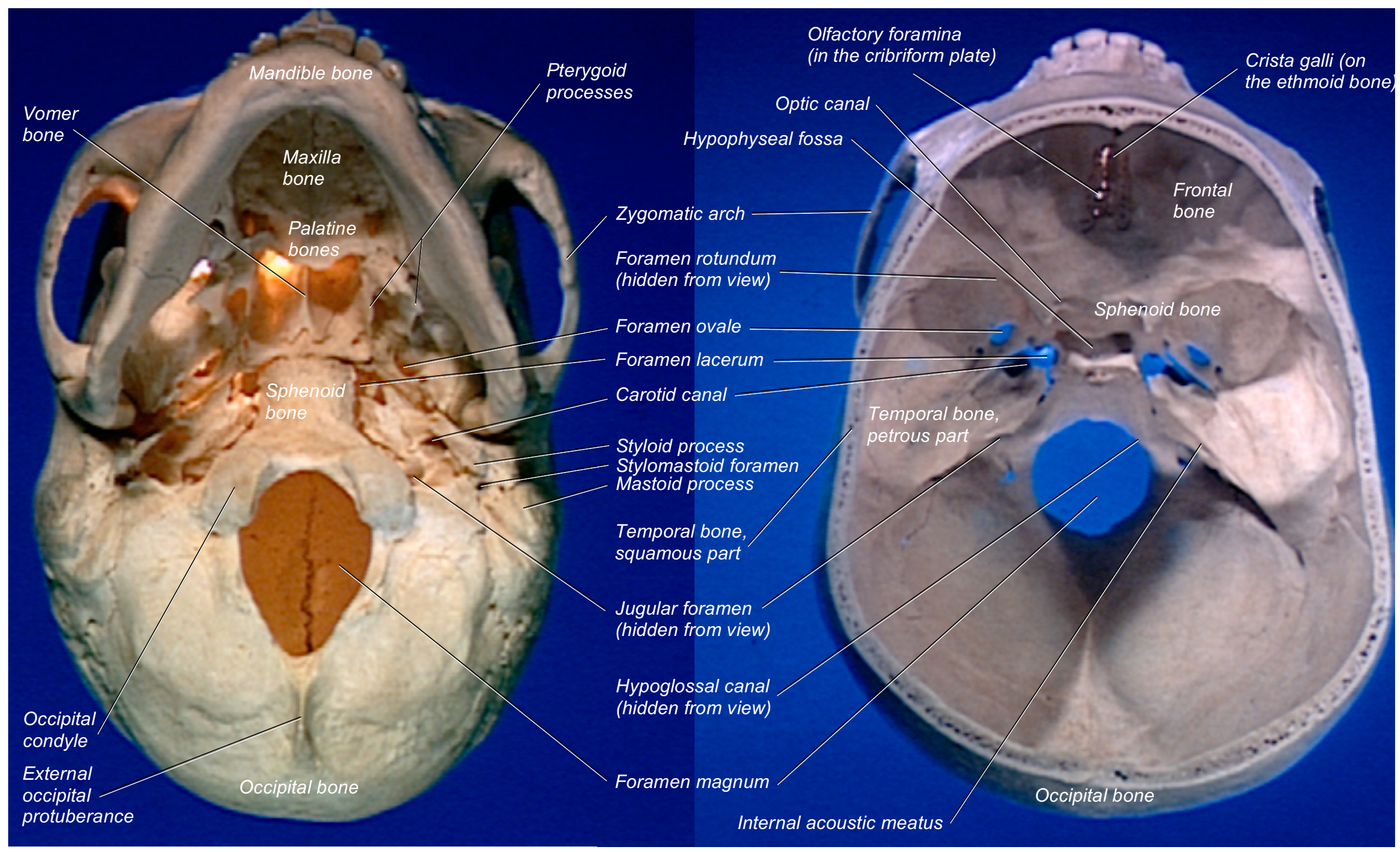

Figure 1.6. Skull, Inferior View

Figure 1.7. Skull, Superior View Inside Cranium 
and self-assess your knowledge.

1) What is a fossa? (LO2)

2) Using the terminology you've learned, describe the structure and location of the infraorbital foramen. (LO2)

3) What does the term petrous mean? (LO2)

4) What bones are labeled in the image below? (LO3)

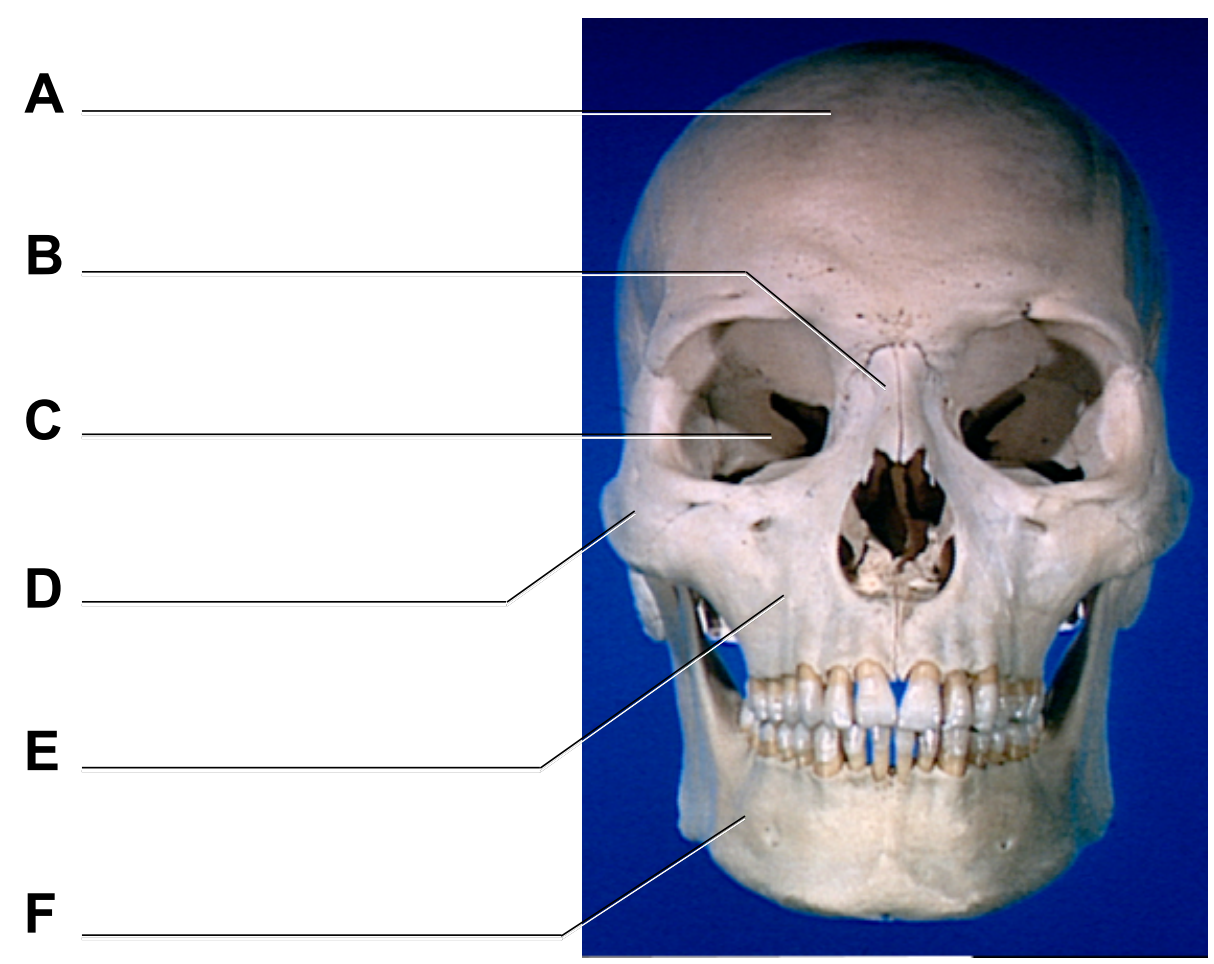

Fig. 1.4.
5) What structure do olfactory foramina pass through? (LO3)

6) What bone is the zygomatic process found on? (LO3)

7) What bony structures are labeled in the image below? (LO3)

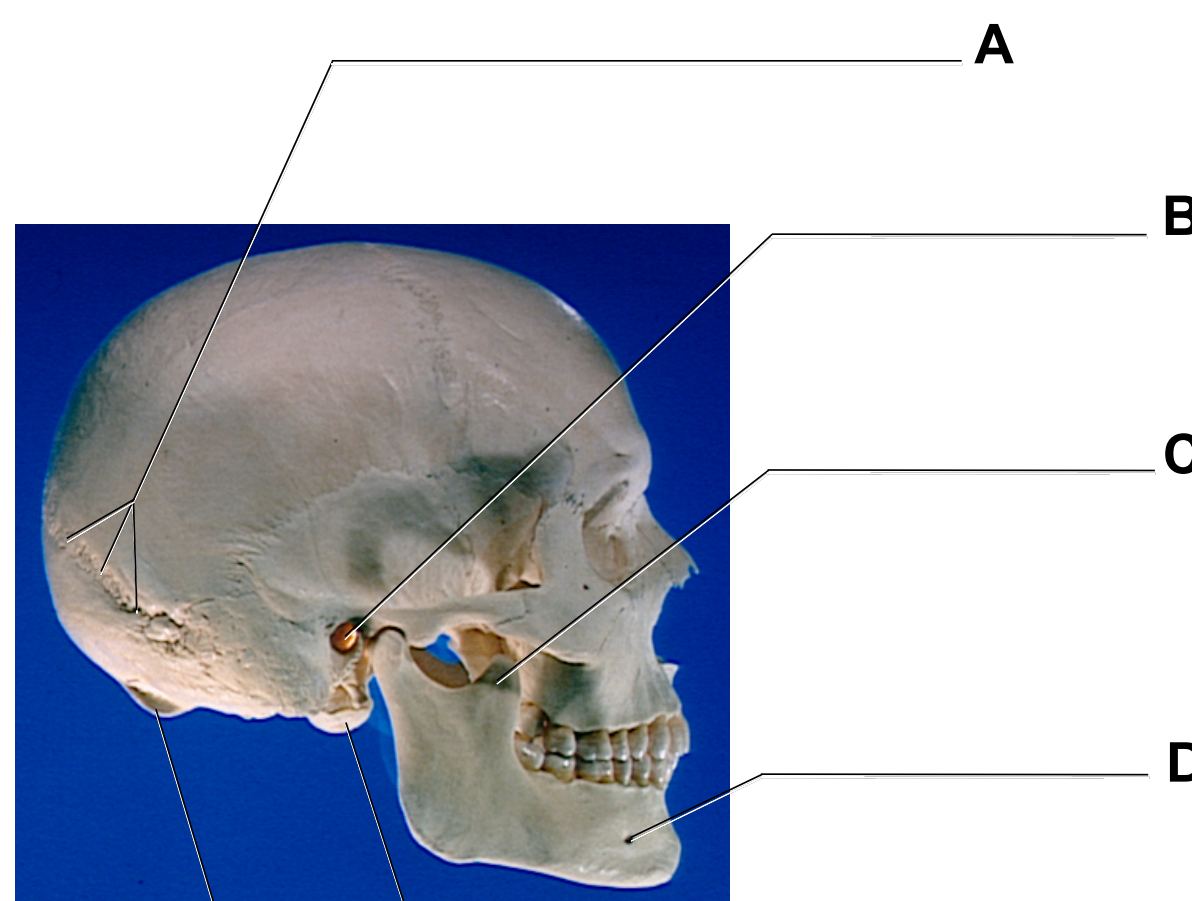

Fig. 1.5

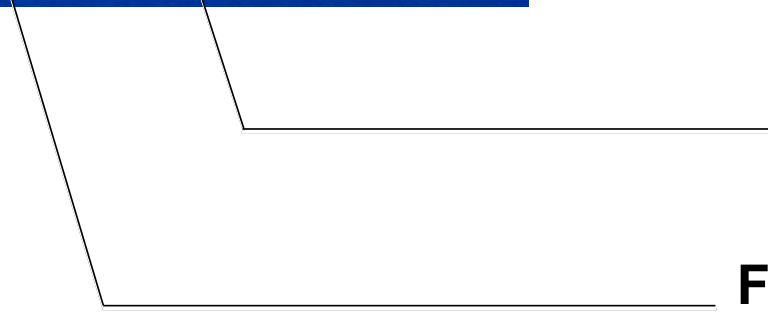

$\mathbf{E}$

$\mathbf{F}$ 


\section{Lab 2: Axial Skeleton (Neck, Thorax, \& Abdomen)}

Instructions: Learn the listed terms using the listed learning objectives, labeled images, lab specimens, and other resources.

LO4: Describe the terminology used to identify structures of the axial skeleton (neck, thorax, and abdomen).

LO5: Identify the bones and associated structures of the axial skeleton (neck, thorax, and abdomen).

\section{Hyoid bone*}

The hyoid bone is located between the base of the mandible and the $3^{\text {rd }}$ cervical vertebra. It is an attachment point for many muscles responsible for tongue movement and swallowing.

\section{Sternum bone}

$\square$ Manubrium (M)

- Jugular notch

O Clavicular notch

$\square$ Body (B)

- Costal notches

Xiphoid process

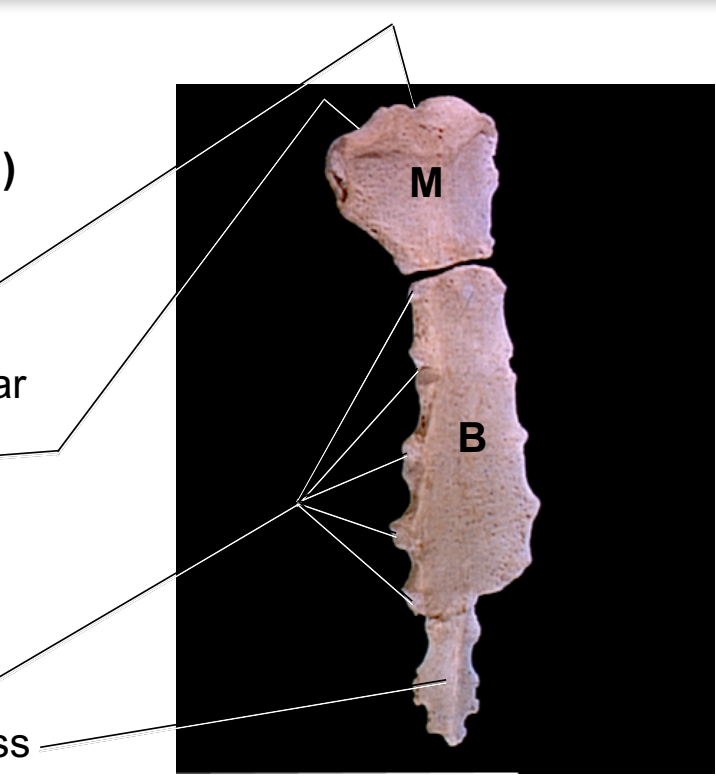

$\underline{X i p h}=$ sword
Figure 2.1. Sternum, Right Anterolateral View

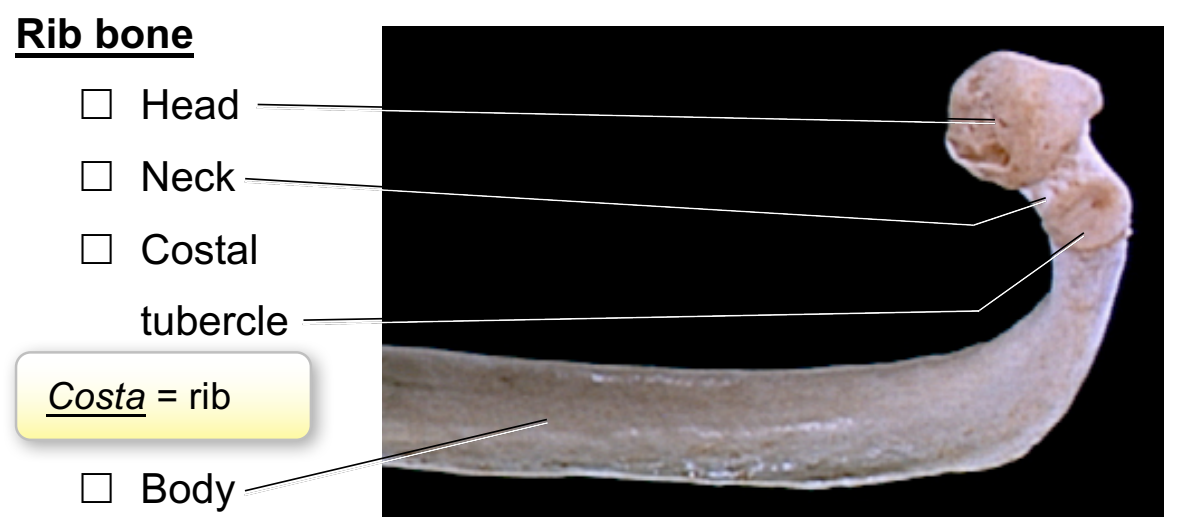

Figure 2.2. Right $6^{\text {th }}$ Rib, Medial View

The articular facets on the head and costal tubercles of the rib articulate posteromedially with facets on the thoracic vertebrae. The anteromedial aspects of the ribs attach to costal cartilages which allow for elasticity of the thorax while linking the ribs to the costal notches on the sternum.

There are 12 pair of ribs. Rib pairs 1-7 are considered "true ribs" because they attach to the sternum via their own separate costal cartilages. Rib pairs 8-12 are considered "false ribs" because they only attach to the sternum by attaching their combined costal cartilages with the costal cartilage of the $7^{\text {th }}$ rib. Lastly, rib pairs 11-12 are considered "floating ribs" because they do not have any costal cartilages attaching them in any way to the sternum. To summarize...

True ribs: ribs 1-7

False ribs: ribs 8-12

Floating ribs: ribs 11-12 


\section{Vertebrae that make up the spine}

$\square 7$ Cervical vertebrae

$\square 12$ Thoracic vertebrae

$\square 5$ Lumbar vertebrae

5 Sacral vertebrae

(all are fused)

4 Coccygeal vertebrae

(usually $\sim 3$ are fused)

\section{TYPICAL VERTEBRAL STRUCTURES}

These are structures that are found on nearly all vertebrae, regardless of the region, except for the noted differences listed under "REGION-SPECIFIC VERTEBRAL STRUCTURES."

\section{Body \\ Pedicle \\ Lamina}

Together, both pedicles and both laminae make up a larger structure known as the vertebral arch.

$\square$ Vertebral foramen

Spinous process

$\square$ Transverse process $\square$ Superior articular facet

Inferior articular facet ${ }^{\star}$

The superior articular facet on one vertebra articulates with the inferior articular facet on the vertebra above it. Similarly, the inferior articular facet on one vertebra articulates with the superior articular facet on the vertebra below it. For example, the superior and inferior articular facets on the $10^{\text {th }}$ thoracic vertebra articulate with the inferior articular facet on the $9^{\text {th }}$ thoracic vertebra and the superior articular facet on the $11^{\text {th }}$ thoracic vertebra, respectively.

\section{Intervertebral foramen}

Inter = between; vertebral = vertebrae; foramen = hole or opening. Therefore, the intervertebral foramina are holes/openings formed between two vertebrae.

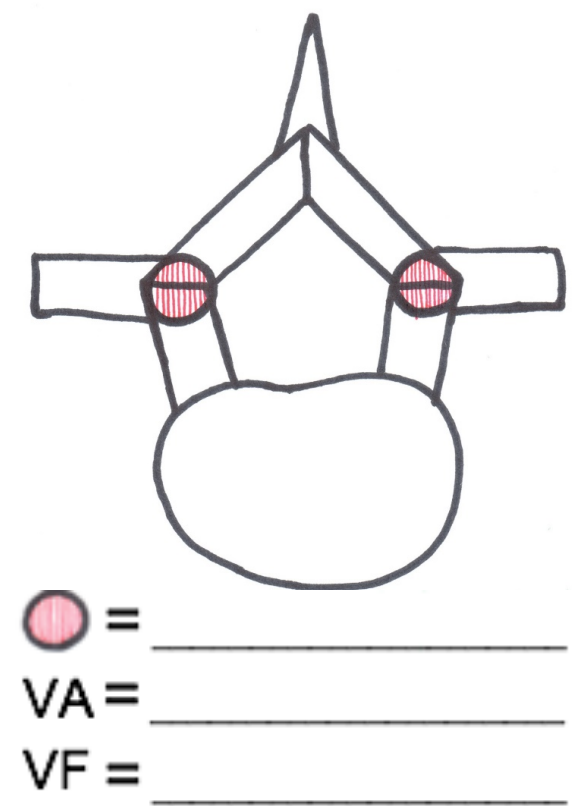

Figure 2.3. Simplified Diagram of Typical Structures on Vertebrae, Superior/Inferior View 


\section{REGION-SPECIFIC VERTEBRAL STRUCTURES}

\section{Cervical vertebrae (C1 - C7)}

\section{Transverse foramen}

Only cervical vertebrae have transverse foramen. These foramen are named accordingly to the process they are on. Later, we will learn that the vertebral artery passes through these foramina.

Cervical vertebrae will often have bifid (divided, or split) spinous process.

Cervical vertebrae will have small bodies because they do not bear much weight (only the weight of the head and neck).

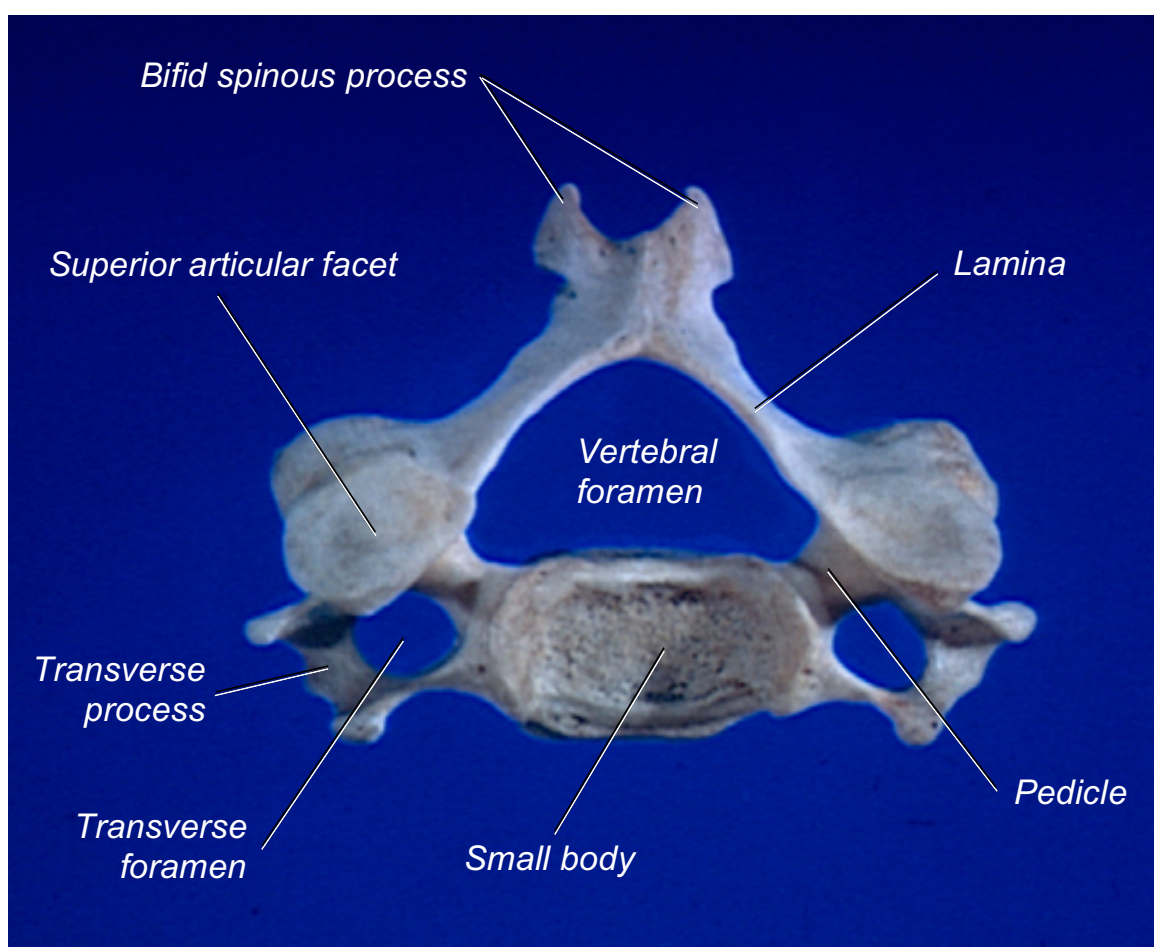

Figure 2.4. Typical Cervical Vertebra, Superior View
Three specifically named cervical vertebrae are especially significant. These are Atlas ( $\mathrm{C} 1$ vertebra), Axis ( $\mathrm{C} 2$ vertebra), and Vertebra Prominens (C7 vertebra).

\section{$\square$ Atlas (C1 vertebra)}

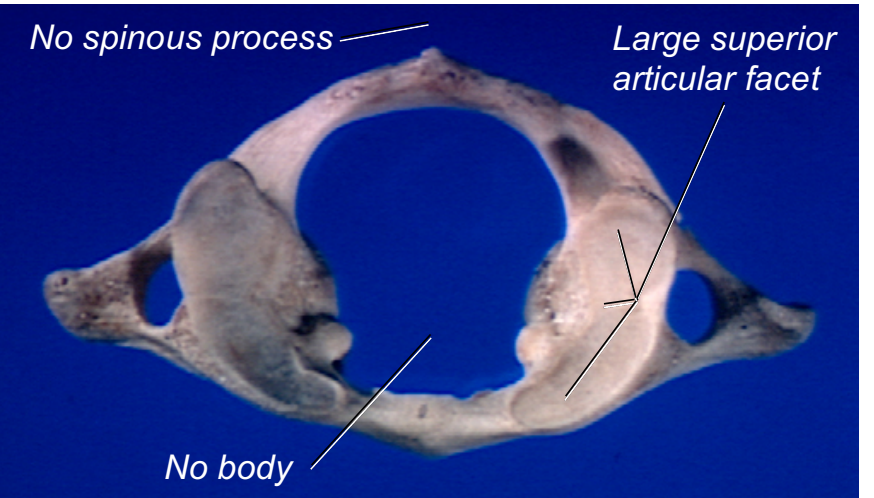

Figure 2.5. Atlas, Superior View

The atlas does not have a body or a spinous process. It also has superior articular facets that are much larger than normal. This is because the occipital condyles on the base of the skull articulate with these facets, connecting the skull to the spine. The joint formed between the articular surfaces of these structures is called the atlanto-occipital joint (named from the two bones forming the joint). The atlanto-occipital joint allows for the main movements necessary to nod your head "yes." 
$\square$ Axis (C2 vertebra)

○ Dens (or Odontoid process)

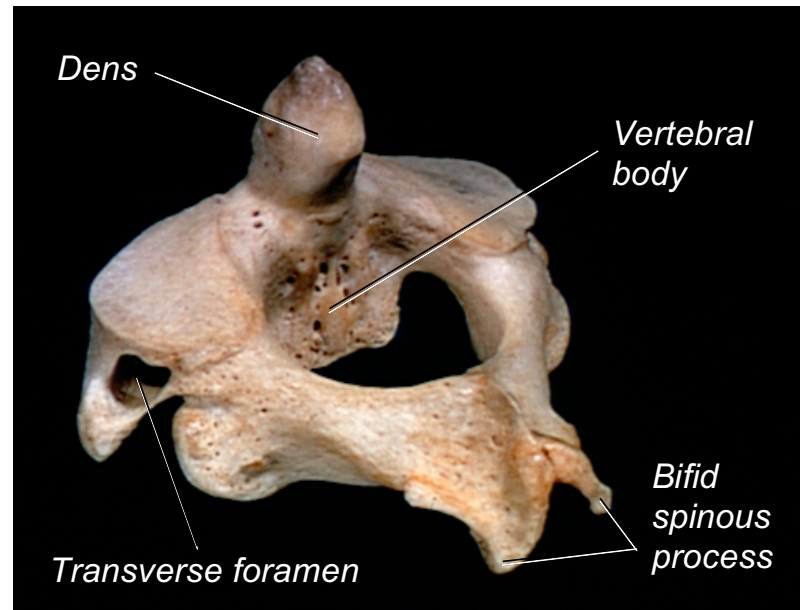

Figure 2.6. Axis, Posterolateral View

The axis is easily identifiable by the dens, a prominent bony structure projecting superiorly from its body. This structure was named "dens" because it resembled the shape of a tooth (den $=$ tooth). The dens was actually once the body of atlas; during development, the body of atlas separates from the rest of the vertebra and fuses to the top of the body of axis. When the atlas and axis are stacked together, the associated facets create the atlantoaxial joint. Because the atlas forms a ring around the superiorly-projecting dens at this joint, the atlas is able to rotate around the dens. Therefore, the atlantoaxial joint allows for the main movements necessary to shake your head "no."

\section{Vertebra prominens (C7 vertebra)}

The vertebra prominens does not have any special structures like the atlas and axis. Instead, its significance lies in its landmark palpability (ability to feel it). When you flex your neck (put your chin to your chest), the spinous process of vertebra prominens "sticks out" and can be palpated at the base of the posterior neck.

\section{Thoracic vertebrae (T1 - T12)}

$\square$ Superior costal facet (or Superior costal demi-facet)

$\square$ Inferior costal facet (or Inferior costal demi-facet)

$\square$ Transverse costal facet

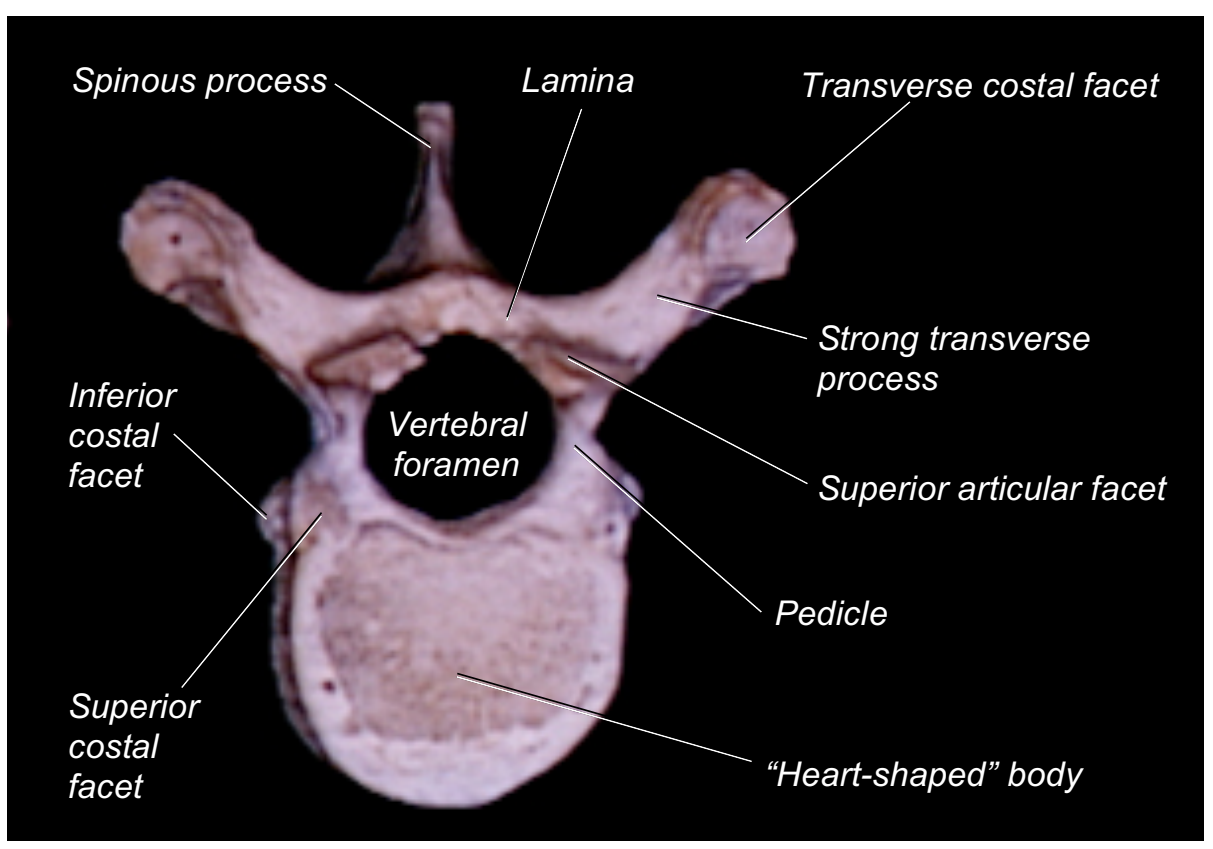

Figure 2.7. Typical Thoracic Vertebra, Superior View

The most significant characteristic of thoracic vertebrae is their articulation with ribs. In most cases, the head of one rib will articulate between two vertebral bodies at the inferior costal facet of the vertebra above and the superior costal facet of the vertebra below. Further, the strong transverse processes of thoracic vertebrae have transverse costal facets on them which articulate with the costal tubercles of the ribs. Thoracic vertebrae also typically have long, angled spinous processes and characteristic "heart-shaped" bodies. 


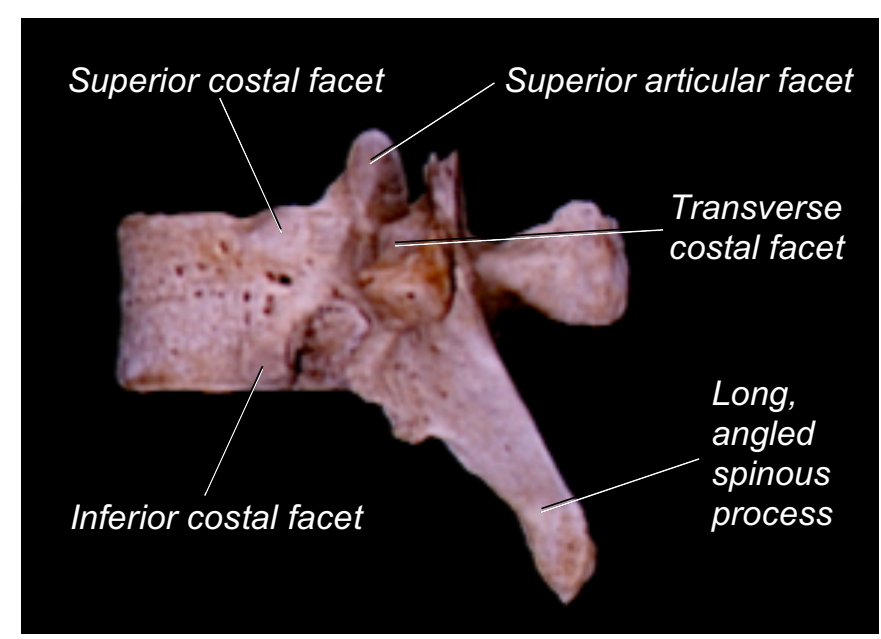

Figure 2.8. Typical Thoracic Vertebra, Posterolateral View

\section{Lumbar vertebrae (L1 - L5)}

$\square$ Mammillary process

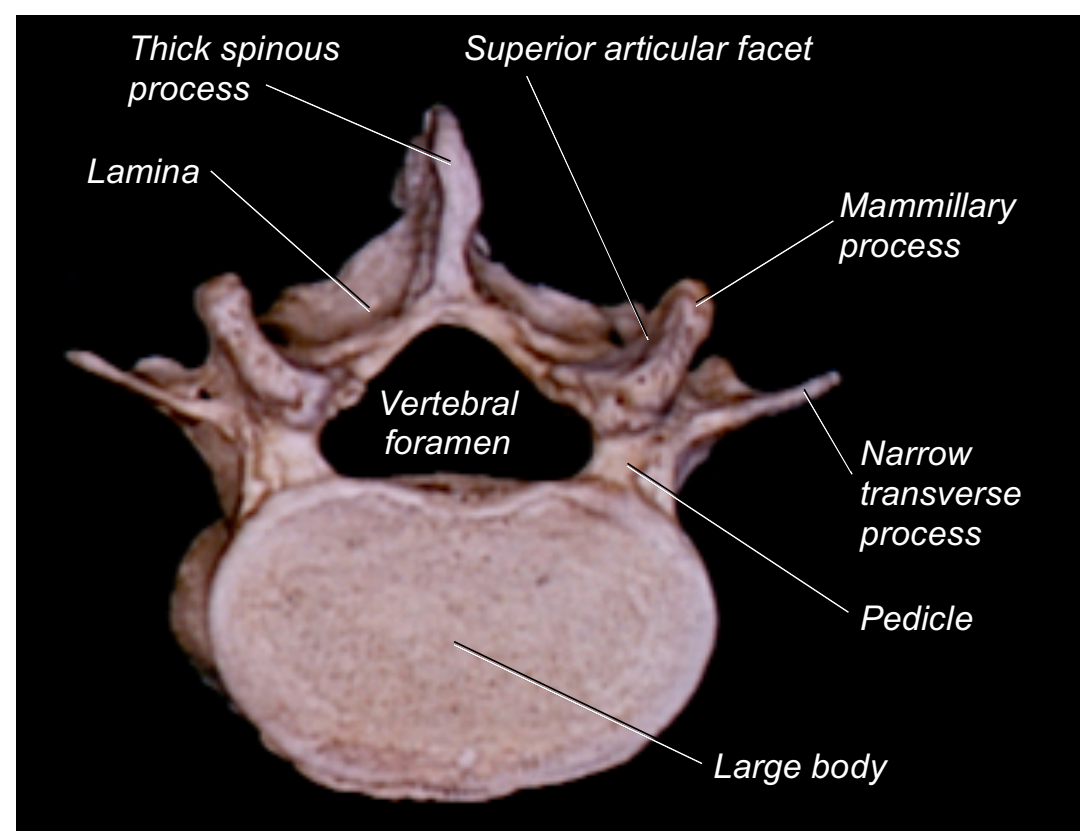

Figure 2.9. Typical Lumbar Vertebra, Superior View
Lumbar vertebrae have mammillary processes. These bony outgrowths project posteriorly from next to the superior articular facets. Lumbar vertebrae also have characteristically large bodies (because they bear more weight than cervical and thoracic vertebrae), narrow transverse processes, and thick, stout spinous processes.

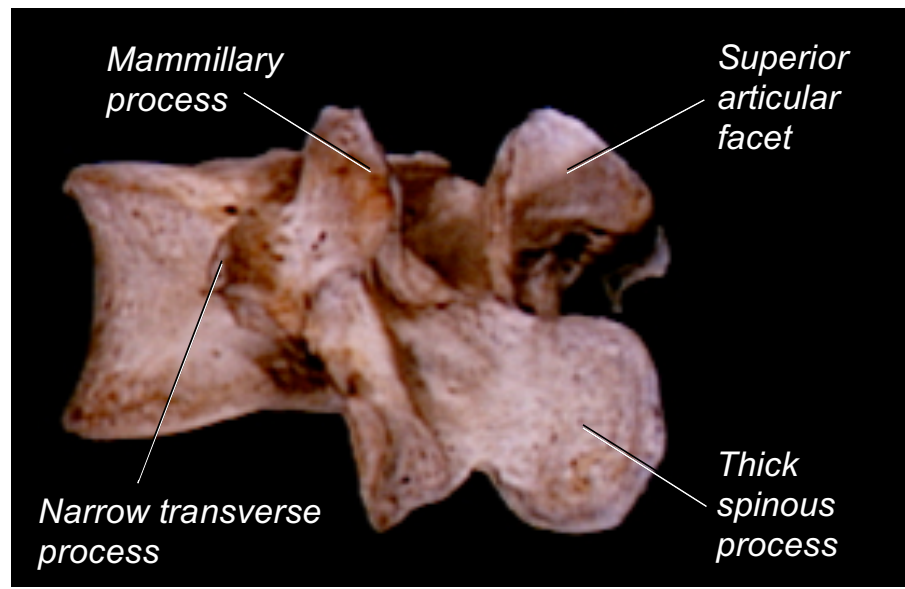

Figure 2.10. Typical Lumbar Vertebra, Posterolateral View

\section{Clinical Application: Scoliosis, Kyphosis, and Lordosis}

Scoliosis (A) is an abnormal lateral curvature of the spine. Kyphosis (B) is an exaggerated thoracic curvature. Lordosis (C) is an exaggerated lumbar curvature.

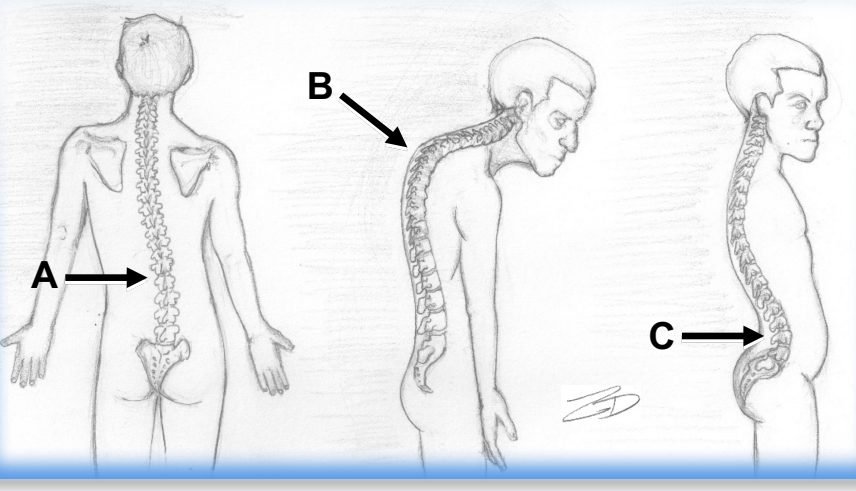




\section{Sacral vertebrae (Sacrum, S1 - S5, fused)}

$\square$ Auricular surface

The auricular surface is roughly in the shape of an ear (auricular = shaped like an ear or earlobe).

$\square$ Sacral canal

$\square$ Sacral hiatus

$\square$ Median sacral crest

$\square$ Posterior sacral foramina

$\square$ Anterior sacral foramina

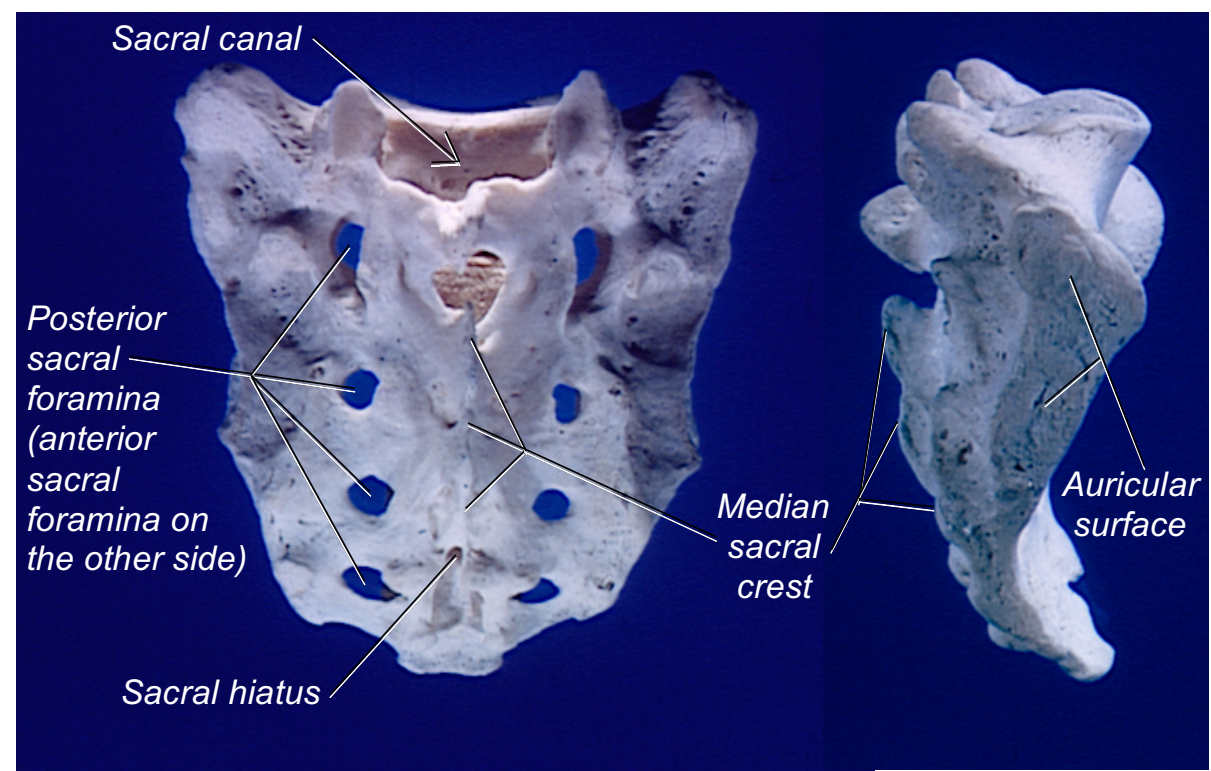

Figure 2.11. Sacrum,

Figure 2.12.

Posterior View

Sacrum,

Lateral View

Five sacral vertebrae fused together to form the sacrum. The fused vertebra have typical vertebral structures, but some of these structures have been altered and renamed because of the fusing (i.e. the fused spinous processes $=$ median sacral crest).

\section{Coccygeal vertebrae (Coccyx, Co1 - Co4, 3 fused)}

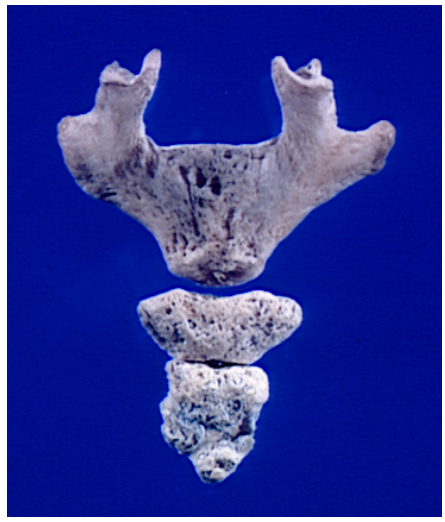

Figure 2.13. Coccyx, Posterior View

\section{Ligaments of the vertebral column}

\section{Nuchal ligament (or Ligamentum nuchae)*}

The nuchal ligament attaches superiorly to the external occipital protuberance and inferiorly to the spinous process of vertebra prominens (and to all the spinous processes in between). This ligament limits flexion of the neck.

\section{Anterior longitudinal ligament*}

The anterior longitudinal ligament attaches to the anterior borders of the vertebral bodies and limits extension of the spine.

\section{Posterior longitudinal ligament*}

The posterior longitudinal ligament attaches to the posterior borders of the vertebral bodies and limits flexion of the spine.

\section{Ligamentum flavum *}

Ligamenta flava (plural form) attach one lamina to another lamina. These elastic ligaments work together to maintain upright posture. 


\section{Clinical Application: Whiplash}

Whiplash is an injury commonly associated with rear-end motor vehicle collisions. In a rear-end collision, the head of the person in the car being rear-ended will be thrown backward, causing hyperextension of the neck. This action can stretch and/or tear the anterior longitudinal ligament, resulting in pain and stiffness in the neck. Following the hyperextension injury in the same accident, the same person's head may then be thrown forward as a result of their vehicle and body being forced forward. This secondary action can cause hyperflexion of the neck and could jeopardize the integrity of the posterior longitudinal ligament and/or the nuchal ligament.

\section{Intervertebral disc}

Nucleus pulposus

Annulus fibrosus

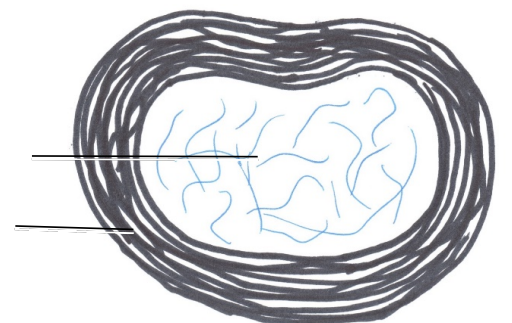

Figure 2.14. Intervertebral Disc Diagram, Superior View

\section{Clinical Application: Herniated Disc}

A herniated disc, also called a slipped disc, results from when a weakened or injured annulus fibrosus is unable to confine the nucleus pulposus anymore. As a result, when pressure is exerted on that intervertebral disc, the nucleus pulposus will bulge out instead of cushioning and supporting the vertebrae. Herniated discs happen most often as a result of intervertebral discs weakening by degeneration from age, but they can also happen as a result of blunt injury or strenuous actions on the back. Spinal nerves exit the vertebral column through intervertebral foramen, which can become compressed as a result of a herniated disc and cause pain in that region.

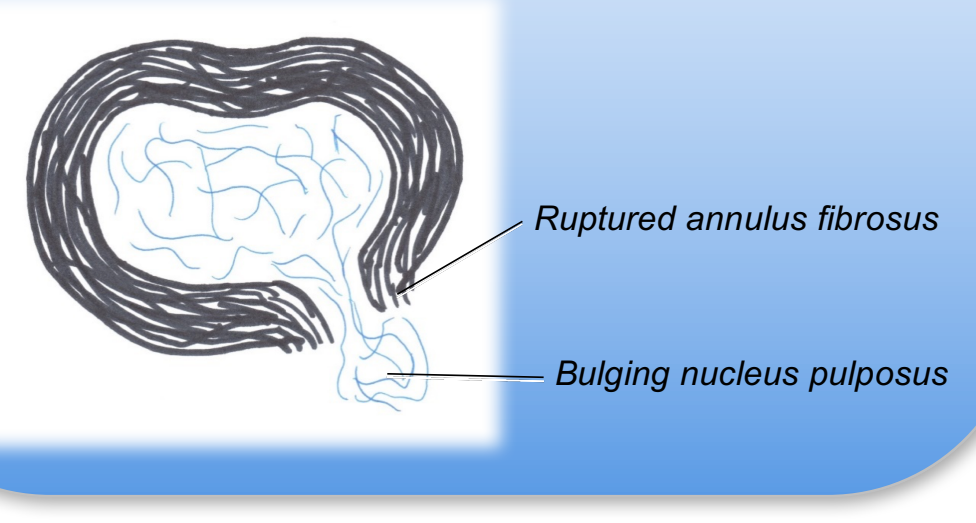

The nucleus pulposus is the inner, jelly-like material of the intervertebral disc that cushions and evenly distributes supportive pressure between vertebral bodies. The annulus fibrosus is the strong outer fibrous component of the intervertebral disc that contains the nucleus pulposus.

There is no intervertebral disc between the skull and atlas or between atlas and axis. There is also no intervertebral discs in between the sacral vertebrae or coccygeal vertebrae.

Intervertebral discs are named according to the vertebra directly above them (i.e. the intervertebral disc between T3 and T4 vertebrae is the T3 intervertebral disc). 
and self-assess your knowledge.

1) What does the term costal mean? (LO4)

2) What does the term xiph mean? (LO4)

3) What structures do the facets on costal tubercles articulate with? (LO5)

4) How many ribs are true ribs? (LO5)

5) What structures make up the vertebral arch? (LO5)

6) What are the superior-most and inferior-most structures that the nuchal ligament attaches to? (LO5)

7) What structures are labeled on this typical thoracic vertebra? (LO5)

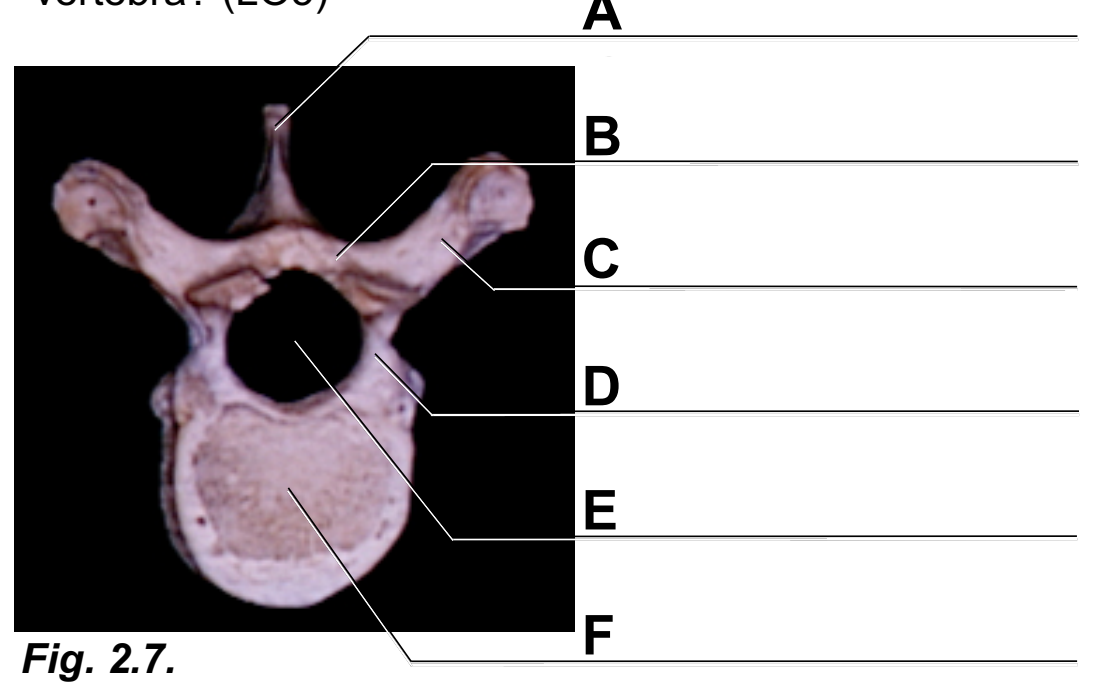

8) Match the following choices with the correct region of the spine that they can be found in. (LO5)
a. 12 vertebrae
b. 5 total vertebrae (fused)
c. 5 total vertebrae (not fused)
d. 4 total vertebrae ( 3 fused)
e. 7 vertebrae
f. Auricular surface
g. Axis
h. Long, angled spinous processes
i. Large vertebral bodies
j. Anterior \& posterior sacral foramina
k. Superior \& inferior costal facets
I. Vertebra prominens
$\mathrm{m}$. Transverse costal facets
n. Transverse foramina
o. Dens
p. Bifid spinous processes
q. Narrow transverse processes
r. "Heart-shaped" vertebral bodies
s. Mammillary processes
t. Atlas
u. Median sacral crest
v. Articulates with the skull
w. Articulates with ribs
$x$. Sacral hiatus
y. Smallest vertebrae
z. Largest vertebrae

Cervical:

Thoracic:

Lumbar:

Sacral:

Coccygeal: 


\section{Lab 3: Appendicular Skeleton (Pectoral Girdle \& Upper Limb}

Instructions: Learn the listed terms using the listed learning objectives, labeled images, lab specimens, and other resources.

LO6: Describe the terminology used to identify structures of the appendicular skeleton (pectoral girdle and upper limb).

LO7: Identify the bones and associated structures of the appendicular skeleton (pectoral girdle and upper limb).

\section{Scapula bone}

$\square$ Acromion

$\square$ Coracoid process

$\square$ Spine

$\underline{\text { Spine }}=$ sharp, slender, ridge-like projection

$\square$ Glenoid fossa (or Glenoid cavity)

$\square$ Supraspinous fossa

$\square$ Infraspinous fossa

$\square$ Subscapular fossa

$\underline{\text { Sub }}=$ under; below

$\square$ Superior border

$\square$ Medial border

$\square$ Lateral border

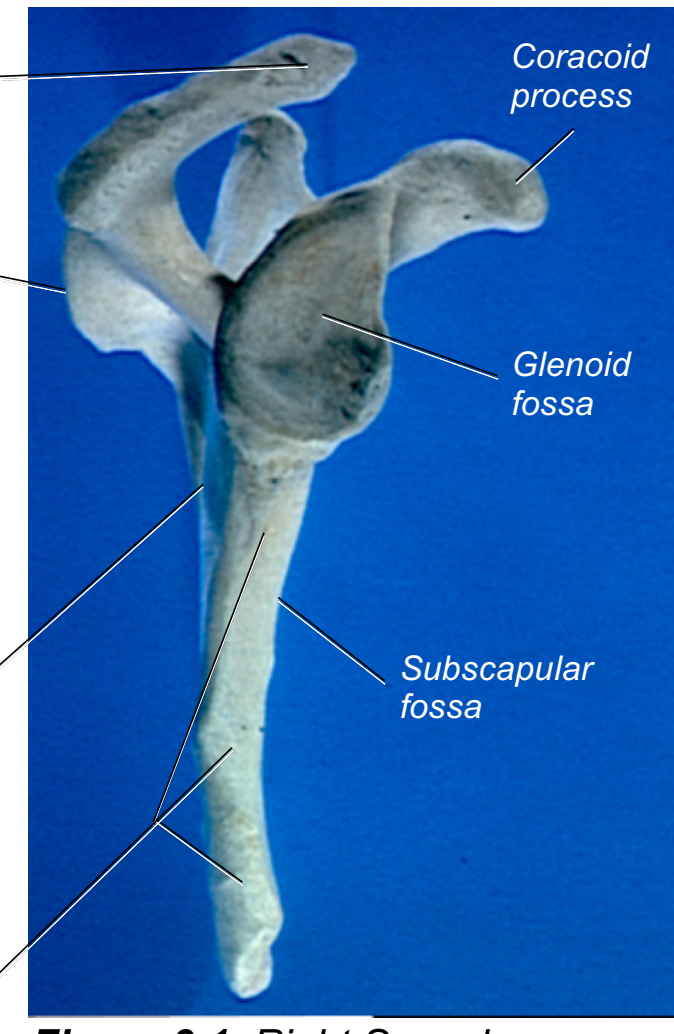

Figure 3.1. Right Scapula, Lateral View

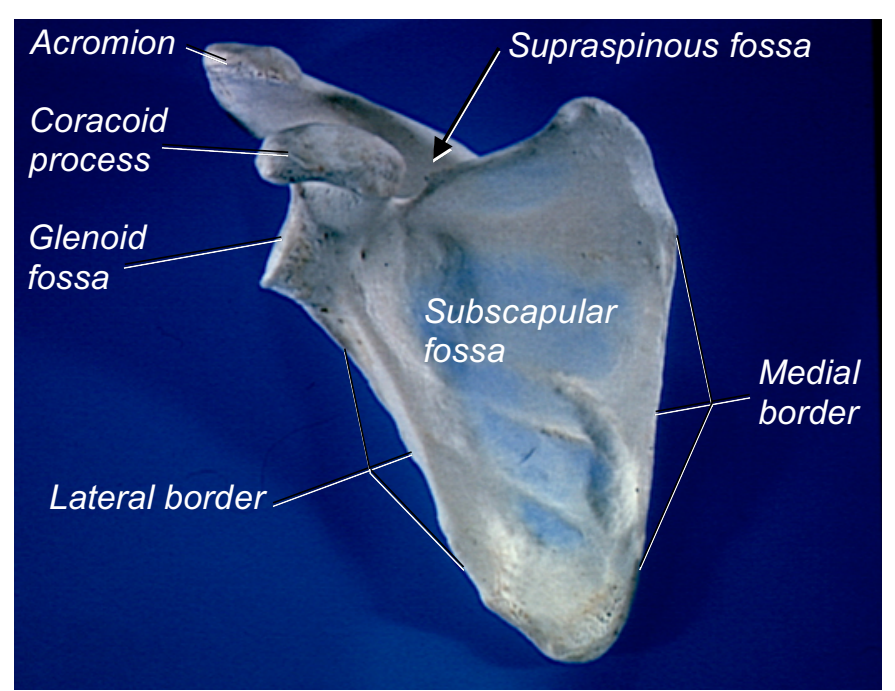

Figure 3.2. Right Scapula, Anterior View

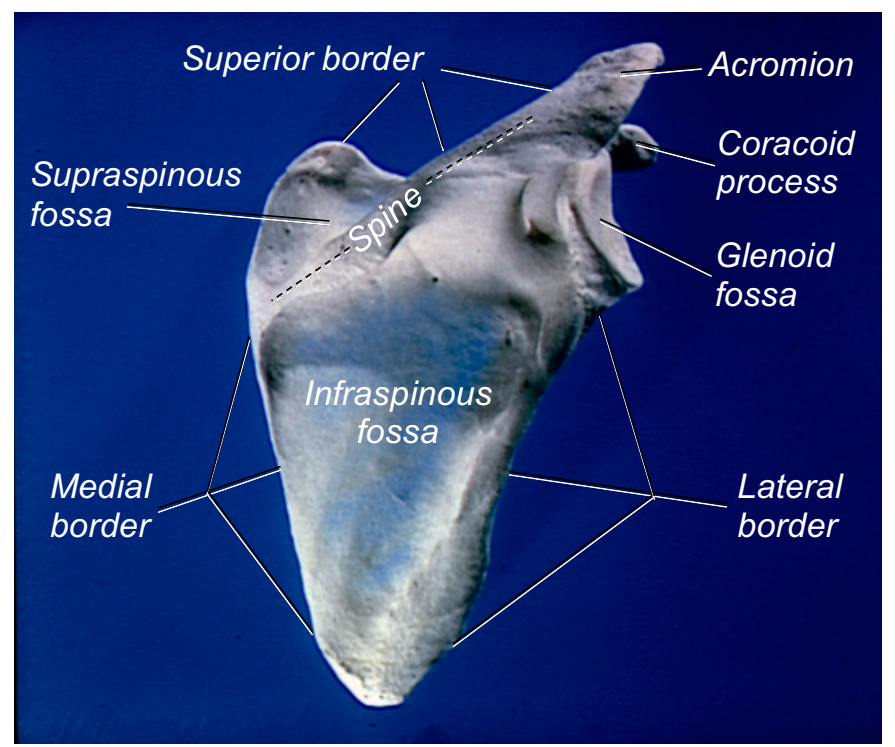

Figure 3.3. Right Scapula, Posterior View 


\section{Clavicle bone}

$\square$ Sternal end

$\square$ Acromial end

$\square$ Conoid tubercle

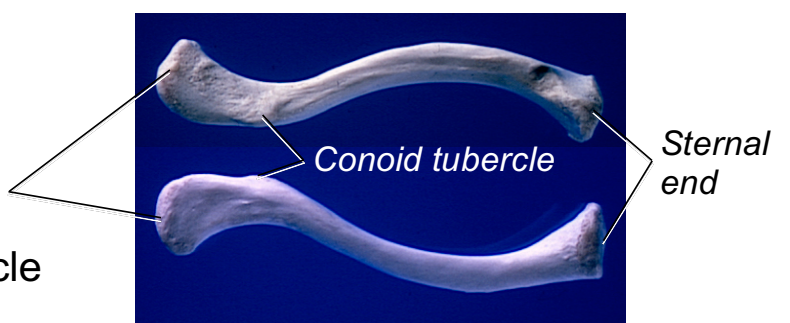

Figure 3.4. (Above) Right Clavicle, Inferior View

Figure 3.5. (Below) Right Clavicle, Superior View

The sternal and acromial ends of the clavicles articulate with the clavicular notches on the manubrium of the sternum and acromions on the scapulae, respectively.

Together, a scapula and clavicle forms a pectoral girdle. The two pectoral girdles function to connect and support the arms to each side of the trunk of the body. The rest of the bones in the upper extremity form joints and regions of the upper limb (i.e. shoulder, arm, elbow, forearm, wrist, and hand).

\section{Humerus bone}

$\square$ Head

The head of the humerus articulates with the glenoid fossa on the scapula to create the shoulder joint.
$\square$ Greater tubercle
$\square$ Lesser tubercle
$\underline{\text { Tubercle }}=$ rounded projection
$\square$ Intertubercular groove
$\square$ Deltoid tuberosity
$\square$ Lateral epicondyle
$\square$ Medial epicondyle
$\square$ Coronoid fossa

$\square$ Olecranon fossa

$\square$ Capitulum (or Capitellum)

$\square$ Trochlea

$\square$ Ulnar groove

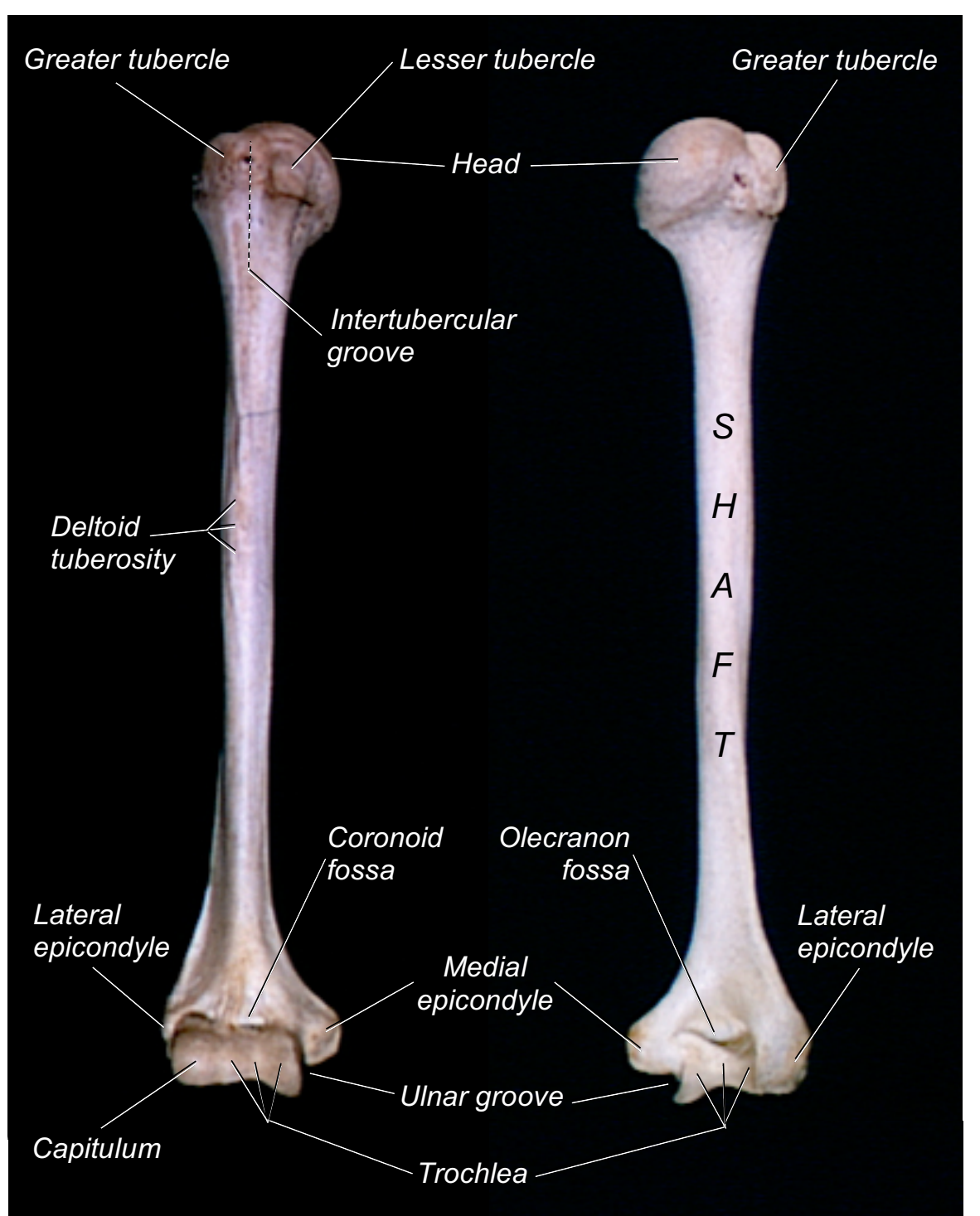

Figure 3.6. Right Humerus, Anterior View

Figure 3.7. Right Humerus, Posterior View 


\section{Clinical Application: "Funny Bone"}

The ulnar nerve passes through the ulnar groove on the humerus. You can feel this on the medial side of your elbow. Although the ulnar groove protects the ulnar nerve, it also provides a bone-hard surface to compress the ulnar nerve against. Compression of the ulnar nerve at the ulnar groove results in a tingling sensation in the $4^{\text {th }}$ and $5^{\text {th }}$ fingers and commonly referred to as "hitting your funny bone."

\section{Ligaments of the shoulder}

$\square$ Glenohumeral ligaments*

\section{Radius bone}

$\square$ Head

$\square$ Radial tuberosity

$\square$ Styloid process

$\square$ Ulnar notch

\section{Ulna bone}

$\square$ Head

$\square$ Olecranon process

Coronoid process

You've now learned a conoid tubercle on the clavicle, a coracoid process on the scapula, a coronoid process on the mandible AND ulna. MAKE SURE TO SPELL THESE VERY SIMILAR TERMS CORRECTLY! $\square$ Trochlear notch

$\square$ Radial notch

$\square$ Ulnar tuberosity

$\square$ Styloid process

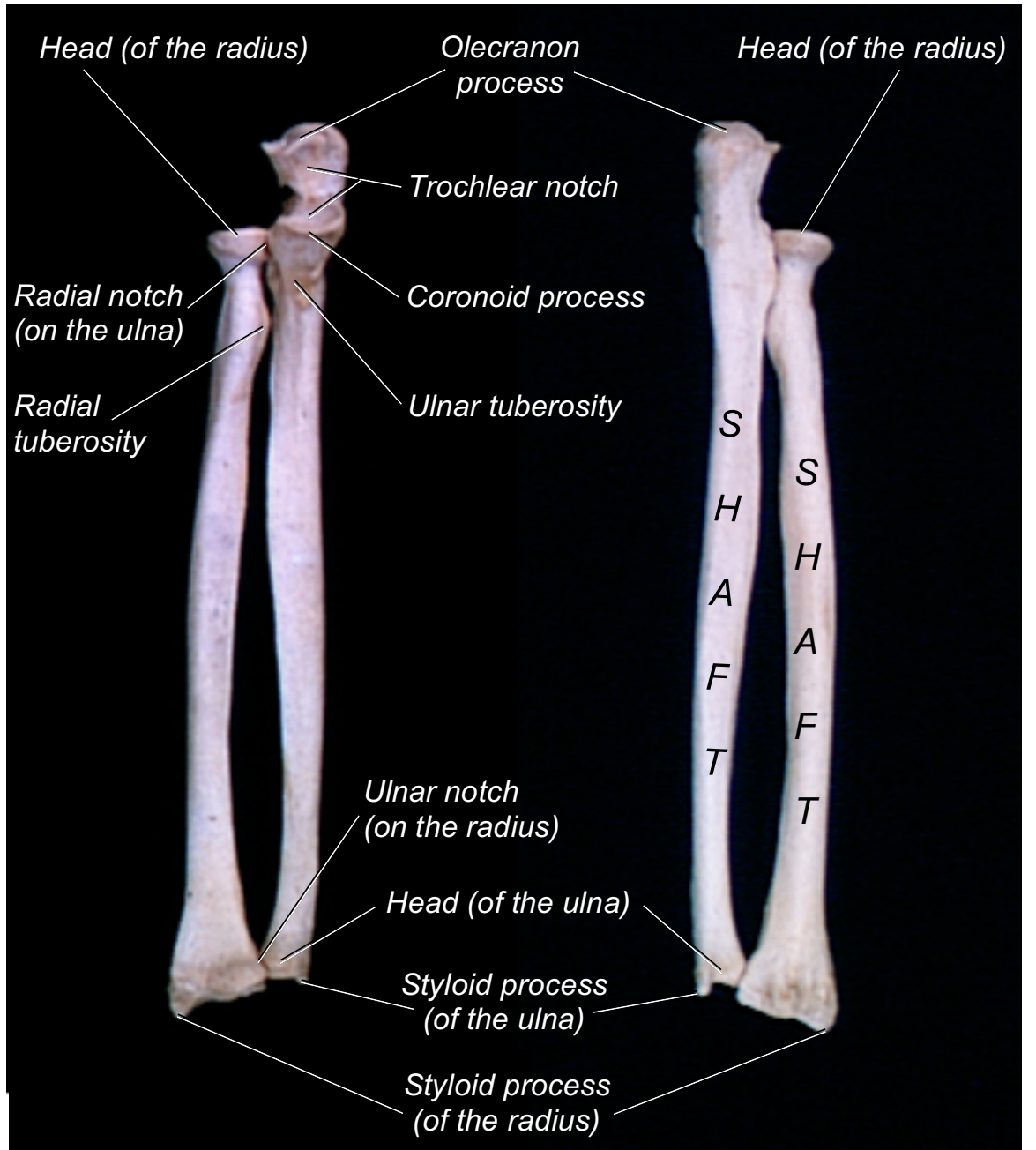

Figure 3.8. Right Radius and Figure 3.9. Right Radius and Ulna, Anterior View
Ulna, Posterior View 
Many articulations are created by the radius and ulna, some between the two bones and others separately with the humerus.

The head of the radius articulates with the capitulum to create the rotating action of the forearm and hand. The action of rotating the forearm and hand so that the palm of the hand is facing posteriorly is called pronation; the action of rotating the forearm and hand so that the palm of the hand is facing anteriorly (as in normal anatomical position) is called supination. Compare these actions to the related anatomical positions of prone and supine that you've already learned.

In order for the radius to rotate, it must also articulate with the ulna next to it. It does this in two places. Aside from articulating with the capitulum, the head of the radius also articulates with the radial notch (on the ulna) as it rotates. Similarly, at the distal end of the radius and ulna, the head of the ulna articulates with the ulnar notch (on the radius).

Remember that anatomical terms are usually named for their purpose, not necessarily what bone they are on (think back to the temporal and zygomatic processes); the radial notch is FOR the radius, so it is located ON the ulna, and the ulnar notch is FOR the ulna, so it is located ON the radius.

As for the ulna, the trochlear notch articulates with the trochlea on the humerus to allow a strong and stable flexing action at the elbow. Further, the coronoid and olecranon processes (on the ulna) articulate into the coronoid and olecranon fossae (on the humerus), respectively.

Finally, the distal-most ends of the radius and ulna will articulate with carpal bones to form the wrist joint.

\section{Ligaments of the elbow and forearm}

$\square$ Ulnar collateral ligament*

Radial collateral ligament*

Collateral $=$ on the side.

The ulnar collateral ligament connects the ulna and the humerus on the ulnar side (medial side) of the elbow joint. The radial collateral ligament connects the radius and the humerus on the radial side (lateral side) of the elbow joint.

\section{Annular ligament*}

\section{$\underline{\text { Annular }}=$ circular; ring-shaped.}

The annular ligament surrounds the head of the radius, stabilizing it as it articulates with the capitulum and radial notch to supinate and pronate the forearm and hand.

\section{Interosseous membrane*}

\section{Carpal bones}

Proximal row, listed lateral to medial

Scaphoid

$\square$ Lunate

Triquetrum

Pisiform

Distal row, listed lateral to medial

Trapezium

Trapezoid

Capitate

Hamate 


\section{Ligaments of the wrist}

$\square$ Radial carpal collateral ligament*

$\square$ Ulnar carpal collateral ligament*

These two ligaments connect the styloid processes of the respective forearm bones to the carpal bones, thus supporting and stabilizing the medial and lateral sides of the wrist.

\section{Metacarpal bones}

Numbered lateral to medial

$\square 1^{\text {st }}, 2^{\text {nd }}, 3^{\text {rd }}, 4^{\text {th }}$, and $5^{\text {th }}$ metacarpals

\section{Phalange bones}

Numbered lateral to medial and named proximal to distal.

$\square 1^{\text {st }}, 2^{\text {nd }}, 3^{\text {rd }}, 4^{\text {th }}$, and $5^{\text {th }}$ proximal phalanges

$\square 2^{\text {nd }}, 3^{\text {rd }}, 4^{\text {th }}$, and $5^{\text {th }}$ middle phalanges

Notice how there is no middle phalanx in your $1^{\text {st }}$ digit!

$\square 1^{\text {st }}, 2^{\text {nd }}, 3^{\text {rd }}, 4^{\text {th }}$, and $5^{\text {th }}$ distal phalanges

\section{Understanding Correct Terminology: Phalanx vs. Phalange.}

Digit $=$ one of 5 typical jointed appendages from your hand. Phalange $=$ one single digit.

$\underline{\text { Phalanx }}=$ one single digit bone.

$\underline{\text { Phalanges }}=$ multiple digit bones OR multiple digits.

Example: The $4^{\text {th }}$ phalange is made up of three phalanges: the $4^{\text {th }}$ proximal phalanx, the $4^{\text {TH }}$ middle phalanx, and the $4^{\text {th }}$ distal phalanx.

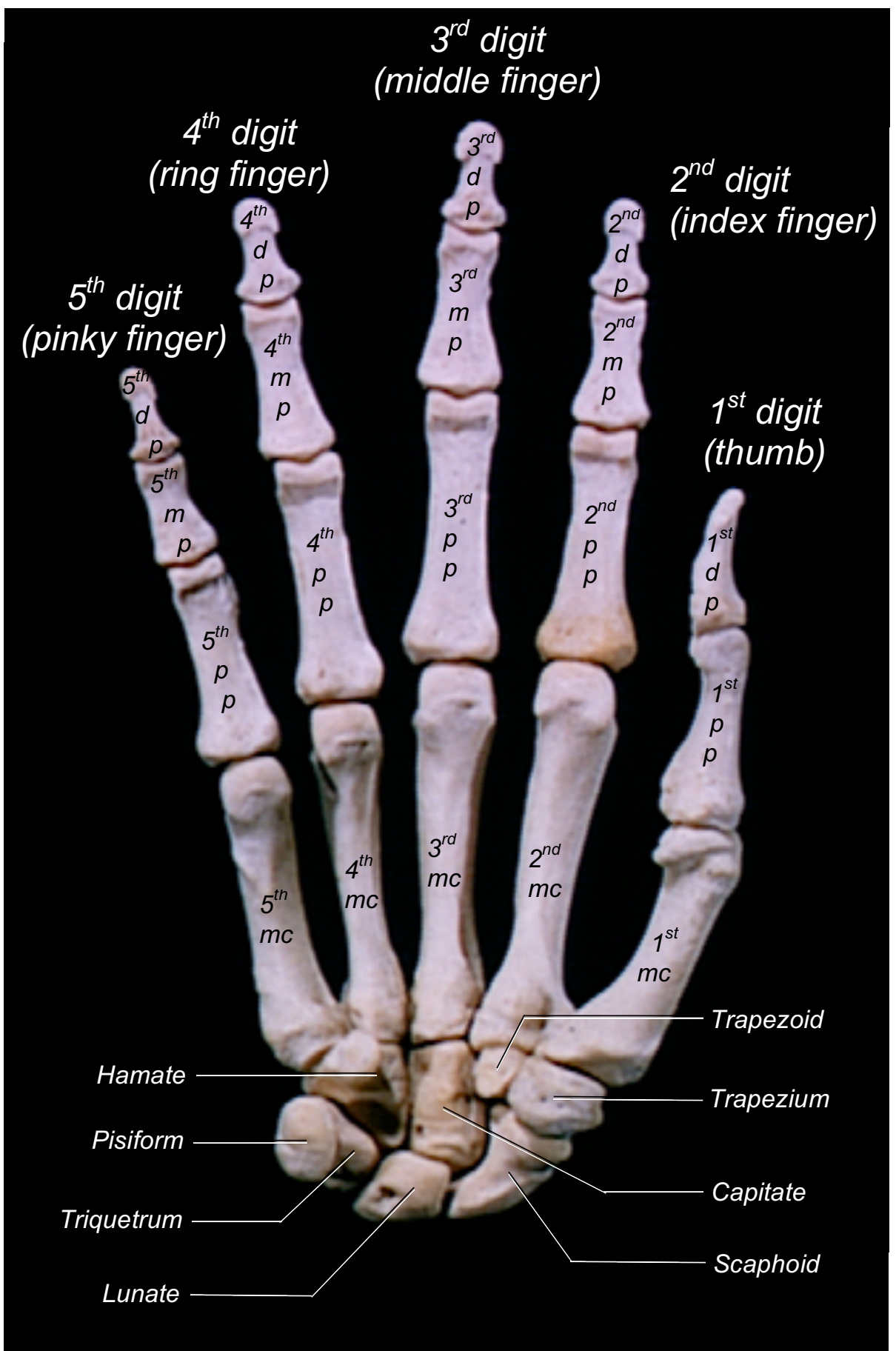

Figure 3.10. Right Wrist and Hand Bones, Anterior View 
1) What region of the upper limb is found distal to the elbow? (LO6)

2) What is the name of the shallow depression found below the spine of the scapula? (hint: use the terminology you know!) (LO6)

3) True or False: the ulna allows for the forearm and hand to supinate and pronate. (LO6, LO7)

4) What ligament supports and stabilizes the medial side of the wrist? (LO7)

5) How many bones make up one entire pectoral girdle and upper limb? (LO7)

6) What bone is located proximal to the $1^{\text {st }}$ distal phalanx? (LO7)

7) What bone is located proximal to the $2^{\text {nd }}$ distal phalanx? (LO7)

8) Using the terminology and the structures you know, describe where you would find the intertubercular groove. (LO6)
9) List the bone(s) that each of the following structures can be found on. (LO7)

a. Conoid tubercle:

b. Coracoid process:

c. Coronoid fossa:

10)What does the term collateral mean? (LO6)

11)Identify the structure that articulates with each of the structures listed below. (LO7)

a. Glenoid cavity -

b. Head of ulna -

c. Trochlear notch -

d. Clavicular notch -

e. Olecranon fossa -

f. Capitulum -

g. Radial notch -

h. Coronoid process -

i. Acromion -

12)Choose the correct answer choices to complete this statement: the head of the radius is located closer to the ( elbow / wrist ), but the head of the ulna is located closer to the ( elbow / wrist ). (LO7) 


\section{Lab 4: Appendicular Skeleton (Pelvic Girdle \& Lower Limb)}

Instructions: Learn the listed terms using the listed learning objectives, labeled images, lab specimens, and other resources.

LO8: Describe the terminology used to identify structures of the appendicular skeleton (pelvic girdle and lower limb).

LO9: Identify the bones and associated structures of the appendicular skeleton (pelvic girdle and lower limb).

\section{Hip bone}

One hip bone consists of three separate, fused bones: the ilium, ischium, and pubis. Some of the listed structures contain these bone names, and therefore, will further help you locate the borders of these bones. Use the internet or another resource to learn where these three bones are located on each hip bone.

Iliac crest

$\square$ lliac fossa

$\square$ Anterior superior iliac spine

$\square$ Anterior inferior iliac spine

$\square$ Posterior superior iliac spine

Posterior inferior iliac spine

Don't be intimidated by these long terms (or others); break them down with terminology and they will become easy to remember. First, all of them are spines on the ilium (from "iliac spine"). Next, determine if the spine you are looking at is on the anterior or posterior side of the hip bone. Once you determine this, then determine if it is the superior or inferior of the two spines on that side. Then put your terms together, and you'll have it! $\square$ Gluteal surface

Greater sciatic notch

$$
\begin{aligned}
& \underline{\text { Sciati }}=\text { hip } \\
& \square \text { Lesser sciatic notch } \\
& \square \text { Ischial spine } \\
& \square \text { Ischial tuberosity } \\
& \square \text { Acetabulum } \\
& \square \text { Obturator foramen } \\
& \square \text { Superior pubic ramus } \\
& \frac{\text { Ramus }=\text { branch }}{\square \text { Inferior pubic ramus }} \\
& \square \text { Pubic symphysis }
\end{aligned}
$$

$\underline{\text { Symphasis }}$ = joint which bony surfaces are firmly united by a plate of fibrocartilage.

\section{Auricular surface}

Much like the scapulae and clavicles form two pectoral girdles, the hip bones, sacrum, and coccyx form one pelvic girdle. The pelvic girdle functions to connect and support the thighs to each side of the trunk of the body. The rest of the bones in the lower extremity form joints and regions of the lower limb (i.e. hip, thigh, knee, leg, ankle, and foot). 


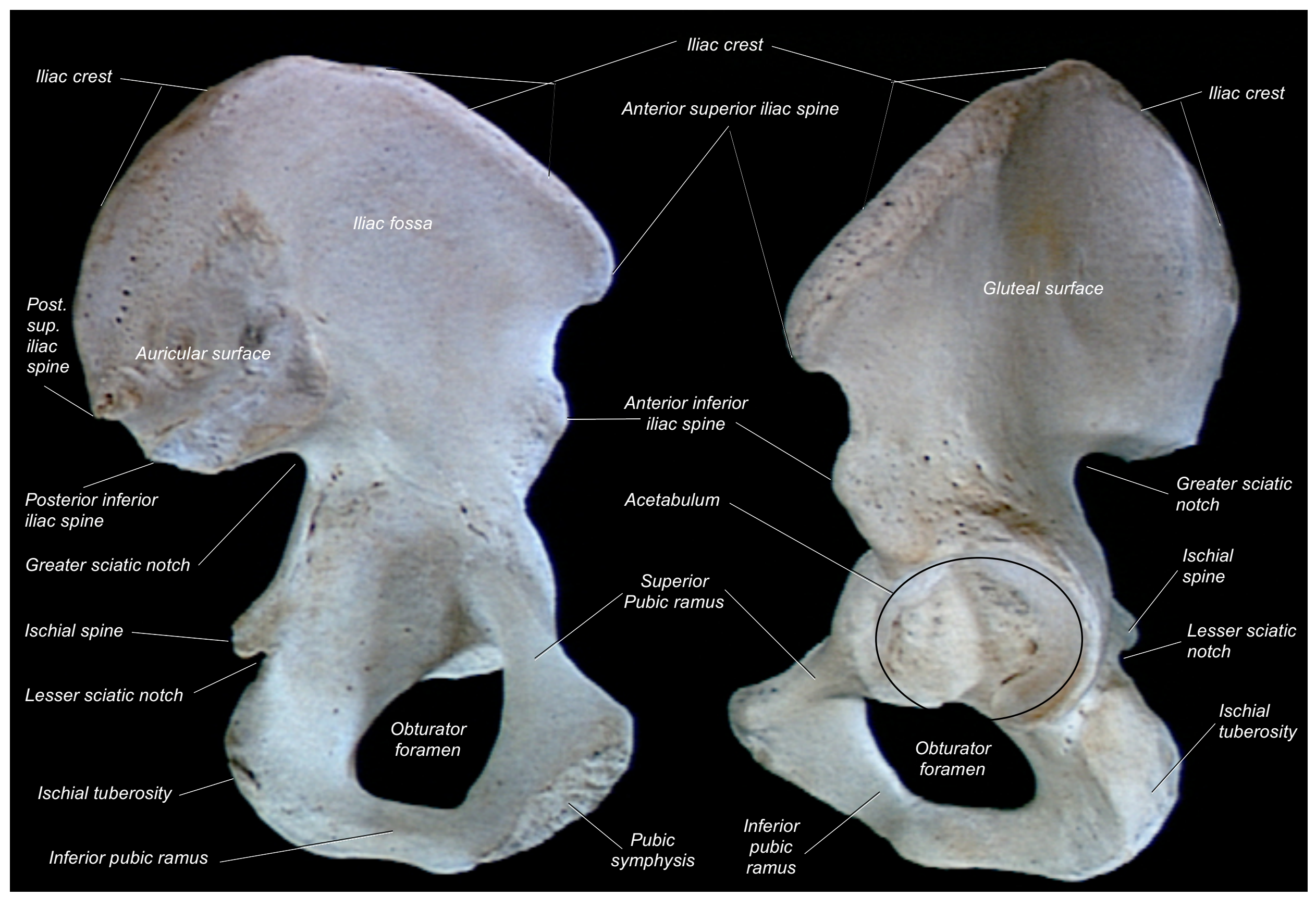

Figure 4.1. Left Hip Bone, Medial View

Figure 4.2. Left Hip Bone, Lateral View 


\section{Femur bone}

$\square$ Head

The head of the femur articulates with the acetabulum.

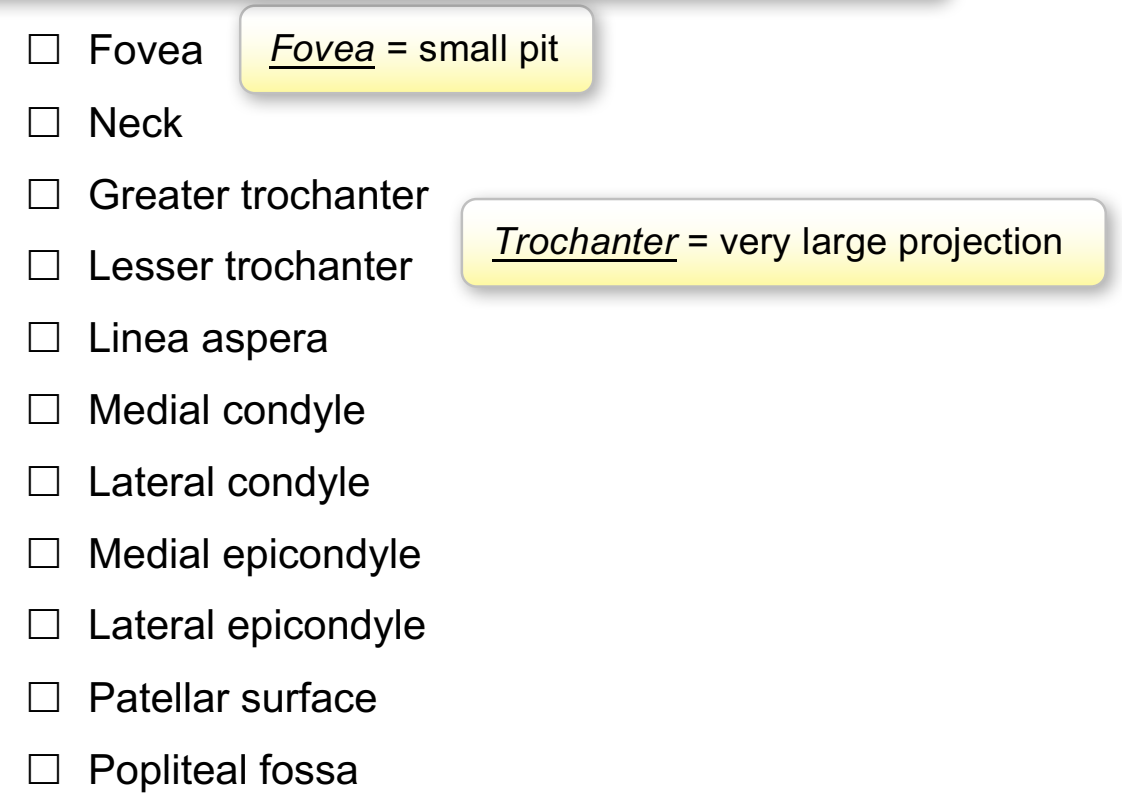

\section{Ligaments of the hip}

$\square$ Iliofemoral ligament* $^{*}$

The iliofemoral ligament is the strongest ligament in the body!

$\square$ Ischiofemoral ligament* $^{*}$

$\square$ Pubofemoral ligament*

$\square$ Capital ligament (or Ligament of head of femur)*

The capital ligament attaches to the fovea and to the inner surface of the acetabulum.

$\square$ Inguinal ligament*

The inguinal ligament attaches to the anterior superior iliac spine and the superomedial part of the superior pubic ramus.

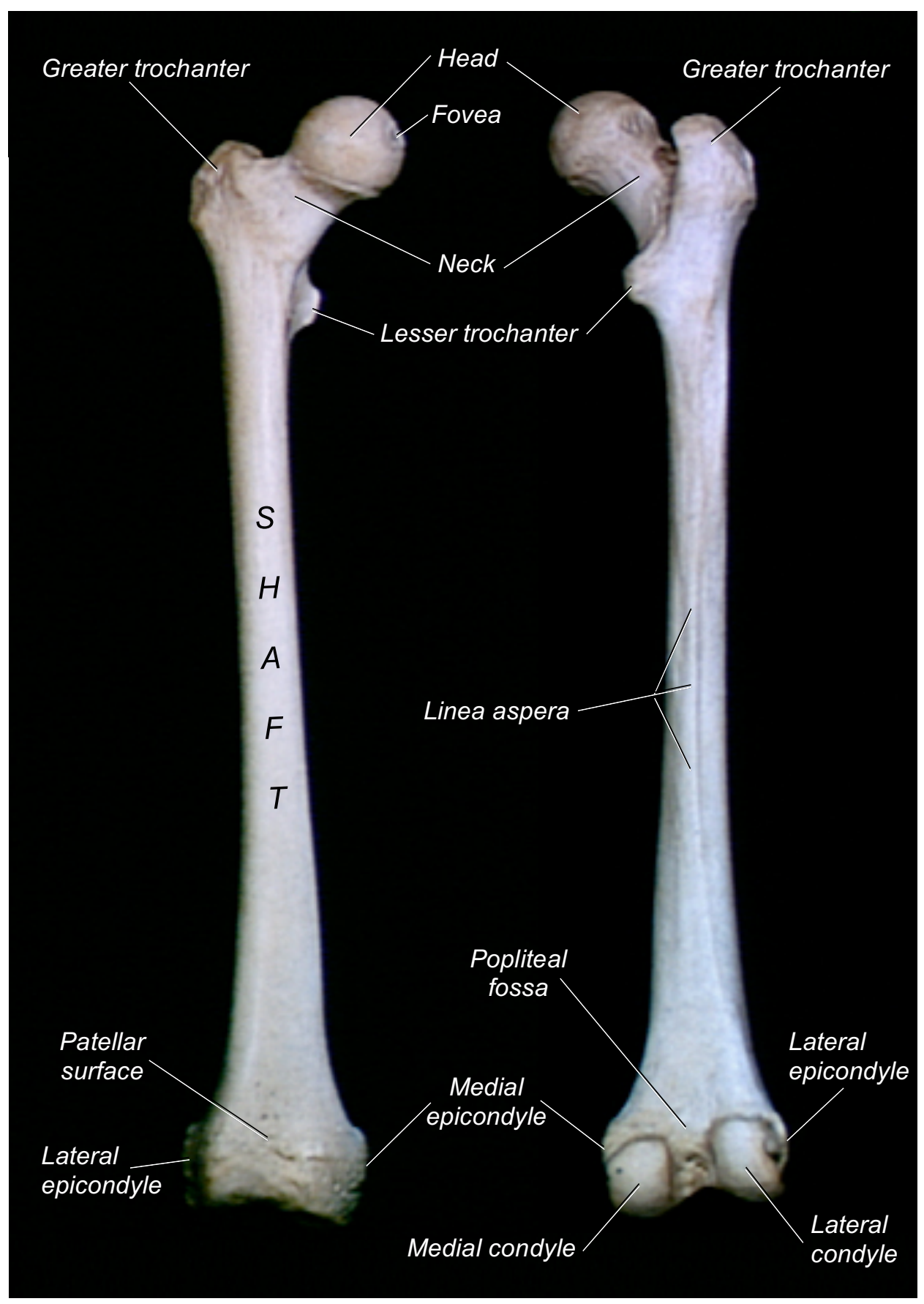

Figure 4.3. Femur,

Figure 4.4. Femur, Anterior View
Posterior View 


\section{Patella bone}

$\square$ Base

$\square$ Apex

$\square$ Medial articular surface

$\square$ Lateral articular surface

The lateral articular surface is typically larger than the medial articular surface. These surfaces articulate with the patellar surface on the femur.

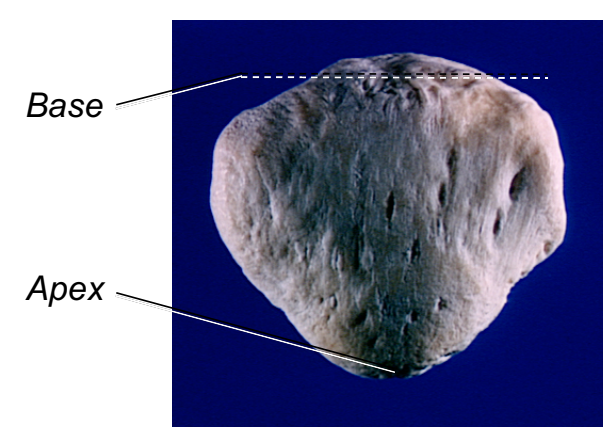

Figure 4.5. Patella, Anterior View

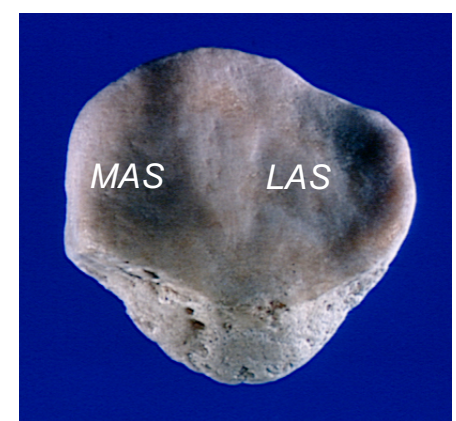

Figure 4.6. Patella, Posterior View

\section{Fibula bone}

$\square$ Head of fibula

$\square$ Lateral malleolus

$\square$ Malleolar fossa

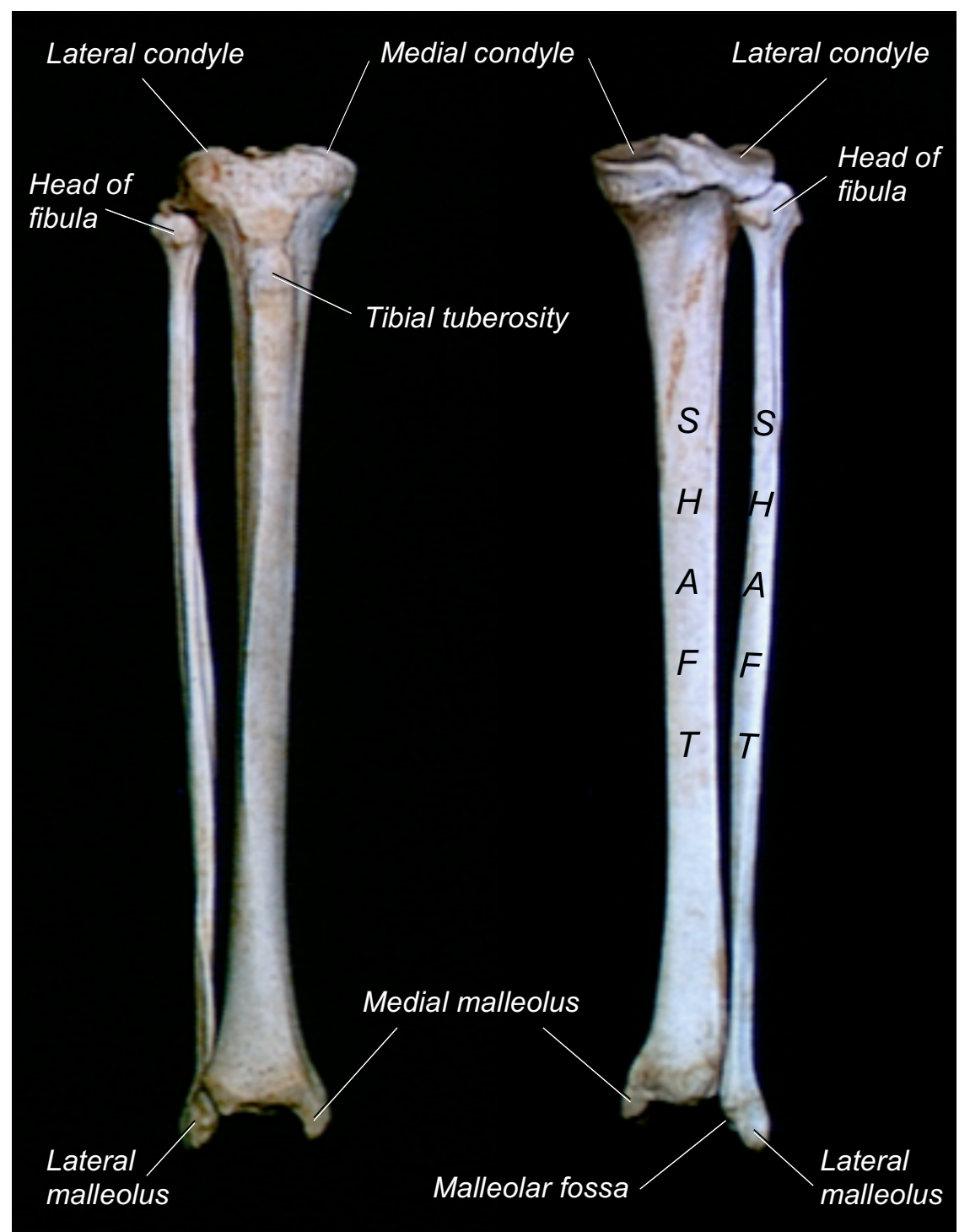

Figure 4.7. Tibia and Fibula, Anterior View

Figure 4.8. Tibia and Fibula, Posterior View 


\section{Ligaments and structures of the knee and leg}

$\square$ Anterior cruciate ligament

$\square$ Posterior cruciate ligament

$\underline{\text { Cruciate }}=$ cross-shaped

$\square$ Medial collateral ligament (or Tibial collateral ligament)

$\square$ Lateral collateral ligament (or Fibular collateral ligament)

$\square$ Patellar ligament

$\square$ Medial meniscus

$\square$ Lateral meniscus

Meniscus $=$ crescent-shaped cartilaginous disk for padding.

$\square$ Interosseous membrane*

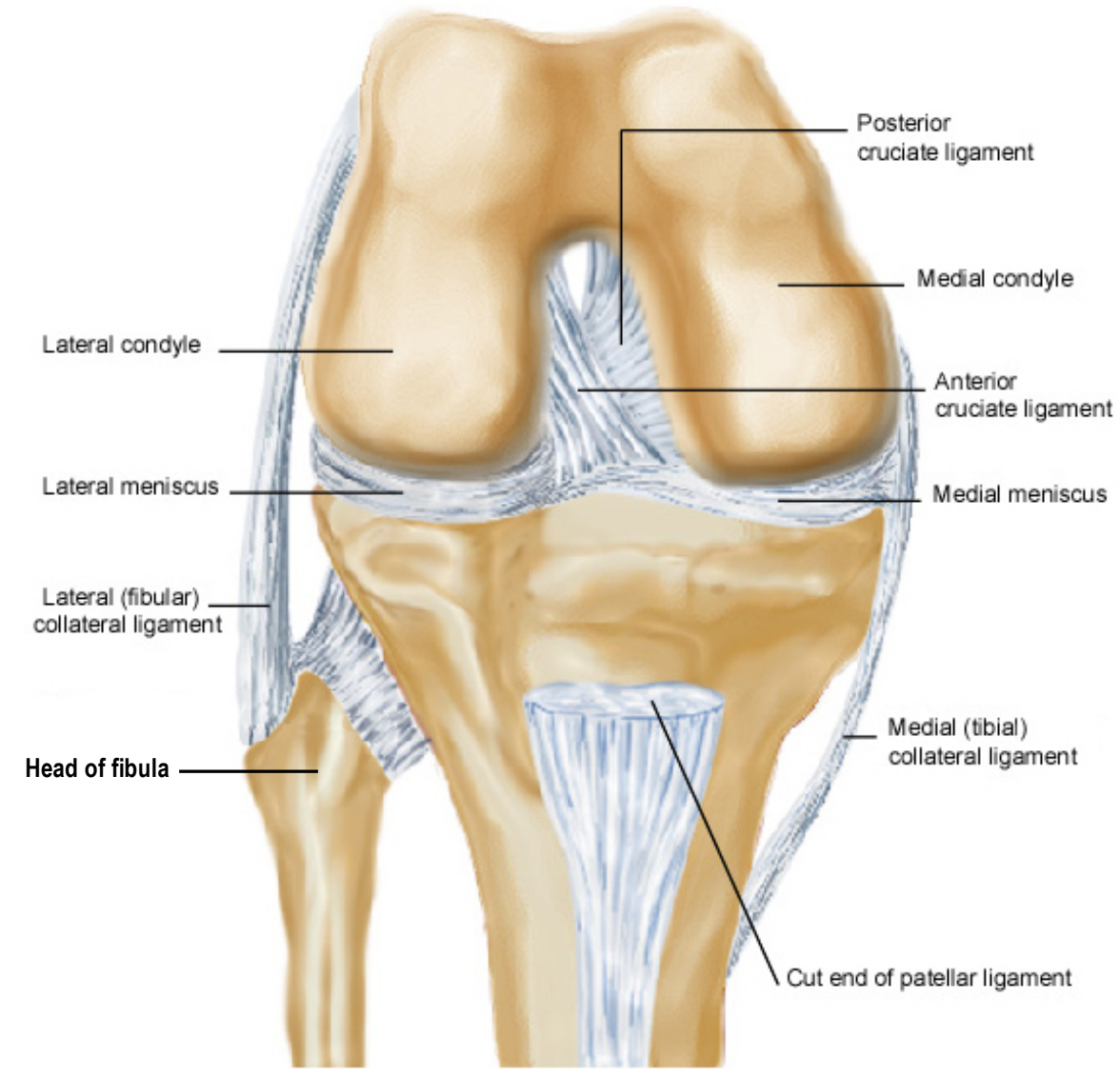

Figure 4.9. Ligaments and Structures of the Knee

\section{Clinical Application: Total Knee Replacement}

Over time, the articular surfaces in the knee may damage from overuse, injury, or just normal wear from bearing the weight of the body over time. Damage to these articular surfaces can cause severe pain. When the damage is severe, surgery may be required to replace the damaged surfaces with artificial surfaces made of plastic and/or metal. In a total knee replacement, all of the articular surfaces and structures of the knee are replaced; the medial and lateral condylar surfaces on the femur and tibia are removed and replaced with a metal plate, and the medial and lateral menisci are removed and replaced with a plastic plate. In a typical total knee replacement, the anterior and posterior cruciate ligaments are removed. Because the shape of the artificial plates add stability to the knee, these ligaments are no longer needed. The medial and lateral collateral ligaments remain in place to continue stabilizing the sides of the knee. The plates are secured with screws and/or adhesive, and depending on the physician's judgment regarding the damage and condition of the knee, the patella is replaced with an artificial patella made of plastic.

\section{Tarsal bones}

$\square$ Talus

$\square$ Navicular $\quad \underline{\text { Navicular }}=$ boat-shaped

$\square$ Calcaneus

Cuboid

Lateral cuneiform

Intermediate cuneiform Cuneiform $=$ wedge-shaped

Medial cuneiform 


\section{Ligaments of the ankle}

$\square$ Deltoid ligament*

$\square$ Calcaneofibular ligament $^{\star}$

\section{Metatarsal bones}

Numbered medial to lateral

$\square 1^{\text {st }}, 2^{\text {nd }}, 3^{\text {rd }}, 4^{\text {th }}$, and $5^{\text {th }}$ metatarsals

\section{Phalange bones}

Numbered medial to lateral and named proximal to distal.

$\square 1^{\text {st }}, 2^{\text {nd }}, 3^{\text {rd }}, 4^{\text {th }}$, and $5^{\text {th }}$ proximal phalanges

$\square 2^{\text {nd }}, 3^{\text {rd }}, 4^{\text {th }}$, and $5^{\text {th }}$ middle phalanges

Notice how there is no middle phalanx in your $1^{\text {st }}$ digit!

\section{$1^{\text {st }}, 2^{\text {nd }}, 3^{\text {rd }}, 4^{\text {th }}$, and $5^{\text {th }}$ distal phalanges}

Remember to use correct phalanx vs. phalange terminology!

The "Phalanx vs. Phalange" terminology you learned for the hand applies to the foot as well. Below is the information if you need to review it. Make sure you are using the terms correctly!

Digit $=$ one of 5 typical jointed appendages from your foot.

Phalange $=$ one single digit.

Phalanx $=$ one single digit bone.

$\underline{\text { Phalanges }}=$ multiple digit bones OR multiple digits

Example: The $4^{\text {th }}$ phalange is made up of three phalanges: the $4^{\text {th }}$ proximal phalanx, the $4^{\text {TH }}$ middle phalanx, and the $4^{\text {th }}$ distal phalanx.

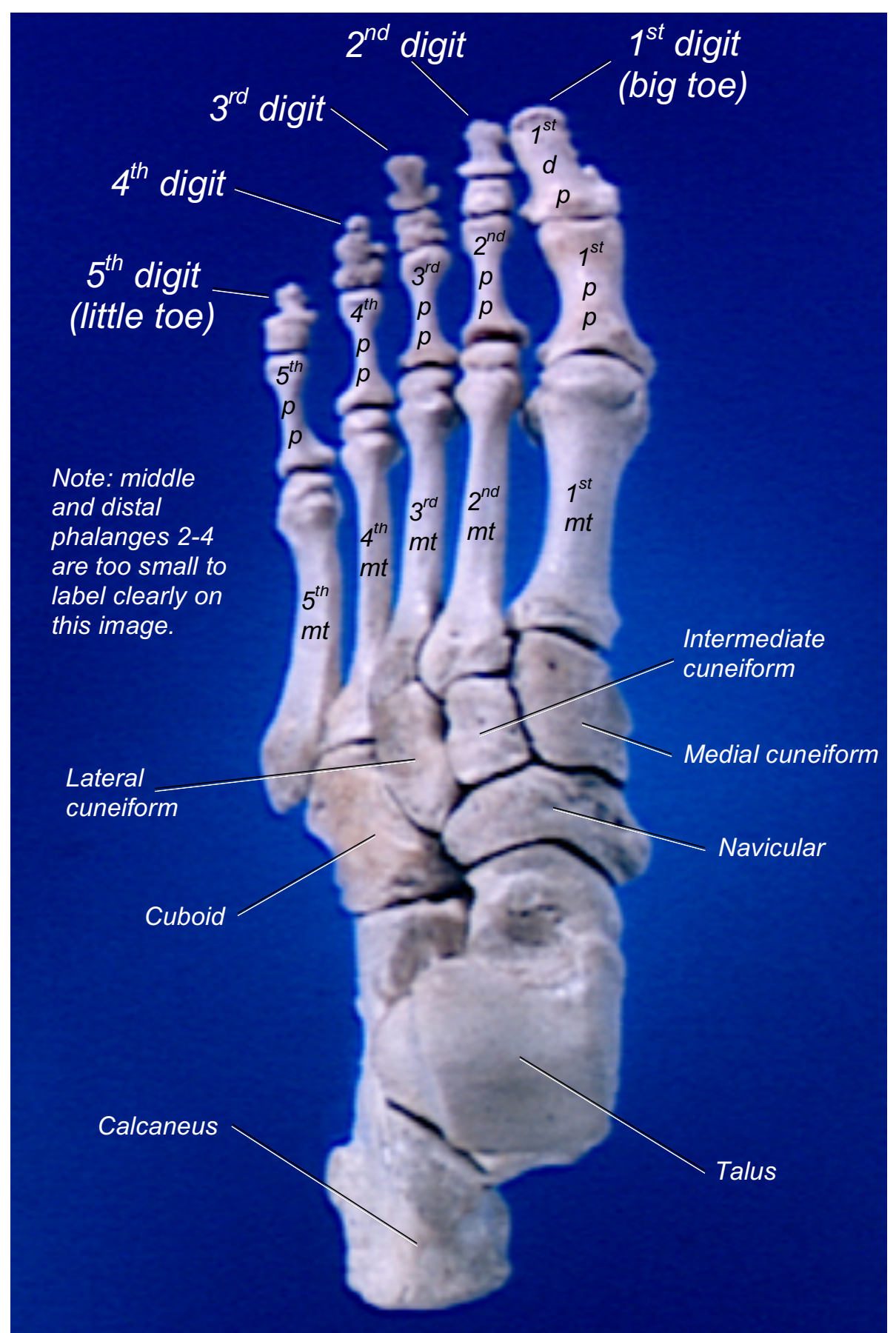

Figure 4.10. Left Ankle and Foot Bones, Superior View 
1) True or False: the number of carpal bones is the same as the number of tarsal bones. (LO9)

2) What two specific bones do each of the following ligaments connect? (hint: break down the terminology) (LO8)

a. lliofemoral ligament:

b. Ischiofemoral ligament:

c. Pubofemoral ligament:

3) Using the terms MEDIAL and LATERAL, fill in the following blanks to make the statements correct. (LO8, LO9)

Digits in the hand are numbered

to

Digits in the foot are numbered

to

4) What region of the lower limb is found proximal to the ankle? (LO8)
5) What does the term fovea mean? (LO8)

6) Identify the structure that articulates with each of the structures listed below. (LO9)

a. Head of femur:

b. Medial condyle of tibia:

c. Lateral condyle of the tibia:

d. Auricular surface of ilium:

e. Patellar surface:

7) What does the term ramus mean? (LO8)

8) How many bones make up one entire lower limb? (LO9) 


\section{Lab 5: Axial Muscles}

Instructions: Learn the listed terms using the listed learning objectives, labeled images, lab specimens, and other resources.

LO10: Describe the terminology used to identify structures of the axial muscles.

L011: Identify the listed axial muscles.

L012. Identify the listed proximal attachments, distal attachments, and actions of the axial muscles.

\section{Important Muscle Terminology:}

As you learn muscles, you should understand these terms and use them correctly:

Innervation: the process of a nerve stimulating a muscle to contract.

Contraction: the process of a muscle becoming shorter and tighter; caused by innervation.

Relaxation: the process of a muscle becoming longer and looser; caused by lack of innervation.

Action: a movement caused by muscle contraction.

Proximal Attachment (PA): the location at which the proximal end of the muscle is attached; also referred to as origin. This attachment typically acts as an anchor to create movement at the distal end of a muscle during contraction.

Distal Attachment (DA): the location at which the distal end of the muscle is attached; also referred to as insertion. This end of a muscle typically moves most during contraction.

Tendon: strong fibrous tissue that attaches muscle to bone. Agonist: a muscle that produces a desired action.
Antagonist: a term used to describe a muscle that causes an opposite (inhibitory) action compared to another muscle.

Synergist: a term used to describe a muscle that causes a same action compared to another muscle.

Uniarticulate: a term used to describe a muscle that spans (crosses) one joint only.

Biarticulate: a term used to describe a muscle that spans (crosses) two joints only.

Multiarticulate: a term used to describe a muscle that spans (crosses) three or more joints.

\section{Muscles of facial expression}

As you practice identifying muscles of facial expression (and others), looking at the direction of the muscle fibers and following them to their places of attachment can help you identify the muscles. Also, use the terminology to help you find the muscles and learn their actions. For example, the frontalis muscle lies over the frontal bone, and the orbicularis oculi muscle is a circular (from orbicular) muscle around the eye (from oculi). This will help you learn the material faster, understand it better, and remember it longer.

\section{Frontalis}

- Action: raises eyebrows and wrinkles forehead

\section{Nasalis}

- Lifts/flares the nostrils

Orbicularis oculi

○ Action: closes the eyelids 
$\square$ Orbicularis oris

- Action: compresses and protrudes the lips

Zygomaticus

- Action: pulls angle of mouth superiorly and laterally

Levator labii superioris

- Action: elevates the upper lip

$\square$ Depressor labii inferioris

- Action: depresses the lower lip

$\square$ Levator anguli oris

○ Action: elevates the angle of the mouth

$\square$ Depressor anguli oris

- Action: depresses the angle of the mouth

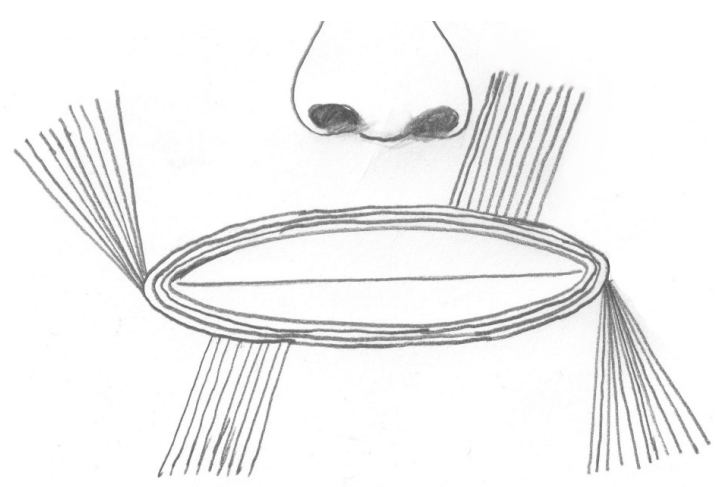

\section{Important Terms:}

Orbicular $=$

$\underline{\text { Oro }}=$

Levator $=$

Depressor $=$

$\underline{L a b i}=$

Anguli $=$

$\underline{\text { Superior }}=$

Inferior $=$

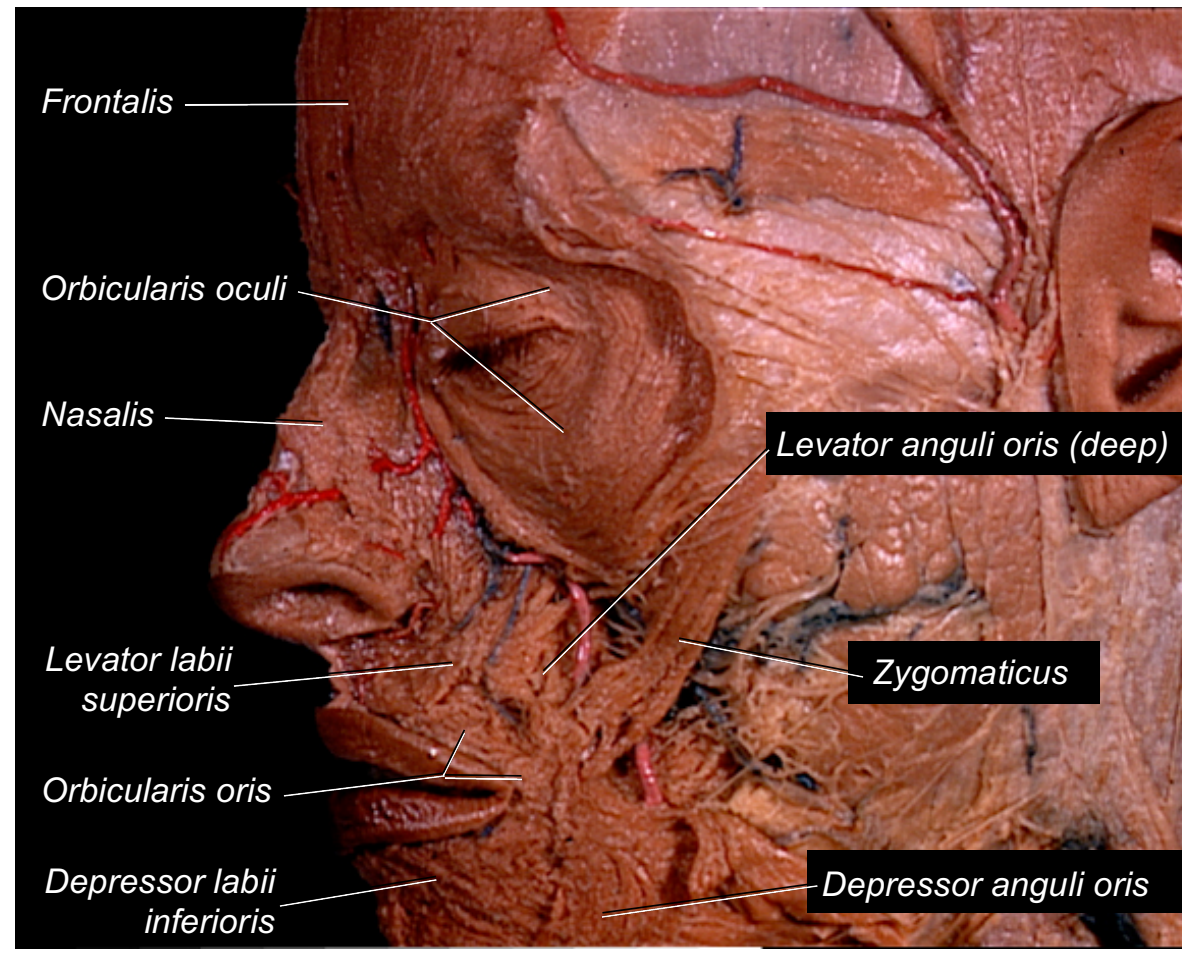

Figure 5.2. Muscles of Facial Expression, Left Lateral View

\section{Clinical Application: The Buccinator Muscle}

The buccinator muscle is a deep muscle of the cheek. Its primary role is to assist in mastication (chewing) by keeping food between upper and lower teeth. If you've ever bitten your cheek bad enough, you may have bitten down on your buccinator (although under most instances, you only bite down on the epithelium and superficial fascia that lines the innermost surfaces of your cheeks). The buccinator muscle also assists infants with suckling and adults with whistling.

Figure 5.1. Simplified Diagram to Help Identify the Muscles that Act on the Mouth and Learn Their Actions 


\section{Muscles of mastication}

$\underline{\text { Mastication }}=$ chewing

$\square$ Temporalis

- PA: lateral surface of cranium

- DA: coronoid process of the mandible

- Action: elevates, retracts, and laterally moves the mandible

$\square$ Masseter*

○ PA: zygomatic arch

retract $=$ posterior movement protract $=$ anterior movement

- DA: mandible

- Action: elevates and protracts the mandible

$\square$ Medial Pterygoid* $^{*}$

$\square$ Lateral Pterygoid*

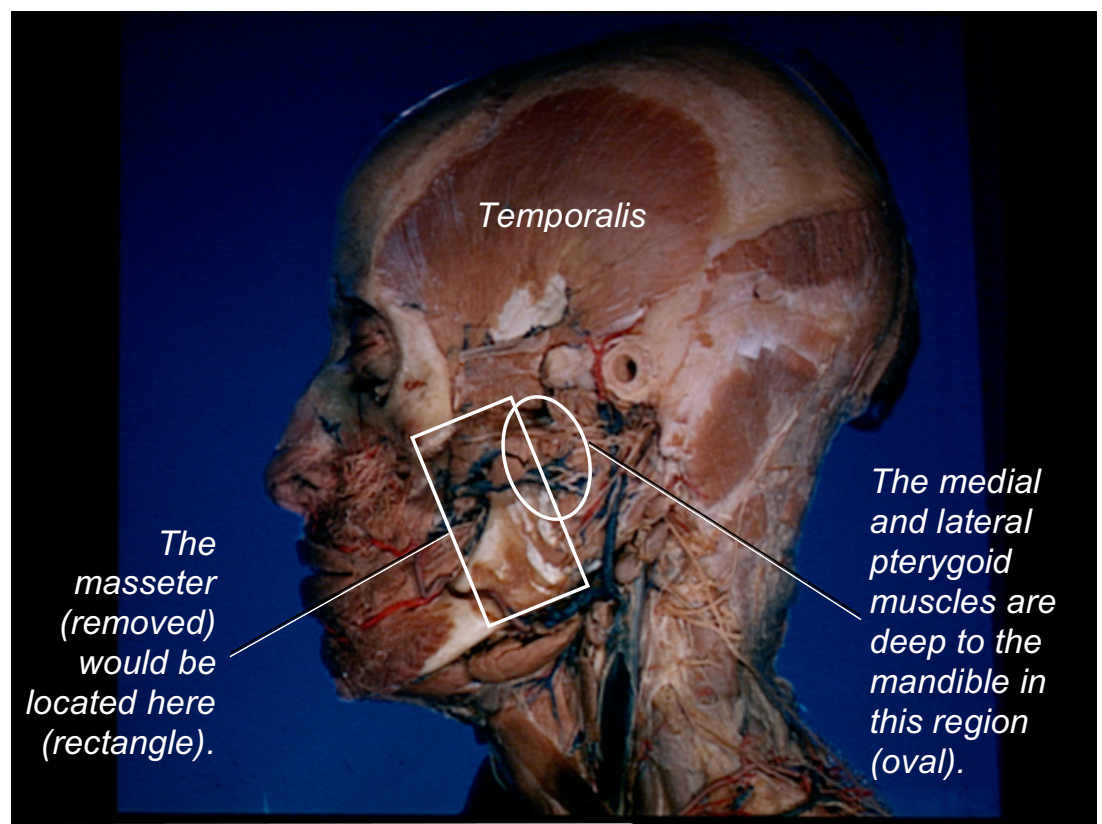

Figure 5.3. Muscles of Mastication, Left Lateral View

\section{Muscles of the neck}

$\square$ Sternocleidomastoid

$\underline{\text { Sterno }}=$ pertaining to the sternum; $\underline{\text { cleido }}=$ pertaining to the clavicle $; \underline{\text { mastoid }}=$ pertaining to the mastoid process.

- PA: sternum and clavicle

○ DA: mastoid process

- Action: tilts head to same side; rotates head to opposite side

Splenius capitis

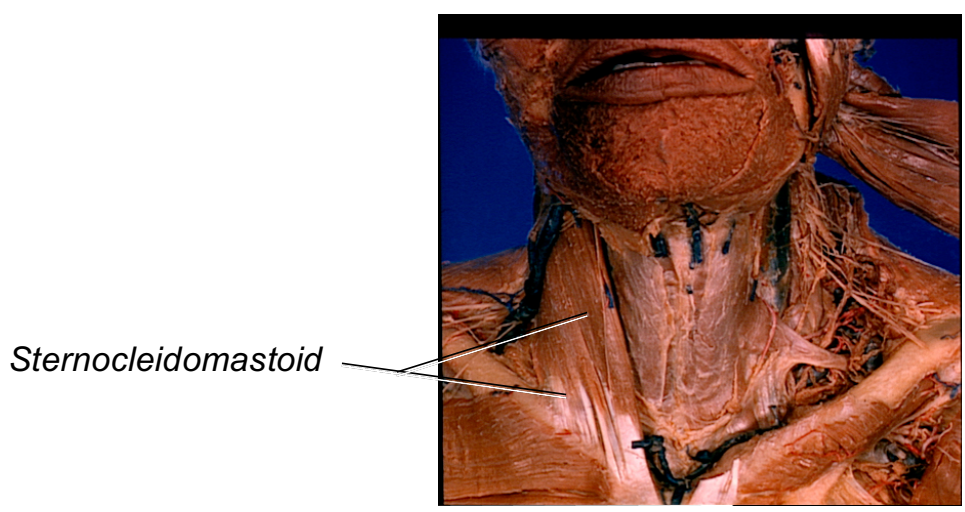

Figure 5.4. Muscles of the Neck, Anterior View

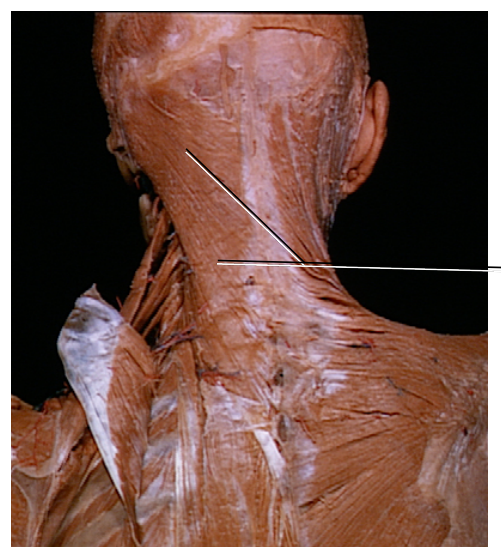

Figure 5.5. Muscles of the Neck, Posterior View 


\section{Muscles of the back}

$\square$ Erector spinae

The erector spinae muscle group consists of three paired muscles (listed from lateral to medial):
$\square$ Iliocostalis
$\square$ Longissimus
$\square$ Spinalis

The erector spinae muscles extend the vertebral column.

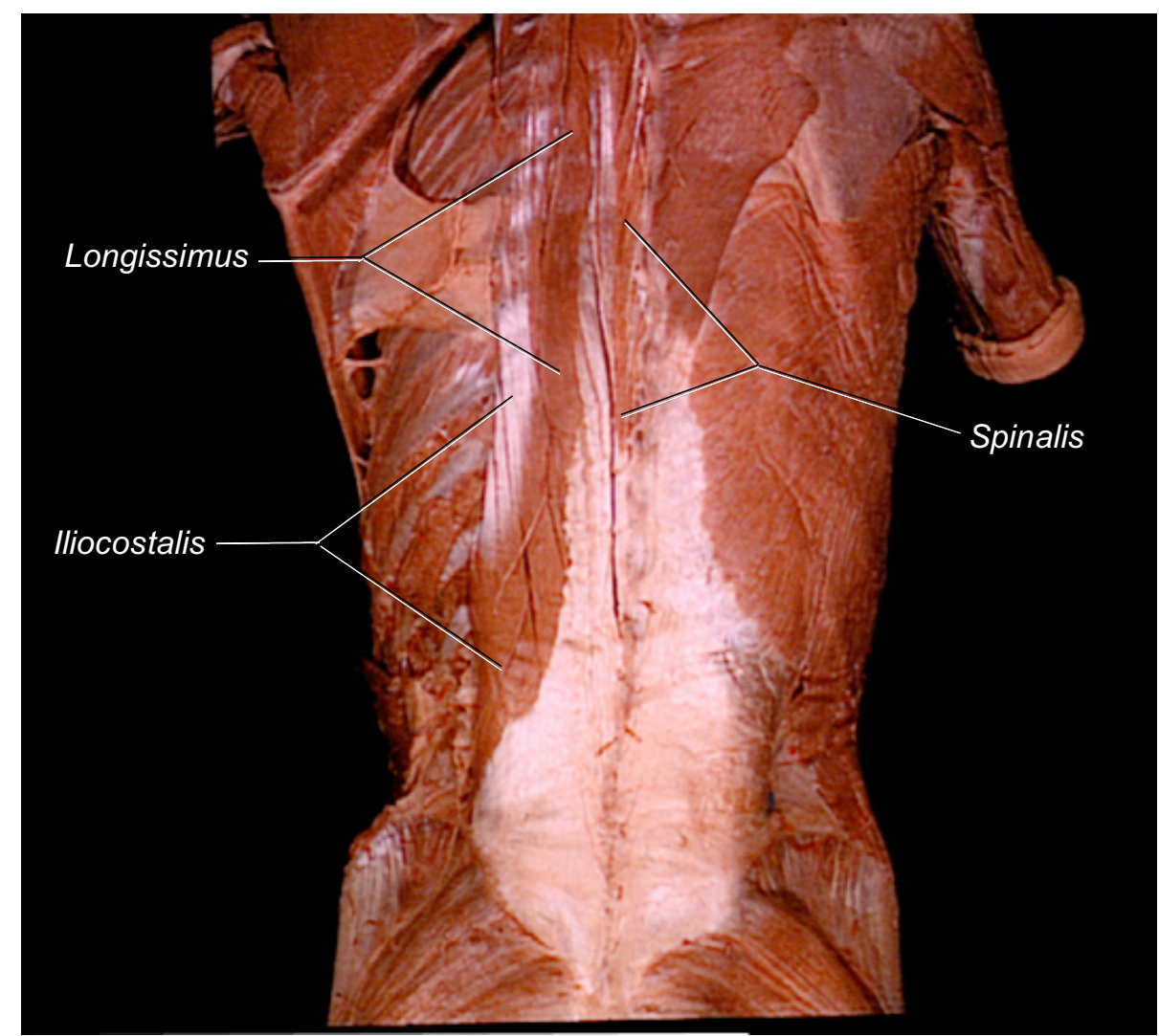

Figure 5.6. Muscles of the Back, Posterior View

\section{Muscles of the thorax}

$\square$ External intercostals

$\square$ Internal intercostals

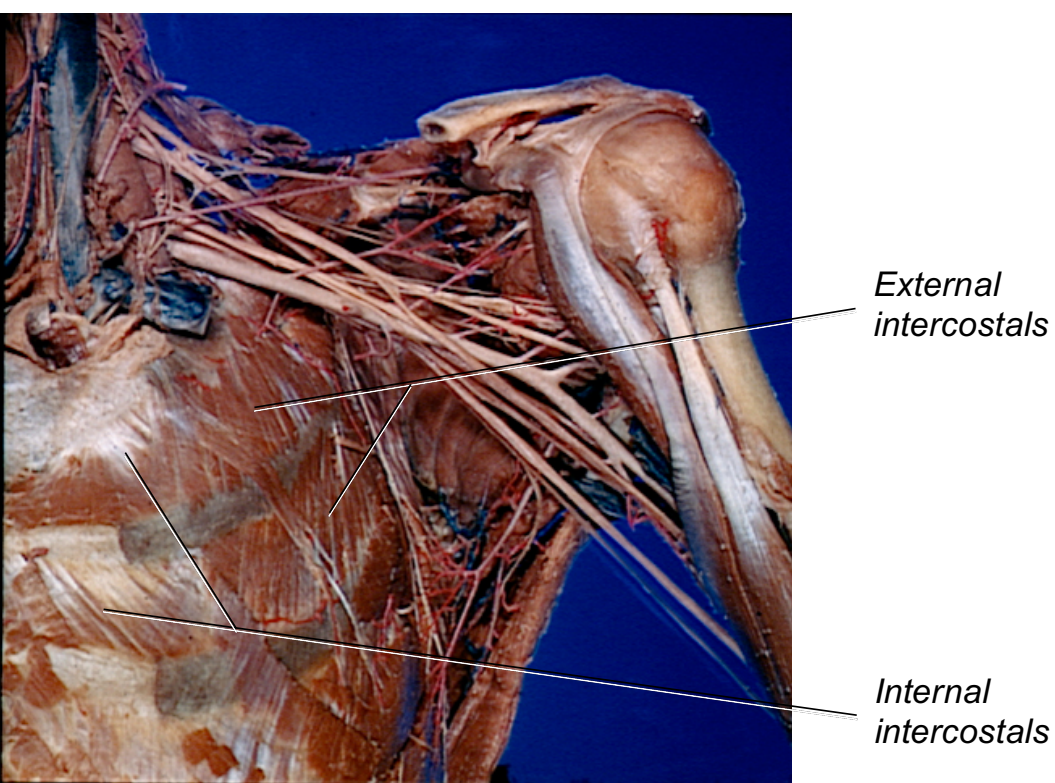

Figure 5.7. Muscles of the Thorax, Anterior View

Although the internal intercostal muscles are deep to the external intercostal muscles, paying attention to the direction of muscle fibers for each muscle can help you identify them correctly. From an anterior view, internal intercostal muscle fibers run in an inferolateral direction from superior to inferior, whereas external intercostal muscle fibers run in an inferomedial direction from superior to inferior. 


\section{Muscles of the abdomen}

$\square$ Rectus abdominis

- Action: flexes the vertebral column

$\square$ External oblique

$\square$ Internal oblique

$\square$ Transversus abdominis*

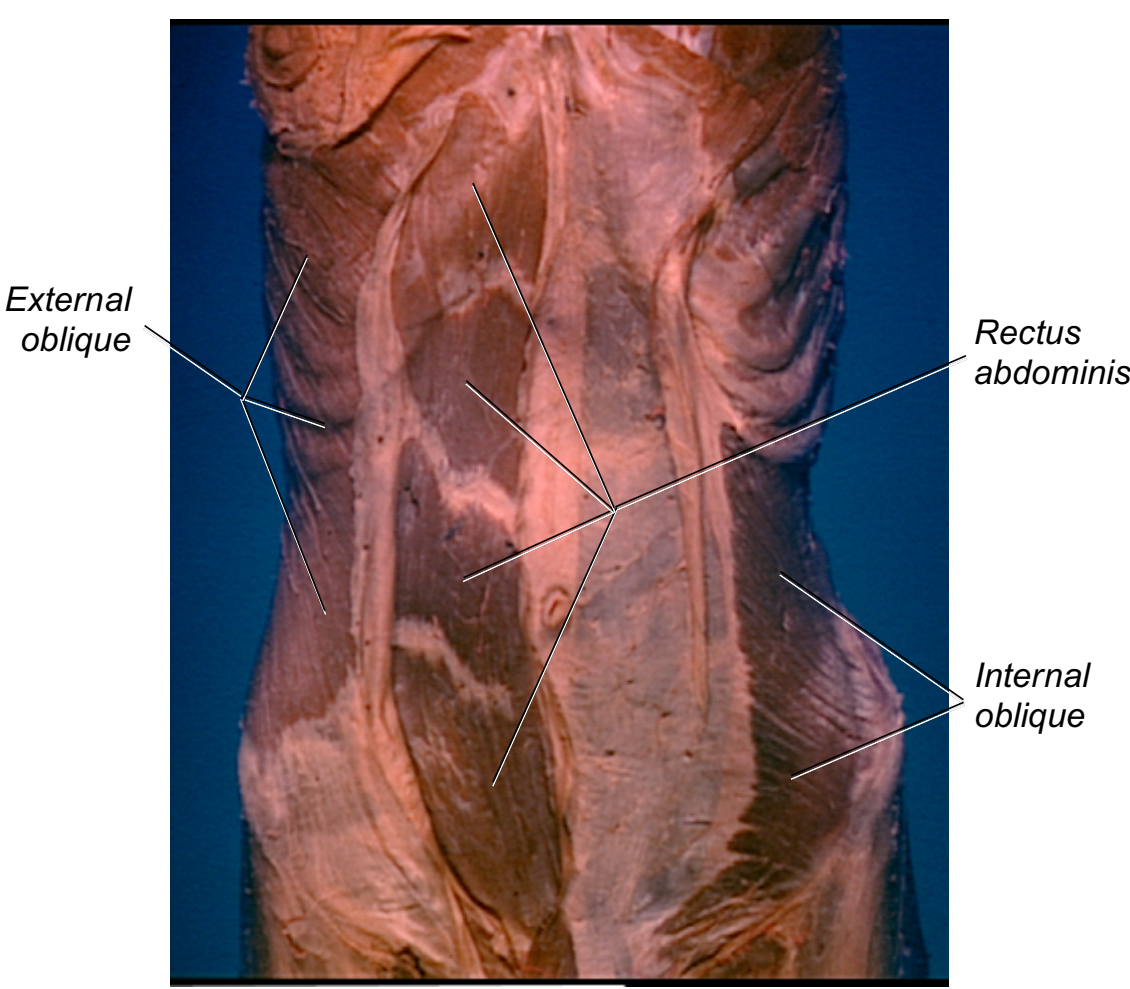

Figure 5.8. Muscles of the Abdomen, Anterior View

The transversus abdominis muscle is not visible in Figure 5.8. because it is the deepest muscle layer of the abdomen. This muscle has horizontal (transverse) muscle fibers deep to the internal oblique and rectus abdominis muscles. 
and self-assess your knowledge.

1) The name of the attachment that acts as an anchor to create movement at the other end of the muscle is called: (LO10)
a. Tendon
b. Proximal attachment
c. Distal attachment

2) What muscle depresses the angle of the mouth? (LO12)

3) What term is used to describe a muscle that spans: (LO10)
a. One joint:
b. Two joints:
c. Three or more joints:

4) What is the proximal attachment (PA) of the masseter muscle? (LO12)

5) Using the terminology you know, what bone does the nasalis muscle cover? (LO10)

6) What is the action of the levator labii superioris muscle? (LO12)

7) What does the term mastication mean? (LO10)
8) Fill in the blanks with the following terms to make the statements correct.

\section{Action Innervation Contraction}

When a muscle receives , it causes of the muscle which, in turn, causes an (LO10)

9) What term would you use to compare the depressor anguli oris and levator anguli oris muscles? (LO10)

10)Identify the following muscles. (LO11)

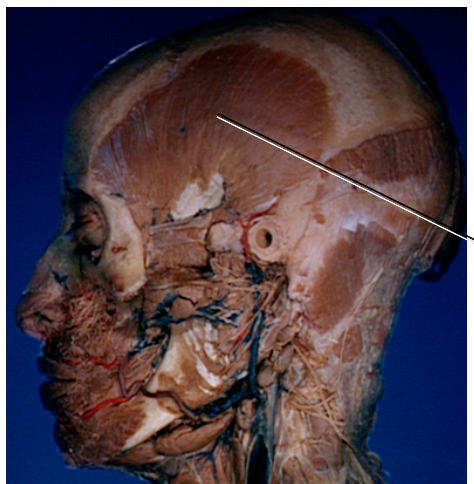

A

Fig. 5.3.

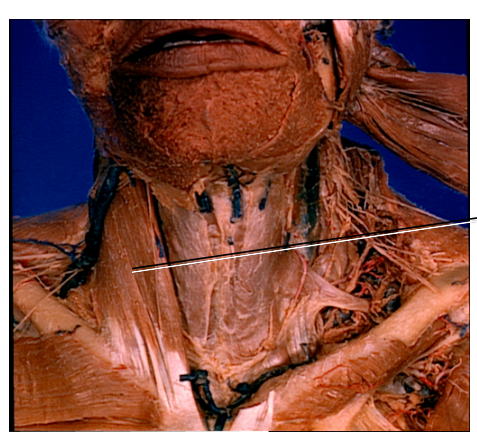

B

Fig. 5.4. 


\section{Lab 6: Appendicular Muscles (Pectoral Girdle \& Upper Limb)}

Instructions: Learn the listed terms using the listed learning objectives, labeled images, lab specimens, and other resources.

L013: Describe the terminology used to identify structures of the appendicular muscles (pectoral girdle and upper limb)

LO14: Identify the listed appendicular muscles (pectoral girdle and upper limb).

L015. Identify the listed proximal attachments, distal attachments, and actions of the appendicular muscles (pectoral girdle and upper limb).

\section{Important Muscle Terminology:}

As you learn muscles, you should understand these terms and use them correctly:

Flexion: movement of a joint that decreases the angle between the respective bones and reference point.

Extension: movement of a joint that increases the angle between the respective bones and reference point.

Adduction: movement toward the midsagittal plane.

Abduction: movement away from the midsagittal plane.

\section{Muscles of the shoulder and arm}

$\square$ Rhomboid major

$\square$ Rhomboid minor

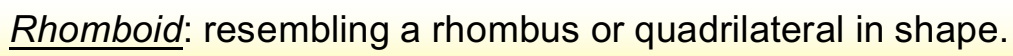

Levator scapulae*

\section{Serratus anterior}

Serratus: resembling the shape of a saw.

Teres major

Teres minor

- PA: lateral border of scapula

- DA: greater tubercle

- Action: laterally rotates the arm

$\square$ Supraspinatus

- PA: supraspinous fossa

- DA: greater tubercle

- Action: abducts the arm

$\square$ Infraspinatus

- PA: infraspinous fossa

- DA: greater tubercle

- Action: laterally rotates the arm

Subscapularis

- PA: subscapular fossa

- DA: lesser tubercle

- Action: adducts and medially rotates the arm

Collectively, the teres minor, supraspinatus, infraspinatus, and subscapularis muscles are a group of muscles known as the rotator cuff muscles. 


\section{Clinical Application: Rotator Cuff Muscles and Shoulder Dislocation}

The rotator cuff muscles (teres minor, supraspinatus, infraspinatus, and subscapularis muscles) provide support and stability to the glenohumeral (shoulder) joint at the following locations: the teres minor and infraspinatus muscles to the posterior side, the supraspinatus to the superior side, and the subscapularis to the anterior side. When any of these muscles are injured, the strength and stability of the glenohumeral joint becomes compromised and may cause the head of the humerus to dislocate from the joint. While the supraspinatus muscle is the most commonly torn rotator cuff muscle, the most common dislocation is an anterior dislocation involving the tendon of the subscapularis muscle. Rotator cuff surgery may be advised if the injury cannot heal on its own or if shoulder dislocations become frequent.

Trapezius

Latissimus dorsi

Pectoralis major

Pectoralis minor

Deltoid

Deltoid: triangular

- PA: lateral one third of clavicle, acromion, and scapular spine

- DA: deltoid tuberosity

○ Action: extends and flexes the shoulder; abducts the arm
Biceps brachii (long head and short head)

Bi: two; ceph: head; brachi: arm. Therefore, you should expect the biceps brachii muscle to be a two-headed muscle of the arm.

- PA (long head): upper lip of glenoid cavity

- PA (short head): tip of coracoid process

- DA (both heads): radial tuberosity

- Action (both heads): flexes the elbow and shoulder; supinates the forearm and the hand

\section{Coracobrachialis}

- PA: coracoid process

- DA: shaft of humerus

- Action: flexes the shoulder and adducts the arm

\section{Brachialis}

- PA: shaft of humerus

- DA: ulnar tuberosity

- Action: flexes the elbow

Triceps brachii (long head, lateral head, and medial head)

- PA (long head): lower lip of glenoid cavity

- PA (lateral head): proximal posterolateral shaft of humerus

- PA (medial head): proximal posteromedial shaft of humerus

- DA: olecranon

- Action: extends the elbow, extends the shoulder, and adducts the arm 


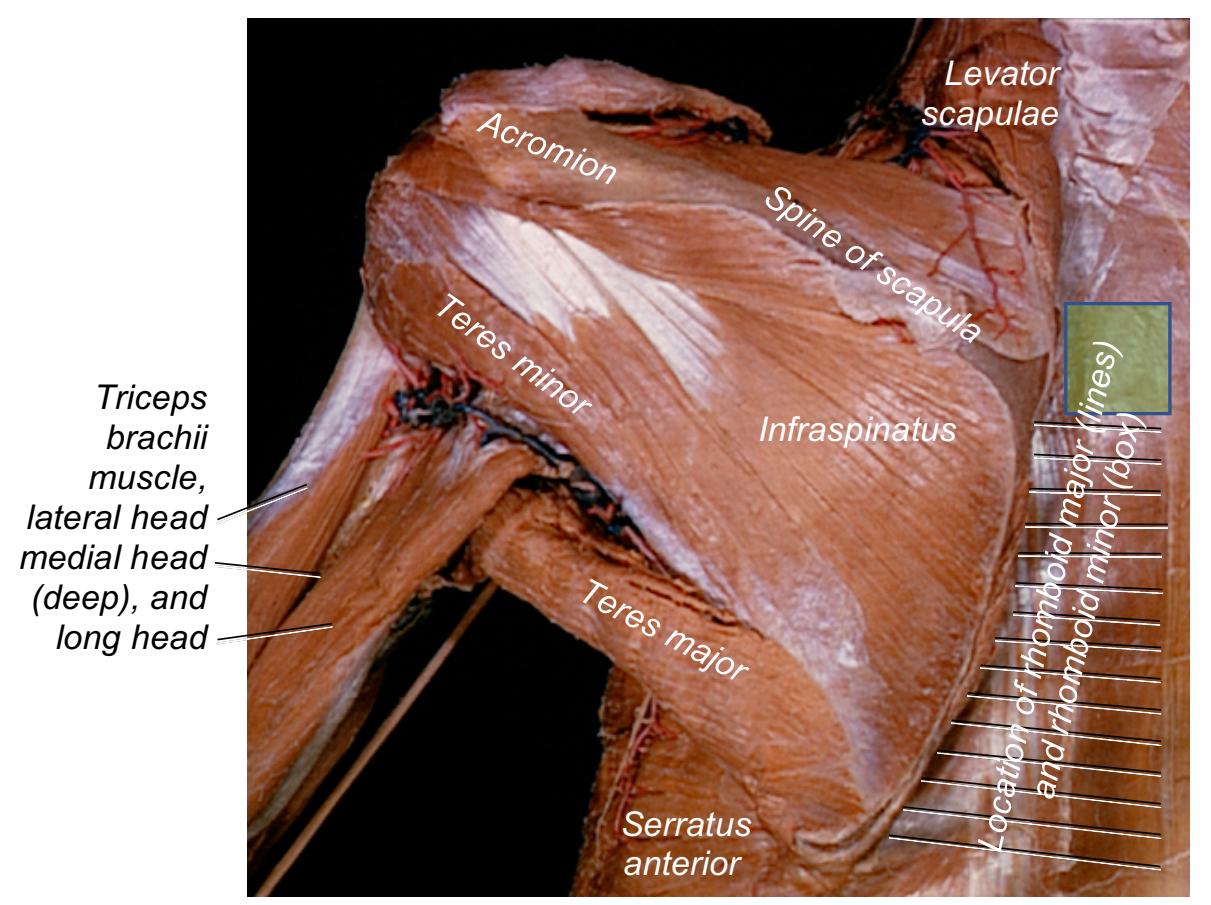

Figure 6.1. Muscles of the Left Arm and Shoulder, Posterior View

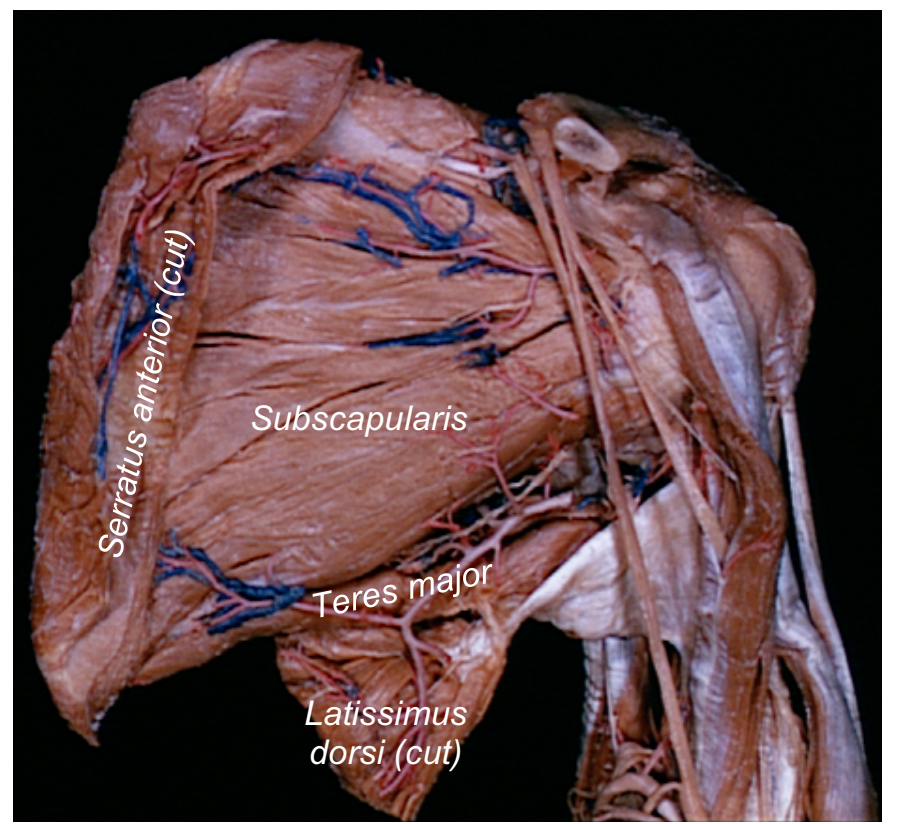

Figure 6.2. Left Subscapularis, Anterior View

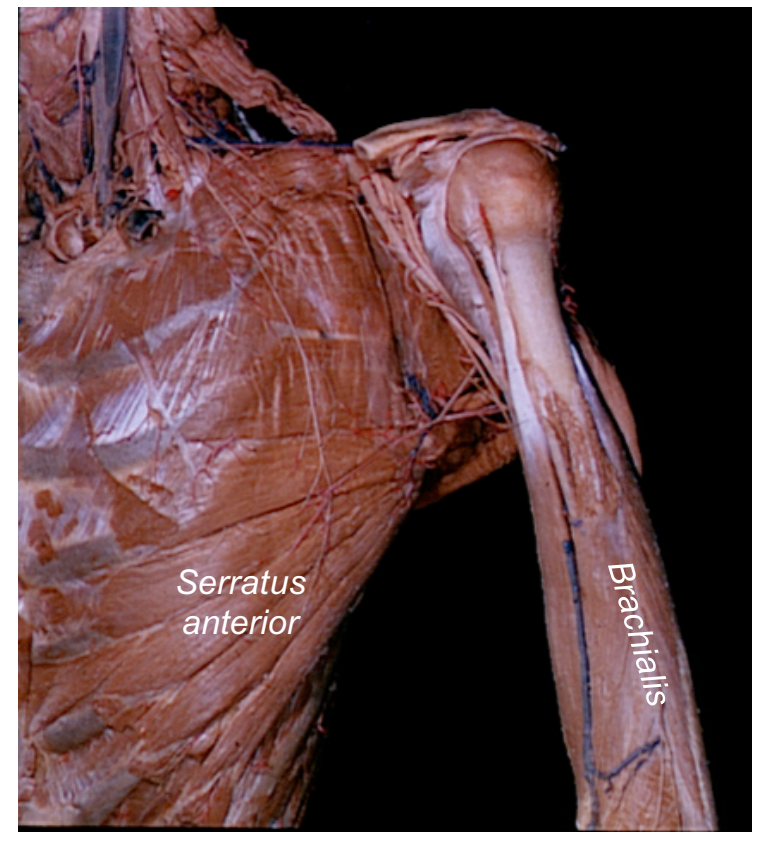

Figure 6.3. Left Serratus Anterior and Brachialis, Anterior View

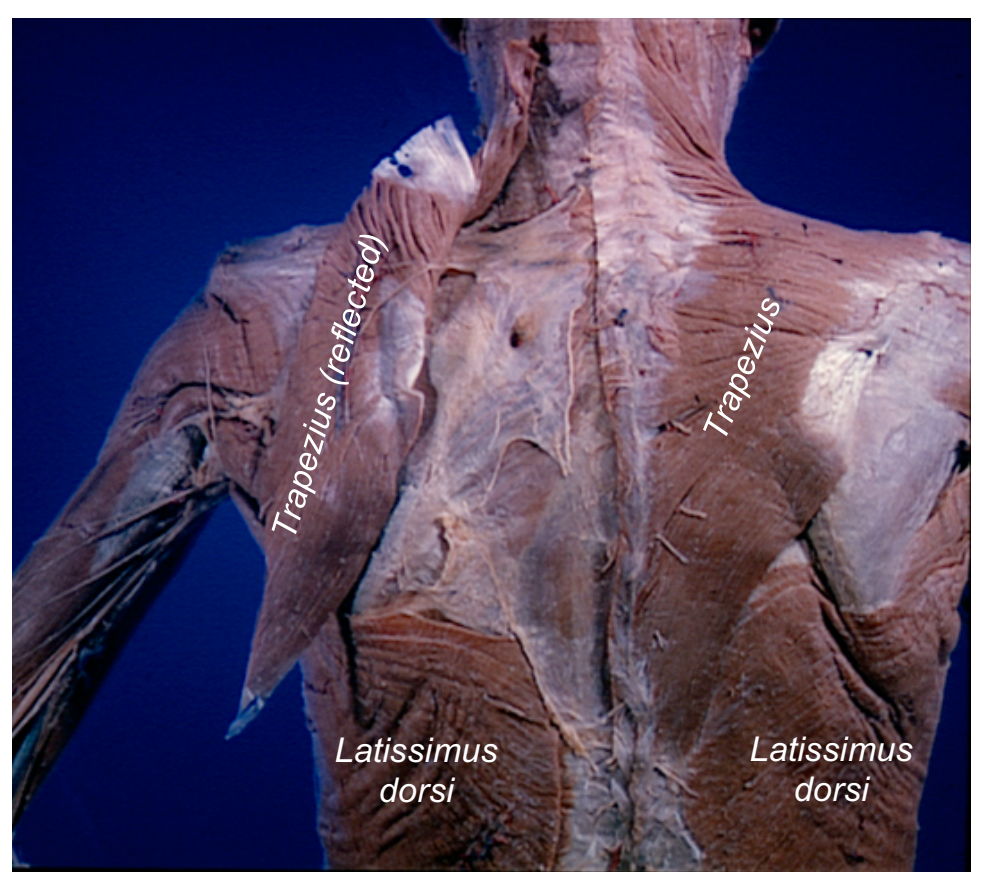

Figure 6.4. Trapezius and Latissimus Dorsi, Posterior View 


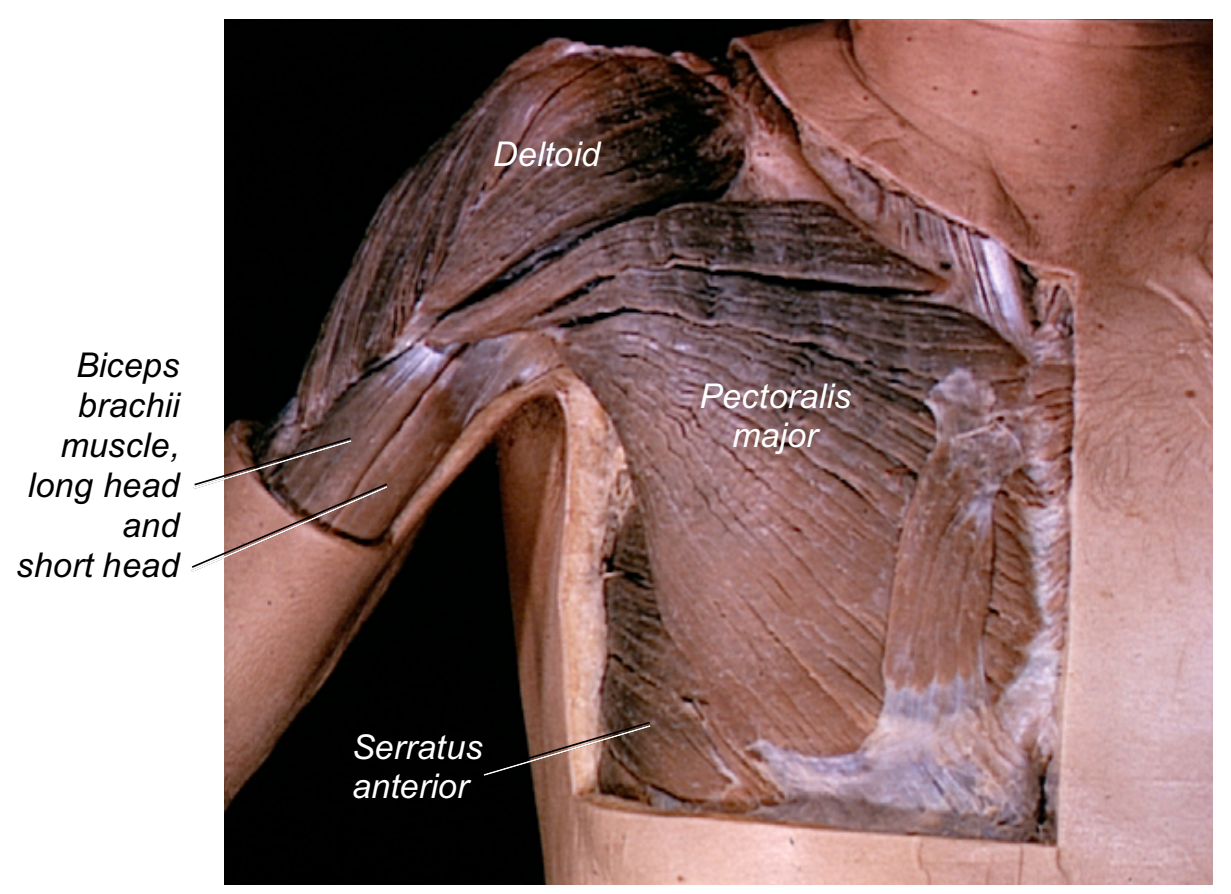

Figure 6.5. Right Pectoralis Major, Anterior View

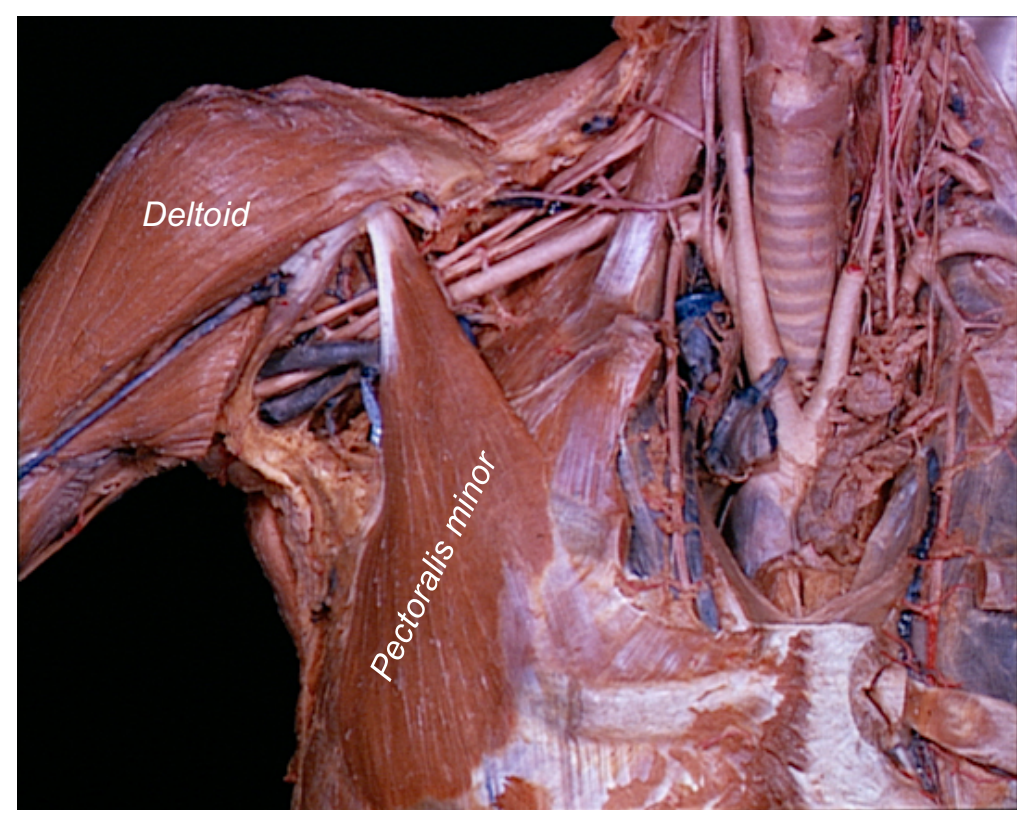

Figure 6.6. Right Pectoralis Minor, Anterior View

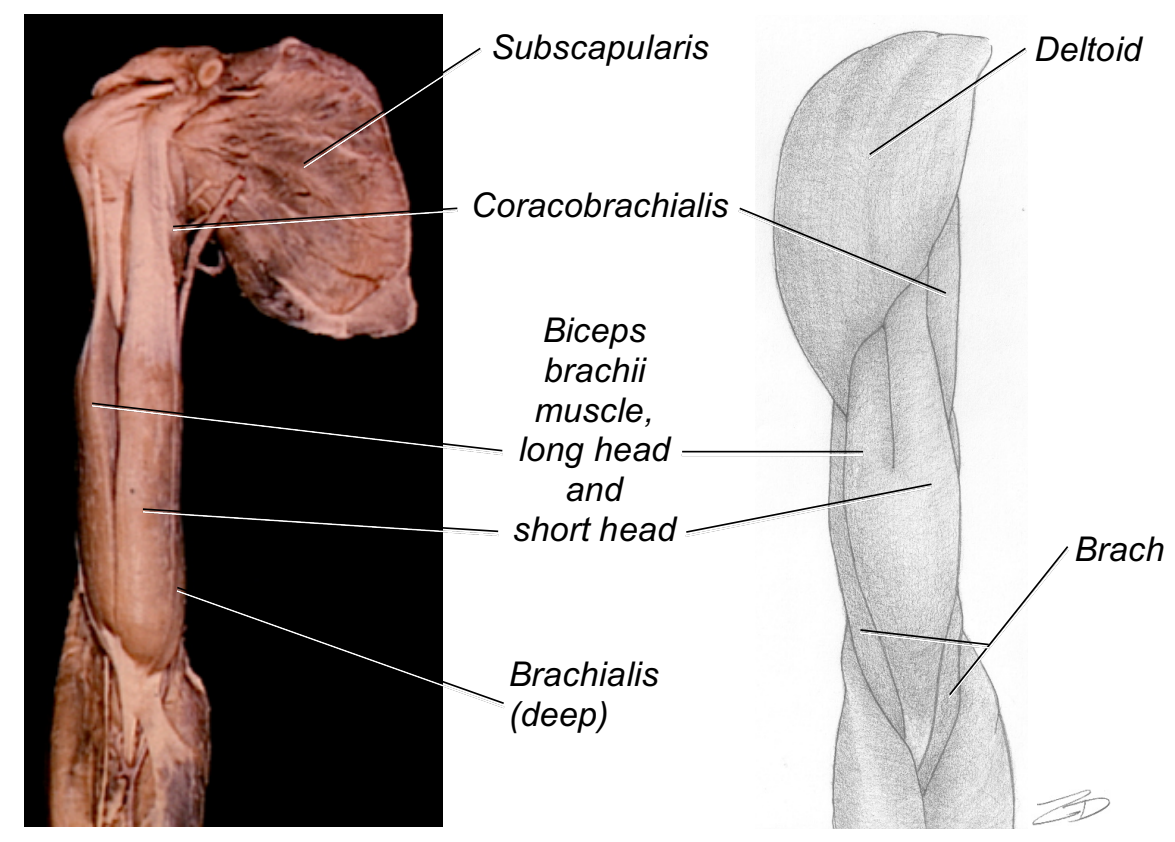

Figure 6.7. Muscles of the Right Arm, Anterior View

Figure 6.8. Muscles (Sketch) o the Right Arm, Anterior View

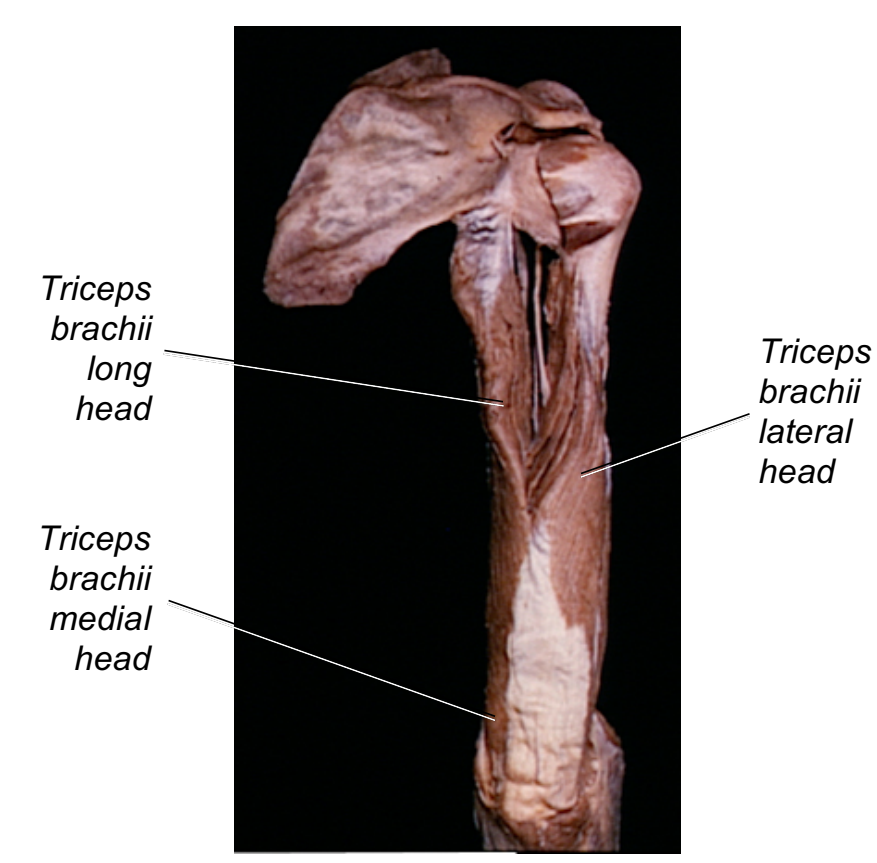

Figure 6.9. Muscles of the Right Arm, Posterior View 


\section{Muscles of the forearm}

$\square$ Brachioradialis

$\square$ Palmaris longus

Flexor digitorum superficialis

Flexor digitorum profundus*

Extensor digitorum

Flexor carpi radialis

- Action: flexes the wrist and abducts the hand

\section{Flexor carpi ulnaris}

- Action: flexes the wrist and adducts the hand

Extensor carpi radialis (longus and brevis)

- Action: extends the wrist and abducts the hand

Extensor carpi ulnaris

- Action: extends the wrist and adducts the hand

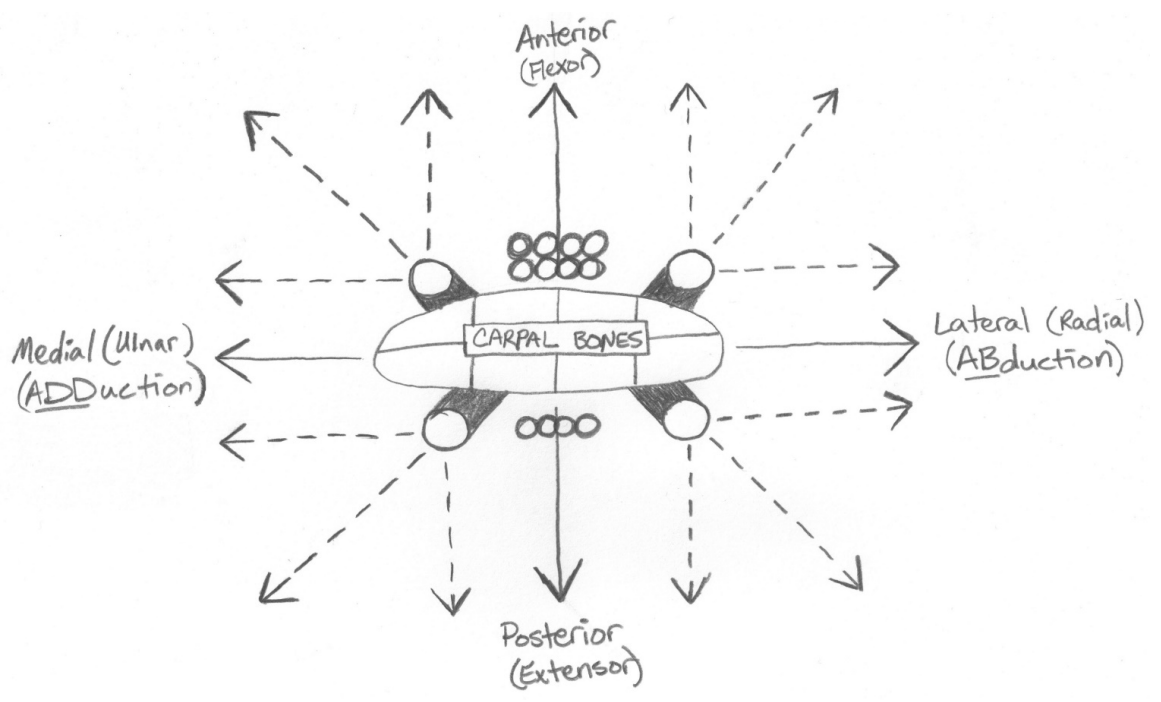

Figure 6.10. Diagram for Learning Actions of Forearm Muscles Passing Through the Wrist, Cross-Section View
Supinator

- Action: supinates the forearm and hand

Pronator teres

- Action: pronates the forearm and hand

Pronator quadratus*

- Action: pronates the forearm and hand

\section{Retinacula of the wrist}

Retinacula are strong, tight, fibrous bands of tissue that hold down tendons at major joints to keep them from bowstringing.

$\square$ Flexor retinaculum

Extensor retinaculum

\section{Muscles of the hand}

$\square$ Thenar muscles (grouped)

Hypothenar muscles (grouped)

$\underline{\text { Hypo }}=$ below

You can see where the thenar and hypothenar muscle groups are located on your own hand. Look for the muscular bulges proximal to your thumb (pollex, or $1^{\text {st }}$ digit) and pinky finger ( $5^{\text {th }}$ digit).

These visible bulges are called the thenar and hypothenar eminences.

$\underline{\text { Eminence }}=$ an elevation

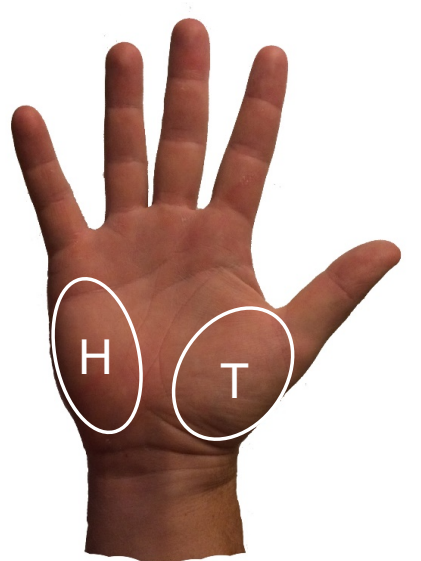

Figure 6.11. Thenar $(T)$ anc Hypothenar $(H)$ Eminences 


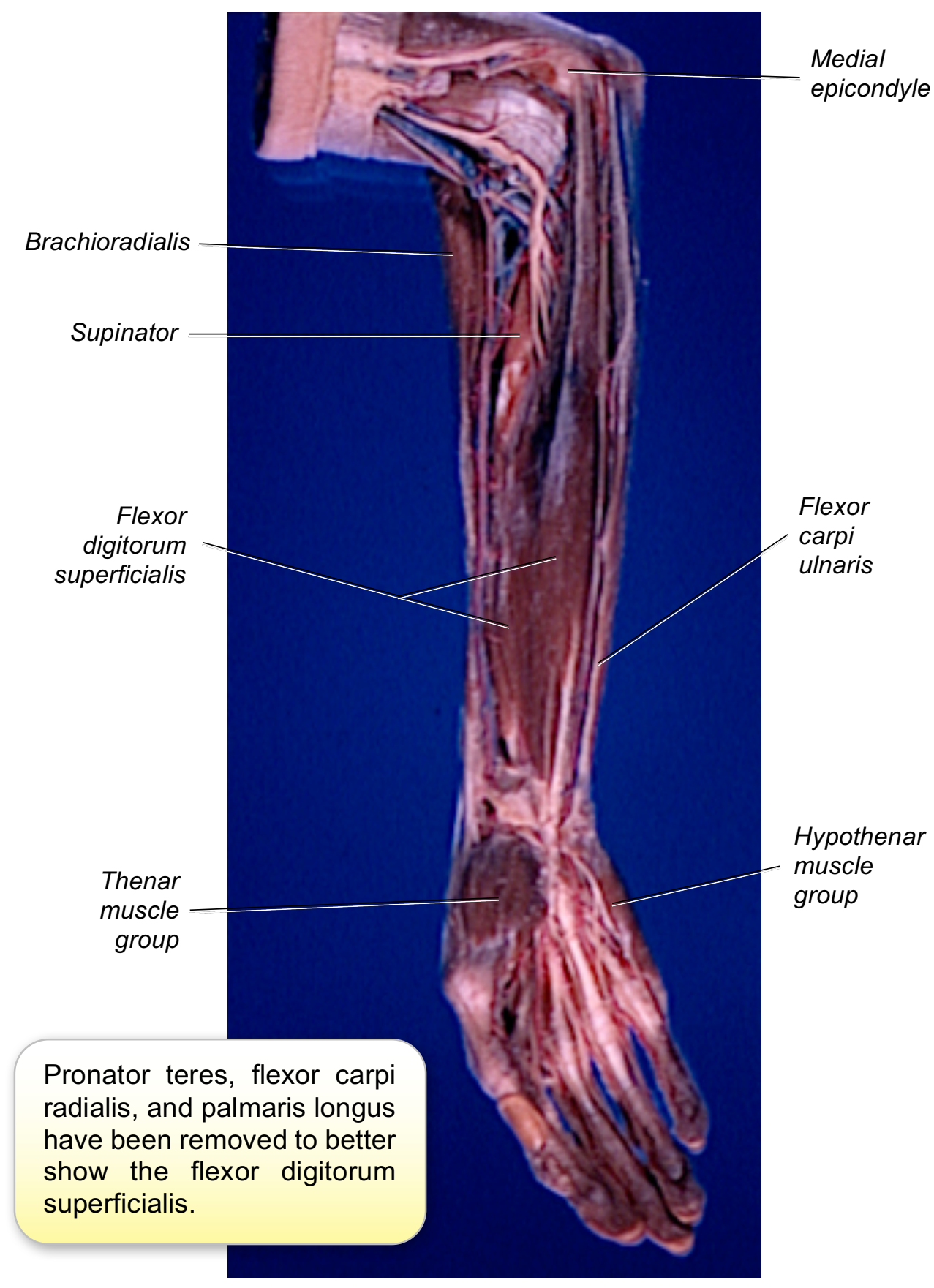

Figure 6.12. Muscles of Right Forearm and Hand, Anterior View

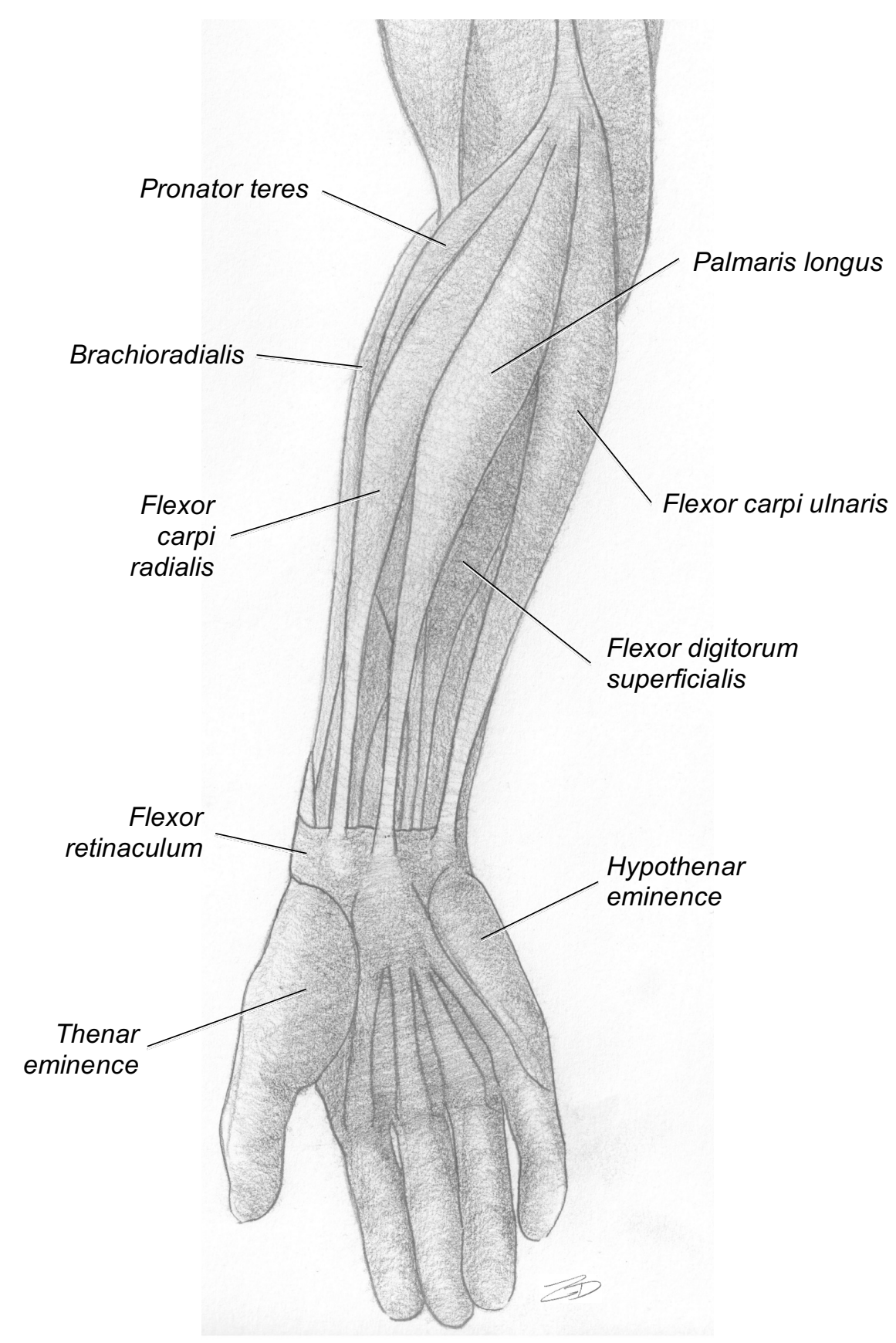

Figure 6.13. Muscles (Sketch) of Right Forearm and Hand, Anterior View 


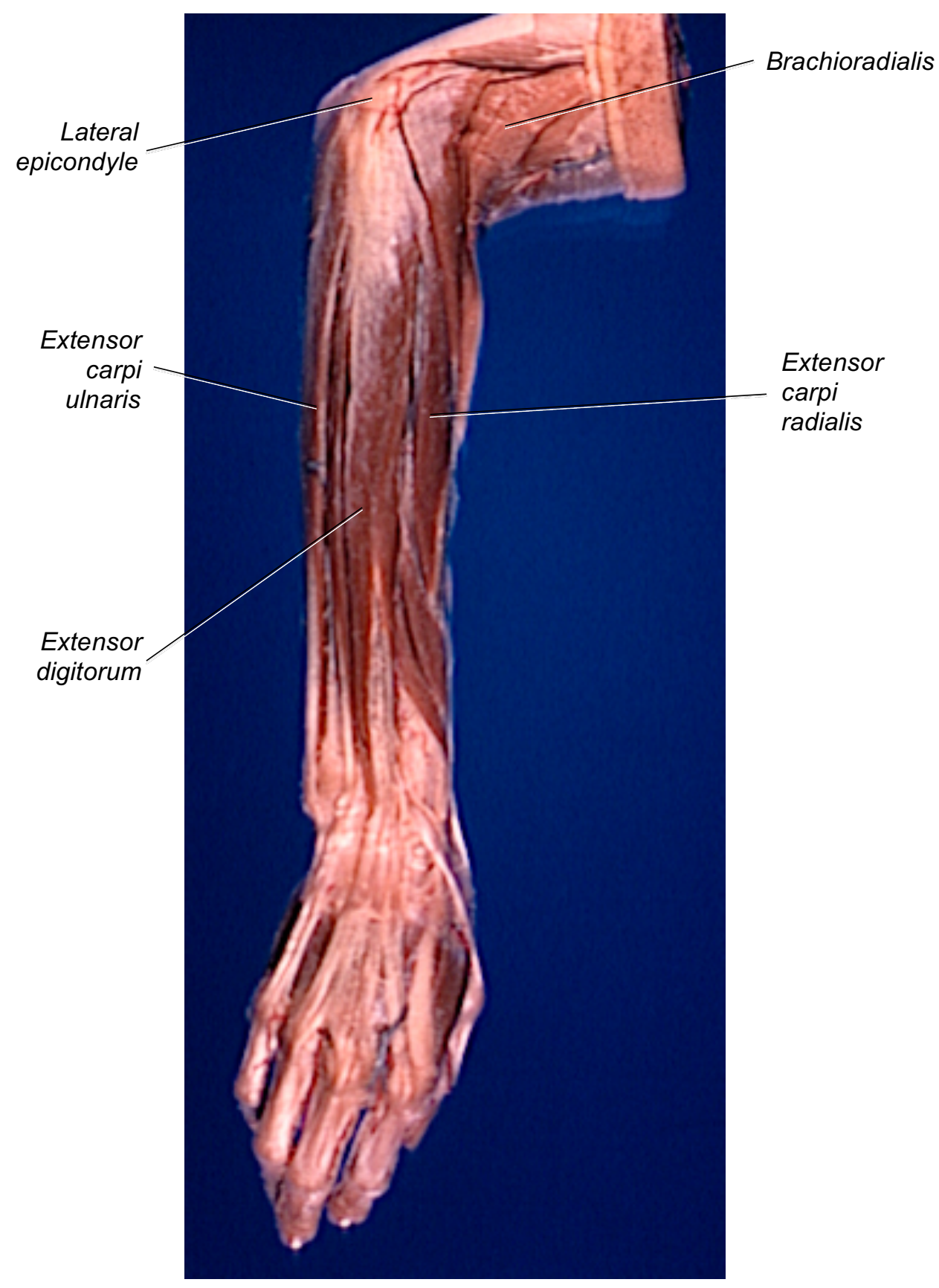

Figure 6.14. Muscles of Right Forearm and Hand, Posterior View

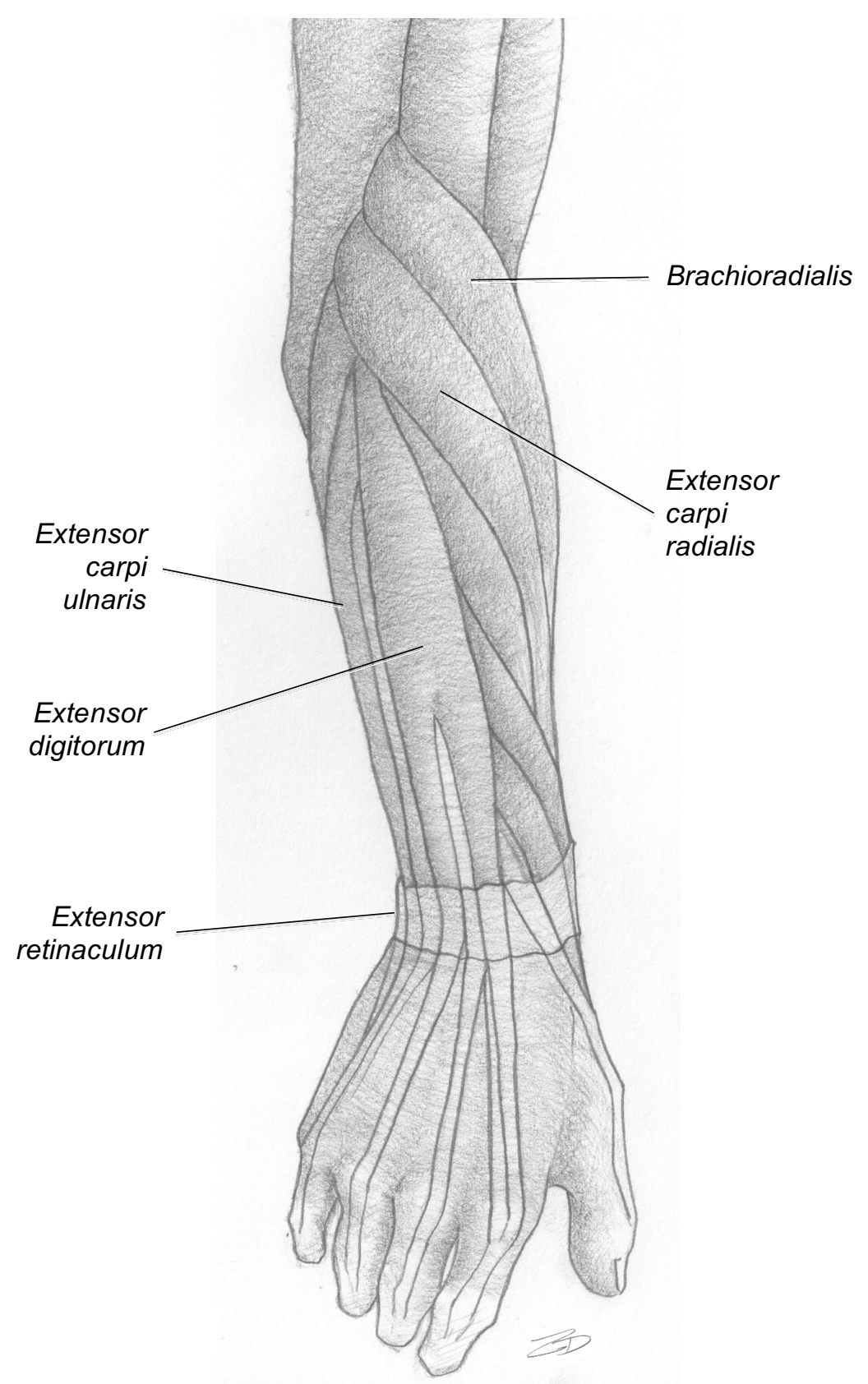

Figure 6.15. Muscles (Sketch) of Right Forearm and Hand, Posterior View 
and self-assess your knowledge.

1) By using the terminology you've learned, and by looking at the attachments and direction of fibers in muscles, you should be able to accurately predict the main action of muscles for which you did not learn the actions. For example, what would you expect the primary action of the following muscles be? (LO13)

Levator scapulae:

Extensor digitorum:

Rhomboid major:

Brachioradialis:

2) Name the synergist(s) to the pronator quadratus muscle. (LO15)

3) Name the antagonist(s) to the pronator quadratus muscle. (LO15)

4) The movement of a joint that increases the angle between the respective bones and reference point is called: (LO13)
a. Flexion
b. Extension
c. Adduction
d. Abduction

5) Identify the action(s) of the flexor carpi ulnaris muscle. (LO15)
6) What is the proximal attachment (PA) of the supraspinatus muscle? (LO15)

7) What is the distal attachment (DA) of the deltoid muscle? (LO15)

8) What is the proximal attachment (PA) of the coracobrachialis muscle? (LO15)

9) What is the distal attachment (DA) of the brachialis muscle? (LO15)

10)Name one muscle that is both a synergist and an antagonist to the extensor carpi radialis muscle. (LO15)

11)Identify the following muscles. (LO14)

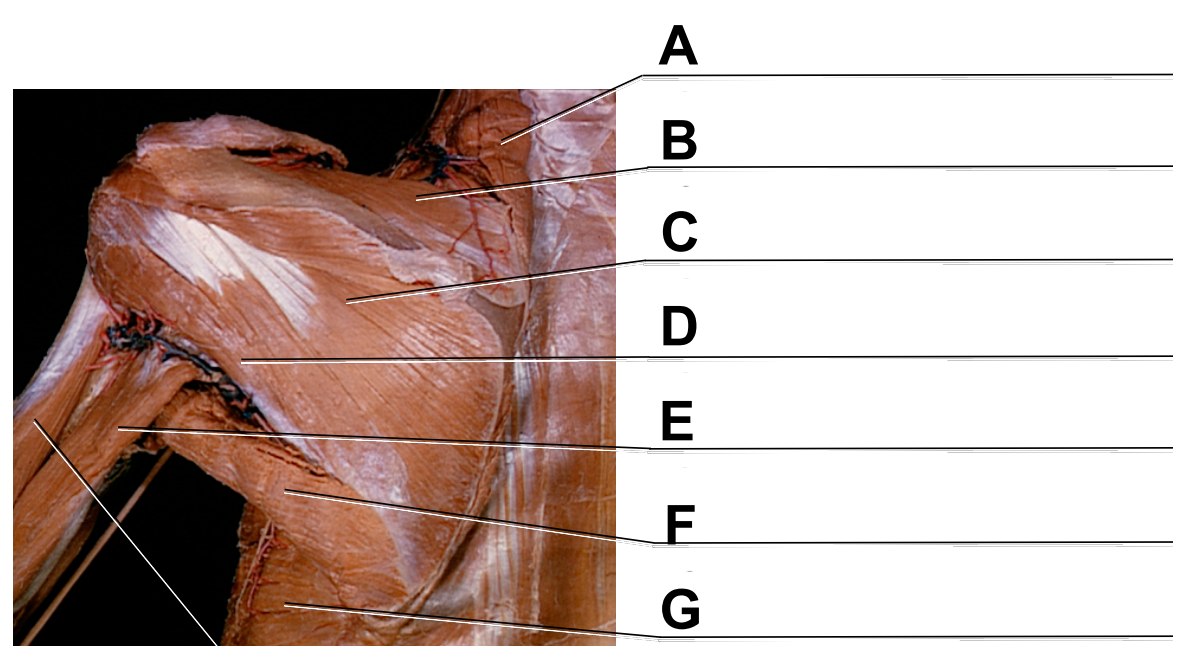

Fig. 6.1. 


\section{Lab 7: Appendicular Muscles (Pelvic Girdle \& Lower Limb)}

Instructions: Learn the listed terms using the listed learning objectives, labeled images, lab specimens, and other resources.

L016: Describe the terminology used to identify structures of the appendicular muscles (pelvic girdle and lower limb)

LO17: Identify the listed appendicular muscles (pelvic girdle and lower limb).

L018. Identify the listed proximal attachments, distal attachments, and actions of the appendicular muscles (pelvic girdle and lower limb).

\section{Important Muscle Terminology:}

As you learn muscles, you should understand these terms and use them correctly:

Dorsiflexion: movement at the ankle that points the foot upward. Plantar flexion: movement at the ankle that points the foot downward.

Inversion: turning inward.

Eversion: turning outward.

\section{Muscles of the hip and thigh}

$\square$ Psoas major

$\square$ Iliacus

$\square$ lliopsoas (psoas major + iliacus)

The psoas major and iliacus muscles combine to form the iliopsoas muscle after they pass under the inguinal ligament.
Gluteus maximus

- Action: extends the hip; laterally rotates the thigh

$\square$ Gluteus medius

$\square$ Gluteus minimus*

Piriformis*

Tensor fasciae latae

The tensor fasciae latae muscle distally attaches to the iliotibial tract, a strong, fibrous reinforcement of the fascia lata (the deep fascia of the thigh that encloses the muscles of the thigh).

$\square$ Gracilis

Pectineus

Sartorius

- PA: anterior superior iliac spine

- DA: medial side of tibial tuberosity

○ Action: flexes the hip; abducts and laterally rotates the thigh; flexes the knee

Adductors (grouped) 


\section{Quadriceps femoris muscles (part of muscles of the thigh)}

$\square$ Rectus femoris

- PA: anterior inferior iliac spine

- DA: patella and tibial tuberosity via patellar ligament

- Action: flexes the hip; extends the knee

Vastus lateralis

- PA: linea aspera

- DA: patella and tibial tuberosity via patellar ligament

- Action: extends the knee

Vastus medialis

- PA: linea aspera

- DA: patella and tibial tuberosity via patellar ligament

- Action: extends the knee

$\square$ Vastus intermedius*

- PA: anterior shaft of femur

- DA: patella and tibial tuberosity via patellar ligament

- Action: extends the knee

\section{Hamstring muscles (part of muscles of the thigh)}

$\square$ Semimembranosus

- PA: ischial tuberosity

- DA: posterior to the medial condyle of the tibia

- Action: extends the hip; flexes the knee

$\square$ Semitendinosus

- PA: ischial tuberosity

- DA: medial side of tibial tuberosity

- Action: extends the hip; flexes the knee

$\square$ Biceps femoris (long head and short head)

- PA (long head): ischial tuberosity

- PA (short head): linea aspera

- DA (both heads): head of fibula

- Action (long head): extends the hip; flexes the knee

- Action (short head): flexes the knee 


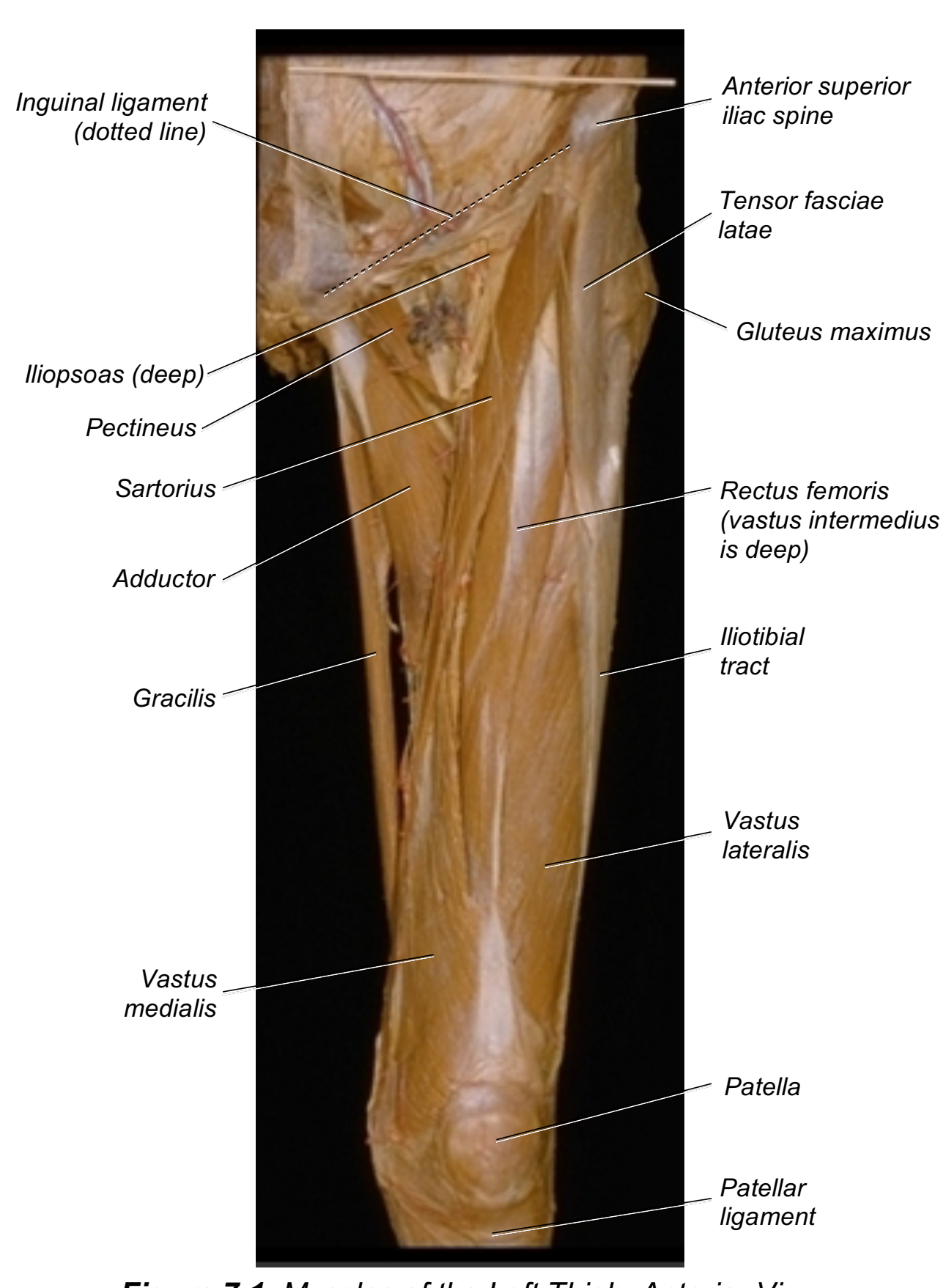

Figure 7.1. Muscles of the Left Thigh, Anterior View

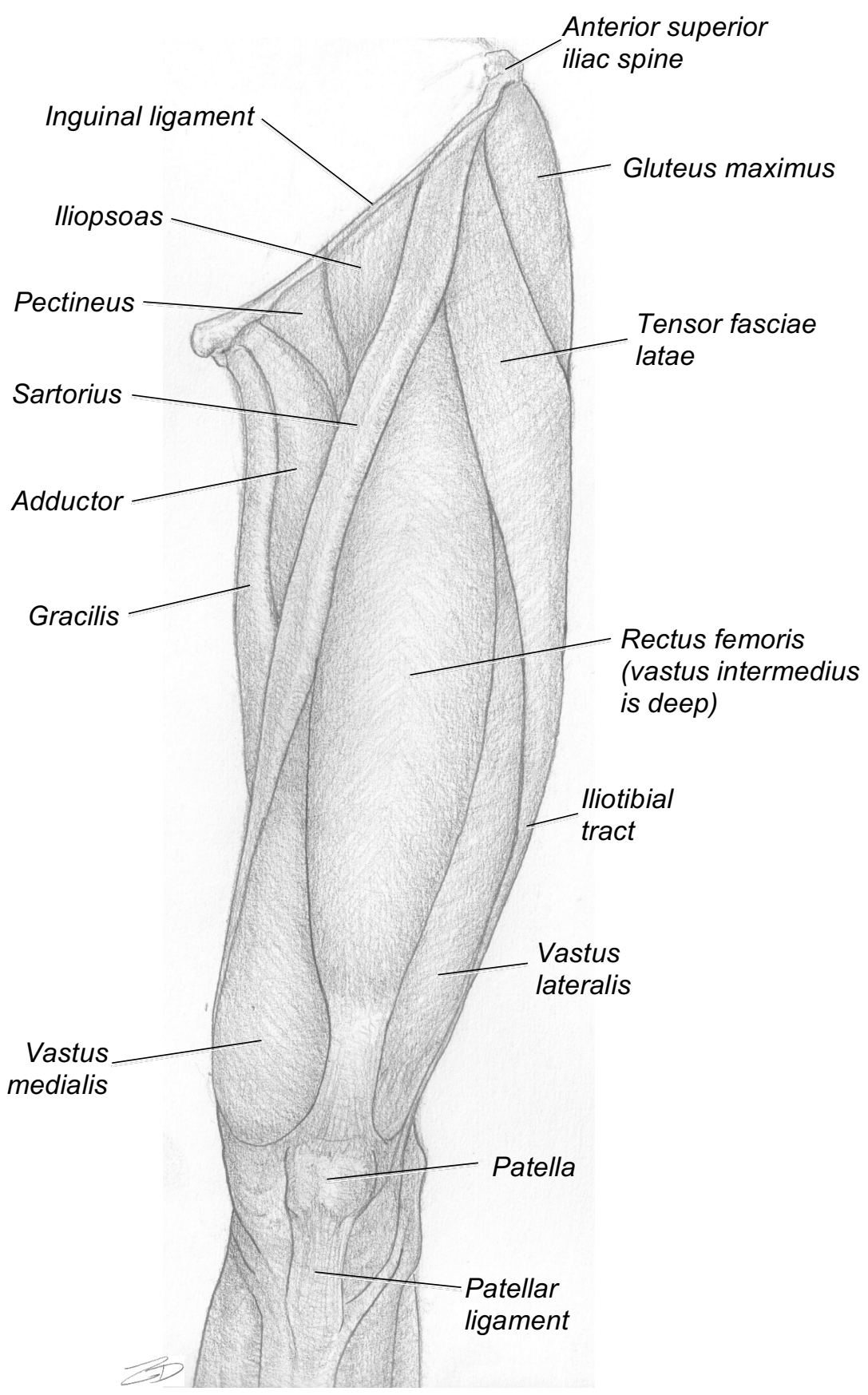

Figure 7.2. Muscles (Sketch) of the Left Thigh, Anterior View 


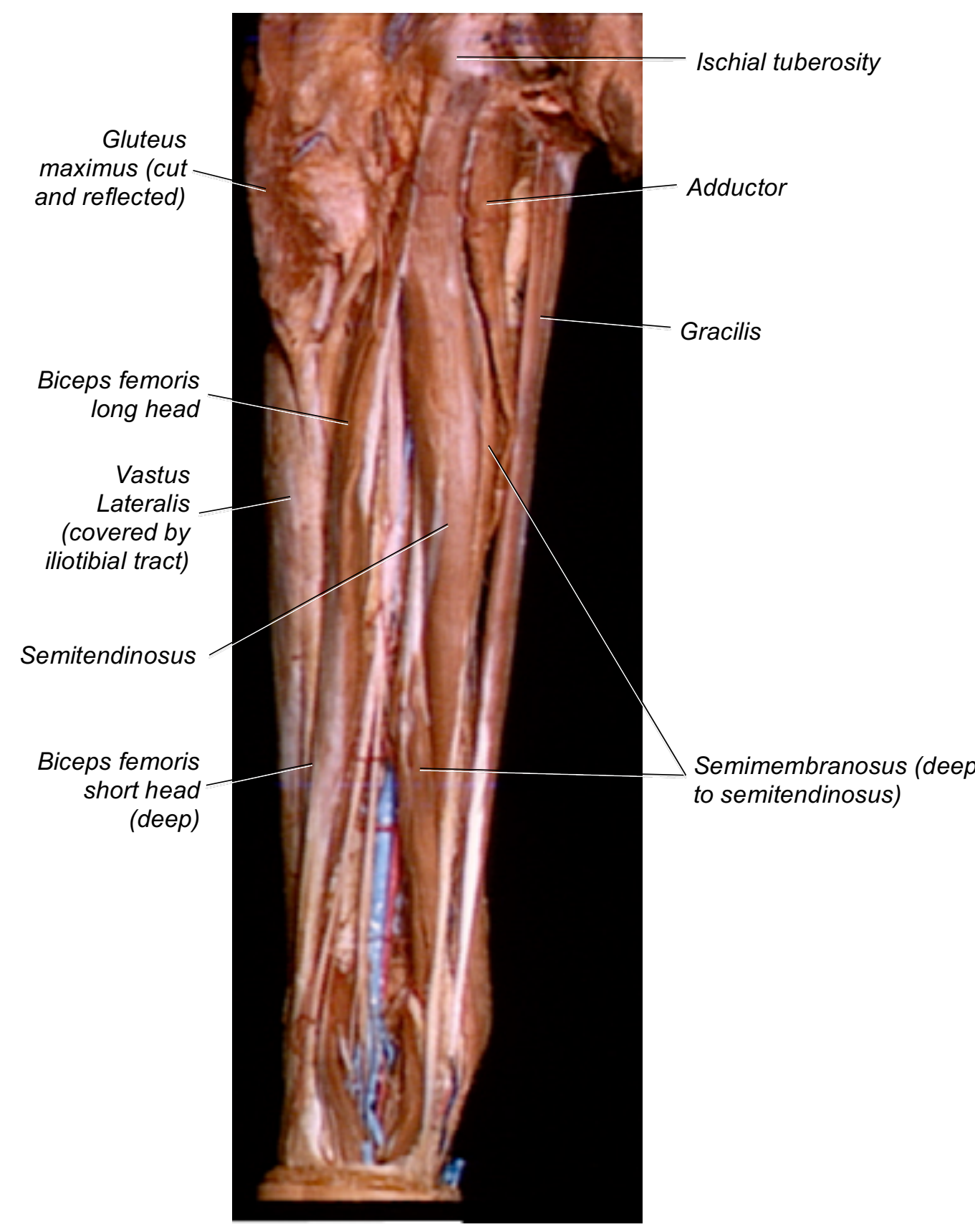

Figure 7.3. Muscles of the Left Gluteal Region and Thigh, Posterior View

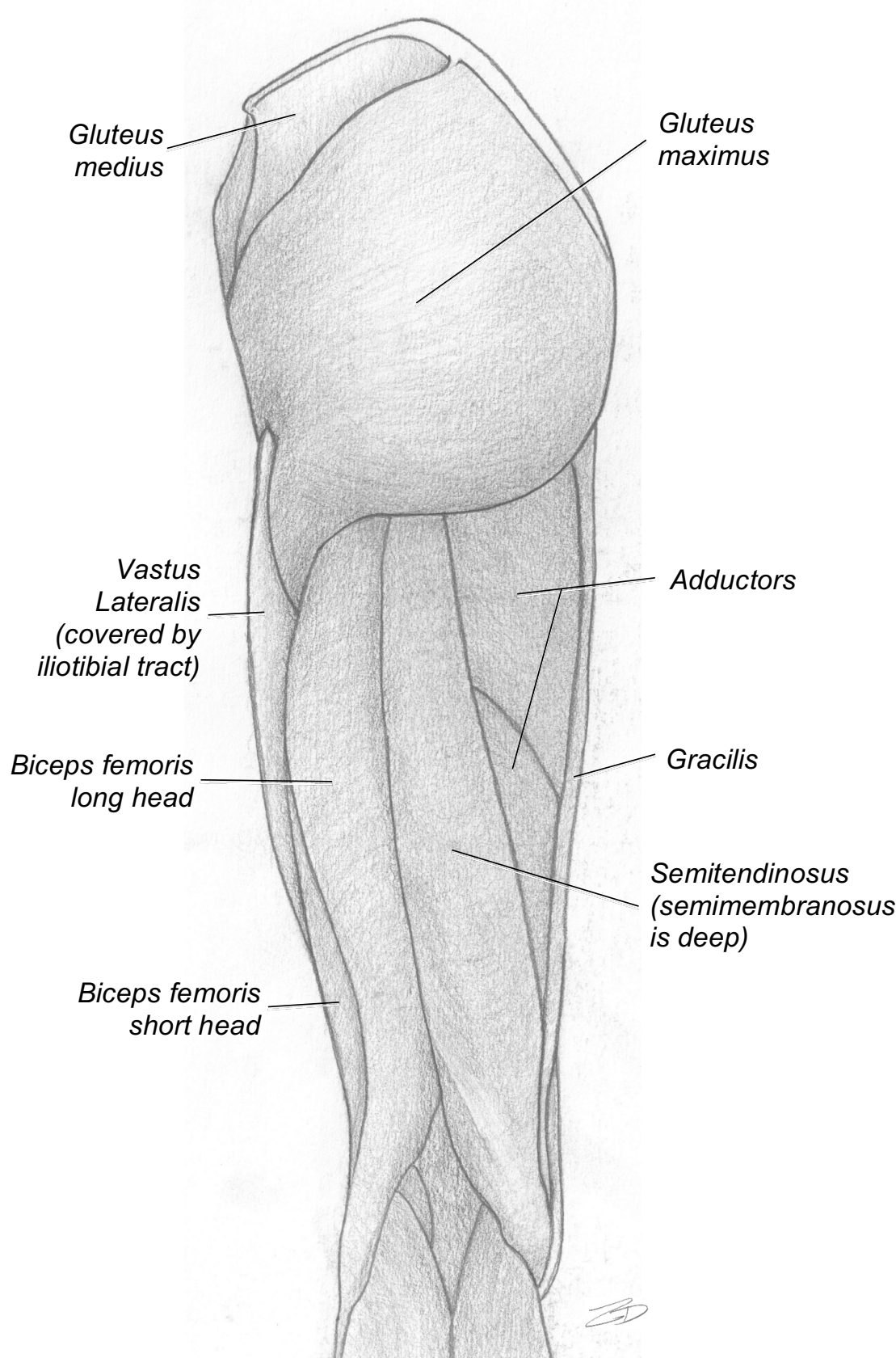

Figure 7.4. Muscles (Sketch) of the Left Gluteal Region and Thigh, Posterior View 

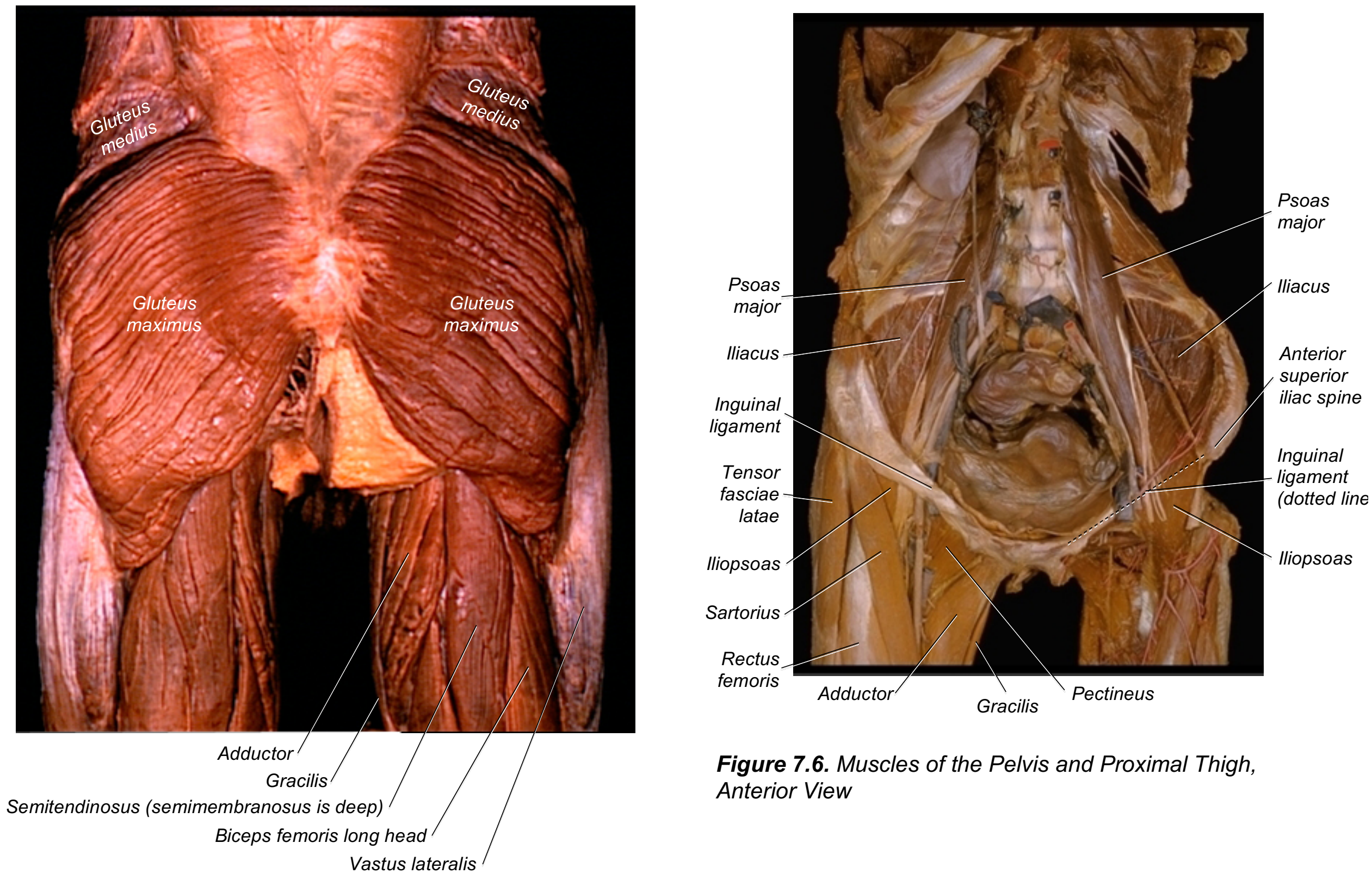

Figure 7.6. Muscles of the Pelvis and Proximal Thigh, Anterior View

Figure 7.5. Muscles of the Gluteal Region and Proximal Thigh, Posterior View 


\section{Clinical Application: "Groin Pull”}

There are many muscles that adduct your thighs, including but not limited to the adductors, gracilis, and pectineus muscles. When these muscles are strained, the resulting pain and discomfort is often diagnosed as a "groin pull." This pain and discomfort can range from mild to severe depending on how strained the muscles are. Severe groin strains can include swelling, bruising, and muscle spasms, making even light walking painful. Groin pulls are especially common among hockey, football, and soccer athletes who generate tremendous force from their adductor muscles. Proper conditioning and stretching of adductor muscles are two of the most effective ways to prevent groin pulls.

\section{Muscles of the leg}

$\square$ Gastrocnemius (medial and lateral heads)

o Action: plantar flexes the ankle; flexes the knee

\section{Soleus}

- Action: plantar flexes the ankle

The tendons of gastrocnemius and soleus muscles merge together to form one thick, strong tendon that attaches to the calcaneus. This tendon is known as the calcaneal tendon.

$\square$ Flexor hallucis longus

$\underline{\text { Hallux }}=$ big toe

$\square$ Flexor digitorum longus

$\square$ Extensor hallucis longus

$\square$ Extensor digitorum longus
Fibularis muscles (grouped)

- Action: everts the foot

\section{Plantaris}

- Action: plantar flexes the ankle; flexes the knee

Although the plantaris has a small muscle belly behind the knee, it has a long, skinny tendon that passes between the gastrocnemius and soleus muscles as it spans distally to attach to the calcaneus.

\section{Tibialis anterior}

- Action: dorsiflexes the ankle; inverts the foot

Tibialis posterior*

○ Action: plantar flexes the ankle; inverts the foot

\section{Muscles of the foot}

$\square$ Extensor hallucis brevis

$\square$ Extensor digitorum brevis

$\square$ Flexor digitorum brevis

$\square$ Abductor hallucis

$\square$ Abductor digiti minimi

\section{Retinacula of the ankle}

$\square$ Flexor retinaculum*

Superior extensor retinaculum

Inferior extensor retinaculum 


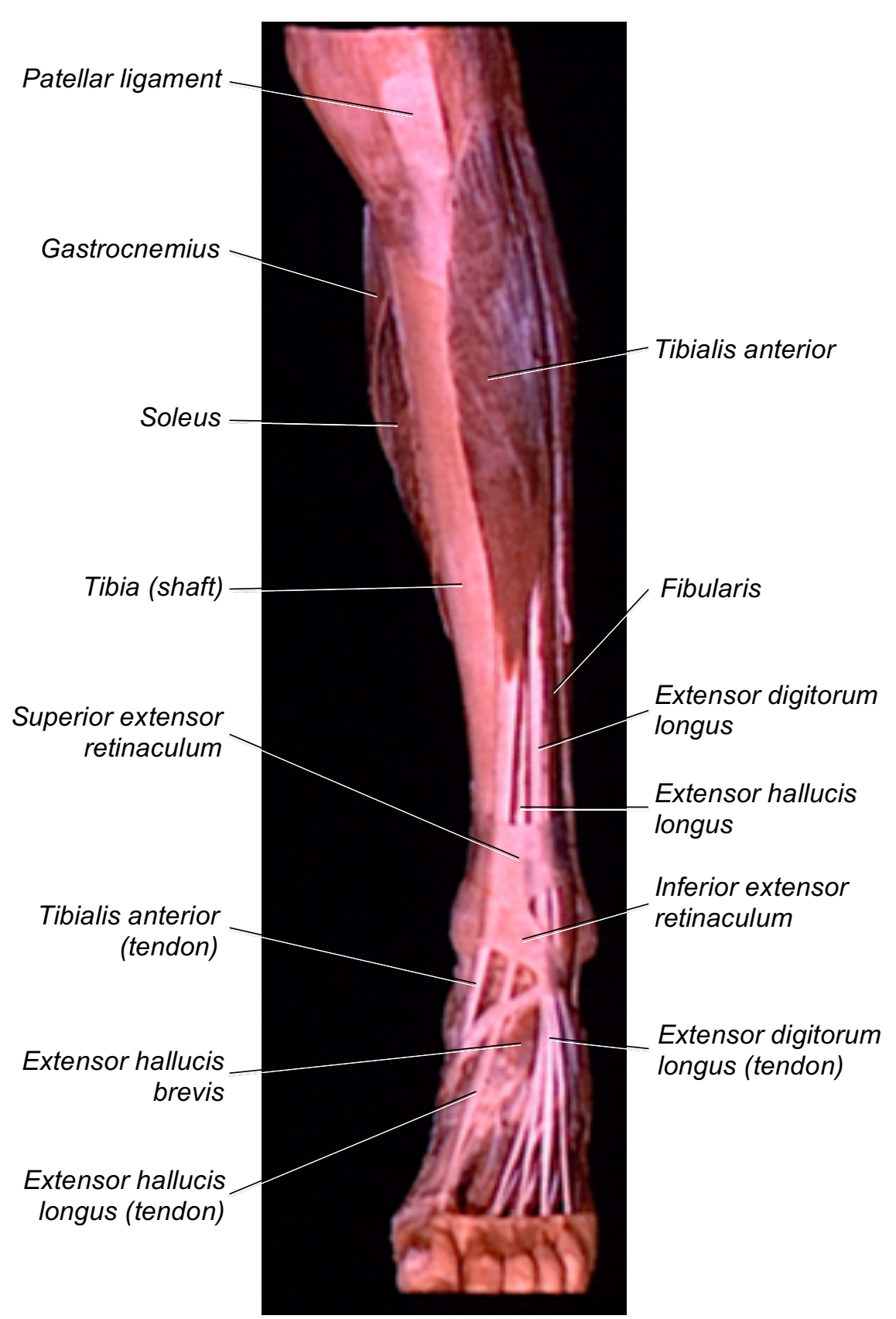

Figure 7.7. Muscles of the Left Leg, Anterior View

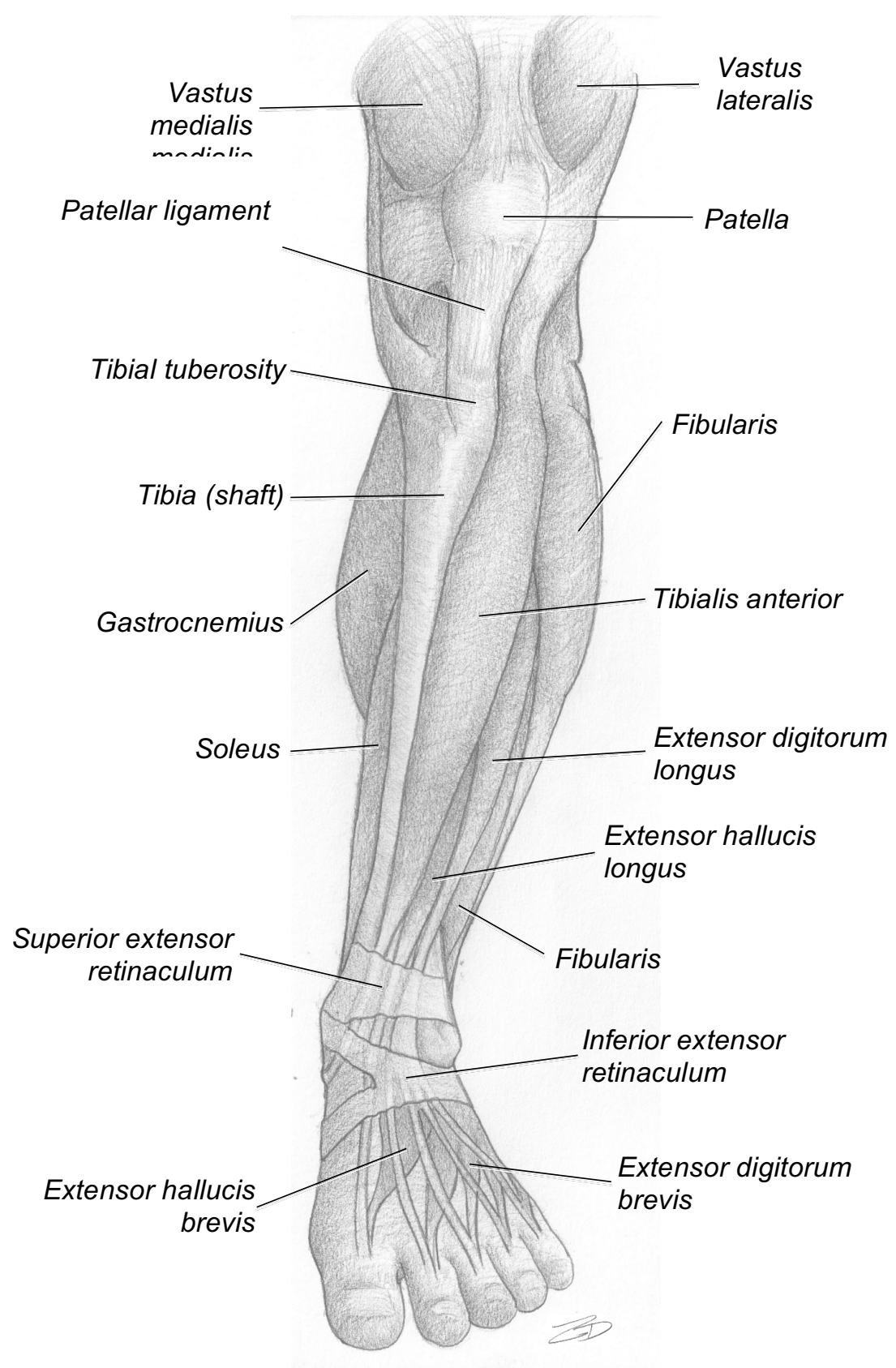

Figure 7.8. Muscles (Sketch) of the Left Leg, Anterior View 


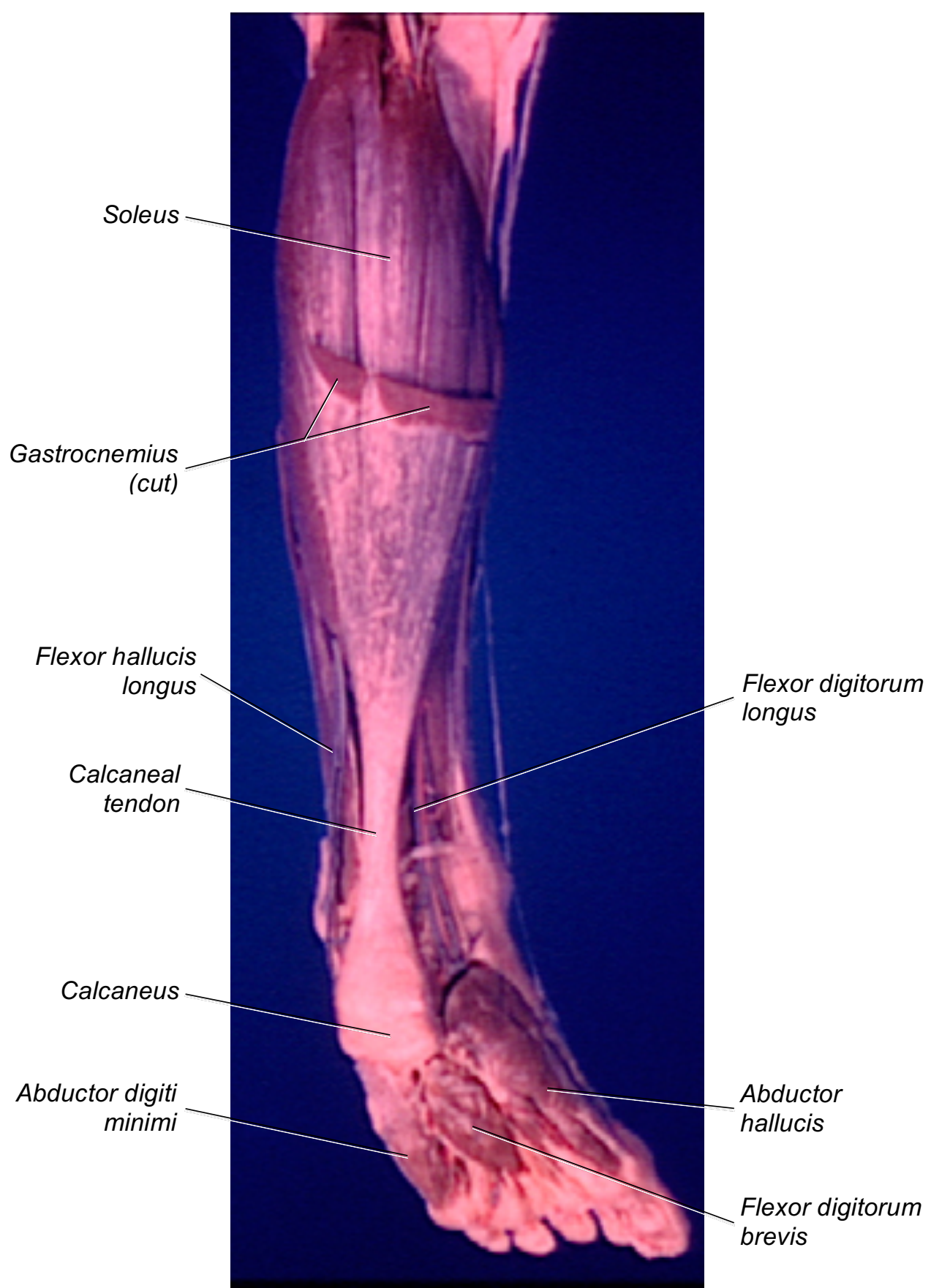

Figure 7.9. Muscles of the Left Leg, Posterior View

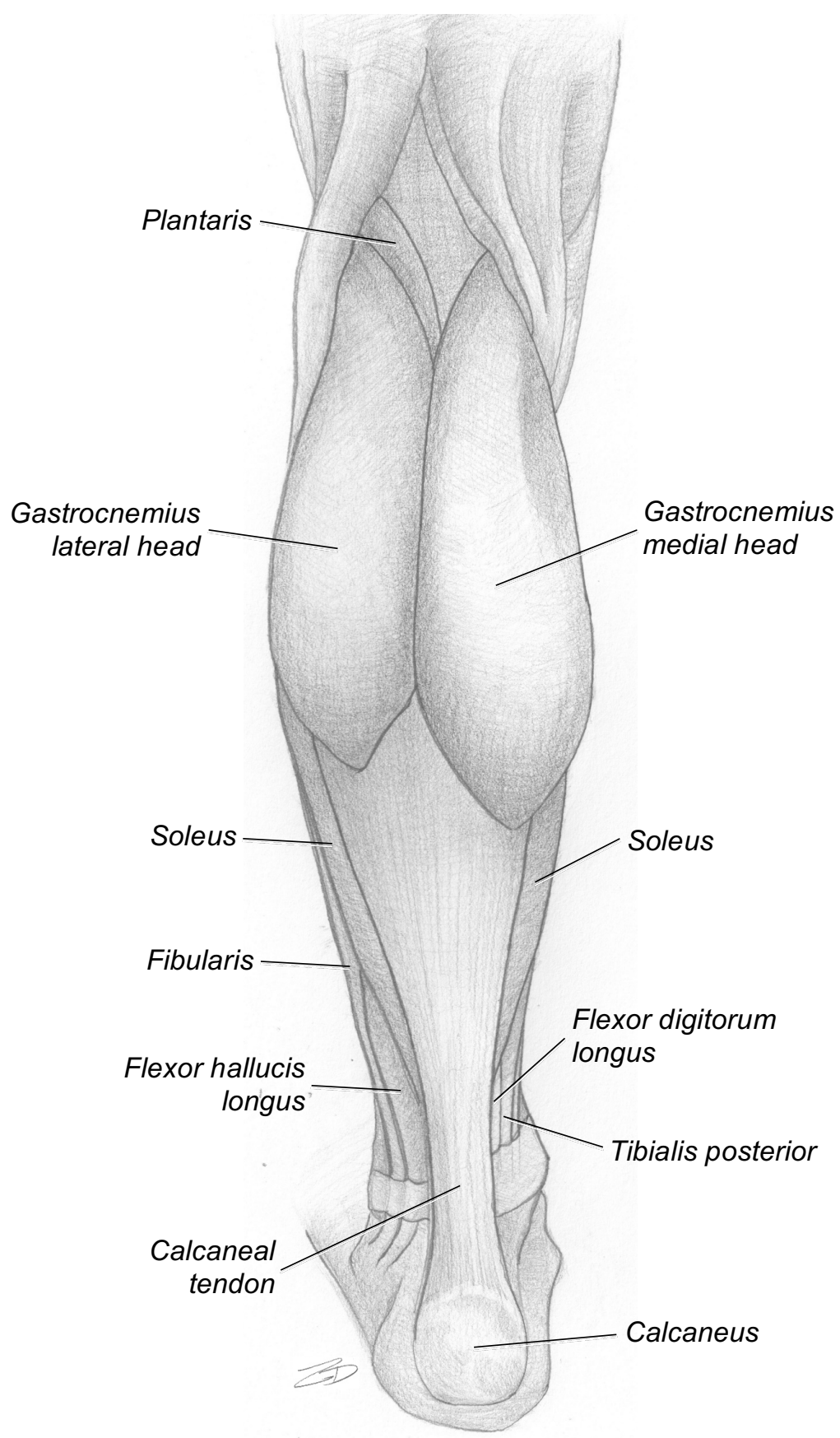

Figure 7.10. Muscles (Sketch) of the Left Leg, Posterior View 
8) What does the term eversion mean? (LO16)

1) Which of the following is NOT true of the rectus femoris muscle? (LO18)
a. It is biarticulate
b. It is a quadriceps femoris muscle
c. It extends the knee
d. It attaches to the anterior superior iliac spine
e. It attaches to the patella

9) Identify the following muscles. (LO17)

2) What is the common action between ALL of the quadriceps femoris muscles? (LO18)

3) What is the common proximal attachment for most of the hamstring muscles? (LO18)

4) Using the terminology you've learned, describe what the muscle name 'biceps femoris' means. (LO16)

5) What is the name of the deep fascia of the thigh that encloses the muscles of the thigh? (LO16)

6) What is the distal attachment of the biceps femoris short head? (LO18)

7) Name a muscle that you would expect to be a synergist to the extensor hallucis longus. (Hint: although you did not learn the actions of these muscles, use the terminology you've learned to predict primary actions of muscles with unknown actions). (LO16)

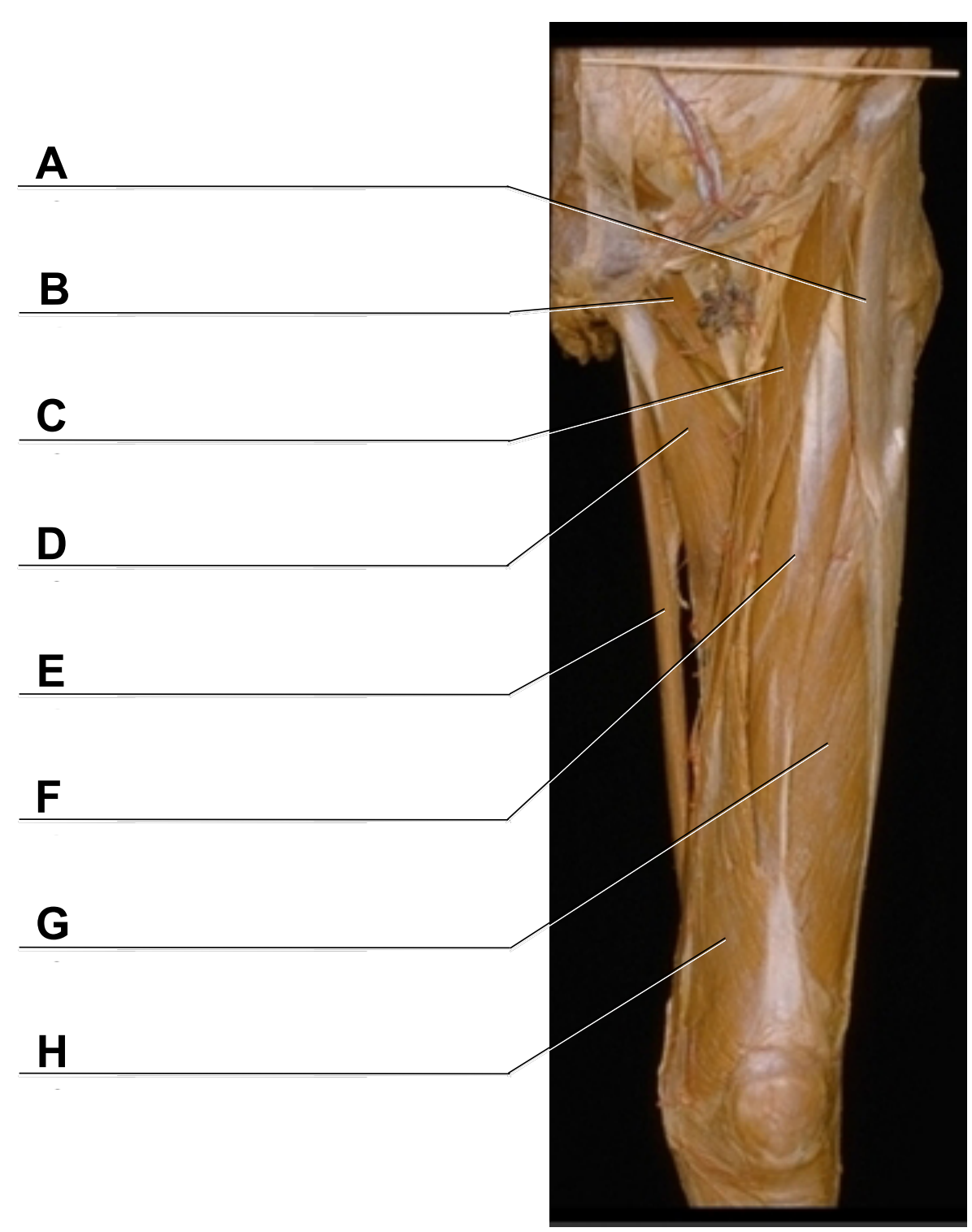

Fig. 7.1. 


\section{Lab 8: Spinal Cord and Peripheral Nerves}

Instructions: Learn the listed terms using the listed learning objectives, labeled images, lab specimens, and other resources.

LO19: Describe the terminology used to identify structures of the spinal cord and peripheral nerves.

LO20: Describe the organization and listed innervation pathways of the nervous system.

LO21: Identify the nerves and associated structures of the spinal cord and peripheral nerves.

\section{Nervous System}

Organization of the Nervous System, Reflex Arc, and Typical Muscle Innervation Pathway

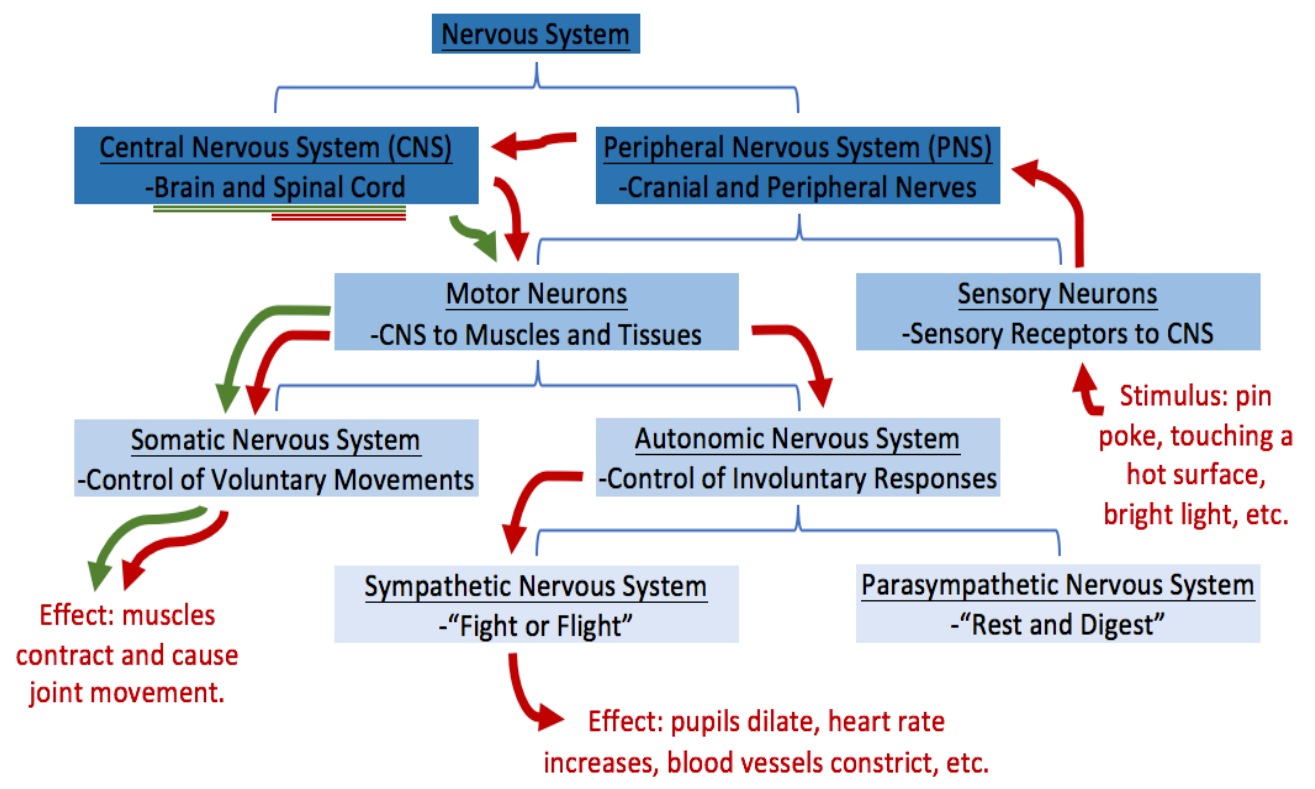

Figure 8.1. Organization of the Nervous System, Reflex Arc, and Typical Muscle Innervation Pathway
Peri $=$ around

\section{Neuron (or Nerve cell)}

$\square$ Nucleus

$\square$ Cell body

Dendrites

Dendrites receive signals from other neurons.

$\square$ Axon

Myelin

Axon terminals

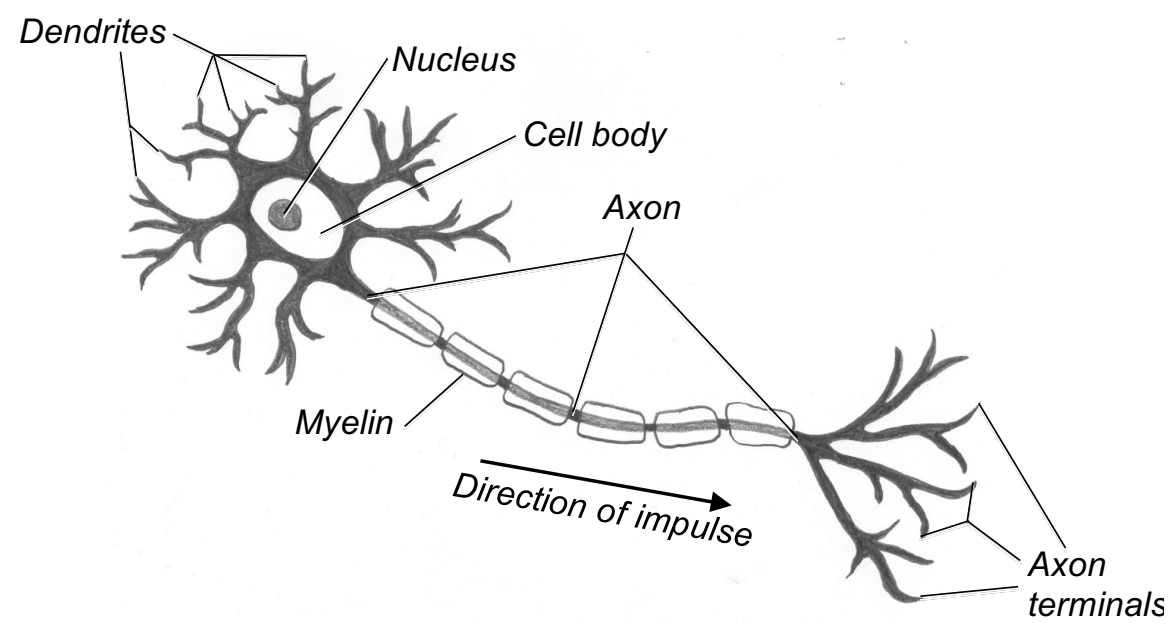

Figure 8.2. Structures of a Neuron 


\section{Meninges}

The central nervous system (CNS), which consists of the brain and spinal cord, is surrounded by three membranes (Meninx $=$ membrane). The outermost, dura mater, is the thickest and toughest meninx that separates the CNS from the surrounding skeleton. The middle, arachnoid mater, is a delicate meninx with a matrix of "spider-like" support structures under it that carry blood vessels and cerebrospinal fluid (CSF) around the central nervous system. The innermost, pia mater, is the thinnest meninx that contains the CSF and is the final barrier before reaching neural tissue. Together, the meninges protect and cushion the brain and spinal cord.

$\underline{\text { Dura }}=$ strong; $\underline{\text { Arac }}=$ spider $; \underline{P i a}=$ tender $; \underline{\text { Mater }}=$ mother

\section{Dura mater}

Arachnoid mater

- Subarachnoid space

$\square$ Pia mater

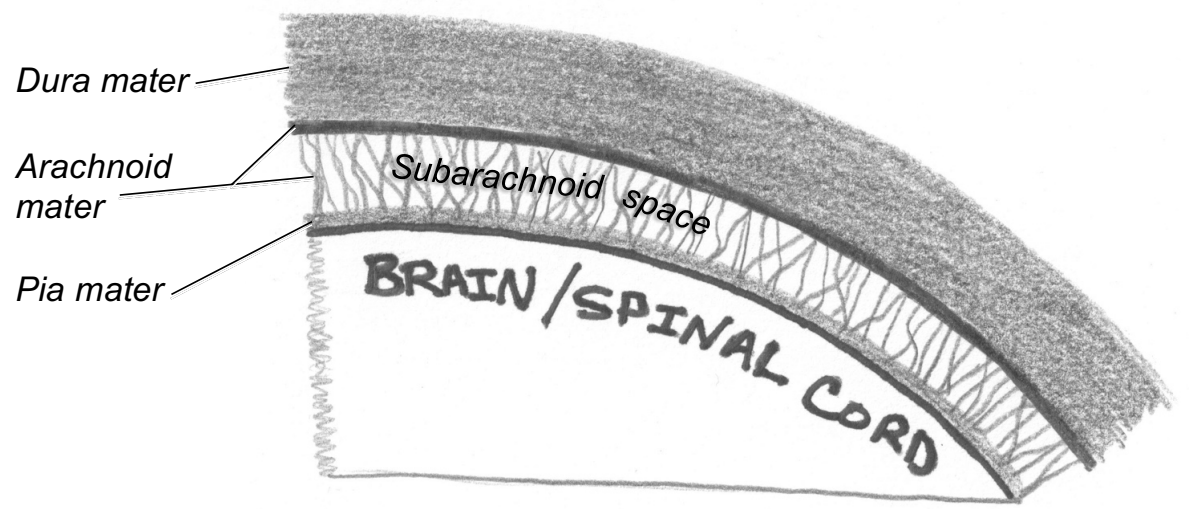

Figure 8.3. Meninges Diagram

\section{Spinal cord and spinal nerves}

\section{Gray matter}

Gray matter is the darker tissue of the spinal cord that consists mostly of nerve cell bodies and dendrites.

\section{White matter}

White matter is the lighter tissue of the spinal cord that consists mostly of myelinated axons.

\section{Anterior median fissure}

Do you remember what fissure means from Lab 1 ?

\section{Posterior sulcus}

$\underline{\text { Sulcus }}=$ a groove on the surface

\section{$\square$ Central canal}

$\square$ Ventral rootlets

$\underline{\text { Ventral }}=$ front

\section{Dorsal rootlets}

$\underline{\text { Dorsal }}=$ back

$\square$ Ventral root

Dorsal root

- Dorsal root ganglion

$\underline{\text { Ganglion }}=$ a cluster of nerve cell bodies

$\square$ Spinal nerve (or Segmental nerve)

$\square$ Ventral ramus

- Rami communicantes

- Sympathetic ganglion

Dorsal ramus

Do you remember what ramus means from Lab 4 ? 
Conus medullaris

Cauda equina

Cauda equina $=$ horse's tail (Latin)

Filum terminale

$\underline{\text { Filum terminale }}=$ terminal thread $($ Latin $)$

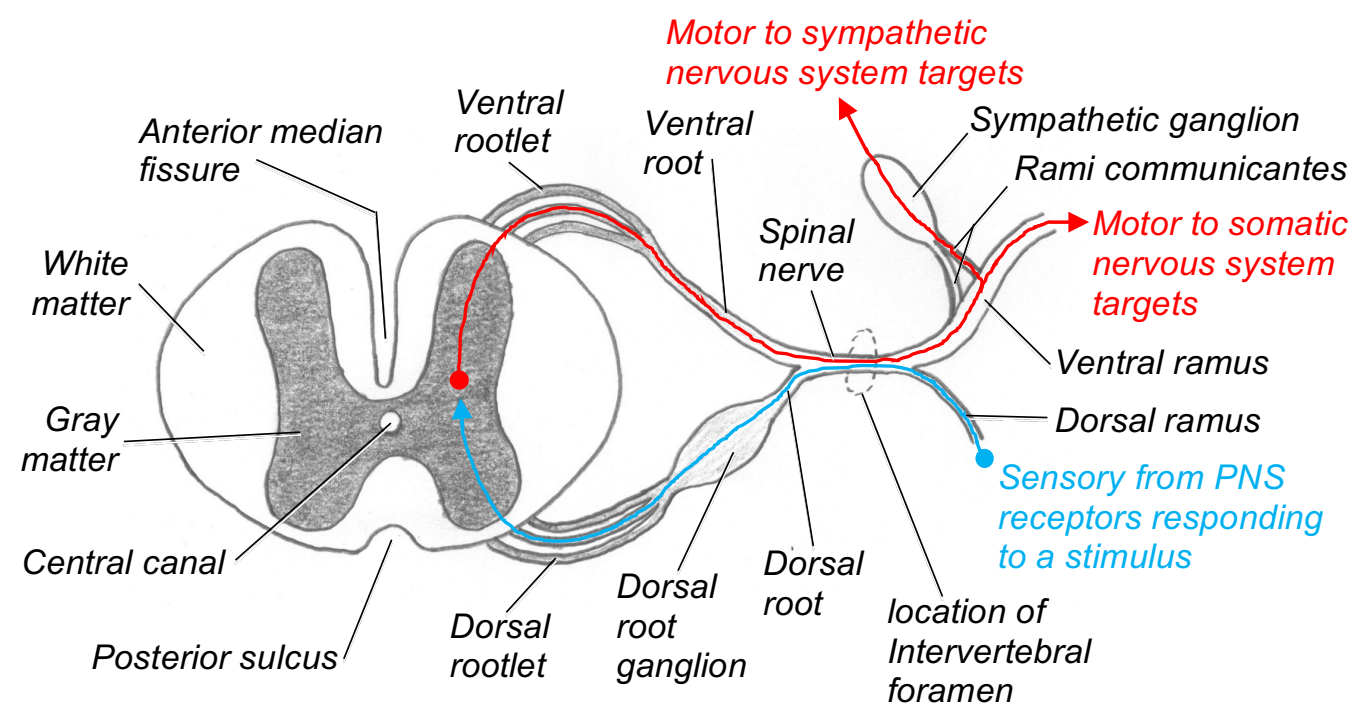

Figure 8.4. Diagram of Transverse Section of Spinal Cord and Spinal Nerve Components with Reflex Arc Pathway

There are 8 pairs of cervical spinal nerves, 12 pairs of thoracic spinal nerves, $\mathbf{5}$ pairs of lumbar spinal nerves, $\mathbf{5}$ pairs of sacral spinal nerves, and 1 pair of coccygeal spinal nerves. All spinal nerves, except for the first pair and last six pairs, exit the vertebral column through intervertebral foramina. The first pair of spinal nerves $(\mathrm{C} 1 \mathrm{n}$.) exits the vertebral column between the occipital bone and atlas. The ventral and dorsal rami of sacral nerves 14 exit the vertebral column through the anterior and posterior sacral foramina respectively, and the $5^{\text {th }}$ pair of sacral nerves and the one pair coccygeal nerves exits the vertebral column through the sacral hiatus.

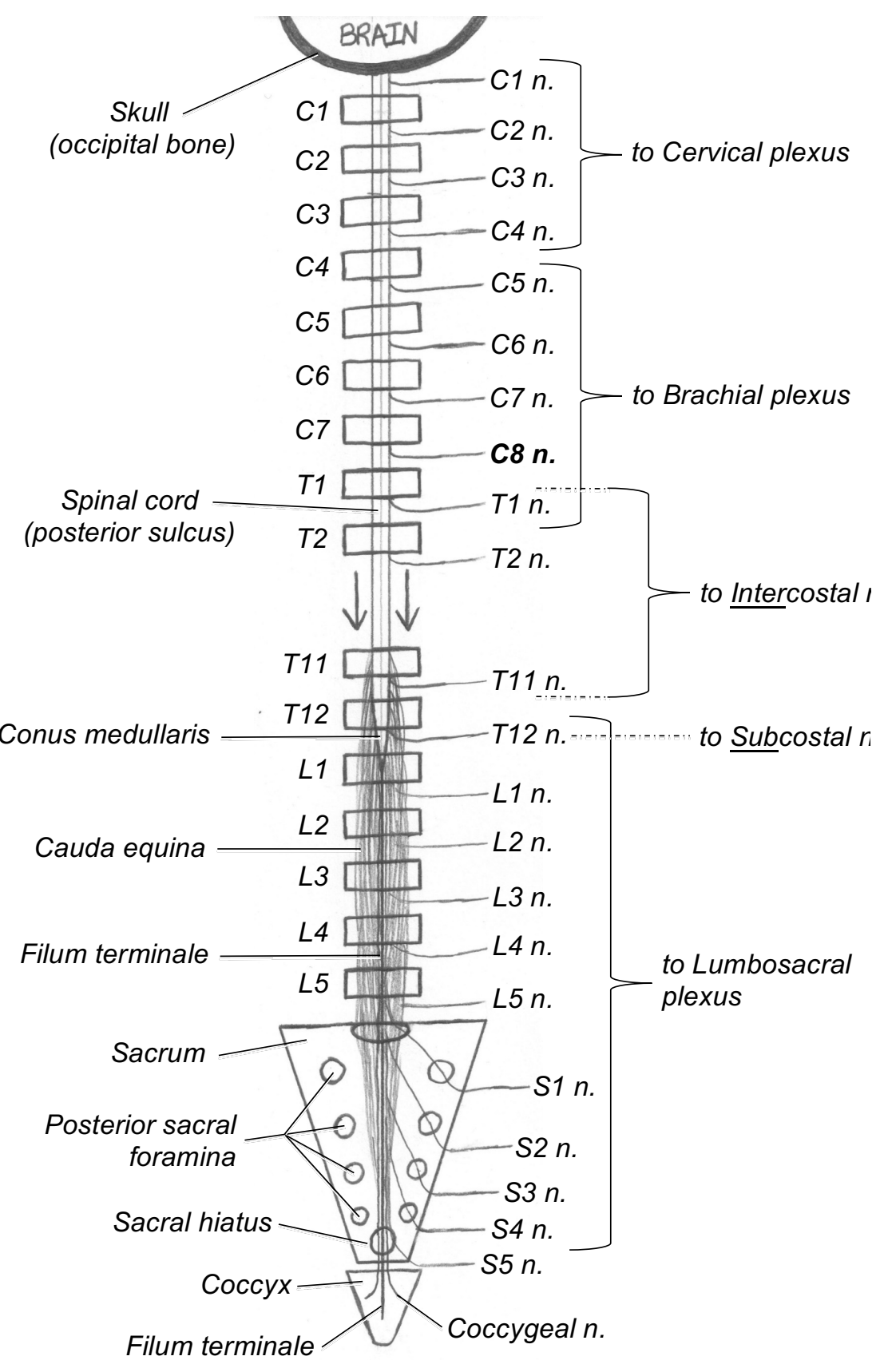

Figure 8.5. Diagram for Identifying Spinal Nerves from Surrounding Structures and Vertebrae 


\section{Clinical Application: Lumbar Puncture}

A lumbar puncture, also called a spinal tap, is a medical procedure done to collect a sample of cerebrospinal fluid (CSF) from a patient. This procedure is typically done for certain diagnostic purposes; since CSF surrounds the entire central nervous system, analysis of the CSF can reveal the health of the environment around the CNS. In this procedure, a needle is inserted between the $3^{\text {rd }}$ and $4^{\text {th }}$ lumbar vertebrae until it punctures the dura mater and arachnoid mater. At this point, the CSF can then be collected from the subarachnoid space. The needle is inserted between the $3^{\text {rd }}$ and $4^{\text {th }}$ lumbar vertebrae so there is less chance for injuring the spinal cord; the spinal cord/conus medullaris ends at around the level of the $1^{\text {st }}$ lumbar vertebrae, and the cauda equina is more likely to "slide around" the needle than be punctured/injured by it.

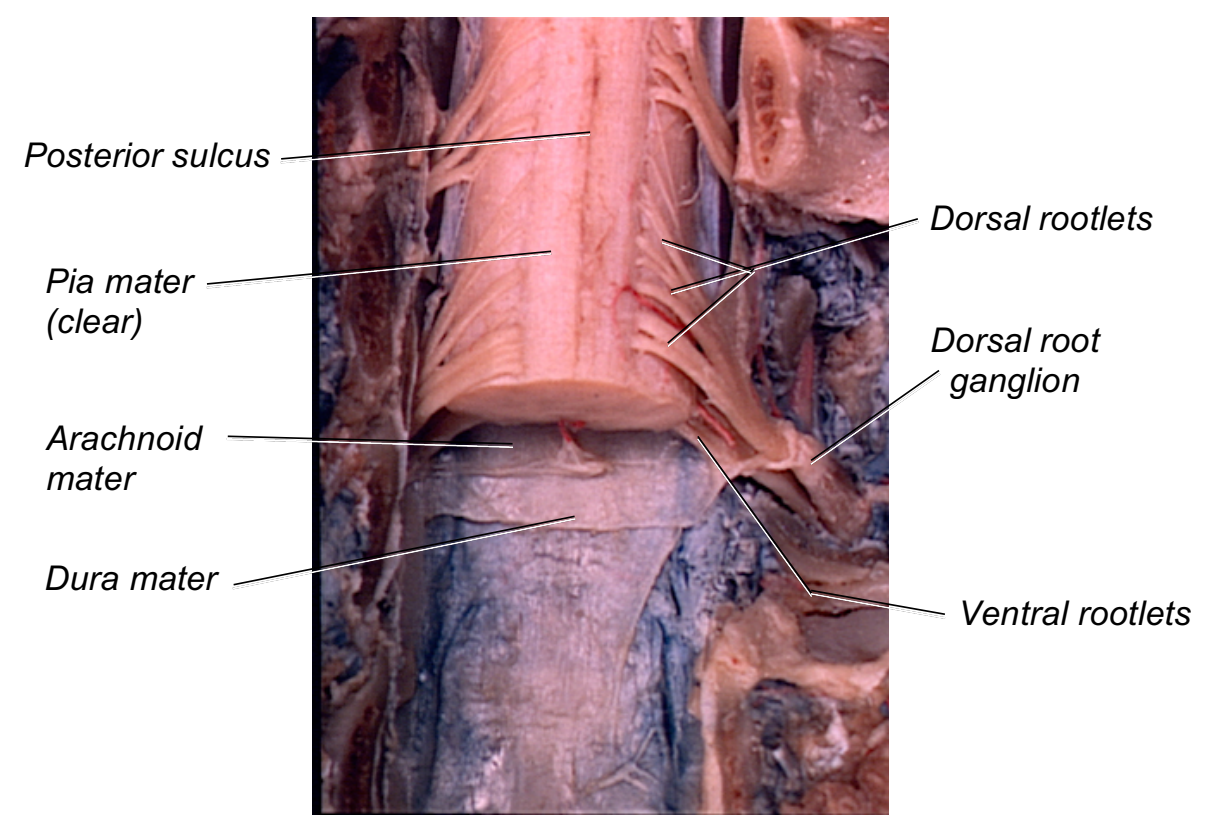

Figure 8.6. Cervical Spinal Cord (cut), Posterior View

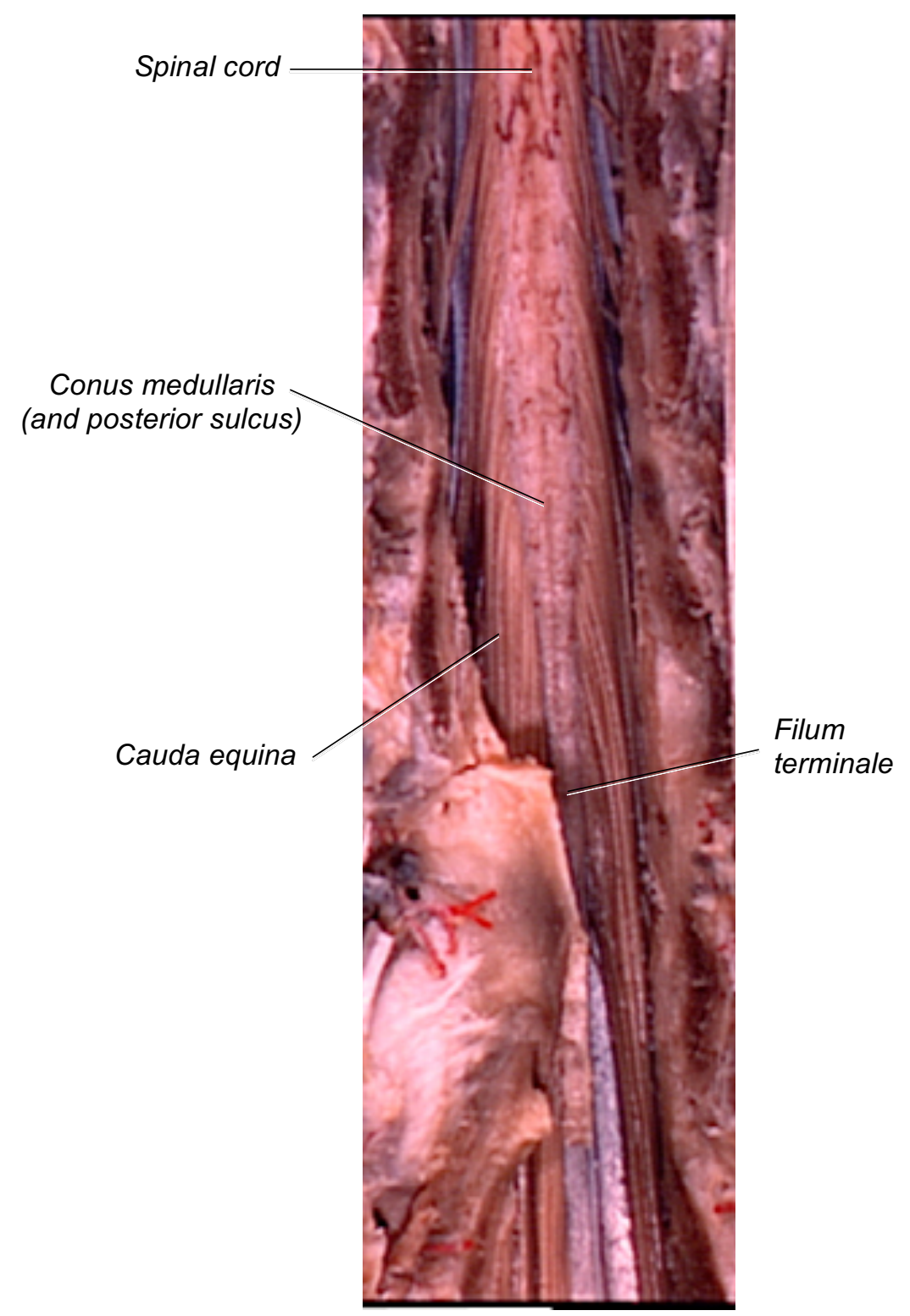

Figure 8.7. Distal Spinal Cord, Posterior View 


\section{Clinical Application: Epidural (for childbirth)}

An epidural is a medical procedure commonly done to relieve pain during labor and delivery. In this procedure, a needle is inserted into the vertebral canal without puncturing the dura mater. With the tip of the needle/catheter above the dura mater (epi = above; dural $=$ of the dura mater), anesthetics are injected into the epidural space. The anesthetics then pass through the meninges and block neuronal impulses, thus inhibiting the transmission of sensation (in this case, pain) to the brain. Just like in a lumbar puncture, the epidural needle can be inserted between L3/L4 vertebrae, but because the pain impulses from the uterus reach the spinal cord at levels between T10-L1, epidurals are more commonly placed more superiorly. A common location for epidural placement is $T 7$, which is easily identifiable at about the level of the inferior border of the scapula. When the drugs are injected, gravity can pull the drugs downward, blocking everything below the epidural location. Therefore, administering an epidural above the level of T10 can relieve labor pain from uterine contractions as well as pain from the pelvic floor transmitted by the pudendal nerve (from S2, S3, and S4 spinal nerves).

A plexus is a network of nerves. The three biggest nerve plexuses in the body are a cervical plexus which serves the head and neck, a brachial plexus which serves the upper limb, and a lumbosacral plexus which serves the pelvis, genitalia, and lower limb. In this course, we will learn the brachial plexus in greater detail than the cervical or lumbosacral plexuses.

Cervical plexus $\left(C_{1}-C_{4}\right.$ ventral rami)

Phrenic $n$.
Brachial plexus $\left(C_{5}-T_{1}\right.$ ventral rami)

The brachial plexus is divided into roots, trunks, divisions, cords, and branches.

\section{Roots}

$\square \mathrm{C}_{5}$ ventral rami

$\square \mathrm{C}_{6}$ ventral rami

$\square \mathrm{C}_{7}$ ventral rami

$\square \mathrm{C}_{8}$ ventral rami

$\square T_{1}$ ventral rami

\section{Trunks}

$\square$ Superior trunk

$\square$ Middle trunk

Inferior trunk

\section{Divisions}

$\square$ Anterior division (x3)

$\square$ Posterior division $(\mathrm{x} 3)$

Each trunk divides into an anterior division and posterior division.

\section{Cords}

$\square$ Lateral cord

Formed from two anterior divisions from the superior and middle trunks.

\section{Posterior cord}

Formed from all three posterior divisions.

\section{Medial cord}

Formed from the one anterior division from the inferior trunk. 


\section{Branches}

The terminal branches of the brachial plexus are the nerves that will innervate the muscles of the upper limb. Some muscles are listed under the nerve that innervates them.

$\square$ Musculocutaneous $\mathrm{n}$.

○ Biceps brachii (both heads)

- Brachialis

- Coracobrachialis

$\square$ Axillary $\mathrm{n}$.

\section{○ Deltoid}

$\square$ Radial $n$.

- Triceps brachii (all heads)

$\square$ Median $\mathrm{n}$.

$\square$ Ulnar $\mathrm{n}$.

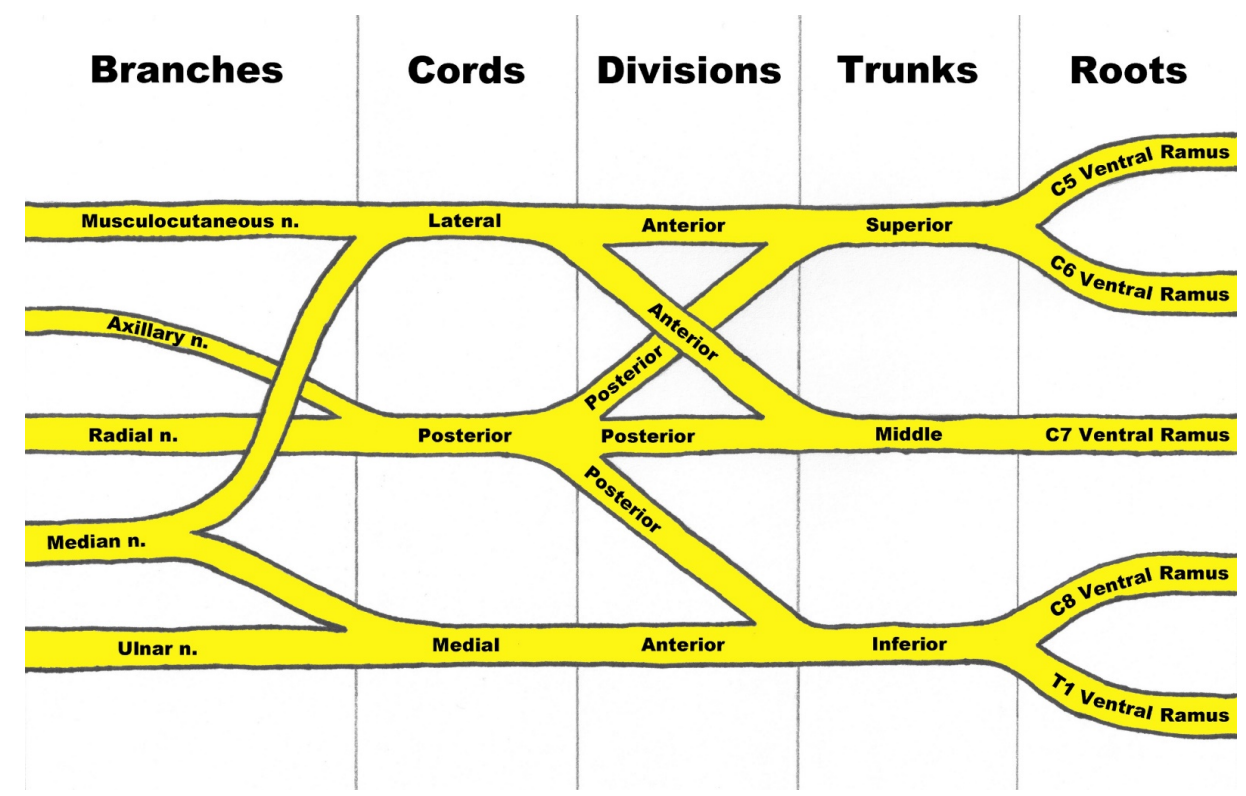

Figure 8.8. Diagram of Right Brachial Plexus, Anterior View

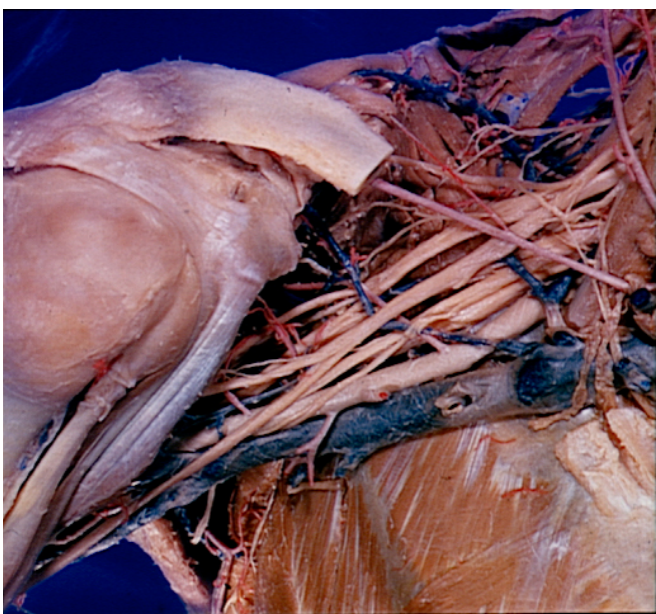

Figure 8.9. Right Brachial Plexus, Anterior View (No Labels)

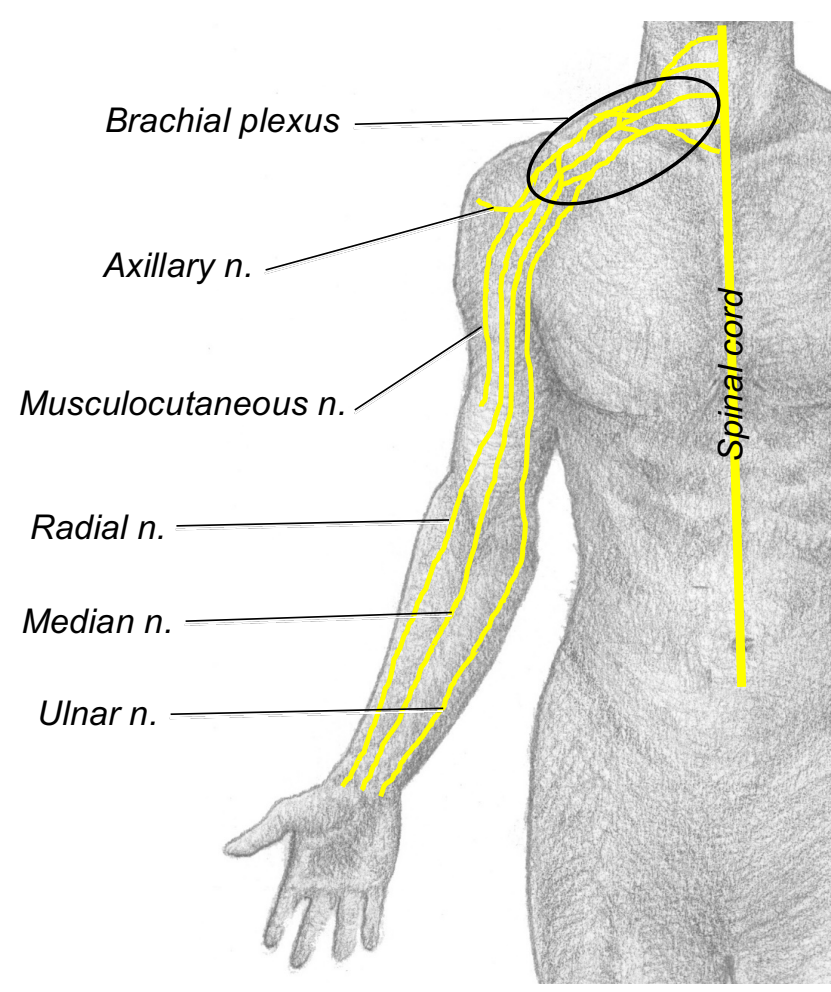

Figure 8.10. Right Upper Limb Nerves Diagram, Anterior View 


\section{Intercostal nerves}

Intercostal nerves are not part of a plexus; they are simply ventral rami from thoracic spinal nerves that travel directly to the region they innervate. Intercostal nerves are found between ribs and between the external and internal intercostal muscles. Notice how there are only 11 intercostal nerves, but there are 12 pair of ribs. This is because for a nerve to be an intercostal nerve, it must be between two ribs. The $12^{\text {th }}$ thoracic spinal nerve is not between two ribs; it is below the last rib, which is why this nerve is called the subcostal nerve.

Also listed are some characteristic dermatomes, or identifiable regions of skin supplied by a single nerve.

Intercostal nerves $\left(\mathrm{T}_{1}-\mathrm{T}_{11}\right.$ ventral rami)

Subcostal nerve ( $T_{12}$ ventral ramus)

$\mathrm{T}_{4}$ ventral rami - sensory innervation at the nipples

$\mathrm{T}_{6-7}$ ventral rami - sensory innervation over the xiphoid process

$\mathrm{T}_{10}$ - sensory innervation at the naval

Lumbosacral plexus $\left(T_{12}-S_{4}\right.$ ventral rami)

$\square$ Superior gluteal $\mathrm{n}$.

- Gluteus medius

- Gluteus minimus

$\square$ Inferior gluteal $\mathrm{n}$.

- Gluteus maximus

Femoral $n$.

- Sartorius

- Rectus femoris
- Vastus lateralis

- Vastus medialis

- Vastus intermedius

\section{Sciatic $n$.}

- Semimembranosus

- Semitendinosus

- Biceps femoris (both heads)

Tibial $n$.

Common fibular $\mathrm{n}$.

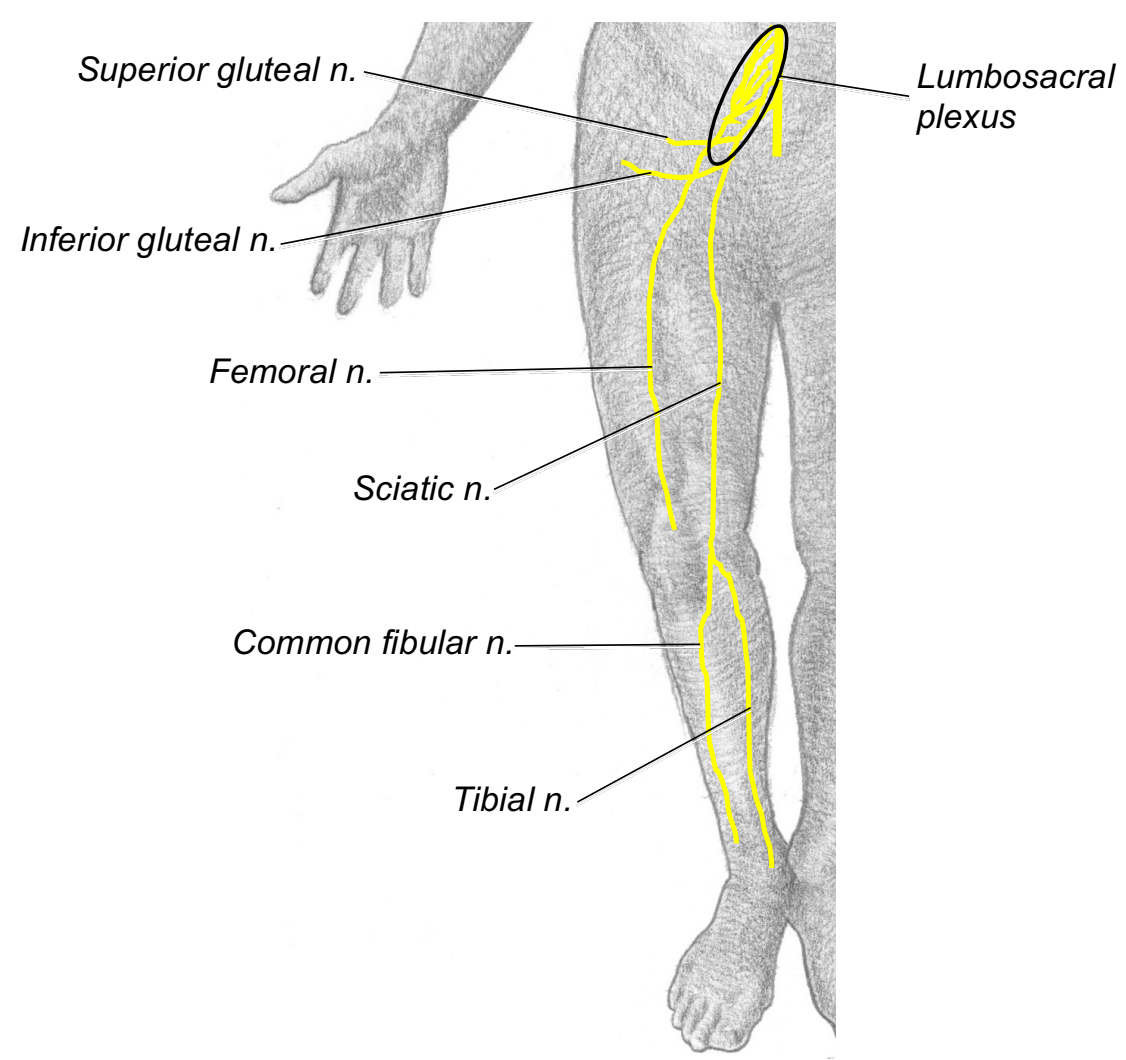

Figure 8.11. Right Lower Limb Nerves Diagram, Anterior View 
and self-assess your knowledge.

1) Fill in the following table with the number of each vertebrae/spinal nerves in each region. (LO21)

\begin{tabular}{|c|c|c|}
\hline & \# Vertebrae & $\begin{array}{c}\text { \# Spinal } \\
\text { nerve pairs }\end{array}$ \\
\hline Cervical & & \\
\hline Thoracic & & \\
\hline Lumbar & & \\
\hline Sacral & & \\
\hline Coccygeal & & \\
\hline
\end{tabular}

2) What named spinal nerve would exit the vertebral column through the intervertebral foramen formed by the $6^{\text {th }}$ and $7^{\text {th }}$ cervical vertebrae? (LO21)

3) Which of the following nervous systems is NOT part of a reflex arc? (LO20)

a. Central nervous system

b. Peripheral nervous system

c. Somatic nervous system

d. Autonomic nervous system

e. Sympathetic nervous system

f. Parasympathetic nervous system

4) What is the difference between a ganglion and a plexus? (LO19)

5) The coccygeal spinal nerves exit the vertebral column through the
6) What does the term ramus mean? (LO19)

7) What part of a neuron receives signals from other neurons? (LO20)

8) What does the term pia mean? (LO19)

9) What two structures does the filum terminale attach? (LO21)

10)What nerve innervates the quadriceps muscles? (LO20)

11)What structures form the posterior cord of the brachial plexus? (LO21)

12) The $C 1$ nerve has another name based on its location below the occipital bone. Using the terminology you've learned, what would you expect the other name of this nerve be? (LO19)

13)In pathway order, list all the structures and components of a reflex arc beginning with touching a hot surface to flexing your forearm to quickly pull back your hand from the hot surface. (LO20) 


\section{Lab 9: Brain}

Instructions: Learn the listed terms using the listed learning objectives, labeled images, lab specimens, and other resources.

LO22: Describe the terminology used to identify structures of the brain.

LO23: Describe the flow pathways of cerebrospinal fluid and venous blood of the brain.

LO24: Identify the regions and associated structures of the brain.

\section{Meninges}

Since the brain is part of the CNS, it is surrounded by the same meninges that surround the spinal cord.

$\square$ Dura mater

$\square$ Arachnoid mater

- Subarachnoid space

Pia mater

\section{Brain}

$\square$ Gray matter

$\square$ White matter

Like the spinal cord, the brain has gray matter and white matter. In the spinal cord, the gray matter was on the inside and the white mater was on the outside. However, in the brain, the gray matter is on the outside and the white matter is on the inside.

\section{Clinical Application: Meningitis}

Meningitis is a condition where the meninges around the brain become inflamed. There are five different types of meningitis: bacterial, viral, fungal, parasitic, and non-infectious. Symptoms of meningitis can include headache, fever, stiff neck, nausea, vomiting, confusion, sleeplessness, sluggishness, skin rash, chills, shivering, lethargy, seizures, light sensitivity, lack of appetite, and/or changes in heart and respiratory rates. Meningitis can be diagnosed by analyzing a sample of cerebrospinal fluid (CSF) collected from a lumbar puncture procedure. Bacterial and viral meningitis are the most common forms of meningitis. Bacterial meningitis is usually severe and can be deadly, even when treated with antibiotics. Fortunately, the most common forms of bacterial meningitis are preventable with vaccines. Viral meningitis is typically mild and resolves after 7-10 days without treatment. Unlike bacterial meningitis, most forms of viral meningitis are not preventable with vaccines. Both bacterial and viral meningitis are contagious and can be spread by airborne droplets or direct contact.

\section{Vesicles of the brain}

There are 5 main vesicles (regions) of the brain: telencephalon, diencephalon, mesencephalon, metencephalon, and myelencephalon. Learn these regions and the structures found within each of them. 


\section{Telencephalon (or Cerebrum)}

$\square$ R. \& L. Cerebral hemispheres
○ Frontal lobe
- Parietal lobe
- Temporal lobe
- Occipital lobe

Gyrus

Gyri are the mounds on the cerebral cortex (outer surface).

\section{$\square$ Sulcus}

Sulci are the crevices on the cerebral cortex (outer surface).

$\square$ Corpus callosum

The corpus callosum is the largest structure that allows signals to travel from one cerebral hemisphere to the other.

$\square$ Septum pellucidum

$\square$ Lateral ventricles (2) (or $1^{\text {st }}$ and $2^{\text {nd }}$ ventricles)

Ventricles are cerebrospinal fluid-filled cavities inside the brain. There are four ventricles in total. The septum pellucidum separates the two lateral ventricles.

\section{$\square$ Choroid plexuses}

A choroid plexus is a group of specialized cells that produce cerebrospinal fluid from the blood in a nearby vessel. Choroid plexuses are found in each ventricle.

\section{Longitudinal fissure}

The longitudinal fissure separates the cerebral hemispheres.

\section{Transverse fissure}

The transverse fissure separates the cerebrum and the cerebellum.

\section{Diencephalon}

$\square$ Fornix

$\square$ Thalamus

\section{Interthalamic adhesion}

The interthalamic adhesion is the second largest structure that allows signals to travel from one cerebral hemisphere to the other.

$\square$ Hypothalamus

$\square$ Pineal gland

\section{Habenula}

The habenula connects the pineal gland to the thalamus.

$\square$ Anterior commissure

\section{Posterior commissure}

The anterior and posterior commissures also allow signals to travel from one cerebral hemisphere to another.

\section{Interventricular foramen}

The two interventricular foramina allow cerebrospinal fluid produced in from the choroid plexuses in each of the lateral ventricles to flow into the third ventricle.

\section{$\square 3^{\text {rd }}$ ventricle \\ $\square$ Choroid plexus \\ $\square$ Hypophysis (or Pituitary gland) \\ Infundibulum}

The infundibulum connects the hypophysis to the hypothalamus.

Optic chiasm

Chiasm $=$ a crossing or intersecting of two tracts.

The optic chiasm is where the two optic nerves intersect.

Mammillary bodies 


\section{Mesencephalon (or Midbrain)}

\section{Corpora quadrigemina}

Corpora quadrigemina $=$ quadruplet (four) bodies (Latin)

- Superior colliculi

- Inferior colliculi

The superior colliculi process sight and the inferior colliculi process sound.

Cerebral aqueduct (or Mesencephalic aqueduct)

The cerebral aqueduct allows cerebrospinal fluid to flow from the $3^{\text {rd }}$ ventricle to the $4^{\text {th }}$ ventricle.

\section{Metencephalon}

Pons

Cerebellum

○ Folia

Folia are the folds on the surface of the cerebellum.

\section{- Arbor vitae}

The arbor vitae is the white matter structure in the cerebellum that resembles the shape of a tree. $\underline{\text { Arbor }}=$ tree; $\underline{\text { vitae }}=$ life.

$\square$ Cerebral aqueduct (or Mesencephalic aqueduct)

$\square 4^{\text {th }}$ ventricle

Cerebellar peduncles (grouped)

The cerebellar peduncles are the structures that attach the cerebellum to the brainstem.

\section{Myelencephalon}

\section{Medulla oblongata}

Recall the brain and spinal cord (CNS) are connected by continuous nerve fibers, yet the gray and white matter in these organs are in opposite locations. The medulla oblongata is the structure where the gray and white matter "switch places" between the two major CNS organs.
$4^{\text {th }}$ ventricle
Choroid plexus
Medial aperture
Lateral apertures
Central canal

Cerebrospinal fluid (CSF) can exit the $4^{\text {th }}$ ventricle through 4 openings: one medial aperture, two lateral apertures, and one opening to the central canal of the spinal cord. After leaving the $4^{\text {th }}$ ventricle through any of these openings, the next place CSF will flow to is the subarachnoid space surrounding the brain and spinal cord.

\section{Clinical Application: Alzheimer's Disease}

Alzheimer's disease began to receive recognition over a century ago when Dr. Alois Alzheimer, a German physician, studied a 51-year-old female who had this disease. After the patient passed away, a brain autopsy revealed the characteristic plaques and tangles that characterize Alzheimer's disease. In many living patients today, the disease is assumed without official diagnosis since the diagnosis requires a brain sample. Symptoms of the disease include memory loss, confusion, and behavioral changes due to the degenerating neural tissue. There is currently no known cure for Alzheimer's Disease. 


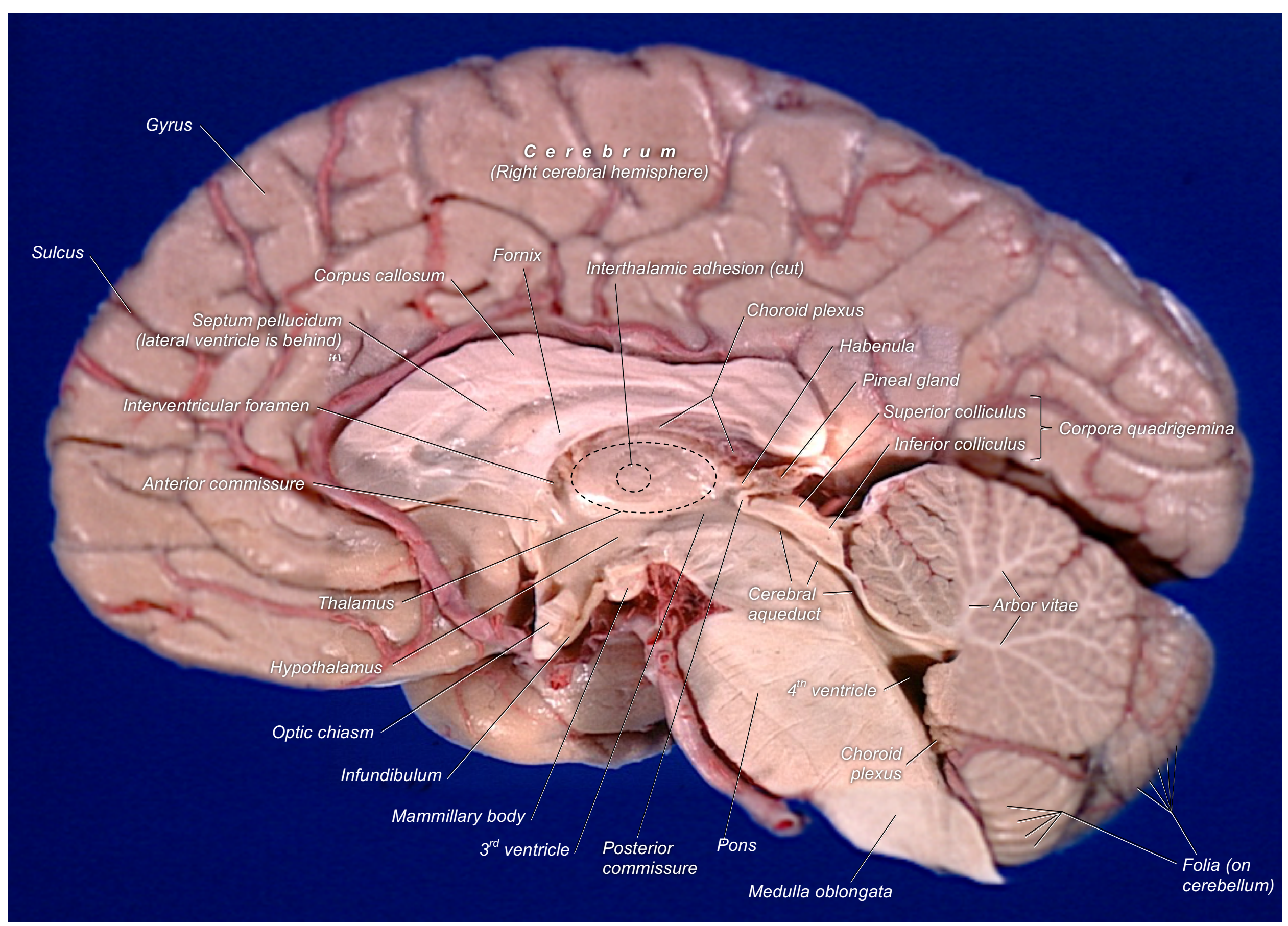

Figure 9.1. Hemisected Brain (Right Half), Midsagittal View. 


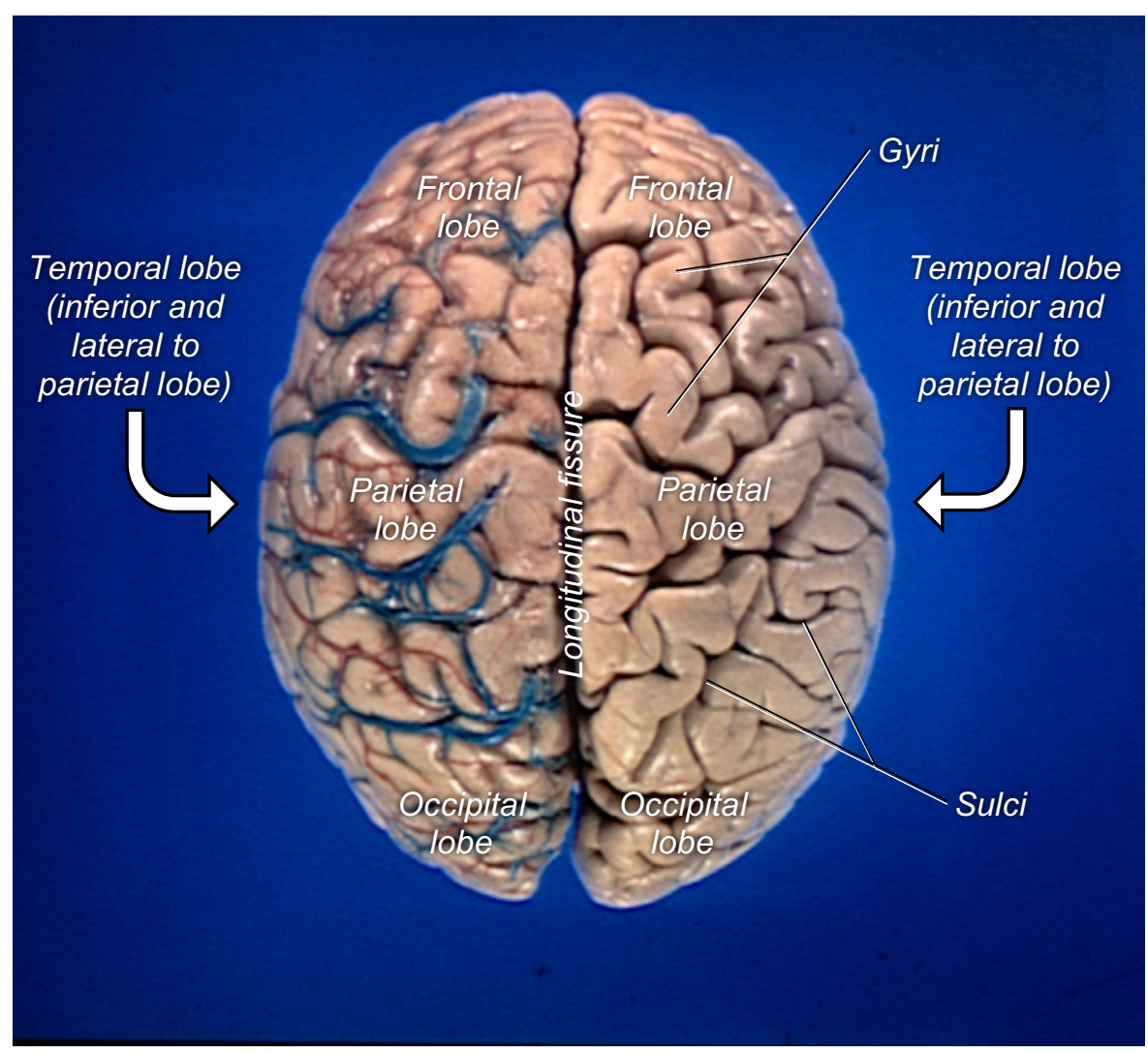

Figure 9.2. Brain (Cerebrum), Superior View

\section{Dural reflections}

Dural reflections are folds of dura mater that extend into the fissures of the brain.

\section{Falx cerebri}

The falx cerebri sits into the longitudinal fissure and physically separates the two cerebral hemispheres.

\section{Tentorium cerebelli}

The tentorium cerebelli sits into the transverse fissure and physically separates the cerebrum and cerebellum.

\section{Dural sinuses}

Dural sinuses, which contain venous blood, are contained within dural reflections and/or dura mater. Dural sinuses collect blood and circulated cerebrospinal fluid and shunt them to through the jugular foramen to be returned back to the heart.

$\square$ Superior sagittal sinus

Inferior sagittal sinus

The superior and inferior sagittal sinuses are contained within the falx cerebri.

\section{$\square$ Straight sinus \\ Confluence of the sinuses}

The straight sinus and confluence of the sinuses are contained within the intersection of the falx cerebri and tentorium cerebelli. The straight sinus receives blood from the inferior sagittal sinus. The confluence of the sinuses collects blood from the superior sagittal and straight sinuses before shunting it laterally to the transverse sinuses.

\section{Transverse sinuses}

The transverse sinuses are contained within the tentorium cerebelli and shunt blood to the sigmoid sinuses.

\section{Sigmoid sinuses}

The sigmoid sinuses are contained within the dura mater lining the base of the skull, and they transport venous blood to the jugular foramen.

\section{Arachnoid granulations}

Arachnoid granulations are projections of the arachnoid mater into the dural sinuses that allow cerebrospinal fluid (CSF) in the subarachnoid space to enter into the dural sinuses. Because cerebrospinal fluid is initially produced from blood by the choroid plexuses, it makes sense to recycle the circulated CSF back into the venous blood. 


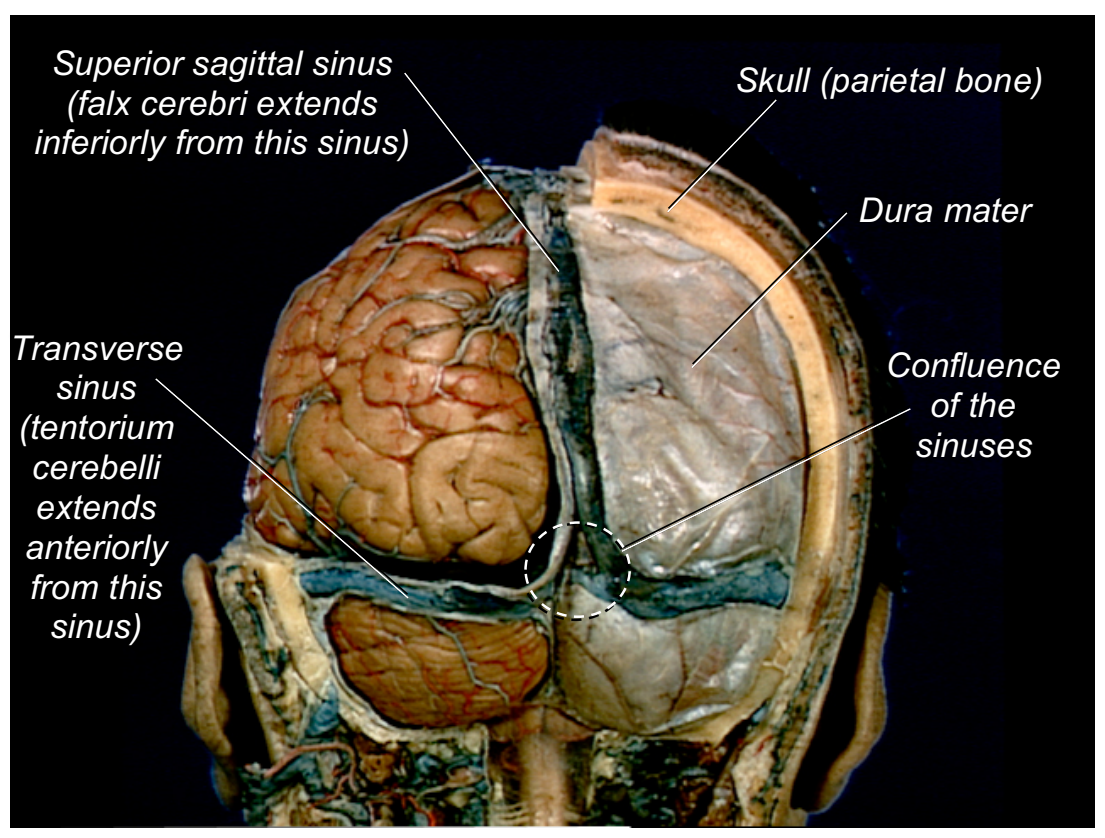

Figure 9.3. Brain and Dural Sinuses, Posterior View

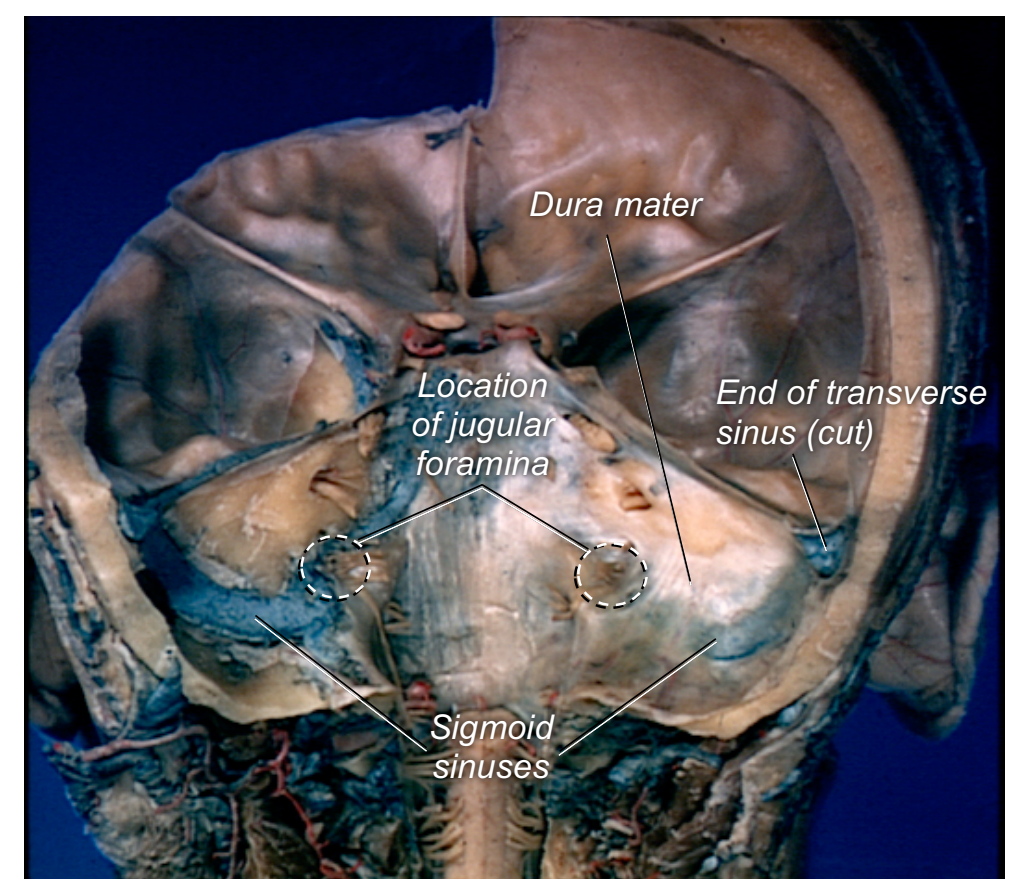

Figure 9.4. Sigmoid Sinuses, Posterosuperior View

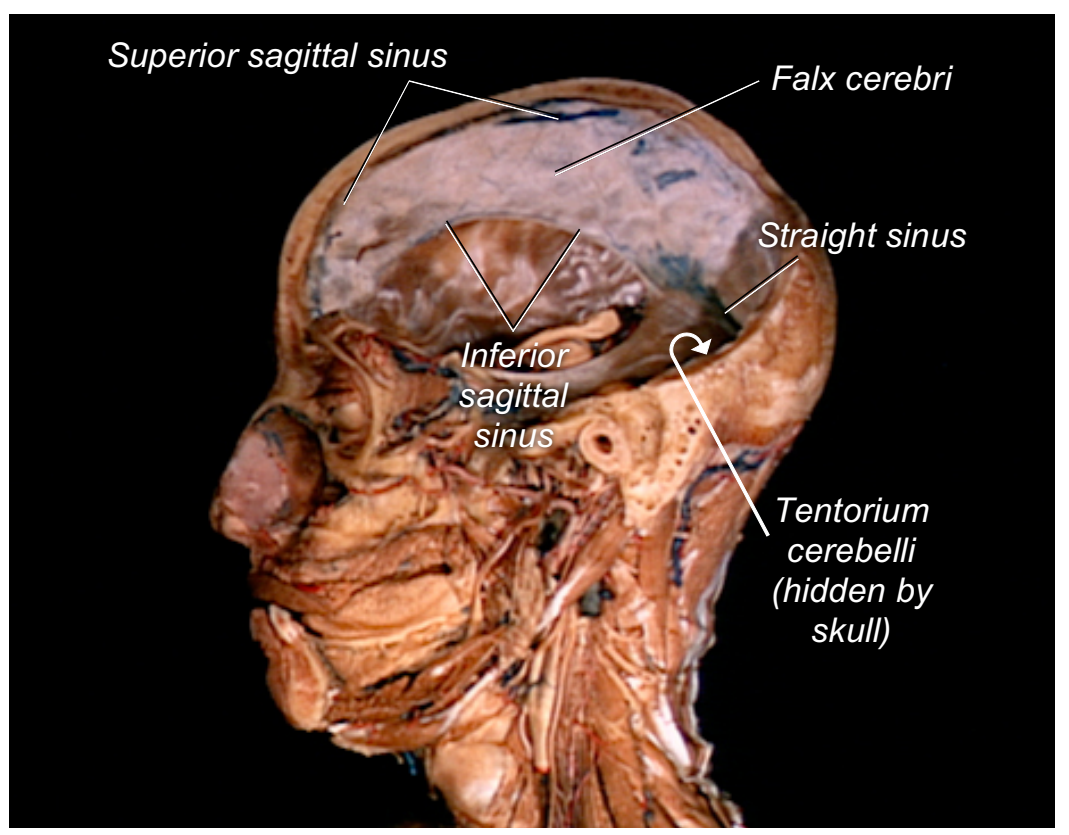

Figure 9.5. Falx Cerebri and Dural Sinuses, Left Lateral View

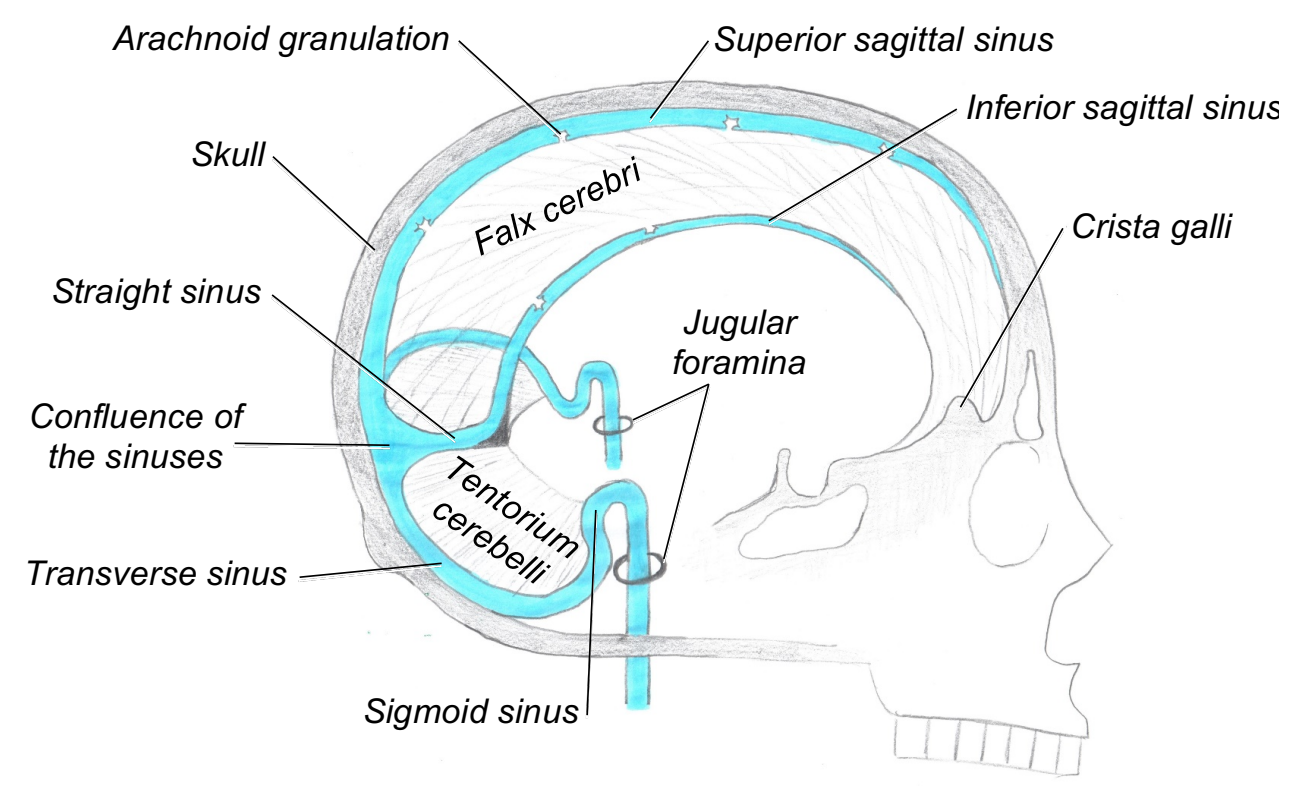

Figure 9.6. Diagram of Dural Reflections and Dural Sinuses, Right Lateral View 
1) What is the difference between a ventricle and a vesicle? (LO 22)

2) Which vesicle is the third ventricle found in? (LO24)

3) Using the terminology association (and some knowledge from Lab 1), what bony structure would you suppose the hypophysis sits into? (LO 22)

4) What structure produces cerebrospinal fluid? (LO24)

5) How many ventricles are there in the brain? (LO24)

6) What structure is in both the mesencephalon and the metencephalon? (LO24)

7) List the structures that allow transmission of signals between the two cerebral hemispheres. (LO24)

8) What does the term hypo mean? (Hint, if you do not remember the meaning of this term from Lab 6, consider the locations of the thalamus and hypothalamus.) (LO 22)

9) List the sinuses that flow directly into the confluence of sinuses. (LO23)
11)Identify the structures labeled in the image below.

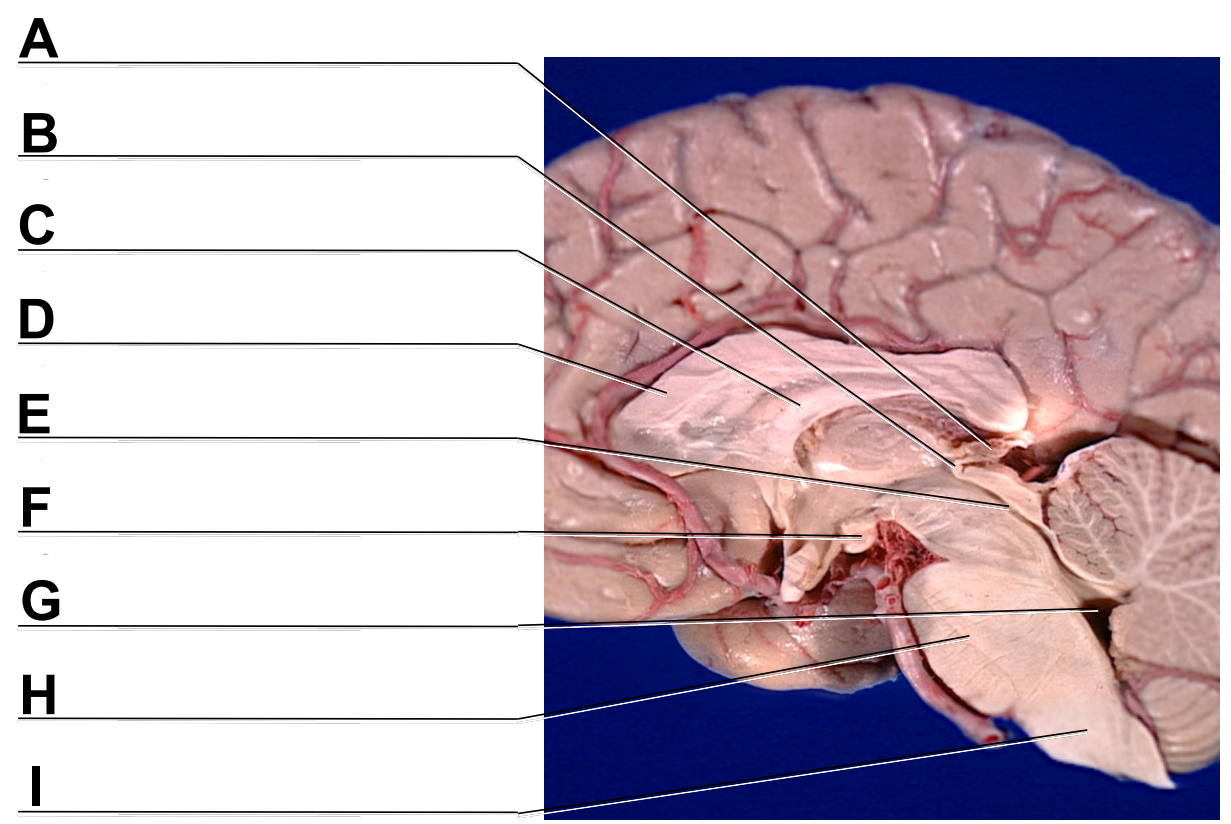

Fig. 9.1.

12)In order, list the structures that cerebrospinal fluid (CSF) and venous blood flows through starting with a choroid plexus in the lateral ventricle and ending at the jugular foramen. (LO23) 


\section{Lab 10: Cranial Nerves}

Instructions: Learn the listed terms using the listed learning objectives, labeled images, lab specimens, and other resources.

LO25: Describe the terminology used to identify structures of the cranial nerves.

LO26: Identify the cranial nerves.

LO27: Describe the cranial nerves (i.e. the openings they pass through, the type of innervation they supply, and their innervation target).

\section{Cranial Nerves}

There are twelve pairs of cranial nerves (CNs). Although you need to know both, you can identify cranial nerves using the "CN \#" method or the name of the cranial nerve. If you use the "CN \#" method, you must use roman numerals to number the cranial nerves. If you are unfamiliar with how roman numerals work, you should familiarize yourself with them (at least for numbers 1-12) since they are commonly named this way.

The cranial nerves carry motor, sensory, and/or special sensory signals. Motor signals innervate muscles/tissues, sensory signals send information to the CNS from sensory receptors, and special sensory signals send information to the CNS from the special senses (vision, hearing and balance, and taste). Some cranial nerves carry more than one type of signal; one cranial nerve even carries all three types of signals.

To enter/exit the skull, cranial nerves pass through openings in the basicranium (base of the skull). Review the openings in the base of the skull that you learned in Lab 1, and learn which opening(s) each nerve passes through (see next page).
The olfactory nerve $(\mathrm{CN} \mathrm{I})$ has distinct parts to it: the tract, bulb, and nerves. Learn these specific structures for this cranial nerve.

The trigeminal nerve $(\mathrm{CN} \mathrm{V})$ has three branches to it: the ophthalmic, maxillary, and mandibular nerves which can be called $\mathbf{C N}$ V1, CN V2, and CN V3 respectively.

Cranial nerves are numbered/named anterior to posterior based on their location. Many students use mnemonic phrases to help them remember the order of the cranial nerves. One popular mnemonic for the order of the nerves is:

Oh Once One Takes The Anatomy Final, Very Good

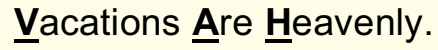

Another popular mnemonic for helping remember the type of innervation is "Some Say Money Matters, But My Brother Says Big Brains Matter More" where S = Sensory or Special Sensory, $\bar{M}=$ Motor, and $B=$ Both (or multiple). This can help you remember the types of innervation, but you still need to remember sensory vs. special sensory and what types are included with "both."

Lastly, here are some terms that may help you learn the material more efficiently: $\underline{t r i}=$ three; $\underline{g l o s s o}=$ tongue; ophth $=$ eye; olfaction $=$ the sense of smell.

Now learn about the cranial nerves on the next page and identify them using the following pages with labeled images. 
Cranial Nerves (CNs) Table

\begin{tabular}{|c|c|c|c|c|c|}
\hline$\checkmark$ & CN\# & CN Name & Associated opening(s) & Type & Innervation Target \\
\hline $\bar{\square}$ & $\mathrm{CNI}$ & Olfactory $\mathrm{n}$. & $\begin{array}{l}\text { Olfactory foramina (in the } \\
\text { cribriform plate) }\end{array}$ & Special Sensory & Olfactory epithelium \\
\hline$\square$ & $\mathrm{CN}$ II & Optic n. & Optic canal & Special Sensory & Retina of the eye \\
\hline$\square$ & CN III & Oculomotor $\mathrm{n}$. & Superior orbital fissure & Motor & $\begin{array}{l}\text { Inferior oblique, medial rectus, superior rectus, and } \\
\text { inferior rectus muscles of the eye }\end{array}$ \\
\hline$\square$ & CN IV & Trochlear $\mathrm{n}$. & Superior orbital fissure & Motor & Superior oblique muscle of the eye \\
\hline$\square$ & $\mathrm{CN} \mathrm{V}$ & Trigeminal $\mathrm{n}$. & $\begin{array}{l}\text { (see individual branches } \\
\text { below) }\end{array}$ & -- & -- \\
\hline$\square$ & CN V1 & Ophthalmic $n$. & $\begin{array}{l}\text { Superior orbital fissure, } \\
\text { supraorbital foramen }\end{array}$ & Sensory & Skin on the upper $1 / 3$ of face \\
\hline$\square$ & $\mathrm{CN}$ V2 & Maxillary $\mathrm{n}$. & $\begin{array}{l}\text { Foramen rotundum, } \\
\text { infraorbital foramen }\end{array}$ & Sensory & Skin on the middle $1 / 3$ of face \\
\hline$\square$ & $\mathrm{CN} V 3$ & Mandibular $\mathrm{n}$. & $\begin{array}{l}\text { Foramen ovale, mandibular } \\
\text { foramen, mental foramen }\end{array}$ & Sensory & Skin on the lower $1 / 3$ of face, pain from tongue \\
\hline$\square$ & $\mathrm{CN}$ V3 & Mandibular $\mathrm{n}$. & Foramen ovale & Motor & $\begin{array}{l}\text { Temporalis, masseter, medial pterygoid, and lateral } \\
\text { pterygoid muscles }\end{array}$ \\
\hline$\square$ & CN VI & Abducens $\mathrm{n}$. & Superior orbital fissure & Motor & Lateral rectus muscle of the eye \\
\hline$\square$ & CN VII & Facial $\mathrm{n}$. & $\begin{array}{l}\text { Internal acoustic meatus, } \\
\text { stylomastoid foramen }\end{array}$ & Motor & Muscles of facial expression \\
\hline$\square$ & CN VII & Facial $n$. & $\begin{array}{l}\text { Internal acoustic meatus, } \\
\text { stylomastoid foramen }\end{array}$ & Special Sensory & Taste-buds on the anterior $2 / 3$ of tongue \\
\hline$\square$ & CN VIII & Vestibulocochlear $\mathrm{n}$. & Internal acoustic meatus & Special Sensory & Inner ear (cochlea and vestibular apparatus) \\
\hline$\square$ & CN IX & Glossopharyngeal $n$. & Jugular foramen & Special Sensory & Taste-buds on the posterior $1 / 3$ of tongue \\
\hline$\square$ & CN IX & Glossopharyngeal n. & Jugular foramen & Motor & Muscles of the pharynx \\
\hline$\square$ & $\mathrm{CN} X$ & Vagus $\mathrm{n}$. & Jugular foramen & Sensory & $\begin{array}{l}\text { Skin across the throat and the external acoustic } \\
\text { meatus }\end{array}$ \\
\hline$\square$ & $\mathrm{CNX}$ & Vagus $\mathrm{n}$. & Jugular foramen & Special Sensory & Taste and gag reflex \\
\hline$\square$ & $\mathrm{CNX}$ & Vagus $n$. & Jugular foramen & Motor & Heart and smooth muscles of pharynx \& gut \\
\hline$\square$ & $\mathrm{CNXI}$ & Accessory $\mathrm{n}$. & $\begin{array}{l}\text { Jugular foramen, foramen } \\
\text { magnum }\end{array}$ & Motor & $\begin{array}{l}\text { Trapezius, sternocleidomastoid, and muscles of the } \\
\text { pharynx }\end{array}$ \\
\hline$\square$ & CN XII & Hypoglossal $n$. & Hypoglossal canal & Motor & Muscles of the tongue \\
\hline
\end{tabular}




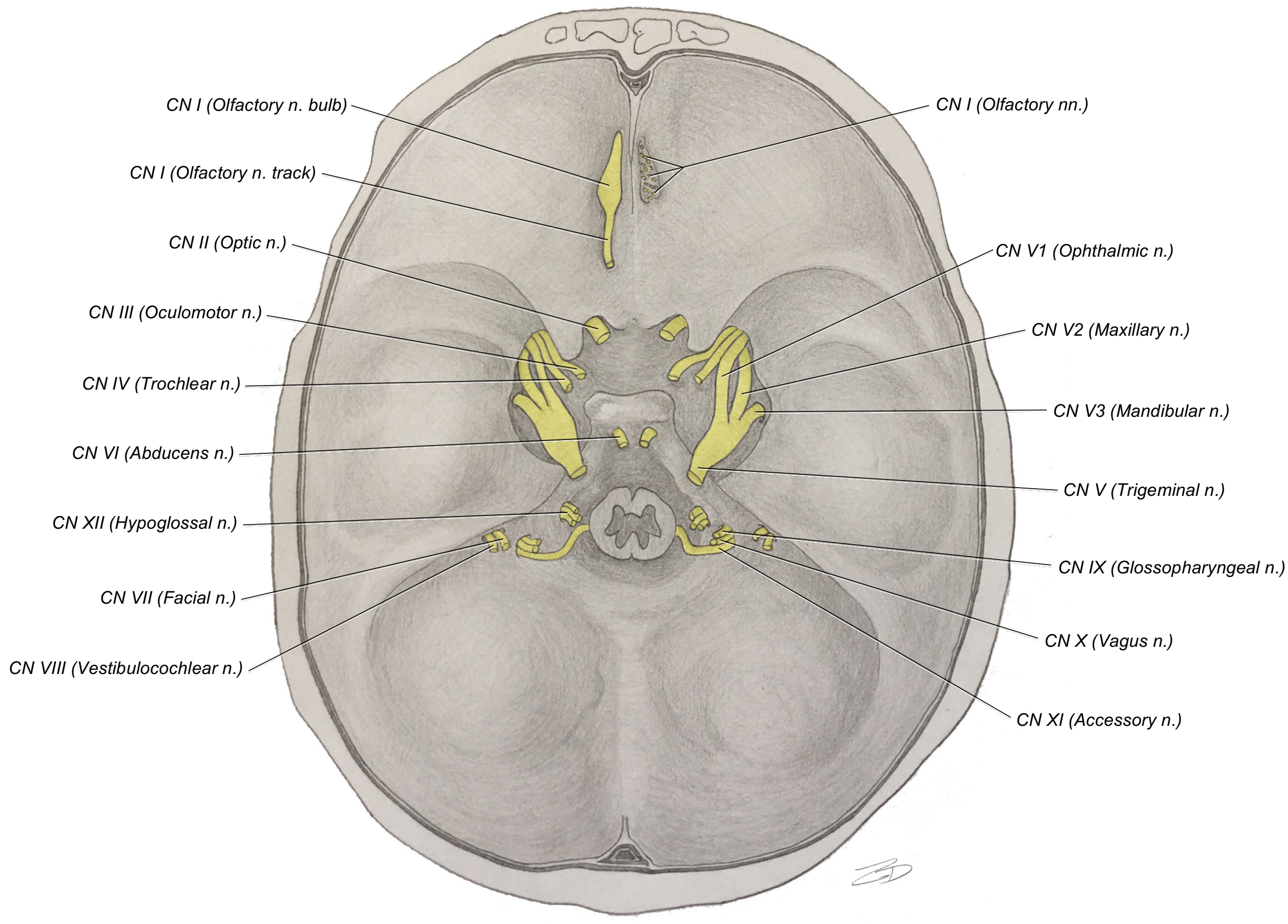

Figure 10.1. Diagram of Cranial Nerves in the Basicranium, Superior View 


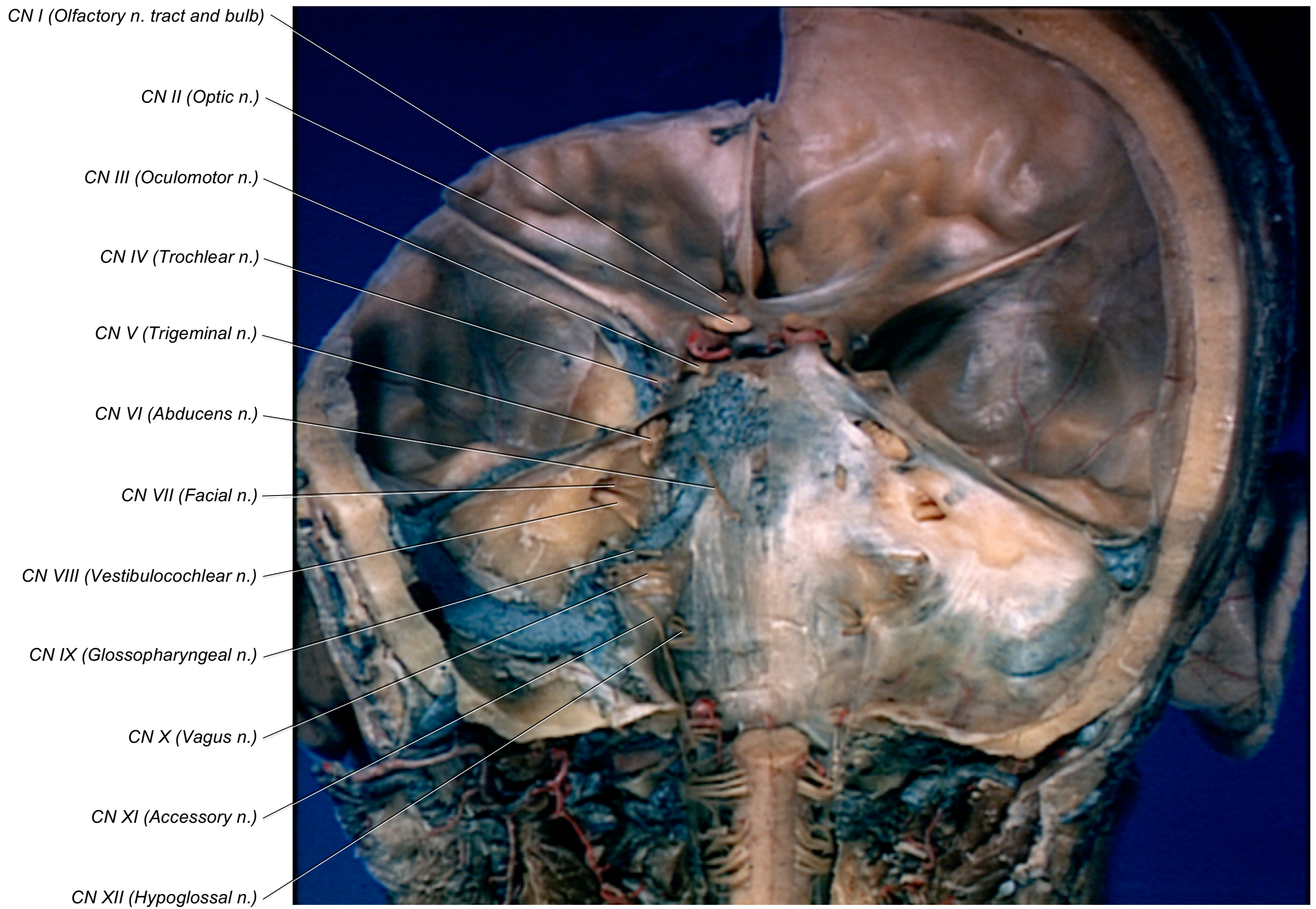

Figure 10.2. Cranial Nerves in the Basicranium, Posterosuperior View 
and self-assess your knowledge.

1) Which of the following is NOT considered special sensory? (LO25)
a. Hearing
b. Vision
c. Touch
d. Balance
e. Taste

2) How many pairs of cranial nerves are there? (LO26)

3) Which cranial nerve is responsible for transmitting the sensory signal from a pin poke on chin to the CNS? (LO27)

4) Name the three branches of $\mathrm{CN} \mathrm{V}$ (trigeminal nerve). (LO26)

5) Which cranial nerve supplies all three types of innervation (motor, sensory, and special sensory)? (LO27)

6) True or False: no cranial nerves pass through the foramen magnum. (LO27)

7) Which cranial nerves transmit signals to our CNS to process taste? (LO27)

8) Which cranial nerve innervates the temporalis muscle? (LO27)
9) Which cranial nerve innervates the levator labii superioris muscle? (LO27)

10)Which cranial nerves pass through the jugular foramen? (LO27)

11)What does the term glosso mean? (LO25)

12)What is olfaction? (LO25)

13)Which cranial nerves innervate muscles of the eye? (LO27)

14)Identify the following cranial nerves. (LO26)

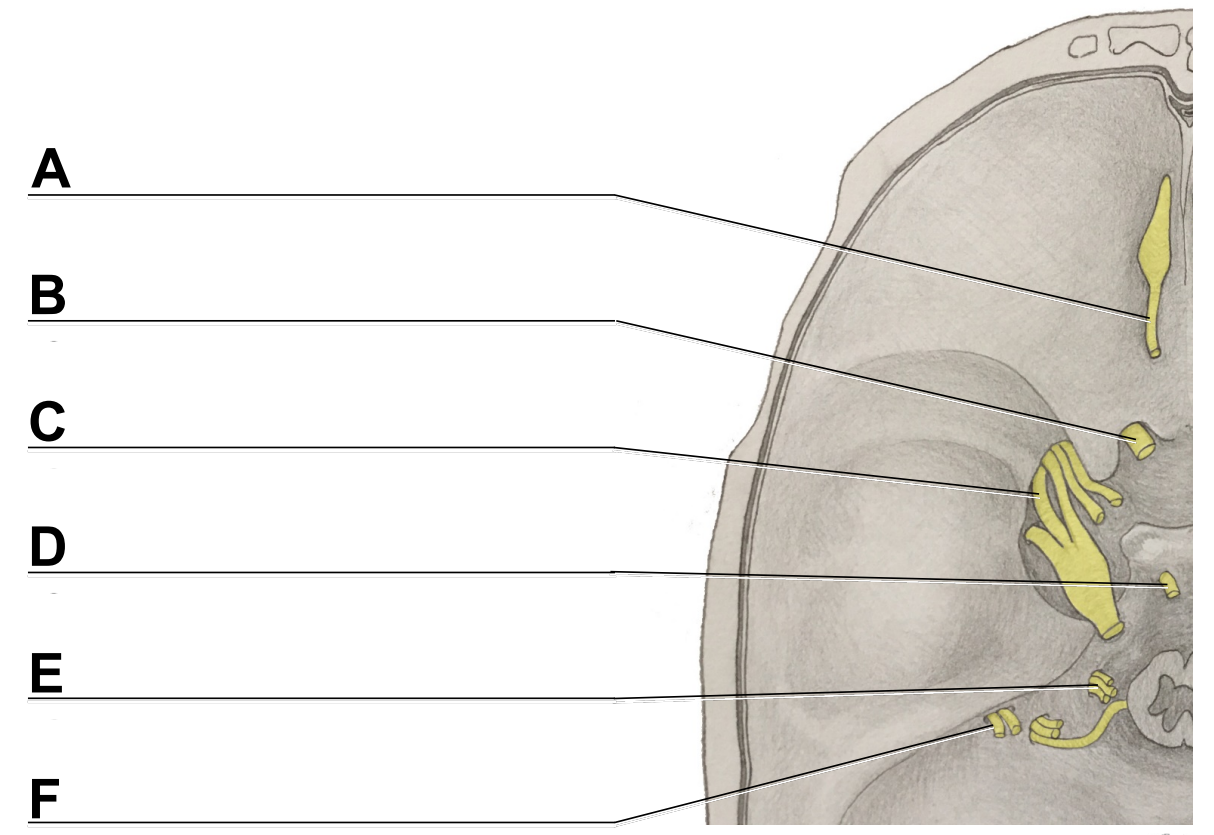

Fig. 10.1. 


\section{Lab 11: Special Senses}

Instructions: Learn the listed terms using the listed learning objectives, labeled images, lab specimens, and other resources.

LO28: Describe the terminology used to identify structures of the special senses.

LO29: Identify the structures of the special senses.

LO30: Describe the flow pathways of sight, tears, and sound, and the actions/innervations of eye muscles.

\section{Eye}

$\square$ CN II (Optic n.)

○ Innervation target: retina of the eye

\section{Sclera}

$\square$ Cornea

$\square$ Iris

\section{Pupil}

The iris is a muscle that constricts/dilates the pupil to control the amount of light that enters the eye.

$\square$ Lens

$\square$ Zonular fibers (or Suspensory ligaments of the lens)

\section{Ciliary body}

The lens is suspended by zonular fibers, which are attached to the ciliary body (a muscle). When the ciliary body contracts, it pulls on the zonular fibers, which then pull/stretch the lens, to direct light onto the fovea on the retina.

\section{Conjunctiva}

\section{Retina}

- Fovea centralis

- Optic disc

\section{Choroid}

Located between the sclera and the retina, the choroid is a vascular layer of the eye that supplies blood to the eye.

\section{Anterior cavity}

- Anterior chamber

- Posterior chamber

\section{Posterior cavity}

The anterior and posterior cavities are separated by the lens, and the anterior and posterior chambers are separated by the iris. Further, the anterior cavity is filled with aqueous humor (a clear, watery fluid), and the posterior cavity is filled with vitreous humor (a transparent, jelly-like tissue).

\section{Clinical Application: Conjunctivitis}

Conjunctivitis (or Pink Eye) is a common inflammation of the conjunctiva of the eye. Symptoms typically include redness, itching, increased tear production, and crusting around the eyes. It is highly contagious, and can be spread by direct contact (i.e. handshake or hug) or indirect contact (i.e. touching a contaminated pencil or doorknob). Although the inflammation is usually not severe and will resolve on its own, the process can be sped up with antibiotic eye drops. 


\section{Structures associated with the eye}

$\square$ Orbicularis oculi muscle

- Action: closes the eyelids

\section{Eyebrow}

$\square$ Upper and lower eyelids

- Upper and lower eyelashes

\section{Lacrimal gland}

The lacrimal gland produces tears.

$\square$ Lacrimal ducts

$\square$ Caruncle

$\square$ Superior and inferior puncta

$\square$ Superior and inferior canaliculi

$\square$ Lacrimal sac

$\square$ Nasolacrimal duct

$\square$ Opening of nasolacrimal duct

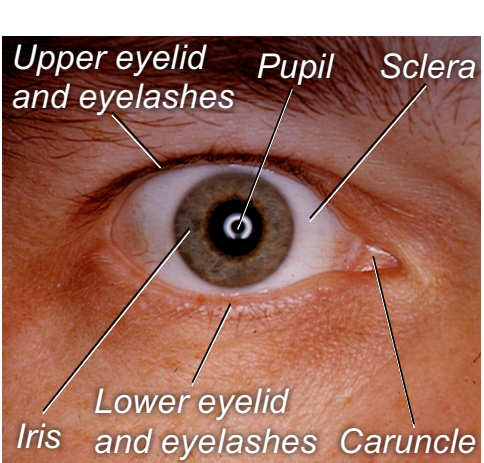

Figure 11.1. Right Eye, Anterior View

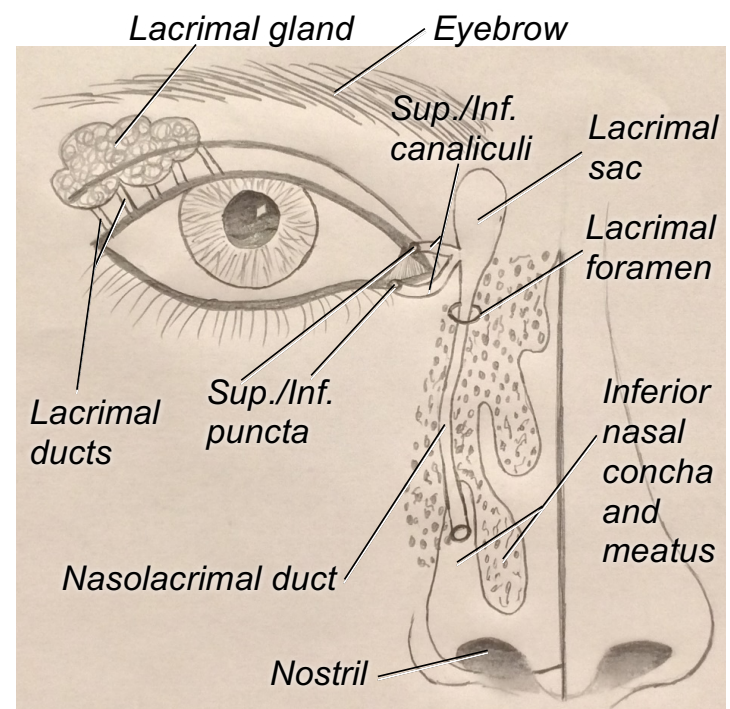

Figure 11.2. Tear structures, Right Anterior View
Do you remember what lacrimal means?

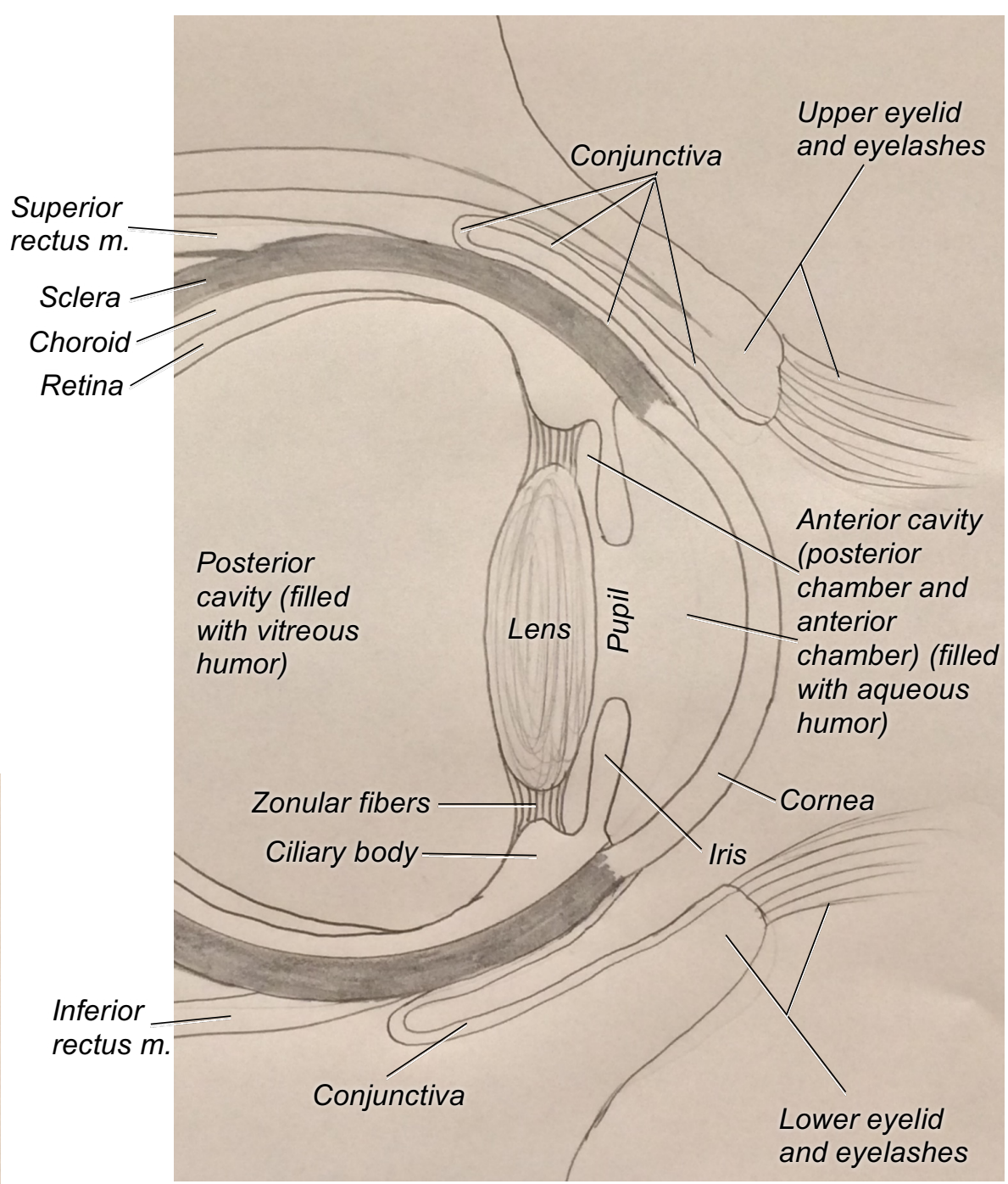

Figure 11.3. Diagram of Eye, Sagittal Section, Lateral View 


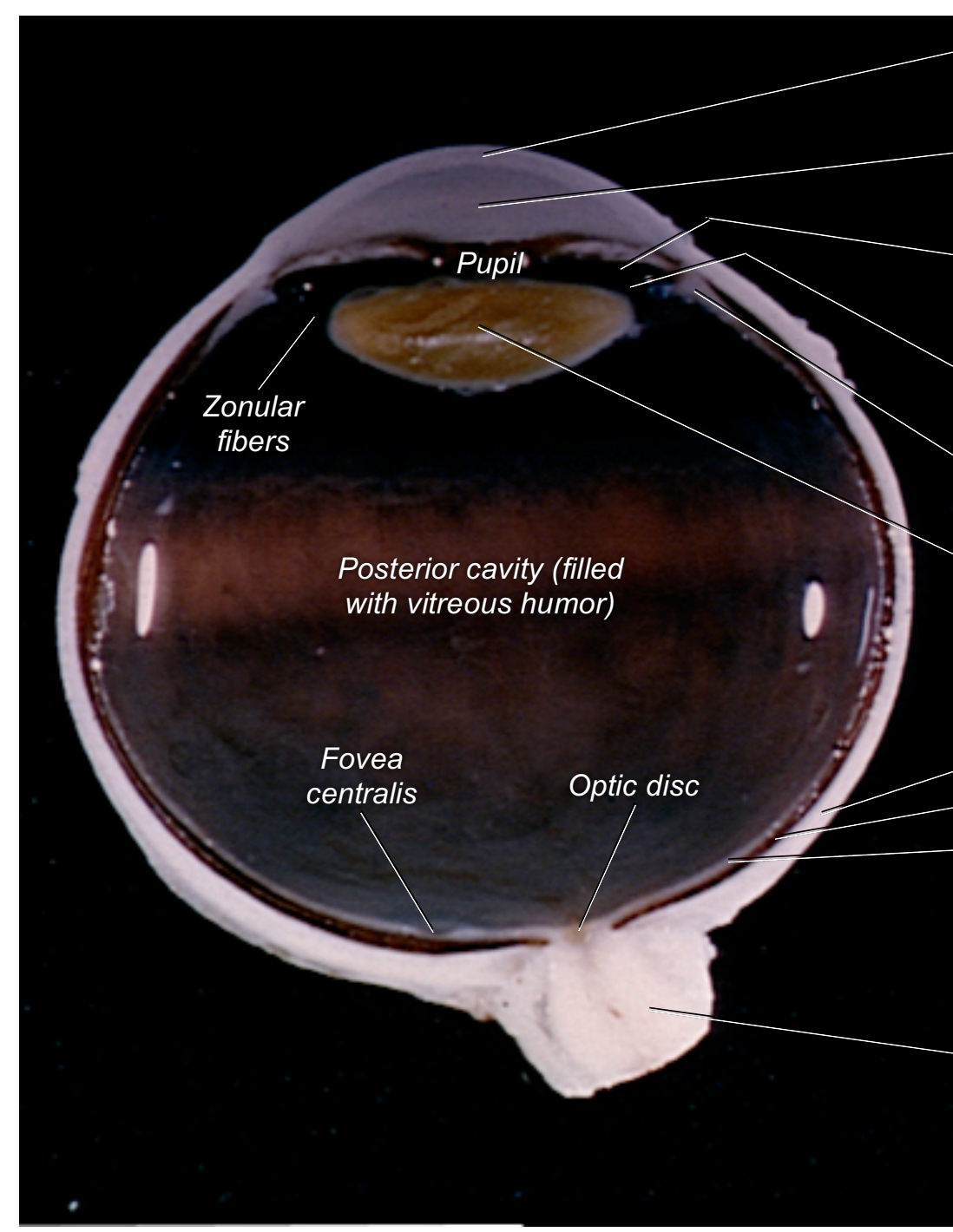

Figure 11.4. Eye, Transverse Section, Superior View

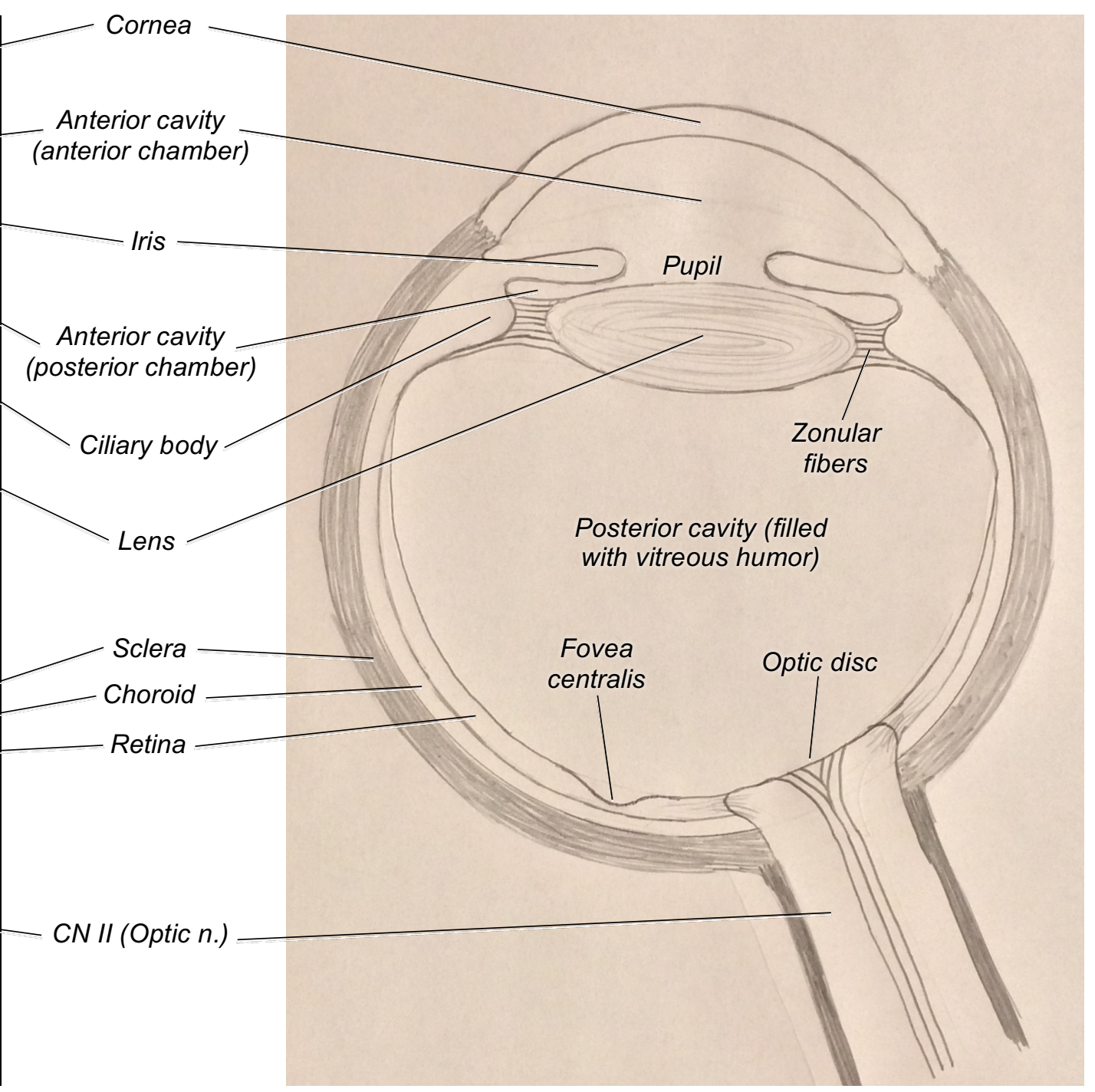

Figure 11.5. Eye (Sketch), Transverse Section, Superior View 


\section{Clinical Application: "Blind Spot"}

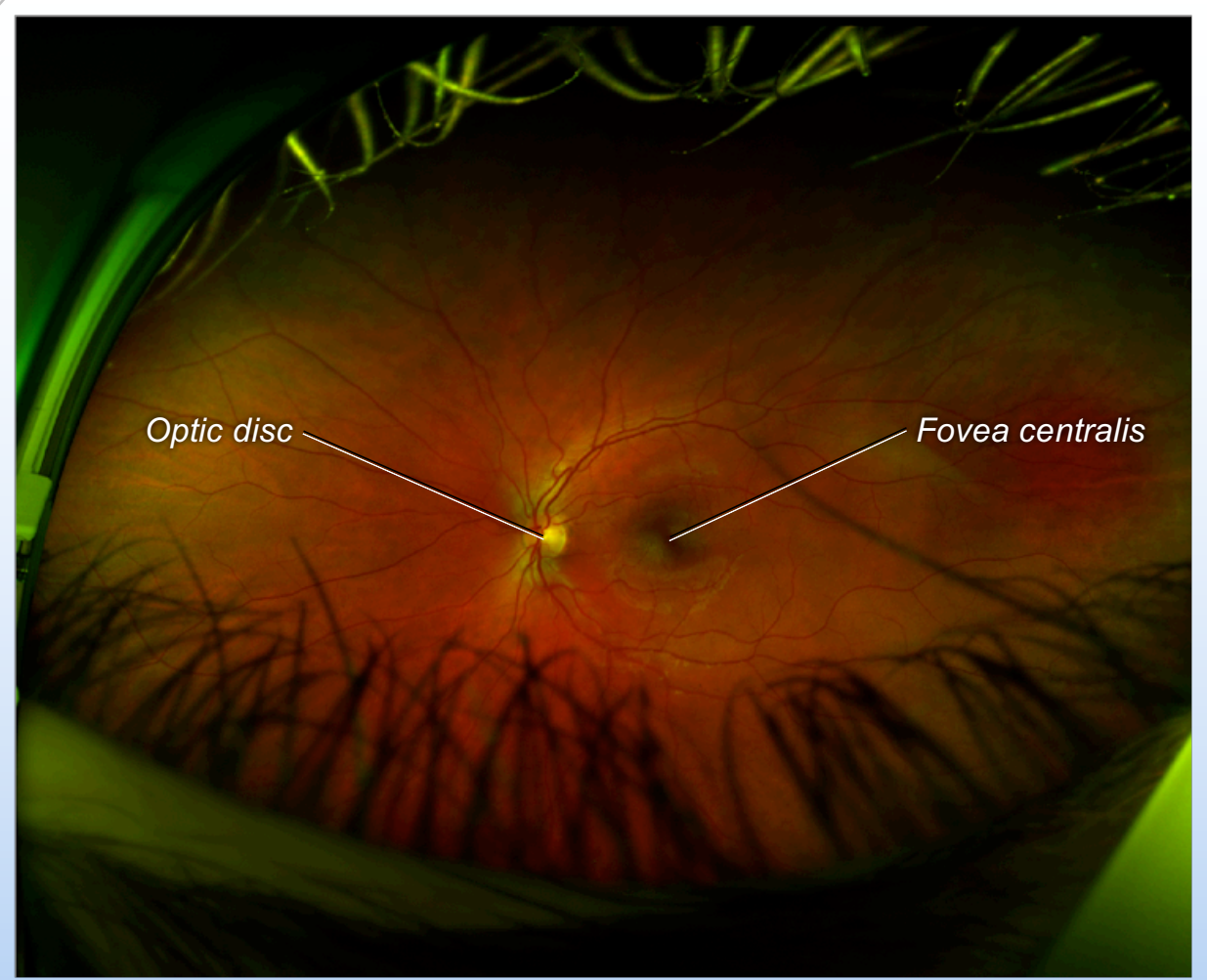

Figure 11.6. Photograph of Left Human Retina, Anterior View
There are no rods or cones (visual receptors) at the optic disc (the structure formed from where CN II (Optic n.) connects to the retina) because that space is being occupied up by the optic nerve fibers and retinal vasculature. Therefore, as light passes through the eye and onto the retina for visual processing, the light that shines on the optic disc is not processed into eyesight, causing a "blind spot" in each eye's normal field of vision. You can "see" your blind spot for yourself using the image below. Close your right eye and look directly at the center of the plus sign with your left eye (don't look away or at the circle). Hold the image about one foot away from your face. While looking directly at the plus sign with your left eye, the white circle should disappear from your peripheral vision because that area in your field of vision is shining on your optic disc. You typically don't notice your blind spots because your brain takes vision from your other eye and "fills in" your blind spots for a full field of view. Unlike the optic disc, the fovea centralis, a structure on the retina near the optic disc, has the highest concentration of cones and processes the most acute vision necessary for reading, driving, etc..
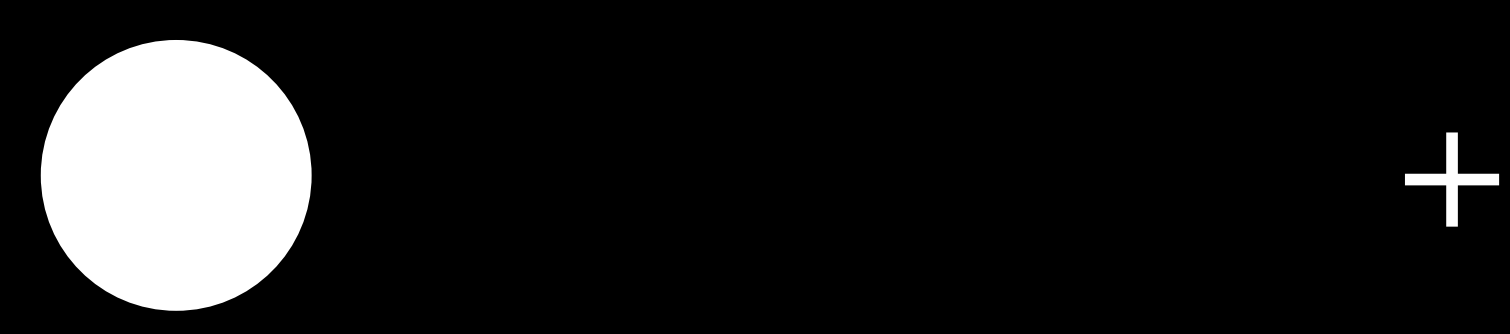


\section{Extrinsic muscles of the eye}

Extrinsic $=$ outside of

Superior rectus

- Innervated by: CN III (Oculomotor n.)

- Action: superior rotation of the eye

$\square$ Inferior rectus

- Innervated by: CN III (Oculomotor n.)

- Action: inferior rotation of the eye

$\square$ Lateral rectus

- Innervated by: CN VI (Abducens n.)

- Action: lateral rotation of the eye

$\square$ Medial rectus

- Innervated by: CN III (Oculomotor n.)

- Action: medial rotation of the eye

$\square$ Superior oblique

- Innervated by: CN IV (Trochlear n.)

- Action: inferior and lateral rotation of the eye

$\square$ Inferior oblique

- Innervated by: CN III (Oculomotor n.)

- Action: superior and lateral rotation of the eye

Do you remember what the terms rectus and oblique mean?

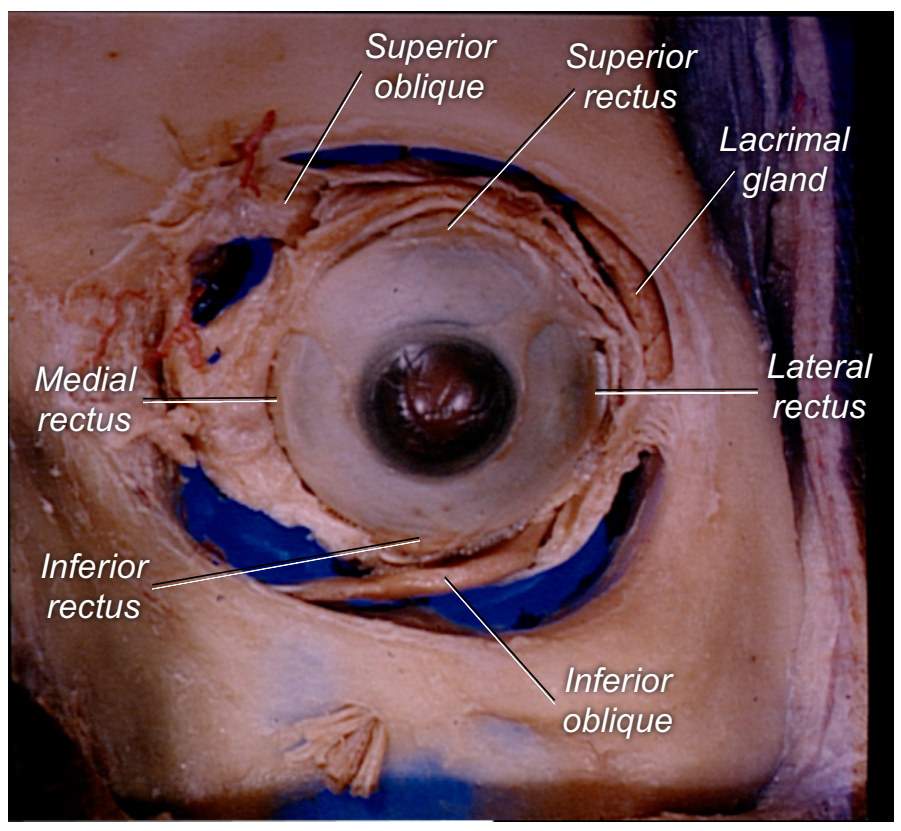

Figure 11.7. Extrinsic Muscles of the Left Eye, Anterior View

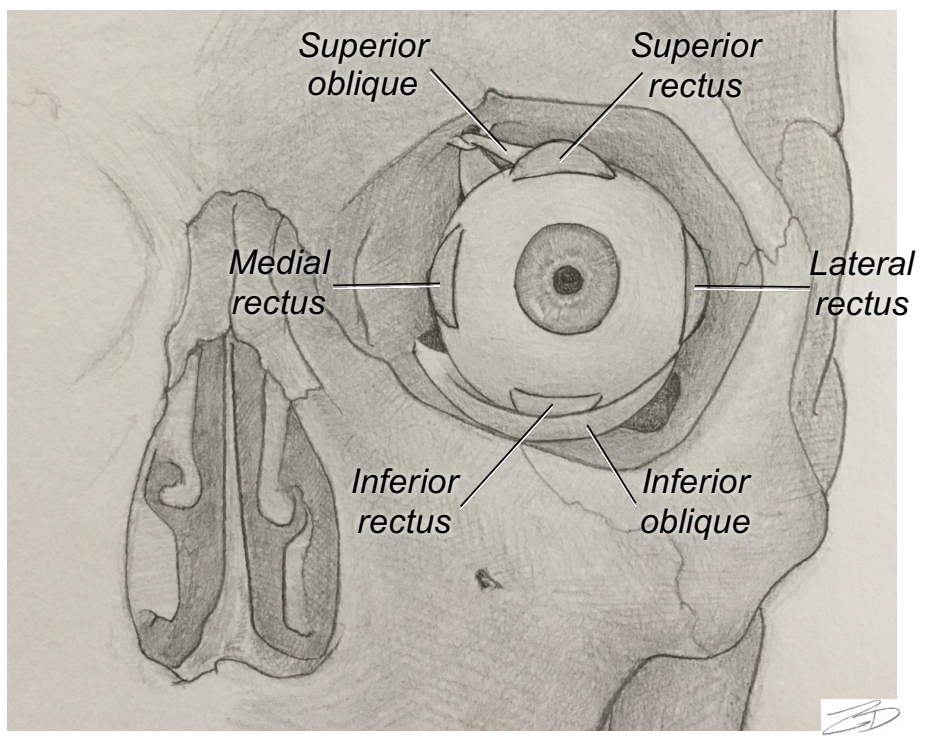

Figure 11.8. Extrinsic Muscles of the Left Eye (Sketch), Anterior View. 


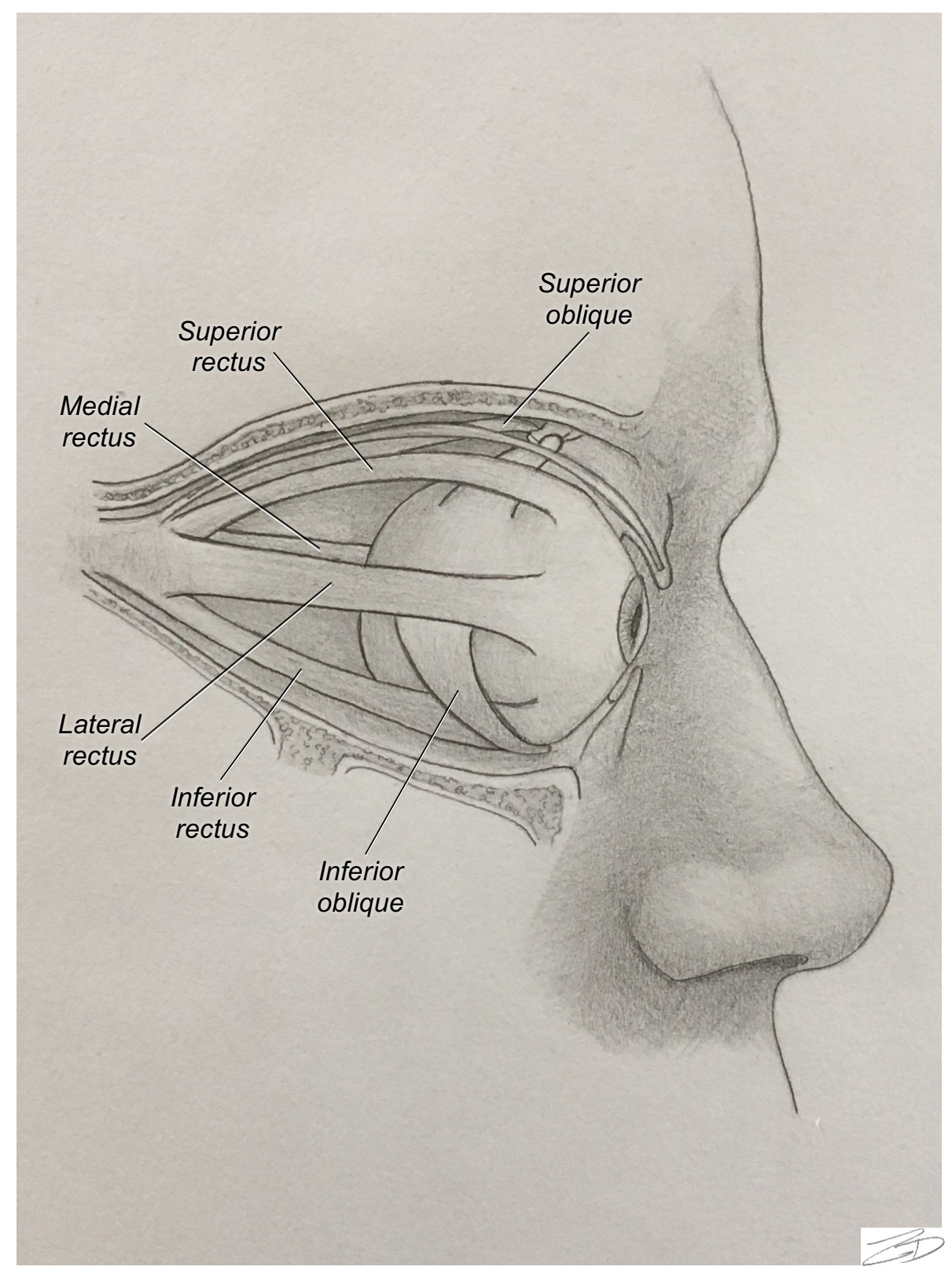

Figure 11.9. Extrinsic Muscles of the Eye (Sketch), Right Lateral View.

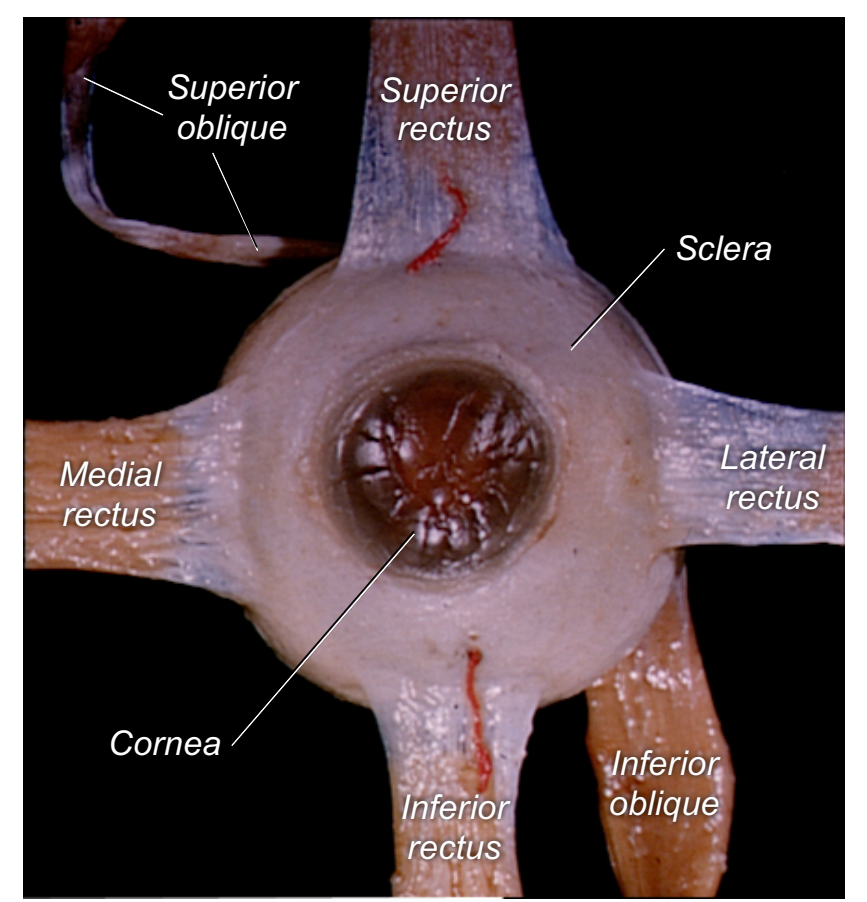

Figure 11.10. Reflected Extrinsic Muscles of the Left Eye (Removed), Anterior View

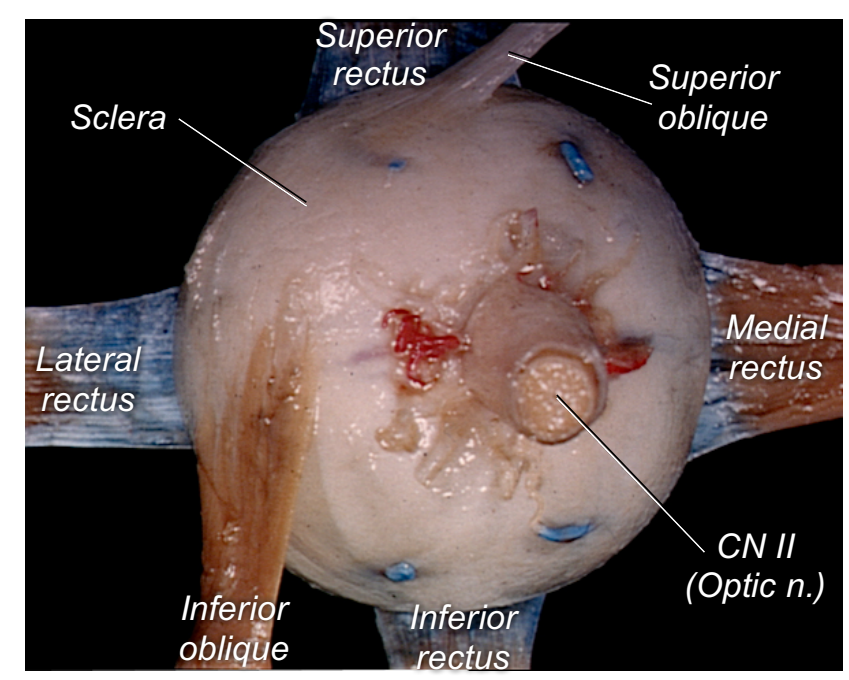

Figure 11.11. Reflected Extrinsic Muscles of the Left Eye (Removed), Posterior View 


\section{Outer ear}

$\square$ Auricle (or pinna)

Do you remember what the term auricular means?

\section{Auditory canal}

The external acoustic meatus is the bony structure opening to the external auditory canal.

Tragus*

\section{Middle ear}

$\square$ Tympanic membrane (or Eardrum)

$\square$ Tympanic cavity

$\square$ Ossicles
- Malleus (or Hammer)
- Incus (or Anvil)
- Stapes (or Stirrup)

$\square$ Pharyngotympanic tube (or Eustachian/auditory tube)

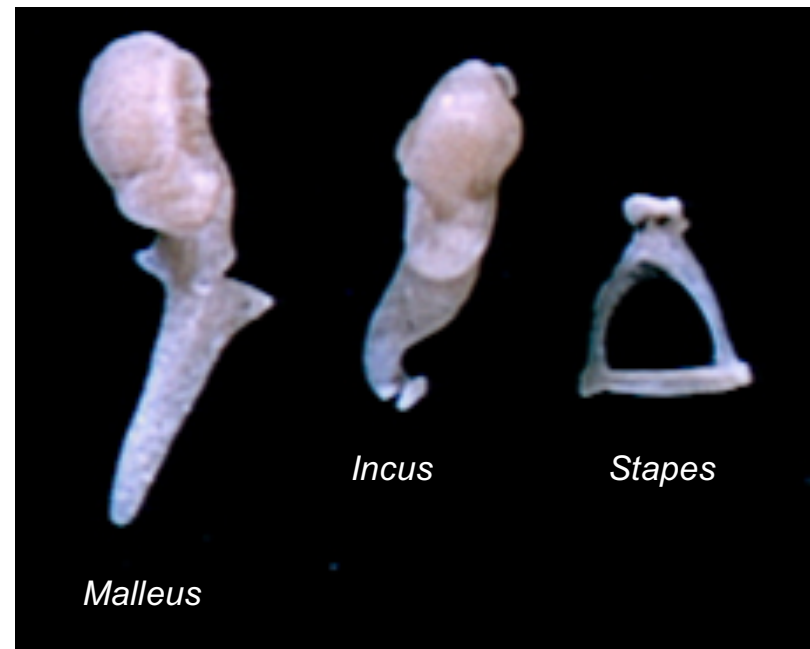

Figure 11.12. Ear Ossicles

\section{Inner ear}

$\square$ Temporal bone (petrous part)

$\square$ Cochlea

$\underline{\text { Cochlea }}=$ snail. Do you remember what petrous means?

\section{Oval window}

The stapes is positioned against the oval window.

\section{Round window}

The round window relieves pressure in the cochlea applied by the stapes. This allows sound waves to move throughout the cochlea.

\section{$\square$ Vestibular apparatus}

- Vestibule

- Semicircular canals

The vestibular apparatus processes the sensation of motion, orientation, and balance.

$\square$ CN VIII (Vestibulocochlear nerve)

$\square$ Internal acoustic meatus

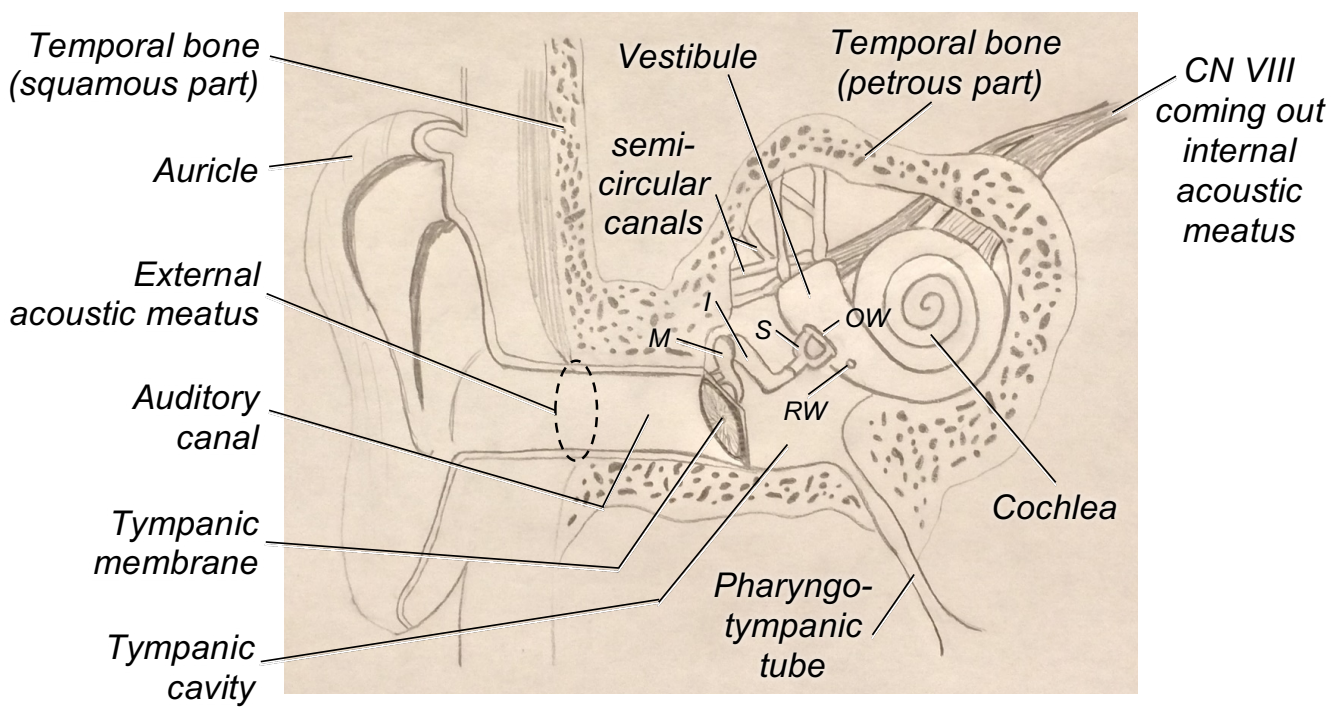

Figure 11.13. Structures of the Outer, Middle, and Inner Ear 


\section{Nose/nasal cavity}

$\square$ Nostrils

$\square$ Cribriform plate

- Olfactory foramina

CN I (Olfactory n.)

- Function: olfactory epithelium

$\square$ Nasal septum

- Septal cartilage

- Perpendicular plate

- Vomer

Superior concha

$\square$ Superior meatus

$\square$ Middle concha

$\square$ Middle meatus

$\square$ Inferior concha

$\square$ Inferior meatus

$\square$ Pharyngeal orifice of pharyngotympanic tube (or Opening for eustachian tube)

\section{Mouth/oral cavity}

$\square$ Tongue

\section{Associated cranial nerves:}

$\square$ CN VII (Facial n.)

- Innervation target: Taste buds on the anterior $2 / 3$ of the tongue $\square$ CN IX (Glossopharyngeal n.)

- Innervation target: Taste buds on the posterior $1 / 3$ of the tongue

CN XII (Hypoglossal n.)

- Innervation target: Muscles of the tongue

$\square \mathrm{CN} X$ (Vagus n.)

- Innervation target: Taste and gag reflex

$\square$ CN V3 (Mandibular n.)

- Innervation target: Skin on the lower $1 / 3$ of face, pain from tongue

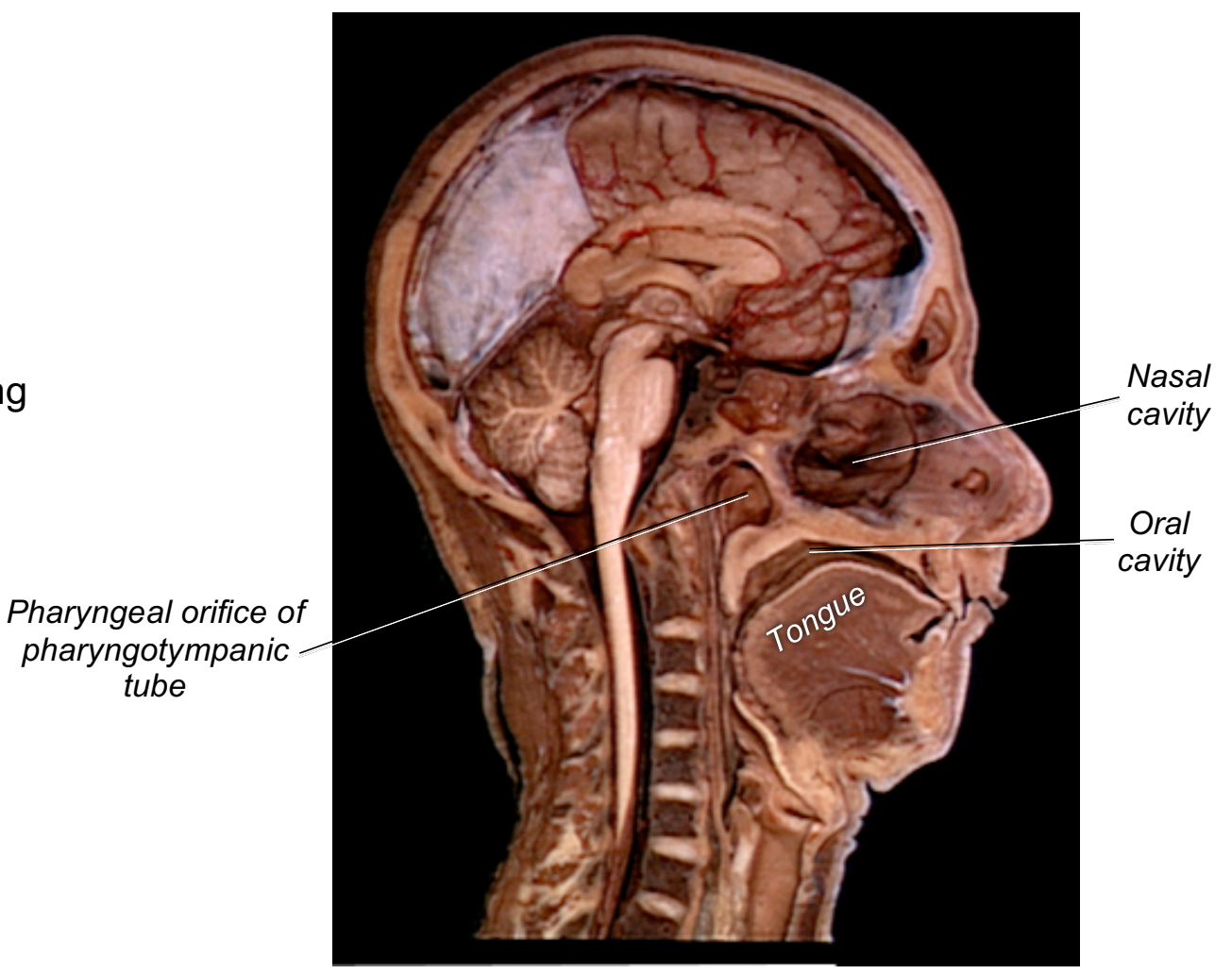

Figure 11.14. Structures of the Nasal and Oral Cavities 
and self-assess your knowledge.

1) What does the term rectus mean? (LO28)

2) What does the term oblique mean? (LO28)

3) In order, list the structures that light passes through before being processed into sight. (LO30)

4) In order, list the structures that sound waves pass through before being interpreted as sound. (LO30)

5) In order, list the structures that tears pass across/through before entering the nasal cavity. (LO30)

6) What nerves are innervating which muscles when a person looks directly left with their eyes only? (LO30)

7) The separates the anterior and posterior cavities, whereas the separates the anterior and posterior chambers. (LO29)
8) What is/are the action(s) of the superior oblique muscle? (LO30)

9) What nerve innervates taste buds on the posterior $1 / 3$ of the tongue? (LO30)

10)The pharyngeal orifice of the pharyngotympanic tube empties into the (LO29)

11)Identify the following structures: (LO29)

$\underline{A}$

B

C

$\underline{D}$

E

$\mathbf{F}$

G

$\underline{H}$

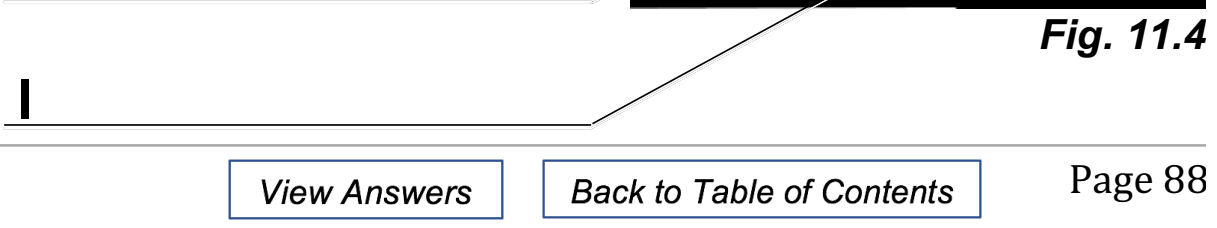




\section{Lab 12: Heart}

Instructions: Learn the listed terms using the listed learning objectives, labeled images, lab specimens, and other resources.

LO31: Describe the terminology used to identify structures of the heart.

LO32: Identify the structures and vessels of the heart.

LO33: Describe the flow pathway of blood through the heart (pulmonary and systemic circulation), blood around the heart (coronary circulation), and electrical impulses through the heart.

\section{Important Cardiovascular Terminology}

The heart has two main functions: 1) the right side pumps deoxygenated blood to the lungs where it will drop off carbon dioxide and pick up oxygen, and 2) the left side pumps the recently oxygenated blood to the body's tissues where it will drop off oxygen and pick up carbon dioxide. These pulmonary and systemic circulations are made possible by thousands of miles of blood vessels called arteries and veins. All arteries carry blood away from the heart, and all veins carry blood toward the heart. Blood changes from arterial blood to venous blood after passing through a respective capillary bed. Arterioles are tiny arteries just before a capillary bed, and venules are tiny veins just after a capillary bed. Usually arteries carry oxygenated blood and veins carry deoxygenated blood, but this is not always true. Red blood cells (RBCs) carry the oxygen and carbon dioxide molecules in blood.

Acceptable abbreviations for this content are as follows:

R. = Right; L. = Left.

a. $=\operatorname{artery}($ aa. $=$ arteries $) ;$ v. = vein (vv. = veins $)$.

\section{Heart and associated structures}

$\square$ Apex

Base

Pericardium (or Pericardial sac)

$\underline{\text { Cardi }}=$ relating to the heart

Pectinate muscle

This muscle makes up the walls of the atria.

Myocardium

This muscle makes up the walls of the ventricles.

R. \& L. auricles

R. atrium

- Opening of superior vena cava

- Opening of inferior vena cava

- Opening of coronary sinus

- Opening of right auricle

Tricuspid valve (or R. atrioventricular valve)

R. ventricle

Pulmonary valve (or R. semilunar valve)

$\underline{\text { Pulm }}=$ relating to the lungs

L. atrium

○ Openings (4) of pulmonary veins

- Opening of left auricle 
L. ventricle

- Bicuspid valve (or mitral/L. atrioventricular valve)

- Aortic valve (or L. semilunar valve)

$\square$ Interatrial septum

- Fossa ovalis

Interventricular septum

Ligamentum arteriosum

Trabeculae carneae

Chordae tendineae

These structures attach the cusps of the tricuspid and bicuspid valves to papillary muscles.

\section{Papillary muscles}

When these muscles contract, they pull on the chordae tendineae which pull on the bicuspid and tricuspid valve cusps. Because ventricular filling/pressure causes the bicuspid and tricuspid valves to close, the tension on the valve cusps from the chordae tendineae/papillary muscles prevents the valves from prolapsing during contraction of the ventricles.

\section{Ascending aorta \\ Superior vena cava \\ Inferior vena cava \\ Pulmonary trunk}

$\underline{\text { Trunk }}$ = a major nerve, vessel, or duct. Trunks are undivided and often short.

○ R. \& L. pulmonary aa.

R. \& L. pulmonary vv.

Be sure to understand how blood flows through the heart and associated structures!

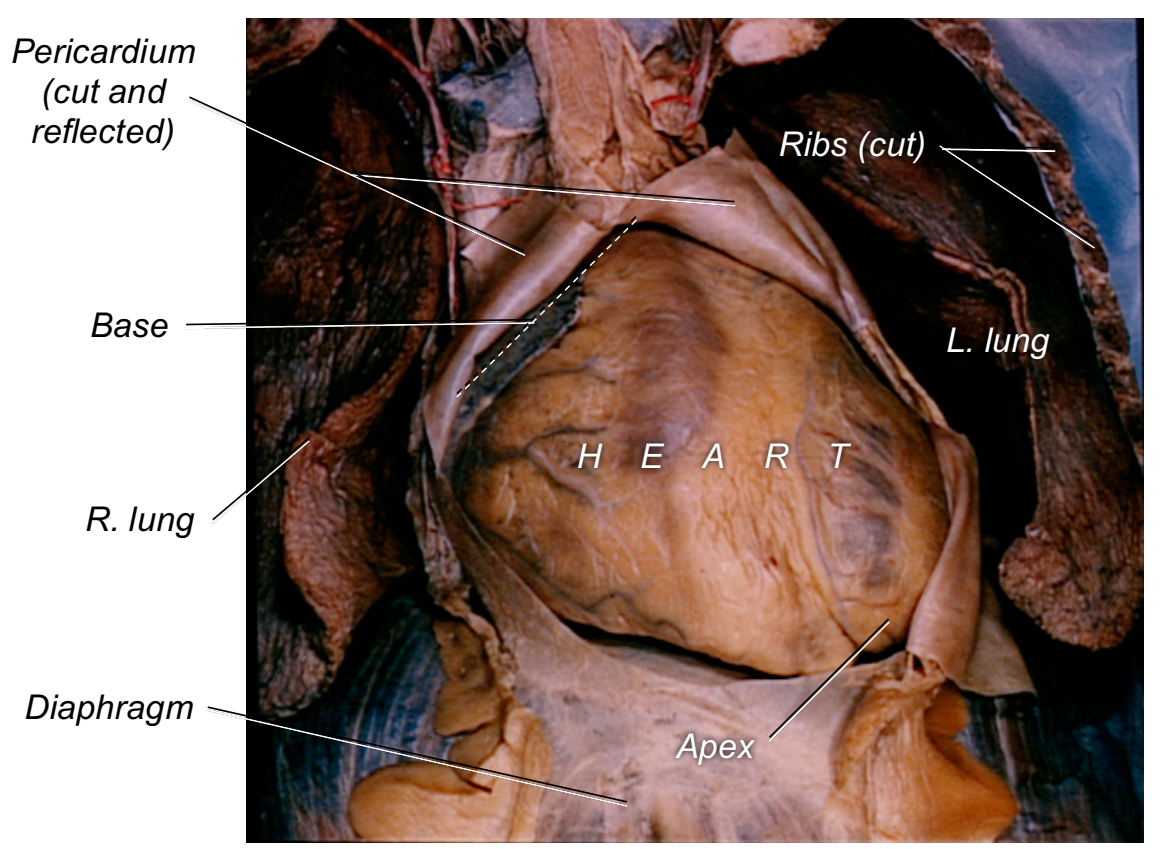

Figure 12.1. Heart in situ, Anterior View.

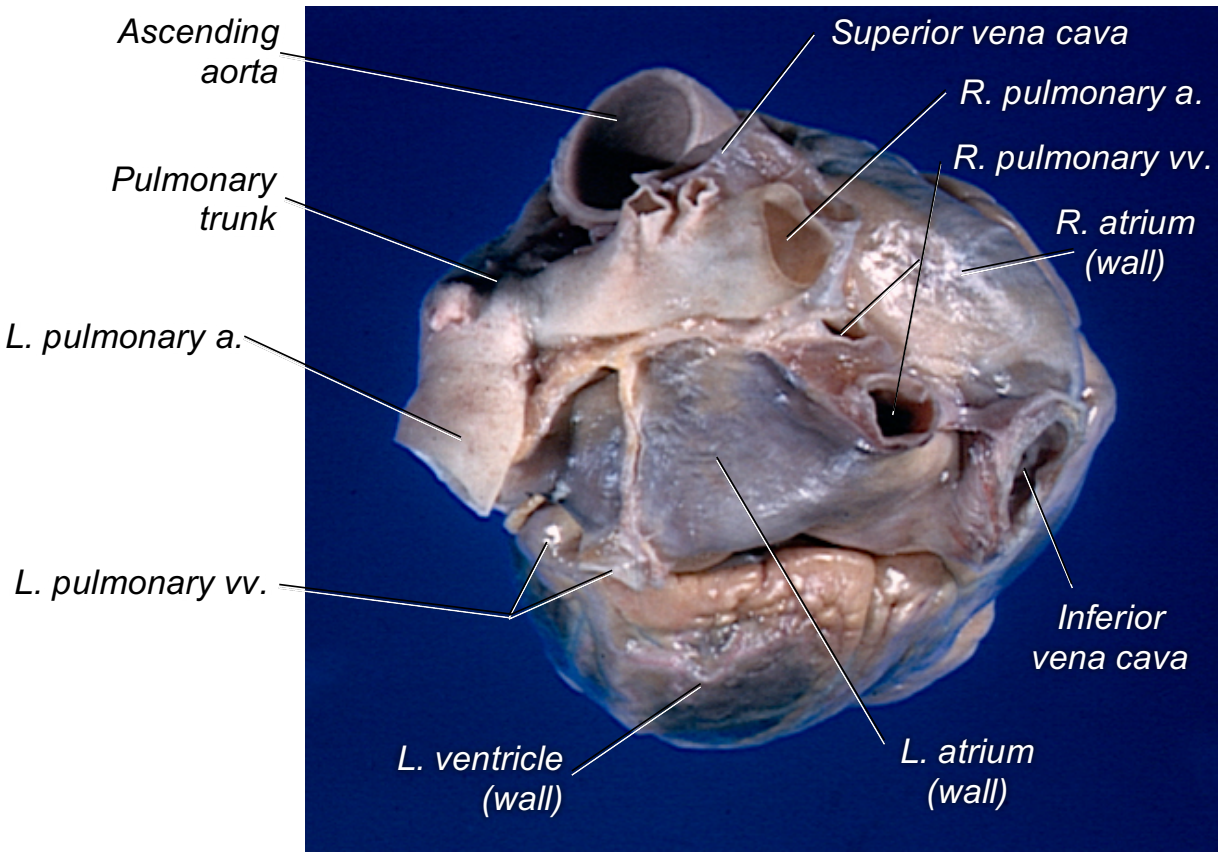

Figure 12.2. Base of Heart, Superior View 


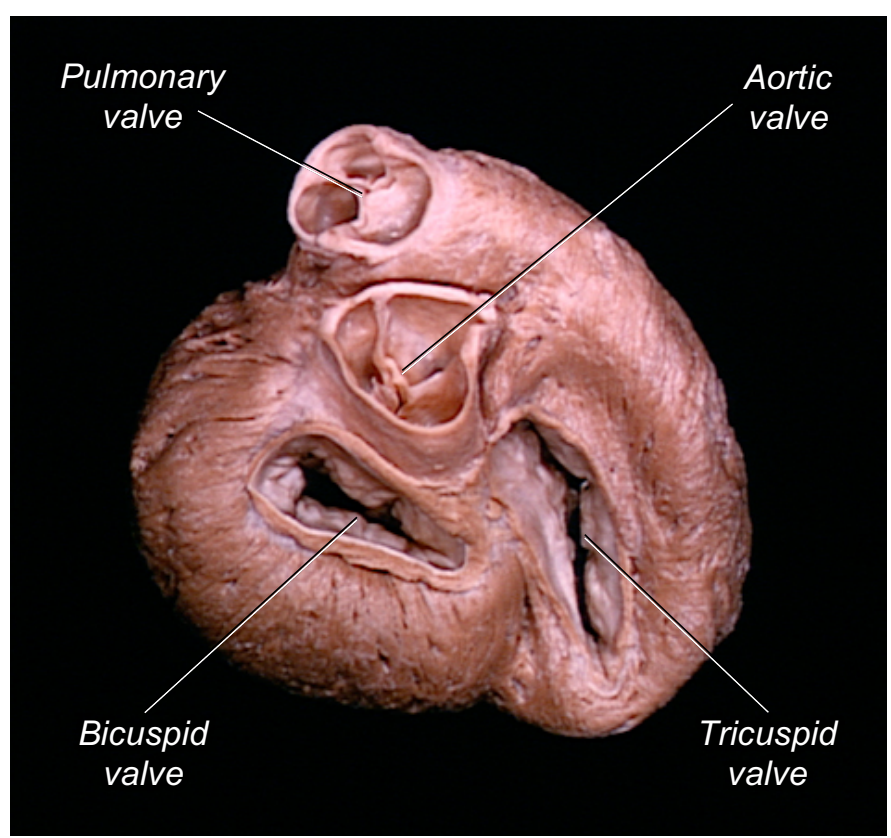

Figure 12.3. Valves of the Heart, Superior View (Atria Removed)

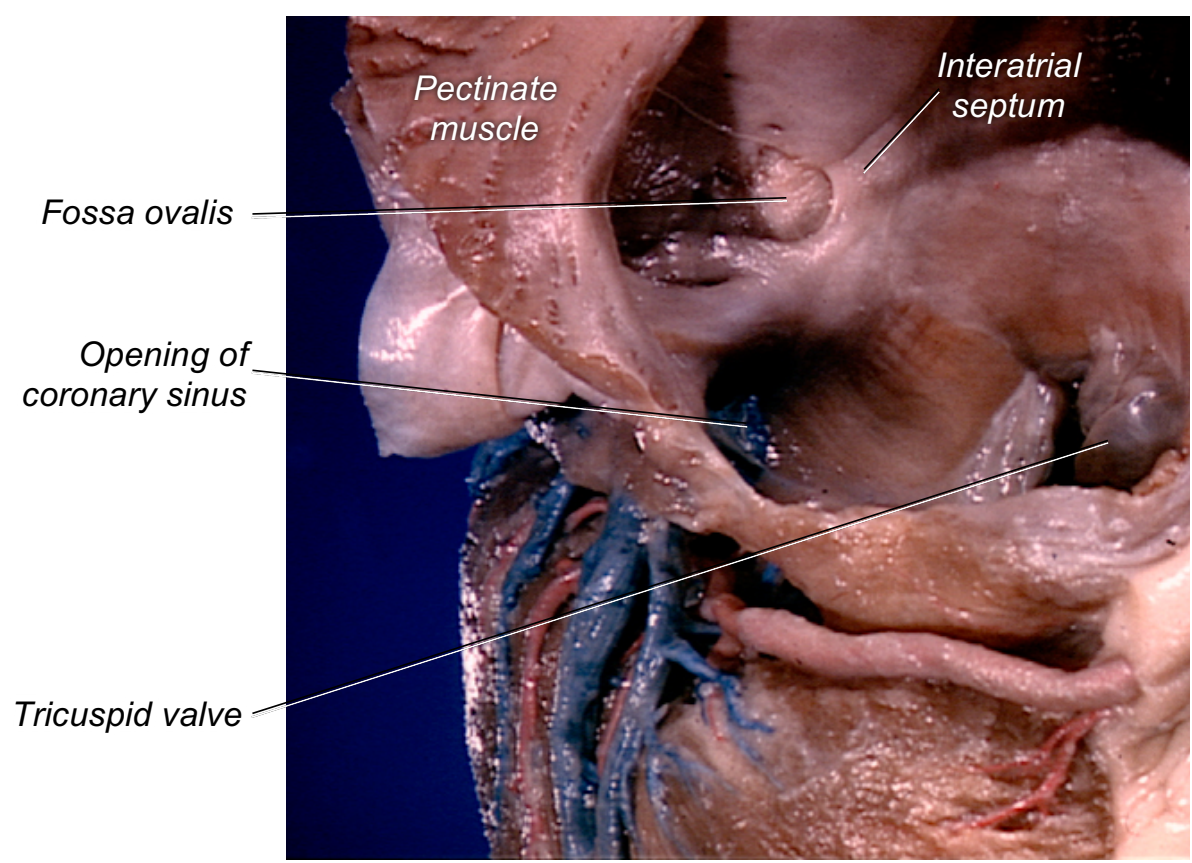

Figure 12.4. Inside the Right Atrium, Superolateral View

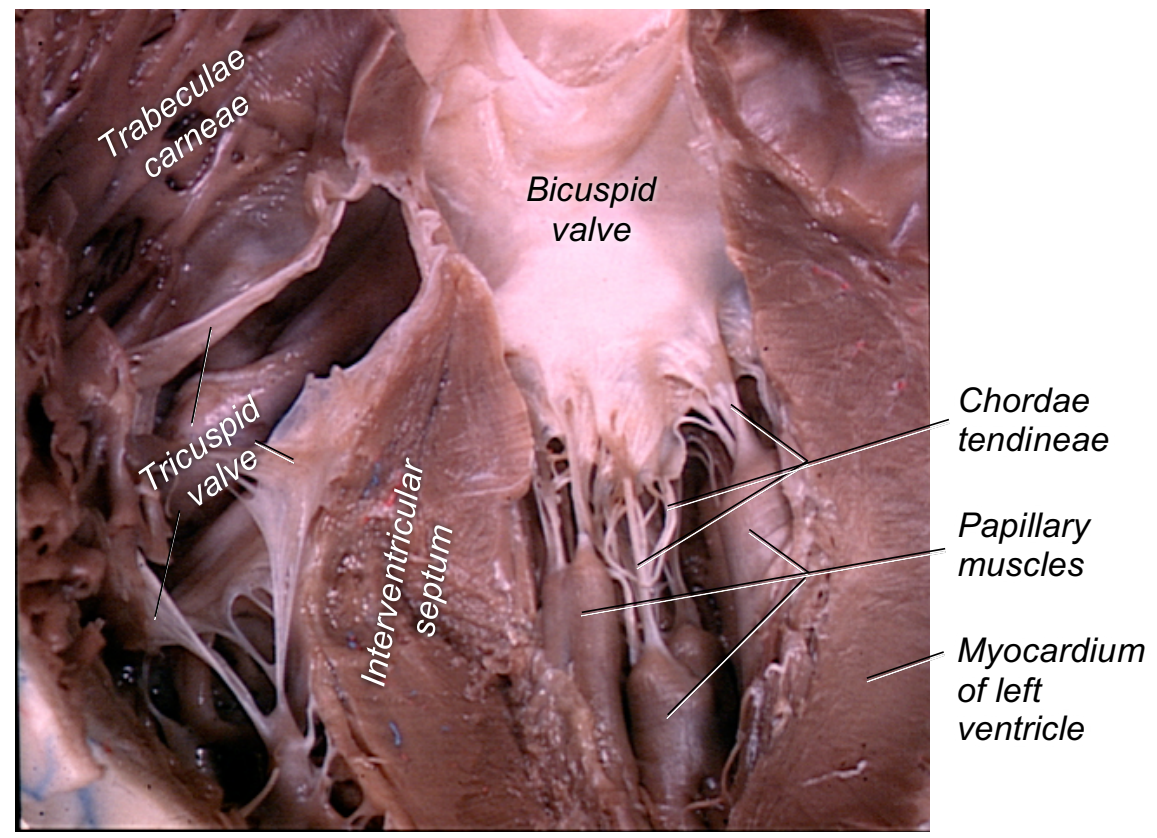

Figure 12.5. Inside the Left Ventricle, Anterior View

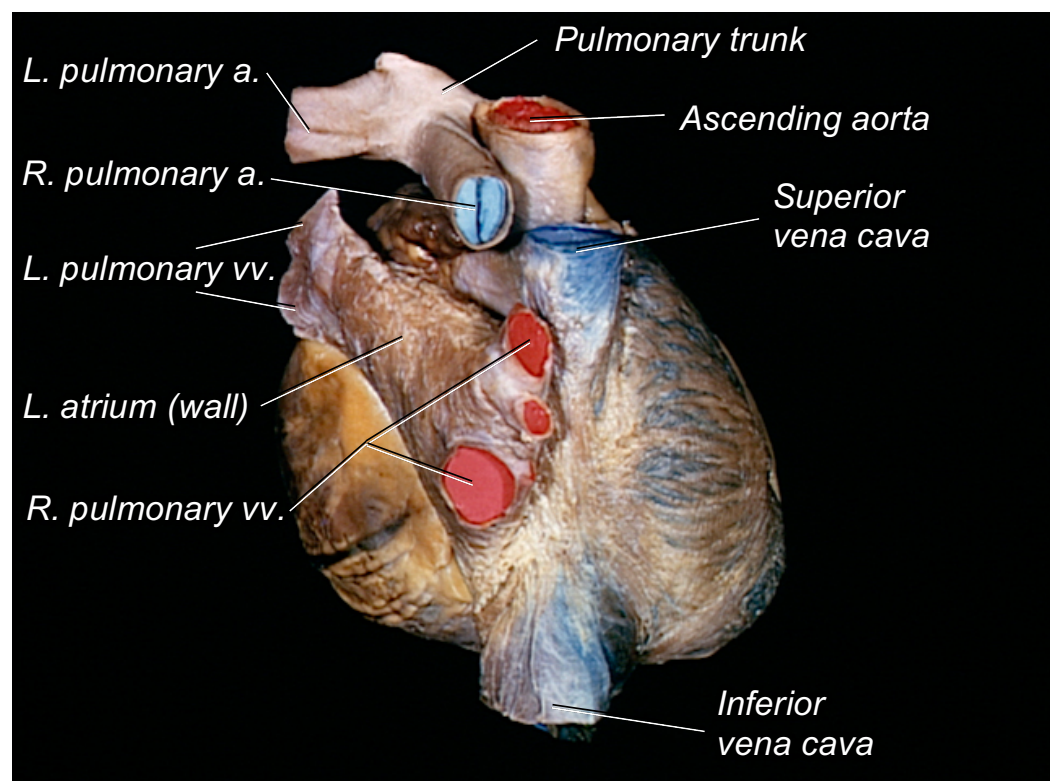

Figure 12.6. Base of Heart, Right Lateral View 


\section{Electrical Conduction of the heart (in pathway order)}

$\square$ Sinoatrial node (or SA node)

The SA node acts as the pacemaker for the heart. This cluster of cells spontaneously stimulates action potentials creating the heart's rhythm.

$\square$ Internodal fibers

These innervate the atria and cause them to contract.

$\square$ Atrioventricular node (or AV node)

The AV node delays the action potential signal to allow the ventricles to fill before contracting.

$\square$ Bundle of His

$\square$ L. and R. bundle branches

$\square$ Purkinje fibers

The Bundle of His, L. and R. bundle branches, and purkinje fibers innervate the interventricular septum and walls of the ventricle.

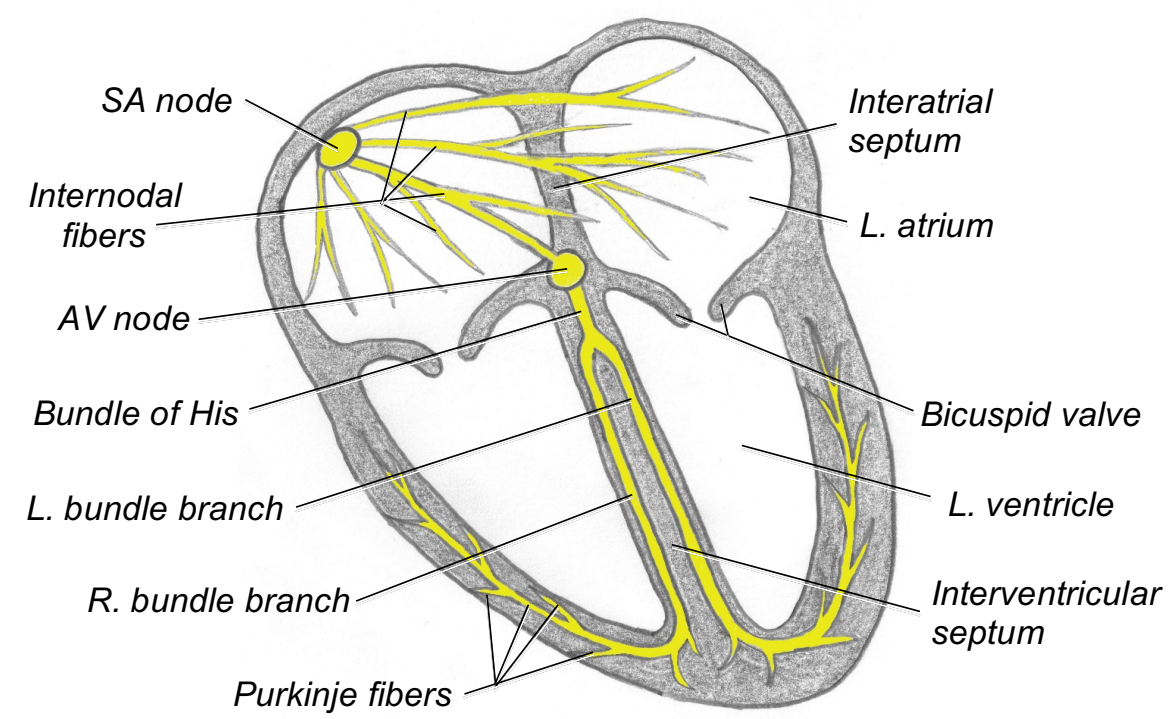

Figure 12.7. Electrical Conduction Structures of the Heart

\section{Clinical Application: Electrocardiogram}

An electrocardiogram, or ECG or EKG, is a procedure that records the electrical activity of a heart. This quick, safe, and painless procedure is non-invasive and only includes attaching electrode patches to the skin. Physicians can use it to monitor heart activity during other procedures, or they can use it in diagnosing a problem with the heart. The result of an ECG is shown below. The $P$ wave is a record of atrial depolarization (contraction) as stimulated by the SA node; the P-R segment is a record of delayed impulse by the AV node; the QRS complex is a record of ventricular depolarization (contraction) and atrial repolarization (relaxation); and the $\mathbf{T}$ wave is a record of ventricular repolarization (relaxation).

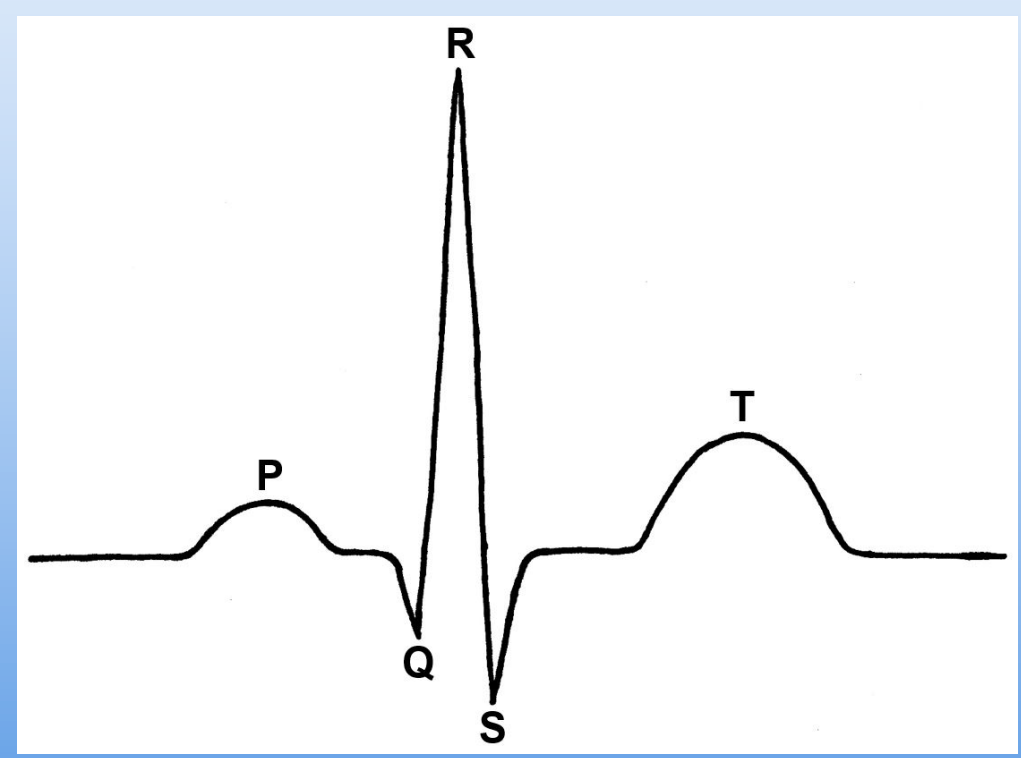




\section{Coronary vessels}

$\underline{\text { Coronary }}=$ referring to the arteries that supply the heart.

R. coronary a.

○ R. marginal a.

- Posterior interventricular a.

L. coronary a.

- Anterior interventricular a.

○ L. marginal a.

$\square$ L. marginal $v$.

$\square$ Small cardiac v. (or R. marginal v.)

$\square$ Great cardiac v. (or Anterior interventricular v.)

$\square$ Middle cardiac v. (or Posterior interventricular v.)

Coronary sinus

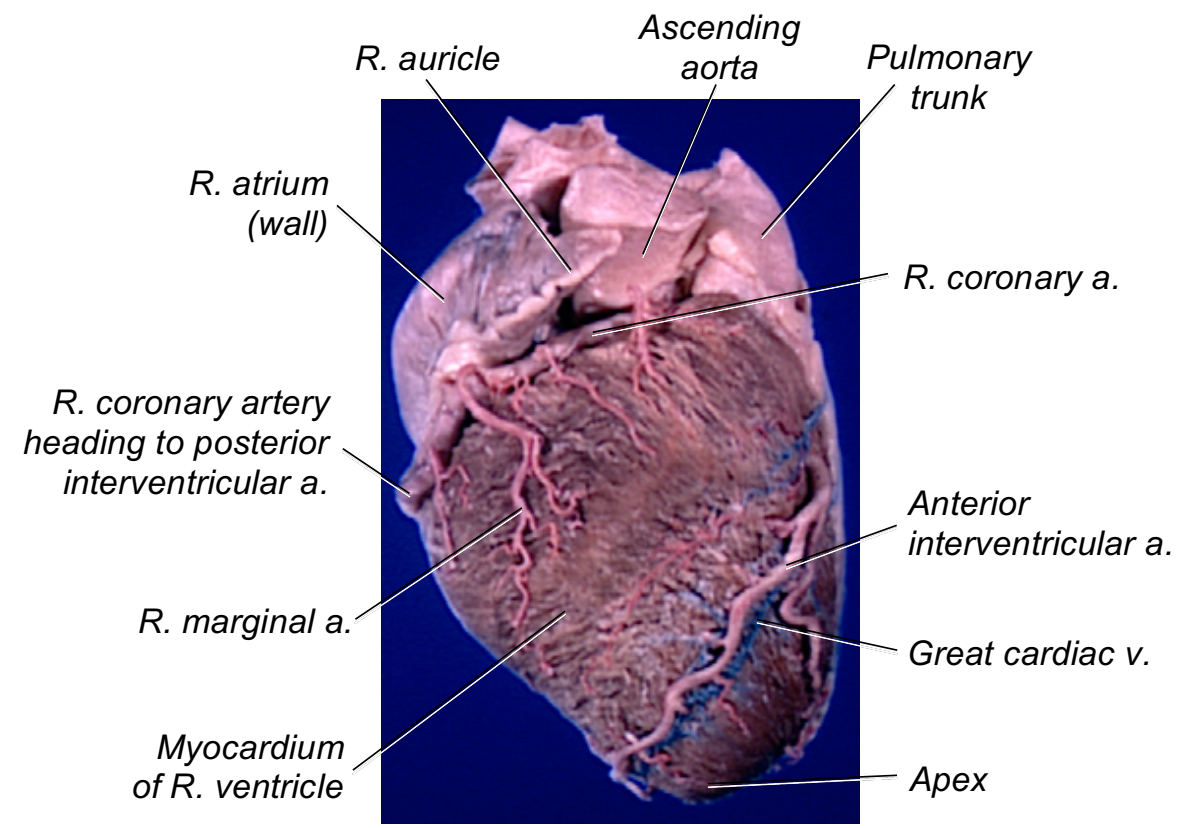

Figure 12.8. Coronary Vessels, Anterior View

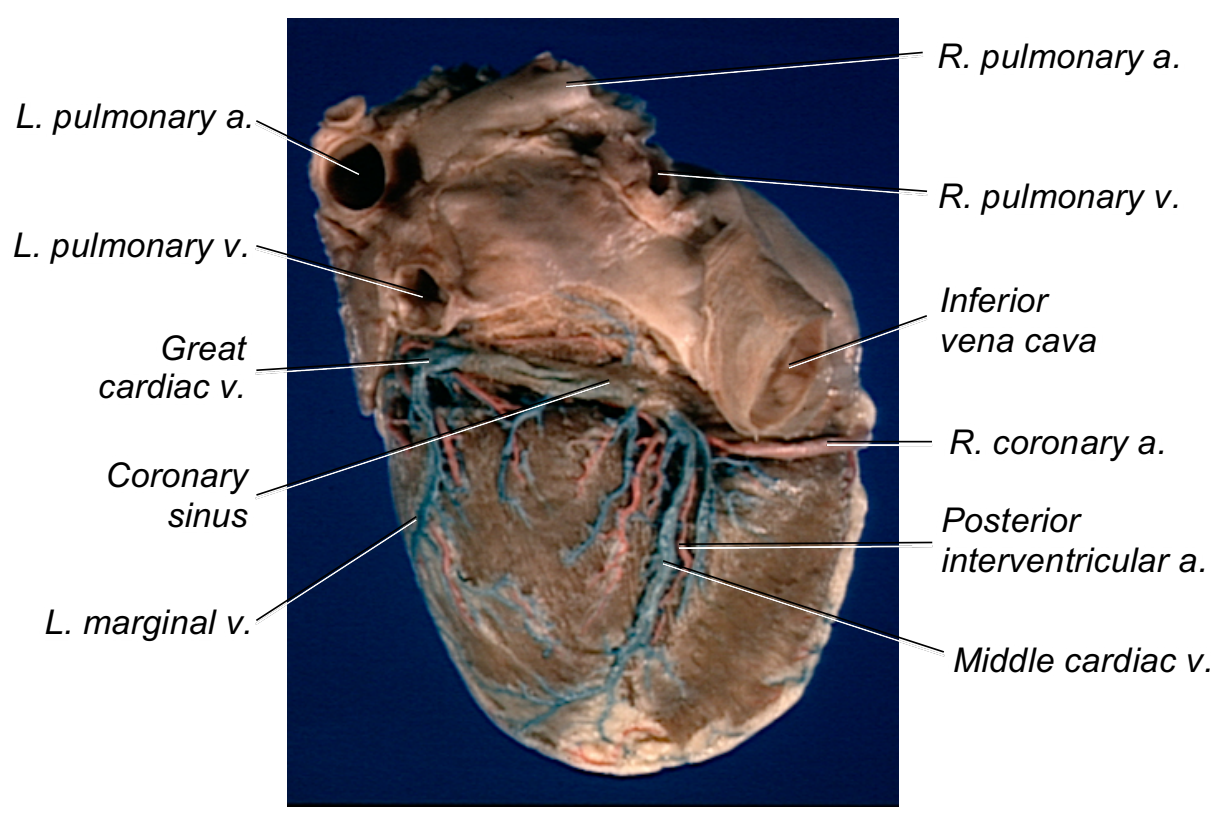

Figure 12.9. Coronary Vessels, Posterior View

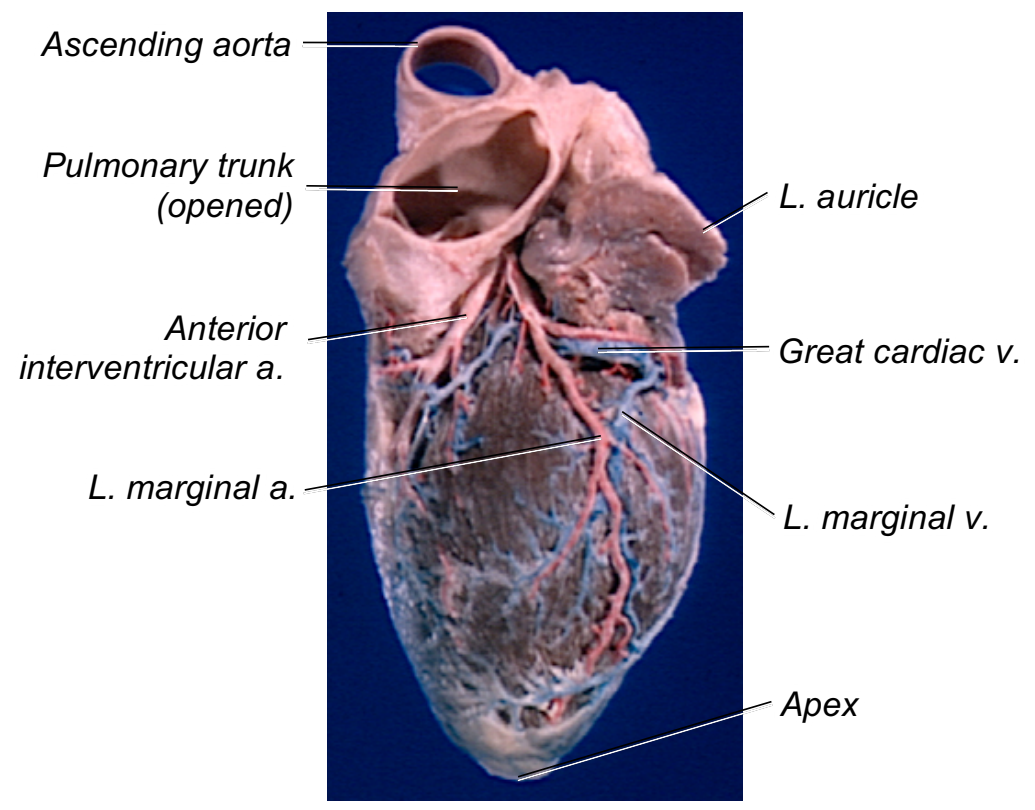

Figure 12.10. Coronary Vessels, Left Lateral View 


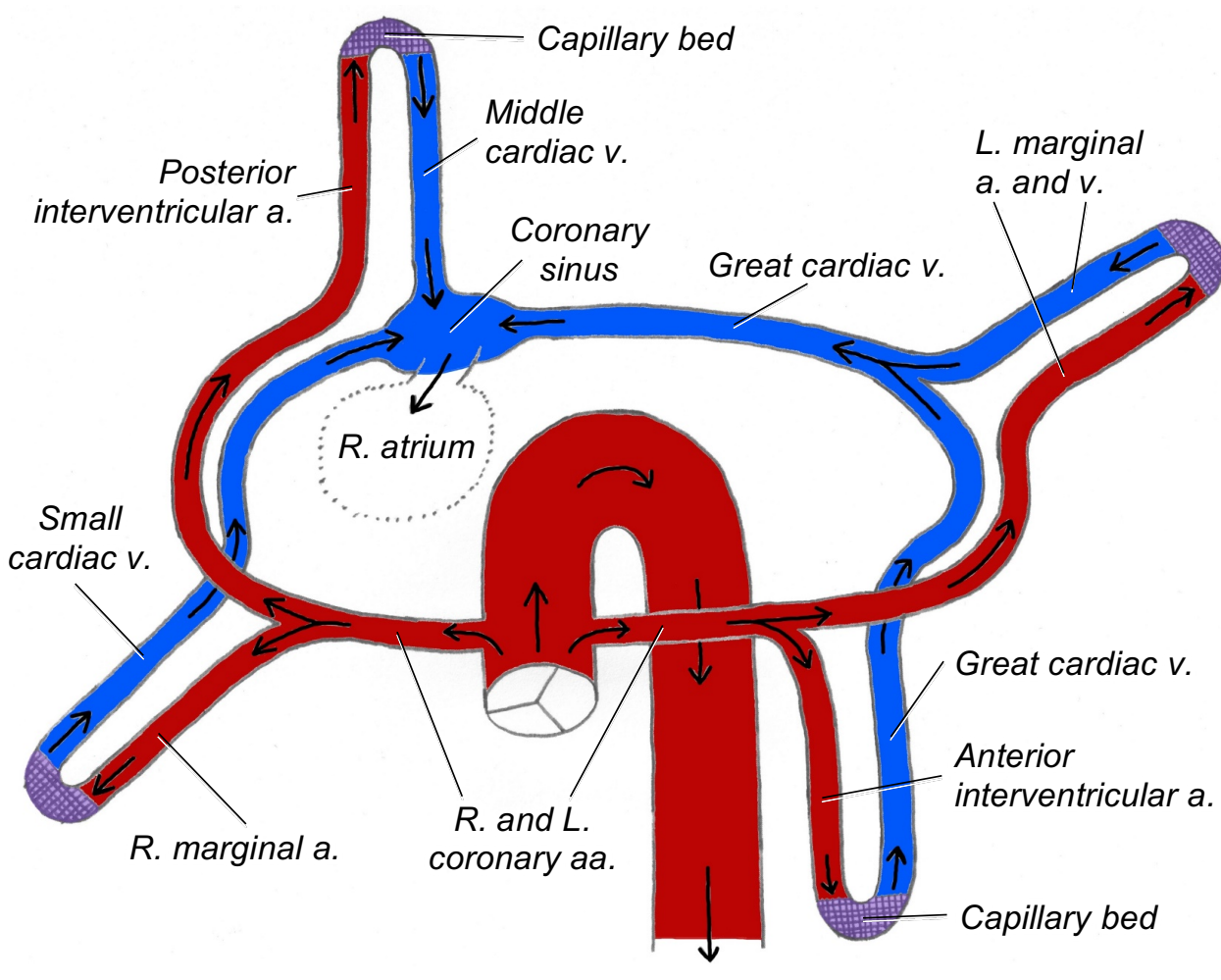

Figure 12.11. Simplified Diagram of Coronary Circulation

\section{Clinical Application: Myocardial Infarction}

Infarction $=$ the obstruction of blood supply to a tissue, causing localized tissue death.

A myocardial infarction, or heart attack, often results from a blockage any one of the many coronary vessels which deliver oxygenated blood to the heart muscle (myocardium). Without oxygen, the affected myocardium dies and therefore can no longer contract properly and pump blood through the heart. Typical symptoms of a myocardial infarction include tightness of the chest, chest pains, fatigue, anxiety, abnormal heart rhythm, lightheadedness, and shortness of breath, among others. Medical help should be sought for a myocardial infarction. Physicians can diagnose a myocardial infarction from an electrocardiogram (ECG), a quick, safe, and painless procedure that records the electrical activity of the heart. Physicians can also use an ECG to detect how much heart damage has occurred, identify the location of the infarction, and monitor progress after treatment. 
and self-assess your knowledge.

1) In order, list the structures that blood flows through as it passes through the heart. (LO33)

2) In order, list the electrical conduction structures that innervate the heart. (LO33)

3) What are the first two branches off the aorta? (LO32)

4) What are the two branches of the right coronary artery? (LO32)

5) How many openings allow blood to flow into or out of an adult right atrium? (LO32)

6) What vein collects blood from the anterior wall of the heart? (LO32)

7) Where did blood just flow from before the left coronary artery? (LO33)

8) Fill in the following blanks with correct numbers:

There are pulmonary veins and

pulmonary arteries. (LO32)
9) Fill in the following blanks with the words arteries or veins: are vessels that carry blood toward the heart, and are vessels that carry blood away from the heart. (LO31)

10)What type of blood (oxygenated or deoxygenated) is found in an adult pulmonary vein? (LO31)

11)What does the term pericardial mean? (LO31)

12)What does the term pulm mean? (LO31)

13)Which structures innervate pectinate muscles? (LO32)

14)Match the following valve movements with their correct causes:

Opening of bicuspid and tricuspid valves:

Closing of bicuspid and tricuspid valves:

Opening of pulmonary and aortic valves:

Closing of pulmonary and aortic valves:

a. Contraction of the atria.

b. Pressure from the blood in the atria.

c. Contraction of the ventricles.

d. Pressure from the blood in the ventricles.

e. Papillary muscles contracting and pulling on chordae tendineae.

f. Pressure from the blood in the pulmonary trunk and aorta. 


\section{Lab 13: Vasculature of the Head, Neck, Thorax, \& Abdomen}

Instructions: Learn the listed terms using the listed learning objectives, labeled images, lab specimens, and other resources.

LO34: Describe the terminology used to identify vasculature of the head, neck, thorax, and abdomen.

LO35: Identify the vessels of the head, neck, thorax and abdomen.

LO36: Describe the flow pathway of blood through the head, neck, thorax, and abdomen.

\section{Regions of the aorta}

$\square$ Thoracic aorta
- Ascending aorta
- Aortic arch
○ Descending thoracic aorta

\section{Abdominal aorta}

- Descending abdominal aorta

The thorax and abdomen are separated by the diaphragm, a bowl-shaped muscle that contracts to cause inhalation of air into the lungs. The ascending aorta primarily supplies oxygenated blood to the heart. The aortic arch primarily supplies oxygenated blood to the head, neck, and upper limbs, and the descending thoracic aorta primarily supplies oxygenated blood to the walls of the thorax (the intercostal spaces). The abdominal aorta primarily supplies oxygenated blood to the abdominal organs, pelvis and pelvic organs, and lower limbs.

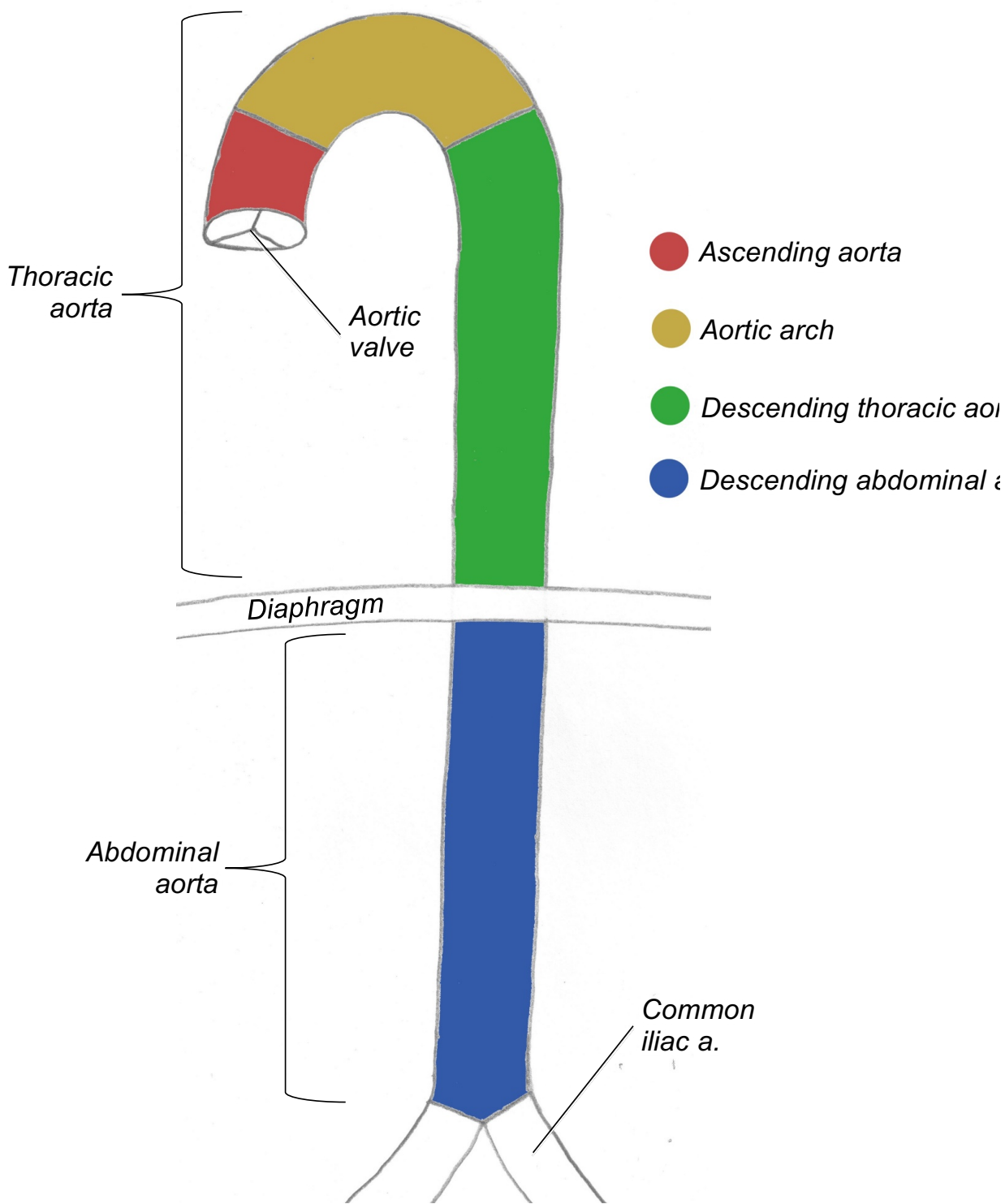

Figure 13.1. Diagram of Regions of the Aorta 


\section{Branches of the thoracic aorta}

$\square$ R. \& L. coronary aa

Brachiocephalic trunk

Do you remember what brachi and ceph mean?

○ R. common carotid a.

- R. subclavian a.

- R. vertebral artery

$\square$ L. common carotid a.

$\square$ L. subclavian a.

\section{- L. vertebral a.}

The vertebral aa. ascend the neck through transverse foramina in the cervical vertebrae before entering the cranium through the foramen magnum.

- Internal thoracic a.

- Anterior intercostal aa.

$\square$ Posterior intercostal aa.

$\square$ Subcostal a.

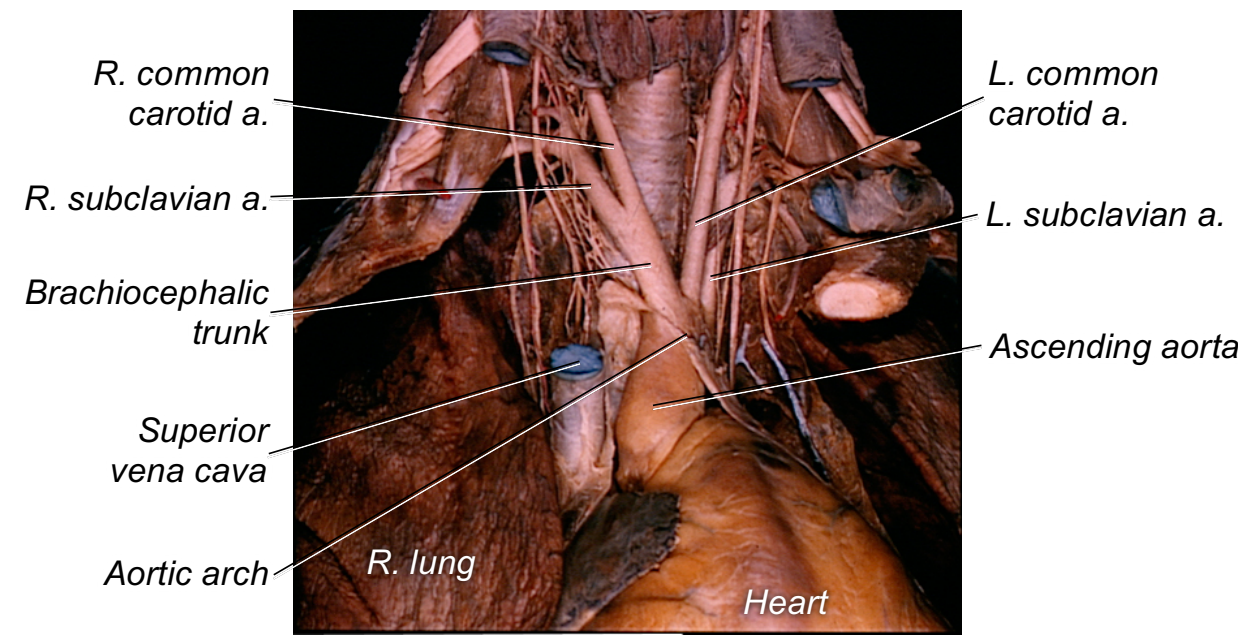

Figure 13.2. Vasculature of the Superior Thorax, Anterior View

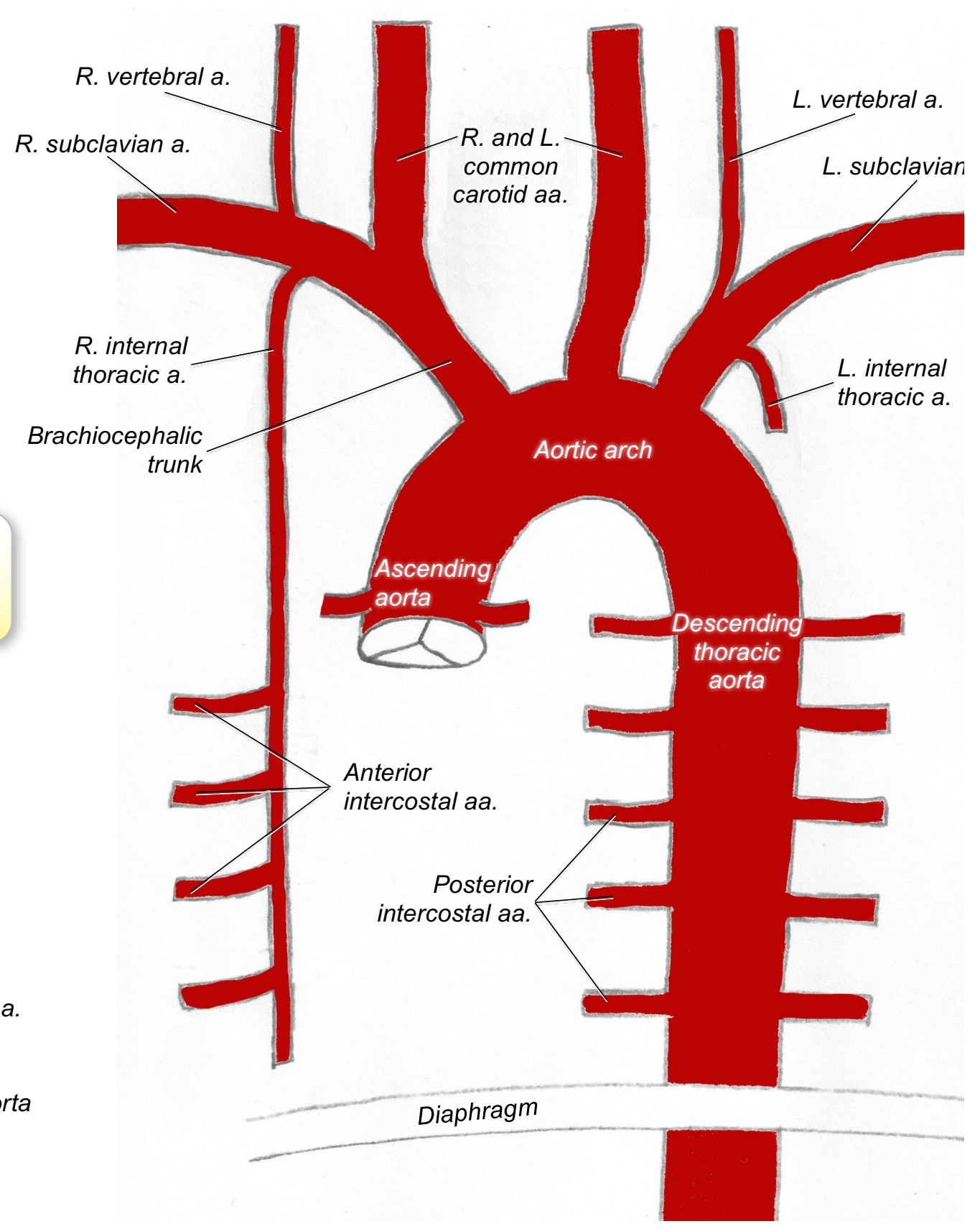

Figure 13.3. Diagram of Branches of the Thoracic Aorta 


\section{Veins of the thorax}

$\square$ R. \& L. Subclavian vv.

$\square$ R. \& L. brachiocephalic vv.

$\square$ Superior vena cava

$\square$ Inferior vena cava

$\square$ Intercostal vv.

Azygos v.

\section{$\underline{\text { Azygos }}=$ unpaired}

$\square$ Hemiazygos $v$.

$\underline{\text { Hemi }}=$ half

$\square$ Accessory hemiazygos $v$.

$\square$ Pulmonary vv.

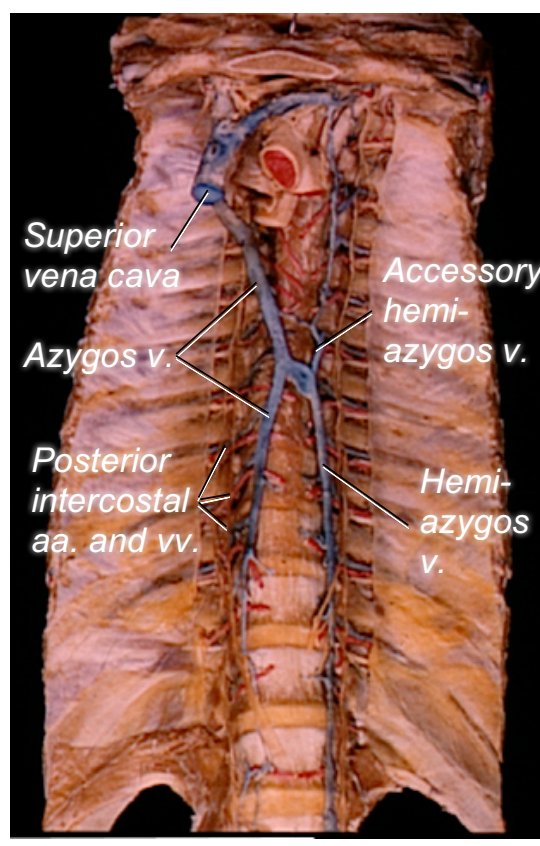

Figure 13.4. Veins of the Posterior Thorax, Anterior View

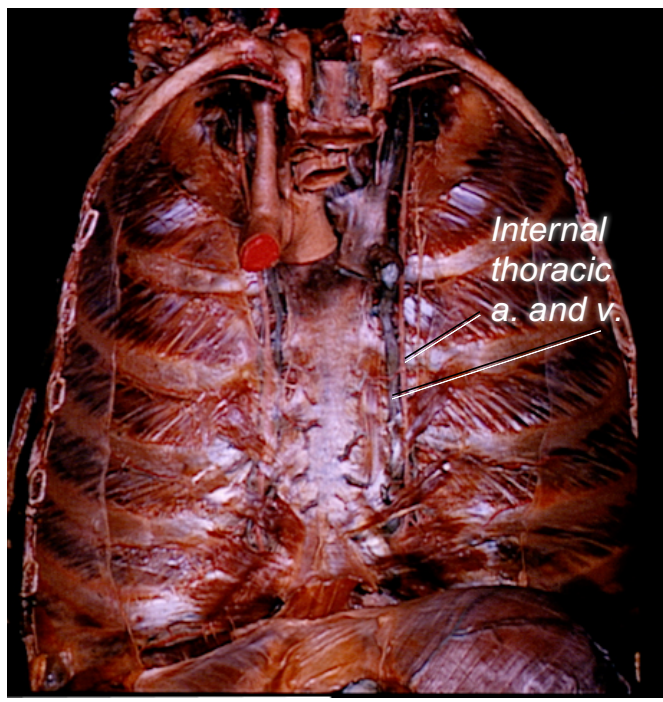

Figure 13.5. Veins of the Anterior Thorax, Posterior View

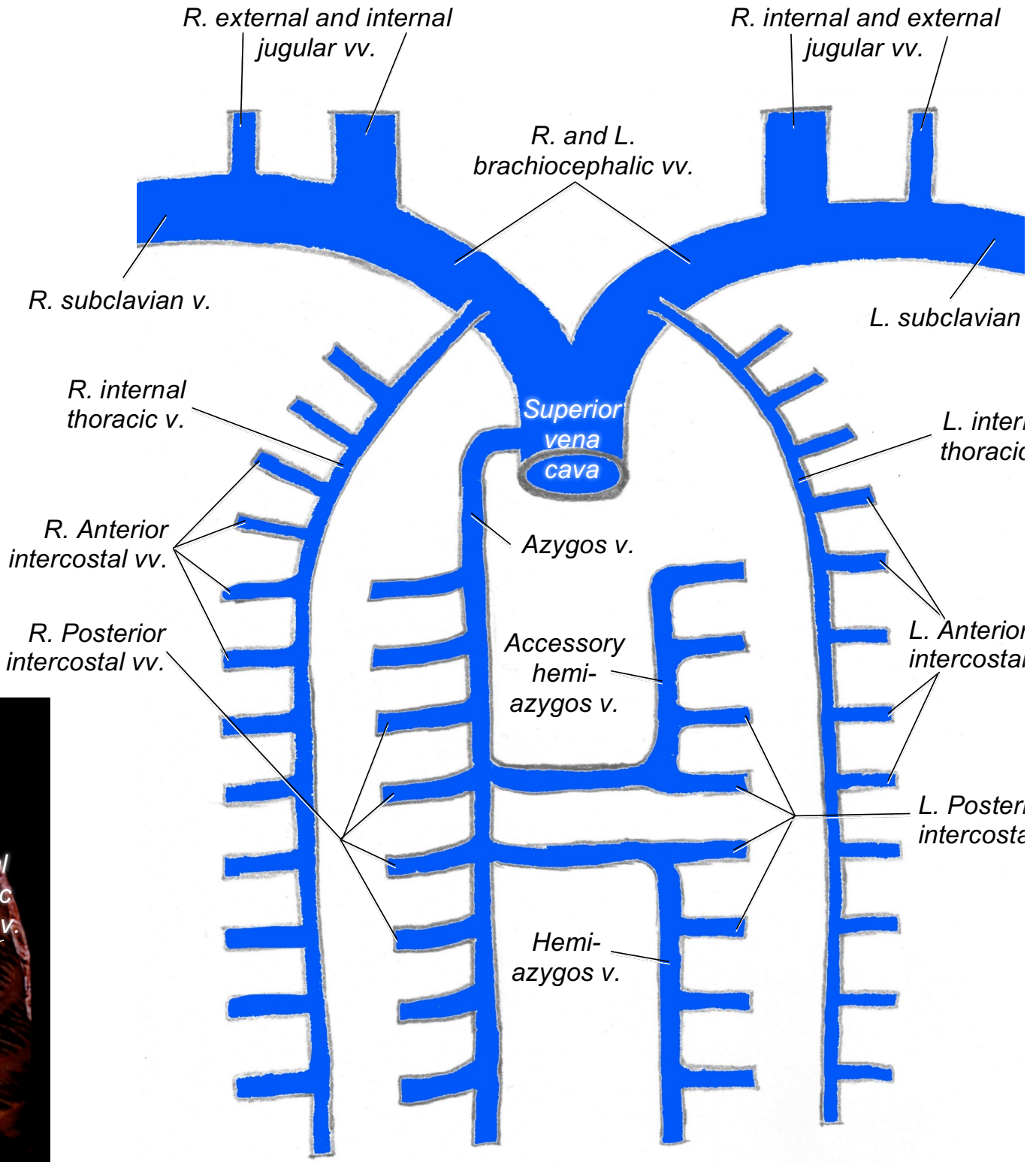

Figure 13.6. Diagram of Veins of the Thorax 


\section{Arteries of the neck and head}

$\square$ R. \& L. common carotid aa.

$\square$ R. \& L. carotid bifurcations (or Carotid sinuses)

$\square$ R. \& L. external carotid aa.

○ R. \& L. facial aa.

$\square$ R. \& L. Internal carotid aa.

$\square \mathrm{R} \& \mathrm{~L}$ vertebral aa.

Arterial circle of Willis

These vessels supply blood to the brain.

○ R. \& L. anterior cerebral aa.

- Anterior communicating a.

○ R. \& L. middle cerebral aa.

- Basilar a.

- R. \& L. posterior cerebral aa.

$\circ$ R. \& L. posterior communicating aa.

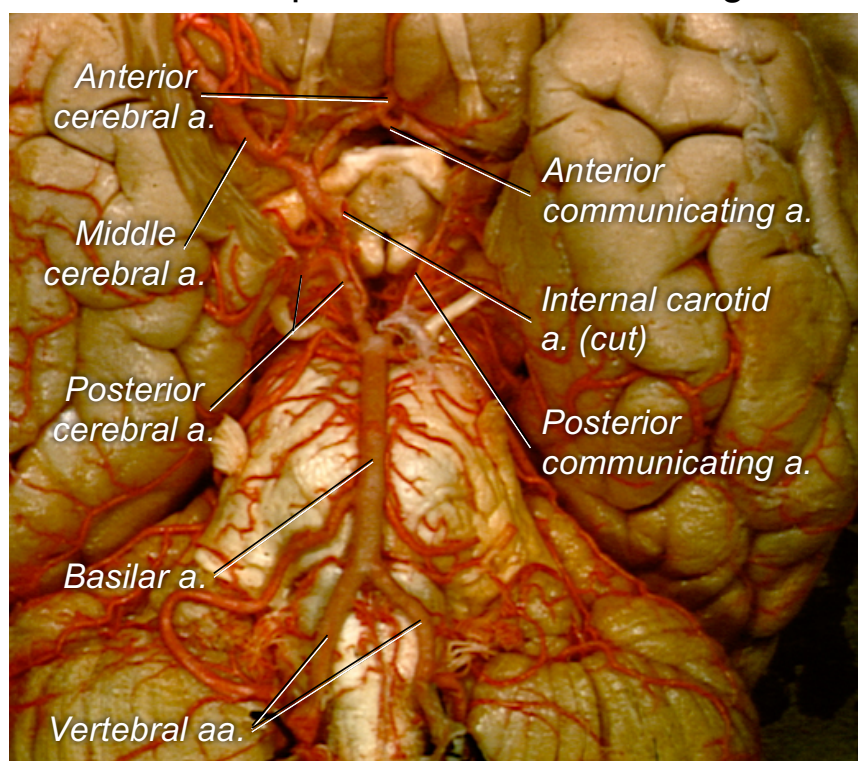

Figure 13.7. Vasculature of the Arterial Circle of Willis

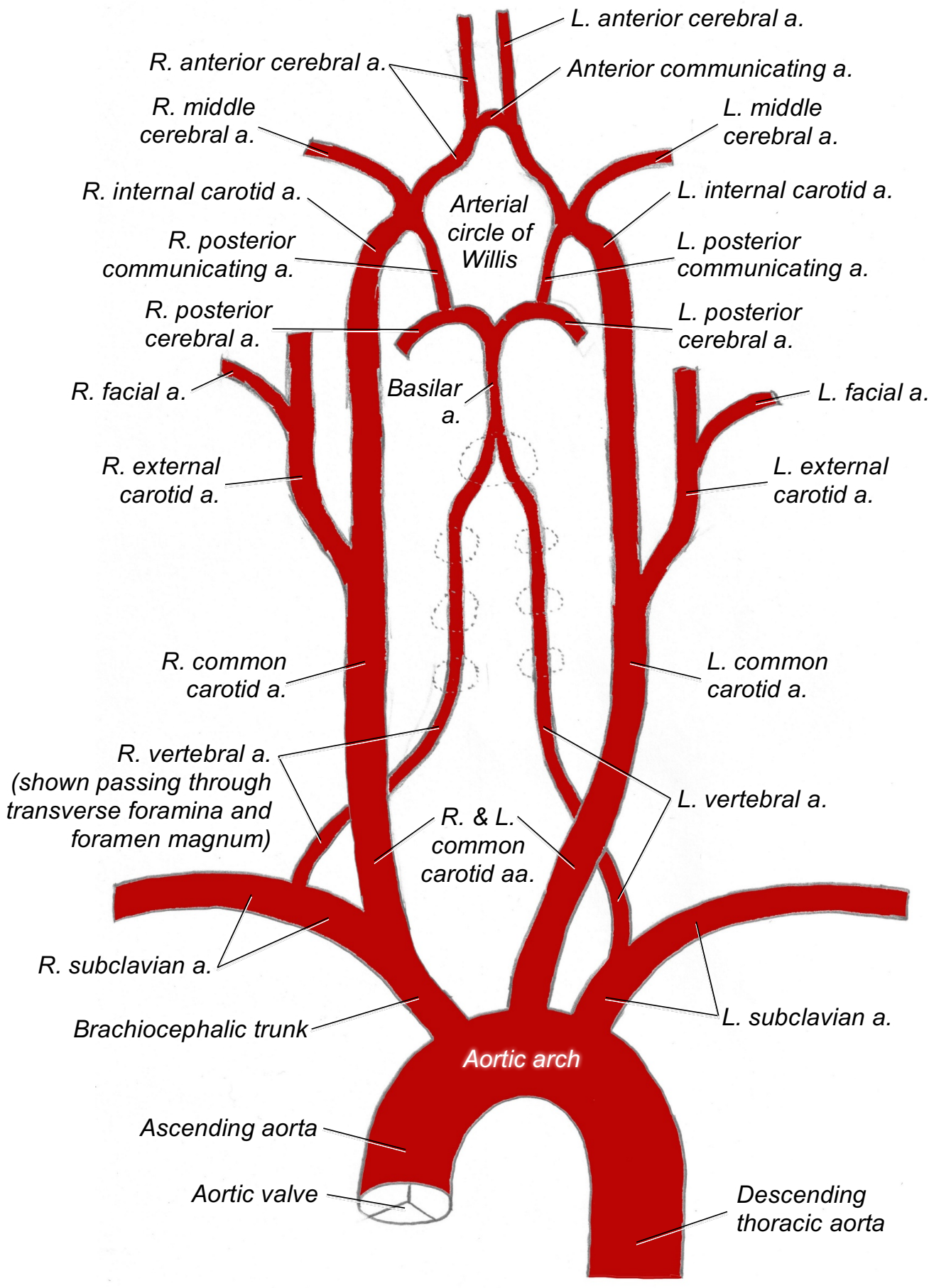

Figure 13.8. Diagram of Arteries of the Neck and Head 


\section{Clinical Application: Cerebrovascular Accident}

A cerebrovascular accident (CVA), or stroke, happens when blood flow is occluded to an area of the brain, causing the death of brain cells (neurons) in that area. There are two main types of CVAs: ischemic stroke and hemorrhagic stroke. In an ischemic stroke, a blood clot blocks the flow of blood in a vessel, preventing blood and oxygen from reaching the brain. In a hemorrhagic stroke, a blood vessel ruptures preventing blood and oxygen from getting to part of the brain. Symptoms vary on location of tissue death, but typical (sudden) symptoms can include dizziness, loss of balance and coordination, difficulty speaking, localized numbness/paralysis, and/or sudden headache accompanied by vomiting. Medical help should be sought right away if a stroke is assumed.

\section{Veins of the neck and head}

$\square$ Cerebral vv. (grouped)

$\square$ Dural sinuses
- Superior sagittal sinus
- Inferior sagittal sinus
- Straight sinus
- Confluence of sinuses
- Transverse sinuses
- Sigmoid sinuses

$\square$ R. \& L. internal jugular vv.

○ R. \& L. facial vv.

R. \& L. external jugular vv.

The external jugular vv. drain into the subclavian vv., and the combination of the subclavian vv. with the internal jugular vv. forms the brachiocephalic vv.

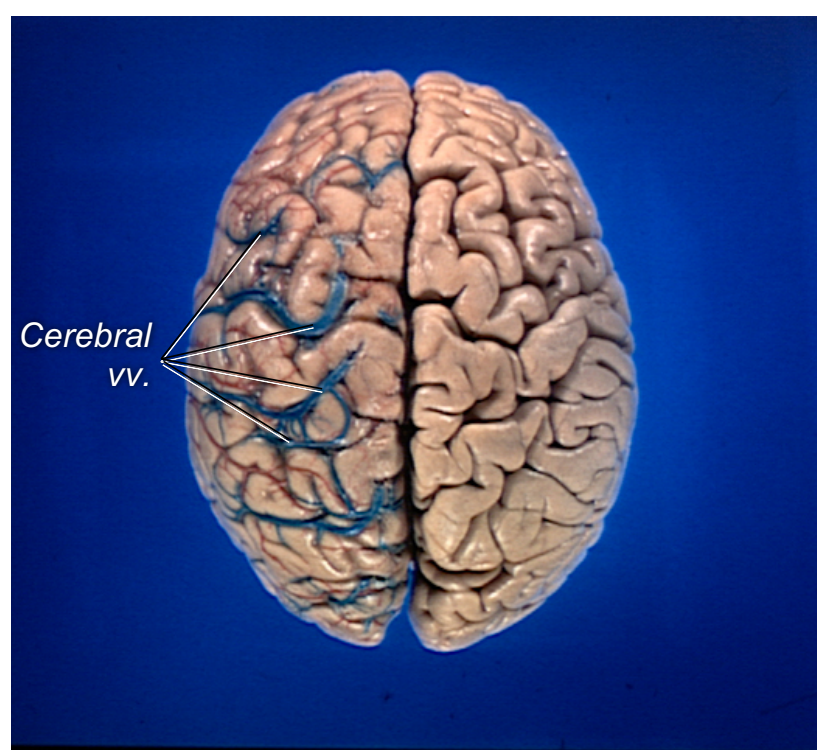

Figure 13.9. Cerebral Veins, Superior View

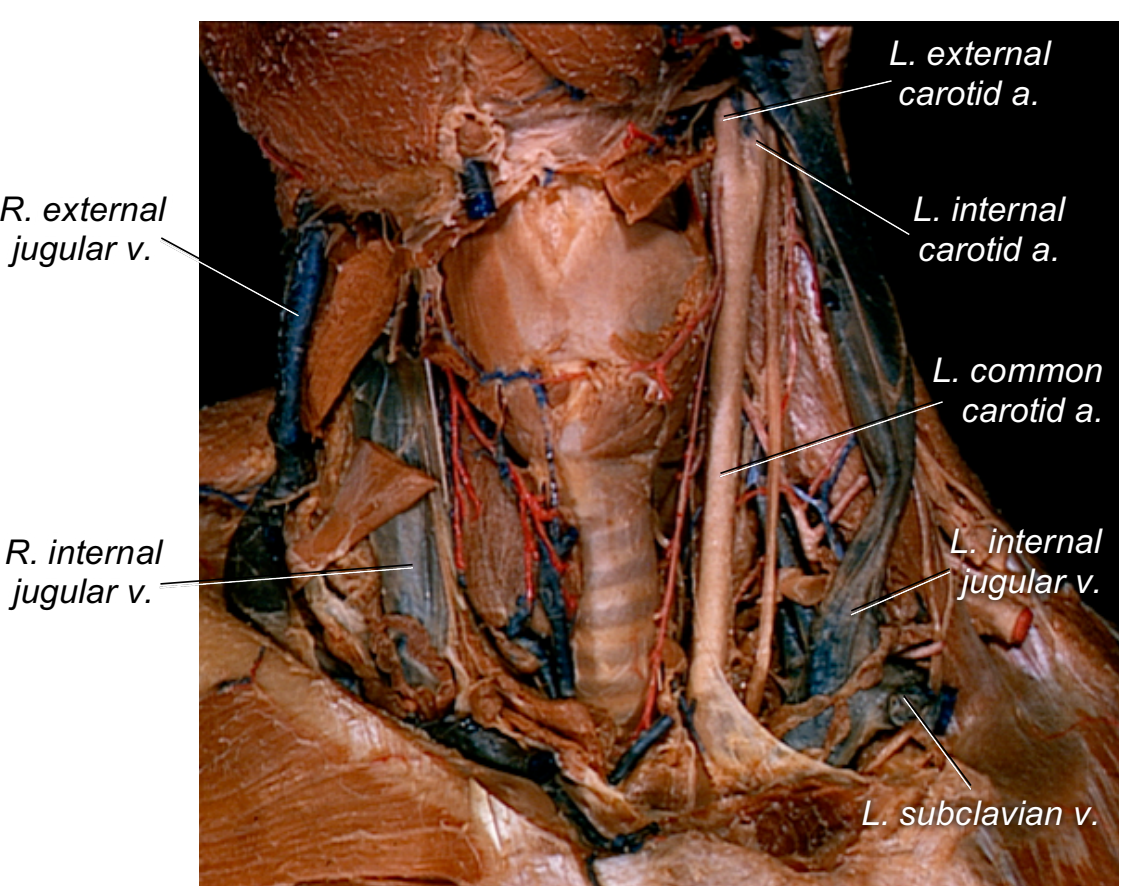

Figure 13.10. Vasculature of the Neck, Anterior View 


\section{Branches of the abdominal aorta}

$\square$ Celiac trunk

- Common hepatic a.

\section{$\underline{\text { Gastr }}=$ stomach}

○ L. gastric a.

$\underline{\text { Hepat }}=$ liver

○ Splenic a.

$\underline{\text { Splen }}=$ spleen

R. \& L. renal aa.

$\underline{R e n}=$ kidney

$\square$ Superior mesenteric a.

○ Middle colic a.

$\mathrm{Col}=$ colon

The colon has 4 main named regions: right colon, middle colon, left colon, and sigmoid colon.

○ R. colic a.

○ Intestinal aa.

$\underline{\text { Intestin }}=$ small intestines

$\square$ Inferior mesenteric a.

○ L. colic a.

○ Sigmoid a.

- Superior rectal a.

$\underline{\text { Rect }}=$ rectum

R. \& L. gonadal aa.

$\underline{\text { Gonad }}=$ related to the testes (in males) or ovaries (in females).

$\square$ R. \& L. common iliac aa.
○ R. \& L internal iliac aa.

These arteries supply the pelvis and gluteal regions.

- R. \& L external iliac aa.

These arteries supply the lower limbs.

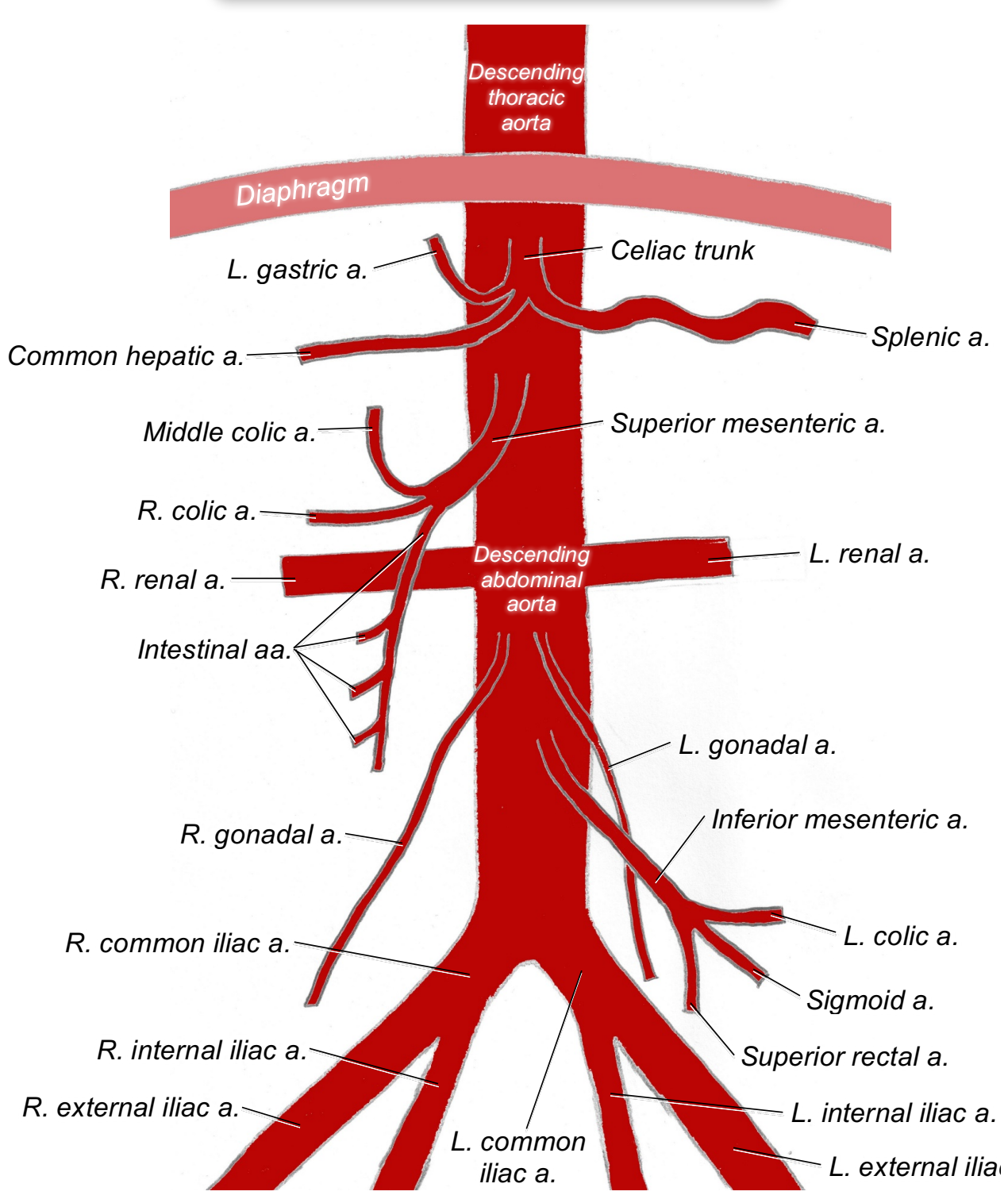

Figure 13.11. Diagram of Branches of the Abdominal Aorta 


\section{Veins of the abdomen}

$\square$ R. \& L. internal iliac vv.

$\square$ R. \& L. external iliac vv.

$\square$ R. \& L. common iliac vv.

R. \& L. gonadal vV.

Notice how the L. gonadal $\mathbf{v}$. drains into the L. renal $\mathbf{v}$. while the R. gonadal $\mathbf{v}$. drains directly into the inferior vena cava.

$\square$ R. \& L. renal vv.

$\square$ Inferior vena cava

\section{Hepatic portal system (part of veins of the abdomen)}

$\square$ Left gastric v.

Splenic v.

Superior mesenteric v.

o Intestinal vv.

○ R. colic v.

- Middle colic $v$.

$\square$ Inferior mesenteric v.

○ L. colic V.

- Sigmoid v.

- Superior rectal v.

\section{Portal v.}

$\square$ Hepatic vv.

$\square$ Inferior vena cava

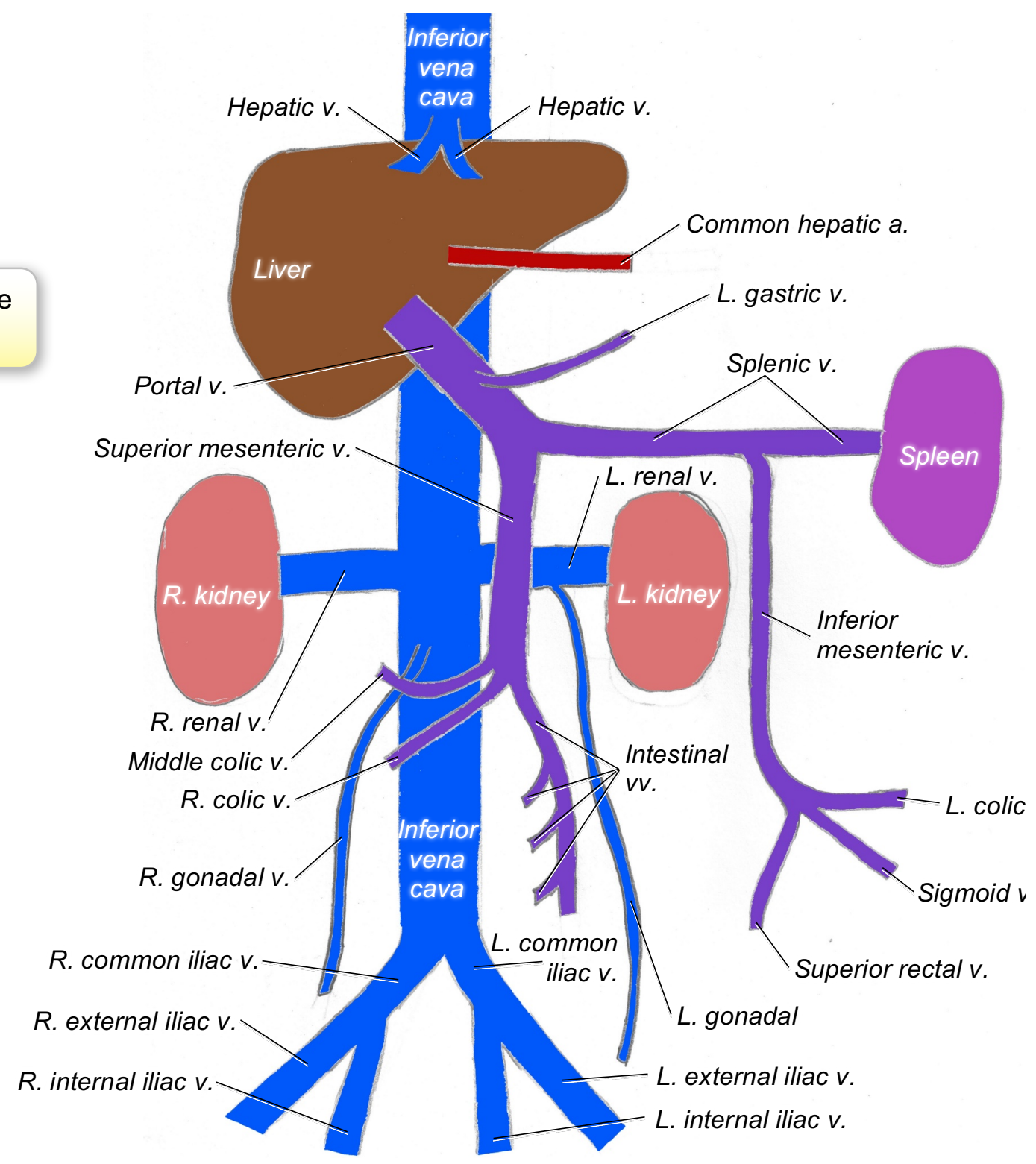

Figure 13.12. Diagram of Abdominal Veins 
Hepatic vv.

Clean, Deoxygenated blood

(heading to heart to be re-oxygenated)

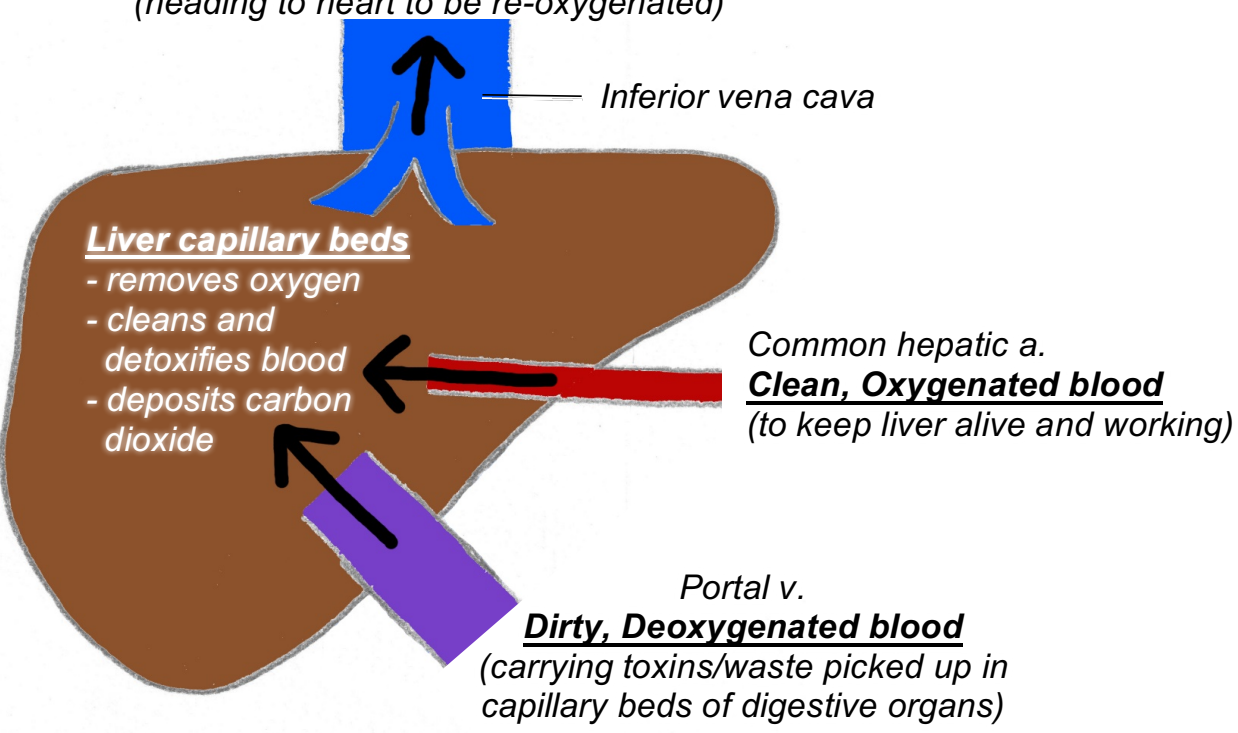

Figure 13.13. Vasculature of the Liver

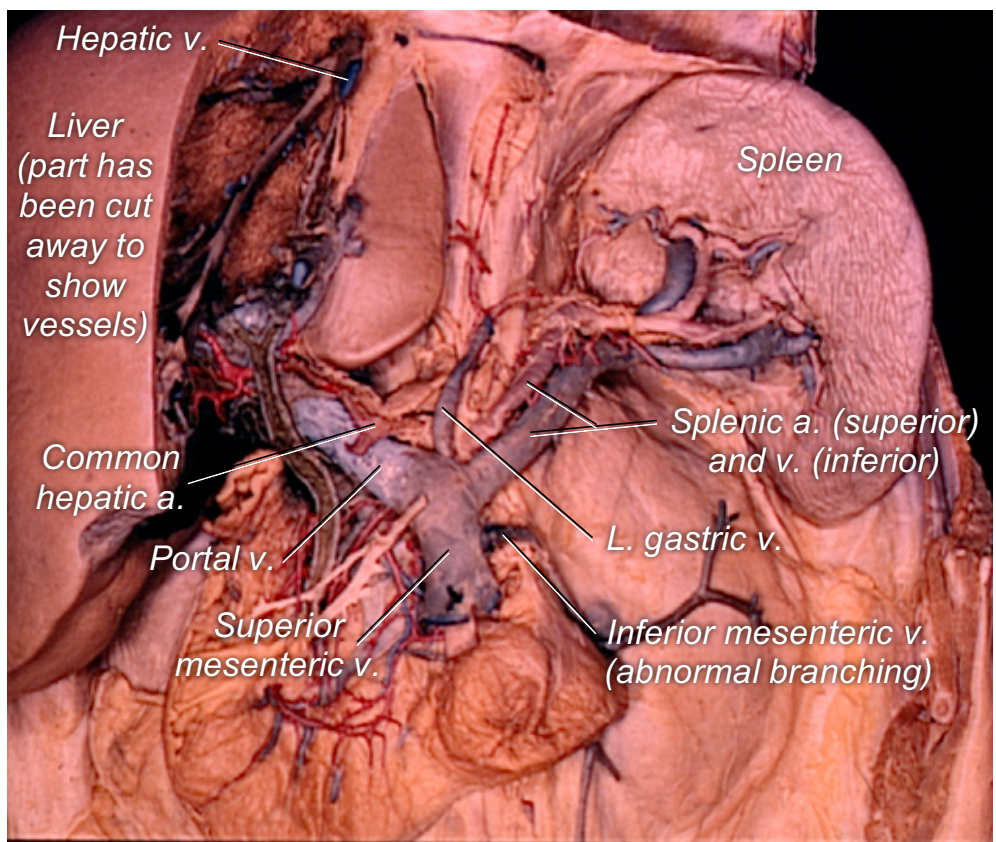

Figure 13.14. Vasculature of the Portal System, Anterior View

\section{Figure 13.13. Vasculature of the Liver}

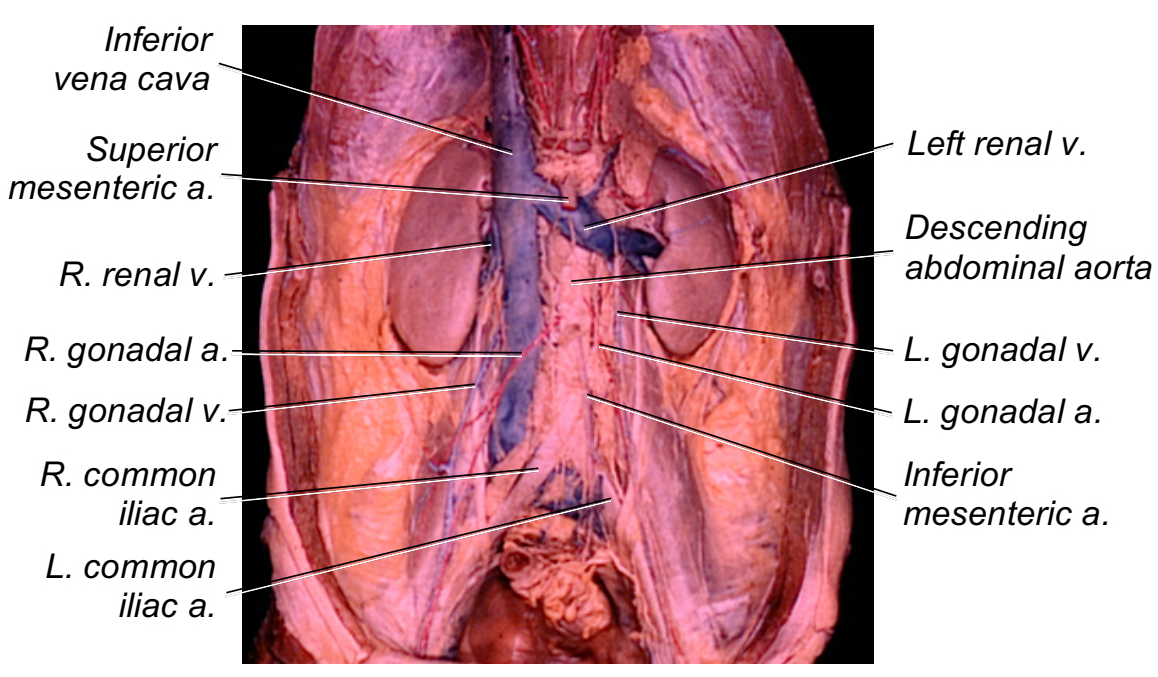

Figure 13.15. Vasculature of the Abdomen, Anterior View 
1) What are the first three branches of the thoracic aorta? (LO35)

2) Using the terminology you've learned, what two regions of the body would the brachiocephalic trunk supply blood to? (LO34)

3) What separates the descending thoracic aorta from the descending abdominal aorta? (LO35)

4) How many brachiocephalic arteries and veins do you have? (LO35)

5) What two vessels combine to form one brachiocephalic vein? (LO35)

6) What vessel does the azygos v. drain into? (LO36)

7) What are the three branches of the celiac trunk? (LO35)

8) What does the term azygos mean? (LO34)

9) What does the term gastr mean? (LO34)

10)What vessel does the R. gonadal v. drain into? (LO36)
12)What are the terminal branches of the aorta? (LO35)

13)The facial $a$. is a branch of the and the facial v. drains into the (LO35)

14)The internal thoracic $a$. is a branch of the , and the internal thoracic $\mathrm{v}$.

drains into the (LO35)

15)In order, list all the structures/vessels that blood will flow through as it flows from the $L$. ventricle to the $L$. anterior cerebral a. to the superior rectal a. to the R. atrium. (LO36) 


\section{Lab 14: Vasculature of the Upper \& Lower Limbs}

Instructions: Learn the listed terms using the listed learning objectives, labeled images, lab specimens, and other resources.

LO37: Describe the terminology used to identify vasculature of the upper and lower limbs.

LO38: Identify the vessels of the upper and lower limbs.

LO39: Describe the flow pathway of blood through the upper and lower limbs.

\section{Arteries of the upper limb}

$\square$ R. \& L. axillary aa.

$\underline{\text { Axilla }}=$ armpit

$\square$ R. \& L. brachial aa.

$\square$ R. \& L. radial aa.

$\square$ R. \& L. ulnar aa.

$\square$ R. \& L. palmar arches aa. (grouped)

$\square$ R. \& L. digital branches aa. (grouped)

\section{Deep veins of the upper limb}

$\square$ R. \& L. Digital branches vv. (grouped)

$\square$ R. \& L. Palmar arches vv. (grouped)

$\square$ R. \& L. Radial vv.

$\square$ R. \& L. Ulnar vv.

$\square$ R. \& L. Brachial vv.

$\square$ R. \& L. Axillary vv.

\section{Superficial veins of the upper limb}

$\square$ R. \& L. cephalic vv.

$\square$ R. \& L. median cubital vv.

$\square$ R. \& L. basilic vv.

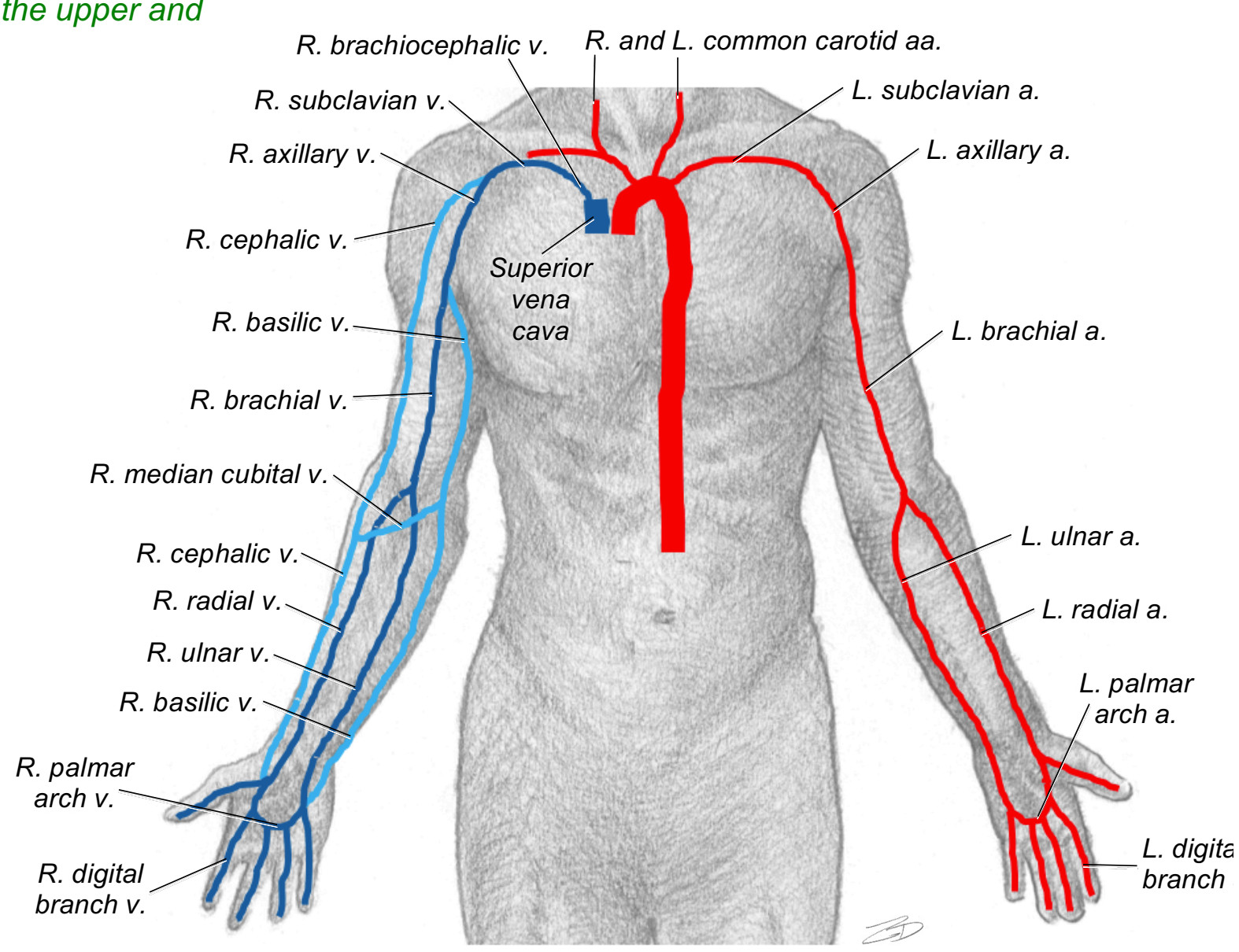

Figure 14.1. Vasculature of the Upper Limb 


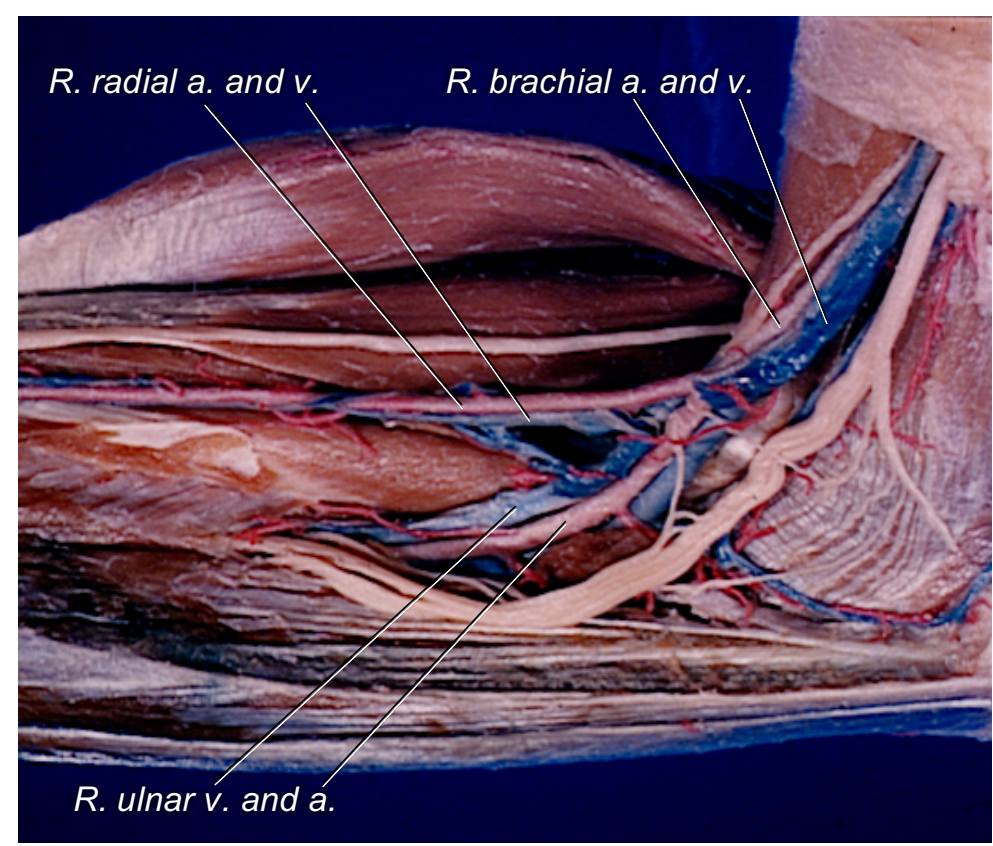

Figure 14.2. Vasculature of the Right Anterior Elbow

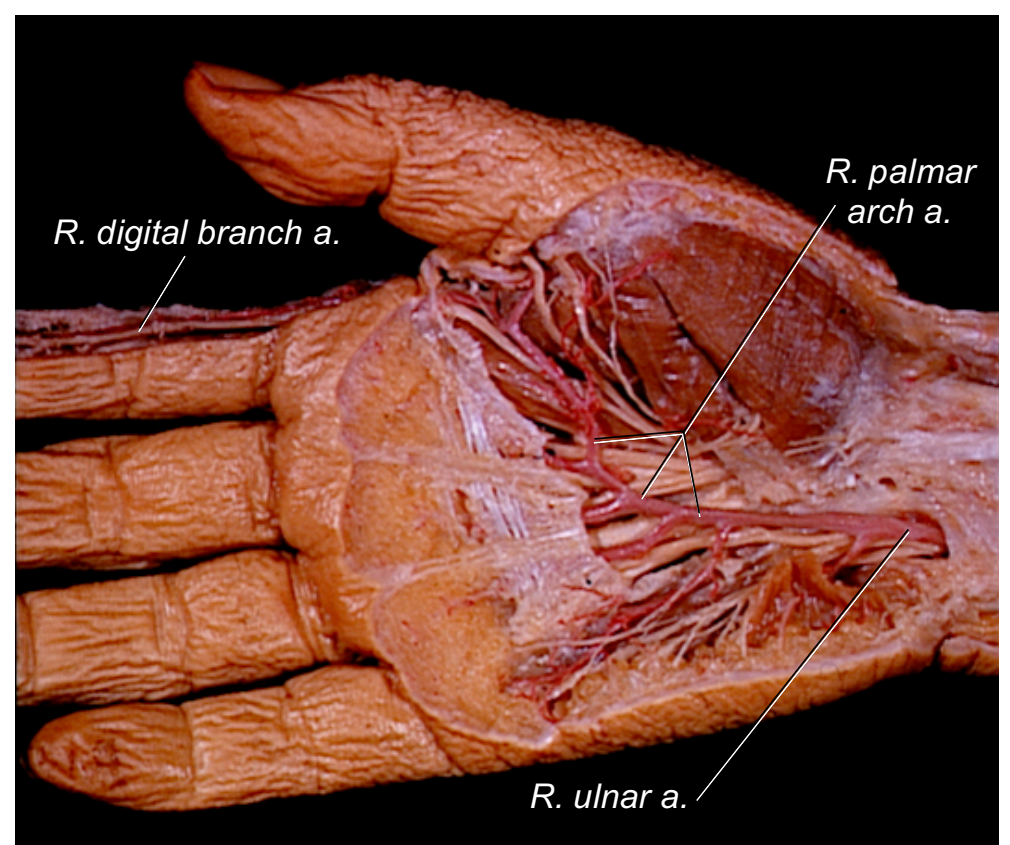

Figure 14.3. Vasculature of the Right Hand, Anterior View

\section{Clinical Application: Blood Pressure}

When the heart's ventricles contract and force blood into the aorta, the pressure of blood increases. That pressure then drops as the ventricles relax and fill and blood passes through the body's capillary beds into lowpressure veins. Having a healthy blood pressure is important for heart health and overall cardiovascular health. Blood pressure can easily be measured with a blood pressure cuff. When the cuff is applied around the arm and inflated, the pressure of the cuff temporarily cuts off the circulation through the brachial artery. As the pressure on the cuff is slowly released allowing blood to slowly flow through the brachial artery again, a sphygmomanometer (blood pressure meter/gauge) is used to record the (high) pressure at which blood begins to move through the brachial artery. This number is the systolic blood pressure, or the pressure which is being exerted on the walls of arteries when the ventricles are contracting. As the cuff is deflated, another (low) pressure number is recorded, known as the diastolic blood pressure, or the pressure which is being exerted on the walls of arteries when the ventricles are relaxed and filling. Normal systolic blood pressure is between $\mathbf{8 0 -}$ $120 \mathrm{~mm}$ HG, and normal diastolic blood pressure is between $\mathbf{6 0 - 8 0 ~} \mathrm{mm} \mathrm{Hg}$. Blood pressure (overall) is reported as systolic blood pressure over diastolic blood pressure (i.e. 120/80). 


\section{Arteries of the lower limb}

$\square$ R. \& L. femoral aa.

$\square$ R. \& L. deep femoral aa.

$\square$ R. \& L. popliteal aa.

$\square$ R. \& L. anterior tibial aa.

Dorsalis pedis a.

$\square$ R. \& L. posterior tibial aa.

$\square$ R. \& L. pedal arches aa. (grouped)

$\square$ R. \& L. digital branches aa. (grouped)

\section{Deep veins of the lower limb}

$\square$ R. \& L. pedal arches vv. (grouped)

$\square$ R. \& L. digital branches vv. (grouped)

$\square$ R. \& L. anterior tibial vv.

$\square$ Dorsalis pedis $\mathrm{v}$.

$\square$ R. \& L. posterior tibial vv.

$\square$ R. \& L. popliteal vv.

$\square$ R. \& L. femoral vv.

\section{Superficial veins of the lower limb}

$\square$ R. \& L. great saphenous v.

$\square$ R. \& L. small saphenous v.

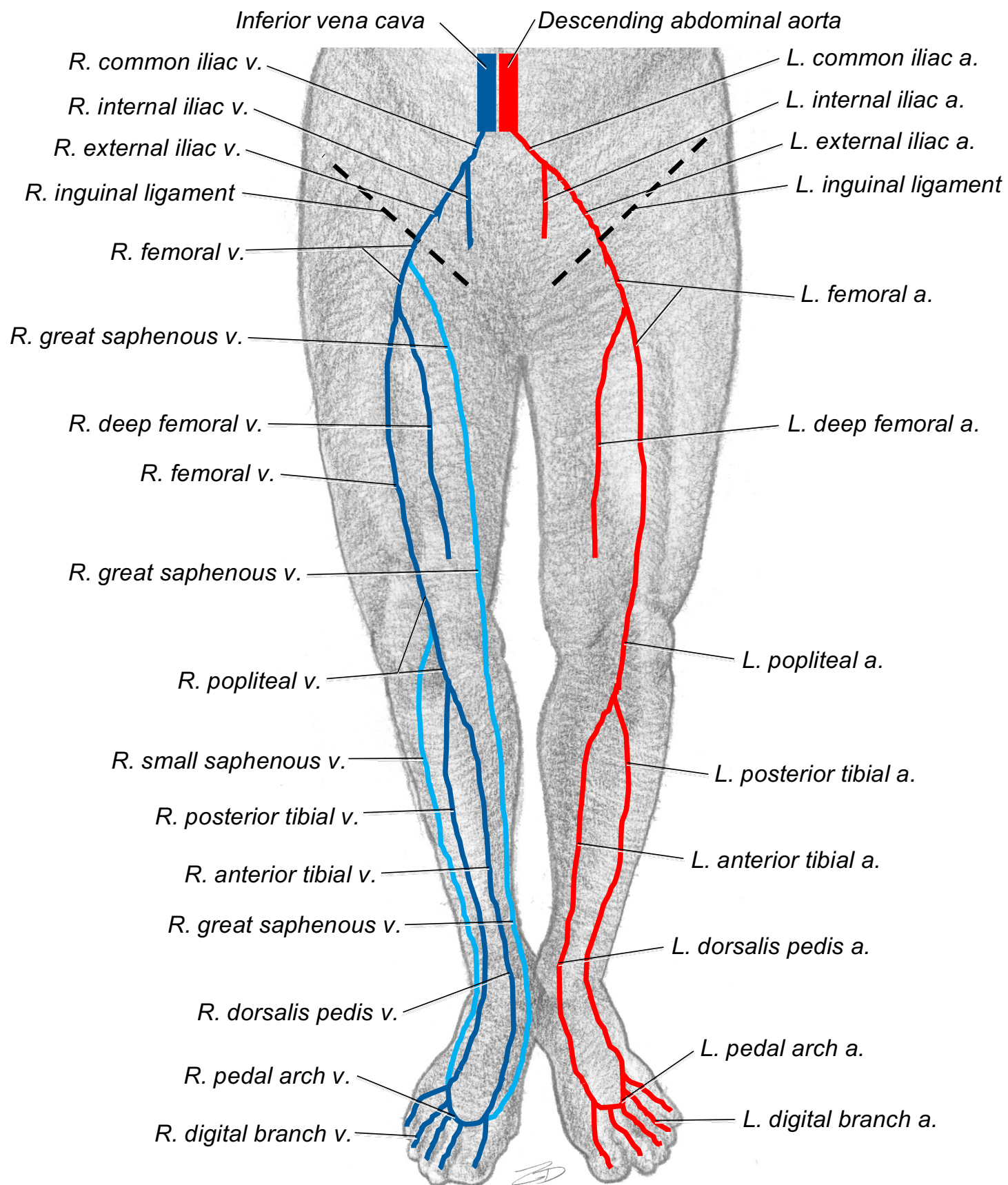

Figure 14.4. Vasculature of the Lower Limb 


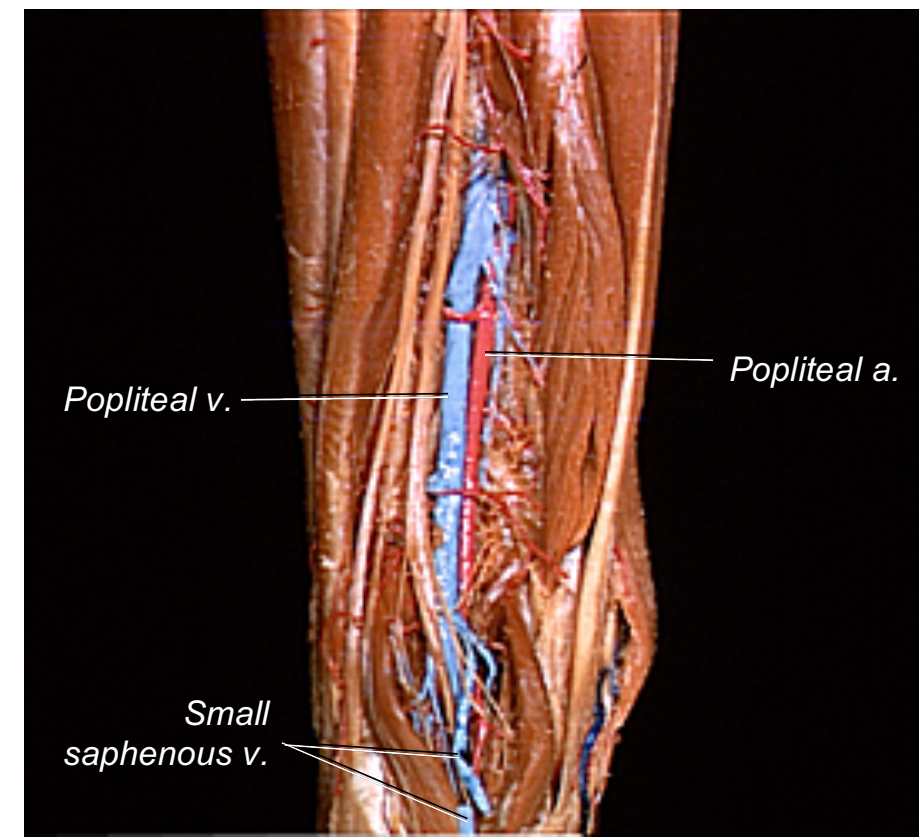

Figure 14.5. Vasculature of the Posterior Knee

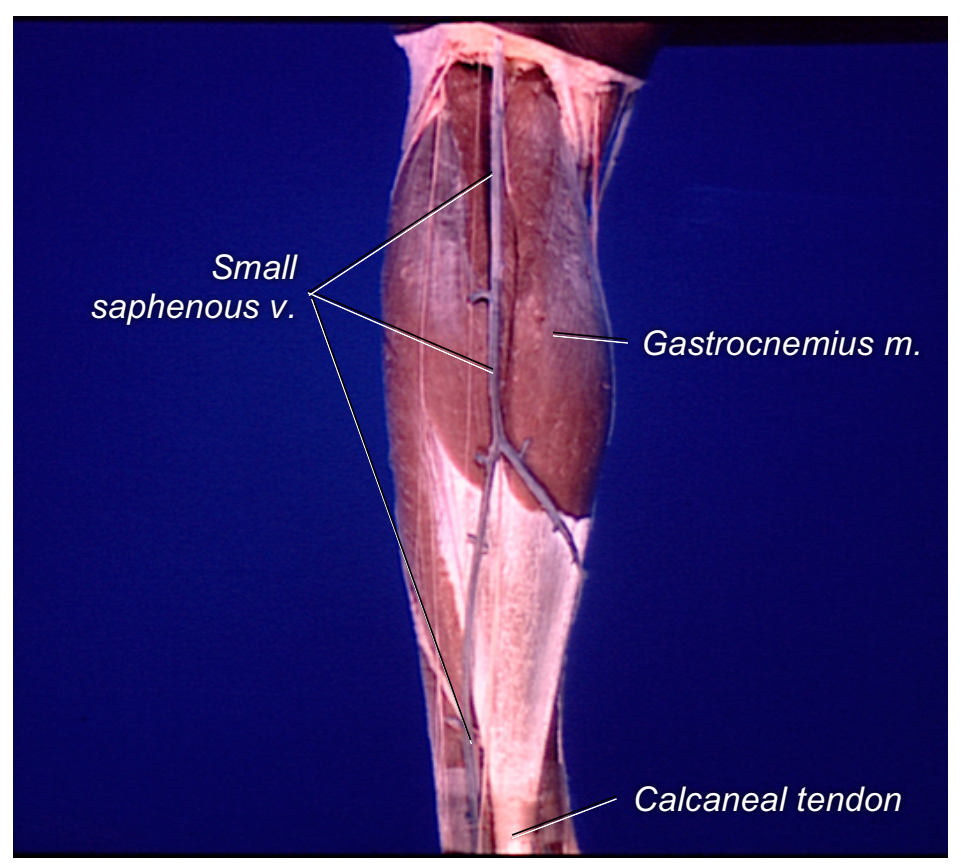

Figure 14.6. Small Saphenous Vein on Posterior Leg

\section{Clinical Application: Blood Transfusions}

Blood types are determined by the presence of $\mathbf{A}$ or $\mathbf{B}$ antigens on the surface of red blood cells (RBCs); Type A blood has A antigens on RBCs, Type B blood has $B$ antigens on RBCs, Type $A B$ blood has both $A$ and $B$ antigens on RBCs, and Type $O$ blood has neither $A$ or $B$ antigens are on RBCs. Further, each blood type has the other blood types' A or B antibodies in the plasma; Type A blood has $B$ antibodies in the plasma, Type B blood has $A$ antibodies in the plasma, Type AB blood has neither $A$ or $B$ antibodies in the plasma, and Type $O$ blood has both $A$ and $B$ antibodies in the plasma. Further, $a$ third antigen known as Rh factor may be present (+) or absent (-). All of these components of blood play important roles in whether or not a body will accept or reject a blood transfusion. Use the information above and supplemental information if necessary to understand the Blood Transfusion Compatibility Table below.

Blood Transfusion Compatability Table

\begin{tabular}{|c|c|c|c|c|c|c|c|c|c|}
\hline & \multicolumn{8}{|c|}{ Donor } \\
\hline & & 0- & $0+$ & A- & A+ & B- & B+ & AB- & AB+ \\
\hline \multirow{4}{*}{$\frac{+\infty}{\Phi}$} & O- & $\sqrt{ }$ & X & X & X & X & X & X & $X$ \\
\hline & $0+$ & $\mathscr{N}$ & $\mathscr{N}$ & $\mathbf{X}$ & $\mathbf{X}$ & $\mathbf{X}$ & $\mathbf{X}$ & $\mathbf{X}$ & $\mathbf{X}$ \\
\hline & A- & $\mathscr{I}$ & $\mathbf{X}$ & $\mathscr{V}$ & $\mathbf{X}$ & X & $\mathbf{X}$ & $\mathbf{X}$ & $\mathbf{X}$ \\
\hline & At & $\sqrt{ }$ & $\sqrt{ }$ & $\sqrt{ }$ & $\sqrt{ }$ & $\mathbf{X}$ & X & $\mathbf{X}$ & $\mathbf{X}$ \\
\hline \multirow{4}{*}{$\stackrel{\bar{d}}{\alpha}$} & B- & $\mathscr{I}$ & $\mathbf{X}$ & $\mathbf{X}$ & $\mathbf{X}$ & $\mathscr{N}$ & $\mathbf{X}$ & $\mathbf{X}$ & $\mathbf{X}$ \\
\hline & B+ & $\sqrt{ }$ & $\sqrt{ }$ & X & X & $\sqrt{ }$ & $\sqrt{ }$ & X & X \\
\hline & AB- & $\mathscr{I}$ & $\mathbf{X}$ & $\sqrt{ }$ & X & $\sqrt{ }$ & $\mathbf{X}$ & $\sqrt{ }$ & $\mathbf{X}$ \\
\hline & $\mathrm{AB}+$ & $\sqrt{ }$ & $\sqrt{ }$ & $\sqrt{ }$ & $\sqrt{ }$ & $\sqrt{ }$ & $\sqrt{ }$ & $\sqrt{ }$ & $\sqrt{ }$ \\
\hline
\end{tabular}


1) What does the term axilla mean? (LO37)

2) List the two main superficial veins of the lower limb. (LO38)

3) What region of the body would the brachial a. be found (hint: use the terminology you've learned)? (LO37)

4) What vessel would you expect to supply the rotator cuff muscles? (LO38)

5) For each vessel listed below, list the vessel(s) that blood will flow into next. (LO39)
a. Cephalic v. $\rightarrow$
b. Basilic v. $\rightarrow$
c. Small saphenous v. $\rightarrow$
d. Great saphenous v. $\rightarrow$
e. Anterior tibial a. $\rightarrow$
f. Femoral a. $\rightarrow$
g. Subclavian v. $\rightarrow$

6) The median cubital v. mainly shunts blood from the vein to the vein.
7) What are the terminal branches of the brachial a.? (LO38)

8) What vessel would you expect to supply the hamstring muscles? (LO38)

9) In order, list the structures blood would flow through to get from your pollex to your hallux.

10)Now that you've learned all of the main blood vessels in the body, create a diagram of all of the ARTERIES together. (LO38, LO39)

11)Now that you've learned all of the main blood vessels in the body, create a diagram of all of the VEINS together. (LO38, LO39) 


\section{Lab 15: Respiratory System}

Instructions: Learn the listed terms using the listed learning objectives, labeled images, lab specimens, and other resources.

LO40: Describe the terminology used to identify structures of the respiratory system.

LO41: Identify the structures of the respiratory system.

LO42: Describe the actions and innervations of respiratory musculature.

LO43: Describe the flow pathway of air and oxygen/carbon dioxide through the respiratory and cardiovascular systems.

\section{Important Respiratory System Terms}

$\square \underline{\text { Respiration }}=$ the process of breathing to taken in oxygen and expel carbon dioxide.

$\square$ Inhalation = breathing in.

$\square \overline{\text { Exhalation }}=$ breathing out.

$\circ$ Air can enter/exit through structures of the oral or nasal cavities.

Pharynx $=$ the cavity behind the nose and mouth leading to the esophagus.

$\square \underline{\text { Larynx }}=$ the hollow organ through which air passes during inhalation and exhalation; it holds the vocal cords and is more commonly known as the "voice box."

\section{Hyoid bone}

$\square$ Body

$\square$ Lesser horn

Greater horn

\section{Nasal cavity}

$\square$ Nostrils (or External nares)

$\square$ Nasal septum

- Vomer bone

- Perpendicular plate (of ethmoid bone)

○ Septal cartilage

Superior, middle, and inferior conchae

Superior, middle, and inferior meati

The meati warm, moisten, and clean air as it heads toward the lungs.

$\square$ Pharyngeal orifice of pharyngotympanic tube (or Opening for eustachian tube)

Opening of nasolacrimal duct

\section{Pharynx}

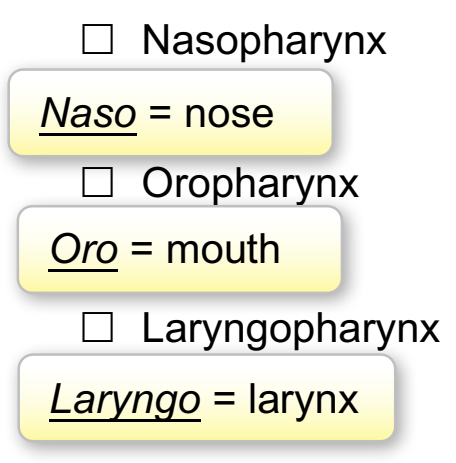




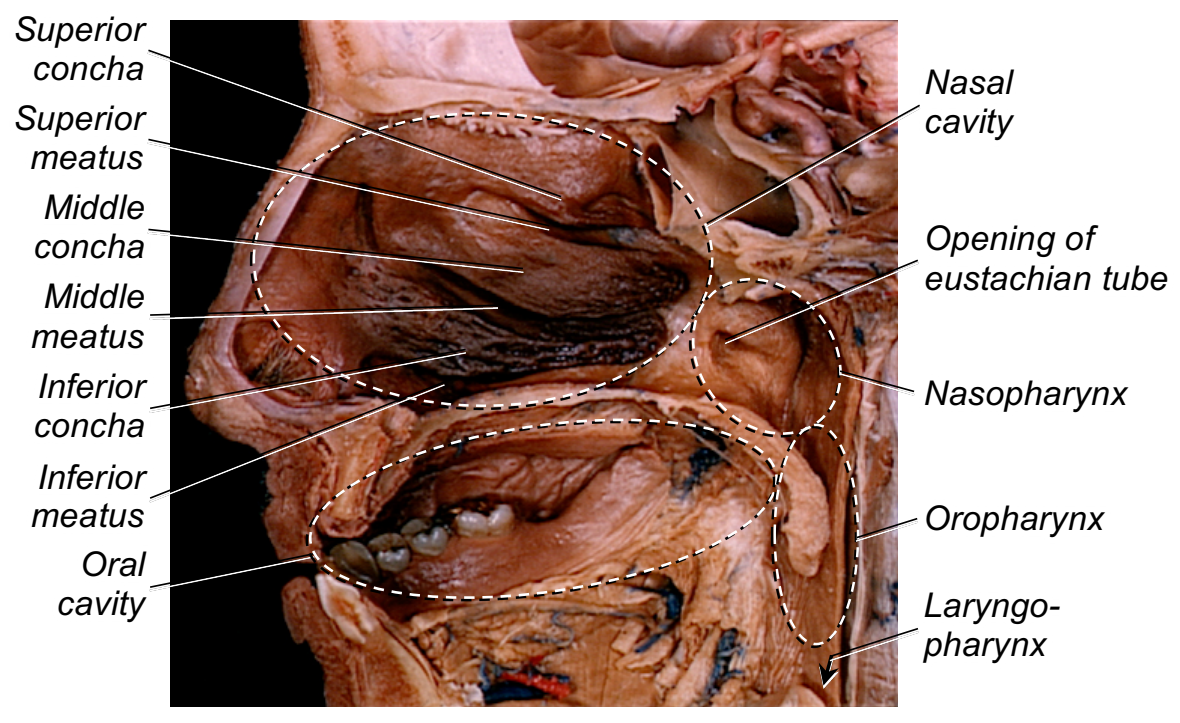

Figure 15.1. Right Head, Midsagittal View

\section{Larynx}

$\square$ False vocal cords

○ Vestibular ligaments + vestibular folds

$\square$ True vocal cords

○ Vocal ligaments + vocal folds

$\square$ Thyroid cartilage

- Laryngeal prominence

Cricoid cartilage

This is the only ring-shaped cartilage of the larynx.

$\square$ Arytenoid cartilage

Vocal ligaments attach to this cartilage.

$\square$ Epiglottis

$\square$ Glottis

The glottis is the superior opening to the larynx.

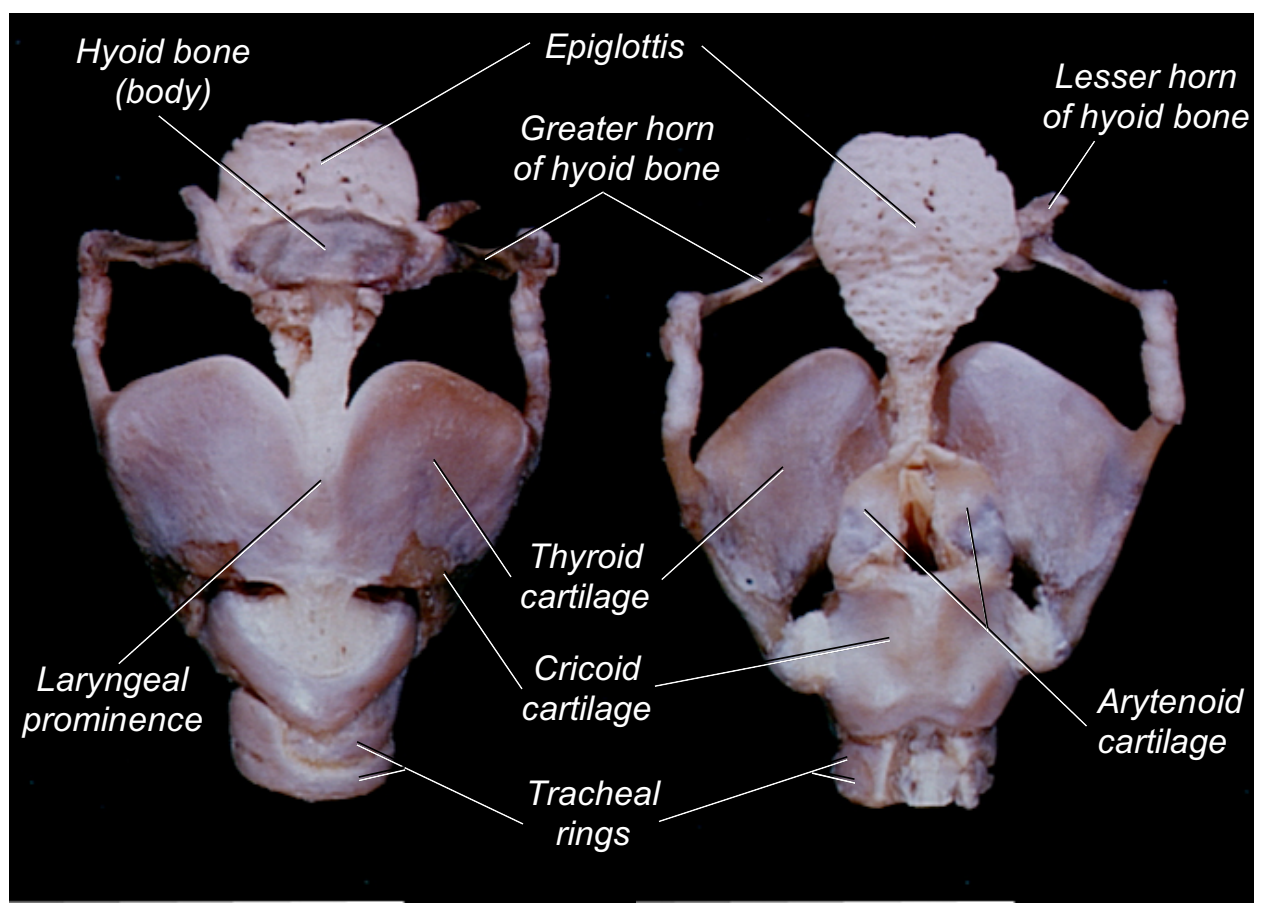

Figure 15.2. Larynx, Anterior View (Left) and Posterior View (Right)

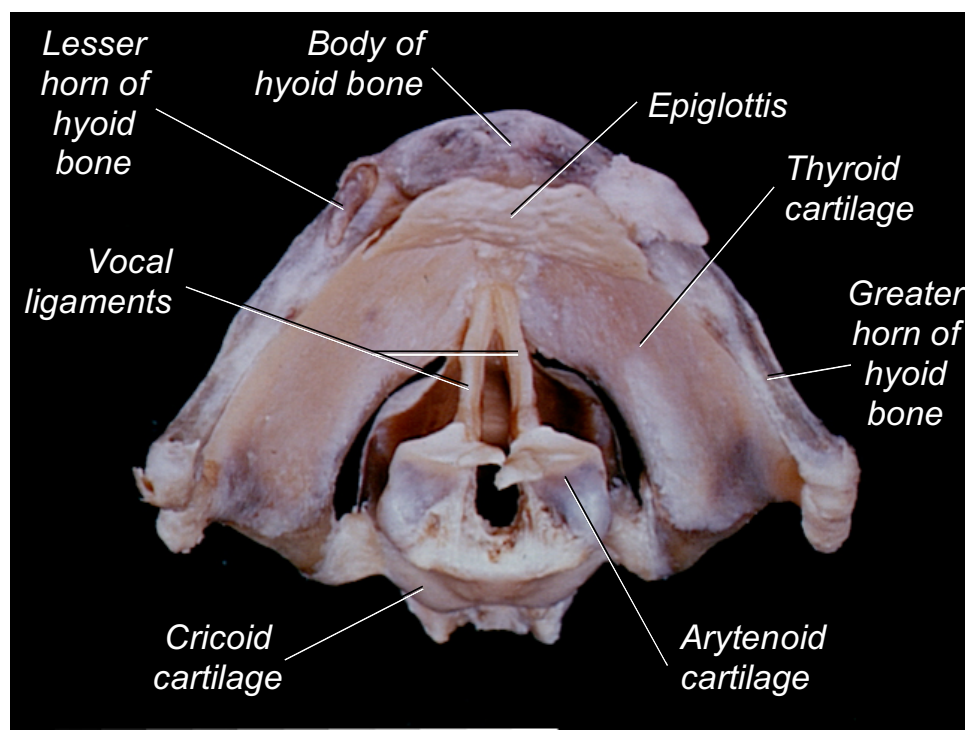

Figure 15.3. Larynx, Superior View 


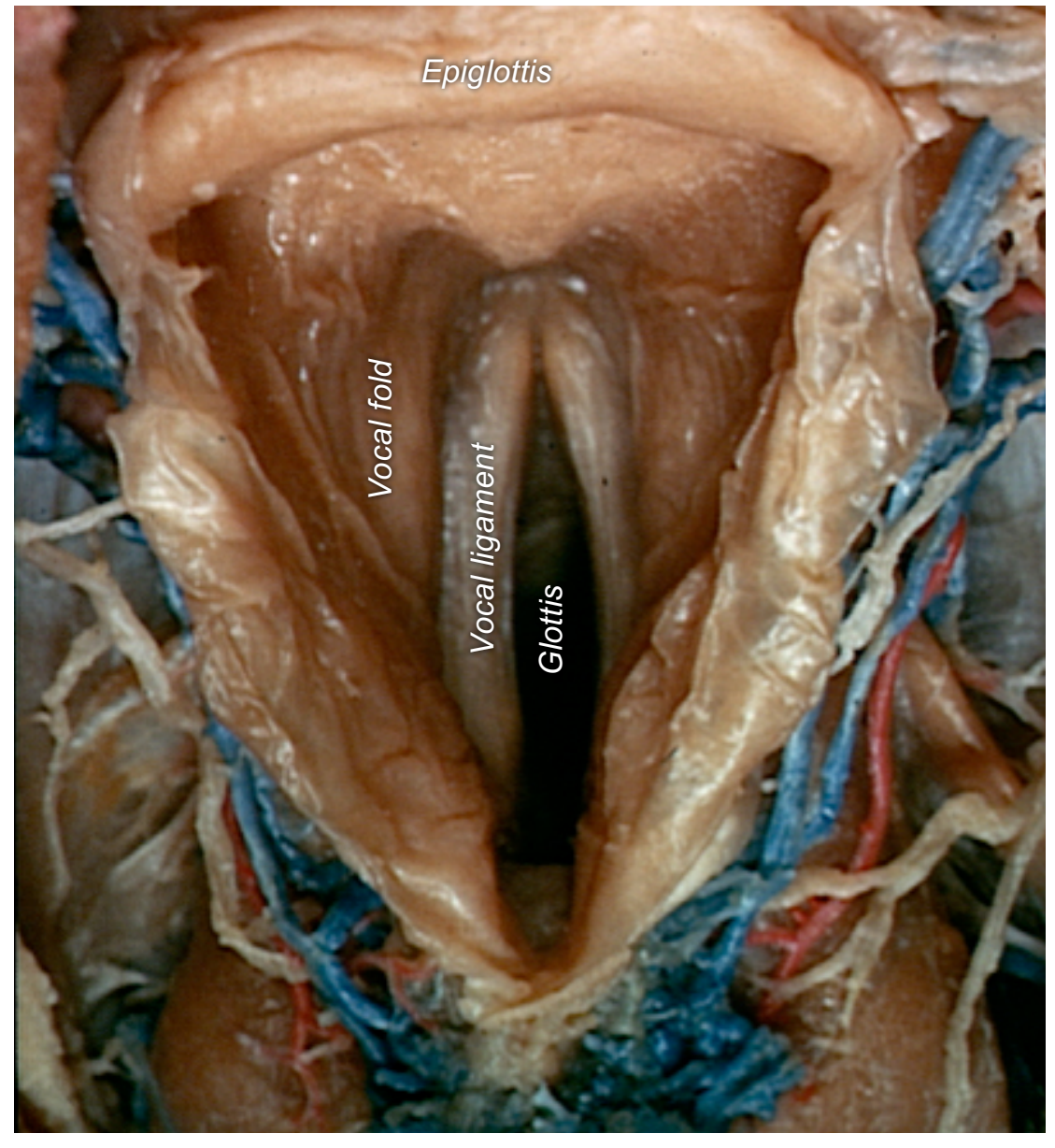

Figure 15.4. Larynx, Superior View in situ

The mediastinum is an area between the lungs where many significant anatomical structures are located. Some structures of the mediastinum include the heart, coronary vessels, esophagus, phrenic nerves, vagus nerves, thymus, thoracic (lymphatic) duct, and lymph nodes.

\section{Trachea and Lungs}

$\square$ Trachea

$\square$ Tracheal rings

$\square$ R. \& L. primary bronchi

$\square$ R. \& L. secondary bronchi

$\square$ R. \& L. tertiary bronchi

$\square$ Bronchioles

$\square$ Alveoli

$\square$ R. Lung

- Hilum

○ 3 lobes

- Superior lobe

- Middle lobe

- Inferior lobe

- Horizontal fissure

- Oblique fissure

The right lung is larger than the left lung because of the location of the heart.

L. lung

○ Hilum

- 2 lobes

- Superior lobe

- Inferior lobe

- Oblique fissure

Do you remember what oblique means? 


\section{Muscles of respiration}

$\square$ Diaphragm

- Innervated by the phrenic nerve (C3 n. + C4 n. + C5 n.)

- Action: inhalation

Inhalation is active, meaning it requires muscles. When the diaphragm and external intercostal muscles contract, they decrease the pressure in the thoracic cavity, causing air to flow in (air flows from high pressure areas to low pressure areas). As the air enters/fills the thoracic cavity, it causes the pressure to increase again until it equals atmospheric pressure, at which point air will stop flowing into the thoracic cavity and your lungs will be full of air.

\section{External intercostals}

- Innervated by intercostal nerves

- Action: inhalation

$\square$ Internal Intercostals

- Innervated by intercostal nerves

○ Action: exhalation (forceful)

Normal exhalation is passive, meaning it does not require muscles. During inhalation, the diaphragm increases abdominal pressure when it contracts and the thoracic wall is stretched by the external intercostal muscles when they contract. When the diaphragm and external intercostal muscles relax, the elasticity of the thoracic walls and pressure in the abdomen put pressure on the thorax, increasing the pressure in the thoracic cavity. Because air flows from high pressure areas to low pressure areas, and the thorax is now under higher pressure than atmospheric pressure, air flows out of the body (exhalation). Forceful exhalation is active and requires the innervation and contraction of the internal intercostal muscles to increase thoracic cavity pressure.

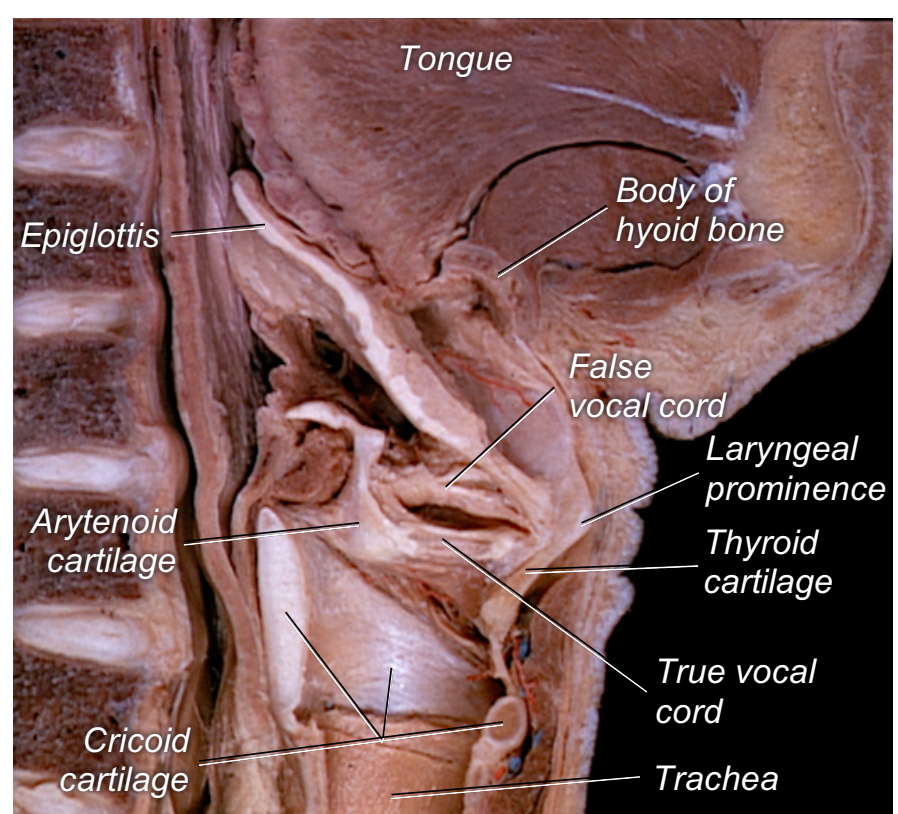

Figure 15.5. Left Larynx, Midsagittal View

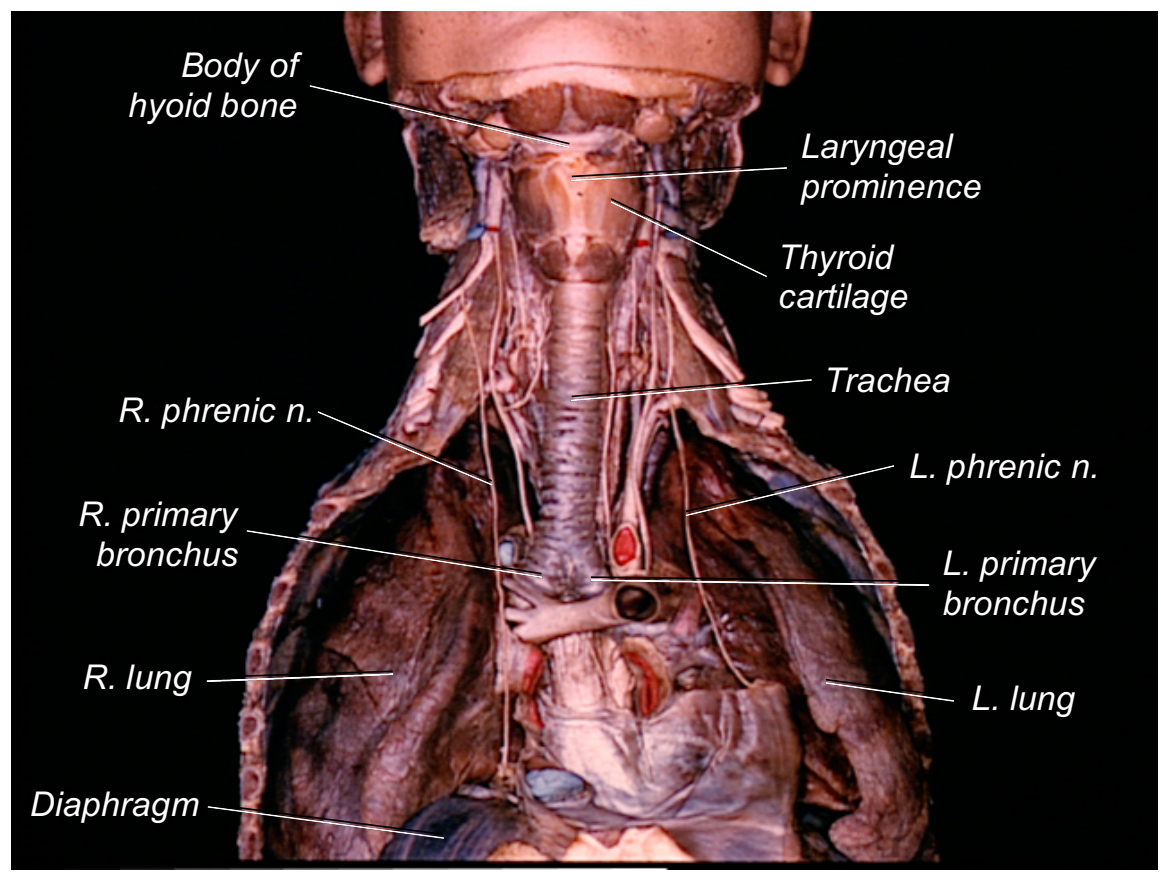

Figure 15.6. Mediastinum and Lungs, Anterior View 


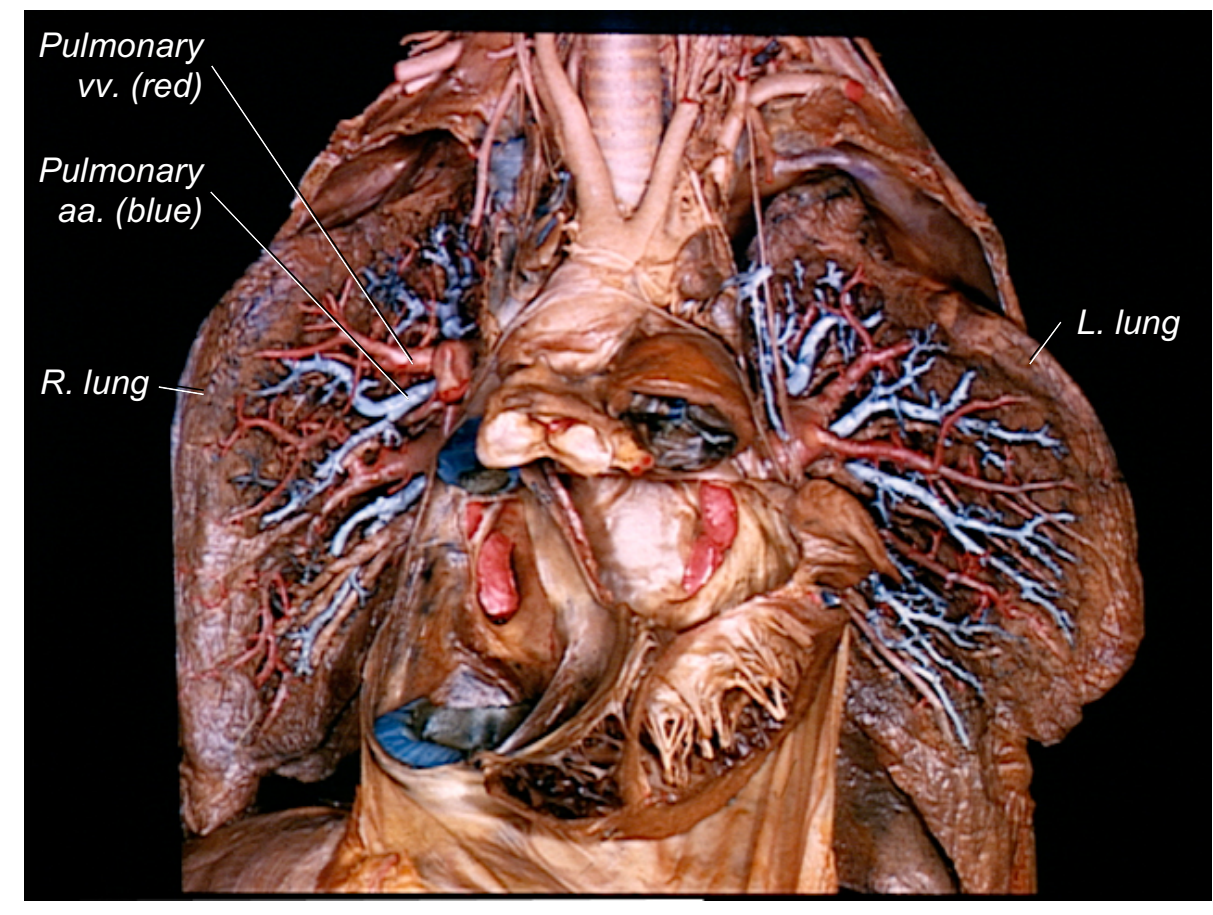

Figure 15.7. Pulmonary Vessels Branching Pattern, Anterior View

\section{Clinical Application: Asthma}

Asthma is a condition in which one's airways become inflamed, causing them to swell and constrict. Chronic asthma can range from mild to severe, but every flare up should be taken seriously. If the airways become too swollen to allow air to pass through them, the body will quickly become deprived of oxygen. Typical symptoms of an asthma attack include coughing, and wheezing, shortness of breath, but a severe asthma attack can close the airways completely and cause asphyxiation (death by suffocation) if not treated. There is no current cure for asthma, but the condition can be managed and treated with inhalers, nebulizers, and/or daily medications.

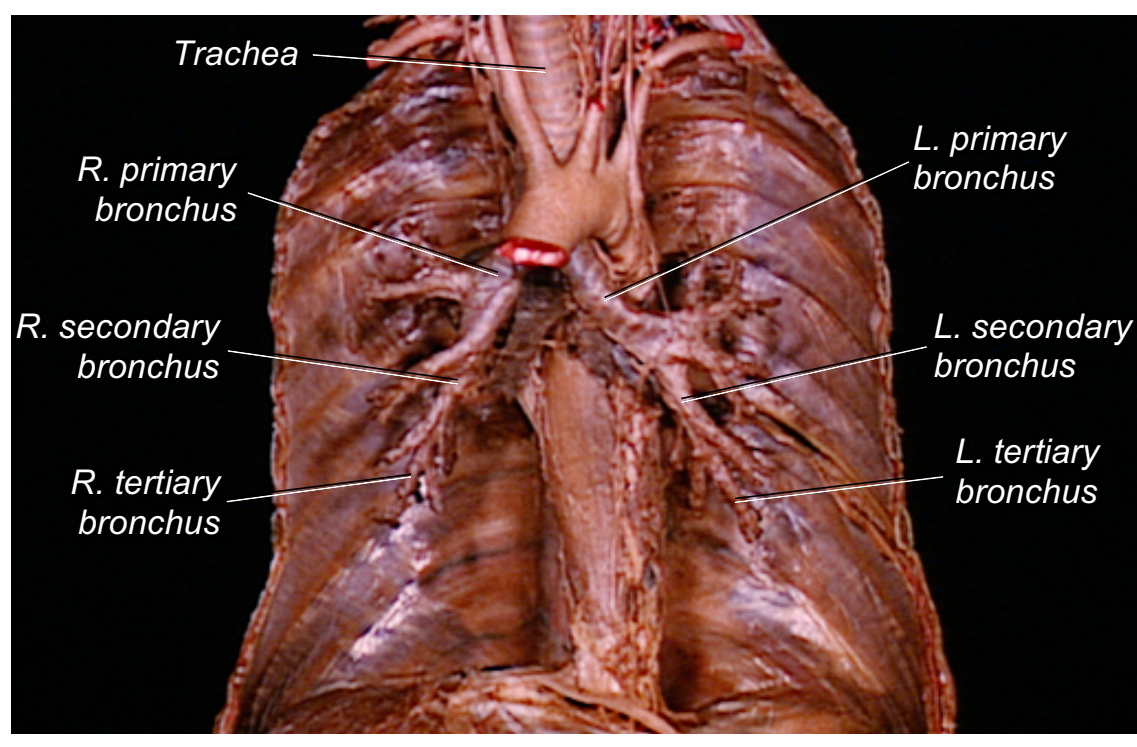

Figure 15.8. Bronchi Branching Pattern, Anterior View

\section{Clinical Application: Pneumonia}

Pneumonia is an infection of the lungs that can be acquired by breathing in bacteria or germs. The infection causes the alveoli to fill with fluid/pus, which can make breathing difficult or laborsome because the body may not be able to get the oxygen it needs from the alveoli walls as easily. Other typical symptoms of pneumonia can include fever, coughing up sputum, chills, nausea, vomiting, and diarrhea, among others. Sometimes symptoms can be mild, causing people to refer to their illness as "walking pneumonia". Because most types of pneumonia are caused by germs or bacteria, antibiotics are commonly used to treat many forms of pneumonia within a reasonable time period. The condition should be taken seriously though; it can be severe and deadly for infants, the elderly, and others with an already compromised immune system. 


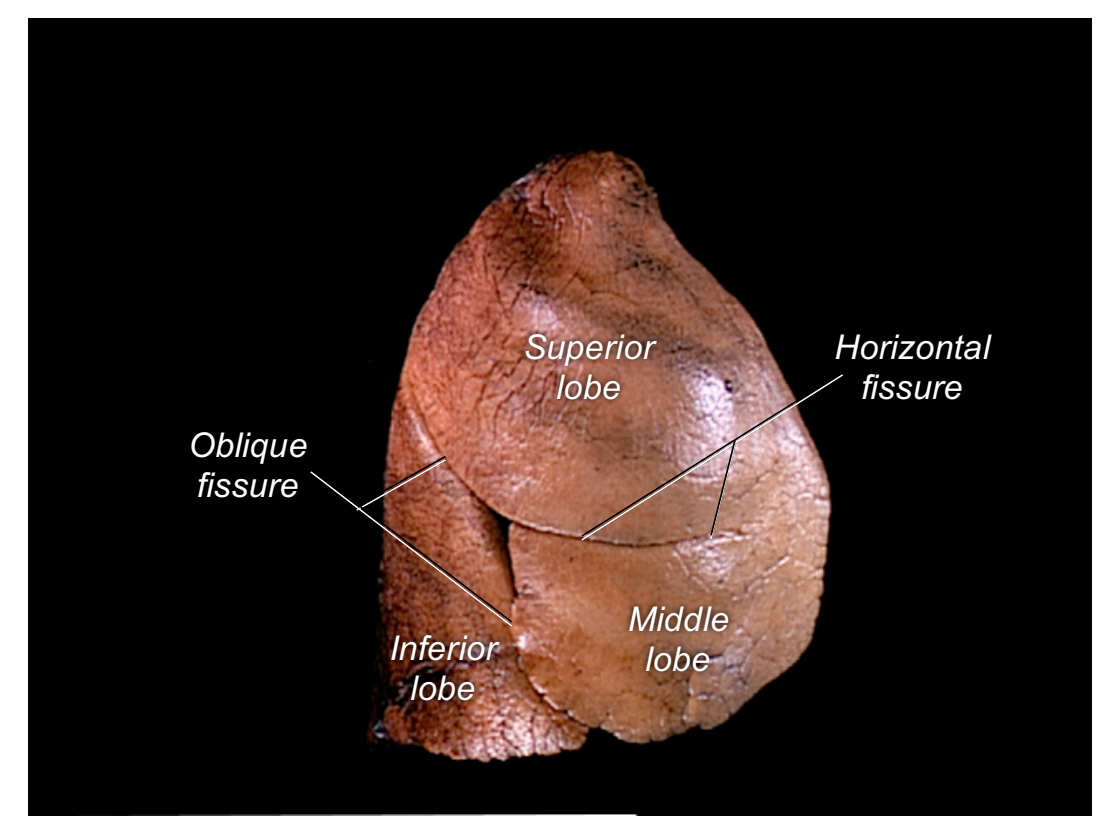

Figure 15.9. Right Lung, Lateral View

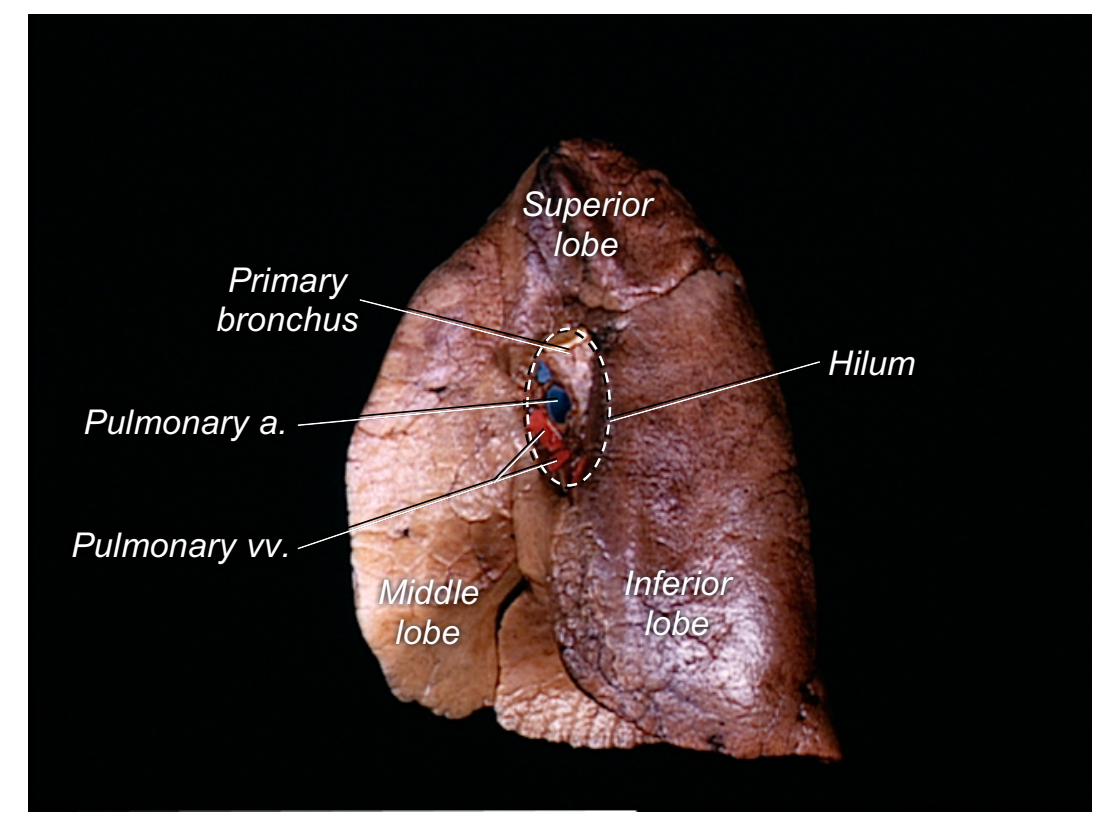

Figure 15.11. Right Lung, Medial View

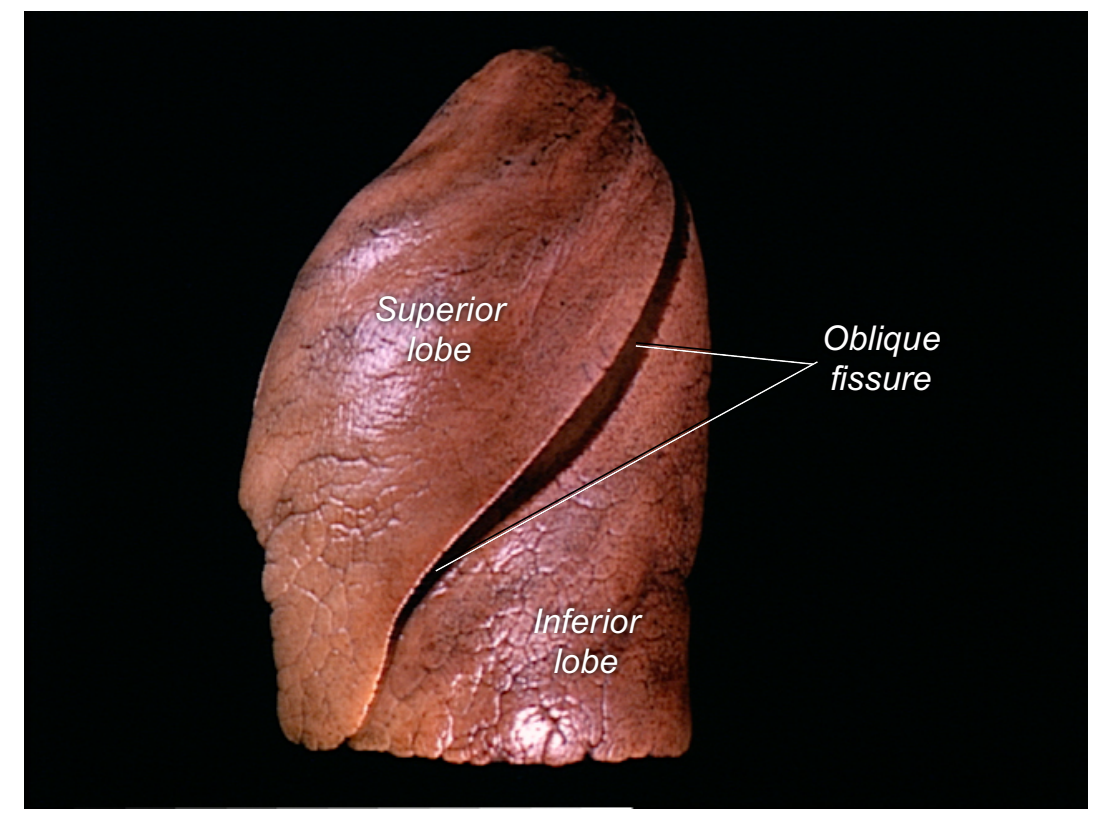

Figure 15.10. Left Lung, Lateral View

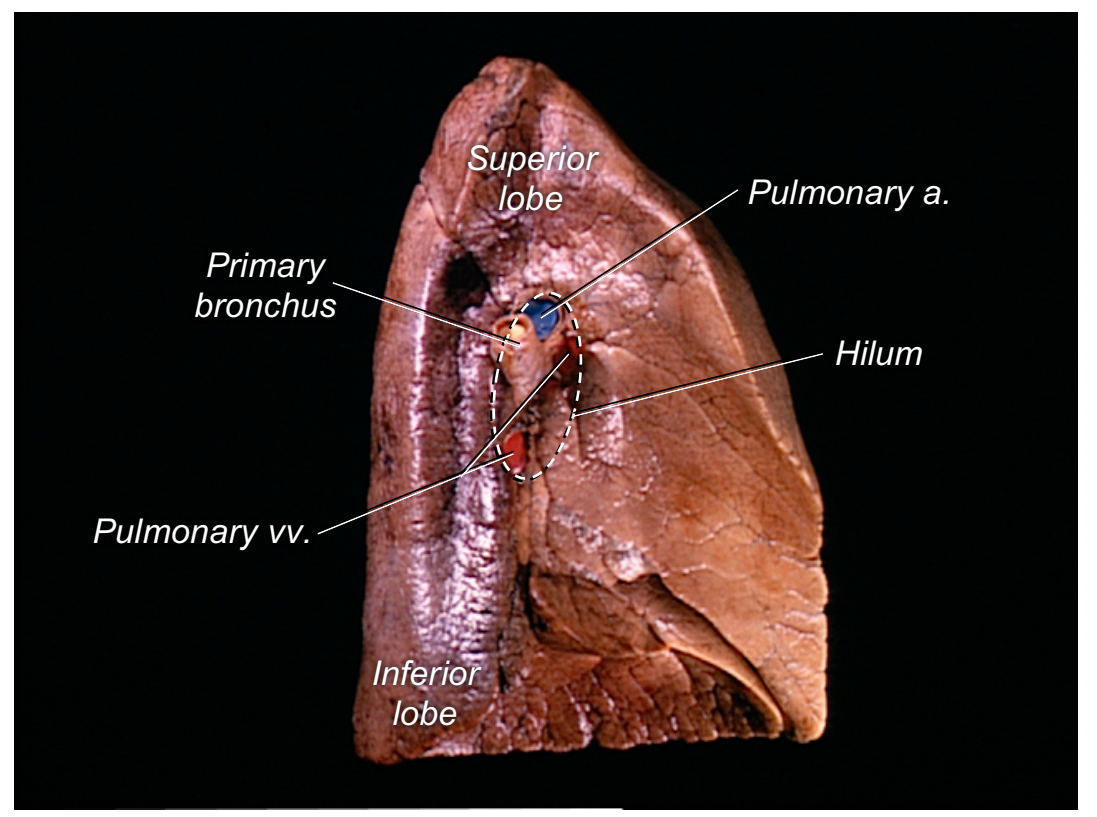

Figure 15.12. Left Lung, Medial View 
1) What muscle(s) is/are necessary for inhalation? (LO42)

2) What muscle(s) is/are necessary for exhalation? (LO42)

3) What is the function of the meati? (LO41)

4) What is the only laryngeal cartilage that is ring-shaped? (LO41)

5) Using the terminology you've learned, describe the location of the epiglottis. (LO40)

6) What vocal cord is more superior? (LO41)

7) What does the term oblique mean? (LO40)

8) Where will air in the primary bronchi flow next if the internal intercostal muscles are being innervated? (LO42, LO43)

9) What spinal nerves contribute to the phrenic n.? (LO41)

10)Where would you look to find the opening of the nasolacrimal duct? (LO41)
11)How many lobes does the right lung have? (LO41)

12)How many lobes does the left lung have? (LO41)

13)What does the term naso mean? (LO40)

14)In order, list the structures that an oxygen molecule would pass through beginning with a nostril and ending in the rectum. (LO43)

15)Describe what causes inhalation. (LO42)

16)Describe what causes exhalation. (LO42) 


\section{Lab 16: Primary Digestive Organs}

Instructions: Learn the listed terms using the listed learning objectives, labeled images, lab specimens, and other resources.

LO44: Describe the terminology used to identify structures of the primary digestive organs.

LO45: Identify the structures of the primary digestive organs.

LO46: Describe the flow pathway of food through the primary digestive organs.

\section{Oral cavity}

$\square$ Mouth

The mouth is the opening to the oral cavity.

Consume or ingest = to eat (and drink) by putting food (and drink) through the mouth and into the oral cavity.

Food $=$ the solid substance that is consumed.

$\underline{B}$ Bolus $=$ a small rounded food mass that has been chewed and mixed with saliva.

\section{Pharynx}

$\square$ Oropharynx

$\square$ Laryngopharynx

\section{Esophagus}

$\square$ Upper esophageal sphincter

$\square$ Lower esophageal sphincter (or cardiac sphincter)

The lower esophageal sphincter relaxes to let a bolus into the stomach and contracts to prevent regurgitation of stomach contents into the esophagus.
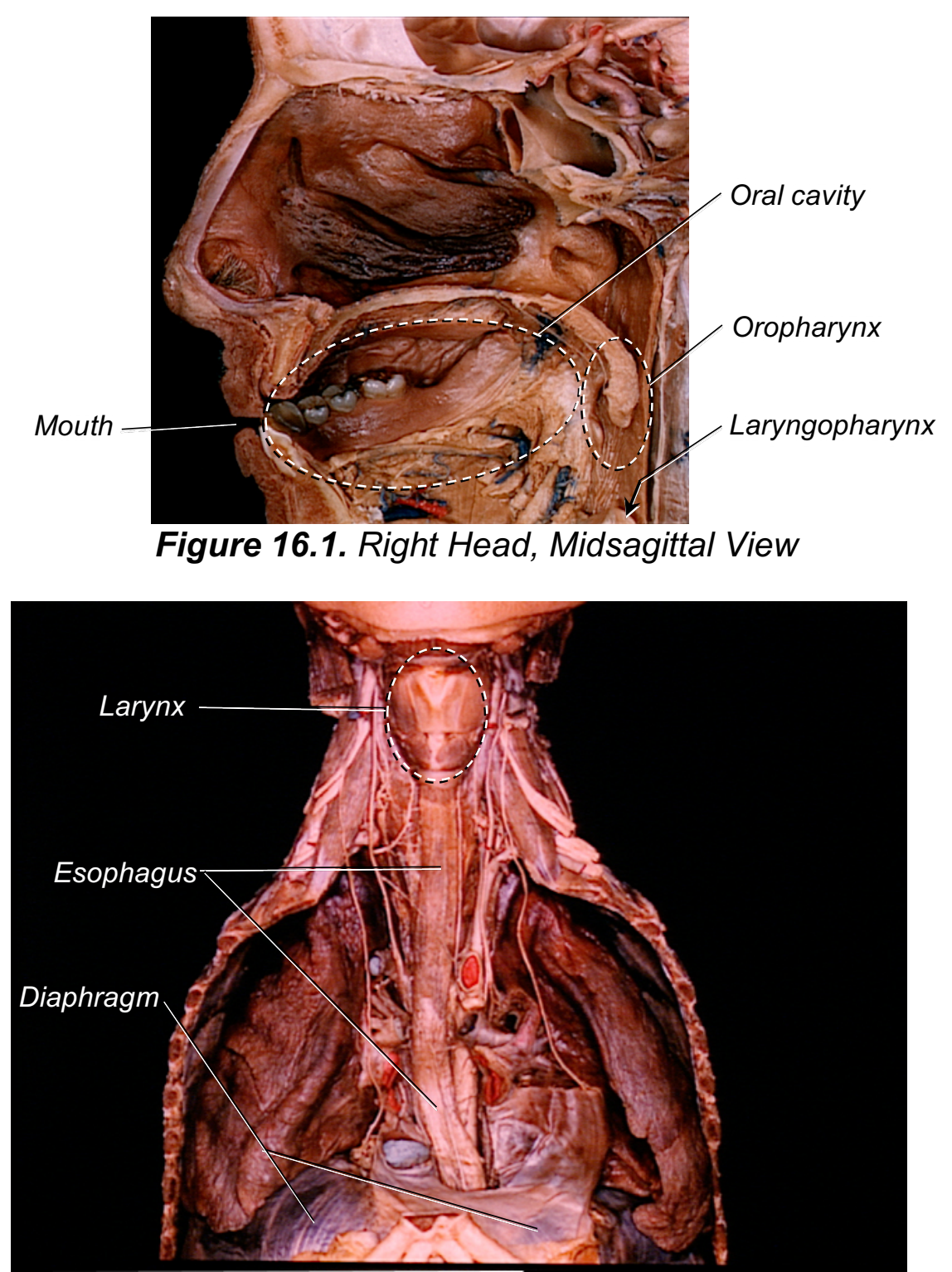

Figure 16.2. Esophagus in Thorax, Anterior View 


\section{Clinical Application: Acid Reflux and Heartburn}

Despite its name, heartburn has nothing to do with the heart. When the lower esophageal sphincter doesn't function properly, stomach acids are able to escape into the lower part of the esophagus and irritate the inner lining of the esophageal wall. Because this acid reflux causes a burning sensation in the thorax near the heart, the symptom is called heartburn.

\section{Stomach}

$\square$ Regions:
- Cardia
- Fundus
- Body
- Pylorus

$\underline{\text { Fundus }}=$ The part of an organ furthest from its opening.

\section{Pyloric sphincter}

This sphincter controls when food is released into the duodenum.

\section{$\square$ Rugae}

$\underline{\text { Rugae }}=$ folds; ridges. Rugae increase surface area and allow for expansion.

$\square$ Lesser curvature

- Lesser omentum

$\square$ Greater curvature

\section{- Greater omentum}

Chyme $=$ the pulpy food substance that has mixed with gastric juices. Do you remember what gastr means?

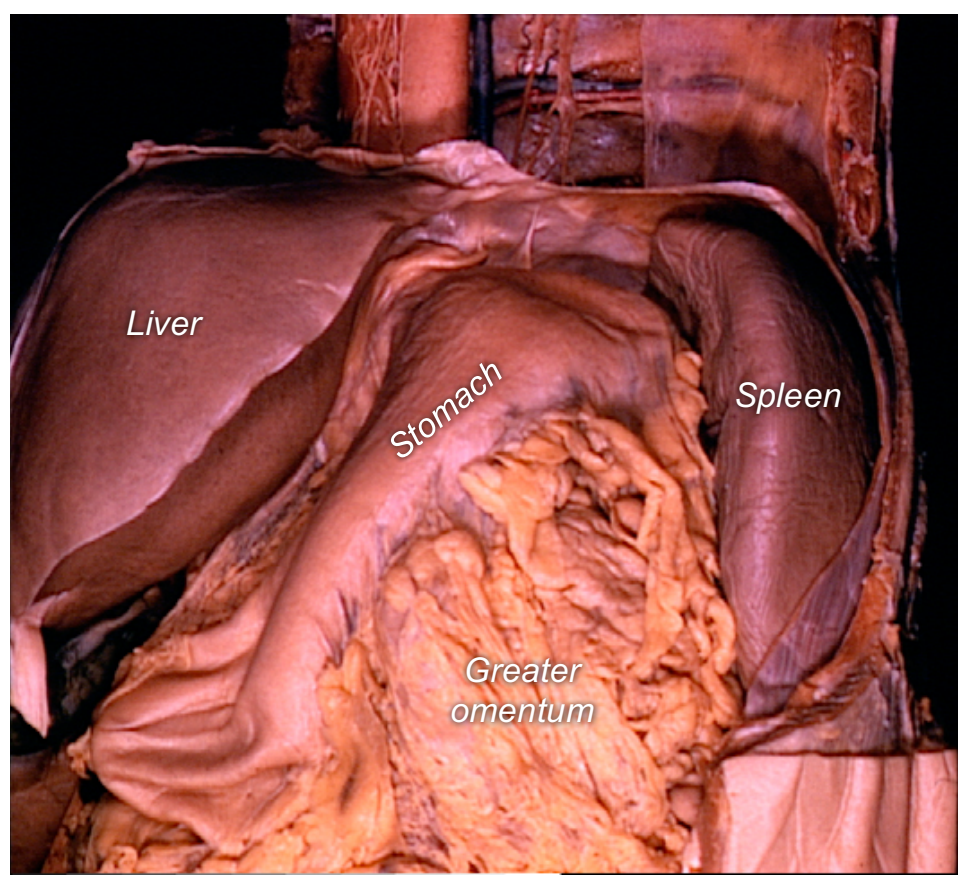

Figure 16.3. Abdominal Viscera in situ, Anteroinferior View

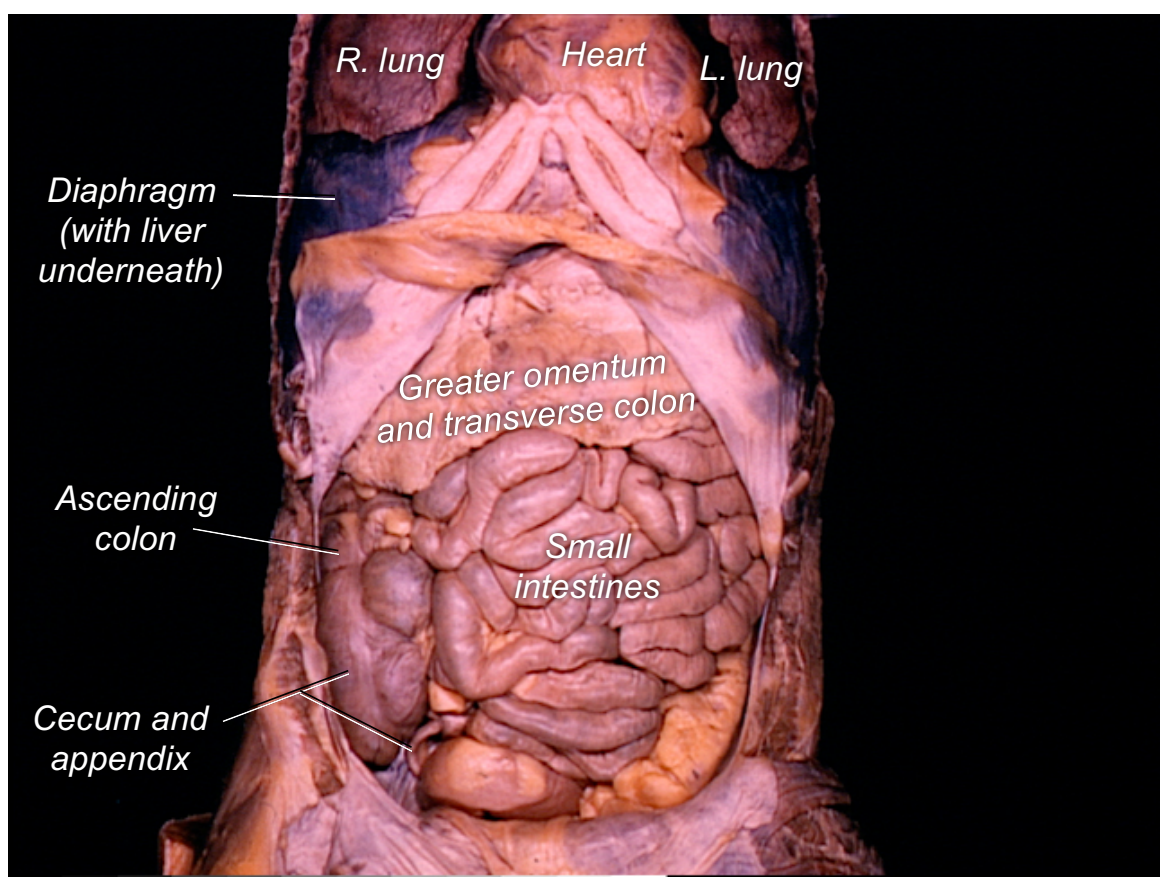

Figure 16.4. Abdominal Viscera in situ, Anterior View \#2 


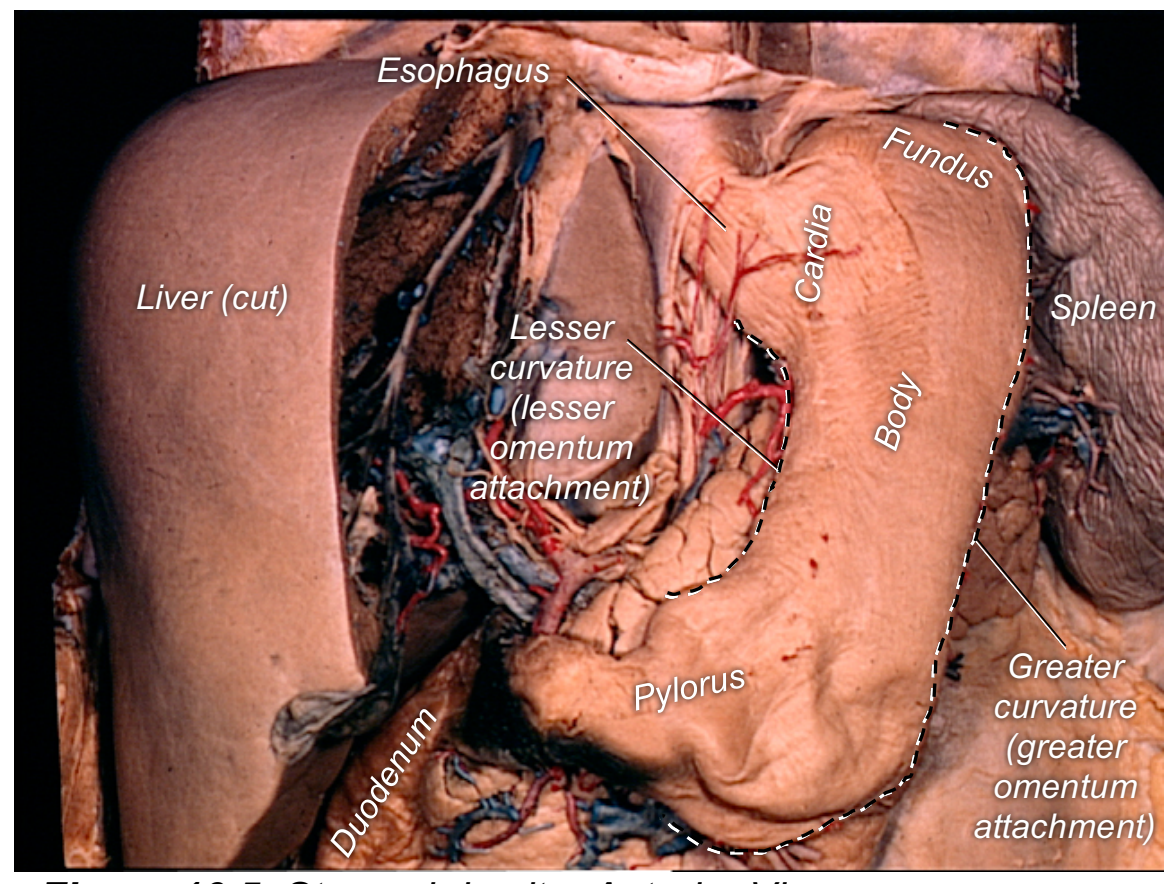

Figure 16.5. Stomach in situ, Anterior View

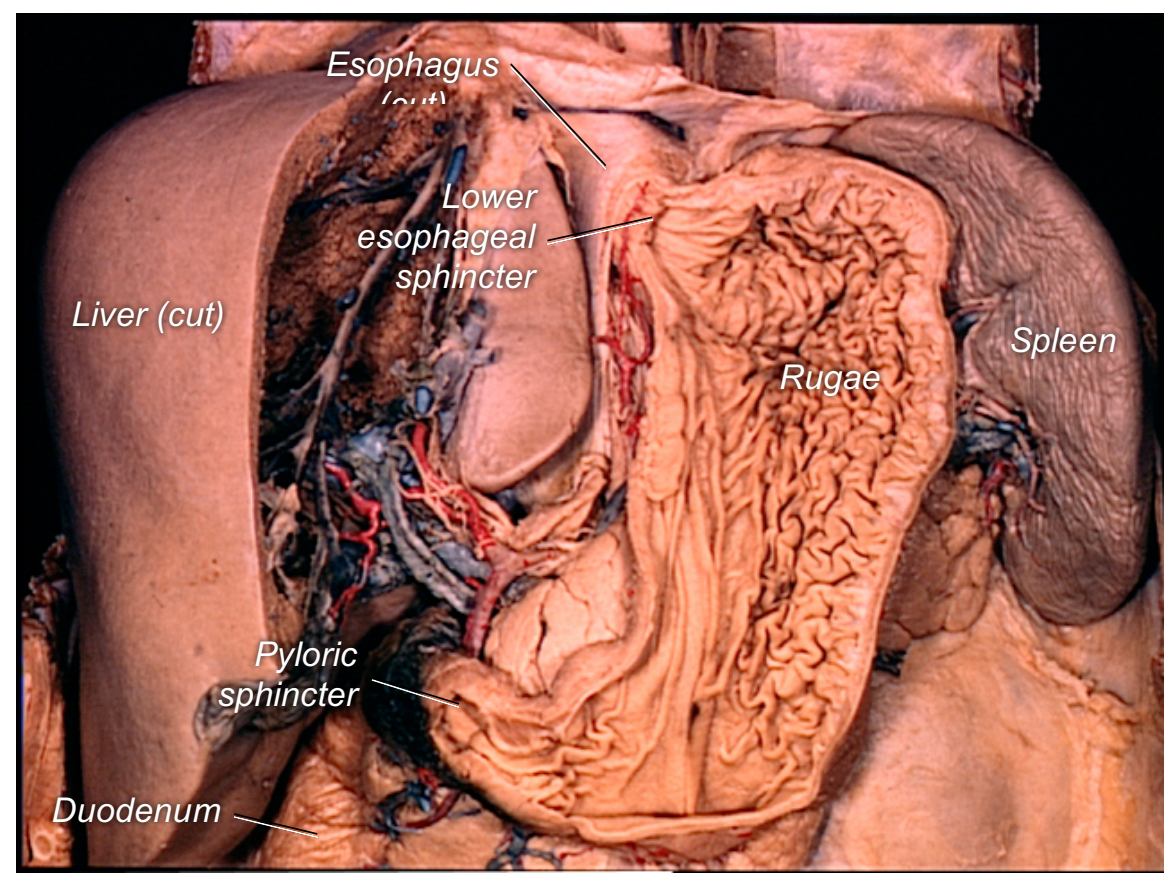

Figure 16.6. Stomach (cut open) in situ, Anterior View

\section{Clinical Application: Gastric Bypass}

Gastric Bypass is a surgical procedure where the stomach is divided into a smaller upper portion and larger lower portion, and then both portions are reconnected to the small intestine. Because the functional volume of the usable portion of the stomach is significantly reduced, the physical and physiological response to consuming food is also altered. In effect, patients will feel full from consuming less food, and less calories will be absorbed by the body because a significant part of the gastrointestinal tract has been bypassed. The need to lose/control weight is the typical reason for gastric bypass surgery, and physicians often prescribe this surgery as treatment for patients fighting and struggling with morbid obesity.

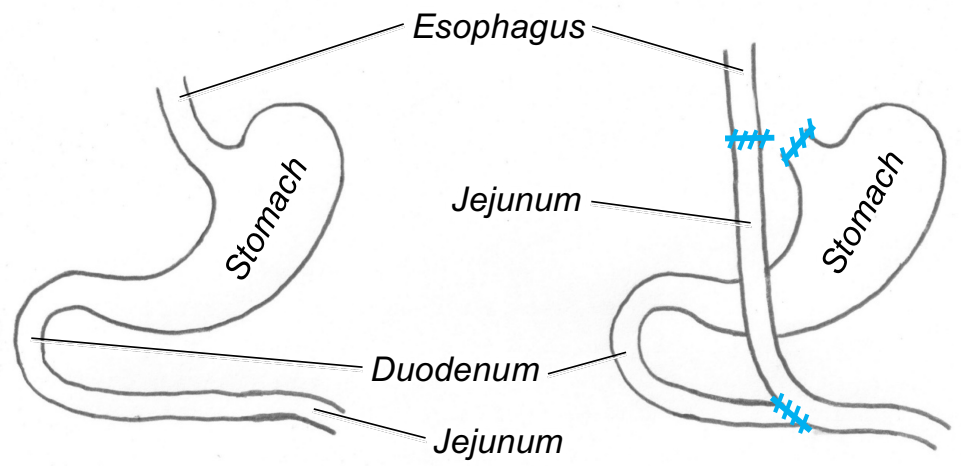

Normal Stomach 


\section{Small intestine}

The small intestine is where the majority of digestion and absorption happens. Digestion $=$ the mechanical and chemical breakdown of food. Absorption $=$ the process of taking molecules from digested food into the body.

\section{$\square$ Regions:}

$$
\begin{aligned}
& \text { - Duodenum } \\
& \text { - Jejunum } \\
& \text { - lleum }
\end{aligned}
$$

The ileum connects to the first part of the large intestine. You must spell ileum correctly, since it is so similar to ilium, a bone you've already learned.

\section{Plicae circulares (or circular folds)}

The plicae circulares increase the surface area of the small intestine. They are most prominent in the duodenum and progressively thin out and transition to the smoother wall of the distal ileum.

\section{Mesentery (proper)}

This double-membraned fold of tissue (called peritoneum) attaches the small intestine to the posterior wall of the abdomen and provides a supportive pathway for blood vessels traveling to and from the small intestine.

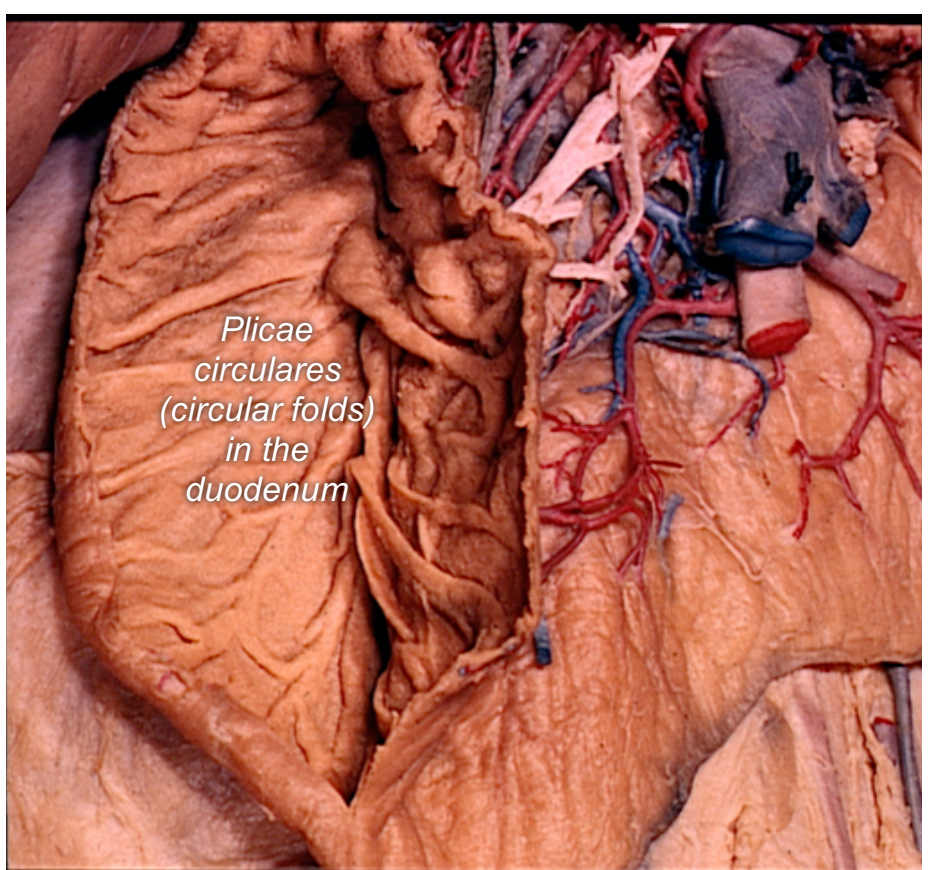

Figure 16.7. Duodenum (cut open), Anterior View

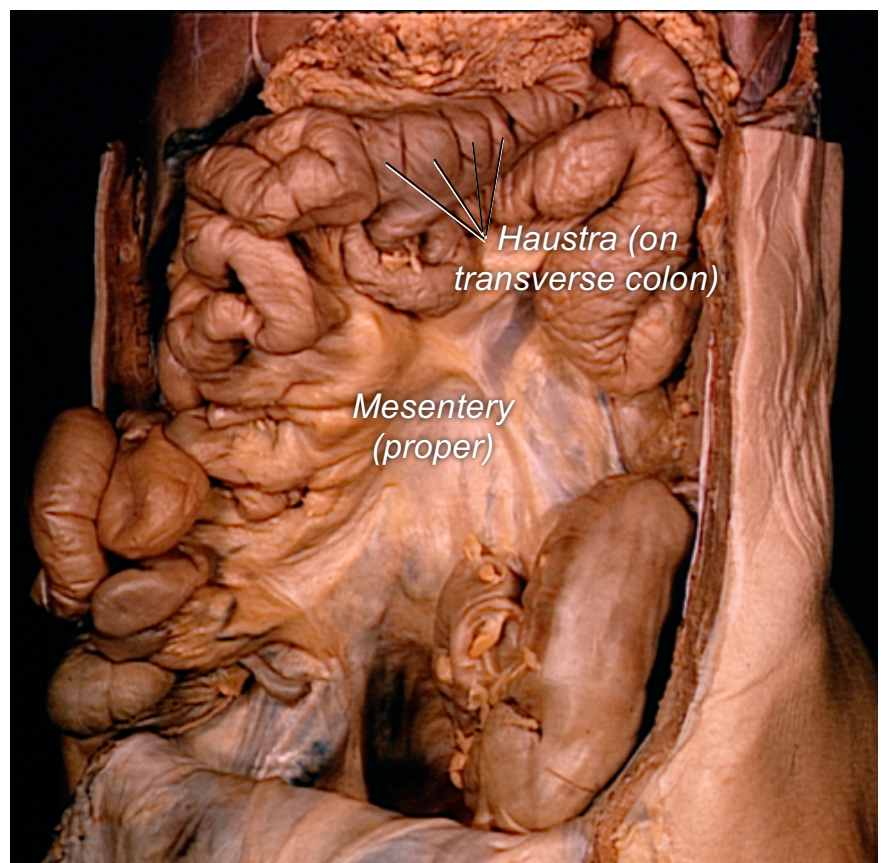

Figure 16.8. Mesentery Proper, Anterior View. 


\section{Large intestine}

The large intestine has three regions: cecum, colon, and rectum. The main function of the large intestine is to dehydrate and compact feces for defecation.

Dehydrate $=$ to remove water to make dryer.

Compact $=$ to make more dense; compress.

Feces $=$ waste matter (excrement) from digested food.

$\underline{\text { Defecation }}=$ the discharge of feces from the bowel.

$\square$ Cecum
○ Appendix
- lleocecal valve

This valve controls the flow of chyme from the ileum into the cecum. Once chyme enters the large intestine, the "food" substance is then called feces.

\section{Colon}

○ Ascending colon (or Right colon)

- Hepatic flexure (or Right colic flexure)

- Transverse colon (or Middle colon)

- Splenic flexure (or Left colic flexure)

○ Descending colon (or Left colon)

○ Sigmoid flexures

- Sigmoid colon

Flexure $=\mathrm{a}$ bend or curve

\section{Tenia coli}

The tenia coli is a longitudinal band of muscle that spans the entire length of the large intestine. It helps move feces through the large intestine. There is a total of three tenia coli.

\section{Haustra}

These are the "pockets" in the colon that help segment feces.

\section{Epiploic appendages}

These are the fatty appendages on the colon.

\section{Mesocolon}

This double-membraned fold of tissue functions just like the mesentery proper, except for the large intestine.

\section{$\square$ Rectum}

$\square$ Internal anal sphincter

External anal sphincter

The autonomic nervous system (involuntarily) controls your internal anal sphincter, and you have voluntary control over your external anal sphincter.

\section{Anus}

The anus is the external opening at the distal end of the rectum.

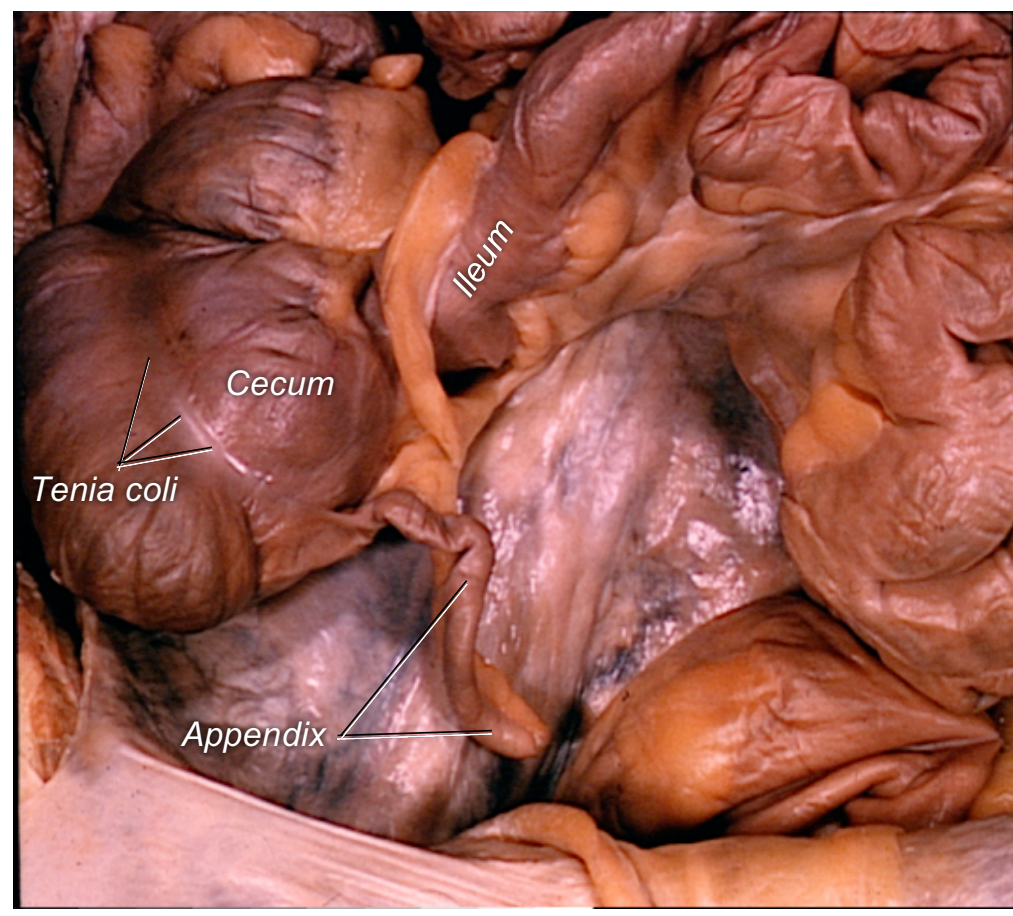

Figure 16.9. Cecum in situ, Anteroinferior View 


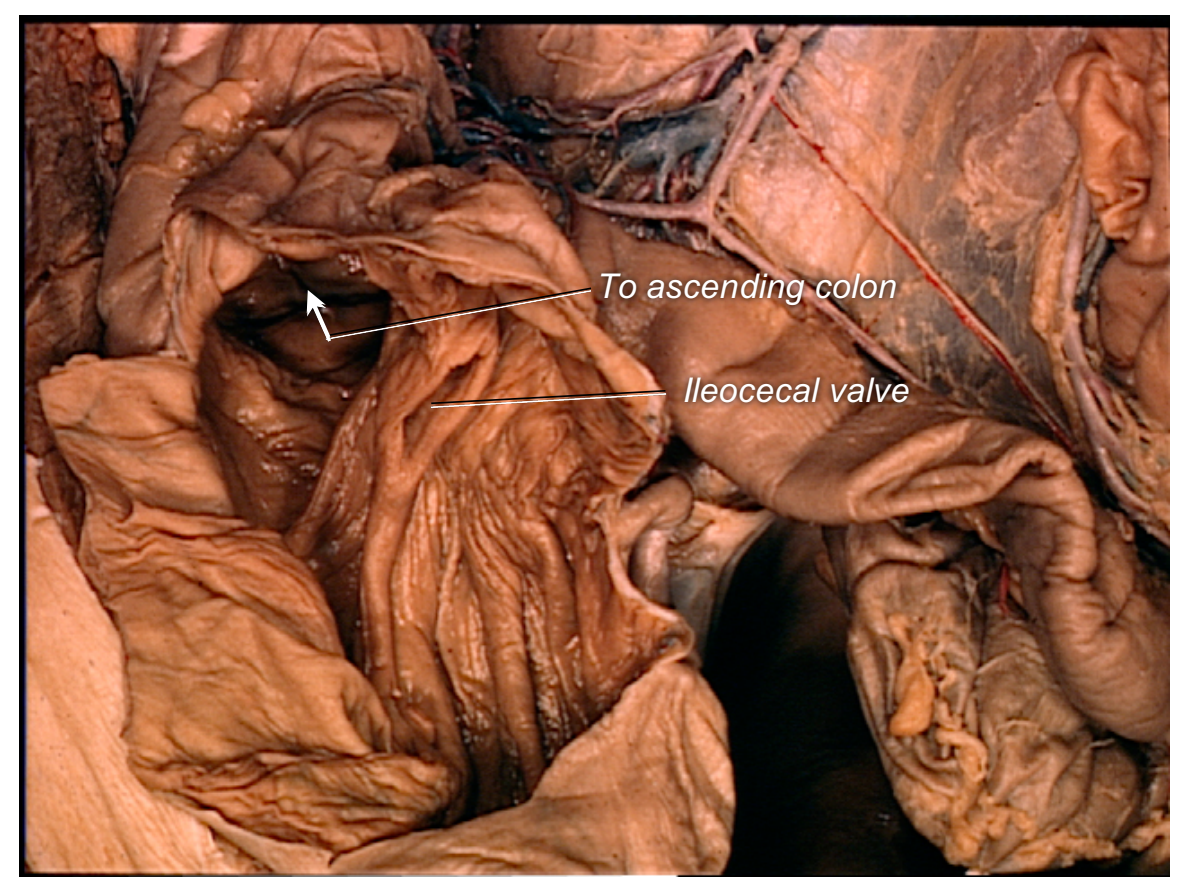

Figure 16.10. Cecum (cut open) in situ, Anterior View

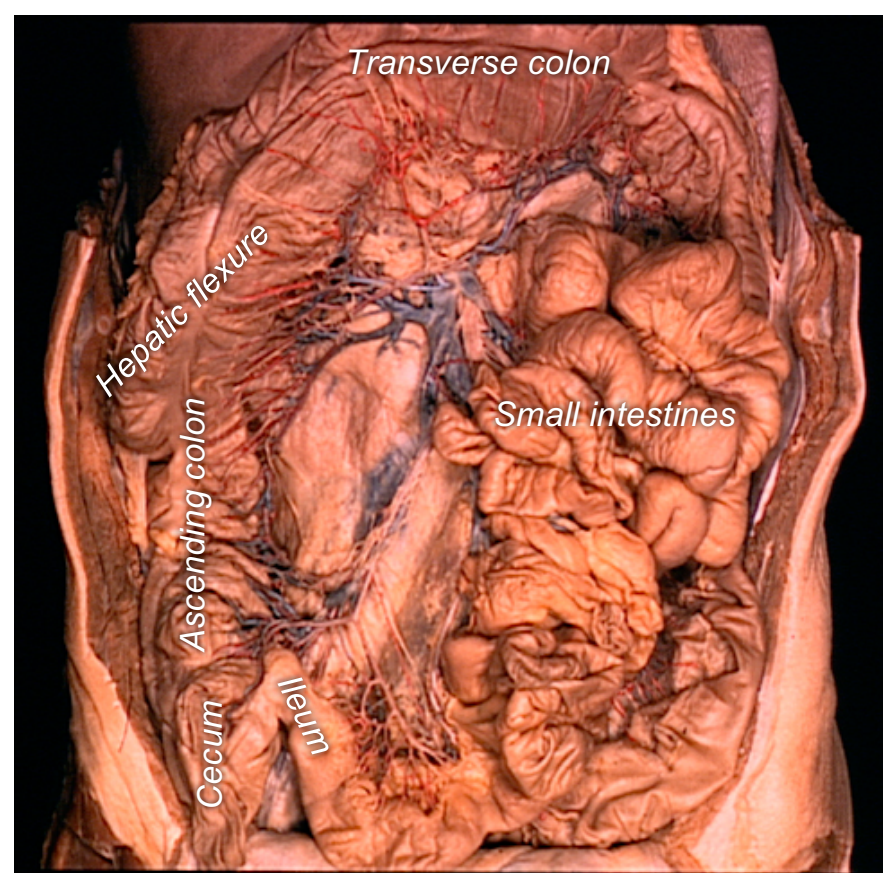

Figure 16.11. Colon, Anterior View \#1

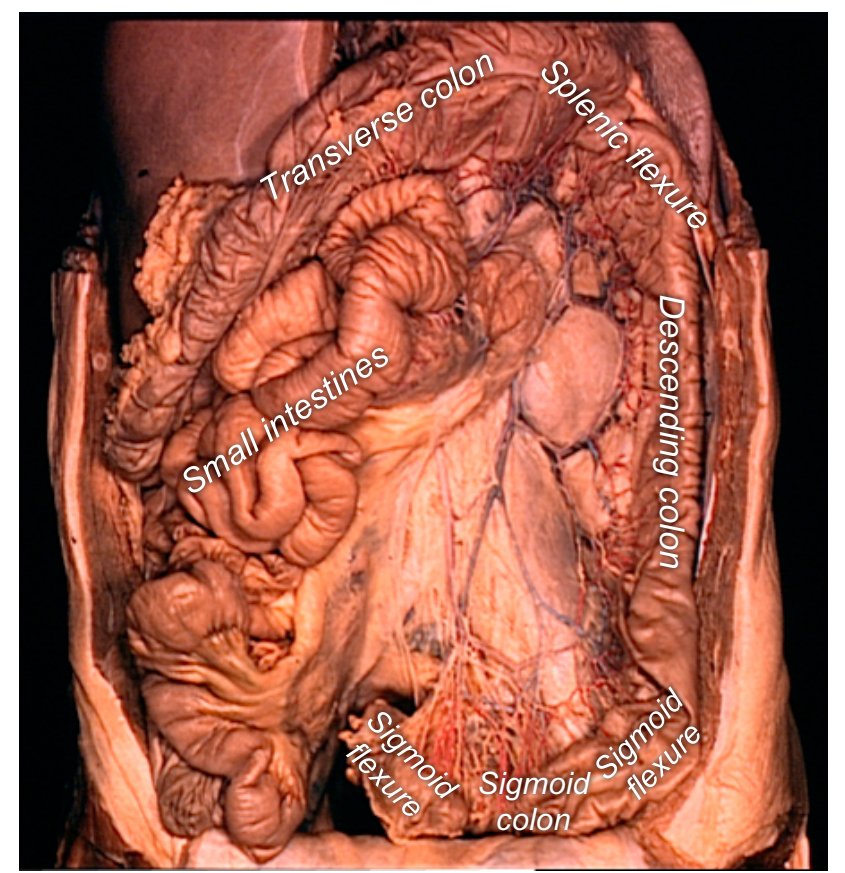

Figure 16.12. Colon, Anterior View \#2

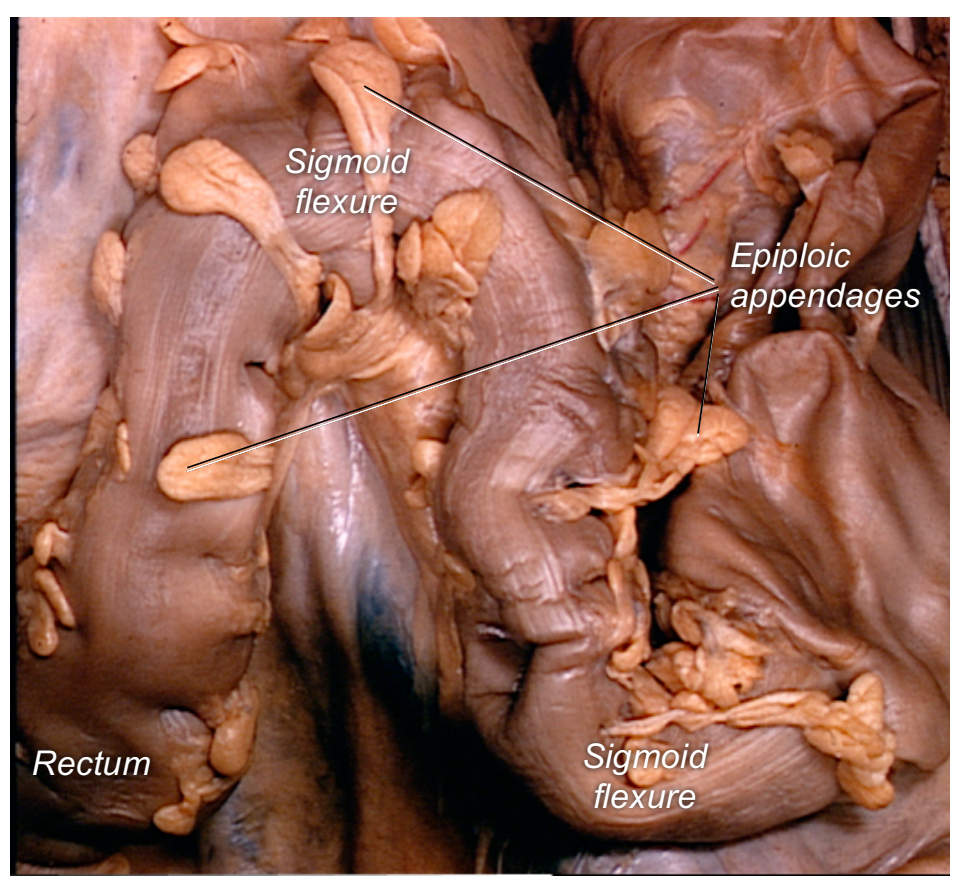

Figure 16.13. Sigmoid Colon, Anterior View 


\section{Clinical Application: Colonoscopy}

A colonoscopy is an endoscopic examination of the large intestine (and sometimes even the distal part of the ileum). This procedure is completed by inserting a colonoscope (a camera on the end of a flexible cable) through the anus and then navigating it through the large intestine. The angle of the cable can be controlled by the physician to navigate around flexures to view more proximal parts of the bowel. To view the walls of the large intestine, is necessary to empty and cleanse the large intestine prior to starting the procedure. This is typically done with a clear-liquid diet for at least $\mathbf{2 4}$ hours before examination. If the colon is not empty and clean, residual feces may block visibility of the bowel walls, and the test may need to be repeated another time after proper preparation. With proper preparation, the walls of the colon can be inspected for polyps, diverticula, ulcers, and various signs of cancer. Most colonoscopes are able to simultaneously collect biopsies for further lab testing.

\section{Clinical Application: Diverticulosis}

Diverticulosis is a condition where small pouches called diverticula form in the wall of the colon. While many people live with asymptomatic diverticula, diverticula can also cause abdominal pain, cramping, constipation, and diarrhea. Inflammation of these pouches, called diverticulitis, can lead to stronger symptoms and include fever and vomiting. Diverticulosis can be diagnosed from a radiographic examination of abdominal viscera, digital rectal examination, or colonoscopy procedure. The most common treatment for diverticulosis is diet modification, but severe cases of diverticulitis may require hospital care and surgery.
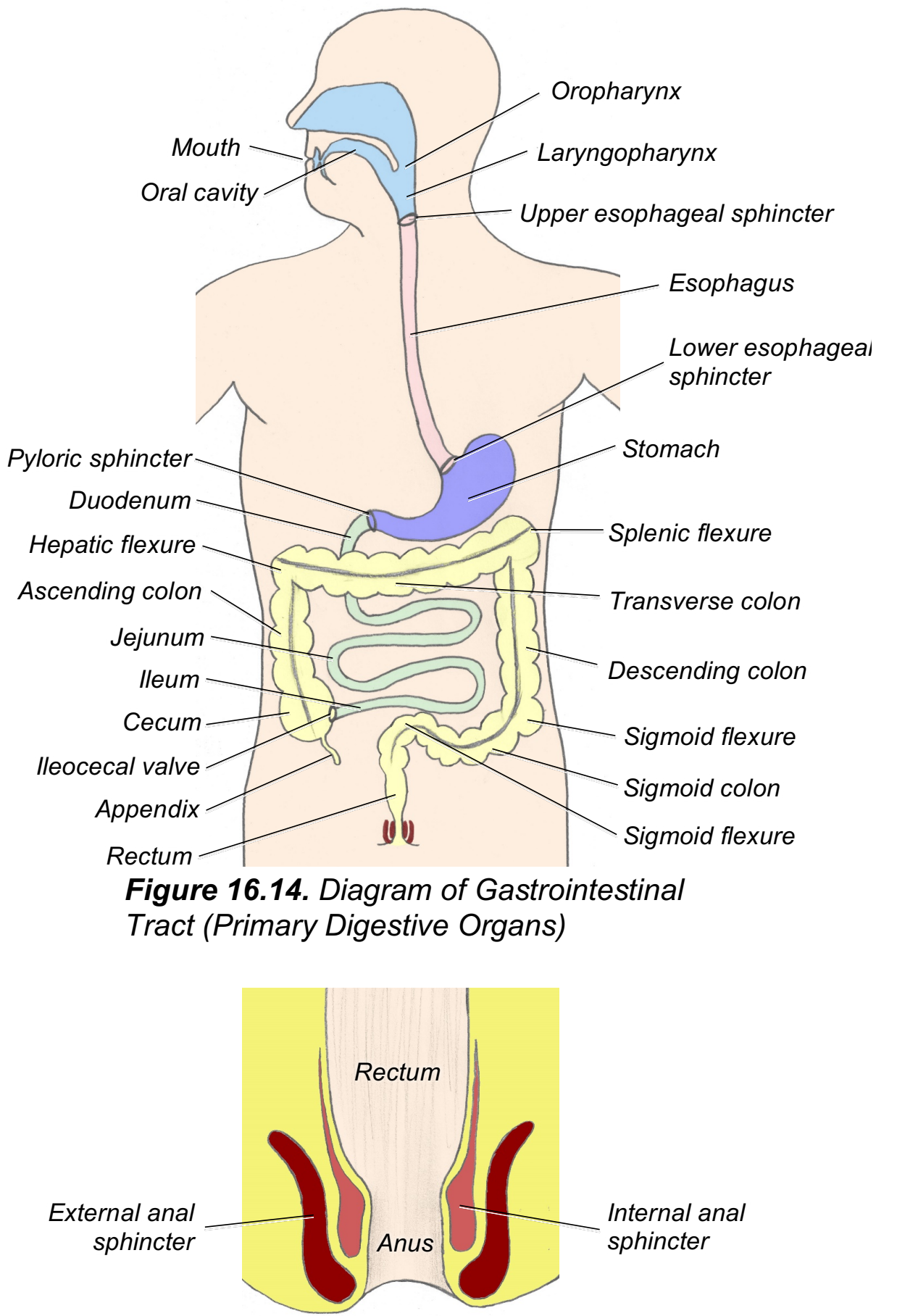

Figure 16.15. Diagram of Anal Sphincters and Anus. 
1) In order, list the structures that a food molecule would pass through as it travels through all of the primary digestive organs. (LO46)
2) True or False: Air passes through the pharynx. (LO43)

3) True or False: Air passes through the larynx. (LO43)

4) True or False: Food (bolus) passes through the pharynx. (LO46)

5) True or False: Food (bolus) passes through the larynx. (LO46)

6) How many sphincters does food pass through as it travels through the entire digestive tract? (LO45, LO46)
7) "Food" has different names as it passes through the digestive tract. What is the name of "food" at the following locations? (LO44)

a. Mouth:

b. Esophagus:

c. Stomach:

d. Small intestine:

e. Colon:

f. Rectum:

8) What does the term flexure mean? (LO44)

9) What is the function of rugae in the stomach? (LO45)

10)Which of the following is NOT a region of the small intestine? (LO45)
a. Duodenum
b. Jejunum
c. Ilium
d. All of the above are regions of the small intestine.

11)What does the term bolus mean? (LO44)

12)How many openings does the cecum have? (LO45) 


\section{Lab 17: Secondary Digestive Organs \& Lymphatic System}

Instructions: Learn the listed terms using the listed learning objectives, labeled images, lab specimens, and other resources.

LO47: Describe the terminology used to identify structures of the secondary digestive organs and lymphatic system.

LO48: Identify the structures of the secondary digestive organs and lymphatic system.

LO49: Describe the flow pathways of bile and pancreatic juices.

In the previous lab, you learned about the primary digestive organs - organs through which food substances traveled as they were digested. In this lab, you will learn about secondary digestive organs - organs which contribute to digestion of food substances but are not part of the primary digestive tract.

\section{Oral cavity}

$\square$ Hard palate

$\square$ Soft palate

$\square$ Tongue
- Dorsum
○ Filiform papillae
- Fungiform papillae
- Foliate papillae
- Circumvallate papillae

Note: "taste buds" are the nerve ending sites within papillae. All papillae are associated with taste buds except filiform papillae. $\square$ Uvula

$\square$ Gingiva

$\square$ Tooth
- Crown
- Occlusal surface
○ Enamel
○ Dentin
○ Pulp cavity
- Occlusal surface
○ Root
- Periodontal ligament
○ Cementum
- Root Canal

Teeth:

- Incisors (8 total)

- Canines (or Cuspids) (4 total)

- Premolars (or Bicuspids) (8 total)

○ Molars (12 total)

Adults typically have $\mathbf{3 2}$ teeth, $\mathbf{1 6}$ that are anchored into alveoli on the mandible, and $\mathbf{1 6}$ are anchored into alveoli on the maxilla. The last four molars are termed "wisdom teeth".

Do you remember what the word den means? 


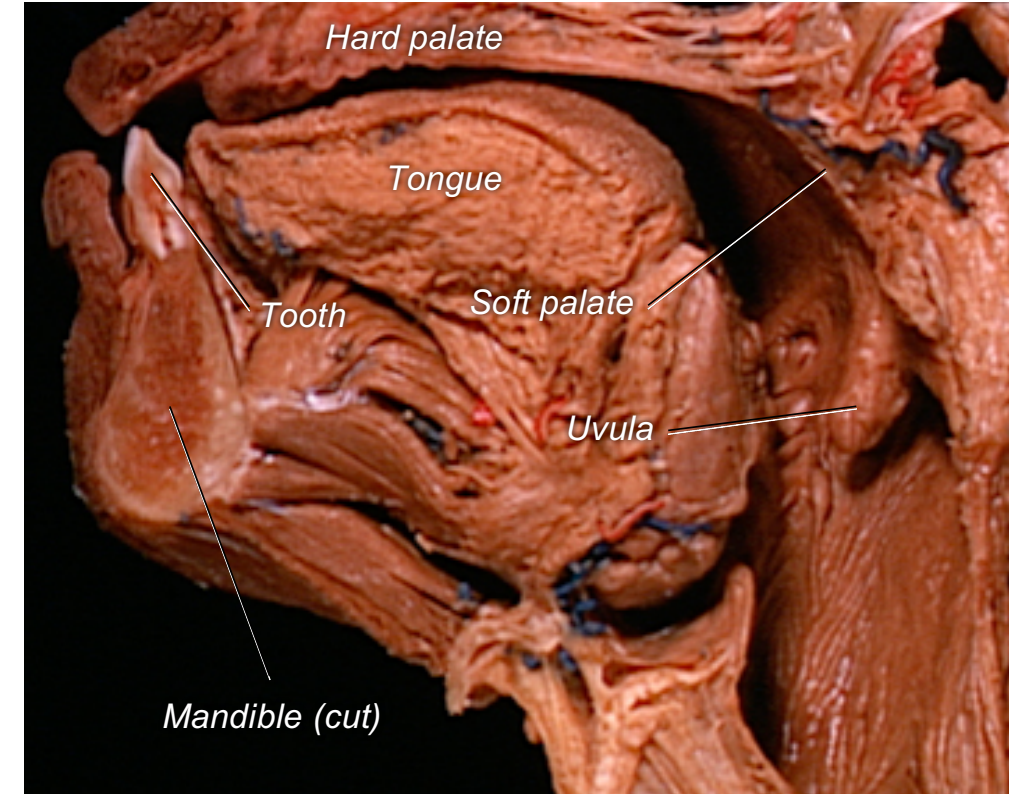

Figure 17.1. Oral Cavity, Midsagittal View

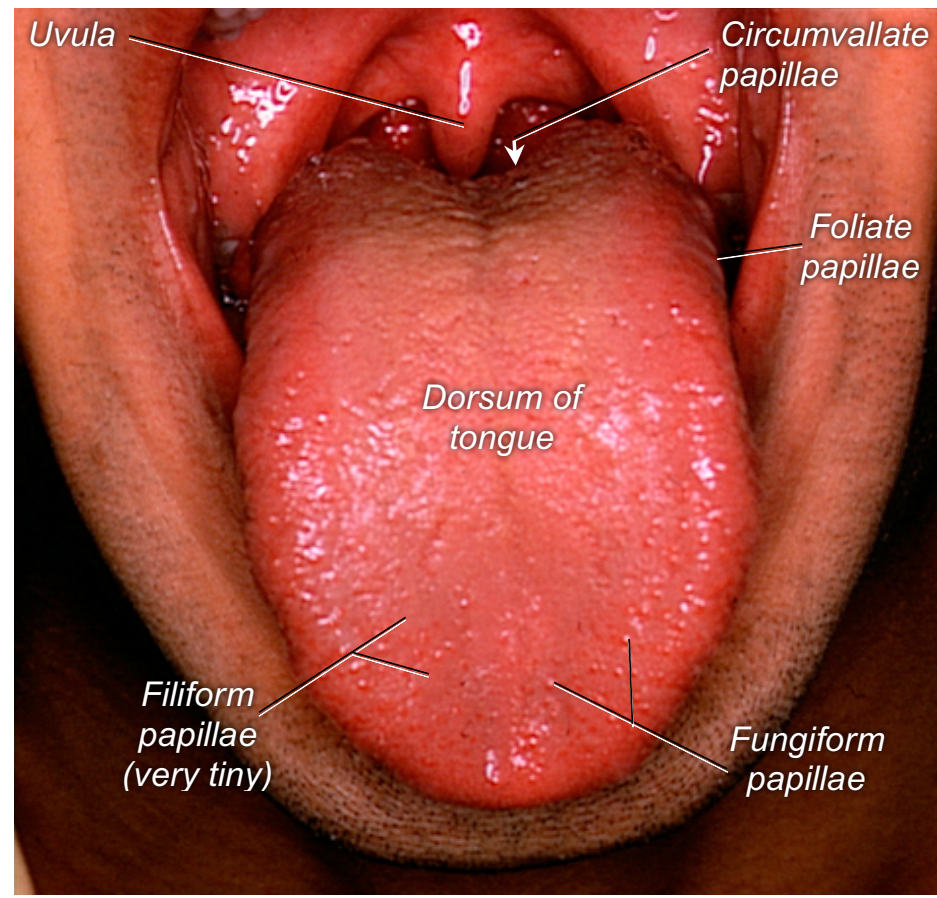

Figure 17.2. Oral Cavity, Anterior View

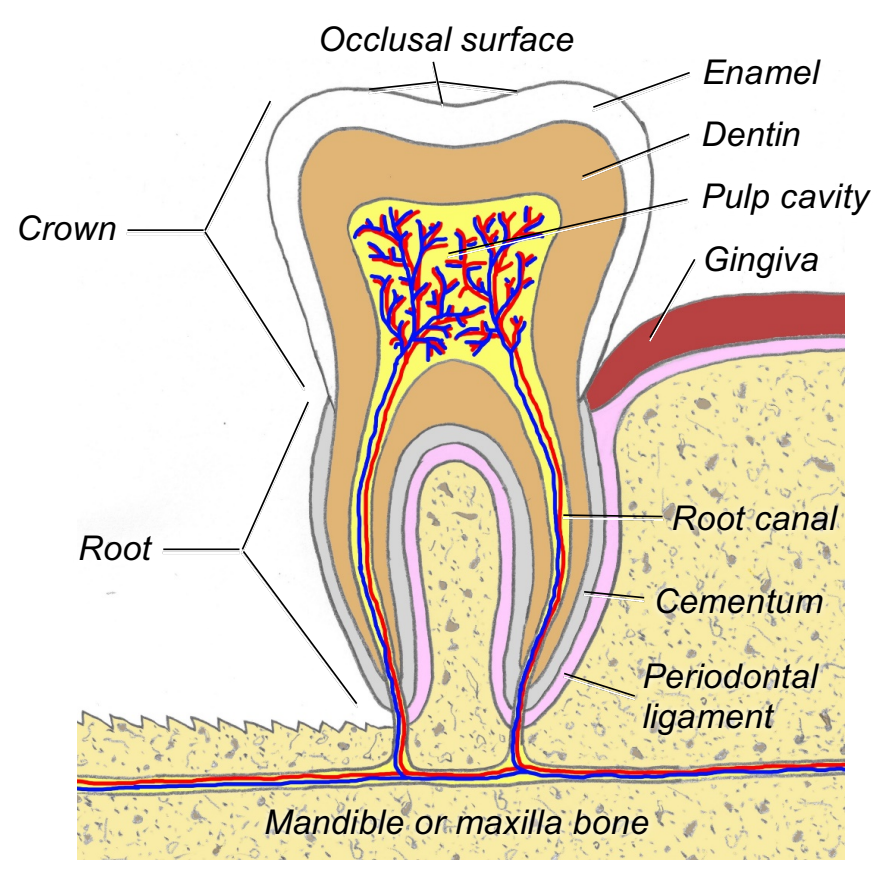

Figure 17.3. Diagram of a Typical Tooth, Anterior View

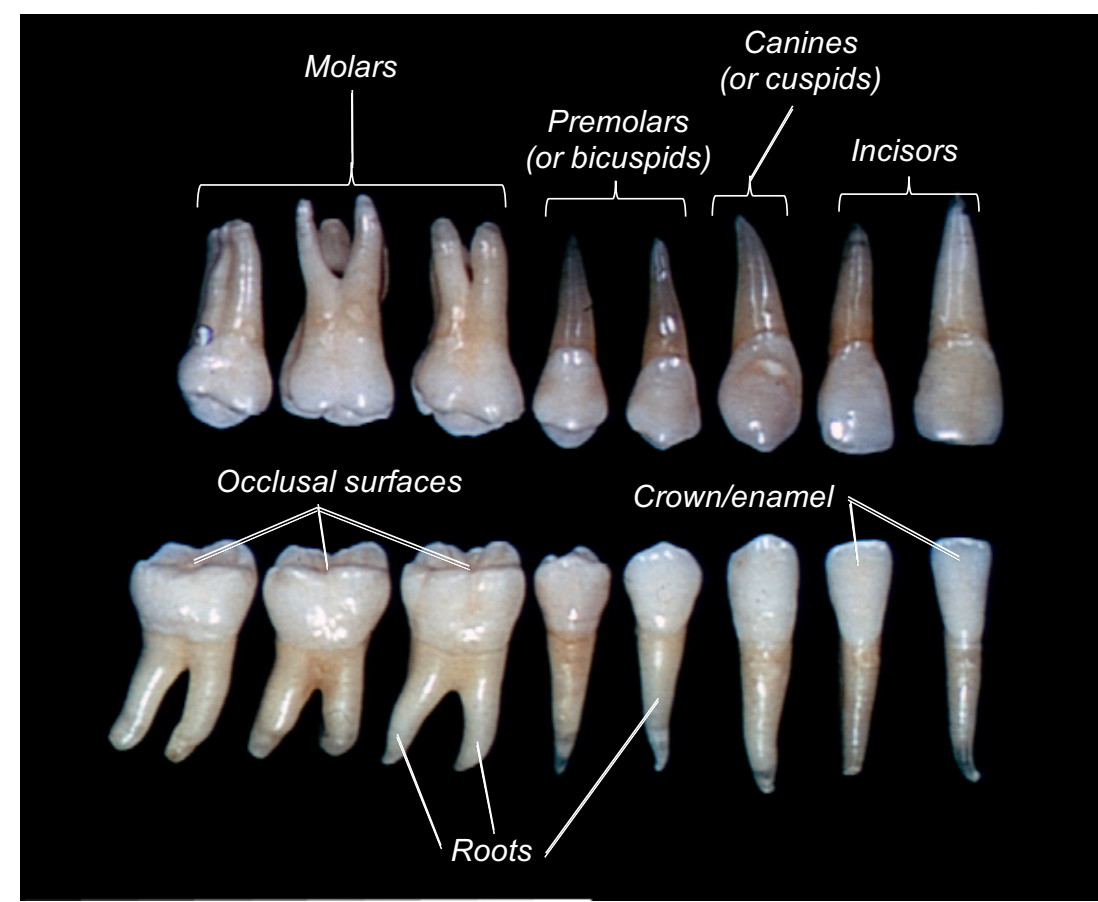

Figure 17.4. Separated Teeth, Anterior View 


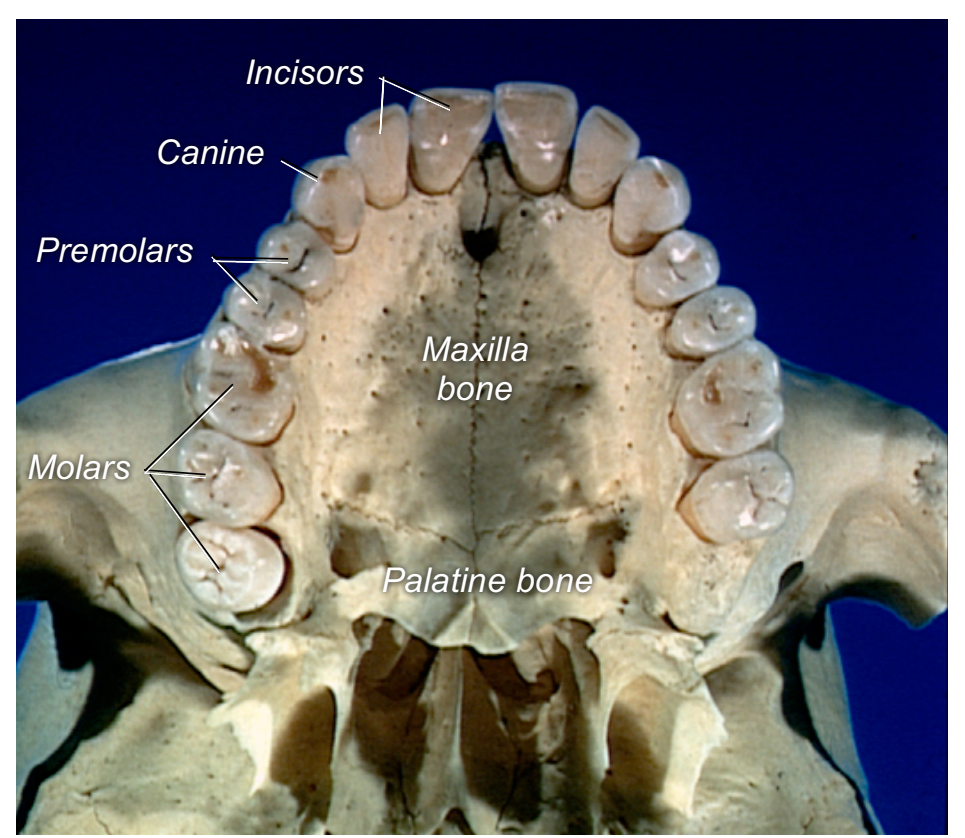

Figure 17.5. Teeth in Maxilla, Inferior View

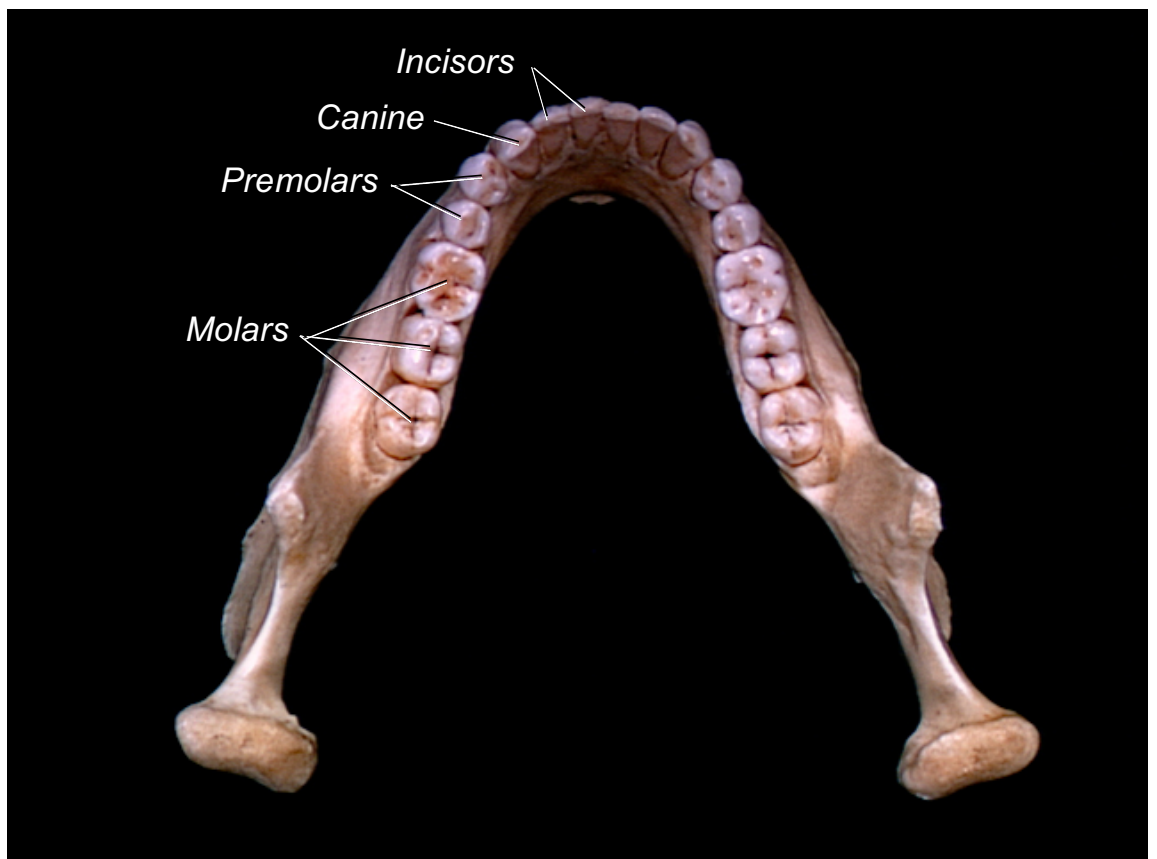

Figure 17.6. Teeth in Mandible, Superior View

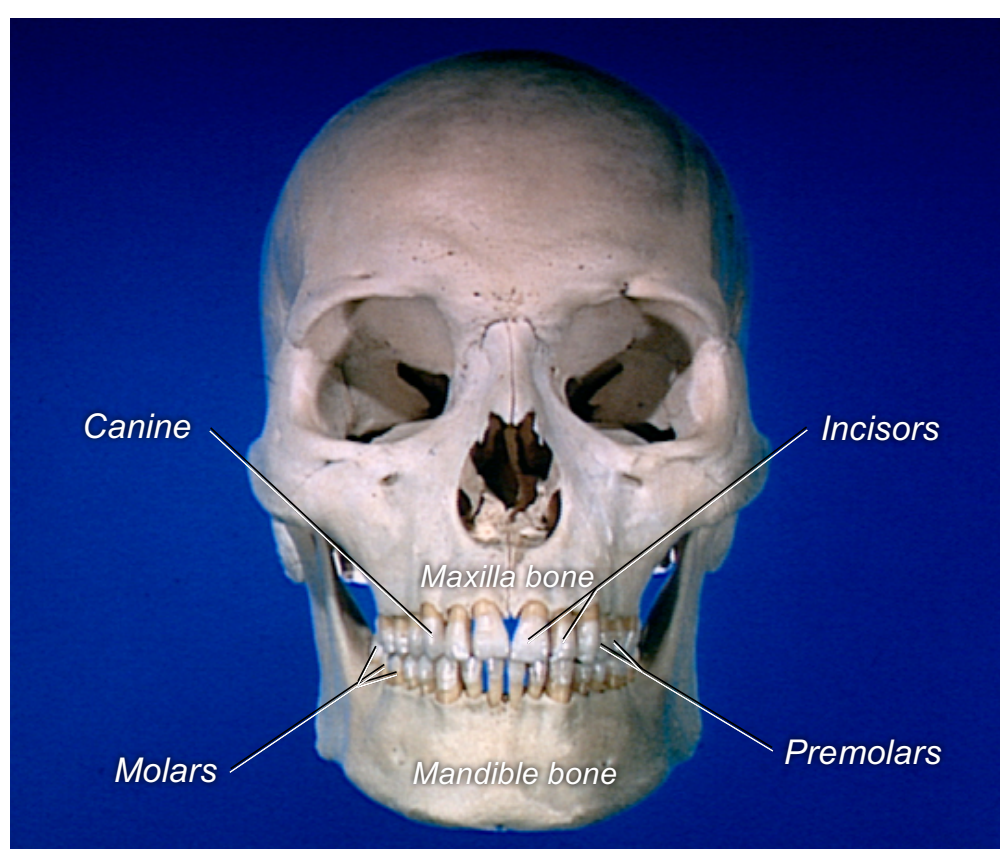

Figure 17.7. Teeth, Anterior View

\section{Clinical Application: Dental Caries}

Dental caries, also called tooth decay or cavities, is a very common oral disease that causes destruction of tooth enamel and creates "cavities" where the tooth has decayed. Progressive dental caries, when left untreated, can cause tooth pain and even tooth loss. Prevention includes brushing/cleaning teeth and a low sugar diet.

\section{Clinical Application: Halitosis}

Halitosis is a chronic condition of "bad breath" caused by odor produced by waste from bacteria and decaying food particles on your tongue, gums, and teeth. Poor oral hygiene is a significant cause of halitosis since bacteria and food particles are normal findings in the oral cavity. 


\section{Salivary glands and ducts}

$\square$ Parotid salivary gland

- Parotid duct

$\square$ Submandibular salivary gland

$\square$ Sublingual salivary gland

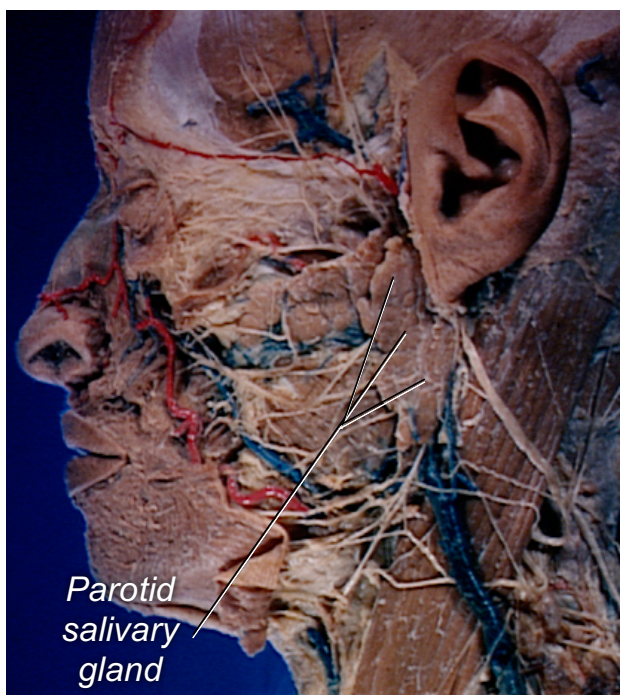

Figure 17.8. Parotid Salivary Gland, Left Lateral View

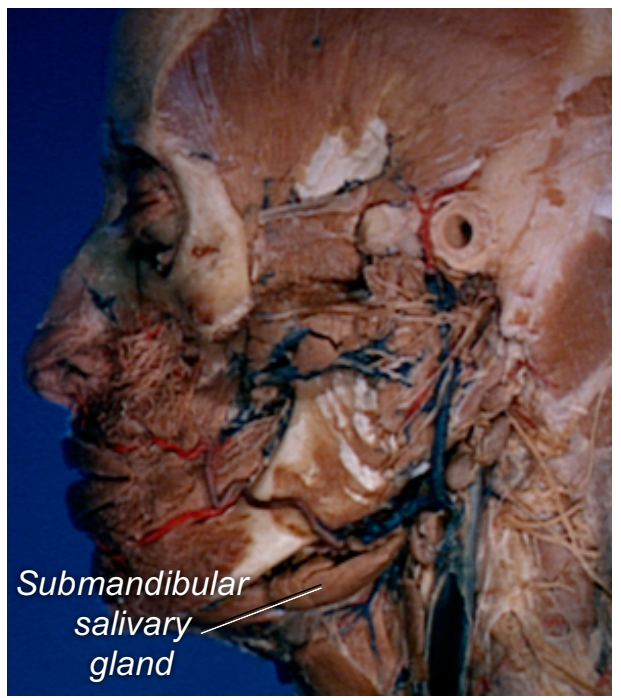

Figure 17.9. Submandibular Salivary Gland, Left Lateral View

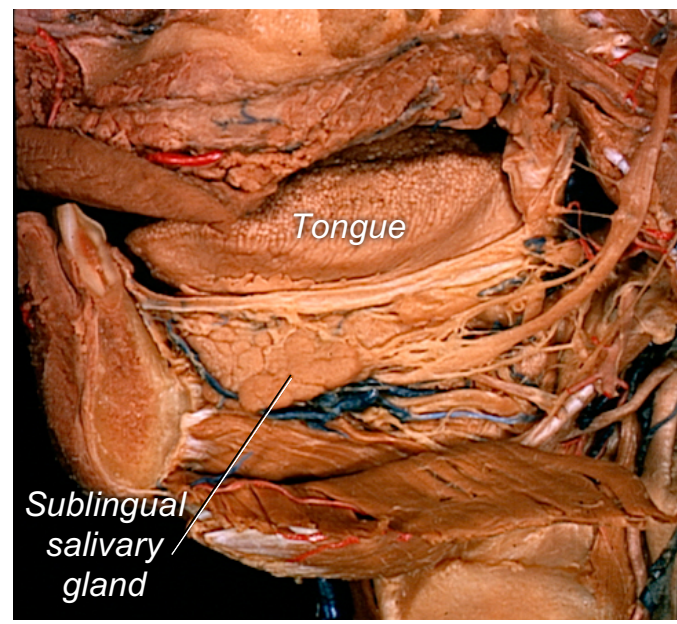

Figure 17.10. Sublingual Salivary Gland, Right Midsagittal View

\section{Small intestine}

$\square$ Major duodenal papilla

$\square$ Minor duodenal papilla

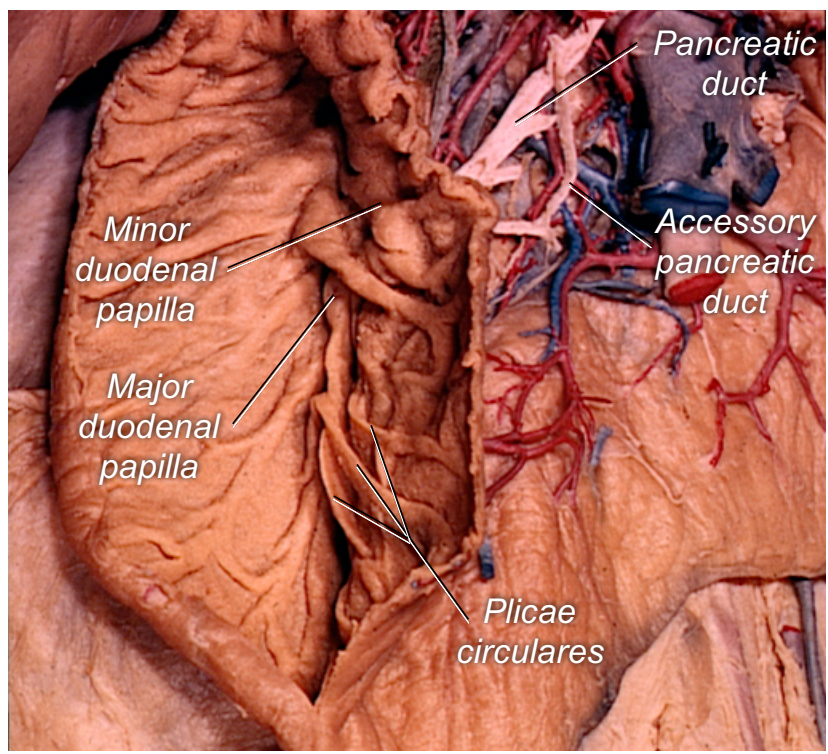

Figure 17.11. Duodenum (cut open), Anterior View

\section{Clinical Application: Celiac Disease}

Celiac disease is an autoimmune disorder caused by ingesting gluten, a protein found in grains. People have varying degrees of this disease, ranging from very mild (even asymptomatic) to severe. If severe inflammation continues over time, it can damage the walls of the small intestine and make absorption of important vitamins and minerals difficult. The classic symptom of celiac disease is diarrhea, but other symptoms (bloating, gas, abdominal discomfort, malnutrition, fatigue, etc.) can result as well. While a gluten-free diet can help manage symptoms (and in severe cases, heal intestinal walls) there is currently no known cure for celiac disease. 


\section{Liver}

Aside from cleaning/detoxifying portal blood, the liver also produces bile. Bile is a greenish-brown alkaline fluid that aids in digestion by emulsifying fats.

$\square$ Lobes:
○ Right lobe
- Left lobe
- Quadrate lobe
- Caudate lobe

$\square$ Coronary ligament

$\square$ Falciform ligament

The coronary ligament attaches the liver to the bottom of the diaphragm, and the falciform ligament attaches the liver to the back of the anterior abdominal wall. These ligaments stabilize and support the liver in its position.

\section{Gallbladder}

The gallbladder stores and concentrates bile.

\section{Biliary tree:}

$\square$ Right hepatic duct

$\square$ Left hepatic duct

$\square$ Common hepatic duct

$\square$ Cystic duct

$\square$ Common Bile duct

$\square$ Hepatopancreatic ampulla

$\square$ Hepatopancreatic sphincter

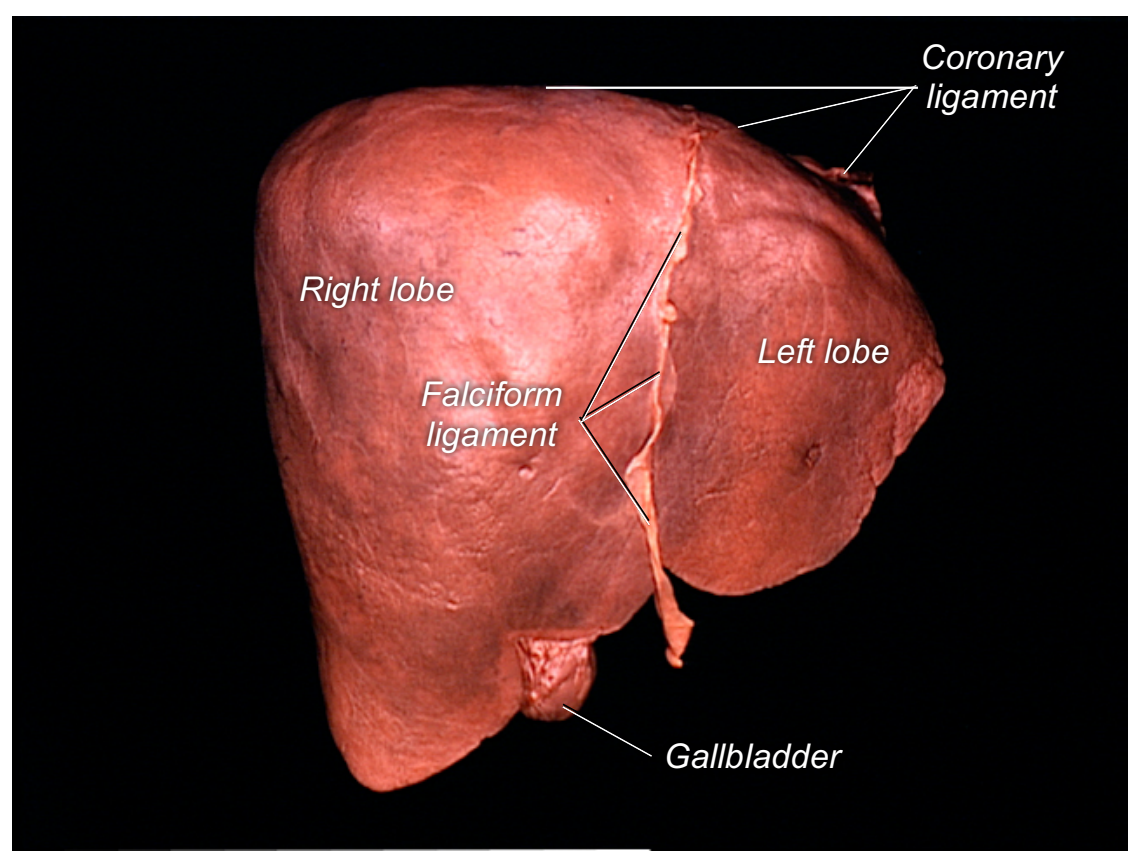

Figure 17.12. Liver and Gallbladder, Anterior View

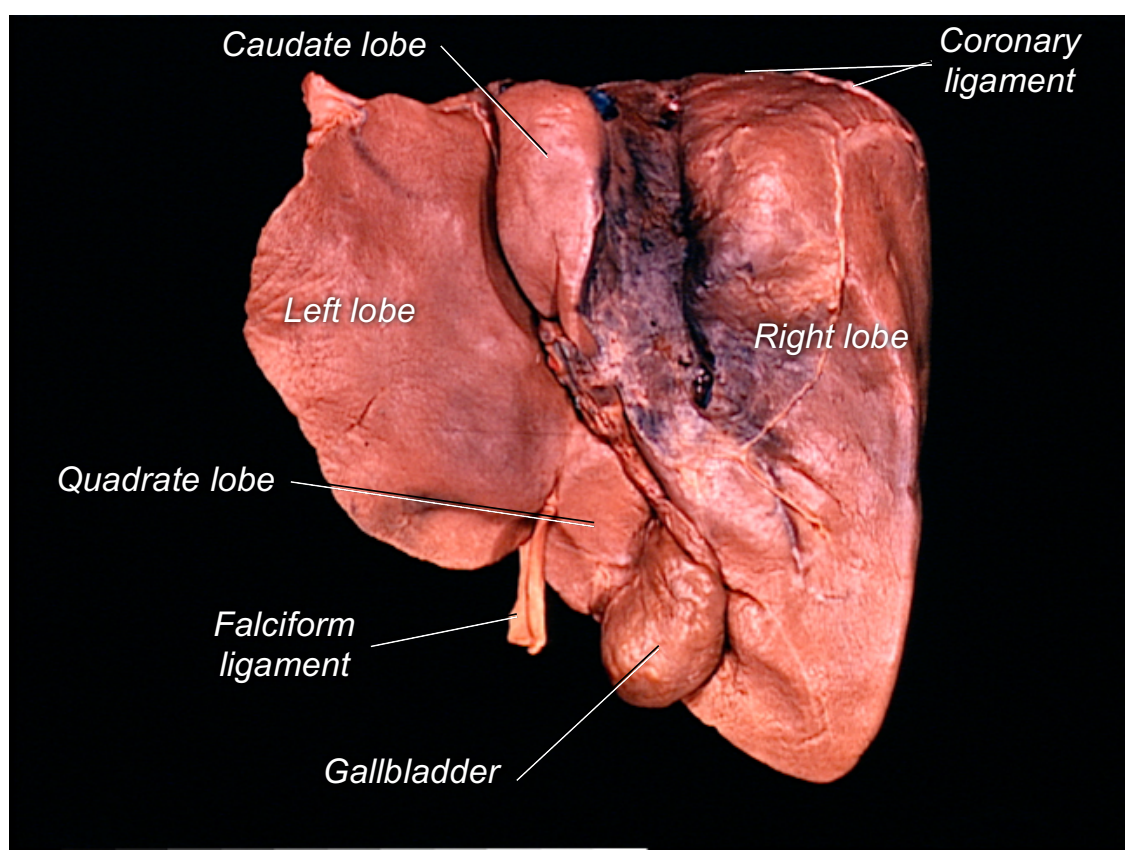

Figure 17.13. Liver and Gallbladder, Posterior View 


\section{Clinical Application: Gallstones}

As the gallbladder concentrates and stores bile, hardened bile deposits can aggregate together and form gallstones. Small gallstones can be asymptomatic and pass through the biliary tree and gastrointestinal tract to be excreted from the body with feces. Large gallstones are unable to enter into the biliary tracts and may require surgery to remove. Medium-sized gallstones can become lodged in biliary tracts, occluding the flow of bile and causing inflammation (known as cholecystitis). The major symptom of cholecystitis is severe pain in the upper right abdominal quadrant. Diagnosis of gallstones requires medical examination and associated tests.

\section{Pancreas}

The pancreas has both endocrine and exocrine functions: it produces glucagon and insulin to regulate blood sugar levels (endocrine) and digestive enzymes to aide in digestion (exocrine).

$\square$ Regions:

$$
\begin{array}{ll}
\circ \text { Head } \\
\circ \text { Body } \\
\circ \text { Tail }
\end{array}
$$

$\square$ Main pancreatic duct

$\square$ Accessory pancreatic duct

The main pancreatic duct combines with the common bile duct to form the hepatopancreatic ampulla. The hepatopancreatic sphincter controls the flow of bile and pancreatic juices through the ampulla and out the major duodenal papilla. The accessory pancreatic duct leads to the minor duodenal papilla.

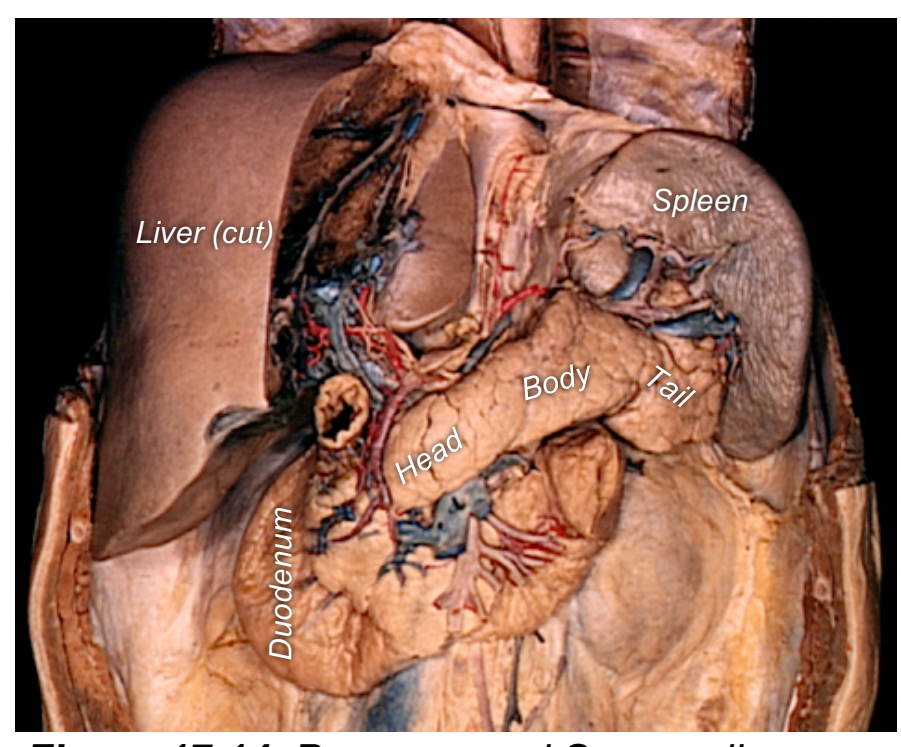

Figure 17.14. Pancreas and Surrounding Viscera, Anterior View

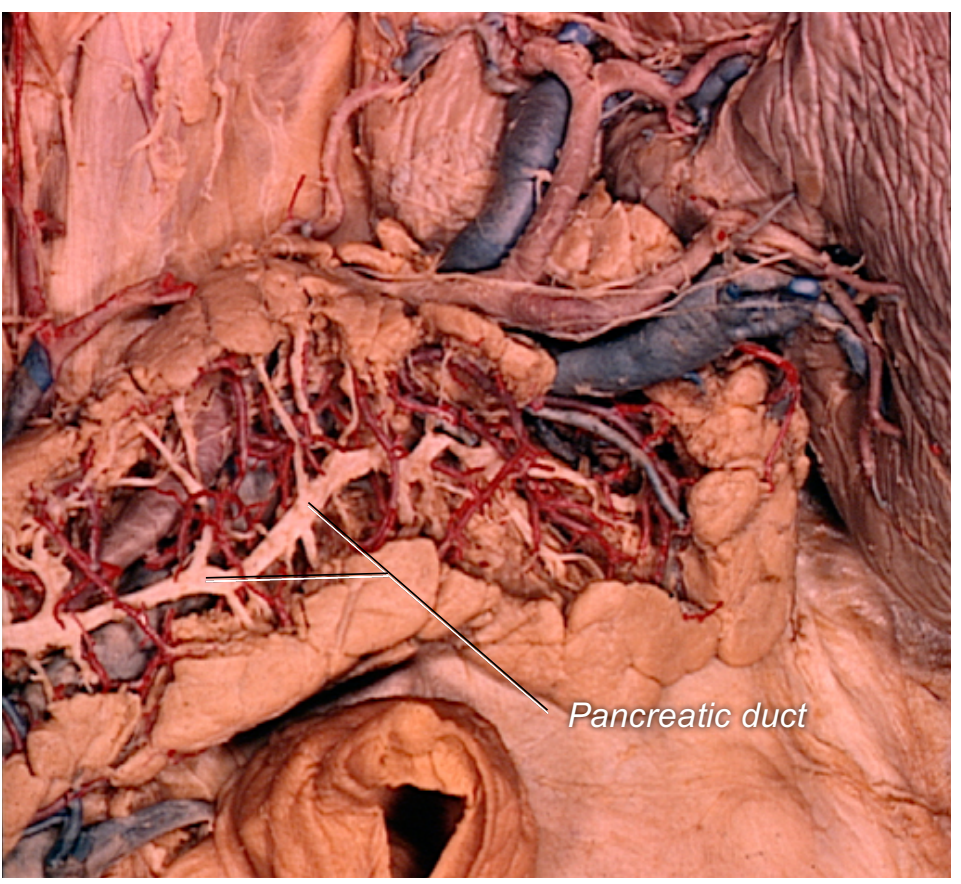

Figure 17.15. Dissected Pancreas and Pancreatic Duct, Anterior View 


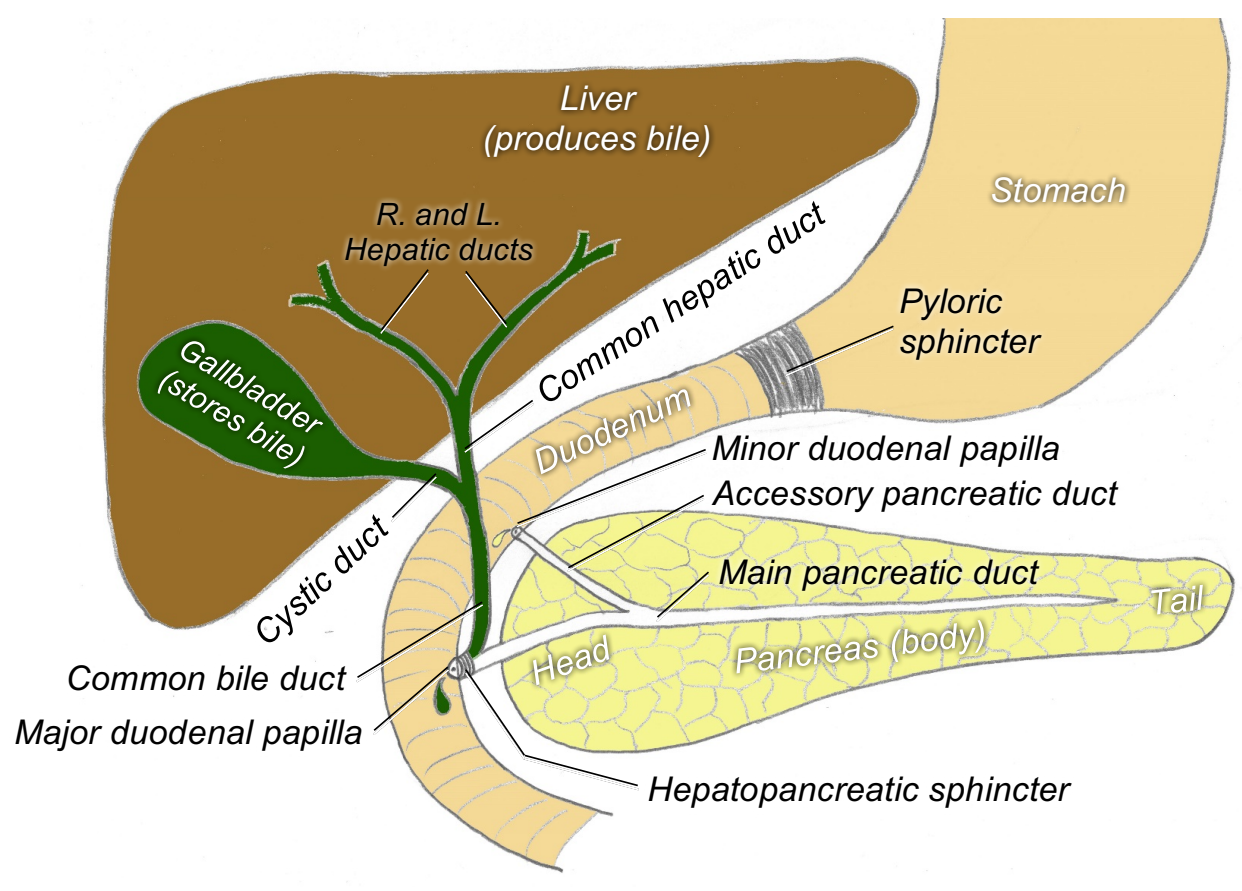

Figure 17.16. Diagram of Biliary Tree, Pancreatic Ducts, and Associated Organs, Anterior View

\section{Clinical Application: Blood Sugar \& Diabetes}

After you consume a meal, your body digests that food to break down the sugars, nutrients, vitamins, minerals, proteins, etc. into usable and readily-absorbed forms. The sugar (in the form of glucose) is absorbed into the circulatory system and taken first to the liver (via the portal v.) to be processed and then to the rest of the body. When the blood sugar level is high, the pancreas produces insulin, a hormone that stimulates the liver to convert glycose to glycogen (the storage form of glucose) and body tissues to uptake glucose from the blood. Both of these actions will cause the level of sugar in the blood to lower. After some time has passed and your body has a low blood sugar level from using the glucose that was in your blood for energy, the pancreas will produce glucagon, a hormone that stimulates the liver to convert previously made glycogen back into glucose and release it into circulation for transportation. This action will cause the level of sugar in the blood to raise. The figure below represents the communication between the pancreas, liver, and tissue cells to maintain homeostatic blood sugar levels.

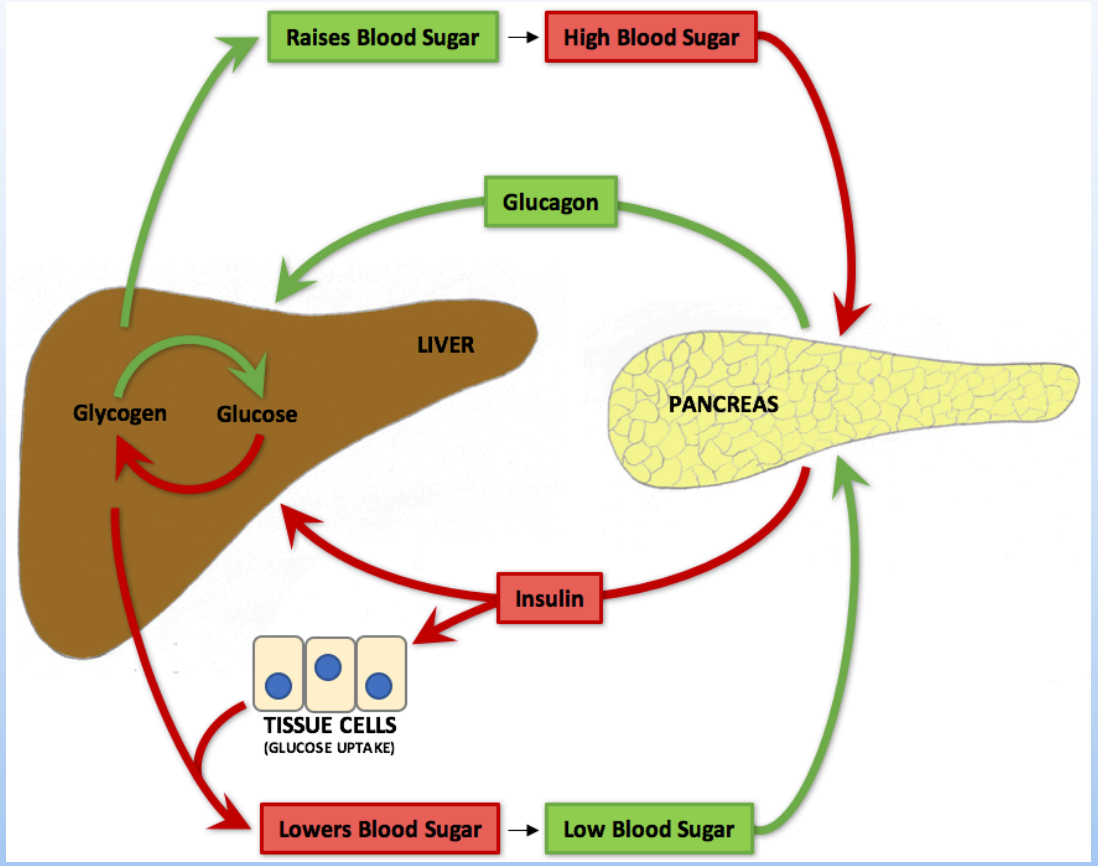

Diabetes is a disease that causes abnormal blood sugar levels. There are three main types of diabetes. Type 1 diabetes is a condition where the pancreas produces little to no insulin. Type 2 diabetes is a condition where the liver is unable to adequately convert glucose into glycogen. Prediabetes is a condition where high blood sugar is caused by hindered glucose processing, but it is not severe enough to be Type 2 diabetes because it can be controllable by diet modification. 


\section{Lymphatic system}

As blood passes through capillaries, the high pressure on the capillary walls allows the capillaries to "leak" fluid. This fluid called interstitial fluid or tissue fluid, is very similar to blood plasma and bathes the tissues. As more interstitial fluid is made, the older fluid collects in lymphatic vessels which transport the fluid (now called "lymph") back to the circulatory system. Because lymph bathes all the tissues, it collects debris from the tissues. As the lymph is transported back to the circulatory system, the debris (bacteria, pathogens, abnormal cells) is cleaned and filtered from the lymph.

\section{Spleen}

The spleen stores platelets and white blood cells as well as recycles old red blood cells, helping maintain the level of debris circulating in the body.

\section{$\square$ Lymph nodes}

- Cervical lymph nodes

- Axillary lymph nodes

○ Inguinal lymph nodes

Lymph nodes filter the lymph of debris as it is en route back to the circulatory system. Immune cells in the lymph nodes attack germs, bacteria, and pathogens that could cause illness. Larger lymph nodes are concentrated in the cervical, axillary, and inguinal regions.

\section{R. and L. lymphatic ducts}

Lymphatic vessels collect to the $R$. and $L$. lymphatic ducts. The R. lymphatic duct collects lymph from the right side of the body superior to the diaphragm, whereas the left lymphatic duct (which is much larger) collects lymph from the entire left side of the body AND the right side of the body inferior to the diaphragm. Both lymphatic ducts drain into the respective subclavian vv.

\section{Tonsils}
- Lingual tonsils
- Palatine tonsils
- Pharyngeal tonsils (or Adenoids)

Tonsils are like lymph nodes that act as a defense mechanism for fighting infection.

\section{Thymus}

The thymus trains and matures T-cells, important white blood cells that fight off germs, infections, and illnesses.

\section{Appendix}

Although the appendix is made of a lot of lymphatic tissue, it is thought to have little to do with the lymphatic system.

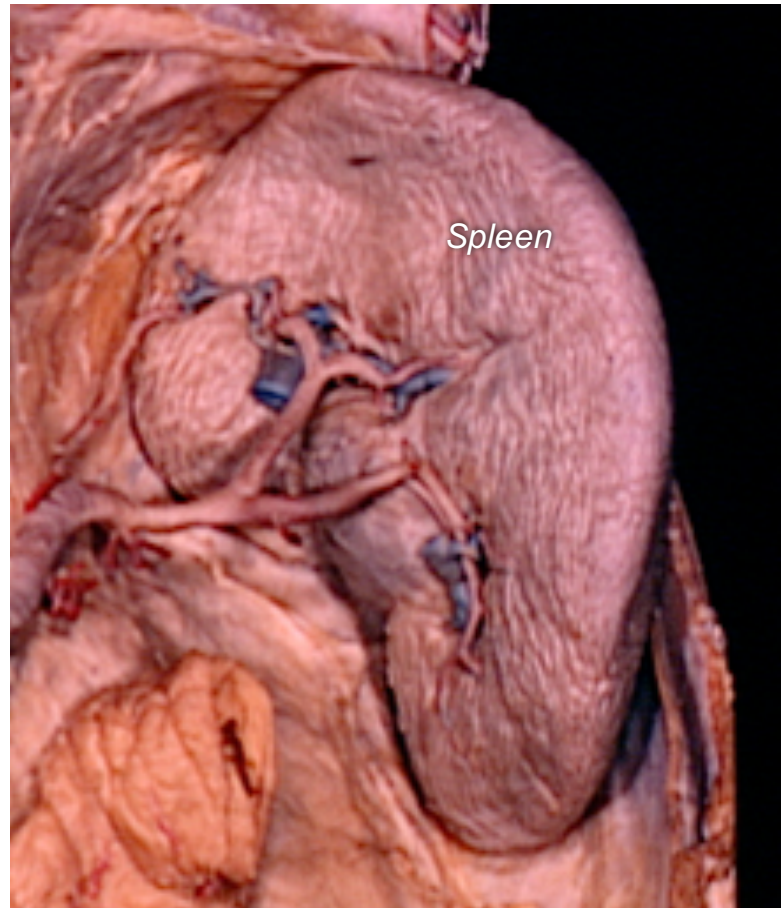

Figure 17.17. Spleen, Anterior View 


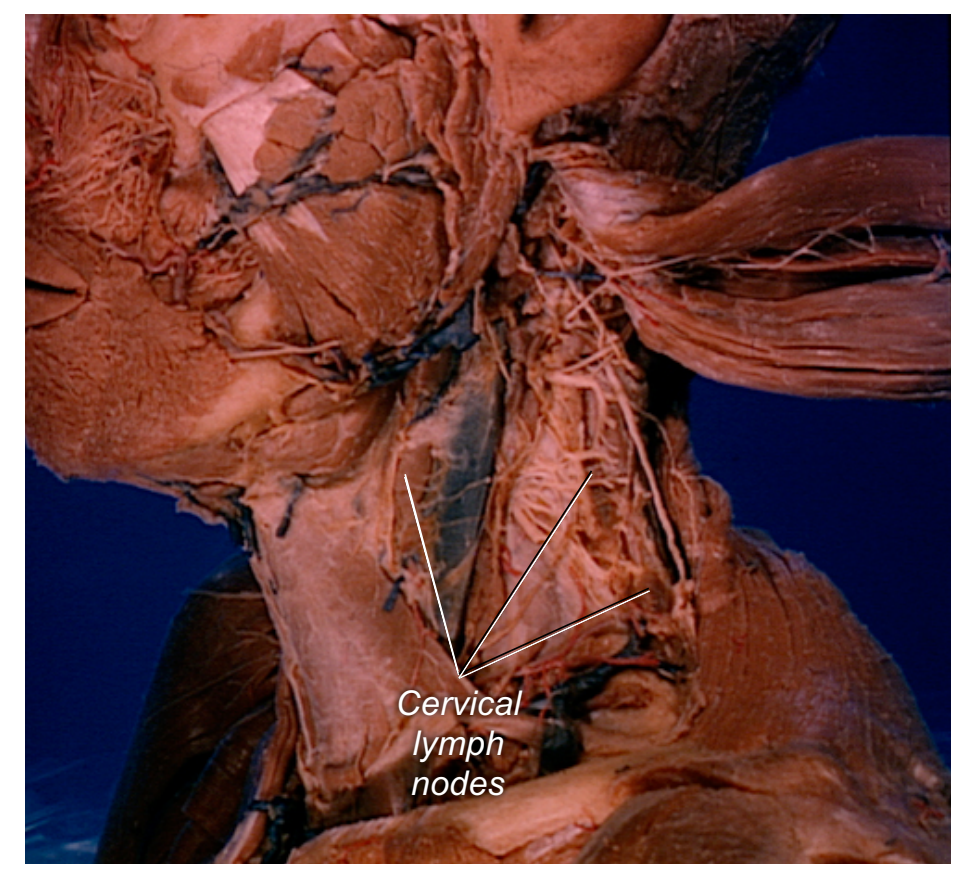

Figure 17.18. Left Cervical Lymph Nodes, Lateral View

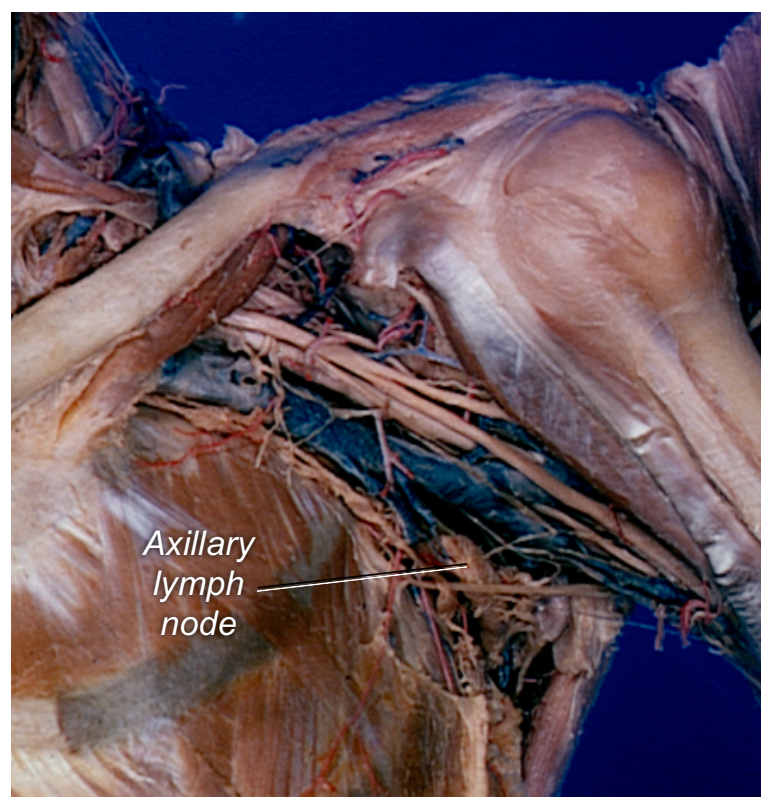

Figure 17.19. Left Axillary Lymph Node, Anterior View

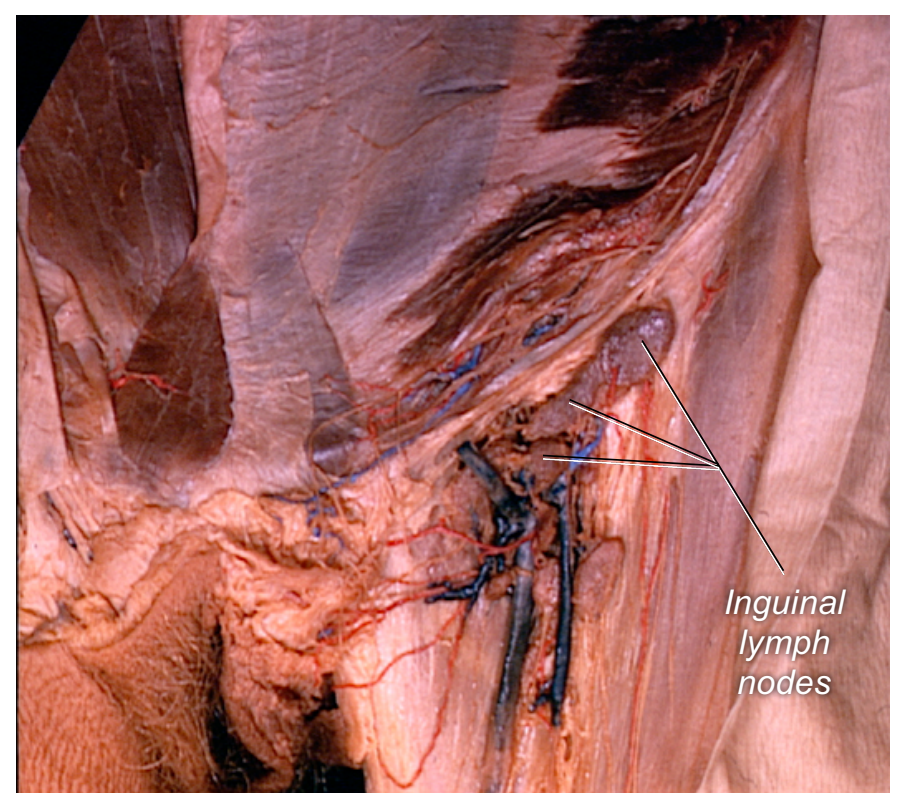

Figure 17.20. Left Inguinal Lymph Nodes, Anterior View

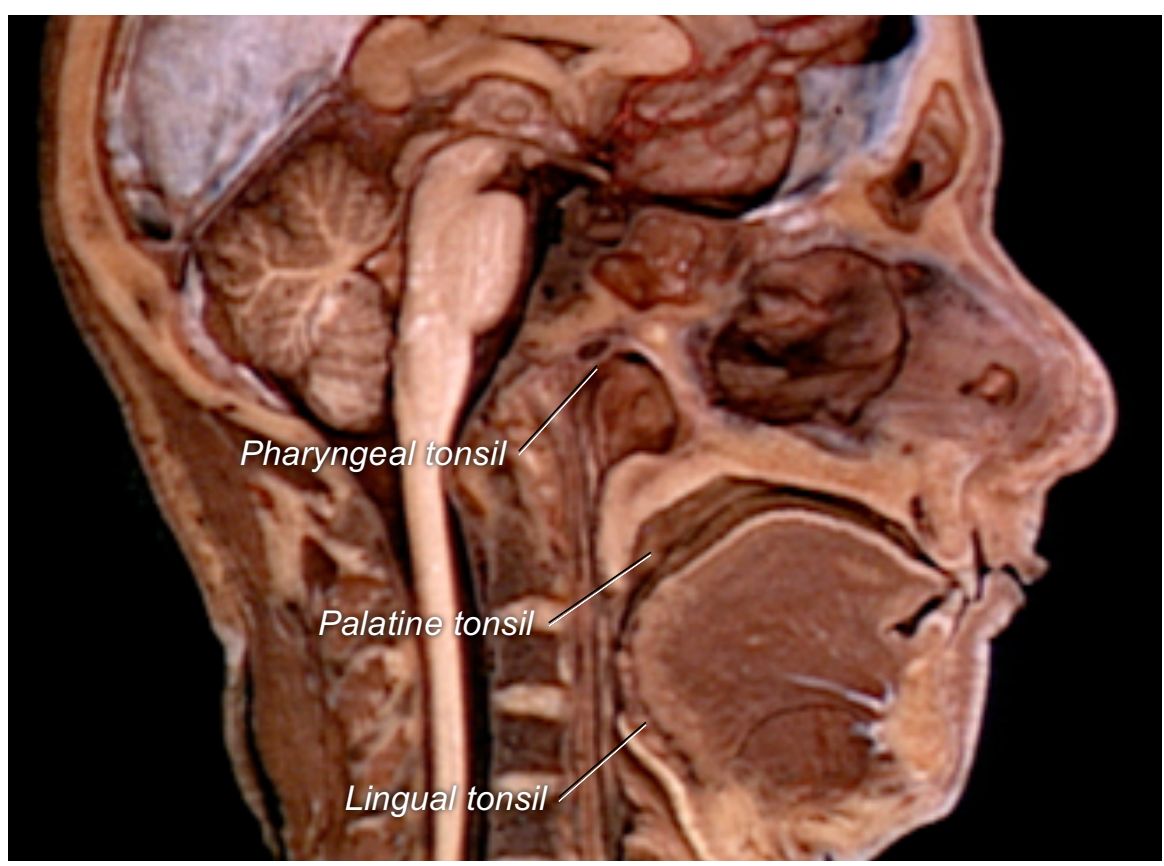

Figure 17.21. Left Tonsils, Midsagittal View 


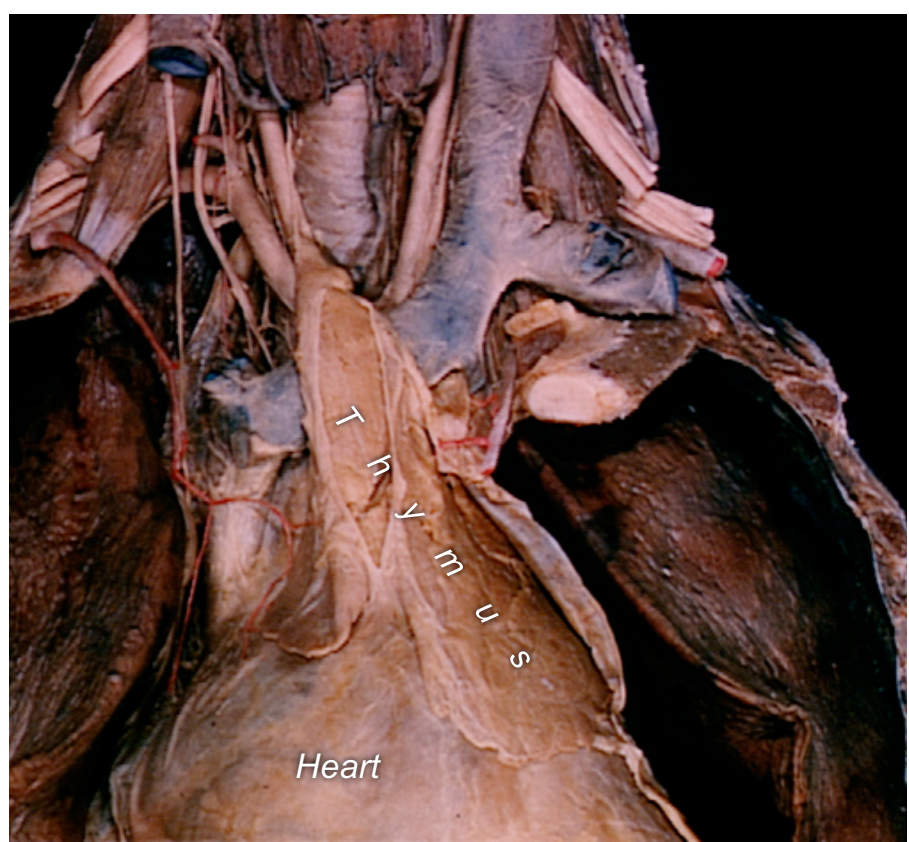

Figure 17.22. Thymus, Anterior View

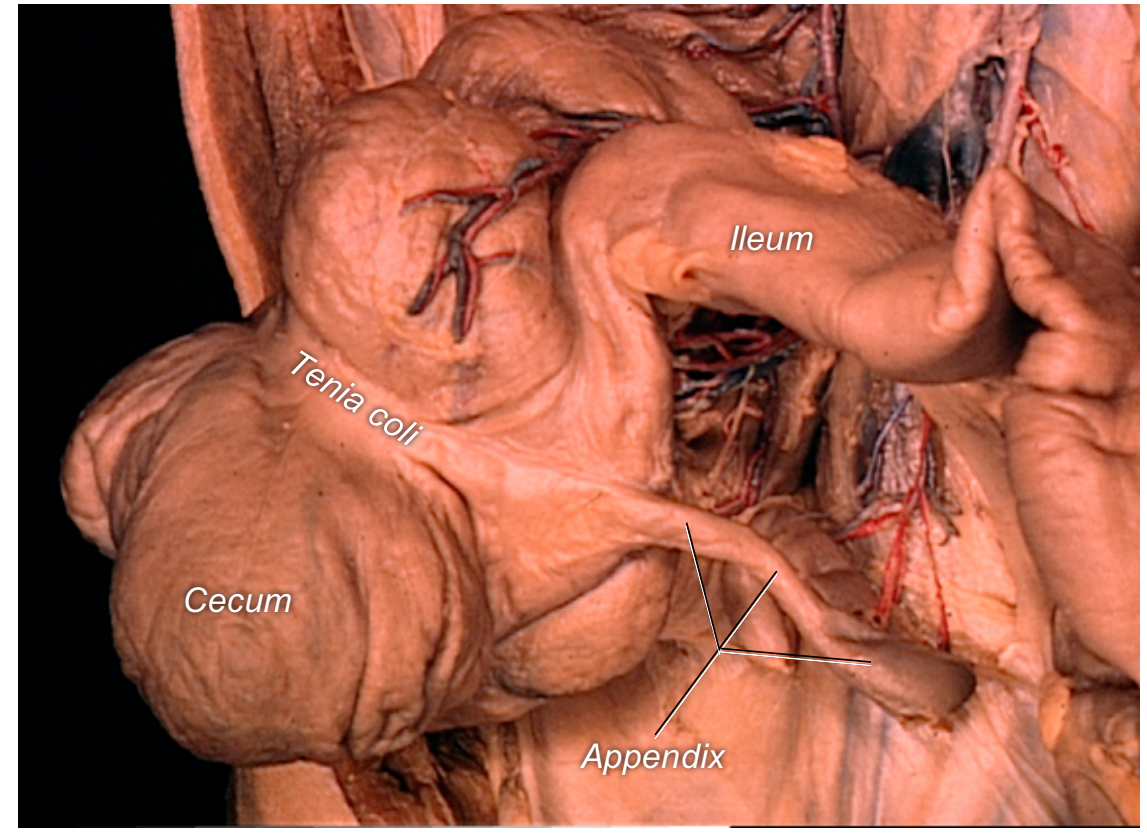

Figure 17.23. Appendix, Anterior View

\section{Clinical Application: Appendicitis}

The appendix is a tube of tissue about the size of a pinky finger (exact thickness and length varies) attached to the cecum. The lumen of the appendix is continuous with that of the cecum, which allows fecal matter to fill the appendix. When the appendix is agitated (typically caused by a component of fecal matter getting stuck in the lumen), it becomes inflamed and is known as appendicitis. Symptoms of appendicitis include severe pain in the lower right quadrant of the abdomen (where the appendix is located), vomiting, and fever. Blood, urine, and radiographic tests can help verify the diagnosis. If left untreated, the inflamed appendix may perforate or rupture, allowing fecal matter and bacteria to spill into the abdominal cavity. This can cause peritonitis (inflammation of the peritoneum), which can be fatal. Therefore, medical attention should be sought immediately if appendicitis is suspected. Typical treatment includes consists of an emergency surgical procedure called an appendectomy to remove the appendix. Because they are sharp and don't digest, one of the most common things that become lodged in the appendix and cause appendicitis is fingernails!

\section{Clinical Application: Edema}

Much like venous blood, interstitial fluid and lymph circulates under very little pressure. Even if you are active during the day, gravity can pool the fluid in the distal portions of your limbs, causing swelling in your forearms, wrists, and hands and legs, ankles, and feet. This swelling and pooling of lymph is called edema. It is normally triggered by pregnancy, but it can also be a sign of an underlying disease such congestive heart failure, kidney disease, or cirrhosis of the liver. 
1) In the song "All I want for Christmas is my two front teeth" what type of teeth is the child referring to? (LO48)

2) What does the term pre mean? (LO47)

3) What does the term den mean? (LO47)

4) Where does bile flow next when the hepatopancreatic sphincter is contracted (closed)? (LO49)

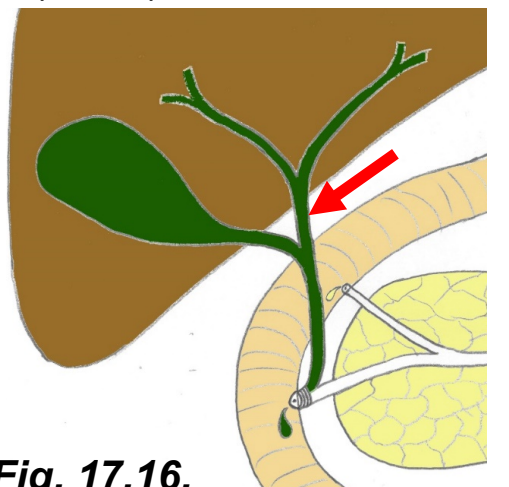

Fig. 17.16.

5) Where does bile flow next when the hepatopancreatic sphincter is relaxed (open)? (LO49)

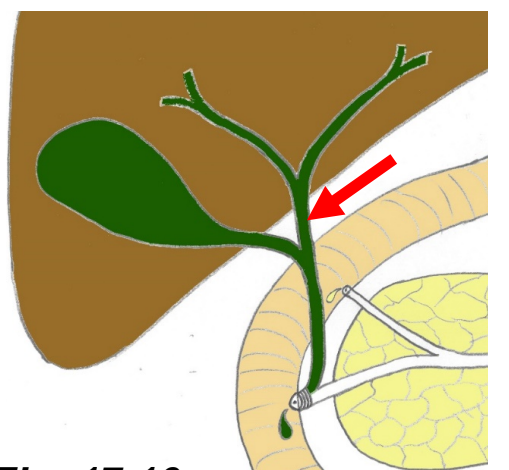

Fig. 17.16.
6) In what three regions are lymph nodes most concentrated? (LO48)

7) How many teeth does a typical adult have in his/her mandible? (LO48)

8) What is the largest lobe of the liver? (LO48)

9) True or False: bile flows through the minor duodenal papilla. (LO49)

10)True or False: pancreatic juices flow through the major duodenal papilla. (LO49)

11)What ligament attaches the liver to the inferior diaphragm? (LO48)

12)Using the terminology, what does the term submandibular salivary gland mean? (LO47)

13)List the papillae of the tongue from smallest to largest. (LO48)

14)What structure is found deep to the enamel on a tooth? (LO48) 


\section{Lab 18: Urinary System}

Instructions: Learn the listed terms using the listed learning objectives, labeled images, lab specimens, and other resources.

LO50: Describe the terminology used to identify structures of the urinary system.

LO51: Identify the structures of the urinary system.

LO52: Describe the flow pathways of blood, waste, and urine through the urinary system.

\section{Kidney}

The kidney filters blood of cellular waste and controls the body's fluid and electrolyte levels. Nephrons are the functional units of kidneys that fulfill these functions. After doing so, the waste (urine) is expelled from the body through the process of micturition ( $\underline{\text { micturition }}=$ urination).

Each kidney has about one million nephrons. Unlike most organs, there are two kidneys located in the abdomen, both of which are more specifically located in the retroperitoneal space. Abdominal viscera are suspended and contained within peritoneum, a membrane that lines the abdominal cavity and many of the abdominal organs. The kidneys are located behind the cavity created by the peritoneum (retro $=$ behind).
Capsule
Cortex
Medulla
Hilum
Suprarenal gland (or Adrenal gland)

Do you remember what the terms supra and ren mean?

\section{Column}

These tissue-filled areas between the pyramids anchor the medulla to the cortex and provide a place for blood vessels to travel.

\section{Renal sinus}

The renal sinus is a cavity within the center of the kidney in which the calyces, pelvis, blood vessels, and adipose are located.

\section{Pyramid}

\section{Renal papilla}

Renal papillae are the tips of the pyramids where the collecting ducts (from nephrons) will deliver urine to the through to the minor calyces.

\section{Major calyx \\ Minor calyx \\ Renal pelvis \\ Ureter}

The ureter will deliver urine to the bladder. 


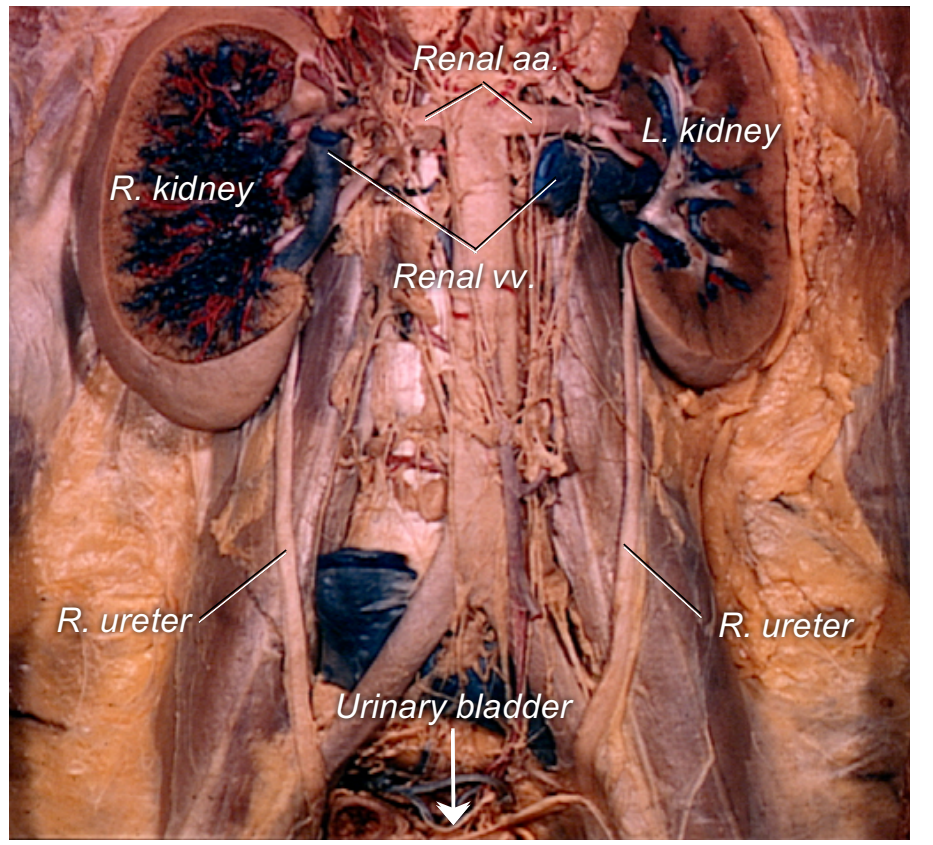

Figure 18.1. Kidneys (dissected) in situ, Anterior View

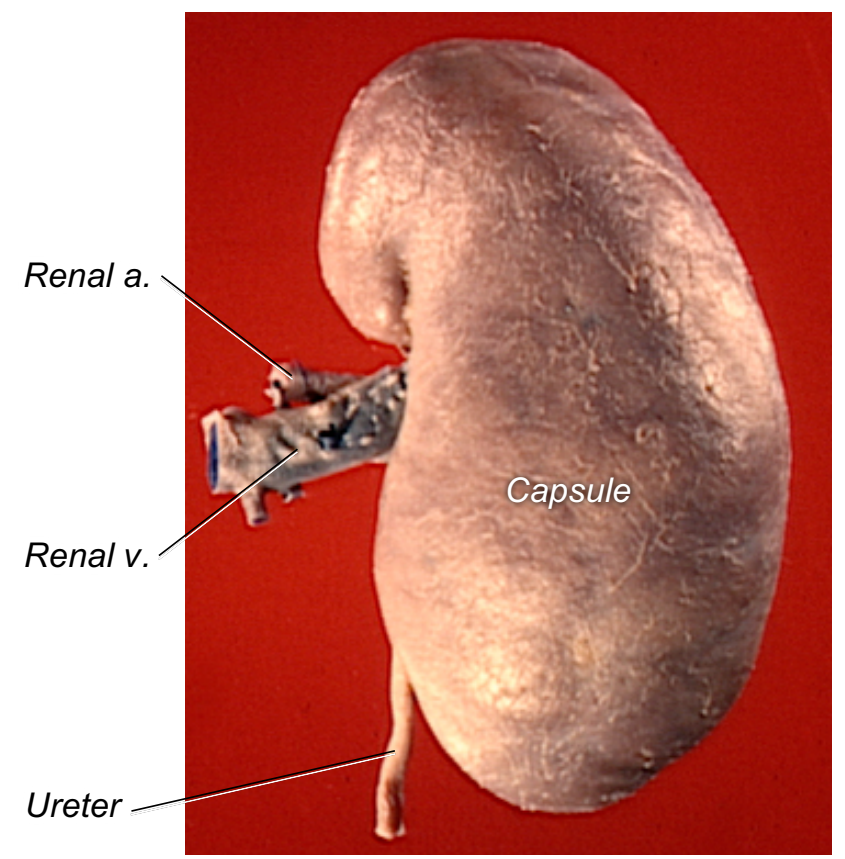

Figure 18.2. Left Kidney, Anterior View

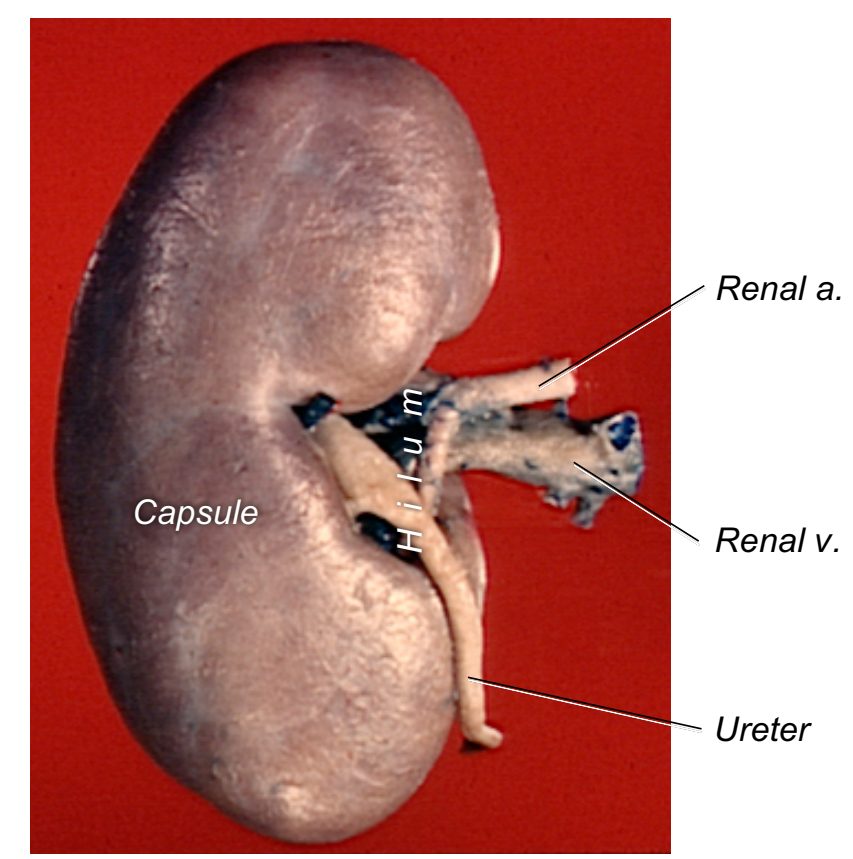

Figure 18.3. Left Kidney, Posterior View

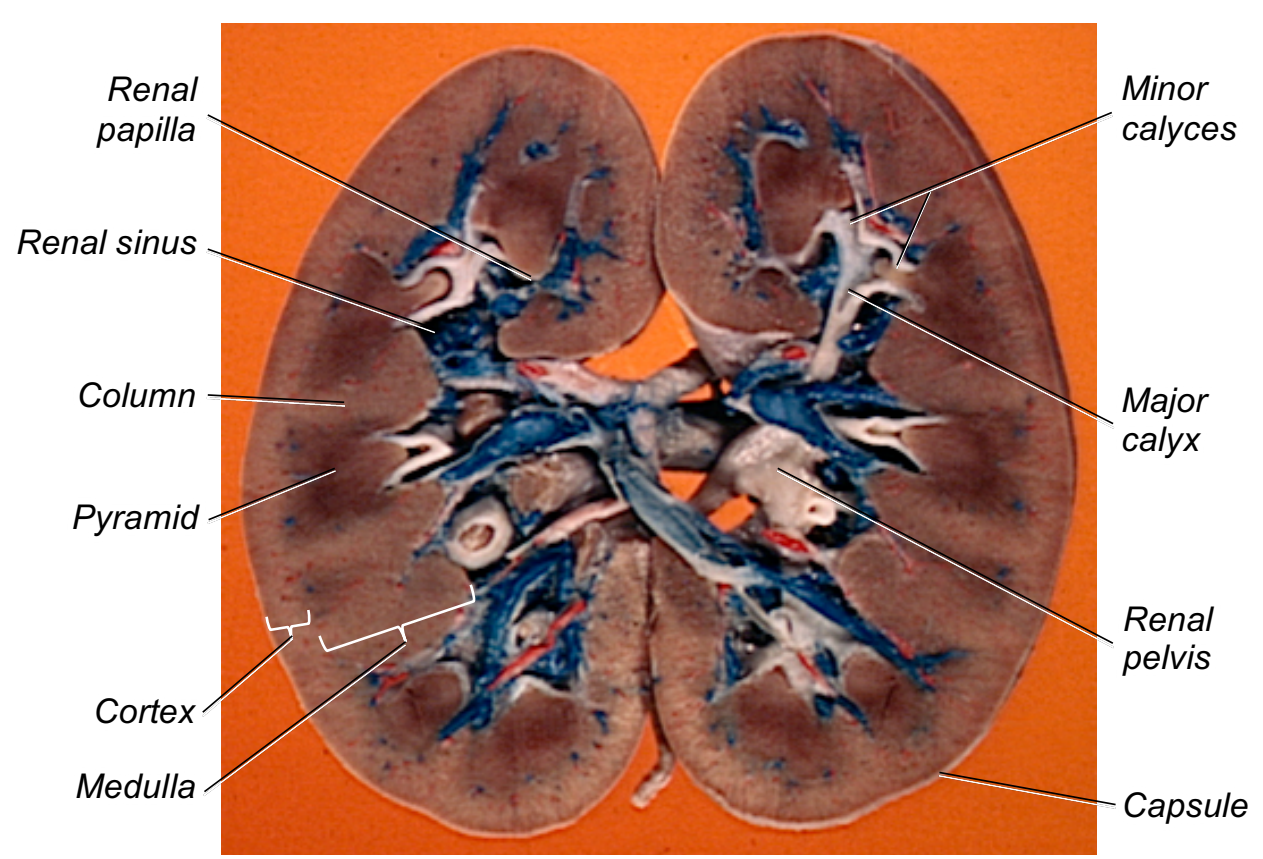

Figure 18.4. Left Kidney (hemisected and reflected) 


\section{Nephron}

$\square$ Afferent arteriole

$\square$ Glomerulus

Glomerulus $=$ a cluster of small blood vessels. This is where the waste is filtered out of the blood.

\section{Efferent arteriole}

\section{Bowman's capsule}

This capsule, which is located in the cortex of the kidney, collects the waste fluid filtered out of the glomerulus. The waste fluid then travels through a series of tubules where water and electrolytes will be reabsorbed into the body.

Proximal convoluted tubule

Convoluted $=$ folded, twisted, or coiled

$\square$ Descending loop of Henle

$\square$ Ascending loop of Henle

The loop of Henle extends into the renal medulla and pyramids.

Distal convoluted tubule

\section{Peritubular capillaries}

The peritubular capillaries surround the loop of Henle to reabsorb water, concentrating the waste fluid.

Collecting duct

Once "waste fluid" has entered the collecting duct it is then considered "urine". Many distal convoluted tubules empty into shared collecting ducts.

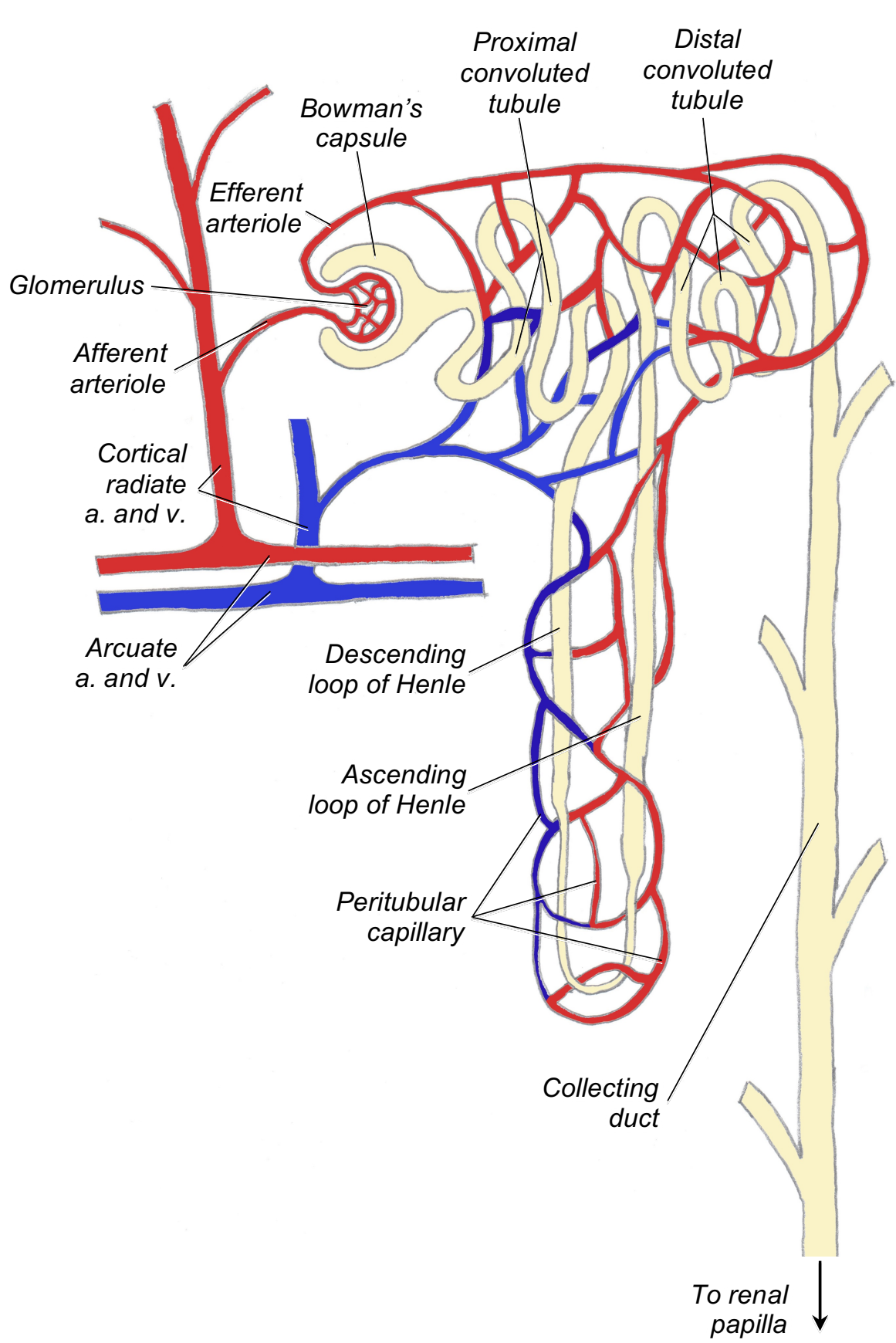

Figure 18.5. Nephron Diagram 
In order for the kidney to work properly, blood vessels must carry blood to the glomerulus for filtration, then to the peritubular capillaries to drop off oxygen (and pick up carbon dioxide and reabsorb water and electrolytes), and then to the renal $v$. to head back to the heart. Below are the vessels that make this special circulation happen. They are listed in the order that blood flows through them.

\section{Renal a.}

Segmental a.

$\square$ Interlobar a.

$\square$ Arcuate a.

Cortical radiate a.

These vessels are named "cortical radiate" because they radiate around the cortex of the kidney.

\section{Afferent arteriole}

Afferent $=$ conducting inward, toward something.

\section{Glomerulus \\ Efferent arteriole}

Efferent $=$ conducting outward, away from something.

Peritubular capillary

Do you remember what peri means?

$\square$ Cortical radiate $v$.

$\square$ Arcuate v.

$\square$ Interlobar v.

$\square$ Segmental v.

$\square$ Renal v.

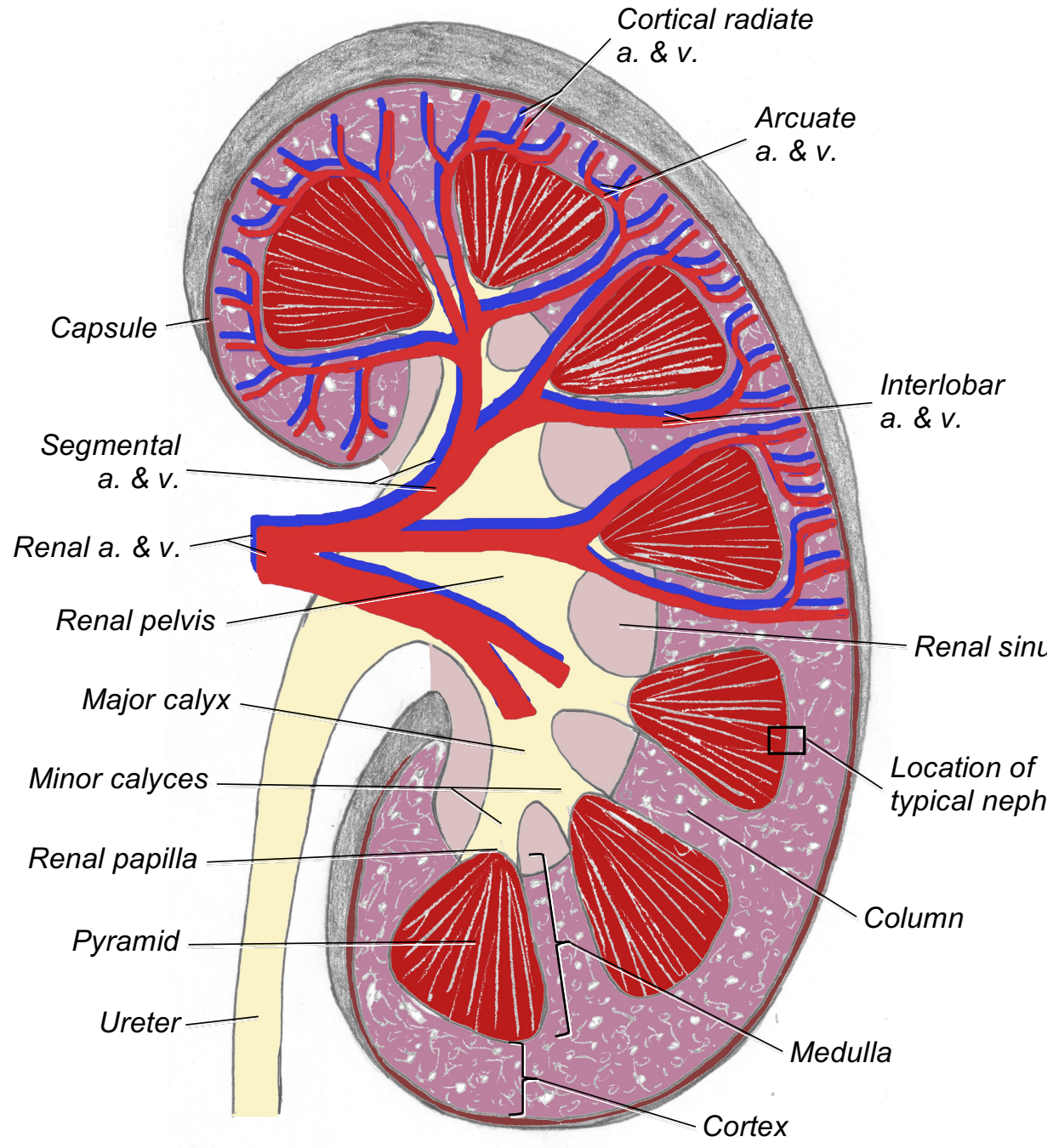

Figure 18.6. Diagram of Kidney Vasculature 
Other than the kidneys, there are many other abdominal viscera are located in the retroperitoneal space. A common mnemonic used to remember what viscera are retroperitoneal is SAD PUCKER:

$\mathbf{S}=$ Suprarenal glands

A = Aorta/inferior vena cava

$\mathbf{D}=$ Duodenum

$\mathbf{P}=$ Pancreas

$\mathbf{U}=$ Ureters

$\square \mathbf{C}=$ Colon (ascending and descending regions only!)

$\square \mathbf{K}=$ Kidneys

$\mathbf{E}=$ Esophagus

$\mathbf{R}=$ Rectum

The other abdominal viscera are considered intraperitoneal (intra $=$ within) with the exception of the urinary bladder. Since the urinary bladder is located below the peritoneal cavity in the subperitoneal space it is referred to as subperitoneal.

\section{Clinical Application: Kidney Stones}

As the kidney transports and concentrates cellular waste, the hard mineral deposits can aggregate and form kidney stones. Small kidney stones may pass through the urinary tract and managed/encouraged with pain medication and a greater intake of fluids. Large kidney stones may require surgical removal or other means of removal. Extracorporeal shock wave lithotripsy is a non-invasive procedure in which high energy shock waves are directed through the body to break up large kidney stones into smaller, passable pieces. Symptoms of kidney stones include waves of severe pain in the lower back (pain may radiate to lower abdomen and groin), abnormally colored urine (pink, red, or brown), nausea/vomiting, and an abnormal micturition pattern.

\section{Clinical Application: Kidney Failure}

Kidney failure is a condition in which the kidneys lose their ability to filter waste from the blood and maintain sufficient fluid levels. As a result, the unfiltered blood contains a build-up of cellular waste, resulting in azotemia (less severe) and uremia (more severe). Similarly, extra fluid volume in the blood causes high blood pressure. Azotemia, uremia, and high blood pressure can severely affect many other areas and tissues in the body, creating a wide spectrum of kidney failure symptoms. If the kidneys have failed completely, the only treatment options are dialysis or transplant. In dialysis, blood is shunted out of the body, filtered of waste in a machine, and then returned to the body. In a kidney transplant, a donor kidney is surgically implanted into the recipient's body in addition to or replacing the nonfunctioning kidney. 


\section{Urinary bladder}

The urinary bladder stores urine until the person is ready to micturate.

$\square$ Detrusor muscle

\section{Rugae}

The rugae in the detrusor muscle act like those in the stomach and allow the urinary bladder to expand as it fills with urine.

\section{Trigone}

The trigone contains sensitive stretch receptors. When the urinary bladder is full and needs to empty itself, these stretch receptors send signals to the brain to trigger the "I need to micturate" sensation.

$\square$ Ureteral openings

Urethral opening

Ureteral and urethral are spelled very similarly. Make sure to spell these terms correctly!

$\square$ Urethra

- External urethra orifice

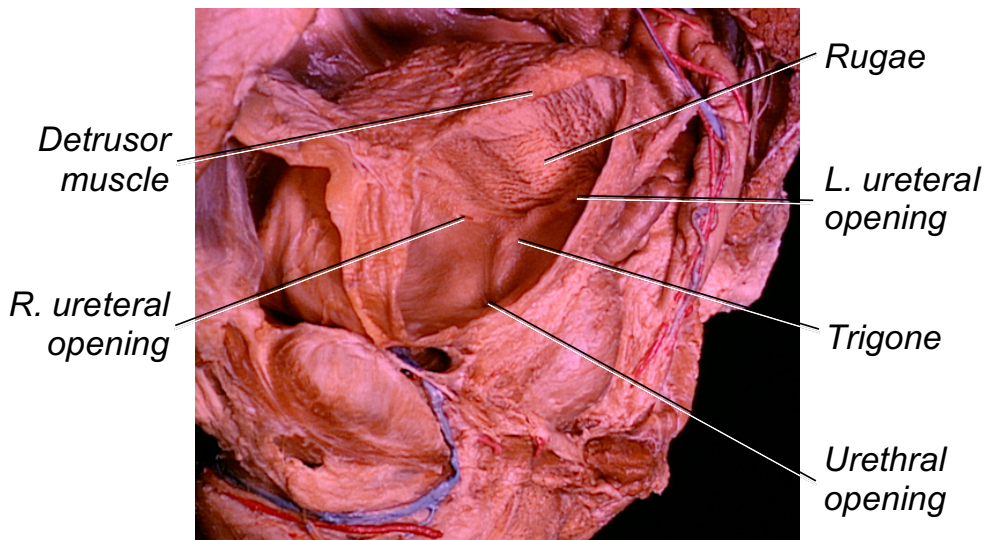

Figure 18.7. Urinary Bladder (cut open) in Hemisected Right Male Pelvis, Parasagittal View

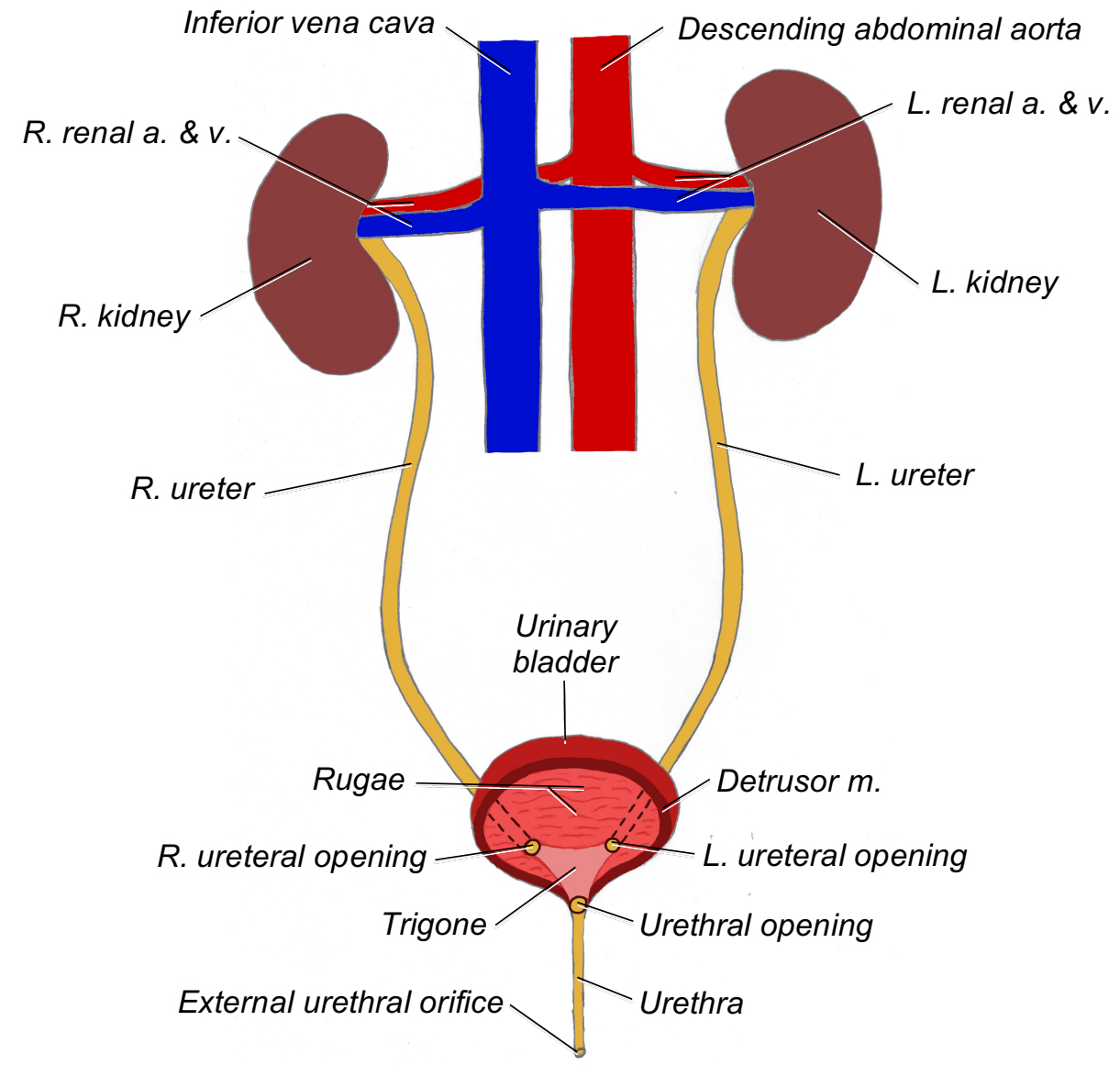

Figure 18.8. Diagram of the Urinary System

\section{Clinical Application: Urinary Tract Infection}

Urinary tract infections (or UTIs) are just that infections of the urinary tract caused by bacteria. Although the infection is typically localized to the lower urinary tract (urethra and bladder), more serious UTIs can affect the upper urinary tract (ureters and kidneys). UTIs are more common in women than men because the female urethra is shorter and the opening to the female urethra is closer to the anus and fecal bacteria. 
1) Using the terminology you've learned, where would you look to find the suprarenal gland? (LO50)

2) In order, list the structures that waste/urine flows through as it is filtered from the blood and expelled from the body. (LO52)

3) In order, list the vessels that blood flows through as it circulates through the kidney. (LO52)

4) How many ureteral openings are there in the urinary bladder? (LO51)

5) How many urethral openings are there in the urinary bladder? (LO51)

6) What structure is formed between the three openings in the urinary bladder? (LO51)

7) What does the term intra mean? (LO50)
8) What is the muscle that makes up the wall of the urinary bladder? (LO51)

9) What structures allow the urinary bladder to expand as it fills with urine? (LO51)

10)For each of the following structures, identify whether each of the following structures is intraperitoneal, retroperitoneal, or subperitoneal. (LO51)

Jejunum:

Esophagus:

Stomach:

Duodenum:

Ascending colon:

Transverse colon:

Descending colon:

Urinary bladder:

Pancreas:

11)What does the term convoluted mean? (LO50)

12)Which of the following is largest? (LO51)
a. Renal pelvis
b. Renal sinus
c. Minor calyces
d. Major calyces 


\section{Lab 19: Male and Female Reproductive Systems}

Instructions: Learn the listed terms using the listed learning objectives, labeled images, lab specimens, and other resources.

LO53: Describe the terminology used to identify structures of the male and female reproductive systems.

LO54: Identify the structures of the male and female reproductive systems.

LO55: Describe the flow pathways of sperm, eggs, and breast milk through the male and female reproductive systems.

\section{Male reproductive system}

$\square$ Testis (or Testicle)

$$
\text { - Tunica albuginea }
$$

The tunica albuginea is a tough, white, fibrous connective tissue that envelopes the testes, the male gonad. (tunic $=a$ membranous sheath enveloping an organ; $\underline{a l b}=$ white).

○ Seminiferous tubules

- Rete testis

The testes produce sperm, the male reproductive cells. Sperm are produced in the seminiferous tubules and collected by the rete testis $(\underline{\text { rete }}=$ net $)$.

$\square$ Epididymis
○ Head
- Body
○ Tail

Sperm travels from the rete testis to the head of the epididymis
Spermatic cord

- Vas deferens

The vas deferens transports sperm from the tail of the epididymis to the prostate gland.
- Gonadal a. (or Testicular a.)
○ Gonadal v. (or Testicular v.)

In order for proper sperm development, the testes must be cooler than core body temperature $\left(95^{\circ} \mathrm{F}\right.$ vs. $\left.98.6^{\circ} \mathrm{F}\right)$. The testes cool some just from being suspended away from the body, but the core temperature arterial blood from the gonadal a. must also be cooled before reaching the testis capillary beds. As blood is cooled in the peripheral capillary beds of the scrotal walls, it collects into a network of gonadal vv. called the pampiniform plexus $($ Pampiniform $=$ shaped like a vine or tendril). As it ascends to the abdomen, this venous plexus wraps around the gonadal a., cooling the arterial blood to sufficient temperature.

\section{Cremaster muscle}

The cremaster muscle is a very thin muscle that surrounds the spermatic cord and testes. Its function (action) is to elevate the testes to warm them when the testes become too cold for optimal sperm production and development.

\section{Scrotum}

The scrotum is the skin sack that contains and suspends the testes. This location allows the testes to be cooler than core body temperature, promoting optimal sperm production and development. 


\section{Dartos muscle}

The dartos muscle is a very thin muscle in the walls of the scrotum. Its function is to elevate the scrotum to warm it when the testes are too cold for optimal sperm production and development.

\section{Seminal vesicles (or Seminal glands)}

The seminal vesicles are two tubular glands that produce fluid that mixes with sperm in the vas deferens just before the prostate gland. Fluid from the seminal vesicles contributes the majority $(\sim 70 \%)$ of the volume of semen (semen $=$ the mixture of sperm and supporting fluids). Fluid from the seminal vesicles contains a lot of fructose, a sugar that is used by sperm as their main energy source.

\section{Prostate gland}

The prostate gland contributes $\sim 26 \%$ to the volume of semen. The prostate gland secretes fluid that stabilizes sperm and protects them from the acidic environment of the vagina. The sperm flow through channels in the prostate gland known as the ejaculatory ducts and the prostatic urethra.

$\square$ Ejaculatory ducts

$\square$ Prostatic urethra

Membranous urethra

The membranous urethra is the short segment of urethra that passes through the floor of the pelvis.

$\square$ Shaft of penis

$\square$ Spongy urethra

Bulbourethral glands (or Cowper's glands)

The bulbourethral glands produce $\sim 1 \%$ of the total volume of semen. They produce a thick mucus that cleans and neutralizes the urethra of residual urine and increases the mobility of sperm in the vagina.
The remaining $\sim 3 \%$ of semen volume is made up of sperm.

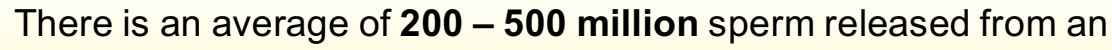
ejaculation (ejaculation $=$ the discharge of semen from the male reproductive tract).

\section{Corpora cavernosa}

The corpora cavernosa are two of the three bodies of erectile tissue bodies in the penis. They engorge with blood to create an erection - a physiological and anatomical condition in which the penis becomes enlarged and firmer (Corpora cavernosa $=$ cavernous bodies) .

\section{Corpus spongiosum}

The corpus spongiosum surrounds the spongy urethra. Its job is to prevent the urethra from being pinched shut during an erection.

\section{Glans penis}

The glans penis is the highly sensitive bulbous structure at the end of the corpus spongiosum and tip of the penis. It contains the external urethral orifice and is covered by the prepuce in uncircumcised males.

External urethral orifice

Prepuce (or foreskin)

\section{Clinical Application: Circumcision}

Males are born with foreskin that surrounds their glans penis. Circumcision is a common procedure for removing the foreskin. Although many parents choose to have their children circumcised for hygienic and preventative care reasons, some families do so based on religious grounds or cultural traditions. Although circumcision is possible at any age, it is most commonly performed on newborns. 


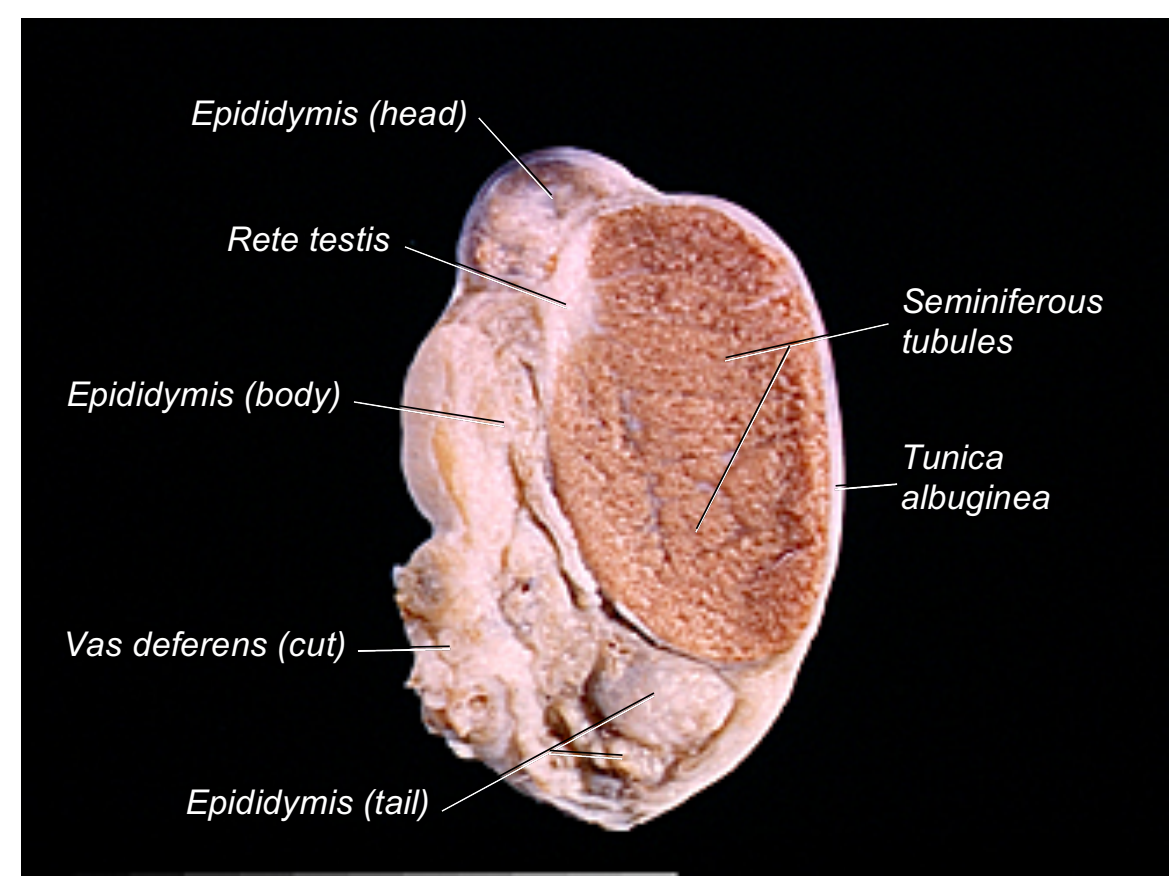

Figure 19.1. Hemisected Testis, Sagittal View

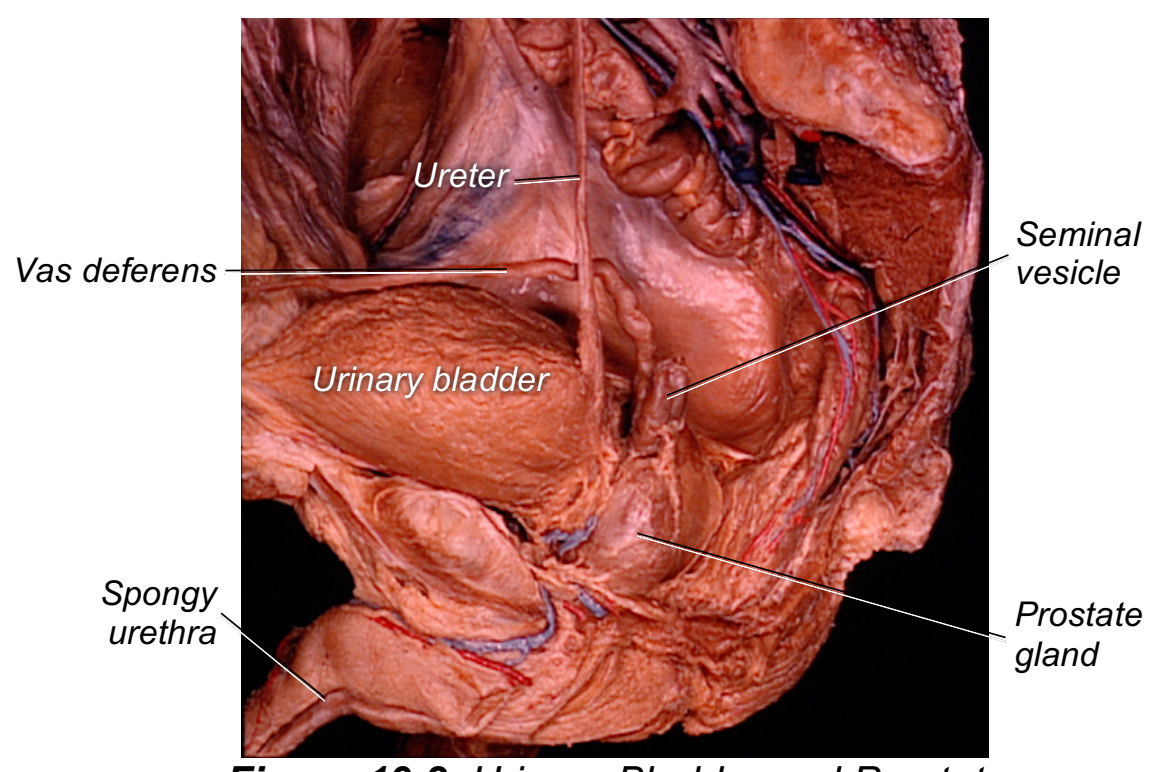

Figure 19.2. Urinary Bladder and Prostate Gland in a Hemisected Pelvis, Sagittal View

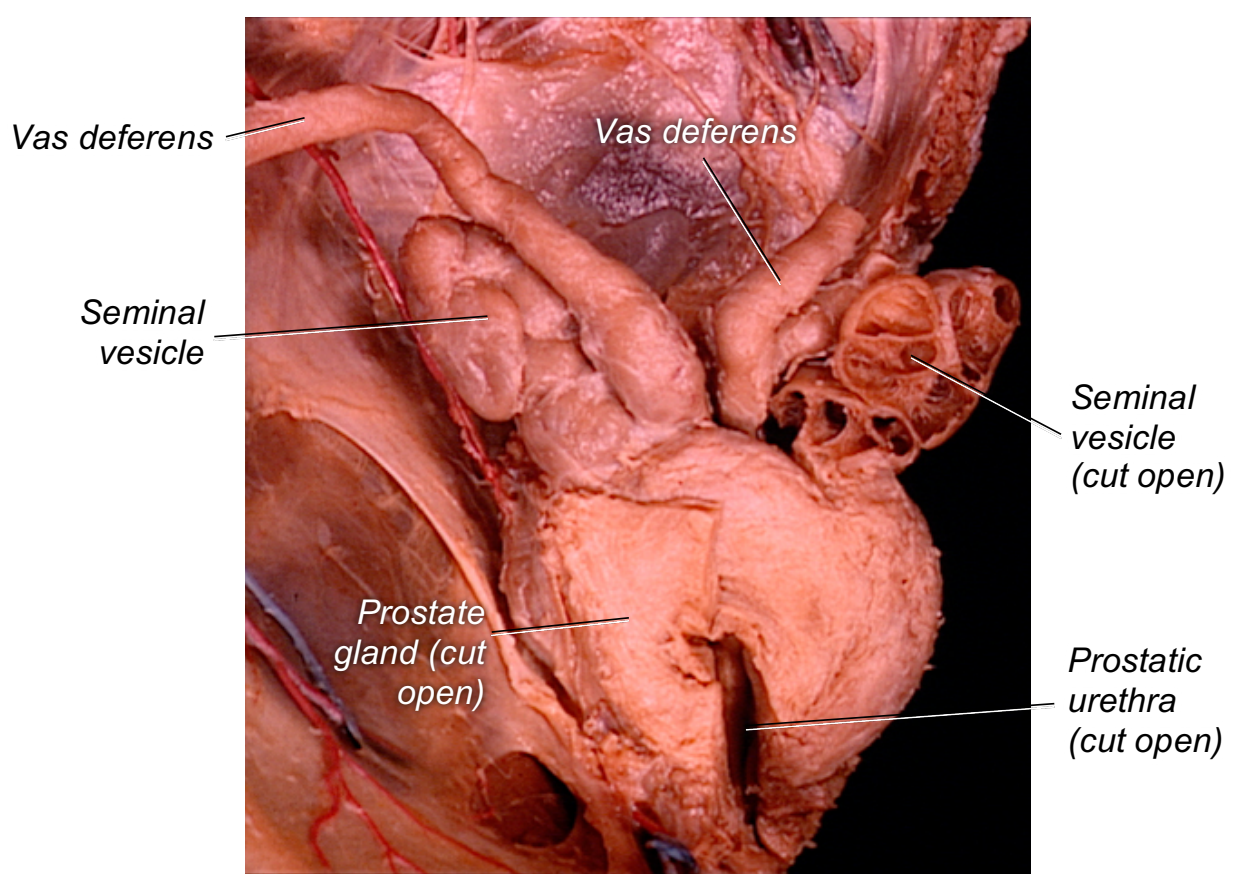

Figure 19.3. Vas Deferens, Seminal Vesicles, and Prostate Gland, Anterosuperior View

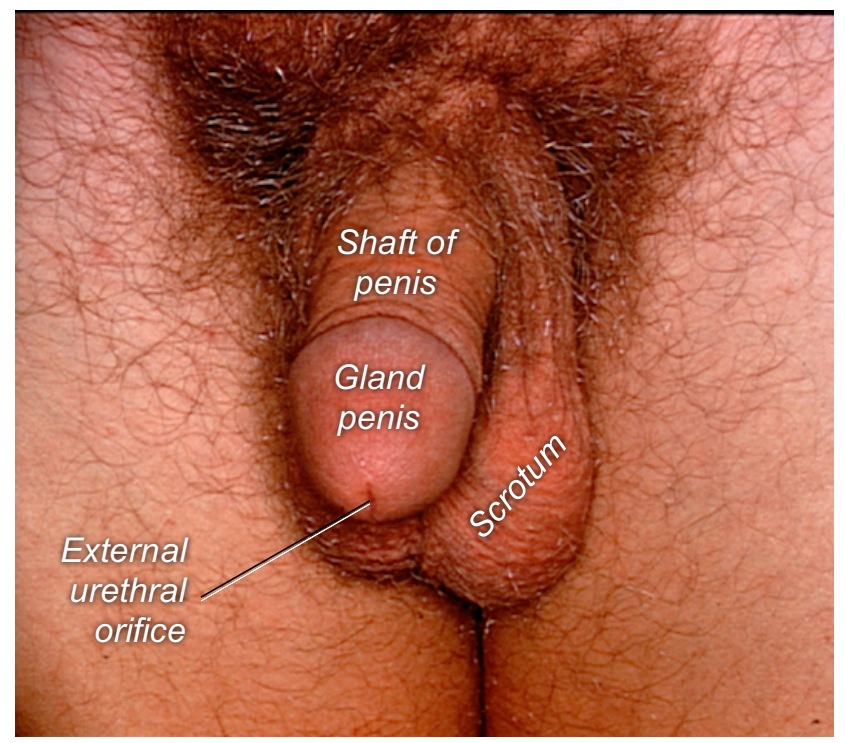

Figure 19.4. Male External Genitalia, Anterior View 


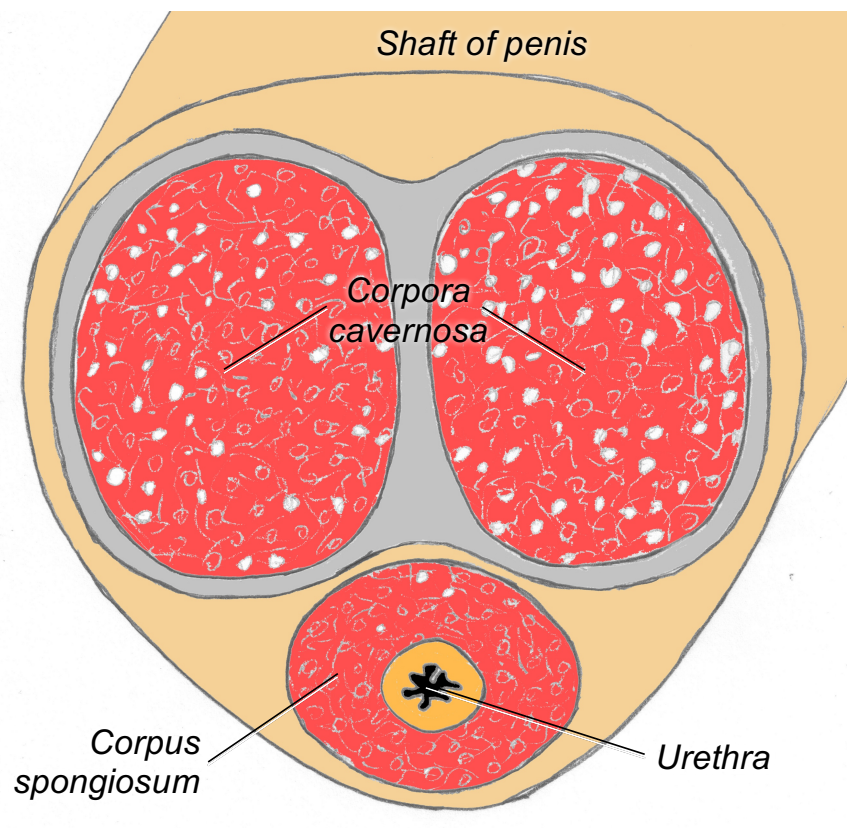

Figure 19.5. Diagram of Trans-sected Penis, Anterior View

\section{Clinical Application: Sexually Transmitted Diseases (STDs)}

Sexually transmitted diseases (STDs), or sexually transmitted infections (STIs), are infections that are transmitted during vaginal, anal, or oral sex. Human papillomavirus (HPV), chlamydia, genital herpes, HIVIAIDS, gonorrhea, and syphilis are some examples of STDs. Symptoms of STDs can include warts, genital pain, genital sores, infertility, and an affected immune system, among others. While some STDs are preventable with vaccinations, contractibility is still possible and there is currently no known cure.

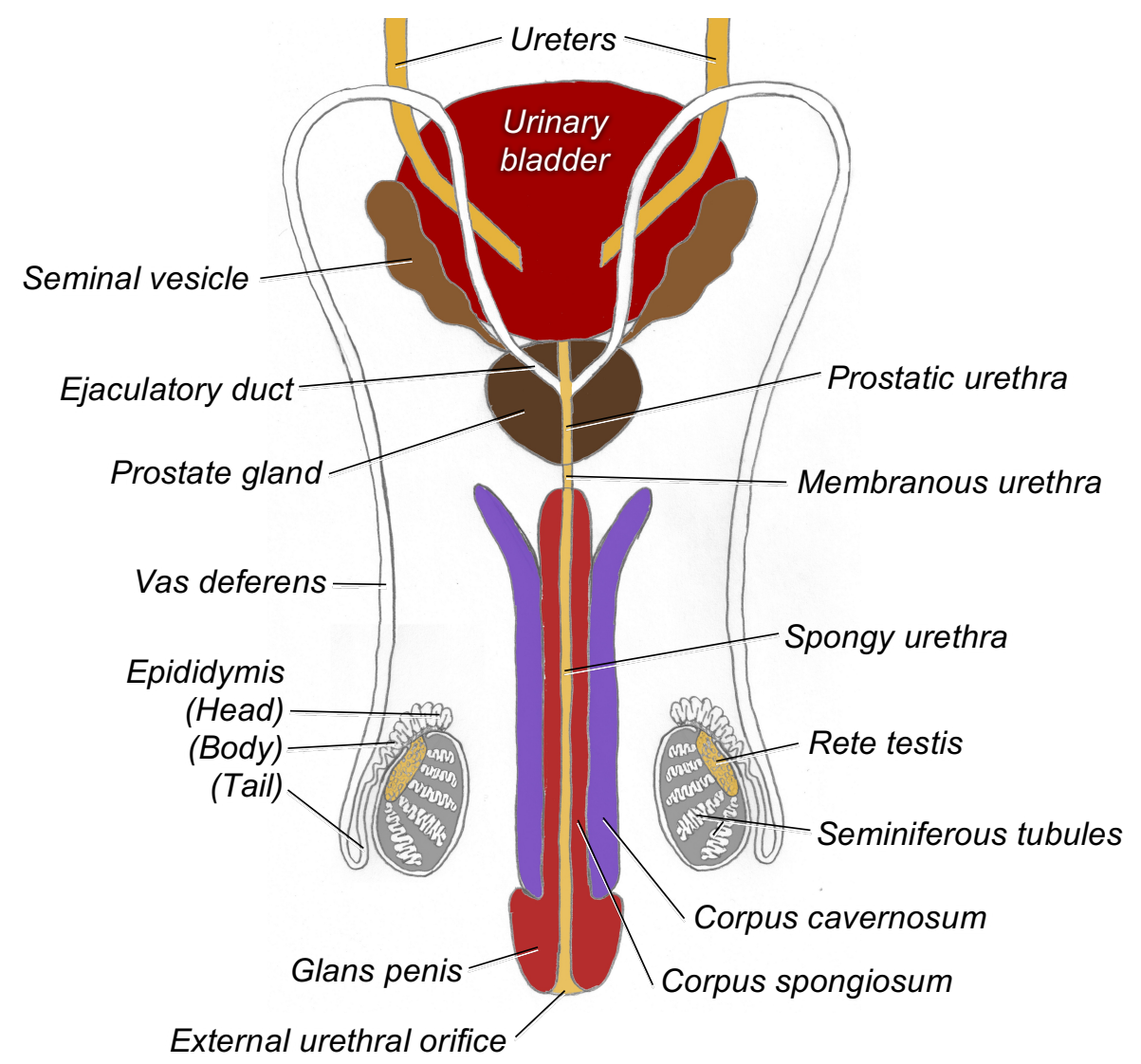

Figure 19.6. Diagram of Male Reproductive Tract

\section{Clinical Application: Vasectomy}

A vasectomy is a surgical procedure to sterilize men. In the procedure, the vas deferens is cut and tied off, preventing sperm from traveling to the urethra and out of the body. Although the flow of sperm is inhibited, ejaculation is still possible; the male sexual response and seminal fluids produced by the seminal vesicles, prostate gland, and bulbourethral glands are unaffected. Although it can be an expensive procedure, a vasectomy is typically reversible. 


\section{Female reproductive system}

$\square$ Breast

- Mammary glands

Mammary glands produce milk for newborn babies.

- Lactiferous ducts

- Lactiferous sinus

○ Nipple

- Areola

○ Suspensory ligaments

Ovaries

Ovaries are the female gonads that contain eggs, the female reproductive cell. Unlike the male testes' ability to continually produce sperm, ovaries contain all the eggs a female will ever have at birth.

$\square$ Tunica albuginea

Like the male testes, the female ovaries are also enveloped in a tunica albuginea.

$\square$ Gonadal a. (or Ovarian a.)

$\square$ Gonadal v. (or Ovarian v.)

Suspensory ligament

This ligament is actually a fold of the peritoneum that "suspends" the ovary from the wall of the pelvis and contains the ovarian a. and v..

\section{Ovarian ligament (or Utero-ovarian ligament)}

This ligament attaches the ovary to the lateral side of the uterus.

\section{Fallopian tubes (or Uterine tubes)}

The fallopian tubes provide a transport eggs released by ovaries to the uterus.

\section{- Fimbriae}

These finger-like projections sweep the released egg into the infundibulum of the fallopian tube.

○ Infundibulum

○ Ampulla

Fertilization (the joining of a sperm and egg) usually occurs in the ampulla.

\section{- Isthmus}

\section{Uterus}

○ Fundus

- Body

\section{Clinical Application: Hysterectomy}

A hysterectomy is a surgical procedure to remove the uterus. A physician may recommend a hysterectomy to a female if she is facing uterine fibroids, a prolapsing uterus, endometriosis, cancer of the uterus, cervix, or ovaries, or other issues. Depending on the issue, the cervix may be removed with the uterus or left in place. A bilateral oophorectomy and/or salpingectomy may also need to be performed if the ovaries and/or fallopian tubes also require removal. 


\section{Broad ligament}

This ligament is another fold of peritoneum provides stabilization to the uterus, fallopian tubes, and ovaries by attaching them to the pelvis.

$\square$ Cervix
- Internal os
- Cervical canal
○ External os

After an egg has traveled through the uterus, internal os (os $=$ opening), cervix, and external os, it enters the vagina, an elastic, muscular tube that will expel the egg from the body.

\section{Vagina}

$$
\text { ○ Rugae }
$$

Vaginal rugae allows for expansion of the vagina during intercourse and childbirth.

Vaginal orifice

Glans clitoris

The glans clitoris is a highly sensitive bulbous erectile structure. It is covered by the prepuce (or Clitoral hood).

$\square$ Prepuce (or Clitoral hood)

$\square$ Urethra

$\square$ External urethral orifice

$\square$ Labia majora

$\square$ Labia minora

Do you remember what labi means?

\section{Mons pubis}

The mons pubis is a fat pad that lies over the joint of the pubic bones. It is typically more prominent in females.

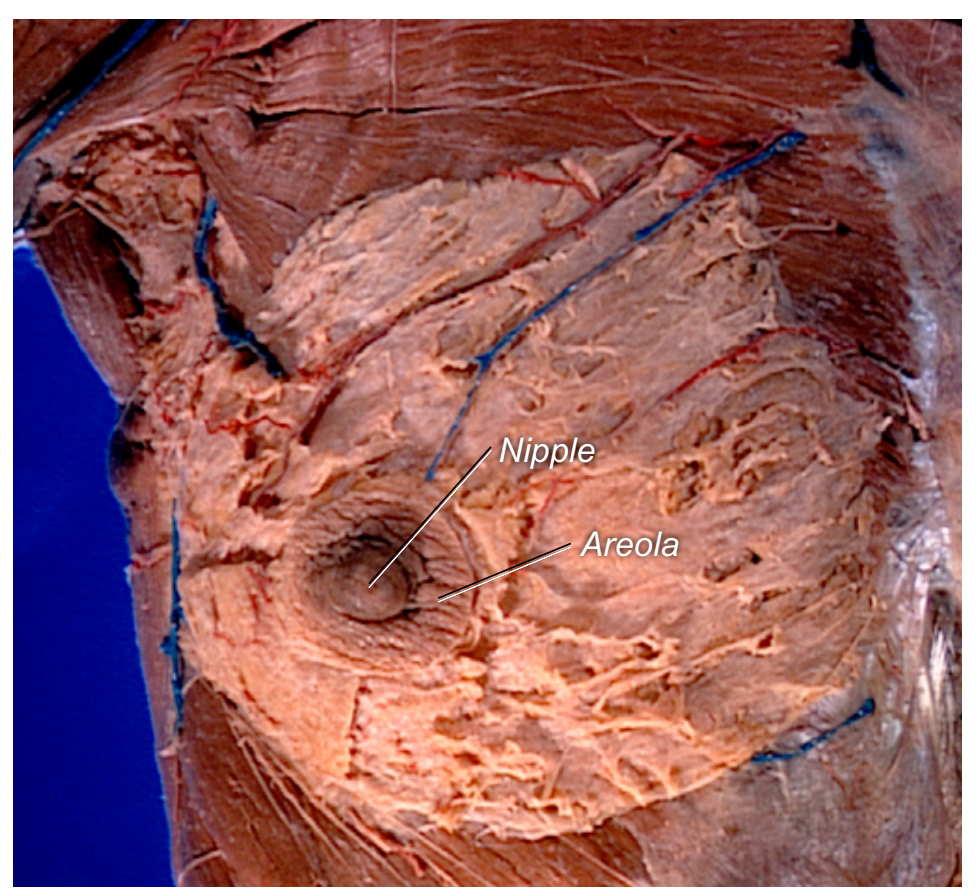

Figure 19.7. Female Breast, Anterior View

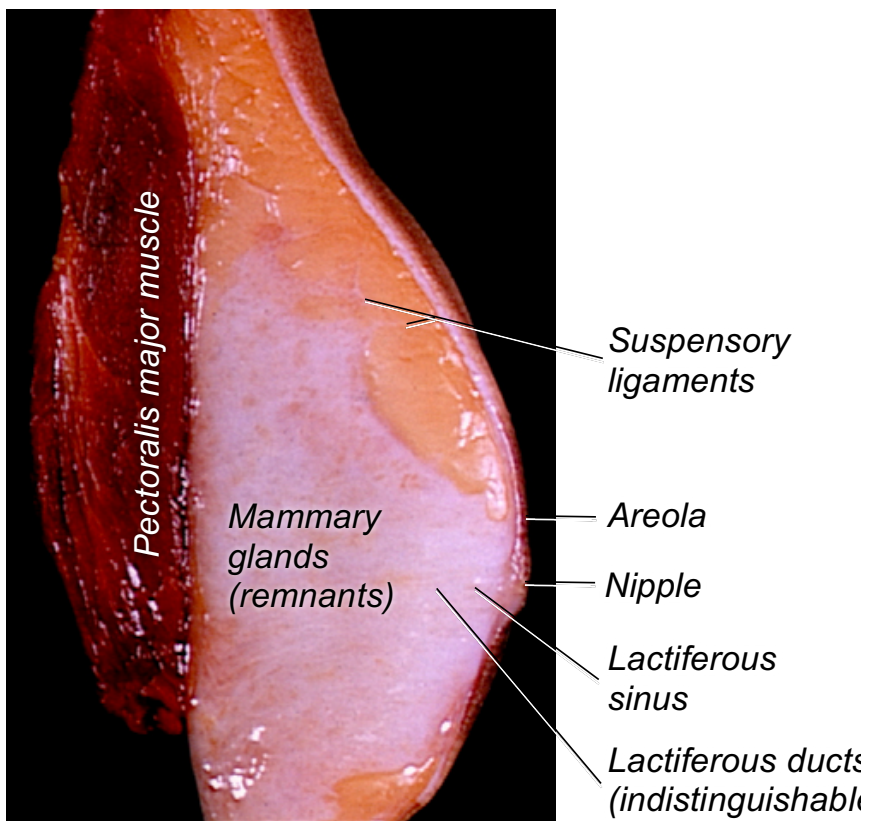

Figure 19.8. Female Breast (Hemisected), Sagittal View 


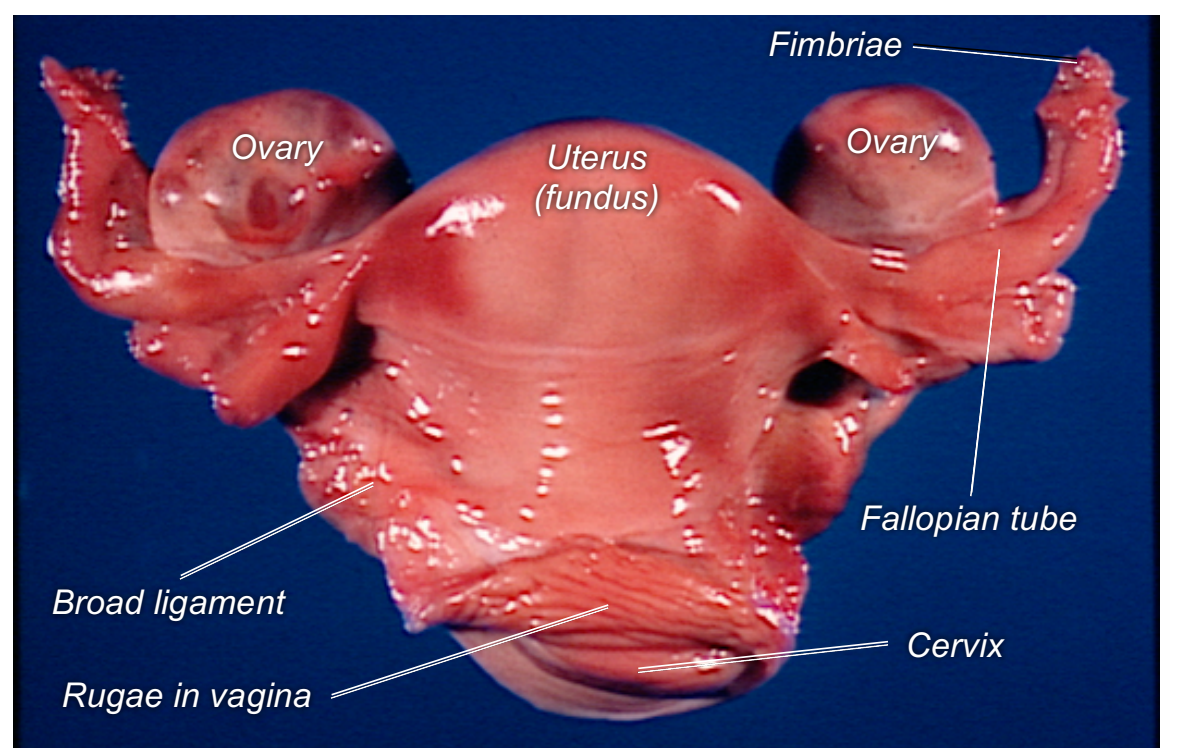

Figure 19.9. Uterus and Ovaries, Anterior View

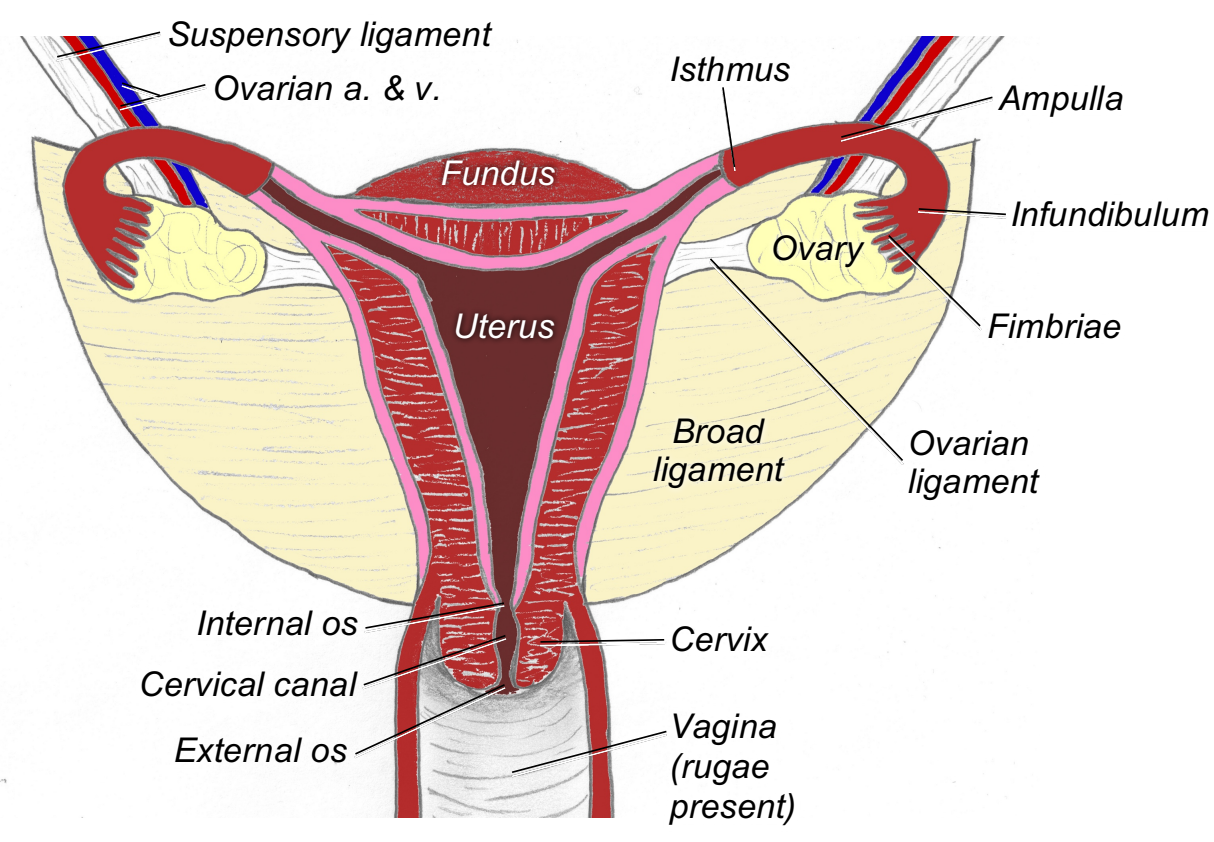

Figure 19.10. Diagram of Female Reproductive Tract

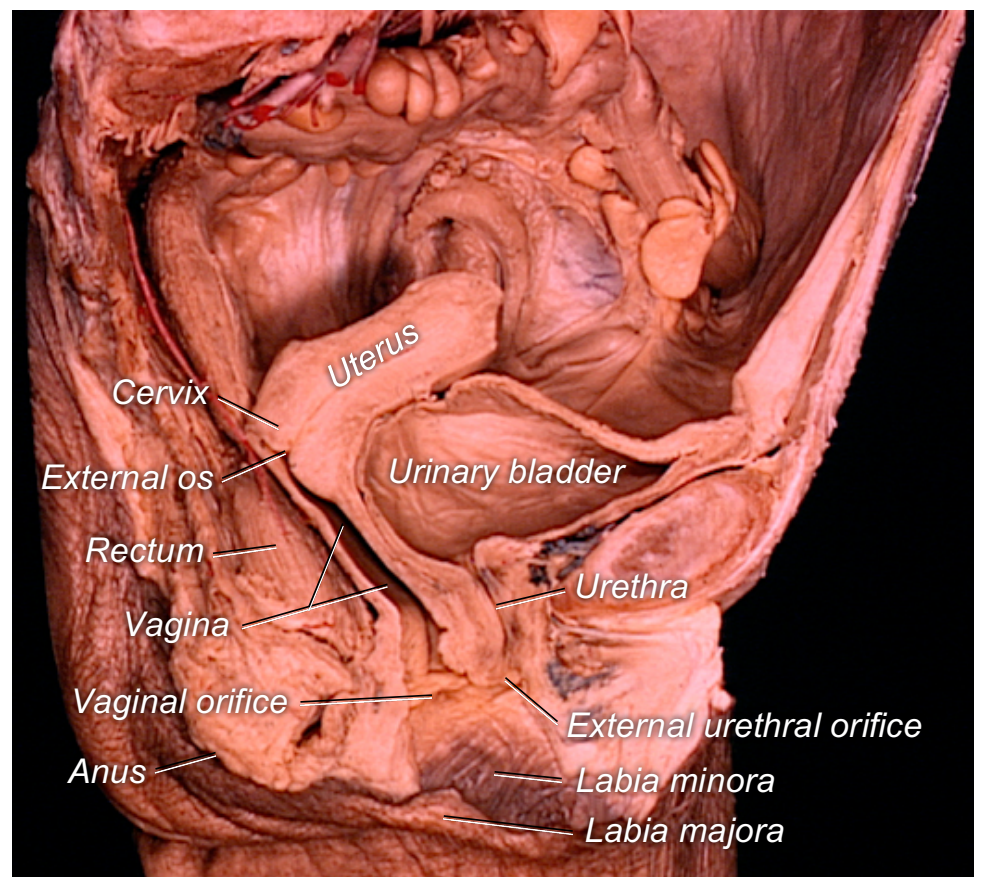

Figure 19.11. Female Hemisected Pelvis, Midsagittal View

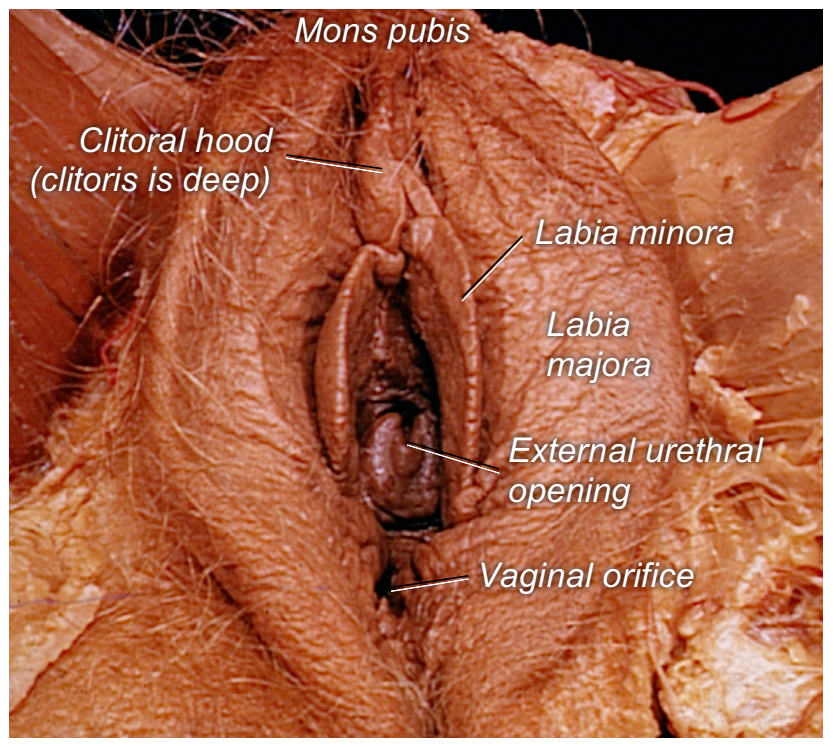

Figure 19.12. Female External Genitalia, Inferior View 
As a zygote develops anatomical structures, the male and female reproductive structures develop from the same (homologous) regions and tissues. For example, males and females each have gonads (testes and ovaries, respectively), and each is enveloped by the tunica albuginea. This is not by coincidence. Below is a table of some more homologous structures.

\section{Homologous structures}

\begin{tabular}{|l|l|}
\hline Female structure & Male structure \\
\hline Ovary & Testis \\
\hline Labia majora & Scrotum \\
\hline Labia minora & Skin on shaft of penis \\
\hline Prepuce (or Clitoral hood) & Prepuce (or Foreskin) \\
\hline Glans clitoris & Glans penis \\
\hline
\end{tabular}

\section{Clinical Application: Menstruation}

Eggs are released from ovaries with the intent for possible fertilization. If a released egg isn't fertilized, the egg will be flushed out along with the inner lining of the uterus. This process is called menstruation and occurs about every 28 days. At the beginning of the menstrual cycle, the inner lining of the uterus sheds if the previously released egg was not fertilized by sperm and implanted to the endometrium. The shedding of endometrium causes vaginal bleeding and is commonly referred to as the "period." After shedding, the endometrium regrows and thickens in preparation for the next egg to be fertilized and implanted. This cycle of menstruation is controlled by luteinizing hormone (LH) and folliclestimulating hormone (FSH) which are produced by the pituitary gland.

\section{Clinical Application: Menopause}

Although females are born with 1-2 million eggs in their ovaries, only about $400-500$ of them will be released for fertilization in their lifetime, and they cannot produce any more eggs. Before puberty, about 11,000 eggs die in the ovaries every month. After puberty, the female body releases an egg every menstrual cycle, but during that process thousands more eggs die off. After the ovaries "run out" of viable eggs to release (typically between the ages of 48-55 years) the body goes through a natural process called menopause. During this process, the ovaries stop releasing eggs, menstruation stops, and levels of sex hormones (mainly estrogen and progesterone) decline. Other common symptoms include vaginal dryness, hot flashes, and mood swings. Since menopause is a natural process, treatment focuses mainly on symptomatic relief.

\section{Clinical Application: Fertilization, Implantation, Pregnancy, and Childbirth}

A zygote is formed when an egg is fertilized by a sperm from intercourse and ejaculation or artificial insemination. Once the zygote divides, it becomes an embryo. Once the embryo divides into a 16-cell spherical mass, it is called a morula. Once the morula grows from more cell division and develops a center cavity, it is called a blastocyst. If the blastocyst implants itself to the inner uterine wall (endometrium), pregnancy will result. The blastocyst continues to grow and becomes a fetus at week 8 of pregnancy onward. Childbirth typically occurs between 38-40 weeks of pregnancy. Once delivered, the baby is called a newborn, neonate, or infant. 
1) In order, list the structures that sperm flow through on its way to exiting the body. (LO55)

2) In order, list the structures that eggs flow through on its way to exiting the body. (LO55)

3) In order, list the structures that breast milk flows through on its way to exiting the body. (LO55)

4) What structures contribute to optimal temperature for sperm production? (LO54)

5) What is the female homologous structure to the male scrotum? (LO54)

6) What is the name for the discharge of semen from the male reproductive tract? (LO53)
7) What ligaments stabilize and support the ovaries? (LO54)

8) What does the term alb mean? (LO53)

9) True or False: the male urinary tract intercedes with the male reproductive tract. (LO54)

10)True or False: the female urinary tract intercedes with the female reproductive tract. (LO54)

11)What is the function of rugae in vagina? (LO54)

12)True or False: both males and females have a tunica albuginea surrounding their gonads. (LO54)

13)From outermost to innermost, what are the three layers of the uterus? (LO54)

14)What ligament connects the ovary to the uterus? (LO54)

15)What does the term labi mean? (LO53)

16)What is semen? (LO54) 


\section{Appendix A: Answers to Self-Assessment Question}

\section{Introductory Material}

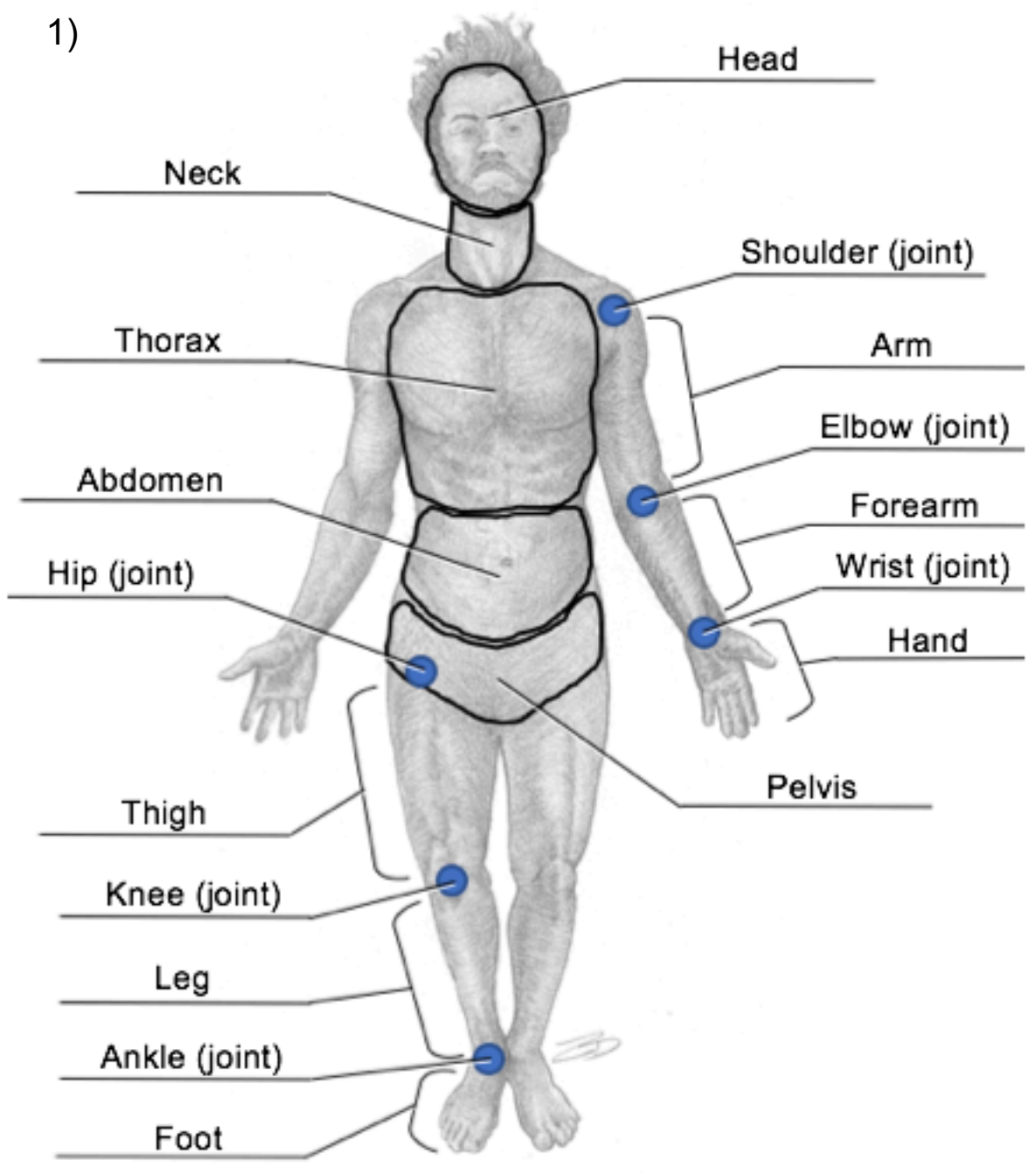

2) Anatomical position.

3) Prone.

4) Away from the midline.

5) Superior.

6) False.

7) True.

8) Coronal suture.

9) Supine = lying face up; prone = lying face down 10)See labeled images on next page.

Anatomical Position,

Anterior View 
Superior

(side/direction)

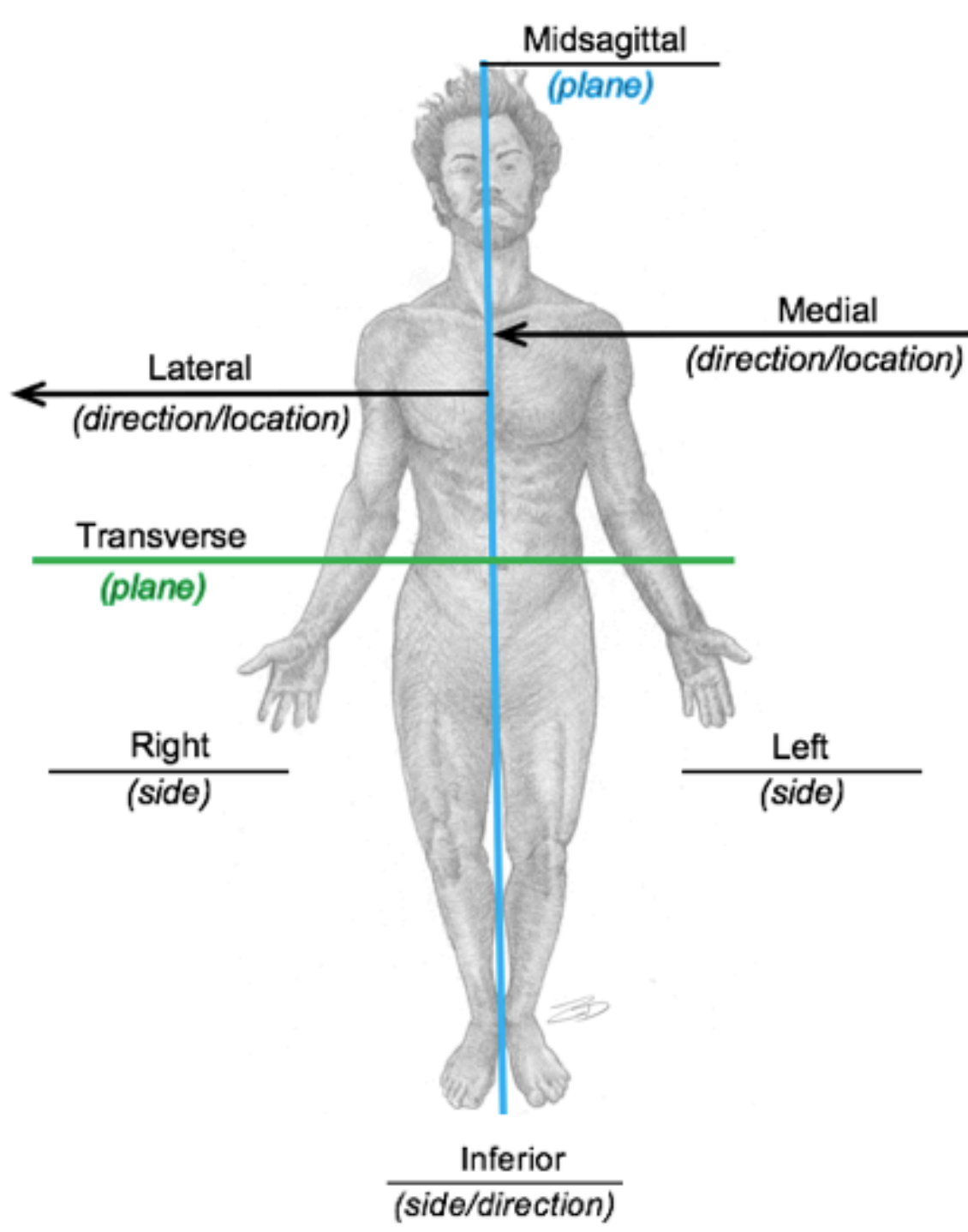

Anatomical Position, Anterior View
Superior

$\overline{\text { (side/direction) }}$

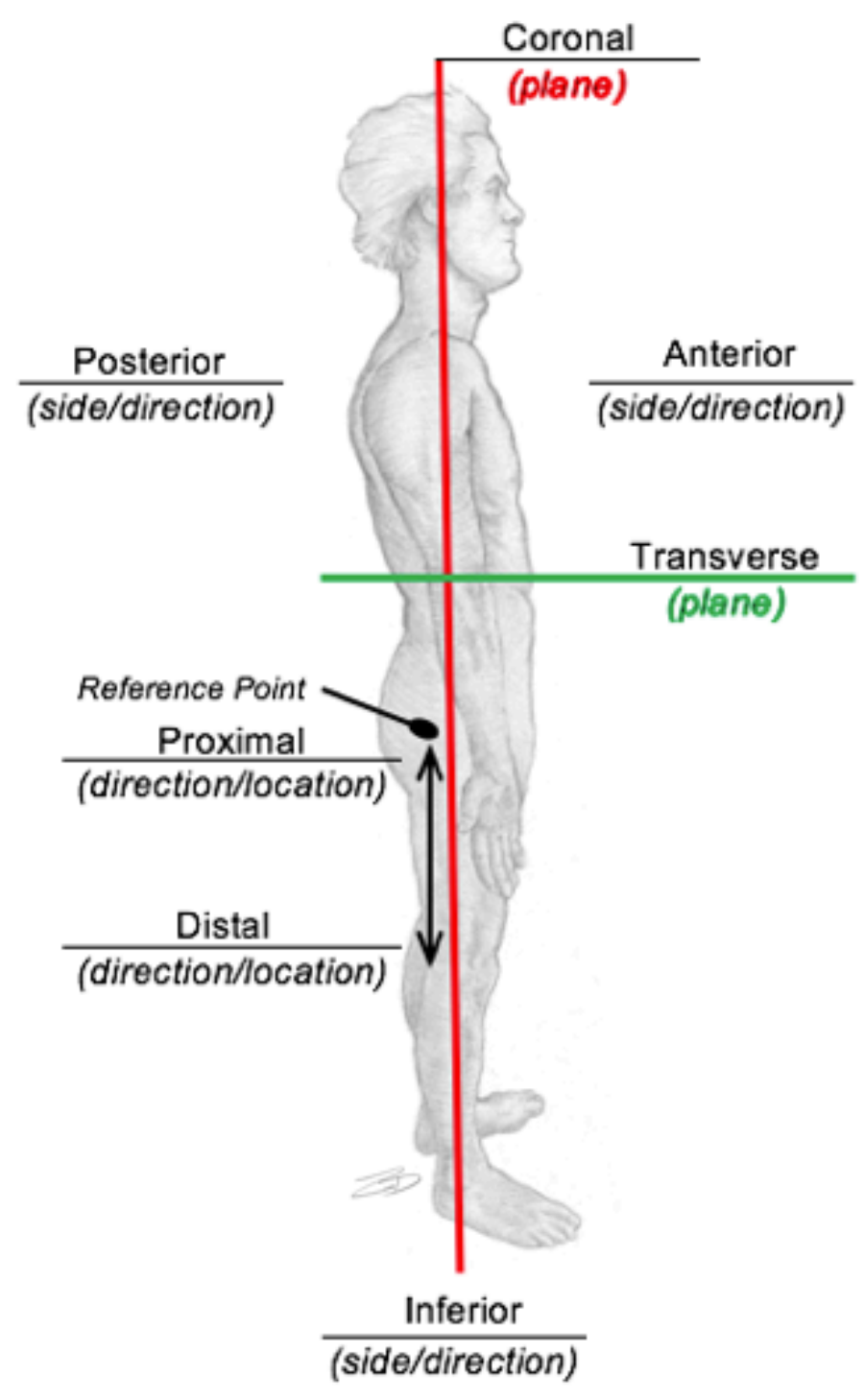

Anatomical Position, Right Lateral View 
Lab 1: Axial Skeleton (Head)

1) Shallow depression.

2) An opening (or hole) below the orbit of the eye.

3) Dense; rock-like.

4) A: Frontal bone; B: Nasal bone; C: Sphenoid Bone; D: Zygomatic bone; E: Maxilla bone; F: Mandible bone.

5) Cribriform plate.

6) Temporal bone.

7) A: Lambdoid suture; B: External acoustic meatus; C: Coronoid process; D: Mental foramen; E: Occipital condyle; F: External occipital protuberance.

\section{Back to Questions}

\section{Lab 2: Axial Skeleton (Neck, Thorax, \& Abdomen)}

1) Rib.

2) Sword.

3) Transverse costal facets.

4) Rib pairs 1-7 (14 ribs).

5) Two pedicles and two laminae.

6) Superior-most = external occipital protuberance; inferiormost $=$ spinous process of vertebra prominens $(\mathrm{C} 7$ vertebra).

7) A: Spinous process; B: Lamina; C: Transverse process; D: Pedicle; E: Vertebral foramen; F: Body

8) Cervical: E, G, L, N, O, P, T, V Thoracic: A, H, K, M, R, W Lumbar: C, I, Q, S, Z Sacral: $\mathbf{B}, \mathbf{F}, \mathbf{J}, \mathbf{U}, \mathbf{X}$ Coccygeal: $\mathbf{D}, \mathbf{Y}$

Back to Questions
Lab 3: Appendicular Skeleton (Pectoral Girdle \& Upper Limb)

1) Forearm.

2) Infraspinous fossa.

3) False.

4) Ulnar carpal collateral ligament.

5) 32.

6) $1^{\text {st }}$ proximal phalanx.

7) $2^{\text {nd }}$ middle phalanx.

8) Between the greater and lesser tubercles on the humerus.

9) (a) Clavicle; (b) Scapula; (c) Humerus.

10) On the side.

11) (a) Head of humerus; (b) Ulnar notch; (c) Trochlea; (d) Sternal end of clavicle; (e) Olecranon process; (f) Head of radius; (g) Head of radius; (h) Coronoid fossa; (i) Acromial end of clavicle.

12)The head of the radius is located closer to the ELBOW but the head of the ulna is located closer to the WRIST.

Back to Questions

\section{Lab 4: Appendicular Skeleton (Pelvic Girdle \& Lower Limb)}

1) False.

2) (a) ilium and femur; (b) ischium and femur; (c) pubis and femur.

3) Digits in the hand are numbered LATERAL to MEDIAL. Digits in the foot are numbered MEDIAL to LATERAL.

4) Leg.

5) Small pit.

6) (a) acetabulum; (b) medial condyle of femur; (c) lateral condyle of femur; (d) auricular surface of sacrum; (e) medial and lateral articular surfaces of patella.

7) Branch.

8) 30 . 


\section{Lab 5: Axial Muscles}

1) B. Proximal attachment.

2) Depressor anguli oris.

3) (a) uniarticulate; (b) biarticulate; (c) multiarticulate.

4) Zygomatic arch.

5) Nasal bone.

6) Elevates the upper lip.

7) Chewing.

8) When a muscle receives innervation, it causes contraction of the muscle which, in turn, causes an action.

9) $\overline{\text { Antagonists. }}$

10) (A) temporalis; (B) sternocleidomastoid.

Back to Questions

\section{Lab 7: Appendicular Muscles (Pelvic Girdle \& Lower Limb)}

1) D. It attaches to the anterior superior iliac spine.

2) Extends the knee.

3) Ischial tuberosity.

4) Two (bi) headed (ceps) muscle of the thigh (femoris).

5) Fascia lata.

6) Head of fibula.

7) Extensor hallucis brevis.

8) Turning outward.

9) (A) tensor fasciae latae; (B) pectineus; (C) sartorius; (D) adductor; (E) gracilis; (F) rectus femoris; (G) vastus lateralis; $(\mathbf{H})$ vastus medialis.

Back to Questions

\section{Lab 6: Appendicular Muscles (Pectoral Girdle \& Upper}

\section{$\underline{\text { Limb) }}$}

1) Levator scapulae would elevate the scapula.

Extensor digitorum would extend the digits.

Rhomboid major would adduct (retract) the scapula.

Brachioradialis would flex the elbow.

2) Pronator teres.

3) Supinator and biceps brachii.

4) B. Extension.

5) Flex wrist and adduct hand.

6) Supraspinous fossa.

7) Deltoid tuberosity.

8) Coracoid process.

9) Ulnar tuberosity.

10)Extensor carpi ulnaris OR flexor carpi radialis.

11)(A) levator scapulae; (B) supraspinatus; (C) Infraspinatus; (D) teres minor; (E) triceps brachii long head; $(\mathbf{F})$ teres major; $(\mathbf{G})$ serratus anterior; $(\mathbf{H})$ triceps brachii lateral head 


\section{Lab 8: Spinal Cord and Peripheral Nerves}

1)

\begin{tabular}{|l|c|c|}
\cline { 2 - 3 } \multicolumn{1}{c|}{} & \# Vertebrae & $\begin{array}{c}\text { \# Spinal } \\
\text { nerve pairs }\end{array}$ \\
\hline Cervical & 7 & 8 \\
\hline Thoracic & 12 & 12 \\
\hline Lumbar & 5 & 5 \\
\hline Sacral & 5 & 5 \\
\hline Coccygeal & 4 & 1 \\
\hline
\end{tabular}

2) $\mathrm{C} 7 \mathrm{n}$.

3) f. Parasympathetic nervous system.

4) A ganglion is a cluster of nerve cell bodies, whereas a plexus is a network of nerves.

5) Sacral hiatus.

6) Branch.

7) Dendrites.

8) Tender

9) The filum terminale connects the distal end of the conus medullaris to the proximal end of the coccyx.

10)Femoral nerve.

11)All three posterior divisions (one from each of the trunks).

12)Suboccipital nerve (the nerve below the occipital bone).

13) Stimulus (hot surface) $\rightarrow$ PNS sensory receptors $\rightarrow$ dorsal ramus $\rightarrow$ spinal nerve $\rightarrow$ dorsal root $\rightarrow$ dorsal root ganglion $\rightarrow$ dorsal rootlets $\rightarrow$ spinal cord $\rightarrow$ ventral rootlets $\rightarrow$ ventral root $\rightarrow$ spinal nerve $\rightarrow$ ventral ramus $\rightarrow$ motor innervation through PNS/somatic nervous to muscles that flex forearm. NOTE: If you wanted to be very detailed in your answer, you could also include components of the brachial plexus and named nerve branches and muscles, and/or you could also include motor innervation responses from the PNS/autonomic/sympathetic nervous systems.

\section{Lab 9: Brain}

1) A ventricle is a cerebrospinal fluid-filled cavity in the brain, whereas a vesicle is a region of the brain.

2) Diencephalon.

3) Hypophyseal fossa (or sella turcica).

4) Choroid plexus.

5) Four.

6) Cerebral aqueduct (or mesencephalic aqueduct)

7) Corpus callosum, interthalamic adhesion, anterior commissure, and posterior commissure.

8) Below.

9) Superior sagittal sinus and straight sinus.

10)Metencephalon.

11)(A) pineal gland; (B) posterior commissure; (C) fornix; (D) corpus callosum; (E) cerebral aqueduct; (F) mammillary body; (G) $4^{\text {th }}$ ventricle; (H) pons; (I) medulla oblongata.

12)Choroid plexuses in lateral ventricles $\rightarrow$ lateral ventricles $\rightarrow$ interventricular foramina $\rightarrow 3^{\text {rd }}$ ventricle (where another choroid plexus is producing more CSF) $\rightarrow$ cerebral aqueduct $\rightarrow 4^{\text {th }}$ ventricle (where another choroid plexus is producing more CSF) $\rightarrow$ medial aperture OR lateral apertures OR central canal of spinal cord $\rightarrow$ subarachnoid space $\rightarrow$ arachnoid granulations $\rightarrow$ superior sagittal sinus (circulated CSF now mixes with venous blood) $\rightarrow$ confluence of sinuses $\rightarrow$ transverse sinuses $\rightarrow$ sigmoid sinuses $\rightarrow$ jugular foramina.

Note: Because arachnoid granulations are on EACH dural sinus, your flow of CSF as it enters the sinuses may differ.

\section{Back to Questions}

Back to Questions 


\section{Lab 10: Cranial Nerves}

1) C. Touch.

2) 12 pairs.

3) CN V3 (Mandibular n.)

4) CN V1 (Ophthalmic n.), CN V2 (Maxillary n.), and CN V3 (Mandibular $\mathrm{n}$.).

5) CN X (Vagus n.)

6) False.

7) CN VII (Facial n.), CN IX (Glossopharyngeal n.), and CN $\mathrm{X}$ (Vagus $\mathrm{n}$.).

8) CN V3 (Mandibular n.)

9) CN VII (Facial n.)

10) CN IX (Glossopharyngeal n.), $C N X$ (Vagus n.), and CN XI (Accessory n.).

11)Tongue.

12) The sense of smell.

13) CN III (Oculomotor n.), CN IV (Trochlear n.), and CN VI (Abducens n.).

14)(A) CN I (Olfactory n. tract); (B) CN II (Optic n.); (C) CN V2 (Maxillary n.); (D) CN VI (Abducens n.); (E) CN XII (Hypoglossal n.); (F) CN VIII (Vestibulocochlear n.).

Back to Questions

\section{Lab 11: Special Senses}

1) Straight.

2) Slanting.

3) Cornea $\rightarrow$ anterior cavity (anterior chamber) filled with aqueous humor $\rightarrow$ pupil $\rightarrow$ anterior cavity (posterior chamber) filled with aqueous humor $\rightarrow$ lens $\rightarrow$ posterior cavity filled with vitreous humor $\rightarrow$ retina $\rightarrow$ optic nerve $\rightarrow$ to brain for processing and interpretation into sight.

4) Sound waves collected by the auricle $\rightarrow$ external acoustic meatus $\rightarrow$ external auditory canal $\rightarrow$ tympanic membrane $\rightarrow$ malleus $\rightarrow$ incus $\rightarrow$ stapes $\rightarrow$ oval window $\rightarrow$ cochlea $\rightarrow$ vestibulocochlear nerve $\rightarrow$ to brain for processing and interpretation into sound.

5) Lacrimal gland $\rightarrow$ lacrimal ducts $\rightarrow$ across the eye (sclera, cornea, and conjunctiva) $\rightarrow$ collects in front of the caruncle $\rightarrow$ superior/inferior lacrimal puncta $\rightarrow$ superior/inferior canaliculi $\rightarrow$ lacrimal sac $\rightarrow$ nasolacrimal duct $\rightarrow$ opening of nasolacrimal duct $\rightarrow$ inferior meatus $\rightarrow$ nasal cavity.

6) The LEFT lateral rectus muscle is being innervated by the LEFT CN VI (Abducens n.) and the RIGHT medial rectus is being innervated by the RIGHT CN III (Oculomotor $n$.).

7) The LENS separates the anterior and posterior cavities, whereas the IRIS separates the anterior and posterior chambers.

8) Inferior and lateral rotation of the eye.

9) CN IX (Glossopharyngeal n.).

10)The pharyngeal orifice of the pharyngotympanic tube empties into the INFERIOR MEATUS (in the nasal cavity).

11)(A) Cornea; (B) iris; (C) ciliary body; (D) lens; (E) retina; (F) choroid; (G) sclera; (H) optic disc; (I) CN II (Optic n.).

Back to Questions 


\section{Lab 12: Heart}

1) Openings of R. atrium $\rightarrow$ R. atrium $\rightarrow$ tricuspid valve $\rightarrow$ R. ventricle $\rightarrow$ pulmonary valve $\rightarrow$ pulmonary trunk $\rightarrow$ (R. and L.) pulmonary aa. $\rightarrow$ lungs $\rightarrow$ (R. and L.) pulmonary vv. $\rightarrow$ L. atrium $\rightarrow$ bicuspid valve $\rightarrow$ L. ventricle $\rightarrow$ aortic valve $\rightarrow$ ascending aorta.

2) SA node $\rightarrow$ internodal fibers $\rightarrow$ AV node $\rightarrow$ Bundle of His $\rightarrow$ L. and R. bundle branches $\rightarrow$ purkinje fibers.

3) R. coronary a. and L. coronary a.

4) R. marginal a. and posterior interventricular a.

5) Four.

6) Great cardiac v.

7) Ascending aorta.

8) There are FOUR pulmonary veins and TWO pulmonary arteries.

9) VEINS are vessels that carry blood toward the heart, and ARTERIES are vessels that carry blood away from the heart. (LO31)

10)Oxygenated.

11)Around the heart (peri = around; $\underline{\text { cardi }}=$ heart).

12)Relating to the lungs.

13) Internodal fibers.

14)Opening of bicuspid and tricuspid valves: $\underline{\mathbf{b}}$. Closing of bicuspid and tricuspid valves: $\underline{\mathbf{d}}$. Opening of pulmonary and aortic valves: $\underline{\mathbf{d}}$. Closing of pulmonary and aortic valves: $\underline{\mathbf{f}}$.

Back to Questions

\section{Lab 13: Vasculature of the Head, Neck, Thorax, and}

\section{Abdomen}

1) R. coronary a., L. coronary a. and brachiocephalic trunk.

2) The arm and head ( $\underline{\text { brachi }}=$ arm; $\underline{\text { ceph }}=$ head).

3) Diaphragm.

4) 1 brachiocephalic a. (trunk) and 2 brachiocephalic vv..

5) Subclavian v. and internal jugular v..

6) Superior vena cava.

7) Common hepatic a., L. gastric a., and splenic a..

8) Unpaired.

9) Stomach.

10)Inferior vena cava.

11)Portal v..

12)R. and L. common iliac aa.

13) The facial a. is a branch of the EXTERNAL CAROTID A. and the facial v. drains into the INTERNAL JUGULAR V..

14)The internal thoracic a. is a branch of the SUBCLAVIAN A., and the internal thoracic $v$. drains into the BRACHIOCEPHALIC V..

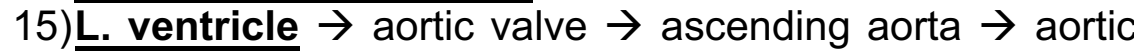
arch $\rightarrow$ L. common carotid a. $\rightarrow$ L. internal carotid a. $\rightarrow$ L. anterior cerebral a. $\rightarrow$ capillary bed in cerebrum $\vec{\rightarrow}$ cerebral v. $\rightarrow$ inferior sagittal sinus $\rightarrow$ straight sinus $\rightarrow$ confluence of the sinuses $\rightarrow$ transverse sinus $\rightarrow$ sigmoid sinus $\rightarrow$ internal jugular v. $\rightarrow$ brachiocephalic v. $\rightarrow$ superior vena cava $\rightarrow$ opening to superior vena cava $\rightarrow \mathrm{R}$. atrium $\rightarrow$ tricuspid valve $\rightarrow R$. ventricle $\rightarrow$ pulmonary valve $\rightarrow$ pulmonary trunk $\rightarrow$ pulmonary a. $\rightarrow$ capillary bed in lung $\rightarrow$ pulmonary v. $\rightarrow$ L. atrium $\rightarrow$ bicuspid valve $\rightarrow \mathrm{L}$. ventricle $\rightarrow$ aortic valve $\rightarrow$ ascending aorta $\rightarrow$ aortic arch $\rightarrow$ descending thoracic aorta $\rightarrow$ descending abdominal aorta $\rightarrow$ inferior mesenteric a. $\rightarrow$ superior rectal a. $\rightarrow$ capillary in rectum $\rightarrow$ superior rectal vein $\rightarrow$ inferior mesenteric v. $\rightarrow$ splenic v. $\rightarrow$ portal v. $\rightarrow$ capillary bed in liver $\rightarrow$ hepatic v. $\rightarrow$ inferior vena cava $\rightarrow$ opening of inferior vena cava $\rightarrow$ right atrium. 


\section{Lab 14: Vasculature of the Upper and Lower Limbs}

1) Armpit.

2) Great saphenous v. and small saphenous v..

3) $\operatorname{Arm}(\underline{\text { brachii }}=$ arm)

4) Axillary a..

5)

a. Cephalic v. $\rightarrow$ AXILLARY V.

b. Basilic v. $\rightarrow$ BRACHIAL V.

c. Small saphenous v. $\rightarrow$ POPLITEAL V.

d. Great saphenous v. $\rightarrow$ FEMORAL V.

e. Anterior tibial a. $\rightarrow$ DORSALIS PEDIS A.

f. Femoral a. $\rightarrow$ DEEP FEMORAL A. or POPLITEAL A. g. Subclavian v. $\rightarrow$ BRACHIOCEPHALIC V.

6) The median cubital v. mainly shunts blood from the CEPHALIC vein to the BASILIC vein.

7) Radial a. and ulnar a..

8) Deep femoral a..

9) Capillary bed in pollex $\rightarrow 1^{\text {st }}$ digital venous branch $\rightarrow$ venous arch $\rightarrow$ ulnar v. $\rightarrow$ brachial v. $\rightarrow$ axillary v. $\rightarrow$ subclavian v. $\rightarrow$ brachiocephalic v. $\rightarrow$ superior vena cava $\rightarrow$ opening to superior vena cava $\rightarrow \mathrm{R}$. atrium $\rightarrow$ tricuspid valve $\rightarrow$ R. ventricle $\rightarrow$ pulmonary valve $\rightarrow$ pulmonary trunk $\rightarrow$ pulmonary a. $\rightarrow$ capillary bed in lung $\rightarrow$ pulmonary v. $\rightarrow$ L. atrium $\rightarrow$ bicuspid valve $\rightarrow$ L. ventricle $\rightarrow$ aortic valve $\rightarrow$ ascending aorta $\rightarrow$ aortic arch $\rightarrow$ descending thoracic aorta $\rightarrow$ descending abdominal aorta $\rightarrow$ common iliac a. $\rightarrow$ external iliac a. $\rightarrow$ femoral a. $\rightarrow$ popliteal a. $\rightarrow$ anterior tibial a. $\rightarrow$ dorsalis pedis a. $\rightarrow$ arterial arch $\rightarrow 1^{\text {st }}$ digital arterial branch $\rightarrow$ capillary bed in hallux.

10) Diagrams may vary.

11)Diagrams may vary.

Back to Questions

\section{Lab 15: Respiratory System}

1) Diaphragm and external intercostal muscles.

2) NONE for passive exhalation; internal intercostals for active (forceful) exhalation.

3) To warm, moisten, and clean air as it heads toward the lungs.

4) Cricoid cartilage.

5) Above the opening to the larynx (epi = above; glottis $=$ opening to the larynx).

6) False vocal cord.

7) Slanting.

8) Trachea.

9) C3 n., C4 n., and C5 n.

10) Under the inferior concha, or in the inferior meatus.

11)3.

12)2.

13)Nose.

14) Nostril $\rightarrow$ meati $\rightarrow$ nasopharynx $\rightarrow$ oropharynx $\rightarrow$ laryngopharynx $\rightarrow$ glottis $\rightarrow$ between the false vocal cords $\rightarrow$ between the true vocal cords $\rightarrow$ trachea $\rightarrow$ primary bronchus $\rightarrow$ secondary bronchus $\rightarrow$ tertiary bronchus $\rightarrow$ bronchiole $\rightarrow$ alveolus $\rightarrow$ capillary bed $\rightarrow$ pulmonary v. $\rightarrow$ L. atrium $\rightarrow$ bicuspid valve $\rightarrow$ L. ventricle $\rightarrow$ aortic valve $\rightarrow$ ascending aorta $\rightarrow$ aortic arch $\rightarrow$ descending thoracic aorta $\rightarrow$ descending abdominal aorta $\rightarrow$ inferior mesenteric a. $\rightarrow$ superior rectal $a$. $\rightarrow$ capillary bed in rectum.

15)ANSWERS MAY VARY. See yellow text box under "Muscles of respiration" heading.

16)ANSWERS MAY VARY. See yellow text box under "Muscles of respiration" heading.

Back to Questions 


\section{Lab 16: Primary Digestive Organs}

1) Mouth $\rightarrow$ oral cavity $\rightarrow$ oropharynx $\rightarrow$ laryngopharynx $\rightarrow$ upper esophageal sphincter $\rightarrow$ esophagus $\rightarrow$ lower esophageal sphincter $\rightarrow$ stomach $\rightarrow$ pyloric sphincter $\rightarrow$ duodenum $\rightarrow$ jejunum $\rightarrow$ ileum $\rightarrow$ ileocecal valve $\rightarrow$ cecum $\rightarrow$ ascending colon $\rightarrow$ hepatic flexure $\rightarrow$ transverse colon $\rightarrow$ splenic flexure $\rightarrow$ descending colon $\rightarrow$ sigmoid flexure $\rightarrow$ sigmoid colon $\rightarrow$ sigmoid flexure $\rightarrow$ rectum $\rightarrow$ internal anal sphincter $\rightarrow$ external anal sphincter $\rightarrow$ anus.

2) True.

3) True.

4) True.

5) False.

6) Seven (Orbicularis oris, upper esophageal sphincter, lower esophageal sphincter, pyloric sphincter, ileocecal 7) valve, internal anal sphincter, and external anal sphincter).
a. Mouth: FOOD
b. Esophagus: BOLUS
c. Stomach: $\mathbf{C H Y M E}$
d. Small intestine: $\underline{\text { CHYME }}$
e. Colon: FECES
f. Rectum: FECES

8) A bend or curve.

9) To increase surface area and allow for expansion.

10)c. Ilium (note the spelling).

11)A small rounded food mass that has been chewed and mixed with saliva.

12) Three (one for the ileocecal valve, one for the appendix, and one for the ascending colon).

\section{Lab 17: Secondary Digestive Organs \& Lymphatic System}

1) Incisors.

2) Before.

3) Tooth.

4) Cystic duct.

5) Common bile duct.

6) Cervical, axillary, and inguinal.

7) 16 .

8) Right lobe.

9) False.

10)True.

11) Coronary ligament.

12)A gland, found under the mandible, that produces saliva (submandibular salivary gland).

13)Filiform, fungiform, foliate, and circumvallate papillae.

14)Dentin.

\section{Back to Questions}

Back to Questions 


\section{Lab 18: Urinary System}

1) Above (from supra) the kidney (from renal).

2) Glomerulus $\rightarrow$ Bowman's capsule $\rightarrow$ proximal convoluted tubule $\rightarrow$ descending loop of Henle $\rightarrow$ ascending loop of Henle $\rightarrow$ distal convoluted tubule $\rightarrow$ collecting duct $\rightarrow$ renal papilla $\rightarrow$ minor calyx $\rightarrow$ major calyx $\rightarrow$ renal pelvis $\rightarrow$ ureter $\rightarrow$ ureteral opening $\rightarrow$ urinary bladder $\rightarrow$ urethral opening $\rightarrow$ urethra.

3) Renal a. $\rightarrow$ segmental a. $\rightarrow$ interlobar a. $\rightarrow$ arcuate a. $\rightarrow$ cortical radiate a. $\rightarrow$ afferent arteriole $\rightarrow$ glomerulus $\rightarrow$ efferent arteriole $\rightarrow$ peritubular capillary $\rightarrow$ cortical radiate v. $\rightarrow$ arcuate v. $\rightarrow$ interlobar v. $\rightarrow$ segmental v. $\rightarrow$ renal v..

4) Two.

5) One.

6) Trigone.

7) Within.

8) Detrusor muscle.

9) Rugae.

10)

Jejunum: INTRAPERITONEAL.

Esophagus: RETROPERITONEAL.

Stomach: INTRAPERITONEAL.

Duodenum: RETROPERITONEAL.

Ascending colon: RETROPERITONEAL.

Transverse colon: INTRAPERITONEAL.

Descending colon: RETROPERITONEAL.

Urinary bladder: SUBPERITONEAL.

Pancreas: RETROPERITONEAL.

11)Folded, twisted, or coiled.

12)b. Renal sinus.

\section{Lab 19: Male and Female Reproductive Systems}

1) Seminiferous tubules (in the testes) $\rightarrow$ rete testis $\rightarrow$ head of epididymis $\rightarrow$ body of epididymis $\rightarrow$ tail of epididymis $\rightarrow$ vas deferens $\rightarrow$ ejaculatory duct (in prostate gland) $\rightarrow$ prostatic urethra $\rightarrow$ membranous urethra $\rightarrow$ spongy urethra $\rightarrow$ external urethral orifice.

2) Ovary $\rightarrow$ Fimbriae (collect egg) $\rightarrow$ infundibulum $\rightarrow$ ampulla $\rightarrow$ isthmus $\rightarrow$ uterus $\rightarrow$ internal os $\rightarrow$ cervical canal $\rightarrow$ external os $\rightarrow$ vagina $\rightarrow$ vaginal orifice.

3) Mammary gland $\rightarrow$ lactiferous ducts $\rightarrow$ lactiferous sinus $\rightarrow$ nipple.

4) Scrotum and testes location, dartos muscle, cremaster muscle, and pampiniform plexus.

5) Labia majora.

6) Ejaculation.

7) Ovarian, suspensory, and broad ligaments.

8) White.

9) True.

10)False.

11)Expansion for intercourse and childbirth.

12)True.

13)Perimetrium, myometrium, and endometrium.

14) Ovarian ligament.

15)Lip.

16)The mixture of sperm and supporting fluids.

Back to Questions

Back to Questions 


\section{Appendix B: Lab Examination Set-Up Guides}

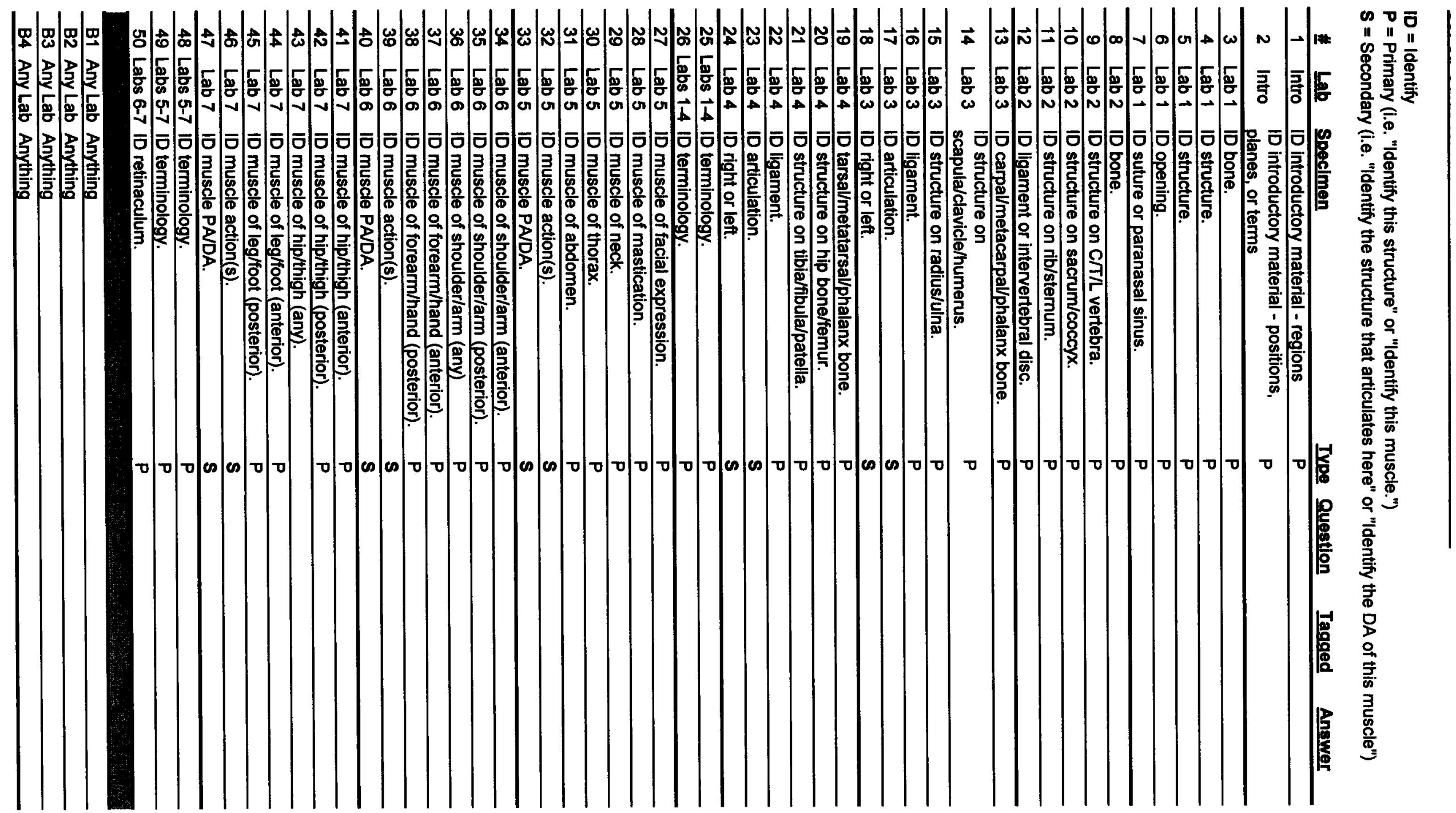




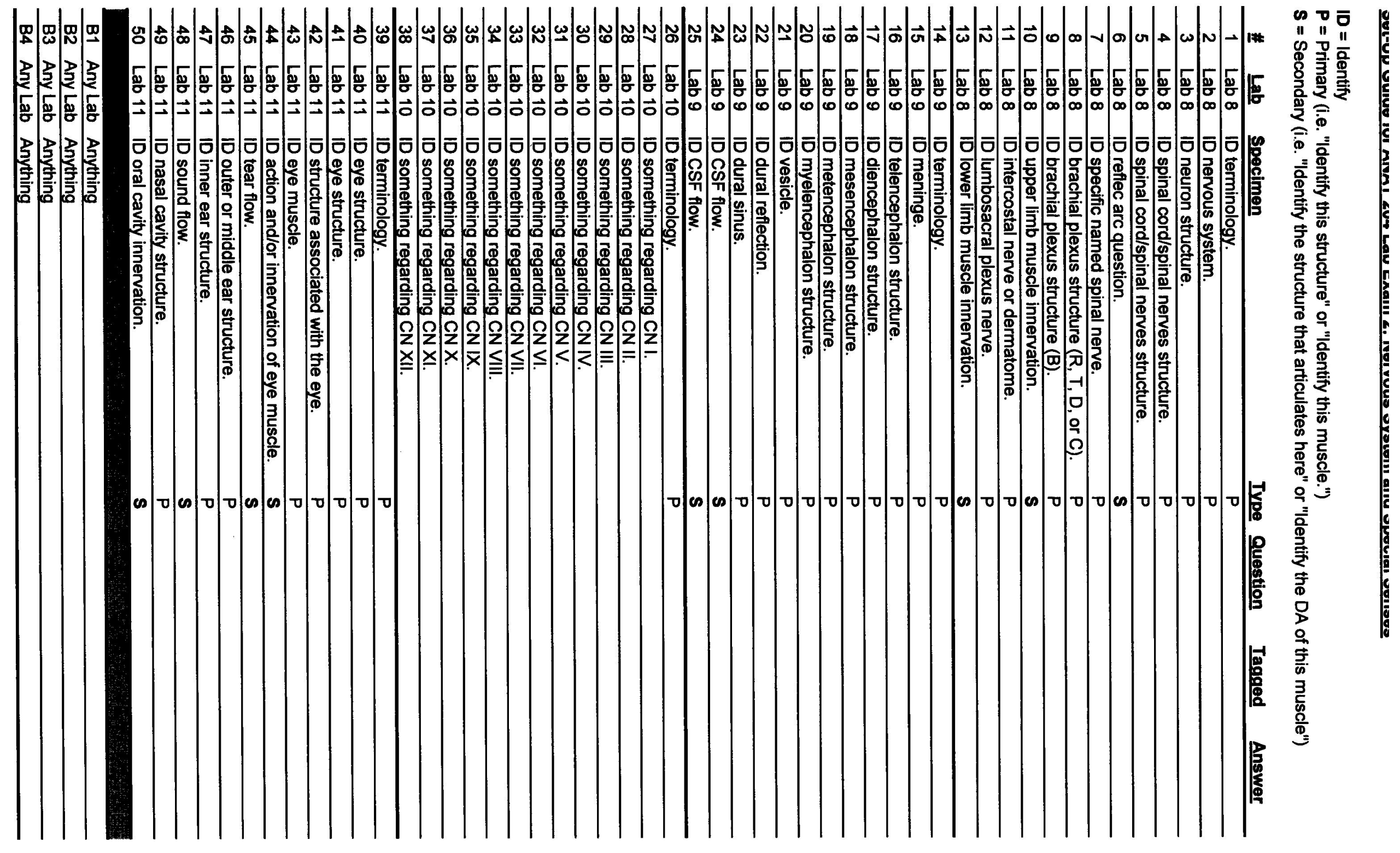




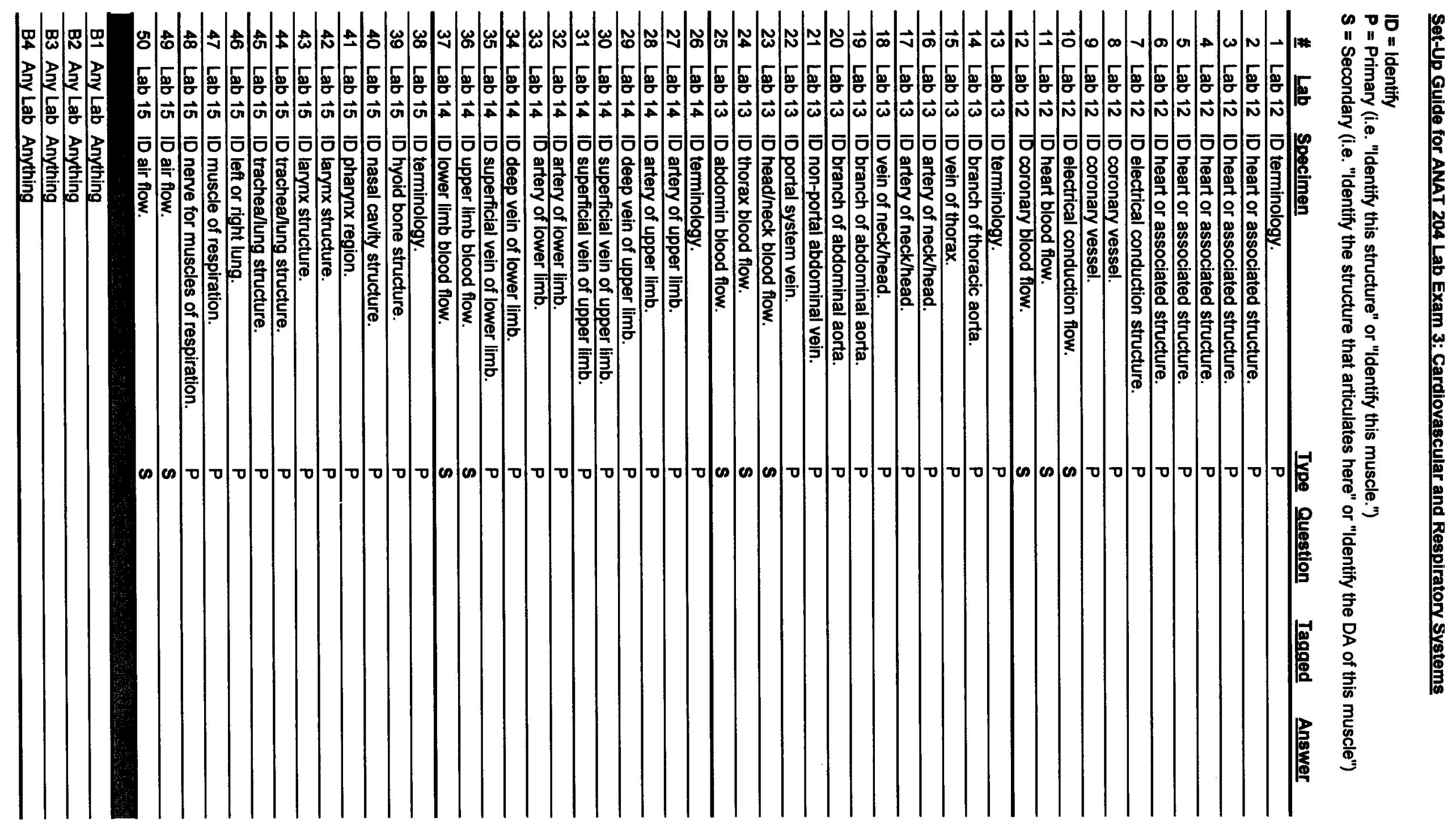




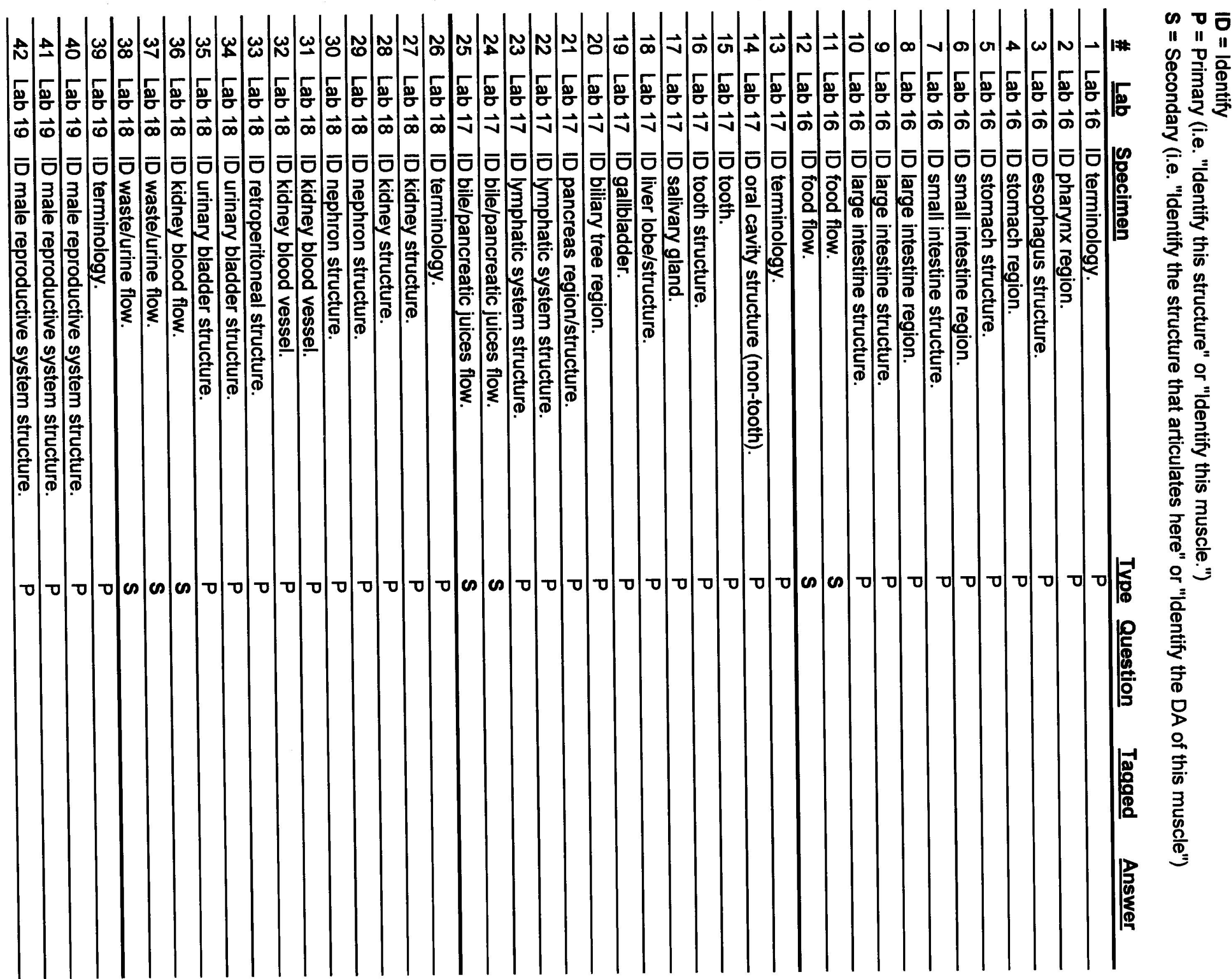




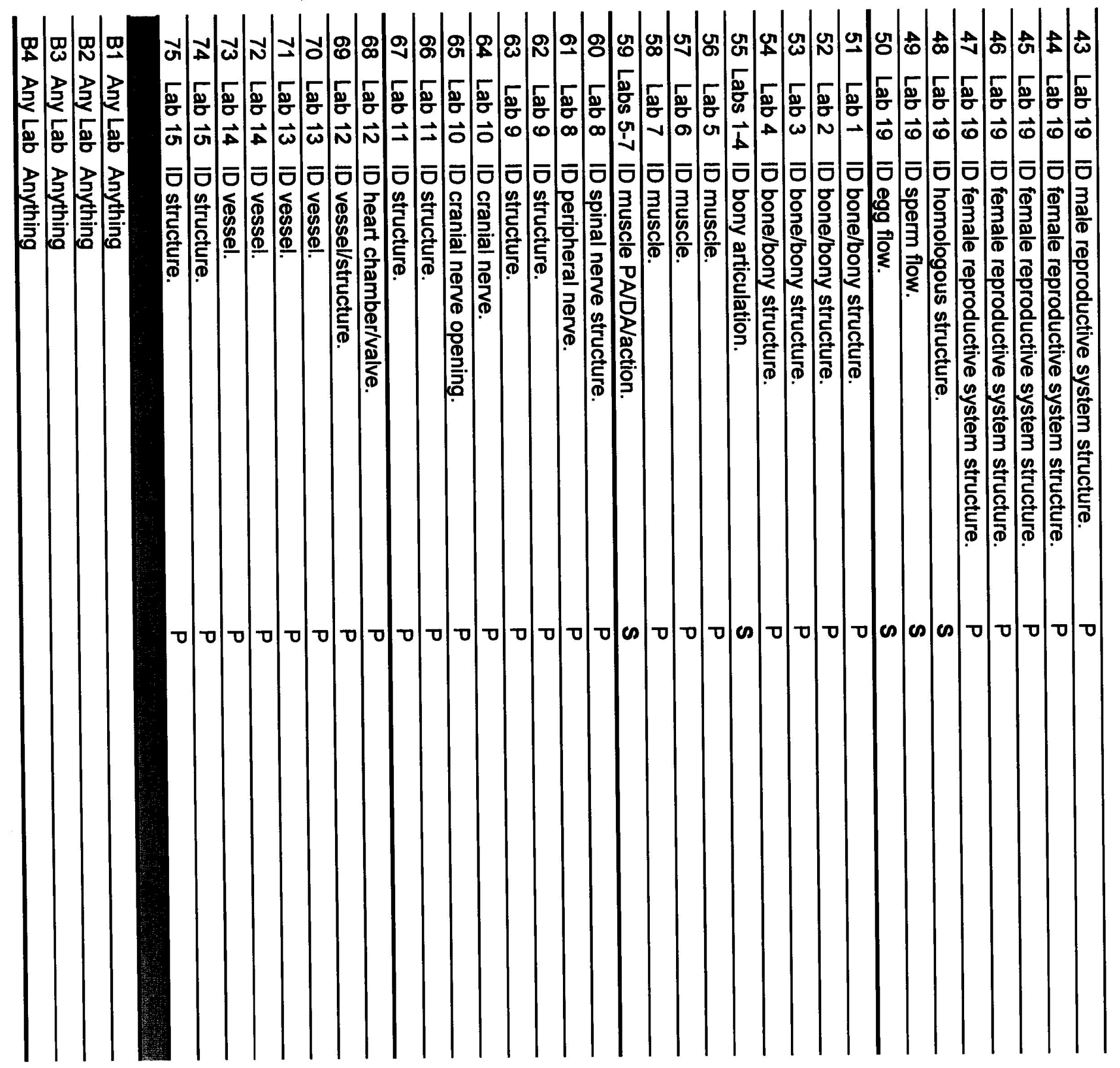




\section{Appendix C: Image and Video Sources (in order by appearance)}

1) Skeleton on cover. Removed background.

a. https://www.flickr.com/photos/liverpoolhls/10818462796/in/photolist-htZs5C-bEUUgb-hu1XgD-htZzau-eHUAjY-huDksd-vayuVg-huENsR-athFZHhtZycC-huEKyV-75jw4G-89SVkC-eHUzWU-FM6v8-bCGjao-hvu23Q-huCWGn-htZxhw-htZvGB-UhQj4w-upaK8E-huEWfe-htZwAk-cdL4NY8E715f-ur3PG7-adSU9m-huDoqB-urGJPz-u3zVKy-7RhS3n-udq1Lt-3c7cn4-tobqQh-jec5yG-FM6xi-u3vnR9-unEco7-u3JA5x-uruuQF-7Rm8614Fhzf8-dvvCWT-FM2zo-89Pzn4-FM6CM-iFiov3-huEF2P-boFZQ1/

2) Video sign for video links

a. made in Microsoft Word.

3) Stop sign for self-assessment questions

a. made in Microsoft Word.

4) Anatomical Position, Anterior View

a. Image drawn by Logan Detweiler and owned by Ethan Snow.

5) Anatomical Position, Right Lateral View

a. Image drawn by Logan Detweiler and owned by Ethan Snow.

6) Zygomatic Arch video.

a. Video made by Ethan Snow and posted on YouTube as unlisted with CC license.

i. https://youtu.be/olXxnDV7n7w

7) Figure 1.1. Simplified Diagrammatic Relationship of Bony Structures of the Ethmoid Bone, Anterior View

a. Image drawn and owned by Ethan Snow.

8) Figure 1.2. Relationship of Perpendicular Plate (of the Ethmoid Bone) to Vomer Bone in the Nasal Septum, Lateral View

a. Image drawn and owned by Ethan Snow.

b. Video made by Ethan Snow and posted on YouTube as unlisted with CC license.

i. https://youtu.be/xsom80qn9SI

9) Clinical Application: Deviated Septum

a. Image drawn by Logan Detweiler and owned by Ethan Snow.

10) Figure 1.3. Simplified Diagrammatic Relationship Between Foramen Lacerum and Carotid Canal

a. Image drawn and owned by Ethan Snow.

11) Figure 1.4. Skull, Anterior View. Cropped and added labels.

a. http://lane.stanford.edu/biomed-resources/bassett/index.html\#bassett=\%2FbassettView.html\%3Fbn\%3D35-4

12) Figure 1.5. Skull, Right Lateral View. Cropped and added labels.

a. http://lane.stanford.edu/biomed-resources/bassett/index.html\#bassett=\%2FbassettView.html\%3Fbn\%3D35-1

13) Figure 1.6. Skull, Inferior View. Cropped and added labels.

a. http://lane.stanford.edu/biomed-resources/bassett/index.html\#bassett=\%2FbassettView.html\%3Fbn\%3D35-6

14) Figure 1.7. Skull, Superior View Inside Cranium. Cropped and added labels.

a. http://lane.stanford.edu/biomed-resources/bassett/index.html\#bassett=\%2FbassettView.html\%3Fbn\%3D36-5

15) Figure 2.1. Sternum, Right Anterolateral View. Cropped and added labels.

a. http://lane.stanford.edu/biomed-resources/bassett/index.html\#bassett=\%2FbassettView.html\%3Fbn\%3D113-7

16) Figure 2.2. Right $6^{\text {th }}$ Rib, Medial View. Cropped and added labels.

a. http://lane.stanford.edu/biomed-resources/bassett/index.html\#bassett=\%2FbassettView.html\%3Fbn\%3D114-3

17) Vertebrae that make up the spine (from "Anatomical Position, Right Lateral View"). 
a. Image drawn by Logan Detweiler and owned by Ethan Snow.

18) Figure 2.3. Simplified Diagram of Typical Structures on Vertebrae, Superior/Inferior View.

a. Image drawn and owned by Ethan Snow.

b. Video made by Ethan Snow and posted on YouTube as unlisted with CC license.

i. https://youtu.be/vd2myXsBWIA

19) Figure 2.4. Typical Cervical Vertebra, Superior View. Cropped and added labels.

a. http://lane.stanford.edu/biomed-resources/bassett/index.html\#bassett=\%2FbassettView.html\%3Fbn\%3D48-7

20) Figure 2.5. Atlas, Superior View. Cropped and added labels.

a. http://lane.stanford.edu/biomed-resources/bassett/index.html\#bassett=\%2FbassettView.html\%3Fbn\%3D48-3

21) Figure 2.6. Axis, Posterolateral View. Cropped and added labels.

a. http://lane.stanford.edu/biomed-resources/bassett/index.html\#bassett=\%2FbassettView.html\%3Fbn\%3D48-6

22) Figure 2.7. Typical Thoracic Vertebra, Superior View. Cropped and added labels.

a. http://lane.stanford.edu/biomed-resources/bassett/index.html\#bassett=\%2FbassettView.html\%3Fbn\%3D113-5

23) Figure 2.8. Typical Thoracic Vertebra, Posterolateral View. Cropped and added labels.

a. http://lane.stanford.edu/biomed-resources/bassett/index.html\#bassett=\%2FbassettView.html\%3Fbn\%3D113-4

24) Figure 2.9. Typical Lumbar Vertebra, Superior View. Cropped and added labels.

a. http://lane.stanford.edu/biomed-resources/bassett/index.html\#bassett=\%2FbassettView.html\%3Fbn\%3D113-5

25) Figure 2.10. Typical Lumbar Vertebra, Posterolateral View. Cropped and added labels.

a. http://lane.stanford.edu/biomed-resources/bassett/index.html\#bassett=\%2FbassettView.html\%3Fbn\%3D113-4

26) Clinical Application: Scoliosis, Kyphosis, and Lordosis

a. Image drawn by Logan Detweiler and owned by Ethan Snow.

27) Figure 2.11. Sacrum, Posterior View.

a. http://lane.stanford.edu/biomed-resources/bassett/index.html\#bassett=\%2FbassettView.html\%3Fbn\%3D154-5

28) Figure 2.12. Sacrum, Lateral View.

a. http://lane.stanford.edu/biomed-resources/bassett/index.html\#bassett=\%2FbassettView.html\%3Fbn\%3D209-2

29) Figure 2.13. Coccyx, Posterior View.

a. http://lane.stanford.edu/biomed-resources/bassett/index.html\#bassett=\%2FbassettView.html\%3Fbn\%3D209-5

30) Figure 2.14. Intervertebral Disc Diagram, Superior View.

a. Image drawn and owned by Ethan Snow.

31) Clinical Application: Herniated Disc.

a. Image drawn and owned by Ethan Snow.

32) Figure 3.1. Right Scapula, Lateral View. Cropped and added labels.

a. http://lane.stanford.edu/search.html?q=scapula\&source=bassett\#bassett=\%2FbassettView.html\%3Fbn\%3D85-5

33) Figure 3.2. Right Scapula, Anterior View. Cropped and added labels.

a. http://lane.stanford.edu/search.html?q=scapula\&source=bassett\#bassett=\%2FbassettView.html\%3Fbn\%3D85-3

34) Figure 3.3. Right Scapula, Posterior View. Cropped and added labels.

a. http://lane.stanford.edu/search.html?q=scapula\&source=bassett\#bassett=\%2FbassettView.html\%3Fbn\%3D85-4

35) Figure 3.4. (Above) Right Clavicle, Inferior View. Cropped and added labels.

a. http://lane.stanford.edu/search.html?q=clavicle\&source=bassett\#bassett=\%2FbassettView.html\%3Fbn\%3D85-2

36) Figure 3.5. (Below) Right Clavicle, Superior View. Cropped and added labels.

a. http://lane.stanford.edu/search.html?q=clavicle\&source=bassett\#bassett=\%2FbassettView.html\%3Fbn\%3D85-1

37) Figure 3.6. Right Humerus, Anterior View. Cropped and added labels.

a. http://lane.stanford.edu/search.html?q=humerus\&source=bassett\#bassett=\%2FbassettView.html\%3Fbn\%3D85-6

38) Figure 3.7. Right Humerus, Posterior View. Cropped and added labels.

a. http://lane.stanford.edu/search.html?q=humerus\&source=bassett\#bassett=\%2FbassettView.html\%3Fbn\%3D85-7

39) Figure 3.8. Right Radius and Ulna, Anterior View. Cropped and added labels. 
a. http://lane.stanford.edu/search.html?q=humerus\&source=bassett\#bassett=\%2FbassettView.html\%3Fbn\%3D85-6

40) Figure 3.9. Right Radius and Ulna, Posterior View. Cropped and added labels.

a. http://lane.stanford.edu/search.html?q=humerus\&source=bassett\#bassett=\%2FbassettView.html\%3Fbn\%3D85-7

41) Figure 3.10. Right Wrist and Hand Bones, Anterior View. Cropped and added labels.

a. http://lane.stanford.edu/search.html?q=hand+bones\&source=bassett\#bassett=\%2FbassettView.html\%3Fbn\%3D87-2

42) Figure 4.1. Left Hip Bone, Medial View. Cropped and added labels.

a. http://lane.stanford.edu/search.html?q=hip+bone\&source=bassett\#bassett=\%2FbassettView.html\%3Fbn\%3D175-2

43) Figure 4.2. Left Hip Bone, Lateral View. Cropped and added labels.

a. http://lane.stanford.edu/search.html?q=coxal+bone\&source=bassett\#bassett=\%2FbassettView.html\%3Fbn\%3D175-1

44) Figure 4.3. Femur, Anterior View. Cropped and added labels.

a. http://lane.stanford.edu/search.html?q=coxal+femur\&source=bassett\#bassett=\%2FbassettView.html\%3Fbn\%3D175-5

45) Figure 4.4. Femur, Posterior View. Cropped and added labels.

a. http://lane.stanford.edu/search.html?q=coxal+femur\&source=bassett\#bassett=\%2FbassettView.html\%3Fbn\%3D175-6

46) Figure 4.5. Patella, Anterior View. Cropped and added labels.

a. http://lane.stanford.edu/search.html?q=patella\&source=bassett\#bassett=\%2FbassettView.html\%3Fbn\%3D176-4

47) Figure 4.6. Patella, Posterior View. Cropped and added labels.

a. http://lane.stanford.edu/search.html?q=patella\&source=bassett\#bassett=\%2FbassettView.html\%3Fbn\%3D176-5

48) Figure 4.7. Tibia and Fibula, Anterior View. Cropped and added labels.

a. http://lane.stanford.edu/search.html?q=coxal+femur\&source=bassett\#bassett=\%2FbassettView.html\%3Fbn\%3D175-5

49) Figure 4.8. Tibia and Fibula, Posterior View. Cropped and added labels.

a. http://lane.stanford.edu/search.html?q=coxal+femur\&source=bassett\#bassett=\%2FbassettView.html\%3Fbn\%3D175-6

50) Figure 4.9. Ligaments and Structure of the Knee. Cropped and added labels.

a. https://collections.lib.utah.edu/details?id=871858\&q=knee\&facet setname s=ehsl heal

51) Figure 4.10. Left Ankle and Foot Bones, Superior View. Cropped and added labels.

a. http://lane.stanford.edu/search.html?q=foot+bones\&source=bassett\#bassett=\%2FbassettView.html\%3Fbn\%3D177-4

52) Figure 5.1. Simplified Diagram to Help Identify the Muscles that Act on the Mouth and Learn Their Actions.

a. Image drawn and owned by Ethan Snow.

b. Video made by Ethan Snow and posted on YouTube as unlisted with CC license. i. https://youtu.be/rBpRtex8S50

53) Figure 5.2. Muscles of Facial Expression, Left Lateral View. Cropped and added labels.

a. http://lane.stanford.edu/biomed-resources/bassett/bassettView.html?bn=63-2

54) Figure 5.3. Muscles of Mastication, Left Lateral View. Cropped and added labels.

a. http://lane.stanford.edu/search.html?q=temporalis\&source=bassett\#bassett=\%2FbassettView.html\%3Fbn\%3D49-5

55) Figure 5.4. Muscles of the Neck, Anterior View. Cropped and added labels.

a. http://lane.stanford.edu/search.html?q=sternocleidomastoid\&source=bassett\#bassett=\%2FbassettView.html\%3Fbn\%3D73-4

56) Figure 5.5. Muscles of the Neck, Posterior View. Cropped and added labels.

a. http://lane.stanford.edu/search.html?q=splenius+capitis\&source=bassett\#bassett=\%2FbassettView.html\%3Fbn\%3D79-1

57) Figure 5.6. Muscles of the Back, Posterior View. Cropped and added labels.

a. http://lane.stanford.edu/search.html?q=longissimus\&source=bassett\#bassett=\%2FbassettView.html\%3Fbn\%3D131-5

58) Figure 5.7. Muscles of the Thorax, Anterior View. Cropped and added labels.

a. http://lane.stanford.edu/search.html?q=internal+intercostal\&source=bassett\#bassett=\%2FbassettView.html\%3Fbn\%3D91-4

59) Figure 5.8. Muscles of the Abdomen, Anterior View. Cropped and added labels.

a. http://lane.stanford.edu/search.html?q=rectus+abdominis\&source=bassett\#bassett=\%2FbassettView.html\%3Fbn\%3D133-6

60) Figure 5.9. Simplified Diagram of Abdominal Muscle Fiber Direction, Anterior View.

a. Image created with Microsoft Word objects by Ethan Snow.

b. Video made by Ethan Snow and posted on YouTube as unlisted with CC license. 
i. https://youtu.be/EbNWY5-Hij5I

61) Figure 6.1. Muscles of the Left Arm and Shoulder, Posterior View. Cropped and added labels.

a. http://lane.stanford.edu/search.html?q=supraspinatus\&source=bassett\#bassett=\%2FbassettView.html\%3Fbn\%3D93-6

62) Figure 6.2. Left Subscapularis, Anterior View. Cropped and added labels.

a. http://lane.stanford.edu/search.html?q=subscapularis\&source=bassett\#bassett=\%2FbassettView.html\%3Fbn\%3D91-7

63) Figure 6.3. Left Serratus Anterior and Brachialis, Anterior View. Cropped and added labels.

a. http://lane.stanford.edu/search.html?q=serrates+anterior\&source=bassett\#bassett=\%2FbassettView.html\%3Fbn\%3D91-5

64) Figure 6.4. Trapezius and Latissimus Dorsi, Posterior View. Cropped and added labels.

a. http://lane.stanford.edu/search.html?q=trapezius\&source=bassett\#bassett=\%2FbassettView.html\%3Fbn\%3D93-1

65) Figure 6.5. Right Pectoralis Major, Anterior View. Cropped and added labels.

a. http://lane.stanford.edu/search.html?q=serrates+anterior\&source=bassett\#bassett=\%2FbassettView.html\%3Fbn\%3D89-2

66) Figure 6.6. Right Pectoralis Minor, Anterior View. Cropped and added labels.

a. http://lane.stanford.edu/search.html?q=pectoralis+minor\&source=bassett\#bassett=\%2FbassettView.html\%3Fbn\%3D89-4

67) Figure 6.7. Muscles of the Right Arm, Anterior View. Cropped and added labels.

a. http://lane.stanford.edu/search.html?q=arm+muscles\&source=bassett\#bassett=\%2FbassettView.html\%3Fbn\%3D95-3

68) Figure 6.8. Muscles (Sketch) of the Right Arm, Anterior View.

a. Image drawn by Logan Detweiler and owned by Ethan Snow.

69) Figure 6.9. Muscles of the Right Arm, Posterior View. Cropped and added labels.

a. http://lane.stanford.edu/search.html?q=arm+muscles\&source=bassett\#bassett=\%2FbassettView.html\%3Fbn\%3D96-2

70) Figure 6.10. Diagram for Learning Actions of Forearm Muscles Passing Through the Wrist, Cross-Section View.

a. Image drawn and owned by Ethan Snow.

b. Video made by Ethan Snow and posted on YouTube as unlisted with CC license.

i. https://youtu.be/DR De50PAxY

71) Figure 6.11. Thenar $(\mathrm{T})$ and Hypothenar $(\mathrm{H})$ Eminences.

a. Photo owned and edited by Ethan Snow.

72) Figure 6.12. Muscles of Right Forearm and Hand, Anterior View. Cropped and added labels.

a. http://lane.stanford.edu/search.html?q=extensor+digitorum\&source=bassett\#bassett=\%2FbassettView.html\%3Fbn\%3D98-1

73) Figure 6.13. Muscles (Sketch) of Right Forearm and Hand, Anterior View.

a. Image drawn by Logan Detweiler and owned by Ethan Snow.

74) Figure 6.14. Muscles of Right Forearm and Hand, Posterior View. Cropped and added labels.

a. http://lane.stanford.edu/search.html?q=extensor+digitorum\&source=bassett\#bassett=\%2FbassettView.html\%3Fbn\%3D106-3

75) Figure 6.15. Muscles of (Sketch) Right Forearm and Hand, Posterior View.

a. Image drawn by Logan Detweiler and owned by Ethan Snow.

76) Figure 7.1. Muscles of the Left Thigh, Anterior View. Cropped and added labels.

a. http://lane.stanford.edu/search.html?q=sartorius\&source=bassett\#bassett=\%2FbassettView.html\%3Fbn\%3D185-5

77) Figure 7.2. Muscles (Sketch) of the Left Thigh, Anterior View.

a. Image drawn by Logan Detweiler and owned by Ethan Snow.

78) Figure 7.3. Muscles of the Left Gluteal Region and Thigh, Posterior View. Cropped and added labels.

a. http://lane.stanford.edu/search.html?q=semitendinosus\&source=bassett\#bassett=\%2FbassettView.html\%3Fbn\%3D183-2

79) Figure 7.4. Muscles (Sketch) of the Left Gluteal Region and Thigh, Posterior View.

a. Image drawn by Logan Detweiler and owned by Ethan Snow.

80) Figure 7.5. Muscles of the Gluteal Region and Proximal Thigh, Posterior View. Cropped and added labels.

a. http://lane.stanford.edu/search.html?q=gluteus+maximus\&source=bassett\#bassett=\%2FbassettView.html\%3Fbn\%3D179-3

81) Figure 7.6. Muscles of the Pelvis and Proximal Thigh, Anterior View. Cropped and added labels.

a. http://lane.stanford.edu/search.html?q=psoas+major\&source=bassett\#bassett=\%2FbassettView.html\%3Fbn\%3D184-6

82) Figure 7.7. Muscles of the Left Leg, Anterior View. Cropped and added labels. 
a. http://lane.stanford.edu/search.html?q=extensor+hallucis+longus\&source=bassett\#bassett=\%2FbassettView.html\%3Fbn\%3D191-7

83) Figure 7.8. Muscles (Sketch) of the Left Leg, Anterior View.

a. Image drawn by Logan Detweiler and owned by Ethan Snow.

84) Figure 7.9. Muscles of the Left Leg, Posterior View. Cropped and added labels.

a. http://lane.stanford.edu/search.html?q=gastrocnemius\&source=bassett\#bassett=\%2FbassettView.html\%3Fbn\%3D195-1

85) Figure 7.10. Muscles (Sketch) of the Left Leg, Posterior View.

a. Image drawn by Logan Detweiler and owned by Ethan Snow.

86) Figure 8.1. Organization of the Nervous System, Reflex Arc, and Typical Muscle Innervation Pathway.

a. Image created with Microsoft Word objects by Ethan Snow.

87) Figure 8.2. Structures of a Neuron.

a. Image drawn and owned by Ethan Snow.

88) Figure 8.3. Meninges Diagram.

a. Image drawn and owned by Ethan Snow.

89) Figure 8.4. Diagram of Transverse Section of Spinal Cord and Spinal Nerve Components with Reflex Arc Pathway.

a. Image drawn and owned by Ethan Snow.

90) Figure 8.5. Diagram for Identifying Spinal Nerves from Surrounding Structures and Vertebrae.

a. Image drawn and owned by Ethan Snow.

91) Figure 8.6. Cervical Spinal Cord (cut), Posterior View. Cropped and added labels.

a. http://lane.stanford.edu/search.html?q=spinal+cord\&source=bassett\#bassett=\%2FbassettView.html\%3Fbn\%3D217-6

92) Figure 8.7. Distal Spinal Cord, Posterior View.

a. http://lane.stanford.edu/search.html?q=conus+medullaris\&source=bassett\#bassett=\%2FbassettView.html\%3Fbn\%3D219-7

93) Figure 8.8. Diagram of Right Brachial Plexus, Anterior View.

a. Image drawn and owned by Ethan Snow.

94) Figure 8.9. Right Brachial Plexus, Anterior View (No Labels). Cropped and flipped.

a. http://lane.stanford.edu/search.html?q=brachial+plexus\&source=bassett\#bassett=\%2FbassettView.html\%3Fbn\%3D91-3

95) Figure 8.10. Right Upper Limb Nerves Diagram, Anterior View (from “Anatomical Position, Anterior View”).

a. Image drawn by Logan Detweiler and owned/edited by Ethan Snow.

96) Figure 8.11. Right Lower Limb Nerves Diagram, Anterior View (from "Anatomical Position, Anterior View").

a. Image drawn by Logan Detweiler and owned/edited by Ethan Snow.

97) Figure 9.1. Hemisected Brain, Right Half, Midsagittal View. Cropped, merged two images (below), and added labels.

a. http://lane.stanford.edu/search.html?q=anterior+commissure\&source=bassett\#bassett=\%2FbassettView.html\%3Fbn \%3D 15-6

b. http://lane.stanford.edu/search.html?q=corpus+callosum\&source=bassett\#bassett=\%2FbassettView.html\%3Fbn\%3D15-7

98) Figure 9.2. Brain (Cerebrum), Superior View. Cropped and added labels.

a. http://lane.stanford.edu/search.html?q=brain+superior\&source=bassett\#bassett=\%2FbassettView.html\%3Fbn\%3D8-3

99) Figure 9.3. Brain and Dural Sinuses, Posterior View. Cropped and added labels.

a. http://lane.stanford.edu/search.html?q=transverse+sinus\&source=bassett\#bassett=\%2FbassettView.html\%3Fbn\%3D1-5

100) Figure 9.4. Sigmoid Sinuses, Posterosuperior View. Cropped and added labels.

a. http://lane.stanford.edu/search.html?q=transverse+sinus\&source=bassett\#bassett=\%2FbassettView.html\%3Fbn\%3D50-2

101) Figure 9.5. Falx Cerebri and Dural Sinuses, Left Lateral View. Cropped and added labels.

a. http://lane.stanford.edu/search.html?q=falx\&source=bassett\#bassett=\%2FbassettView.html\%3Fbn\%3D49-7

102) Figure 9.6. Diagram of Dural Reflections and Dural Sinuses, Right Lateral View.

a. Image drawn and owned by Ethan Snow.

103) Figure 10.1. Diagram of Cranial Nerves in the Basicranium, Superior View.

a. Image drawn and owned by Ethan Snow.

104) Figure 10.2. Cranial Nerves in the Basicranium, Posterosuperior View. Cropped and added labels.

a. http://lane.stanford.edu/search.html?q=abducens\&source=bassett\#bassett=\%2FbassettView.html\%3Fbn\%3D50-2 
105) Figure 11.1. Right Eye, Anterior View. Cropped and added labels.

a. http://lane.stanford.edu/search.html?q=eye\&source=bassett\#bassett=\%2FbassettView.html\%3Fbn\%3D52-1

106) Figure 11.2. Tear structures, Right Anterior View.

a. Image drawn and owned by Ethan Snow.

107) Figure 11.3. Diagram of Eye, Sagittal Section, Lateral View

a. Image drawn and owned by Ethan Snow.

108) Figure 11.4. Eye, Transverse Section, Superior View. Cropped and added labels.

a. http://lane.stanford.edu/search.html?q=eye\&source=bassett\#bassett=\%2FbassettView.html\%3Fbn\%3D58-2

109) Figure 11.5. Eye (Sketch), Transverse Section, Superior View.

a. Image drawn and owned by Ethan Snow.

110) Figure 11.6. Photograph of Left Human Retaina, Anterior View.

a. Image owned by Ethan Snow.

111) Figure 11.7. Extrinsic Muscles of the Left Eye, Anterior View. Cropped and added labels.

a. http://lane.stanford.edu/search.html?q=eye\&source=bassett\#bassett=\%2FbassettView.html\%3Fbn\%3D57-1

112) Figure 11.8. Extrinsic Muscles of the Left Eye (Sketch), Anterior View.

a. Image drawn by Logan Detweiler and owned by Ethan Snow.

113) Figure 11.9. Extrinsic Muscles of the Eye (Sketch), Right Lateral View.

a. Image drawn by Logan Detweiler and owned by Ethan Snow.

114) Figure 11.10. Reflected Extrinsic Muscles of the Left Eye (Removed), Anterior View. Cropped and added labels.

a. http://lane.stanford.edu/search.html?q=eye\&source=bassett\#bassett=\%2FbassettView.html\%3Fbn\%3D57-5

115) Figure 11.11. Reflected Extrinsic Muscles of the Left Eye (Removed), Posterior View. Cropped and added labels.

a. http://lane.stanford.edu/search.html?q=eye\&source=bassett\#bassett=\%2FbassettView.html\%3Fbn\%3D57-6

116) Figure 11.12. Ear Ossicles. Cropped and added labels.

a. http://lane.stanford.edu/search.html?q=ear\&source=bassett\#bassett=\%2FbassettView.html\%3Fbn\%3D63-1

117) Figure 11.13. Structures of the Outer, Middle, and Inner Ear.

a. Images drawn by Heather Jaeche and owned/edited by Ethan Snow.

118) Figure 11.14. Structures of the Nasal and Oral Cavities. Cropped and added labels.

a. http://lane.stanford.edu/search.html?q=tongue\&source=bassett\#bassett=\%2FbassettView.html\%3Fbn\%3D50-1

119) Figure 12.1. Heart in situ, Anterior View. Cropped and added labels.

a. http://lane.stanford.edu/search.html?q=heart\&source=bassett\#bassett=\%2FbassettView.html\%3Fbn\%3D116-7

120) Figure 12.2. Base of Heart, Superior View. Cropped and added labels.

a. http://lane.stanford.edu/search.html?q=heart\&source=bassett\#bassett=\%2FbassettView.html\%3Fbn\%3D120-1

121) Figure 12.3. Valves of the Heart, Superior View (Atria Removed). Cropped and added labels.

a. http://lane.stanford.edu/search.html?q=heart\&source=bassett\#bassett=\%2FbassettView.html\%3Fbn\%3D120-6

122) Figure 12.4. Inside the Right Atrium, Superolateral View. Cropped and added labels.

a. http://lane.stanford.edu/search.html?q=heart\&source=bassett\#bassett=\%2FbassettView.html\%3Fbn\%3D121-2

123) Figure 12.5. Inside the Left Ventricle, Anterior View. Cropped and added labels.

a. http://lane.stanford.edu/search.html?q=heart\&source=bassett\#bassett=\%2FbassettView.html\%3Fbn\%3D122-5

124) Figure 12.6. Base of Heart, Right Lateral View. Cropped and added labels.

a. http://lane.stanford.edu/search.html?q=heart\&source=bassett\#bassett=\%2FbassettView.html\%3Fbn\%3D120-2

125) Figure 12.7. Electrical Conduction Structures of the Heart

a. Image drawn and owned by Ethan Snow.

126) Clinical Application: Electrocardiogram

a. Image drawn and owned by Ethan Snow.

127) Figure 12.8. Coronary Vessels, Anterior View. Cropped and added labels.

a. http://lane.stanford.edu/search.html?q=heart\&source=bassett\#bassett=\%2FbassettView.html\%3Fbn\%3D119-2 
128) Figure 12.9. Coronary Vessels, Posterior View. Cropped and added labels.

a. http://lane.stanford.edu/search.html?q=heart\&source=bassett\#bassett=\%2FbassettView.html\%3Fbn\%3D119-6

129) Figure 12.10. Coronary Vessels, Left Lateral View. Cropped and added labels.

a. http://lane.stanford.edu/search.html?q=heart\&source=bassett\#bassett=\%2FbassettView.html\%3Fbn\%3D119-4

130) Figure 12.11. Simplified Diagram of Coronary Circulation.

a. Image drawn and owned by Ethan Snow.

131) Figure 13.1. Diagram of Regions of the Aorta

a. Image drawn and owned by Ethan Snow.

132) Figure 13.2. Vasculature of the Superior Thorax, Anterior View. Cropped and added labels.

a. http://lane.stanford.edu/search.html?q=aortic+arch\&source=bassett\#bassett=\%2FbassettView.html\%3Fbn\%3D117-2

133) Figure 13.3. Diagram of Branches of the Thoracic Aorta

a. Image drawn and owned by Ethan Snow.

134) Figure 13.4. Veins of the Posterior Thorax, Anterior View. Cropped and added labels.

a. http://lane.stanford.edu/search.html?q=azygos\&source=bassett\#bassett=\%2FbassettView.html\%3Fbn\%3D126A-5

135) Figure 13.5. Veins of the Anterior Thorax, Posterior View. Cropped and added labels.

a. http://lane.stanford.edu/search.html?q=internal+thoracic\&source=bassett\#bassett=\%2FbassettView.html\%3Fbn\%3D116-1

136) Figure 13.6. Diagram of Veins of the Thorax

a. Image drawn and owned by Ethan Snow.

137) Figure 13.7. Vasculature of the Arterial Circle of Willis. Cropped and added labels.

a. http://lane.stanford.edu/search.html?q=circle+of+willis\&source=bassett\#bassett=\%2FbassettView.html\%3Fbn\%3D4-4

138) Figure 13.8. Diagram of Arteries of the Neck and Head

a. Image drawn and owned by Ethan Snow.

139) Figure 13.9. Cerebral Veins, Superior View. Cropped and added labels.

a. http://lane.stanford.edu/search.html?q=brain+superior\&source=bassett\#bassett=\%2FbassettView.html\%3Fbn\%3D8-3

140) Figure 13.10. Vasculature of the Neck, Anterior View. Cropped and added labels.

a. http://lane.stanford.edu/search.html?q=common+carotid+artery\&source=bassett\#bassett=\%2FbassettView.html\%3Fbn\%3D76-1

141) Figure 13.11. Diagram of Branches of the Abdominal Aorta

a. Image drawn and owned by Ethan Snow.

142) Figure 13.12. Diagram of Abdominal Veins.

a. Image drawn and owned by Ethan Snow.

143) Figure 13.13. Vasculature of the Liver.

a. Image drawn and owned by Ethan Snow.

144) Figure 13.14. Vasculature of the Portal System, Anterior View. Cropped and added labels.

a. http://lane.stanford.edu/search.html?q=portal+vein\&source=bassett\#bassett=\%2FbassettView.html\%3Fbn\%3D147-6

145) Figure 13.15. Vasculature of the Abdomen, Anterior View. Cropped and added labels.

a. http://lane.stanford.edu/search.html?q=superior+mesenteric\&source=bassett\#bassett=\%2FbassettView.html\%3Fbn\%3D149-2

146) Figure 13.16. Facial Artery and Vein, Left Lateral View. Cropped and added labels.

a. http://lane.stanford.edu/search.html?q=facial+artery\&source=bassett\#bassett=\%2FbassettView.html\%3Fbn\%3D49-3

147) Figure 14.1. Vasculature of the Upper Limb (from "Anatomical Position, Anterior View").

a. Image drawn by Logan Detweiler and owned/edited by Ethan Snow.

148) Figure 14.2. Vasculature of the Right Anterior Elbow. Cropped and added labels.

a. http://lane.stanford.edu/search.html?q=cubital+fossa\&source=bassett\#bassett=\%2FbassettView.html\%3Fbn\%3D98-2

149) Figure 14.3. Vasculature of the Right Hand, Anterior View. Cropped and added labels.

a. http://lane.stanford.edu/search.html?q=hand+artery\&source=bassett\#bassett=\%2FbassettView.html\%3Fbn\%3D101-4

150) Figure 14.4. Vasculature of the Lower Limb (from "Anatomical Position, Anterior View").

a. Image drawn by Logan Detweiler and owned/edited by Ethan Snow. 
151) Figure 14.5. Vasculature of the Posterior Knee. Cropped and added labels.

a. http://lane.stanford.edu/search.html?q=popliteal+vein\&source=bassett\#bassett=\%2FbassettView.html\%3Fbn\%3D183-5

152) Figure 14.6. Small Saphenous Vein on Posterior Leg. Cropped and added labels.

a. http://lane.stanford.edu/search.html?q=small+saphenous+v.\&source=bassett\#bassett=\%2FbassettView.html\%3Fbn\%3D194-4

153) Clinical Application: Blood Transfusions

a. Table image created with Microsoft Excel objects by Ethan Snow.

154) Figure 15.1. Right Head, Midsagittal View. Cropped and added labels.

a. http://lane.stanford.edu/search.html?q=middle+concha\&source=bassett\#bassett=\%2FbassettView.html\%3Fbn\%3D72-2

155) Figure 15.2. Larynx, Anterior View (Left) and Posterior View (Right). Cropped, merged two images (below), and added labels.

a. http://lane.stanford.edu/search.html?q=larynx\&source=bassett\#bassett=\%2FbassettView.html\%3Fbn\%3D84-3

b. http://lane.stanford.edu/search.html?q=larynx\&source=bassett\#bassett=\%2FbassettView.html\%3Fbn\%3D84-2

156) Figure 15.3. Larynx, Superior View. Cropped and added labels.

a. http://lane.stanford.edu/search.html?q=larynx\&source=bassett\#bassett=\%2FbassettView.html\%3Fbn\%3D84-1

157) Figure 15.4. Larynx, Superior View in situ. Cropped and added labels.

a. http://lane.stanford.edu/biomed-resources/bassett/bassettView.html?bn=83-4

158) Figure 15.5. Left Larynx, Midsagittal View. Cropped and added labels.

a. http://lane.stanford.edu/search.html?q=larynx\&source=bassett\#bassett=\%2FbassettView.html\%3Fbn\%3D83-7

159) Figure 15.6. Mediastinum and Lungs, Anterior View. Cropped and added labels.

a. http://lane.stanford.edu/search.html?q=phrenic+nerve\&source=bassett\#bassett=\%2FbassettView.html\%3Fbn\%3D123-5

160) Figure 15.7. Pulmonary Vessels Branching Pattern, Anterior View. Cropped and added labels.

a. http://lane.stanford.edu/search.html?q=lung\&source=bassett\#bassett=\%2FbassettView.html\%3Fbn\%3D123-7

161) Figure 15.8. Bronchi Branching Pattern, Anterior View. Cropped and added labels.

a. http://lane.stanford.edu/biomed-resources/bassett/bassettView.html?bn=129-1

162) Figure 15.9. Right Lung, Lateral View. Cropped and added labels.

a. http://lane.stanford.edu/search.html?q=lung\&source=bassett\#bassett=\%2FbassettView.html\%3Fbn\%3D123-2

163) Figure 15.10. Right Lung, Medial View. Cropped and added labels.

a. http://lane.stanford.edu/search.html?q=lung\&source=bassett\#bassett=\%2FbassettView.html\%3Fbn\%3D123-1

164) Figure 15.11. Left Lung, Lateral View. Cropped and added labels.

a. http://lane.stanford.edu/search.html?q=lung\&source=bassett\#bassett=\%2FbassettView.html\%3Fbn\%3D123-4

165) Figure 15.12. Left Lung, Medial View. Cropped and added labels.

a. http://lane.stanford.edu/search.html?q=lung\&source=bassett\#bassett=\%2FbassettView.html\%3Fbn\%3D123-3

166) Figure 16.1. Right Head, Midsagittal View. Cropped and added labels.

a. http://lane.stanford.edu/search.html?q=concha\&source=bassett\#bassett=\%2FbassettView.html\%3Fbn\%3D72-2

167) Figure 16.2. Esophagus in Thorax, Anterior View. Added labels.

a. http://lane.stanford.edu/search.html?q=esophagus\&source=bassett\#bassett=\%2FbassettView.html\%3Fbn\%3D128-7

168) Figure 16.3. Abdominal Viscera in situ, Anteroinferior View. Added labels.

a. http://lane.stanford.edu/search.html?q=omentum\&source=bassett\#bassett=\%2FbassettView.html\%3Fbn\%3D138-7

169) Figure 16.4. Abdominal Viscera in situ, Anterior View. Added labels.

a. http://lane.stanford.edu/search.html?q=omentum\&source=bassett\#bassett=\%2FbassettView.html\%3Fbn\%3D138-3

170) Figure 16.5. Stomach in situ, Anterior View. Added labels.

a. http://lane.stanford.edu/search.html?q=stomach\&source=bassett\#bassett=\%2FbassettView.html\%3Fbn\%3D142-5

171) Figure 16.6. Stomach (cut open) in situ, Anterior View. Added labels.

a. http://lane.stanford.edu/search.html?q=stomach\&source=bassett\#bassett=\%2FbassettView.html\%3Fbn\%3D142-6

172) Clinical Application: Gastric Bypass.

a. Images drawn and owned by Ethan Snow.

173) Figure 16.7. Duodenum (cut open), Anterior View. Cropped and added labels. 
a. http://lane.stanford.edu/search.html?q=duodenum\&source=bassett\#bassett=\%2FbassettView.html\%3Fbn\%3D147-3 174) Figure 16.8. Mesentery Proper, Anterior View. Cropped and added labels.

a. http://lane.stanford.edu/search.html?q=mesentery\&source=bassett\#bassett=\%2FbassettView.html\%3Fbn\%3D140-2

175) Figure 16.9. Cecum in situ, Anteroinferior View. Cropped and added labels.

a. http://lane.stanford.edu/search.html?q=cecum\&source=bassett\#bassett=\%2FbassettView.html\%3Fbn\%3D139-6

176) Figure 16.10. Cecum (cut open) in situ, Anterior View. Added labels.

a. http://lane.stanford.edu/search.html?q=cecum\&source=bassett\#bassett=\%2FbassettView.html\%3Fbn\%3D143-4

177) Figure 16.11. Colon, Anterior View \#1. Cropped and added labels.

a. http://lane.stanford.edu/search.html?q=colon\&source=bassett\#bassett=\%2FbassettView.html\%3Fbn\%3D143-1

178) Figure 16.12. Colon, Anterior View \#2. Cropped and added labels.

a. http://lane.stanford.edu/search.html?q=colon\&source=bassett\#bassett=\%2FbassettView.html\%3Fbn\%3D144-1

179) Figure 16.13. Sigmoid Colon, Anterior View. Cropped and added labels.

a. http://lane.stanford.edu/search.html?q=sigmoid+colon\&source=bassett\#bassett=\%2FbassettView.html\%3Fbn\%3D140-3

180) Figure 16.14. Diagram of Gastrointestinal Tract (Primary Digestive Organs).

a. Image drawn and owned by Ethan Snow.

181) Figure 16.15. Diagram of Anal Sphincters and Anus.

a. Image drawn and owned by Ethan Snow.

182) Figure 17.1. Oral Cavity, Midsagittal View. Cropped and added labels.

a. http://lane.stanford.edu/search.html?q=tongue\&source=bassett\#bassett=\%2FbassettView.html\%3Fbn\%3D78-1

183) Figure 17.2. Oral Cavity, Anterior View. Cropped and added labels.

a. http://lane.stanford.edu/search.html?q=tongue\&source=bassett\#bassett=\%2FbassettView.html\%3Fbn\%3D70-5

184) Figure 17.3. Diagram of a Typical Tooth, Anterior View.

a. Image drawn and owned by Ethan Snow.

185) Figure 17.4. Separated Teeth, Anterior View. Cropped and added labels.

a. http://lane.stanford.edu/search.html?q=teeth\&source=bassett\#bassett=\%2FbassettView.html\%3Fbn\%3D47-2

186) Figure 17.5. Teeth in Maxilla, Inferior View. Cropped and added labels.

a. http://lane.stanford.edu/search.html?q=maxilla\&source=bassett\#bassett=\%2FbassettView.html\%3Fbn\%3D38-3

187) Figure 17.6. Teeth in Mandible, Superior View. Added labels.

a. http://lane.stanford.edu/search.html?q=teeth\&source=bassett\#bassett=\%2FbassettView.html\%3Fbn\%3D46-4

188) Figure 17.7. Teeth, Anterior View. Cropped and added labels.

a. http://lane.stanford.edu/search.html?q=maxilla\&source=bassett\#bassett=\%2FbassettView.html\%3Fbn\%3D35-4

189) Figure 17.8. Parotid Salivary Gland, Left Lateral View. Cropped and added labels.

a. http://lane.stanford.edu/search.html?q=parotid\&source=bassett\#bassett=\%2FbassettView.html\%3Fbn\%3D49-3

190) Figure 17.9. Submandibular Salivary Gland, Left Lateral View. Cropped and added labels.

a. http://lane.stanford.edu/search.html?q=parotid\&source=bassett\#bassett=\%2FbassettView.html\%3Fbn\%3D49-5

191) Figure 17.10. Sublingual Salivary Gland, Right Midsagittal View. Cropped and added labels.

a. http://lane.stanford.edu/search.html?q=sublingual\&source=bassett\#bassett=\%2FbassettView.html\%3Fbn\%3D69-6

192) Figure 17.11. Duodenum (cut open), Anterior View. Cropped and added labels.

a. http://lane.stanford.edu/search.html?q=duodenal+papilla\&source=bassett\#bassett=\%2FbassettView.html\%3Fbn\%3D147-3

193) Figure 17.12. Liver and Gallbladder, Anterior View. Added labels.

a. http://lane.stanford.edu/search.html?q=liver\&source=bassett\#bassett=\%2FbassettView.html\%3Fbn\%3D144-5

194) Figure 17.13. Liver and Gallbladder, Posterior View. Added labels.

a. http://lane.stanford.edu/search.html?q=liver\&source=bassett\#bassett=\%2FbassettView.html\%3Fbn\%3D144-7

195) Figure 17.14. Pancreas and Surrounding Viscera, Anterior View. Cropped and added labels.

a. http://lane.stanford.edu/search.html?q=liver\&source=bassett\#bassett=\%2FbassettView.html\%3Fbn\%3D145-6

196) Figure 17.15. Dissected Pancreas and Pancreatic Duct, Anterior View. Cropped and added labels. 
a. http://lane.stanford.edu/search.html?q=liver\&source=bassett\#bassett=\%2FbassettView.html\%3Fbn\%3D146-5

197) Figure 17.16. Diagram of Biliary Tree and Pancreatic Ducts, Anterior View.

a. Image drawn and owned by Ethan Snow.

198) Clinical Application: Blood Sugar and Diabetes.

a. Image drawn and created with Microsoft Word objects by Ethan Snow.

199) Figure 17.17. Spleen, Anterior View. Cropped and added labels.

a. http://lane.stanford.edu/search.html?q=spleen\&source=bassett\#bassett=\%2FbassettView.html\%3Fbn\%3D148-2

200) Figure 17.18. Left Cervical Lymph Nodes, Lateral View. Cropped and added labels.

a. http://lane.stanford.edu/search.html?q=cervical+lymph+node\&source=bassett\#bassett=\%2FbassettView.html\%3Fbn\%3D73-5

201) Figure 17.19. Left Axillary Lymph Node, Anterior View. Cropped and added labels.

a. http://lane.stanford.edu/search.html?q=cervical+lymph+node\&source=bassett\#bassett=\%2FbassettView.html\%3Fbn\%3D90-4

202) Figure 17.20. Left Inguinal Lymph Nodes, Anterior View. Cropped and added labels.

a. http://lane.stanford.edu/search.html?q=inguinal+lymph\&source=bassett\#bassett=\%2FbassettView.html\%3Fbn\%3D137-4

203) Figure 17.21. Left Tonsils, Midsagittal View. Cropped and added labels.

a. http://lane.stanford.edu/search.html?q=tongue\&source=bassett\#bassett=\%2FbassettView.html\%3Fbn\%3D50-1

204) Figure 17.22. Thymus, Anterior View. Cropped and added labels.

a. http://lane.stanford.edu/search.html?q=thymus\&source=bassett\#bassett=\%2FbassettView.html\%3Fbn\%3D116-6

205) Figure 17.23. Appendix, Anterior View. Cropped and added labels.

a. http://lane.stanford.edu/search.html?q=appendix\&source=bassett\#bassett=\%2FbassettView.html\%3Fbn\%3D143-3

206) Figure 18.1. Kidneys (dissected) in situ, Anterior View. Cropped and added labels.

a. http://lane.stanford.edu/search.html?q=kidney\&source=bassett\#bassett=\%2FbassettView.html\%3Fbn\%3D151-4

207) Figure 18.2. Left Kidney, Anterior View. Cropped and added labels.

a. http://lane.stanford.edu/search.html?q=kidney\&source=bassett\#bassett=\%2FbassettView.html\%3Fbn\%3D149-3

208) Figure 18.3. Left Kidney, Posterior View. Cropped and added labels.

a. http://lane.stanford.edu/search.html?q=kidney\&source=bassett\#bassett=\%2FbassettView.html\%3Fbn\%3D149-4

209) Figure 18.4. Left Kidney (hemisected and reflected). Cropped and added labels.

a. http://lane.stanford.edu/search.html?q=kidney\&source=bassett\#bassett=\%2FbassettView.html\%3Fbn\%3D149-5

210) Figure 18.5. Nephron Diagram

a. Image drawn and owned by Ethan Snow.

211) Figure 18.6. Diagram of Kidney Vasculature

a. Image drawn and owned by Ethan Snow.

212) Figure 18.7. Urinary Bladder (cut open) in Hemisected Right Male Pelvis, Parasagittal View. Cropped and added labels.

a. http://lane.stanford.edu/search.html?q=bladder\&source=bassett\#bassett=\%2FbassettView.html\%3Fbn\%3D170-6

213) Figure 18.8. Diagram of the Urinary System

a. Image drawn and owned by Ethan Snow.

214) Figure 19.1. Hemisected Testis, Sagittal View. Added labels.

a. http://lane.stanford.edu/search.html?q=testis\&source=bassett\#bassett=\%2FbassettView.html\%3Fbn\%3D166-1

215) Figure 19.2. Urinary Bladder and Prostate Gland in a Hemisected Pelvis, Sagittal View. Cropped and added labels.

a. http://lane.stanford.edu/search.html?q=prostate\&source=bassett\#bassett=\%2FbassettView.html\%3Fbn\%3D170-3

216) Figure 19.3. Vas Deferens, Seminal Vesicles, and Prostate Gland, Anterosuperior View. Cropped and added labels.

a. http://lane.stanford.edu/search.html?q=prostate\&source=bassett\#bassett=\%2FbassettView.html\%3Fbn\%3D171-3

217) Figure 19.4. Male External Genitalia, Anterior View. Cropped and added labels.

a. http://lane.stanford.edu/search.html?q=genitalia\&source=bassett\#bassett=\%2FbassettView.html\%3Fbn\%3D165-1

218) Figure 19.5. Diagram of Trans-sected Penis, Anterior View.

a. Image drawn and owned by Ethan Snow.

219) Figure 19.6. Diagram of Male Reproductive Tract. 
a. Image drawn and owned by Ethan Snow.

220) Figure 19.7. Female Breast, Anterior View. Cropped and added labels.

a. http://lane.stanford.edu/search.html?q=breast\&source=bassett\#bassett=\%2FbassettView.html\%3Fbn\%3D114-6

221) Figure 19.8. Female Breast (Hemisected), Sagittal View. Cropped and added labels.

a. http://lane.stanford.edu/search.html?q=breast\&source=bassett\#bassett=\%2FbassettView.html\%3Fbn\%3D114-7

222) Figure 19.9. Uterus and Ovaries, Anterior View. Cropped and added labels.

a. http://lane.stanford.edu/search.html?q=uterus\&source=bassett\#bassett=\%2FbassettView.html\%3Fbn\%3D164-2

223) Figure 19.10. Diagram of Female Reproductive Tract.

a. Image drawn and owned by Ethan Snow.

224) Figure 19.11. Female Hemisected Pelvis, Midsagittal View. Cropped and added labels.

a. http://lane.stanford.edu/search.html?q=uterus\&source=bassett\#bassett=\%2FbassettView.html\%3Fbn\%3D161-3

225) Figure 19.12. Female External Genitalia, Inferior View. Cropped and added labels.

a. http://lane.stanford.edu/search.html?q=genitalia\&source=bassett\#bassett=\%2FbassettView.html\%3Fbn\%3D156-7 